\title{
UCRL-TR-216049
}

LAW RENCE LIVERMORE N A TIO NAL LABORATORY

Tables of specifications and requirements for 1.0 MJ targets for 2010 ignition on NIF

S. W. Haan, G. Deis, J. Klingmann, D. Wilson

October 11, 2005 
This document was prepared as an account of work sponsored by an agency of the United States Government. Neither the United States Government nor the University of California nor any of their employees, makes any warranty, express or implied, or assumes any legal liability or responsibility for the accuracy, completeness, or usefulness of any information, apparatus, product, or process disclosed, or represents that its use would not infringe privately owned rights. Reference herein to any specific commercial product, process, or service by trade name, trademark, manufacturer, or otherwise, does not necessarily constitute or imply its endorsement, recommendation, or favoring by the United States Government or the University of California. The views and opinions of authors expressed herein do not necessarily state or reflect those of the United States Government or the University of California, and shall not be used for advertising or product endorsement purposes.

This work was performed under the auspices of the U.S. Department of Energy by University of California, Lawrence Livermore National Laboratory under Contract W-7405-Eng-48. 


\title{
Tables of specifications and requirements for 1.0 MJ targets for 2010 ignition on NIF
}

\author{
Compiled by Steve Haan and Gary Deis \\ Drawings compiled by Jeff Klingmann \\ Lawrence Livermore National Laboratory
Uniform $\mathrm{Be}(\mathrm{Cu})$ requirements compiled by Doug Wilson
Los Alamos National Laboratory

September 30, 2005

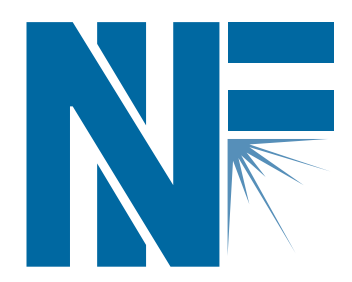




\section{Notes on target requirements}

Requirements for point design in "Point design requirements" and supporting tables and figures

Requirements for $\mathrm{CH}$ alternate capsule in "Alt Cap - $\mathrm{CH}$ " and supporting tables and figures

Requirements for Uniform $\mathrm{Be}(\mathrm{Cu})$ alternate capsule in "Alt Cap - Uniform $\mathrm{Be}(\mathrm{Cu})$ " and supporting tables and figures

Requirements for Foam Filled alternate hohlraum in "Alt Hohlraum - Foam" and supporting figures

Requirements for Lined alternate hohlraum in "Alt Hohlraum - Lined" and supporting figures

Any capsule, point design or alternate, can be fielded in any hohlraum, point design or alternate, with the requirements unchanged 
Ignition Point Design Requirements - Be Capsule in Gas-Filled Hohlraum

\begin{tabular}{|c|c|c|c|c|c|c|}
\hline Number & $\operatorname{Rev}$ & WBS & Component & Title & short req't & Requirement Text \\
\hline 1 & 0 & 1.4 .1 & Target & Requirements apply at shot temperature & $\begin{array}{l}\text { Requirements apply at } \\
\text { shot temperature }\end{array}$ & $\begin{array}{l}\text { All requirements on targets shall apply at the temperature of the subject component at the } \\
\text { time of the shot }\end{array}$ \\
\hline 2 & 0 & 1.4 .1 & Target & Tolerances are specified as hard limits & Tolerances are hard limits & $\begin{array}{l}\text { Unless specifically stated otherwise, all requirements on targets shall be hard limits (as } \\
\text { opposed to statistical values such as } 1 \text { sigma, } 3 \text { sigma, or RMS). If targets are produced } \\
\text { that do not meet hard-limit requirements, they shall be dispositioned on a case-by-case } \\
\text { basis by an MRB. }\end{array}$ \\
\hline 3 & 0 & I.4.1.1 & Capsule & $\begin{array}{l}\text { Capsule outer radius, range of possible } \\
\text { requested values and point-design value }\end{array}$ & $900-1100 \mu \mathrm{m}$ & $\begin{array}{l}\text { Capsules shall be producable with outer radii within the range } 900-1100 \mu \mathrm{m} \text {. The outer } \\
\text { radius of each capsule will be specified within this range. The point design value shall be } \\
1.0 \mathrm{~mm} \text {. }\end{array}$ \\
\hline 4 & 0 & 1.4 .1 .1 & Capsule & Capsule outer radius, tolerance & $\pm 5 \mu \mathrm{m}$ & The outer radius of the capsule shall be within $+/-5 \mu \mathrm{m}$ of the specified value. \\
\hline 5 & 0 & 1.4.1.1 & Capsule & Ablator - Low level impurities & $\begin{array}{l}\operatorname{sum}(\text { atomic fraction })^{*} Z^{\wedge} 2 \\
<0.1\end{array}$ & $\begin{array}{l}\text { Except for allowed ingredients as listed in the ablator composition entries, the ablator } \\
\text { material in all layers shall contain suffieciently low impurity levels that the sum over all } \\
\text { impurities of atom fraction * } Z^{\wedge} 2 \text { shall be less than or equal to } 0.20 \text {, i.e. } 20 \% \text {. For example, } \\
\text { a barely allowed impurity set would be [C,Al,Si,Mn,Fe,Ni] with atomic fractions } \\
0.01^{*}[.015, .007, .006, .0005, .021, .0031] \text { giving Fe-dominated weighted sum } \\
0.01^{*}(.54+1.18+1.18+0.31+14.20+2.43)=0.20\end{array}$ \\
\hline 6 & 0 & 1.4.1.1 & Capsule & $\begin{array}{l}\text { Ablator - High level impurities with known } \\
\text { acceptable presence }\end{array}$ & & $\begin{array}{l}\text { Ablators may contain specified materials as described in individual albator composition } \\
\text { requirments. }\end{array}$ \\
\hline 7 & 0 & 1.4 .1 .1 & Capsule & $\begin{array}{l}\text { Ablator - measurement of } x \text {-ray optical depth } \\
\text { variations }\end{array}$ & accuracy $<0.01 \%$ & $\begin{array}{l}\text { Lateral variations in optical depth through the ablator shall be measured with accuracy } \\
\text { better than } 0.01 \% \text {, at lateral scales between } 120 \text { and } 3000 \mu \mathrm{m} \text { (modes } 2-25 \text { ), at } 5.4 \mathrm{keV} \text { x- } \\
\text { ray energy. Entire capsule shall be measured down to } 120 \mu \mathrm{m} \text { lateral scale (mode } 25 \text { ). } \\
\text { These measurements will constrain porosity, density variations, isolated defects, and } \\
\text { variations in thickness of the internal layers. It will be very valuable to have scans of } \\
\text { representative patches of representative capsules at lateral scans down to } 20 \mu \mathrm{m} \text { (mode } \\
\text { 150). }\end{array}$ \\
\hline 8 & 0 & 1.4 .1 .1 & Capsule & Ablator - average mass density & $\begin{array}{l} \pm 3 \% \text { absolute } \\
\pm 1.5 \% \text { relative to } \\
\text { campaign average } \\
\end{array}$ & $\begin{array}{l}\text { The average ablator density of each target shall be within } \pm 3 \% \text { absolute of the specified } \\
\text { value, and within } \pm 1.5 \% \text { of the average of all targets in each ignition campaign with given } \\
\text { ablator }\end{array}$ \\
\hline 9 & 0 & 1.4.1.1 & Capsule & Ablator - voids & $\begin{array}{l}<3 \% \text { void fract, } \\
<0.1 \mu \mathrm{m} 3 \text { void volume }\end{array}$ & $\begin{array}{l}\text { Transverse variations in the column density of the ablator due to voids and/or density } \\
\text { nonuniformity shall be less than that corresponding to randomly located voids of volume } \\
0.1 \mu \mathrm{m} 3 \text { summing to void fraction of } 3 \% \text {. }\end{array}$ \\
\hline 10 & 0 & 1.4.1.1 & Capsule & Ablator oxide layers & & $\begin{array}{l}\text { Oxide layers on the inside and outside of the ablator shall have oxygen less than or equal } \\
\text { to that of a } 100 \mathrm{~nm} \text { layer of } 50 \% \mathrm{O} \text {, at density less than twice the bulk density. If present, } \\
\text { oxide layers internal to the ablator shall be small enough that the ablator satisfies the } \\
\text { requirements on impurities and on optical depth when averaged over any } 3 \mu \mathrm{m} \text { layer. } \\
\text { Requires an internal layer of } \mathrm{BeO} \text { at density } 3 \mathrm{~g} / \mathrm{cc} \text { to be thinner than } 20 \mathrm{~nm} \text {. }\end{array}$ \\
\hline 11 & 0 & 1.4 .1 .1 & Capsule & Capsule surface isolated defects & see figure & $\begin{array}{l}\text { There shall be no isolated defects on the outside of the capsule of size larger than the } \\
\text { maximum size shown in the figure "Isolated Surface Defects - Rev } 0 " \text {. There shall be no } \\
\text { more than } 20 \text { isolated defects whose size is within a factor of three of the limit shown in the } \\
\text { figure, and no more than } 100 \text { within a factor of } 10 \text {. An isolated defect is defined as a } \\
\text { departure from a surface, smooth at modes }>20 \text {, that matches the nearby capsule surface } \\
\text { within } 10 \mathrm{~nm} \text {. The defect's "size" is the maximum deviation from that smooth surface. }\end{array}$ \\
\hline 12 & 0 & I.4.1.1 & Capsule & Capsule cleanliness & See isolated defects & $\begin{array}{l}\text { The surface of the capsule shall have cleanliness such that no surface contaminants } \\
\text { exceed the isolated defect spec or have atomic number } Z \text { greater than that of Si. For film } \\
\text { contamination, requirement is same as oxide layers. Contaminants other than oxygen have } \\
\text { requirement tightened proportional to } 1 / Z^{\wedge} 2 \text {. }\end{array}$ \\
\hline 13 & 0 & I.4.1.12.4 & $\begin{array}{l}\text { Be(layered dopant) } \\
\text { Capsule }\end{array}$ & Ablator composition & $\mathrm{Be}$ & $\begin{array}{l}\text { The ablator material shall be beryllium, doped with } \mathrm{Cu} \text { as described in Table "Ablator layer } \\
\text { requirements for layered dopant Be capsule - Rev } 0 \text { ". Oxygen shall be nominally } 0.4 \text { at } \% \text {, } \\
<0.8 \text { at } \% \text { required. Ar shall be }<0.1 \text { at } \% \text {. }\end{array}$ \\
\hline 14 & 0 & I.4.1.12.4 & $\begin{array}{l}\text { Be(layered dopant) } \\
\text { Capsule }\end{array}$ & $\begin{array}{l}\text { Ablator total thickness - range of possible } \\
\text { requested values }\end{array}$ & $160 \mu \mathrm{m}$ & $\begin{array}{l}\text { Capsule ablator shall be producable with total ablator thickness within the range 150- } \\
170 \mu \mathrm{m} \text {. The ablator thickness for each capsule will be specified within that range. Current } \\
\text { point design } 160 \mu \mathrm{m} \text {. }\end{array}$ \\
\hline
\end{tabular}

Page 1 of 5 


\begin{tabular}{|c|c|c|c|c|c|c|}
\hline Number & Rev & WBS & Component & Title & short req't & Requirement Text \\
\hline 15 & 0 & I.4.1.12.4 & $\begin{array}{l}\text { Be(layered dopant) } \\
\text { Capsule }\end{array}$ & Ablator Dopant & $\mathrm{Cu}$ & The dopant material for all doped layers shall be $\mathrm{Cu}$ \\
\hline 16 & 0 & I.4.1.12.4 & $\begin{array}{l}\text { Be(layered dopant) } \\
\text { Capsule }\end{array}$ & Ablator layer thicknesses & see table & $\begin{array}{l}\text { The thickness of the layers within the ablator shall be as listed in Table "Ablator layer } \\
\text { requirements for layered dopant Be capsule - Rev 0" }\end{array}$ \\
\hline 17 & 0 & I.4.1.12.4 & $\begin{array}{l}\text { Be(layered dopant) } \\
\text { Capsule }\end{array}$ & Ablator layer dopant concentration & see table & $\begin{array}{l}\text { The dopant concentration in each of the layers within the ablator shall be as listed in Table } \\
\text { "Ablator layer requirements for layered dopant Be capsule - Rev 0" }\end{array}$ \\
\hline 18 & 0 & I.4.1.12.4 & $\begin{array}{l}\text { Be(layered dopant) } \\
\text { Capsule }\end{array}$ & Ablator layer density & see table & $\begin{array}{l}\text { The density of each of the layers within the ablator shall be as listed in Table "Ablator layer } \\
\text { requirements for layered dopant Be capsule - Rev } 0 . \text {." The current assumption is that } \\
\text { density will be } 1.75 \text {, but higher density would provide better performance. Density is to be } \\
\text { known within } 3 \% \text { and reproducible within } 1.5 \% \text {. }\end{array}$ \\
\hline 19 & 0 & I.4.1.12.4 & $\begin{array}{l}\text { Be(layered dopant) } \\
\text { Capsule }\end{array}$ & Ablator inner surface figure & see table & $\begin{array}{l}\text { The power spectrum of the deviation of the inner surface of the Be ablator from a perfect } \\
\text { sphere of the same average radius shall not exceed the spectrum in Table "Surface } \\
\text { roughness and thickness power spectra for layered dopant Be capsule }- \text { Rev } 0 . " \text { "Shall not } \\
\text { exceed" means that the } 2 D \text { power in modes } 1-12 \text { shall be less than that tabulated, and the } \\
1 D \text { power in a trace, summed over any }+/-15 \% \text { bandwidth above mode } 12 \text { shall be less } \\
\text { than that of the same bandwidth for the reference power spectra. }\end{array}$ \\
\hline 20 & 0 & I.4.1.12.4 & $\begin{array}{l}\text { Be(layered dopant) } \\
\text { Capsule }\end{array}$ & Ablator thickness non-uniformity & see table & $\begin{array}{l}\text { The power spectrum of the non-uniformity of the cumulative thickness of each layer shall } \\
\text { not exceed the spectrum in Table "Surface roughness and thickness power spectra for } \\
\text { layered dopant Be capsule - Rev } 0 . " \text { "Shall not exceed" means that the } 2 \mathrm{D} \text { power in modes } \\
1-12 \text { shall be less than that tabulated, and the } 1 \mathrm{D} \text { power in a trace, summed over any }+/- \\
15 \% \text { bandwidth above mode } 12 \text { shall be less than that of the same bandwidth for the } \\
\text { reference power spectra. }\end{array}$ \\
\hline 21 & 0 & I.4.1.1 & $\begin{array}{l}\text { Capsule fill tube and } \\
\text { hole }\end{array}$ & Fill tube orientation wrt/ Hohlraum & $\begin{array}{l} \pm 10^{\circ} \text { of waist, } \pm 10^{\circ} \text { of } \\
\text { perpendicular to the } \\
\text { theta }=90 \text { phi }=340 \text { LOS }\end{array}$ & $\begin{array}{l}\text { The capsule fill tube shall be oriented within }+/-10^{\circ} \text { of waist, azimuth within }+/-10^{\circ} \text { of } \\
\text { perpendicular to the theta }=90 \text { phi }=340 \text { LOS }\end{array}$ \\
\hline 22 & 0 & I.4.1.1 & $\begin{array}{l}\text { Capsule fill tube and } \\
\text { hole }\end{array}$ & Fill hole through ablator - configuration & see figure & $\begin{array}{l}\text { The fill hole through the ablator shall have mass deficit less than or equal to } 1.25^{*} \text { the mass } \\
\text { deficit of the hole in Figure "Fill tube configuration - Rev } 0 " \text {. The figure is representative of } \\
\text { one allowed configuration but is not required. The actual values of dimensions such as } \\
\text { those shown in the figure shall be measured with an accuracy better than } 20 \% \text { for each } \\
\text { capsule. }\end{array}$ \\
\hline 23 & 0 & 1.4 .1 .1 & $\begin{array}{l}\text { Capsule fill tube and } \\
\text { hole }\end{array}$ & Fill tube configuration at capsule & see figure & $\begin{array}{l}\text { The fill tube shall have OD less than } 15 \mu \mathrm{m} \text {. The OD< } 15 \mu \mathrm{m} \text { shall be maintained for at } \\
\text { least } 100 \text { microns from the capsule surface. The current point design, which is not a } \\
\text { requirement, is that the tube be configured and installed in conformance with Figure "Fill } \\
\text { tube configuration - Rev } 0 \text { ". The actual values of dimensions such as those shown in the } \\
\text { figure shall be measured with an accuracy better than } 20 \% \text { for each capsule. }\end{array}$ \\
\hline 24 & 0 & I.4.1.1 & $\begin{array}{l}\begin{array}{l}\text { Capsule fill tube and } \\
\text { hole }\end{array} \\
\end{array}$ & Fill tube material composition & $\mathrm{Z}<=\mathrm{Si}$ & $\begin{array}{l}\text { The fill tube shall be composed of material with Z less than or equal to } \mathrm{Si} \text {, and shall contain } \\
\text { less than } 100 \% / \mathrm{Z}^{\wedge} 2 \text { of other-than-nominal elements. Its density shall be less than } 2.5 \mathrm{~g} / \mathrm{cc} \text {, } \\
\text { and known to }+/-10 \% \text {. Current point design material is } \mathrm{SiO} 2 \text {, density } 2.2 \mathrm{~g} / \mathrm{cc} \text {. }\end{array}$ \\
\hline 25 & 0 & I.4.1.1 & \begin{tabular}{l|}
$\begin{array}{l}\text { Capsule fill tube and } \\
\text { hole }\end{array}$ \\
\end{tabular} & Fill tube glue composition & known & $\begin{array}{l}\text { The composition of the glue attaching the fill tube to the capsule shall be known with an } \\
\text { accuracy better than } 100 \% / Z^{\wedge} 2 \text { atomic fraction }\end{array}$ \\
\hline 26 & 0 & I.4.1.1 & $\begin{array}{l}\text { Capsule fill tube and } \\
\text { hole }\end{array}$ & Fill tube glue fillet mass & $<2.5 \mathrm{e}-9 \mathrm{~g}$ & $\begin{array}{l}\text { The mass of the glue fillet shall be }<2.5 \mathrm{e}-9 \mathrm{~g} \text {, and shall be known with an accuracy better } \\
\text { than } 1 \mathrm{e}-9 \mathrm{~g} \text {. The glue fillet is defined as the glue that extends outside the average outer } \\
\text { radius of the capsule. In addition, the mass of glue that extends inside the average outer } \\
\text { radius of the capsule shall be known with an accuracy better than } 1 \mathrm{e}-9 \mathrm{~g} \text {. }\end{array}$ \\
\hline 27 & 0 & 1.4 .1 .1 & $\begin{array}{l}\text { Capsule fill tube and } \\
\text { hole }\end{array}$ & Fill tube in hohlraum & $<2 \mathrm{e}-6 \mathrm{~g}$ & $\begin{array}{l}\text { The mass of the fill tube in the hohlraum shall be less than } 2 \mathrm{e}-6 \mathrm{~g} \text {. Note that a } 10 \text { micron } \\
\text { rod of glass } 1.6 \mathrm{~mm} \text { long has mass } 3.1 \mathrm{e}-7 \text {, and a tube with radius proportional to distance } \\
\text { from the hohlraum center has mass } 1.1 \mathrm{e}-6 \text {. }\end{array}$ \\
\hline 28 & 0 & I.4.1.1.5 & DT fuel & $\mathrm{D} / \mathrm{T}$ ratio & D/T $47-53 \%$ & $\begin{array}{l}\text { The fuel shall be a mixture of deuterium and tritium, consisting of } 47-53 \% \text { deuterium at shot } \\
\text { time. The } D / T \text { ratio shall be known for each target with an accuracy better than } 3 \%\end{array}$ \\
\hline
\end{tabular}

Page 2 of 5 


\begin{tabular}{|c|c|c|c|c|c|c|}
\hline Number & $\operatorname{Rev}$ & WBS & Component & Title & short req't & Requirement Text \\
\hline 29 & 0 & 1.4 .1 .1 .5 & DT fuel & Protium concentration & $<0.1 \%$ & $\begin{array}{l}\text { The concentration of protium in the DT fuel shall be less than } 0.1 \% \text {, and shall be known for } \\
\text { each target with an accuracy of better than } 0.05 \%\end{array}$ \\
\hline 30 & 0 & I.4.1.1.5 & DT fuel & He3 concentration & $\begin{array}{l}<36 \text { hours } \\
\text { goal } 24 \text { hours }\end{array}$ & $\begin{array}{l}\text { The He } 3 \text { fraction in the target at shot time shall be less than the fraction that would result } \\
\text { from } 36 \text { hours of decay from pure DT. The design goal shall be } 24 \text { hours. Current assumed } \\
\text { nominal is } 31 \text { hours. }\end{array}$ \\
\hline 31 & 0 & I.4.1.1.5 & DT fuel & Concentration of other impurities & $<0.003 \%$ & $\begin{array}{l}\text { The concentration of all impurities in the DT fuel other than } \mathrm{H} \text { and } \mathrm{He} 3 \text { shall be less than } \\
0.003 \text { at } \%\end{array}$ \\
\hline 32 & 0 & I.4.1.1.5 & DT fuel & Ice average density & \begin{tabular}{|l}
$0.25 \pm 0.015 \mathrm{~g} / \mathrm{cc}$ \\
absolute, \\
$\pm 0.005 \mathrm{~g} / \mathrm{cc}$ relative \\
\end{tabular} & $\begin{array}{l}\text { The average density of the DT ice shall be } 0.25 \pm 0.015 \mathrm{~g} / \mathrm{cc} \text {. The average density of the } \\
\text { DT ice for all targets in a campaign shall vary by less than } 0.005 \mathrm{~g} / \mathrm{cc} \text { RMS. }\end{array}$ \\
\hline 33 & 0 & I.4.1.1.5 & DT fuel & Ice thickness & see table & $\begin{array}{l}\text { For the DT ice layer, the range of possible thicknesses, the point design thickness, and the } \\
\text { tolerance shall be as shown in table "DT layer data - Rev 0" }\end{array}$ \\
\hline 34 & 0 & I.4.1.1.5 & DT fuel & Ice thickness nonuniformities & see figure & $\begin{array}{l}\text { The power spectrum of DT ice thickness nonuniformities shall be less than the spectra in } \\
\text { Table "DT Ice Thickenss Power Spectrum - Rev } 0 . " \text { Modes } 1-18 \text { shall have } 2 \mathrm{D} \text { power less } \\
\text { than the 2D power spectrum, and modes }>18 \text { shall be such that } 1 \mathrm{D} \text { trace power summed } \\
\text { over any } 15 \% \text { bandwidth is less than the corresponding sum of the tabulated trace } \\
\text { spectrum. }\end{array}$ \\
\hline 35 & 0 & |.4.1.1.5 & DT fuel & $\begin{array}{l}\text { Cracks, void, defects in inner half of DT layer } \\
\text { - void size }\end{array}$ & $<1.5 \mu \mathrm{m} 3$ & $\begin{array}{l}\text { In the inner half of the fuel layer, there shall be no voids, cracks, or defects larger than } 1.5 \\
\mu m 3 . \text { The inner half of the fuel layer is defined as the volume from the average radius to } \\
\text { inner radius of the DT layer. }\end{array}$ \\
\hline 36 & 0 & I.4.1.1.5 & DT fuel & $\begin{array}{l}\text { Cracks, void, defects in inner half of DT layer } \\
\text { - void density }\end{array}$ & $<4 \mathrm{vol} \%$ & $\begin{array}{l}\text { In the inner half of the fuel layer, the density of voids, cracks, or defects shall be less than } 4 \\
\text { vol\%. The inner half of the fuel layer is defined as the volume from the average radius to } \\
\text { inner radius of the DT layer. }\end{array}$ \\
\hline 37 & 0 & I.4.1.1.5 & DT fuel & $\begin{array}{l}\text { Cracks, void, defects in outer half of DT layer } \\
\text { - void size }\end{array}$ & $<0.3 \mu \mathrm{m} 3$ & $\begin{array}{l}\text { In the outer half of the fuel layer, there shall be no voids, cracks, or defects larger than } 0.3 \\
\mu m 3 \text {. The outer half of the fuel layer is defined as the volume from the average radius to } \\
\text { outer radius of the DT layer. }\end{array}$ \\
\hline 38 & 0 & I.4.1.1.5 & DT fuel & $\begin{array}{l}\text { Cracks, void, defects in outer half of DT layer } \\
\text { - void density }\end{array}$ & $<4 \mathrm{vol} \%$ & $\begin{array}{l}\text { In the outer half of the fuel layer, there density of voids, cracks, or defectsshall be less than } \\
4 \text { vol\%. The outer half of the fuel layer is defined as the volume from the average radius to } \\
\text { outer radius of the DT layer. }\end{array}$ \\
\hline 39 & 0 & I.4.1.1.5 & DT fuel & $\begin{array}{l}\text { Central temperature, as equivalent to DT gas } \\
\text { density }\end{array}$ & $0.3 \pm 0.05 \mathrm{mg} / \mathrm{cc}$ & $\begin{array}{l}\text { The central temperature of the DT fuel shall correspond to an equilibrium gas density of } 0.3 \\
\mathrm{mg} / \mathrm{cc} \pm 0.05 \mathrm{mg} / \mathrm{cc} \text {. The corresponding gas density shall be known with an accuracy better } \\
\text { than } 0.02 \mathrm{mg} / \mathrm{cc} \text {. This corresponds to knowledge of the temperature inside capsule with an } \\
\text { accuracy better than }+/-85 \mathrm{mK}\end{array}$ \\
\hline 40 & 0 & I.4.1.1.5 & DT fuel & Gap between ice and ablator & $\begin{array}{l}\text { variations due to } \\
\text { ice+gaps }<20 \% \text { RMS of } \\
\text { total variation }\end{array}$ & $\begin{array}{l}\text { Lateral variations in the column density of the ice and gaps from R(ablator inner)- } 2 \mu \mathrm{m} \text { to } \\
\mathrm{R}(\text { ablator, inner) }+2 \mu \mathrm{m} \text { shall be less than } 20 \% \text { RMS of the total variation in column density } \\
\text { (of ice, gaps, and ablator) over the same range. This is equivalent to the requirement that } \\
\text { the variations in column density over that range are at least } 80 \% \text { RMS due to column } \\
\text { density variations within the ablator alone. }\end{array}$ \\
\hline 41 & 0 & 1.4 .1 .1 .5 & \begin{tabular}{|l|} 
DT fuel \\
\end{tabular} & Fractionation of DT & $T$ fract $=0.5+/-0.1$ & The tritium fraction in any cubic volume $50 \mu \mathrm{m}$ on each side shall be $0.5+/-0.1$ \\
\hline 42 & 0 & 1.4 .1 .2 & Hohlraum & $\begin{array}{l}\text { Hohlraum configuration - range of requested } \\
\text { values }\end{array}$ & see dwg & $\begin{array}{l}\text { Hohlraums shall be producable with features in the dimensional ranges shown in Figure } \\
\text { "Range of possible hohlraum dimensions" }\end{array}$ \\
\hline 43 & 0 & I.4.1.2 & Hohlraum & LEH stay-out zone & see dwg & $\begin{array}{l}\text { Any object that protrudes into the LEH stayout zone shown in Figure "LEH Stayout Zone - } \\
\text { Rev 0" shall be reviewed and approved by the physics group as a part of the approval of } \\
\text { the overall target design. As a design goal, there shall be no objects in the stayout zone. }\end{array}$ \\
\hline 44 & 0 & I.4.1.2.1 & Hohlraum & Hohlraum wall composition & $\begin{array}{l}3 \text { layers } \\
\mathrm{Au} \\
\text { U0.75/Au0.25 } \\
\text { low-Z@waistband }\end{array}$ & $\begin{array}{l}\text { The hohlraum shall be composed of several layers, with configuration as shown in Figures } \\
\text { "Gas filled hohlraum point design (b)", Details A and D. The inner layer shall be Au, } 0.2 \\
\text { microns thick. The second layer shall be } 75 \text { at } \% \cup, 25 \text { at } \% \text { Au, with less than } 5 \text { at } \% \text { O, with } \\
\text { thickness at least } 7 \text { microns. If this second layer is less than } 10 \text { microns thick, it shall be } \\
\text { backed with a gold layer so that the the total thickness is at least } 10 \text { microns. The high-Z } \\
\text { layers may be backed with a backing layer. In the waistband area, the backing layer shall } \\
\text { be a material with } Z<<=13 \text { (Al), with maximum thickness } 150 \text { microns. The material in the } \\
\text { backing layer outside the waistband is not specified. For each hohlraum, the composition } \\
\text { of the inner Au layer and of the Au: } U \text { layer shall be known to }<5 \% \text { for each ingredient. }\end{array}$ \\
\hline
\end{tabular}

Page 3 of 5 


\begin{tabular}{|c|c|c|c|c|c|c|}
\hline Number & $\operatorname{Rev}$ & WBS & Component & Title & short req't & Requirement Text \\
\hline 45 & 0 & 1.4.1.2.2 & Hohlraum & LEH shield material & same as hohlraum & $\begin{array}{l}\text { The LEH shields shall be composed of the same material as the hohlraum, cocktail material } \\
\text { coated on a gold substrate, with an outermost gold coating } 0.2 \mu \mathrm{m} \text { thick. Cocktail shall be at } \\
\text { least } 7 \text { microns thick, and the gold substrate at least } 11 \text { microns thick. }\end{array}$ \\
\hline 46 & 0 & I.4.1.2.2 & Hohlraum & LEH shield low-z overcoat material & $\mathrm{CH}$ full density & $\begin{array}{l}\text { The overcoat on the LEH shield shall be composed of } \mathrm{CH} \text {, density } 1.05+/-5 \% \text {, composition } \\
\text { characterized to }+/-10 \% \text { on all known materials }(\mathrm{C}, \mathrm{H},<10 \% \mathrm{O}) \text { and }<100 \% / \mathrm{Z}^{\wedge} 2 \text { for all } \\
\text { impurities. }\end{array}$ \\
\hline 47 & 0 & 1.4.1.2.2 & Hohlraum & LEH shield support material & Kapton, $200 \mathrm{~nm} \pm 35 \mathrm{~nm}$ & $\begin{array}{l}\text { The material used for supporting the LEH shields shall be Kapton polyimide C22H10N2O5, } \\
\text { with thickness of } 200 \mathrm{~nm} \pm 35 \mathrm{~nm} \text {, density } 1.42 \mathrm{~g} / \mathrm{cc}+/-10 \% \text {. }\end{array}$ \\
\hline 48 & 0 & |.4.1.2.4 & Hohlraum & LEH window - composition & Upliex + 20nm Al & $\begin{array}{l}\text { The LEH window shall be composed of Upilex polyimide C22H10N2O4, coated with } 20+/- \\
5 \mathrm{~nm} \text { Al. The content of impurities shall be less than than } 100 \% / \mathrm{Z}^{\wedge} 2\end{array}$ \\
\hline 49 & 0 & I.4.1.2.4 & Hohlraum & LEH window - density & $1.47 \mathrm{~g} / \mathrm{cc}$ & $\begin{array}{l}\text { The density of the LEH window material (not including the Al coating) shall be } 1.47 \mathrm{~g} / \mathrm{cc}+/- \\
10 \%\end{array}$ \\
\hline 50 & 0 & $\mid .4 .1 .2 .4$ & Hohlraum & LEH window - glue & $<50 \mu \mathrm{m}$ & $\begin{array}{l}\text { Glue to afix LEH window shall be }<50 \text { microns thick and end within } 100 \text { microns of the LEH } \\
\text { liner, as shown in hohlraum drawing (b) }\end{array}$ \\
\hline 51 & 0 & I.4.1.2.1 & Hohlraum & Neutron line of sight window thickness & see hohlraum wall & $\begin{array}{l}\text { The window through the hohlraum for the neutron line of sight shall have arial density less } \\
\text { than or equal to that specified in "hohlraum wall composition", with variations in arial density } \\
<50 \% \text { over a field of view with diameter }>=400 \mu \mathrm{m} \text {. The current configuration of an entire } \\
\text { band for the } x \text {-ray line of sight provides for the neutron line of sight. }\end{array}$ \\
\hline 52 & 0 & I.4.1.2.1 & Hohlraum & X-ray line of sight & see hohlraum wall & $\begin{array}{l}\text { For the } x \text {-ray line of sight there shall be a band around the waist of the hohlraum, at least } \\
400 \mu \mathrm{m} \text { wide, with composition as specified in "hohlraum wall composition." Variations in } \\
\text { arial density of the high Z material shall be }<1.5 \% \text { over a field of view with diameter }>/=400 \\
\mu \mathrm{m} \text {. }\end{array}$ \\
\hline 53 & 0 & $\mid .4 .1 .2 .4$ & Hohlraum & Tent - uniformity/roughness & same as ablator & $\begin{array}{l}\text { The thickness uniformity of the tent where it contacts the ablator shall provide a surface } \\
\text { smoothness that meets capsule roughness requirement }\end{array}$ \\
\hline 54 & 0 & I.4.1.2.4 & Hohlraum & Tent - composition & Kapton & $\begin{array}{l}\text { The tent shall be composed of Kapton polyimide C22H10N2O5. The material composition } \\
\text { shall be known with an accuracy of }+/-5 \% \text { on all ingredients known to be present, and less } \\
\text { than } 100 \% / Z^{\wedge} 2 \text { for all impurities }\end{array}$ \\
\hline 55 & 0 & $\mid .4 .1 .2 .4$ & Hohlraum & Tent - density & $1.42 \mathrm{~g} / \mathrm{cc}$ & The tent material shall have a density of $1.42 \mathrm{~g} / \mathrm{cc}+/-5 \%$ \\
\hline 56 & 0 & 1.4.1.2.1 & Hohlraum & Starburst pattern & see dwg & $\begin{array}{l}\text { The feature size and total area of the starburst pattern shall be less than } 120 \% \text { of the } \\
\text { design in Figure "Hohlraum Starburst Pattern" }\end{array}$ \\
\hline 57 & 0 & I.4.1.2 & Hohlraum & Hohlraum cleanliness & $<2.5 \mathrm{e}-6 \mathrm{~g}$ & $\begin{array}{l}\text { The interior surface of the hohlraum and LEH shield shall have cleanliness such that total } \\
\text { mass of contaminants is less than } 2.5 \mathrm{e}-6 \mathrm{~g} \text { ( } 1 \% \text { of mass of foam or gas fill) }\end{array}$ \\
\hline 58 & 0 & 1.4 .1 .2 & Gas-filled hohlraum & $\begin{array}{l}\text { Hohlraum configuration - dimensions and } \\
\text { tolerances }\end{array}$ & see dwg & $\begin{array}{l}\text { The hohlraum configuration shall be as shown in Figures "Gas filled hohlraum design" (a) } \\
\text { and (b). For parameters which may be requested over a specified range of values, the } \\
\text { tolerances shown will apply at the requested dimension }\end{array}$ \\
\hline 59 & 0 & 1.4 .1 .2 .2 & Gas-filled hohlraum & Hohlraum gas fill - composition & $\mathrm{He}$ & $\begin{array}{l}\text { The hohlraum shall be filled with a mixture of } \mathrm{He} \text { and } \mathrm{H} \text {, with a } \mathrm{H} \text { density specified in the } \\
\text { range from } 0 \text { to } 0.65 \mathrm{mg} / \mathrm{cc} \text {. The point design value of } \mathrm{H} \text { density shall be zero. }\end{array}$ \\
\hline 60 & 0 & 1.4 .1 .2 .2 & Gas-filled hohlraum & Hohlraum gas fill - density & $1.3 \mathrm{e}-3 \pm 1.0 \mathrm{e}-4 \mathrm{~g} / \mathrm{cm} 3$ & The density of the gas filling the hohlraum shall be $1.3 \mathrm{e}-3 \pm 1.0 \mathrm{e}-4 \mathrm{~g} / \mathrm{cm} 3$ \\
\hline 61 & 0 & 1.4 .1 .2 .2 & Gas-filled hohlraum & LEH liner - composition & $\mathrm{CH}$ full density & $\begin{array}{l}\text { The lining on the LEH shall be composed of } \mathrm{CH} \text {, density } 1.05+/-5 \% \text {, composition } \\
\text { characterized to }+/-10 \% \text { on all known materials }(\mathrm{C}, \mathrm{H},<10 \% \mathrm{O}) \text { and }<100 \% / \mathrm{Z}^{\wedge} 2 \text { for all } \\
\text { impurities. }\end{array}$ \\
\hline 62 & 0 & 1.4.1.2.4 & Gas-filled hohlraum & Convection baffles & same as tent & $\begin{array}{l}\text { Convection baffles shall be meet the same thickness and composition requirements as the } \\
\text { shield supports. }\end{array}$ \\
\hline 63 & 0 & 1.4 .1 & Target & $\begin{array}{l}\text { Target orientation in target chamber - } \\
\text { hohlraum axis }\end{array}$ & vertical $\pm 4 \mathrm{mrad}$ & $\begin{array}{l}\text { The angle bewteen the hohlraum axis and the target chamber axis shall be less than }+/-4 \\
\text { mrad. }\end{array}$ \\
\hline 64 & 0 & N.L & Laser & Beam alignment with target LEHs & $<10 \mu \mathrm{m}$ RMS & $\begin{array}{l}\text { The RMS distance from the centroids of the laser beams to the centroid of the respective } \\
\text { LEH shall be less than } 10 \mu \mathrm{m} \text {. The distance is measured in } 3 D \text {, including } R \text { and } Z \\
\text { components. }\end{array}$ \\
\hline 65 & 0 & N.L & Laser & Laser pulse shape, energy, peak power & See Table & $\begin{array}{l}\text { The laser pulses for the } 4 \text { cones shall be as specified in Table "Laser pulse shape - Rev } 0 " \text { ". } \\
\text { The peak power and total energy associated with these specified pulse shapes are shown } \\
\text { in Table "Laser peak power and total energy for required pulse shapes." }\end{array}$ \\
\hline 66 & 0 & N.L & Laser & Reproducibility of total laser power $\mathrm{P}(\mathrm{t})$ & $3 \%$ RMS & $\begin{array}{l}\text { The total laser power incident on the hohlraum as a function of time, smoothed over any } \\
\text { 2ns window, shall be reproducible shot to shot to better than } 3 \% \text { RMS }\end{array}$ \\
\hline
\end{tabular}




\begin{tabular}{|c|c|c|c|c|c|c|}
\hline Number & $\operatorname{Rev}$ & WBS & Component & Title & short req't & Requirement Text \\
\hline 67 & 0 & N.L & Laser & Laser power imbalance & TBD & TBD. Currently assumed to be per Jones memo. \\
\hline 68 & 0 & N.L & Laser & Spot shape & See Table & $\begin{array}{l}\text { The shape of the laser spot shall be as defined in Table "Laser Spot Shapes - Rev 0". The } \\
\text { position-dependent relative intensity at any point shall be either (i) within }+/-10 \% \text { of the } \\
\text { specified values, or (ii) less than } 0.5 \% \text { of the central intensity. }\end{array}$ \\
\hline 69 & 0 & N.L & Laser & SSD bandwidth & 90-150 GHz @1w & The SSD bandwidth shall be $90 \mathrm{GHz} @ 1$ w, with the capability to go to $150 \mathrm{GHz}$ \\
\hline 70 & 0 & N.L & Laser & Polarization smoothing & Checkerboard & $\begin{array}{l}\text { The upper and lower beams in each laser quad (as viewed from target chamber center) } \\
\text { shall have orthogonal polarizations. }\end{array}$ \\
\hline 71 & 0 & N.L & Laser & 2-color separation & $\begin{array}{l}1.9+/-10 \% \text { angstrom @ } \\
1 \mathrm{w}, \text { adjustable }+/-0.7 \\
\text { angstrom }\end{array}$ & $\begin{array}{l}\text { The separation of the two colors shall be adjustable between } 1.2 \text { and } 2.6 \text { angstroms at } 1 \mathrm{w} \text {. } \\
\text { The point design value shall be } 1.9+/-10 \% \text { angstrom }\end{array}$ \\
\hline 72 & 0 & N.L & Laser & Beam pointing & see table & $\begin{array}{l}\text { Each individual beam (not quad) shall be pointed to place the best focus of the beam in the } \\
\text { plane of the LEH, in the phi plane of the lens, at the radius from the hohlraum axis shown in } \\
\text { Table "Beam pointing - Rev 0" }\end{array}$ \\
\hline 73 & 0 & N.L & Laser & Beam positioning errors & $<50 \mu \mathrm{m} \mathrm{rms}$ & $\begin{array}{l}\text { The deviations of the individual beam positions from their aim points (on each shot) shall be } \\
\text { less than } 50 \mu \mathrm{m} \text { RMS. }\end{array}$ \\
\hline 73 & $<==$ hig & hest $\mathrm{s} / \mathrm{n}$ usec & & Iast line & & \\
\hline & & & & & & \\
\hline
\end{tabular}


Ablator layer requirements for graded doped Be capsule - Rev 0

\begin{tabular}{|c|c|c|c|c|c|}
\hline Layer no & \begin{tabular}{|c|} 
Point Design \\
Inner Radius, $\mu \mathrm{m}$ \\
(ref)
\end{tabular} & $\begin{array}{c}\text { Point Design Outer } \\
\text { Radius, } \mu \mathrm{m} \text { (ref) }\end{array}$ & Required Thickness, $\mu \mathrm{m}$ & $\begin{array}{c}\text { Required Dopant } \\
\text { Concentration, at \% }\end{array}$ & $\begin{array}{c}\text { Required } \\
\text { Density, g/cc } \\
\text { (note 1) }\end{array}$ \\
\hline Total, all layers & \begin{tabular}{|r|}
840 \\
\end{tabular} & 1000 & $160+/-3$ & $n / a$ & $n / a$ \\
\hline \multirow[t]{2}{*}{5} & \multirow[t]{2}{*}{915} & \multirow[t]{2}{*}{1000} & \multirow[t]{2}{*}{ as required (nominally 85 ) } & Outer $70 \mu \mathrm{m}<0.05$ & $1.75+/-3 \%$ \\
\hline & & & & Outer $80 \mu \mathrm{m}<0.10$ & \\
\hline 4 & 900 & 915 & $15+/-3$ & $0.35+/-0.1$ & $1.785+/-3 \%$ \\
\hline 3 & 850 & 900 & $50+/-3$ & $0.7+/-0.1$ & $1.82+/-3 \%$ \\
\hline 2 & 845 & 850 & $5+/-1.5$ & $0.35+/-0.1$ & $1.785+/-3 \%$ \\
\hline 1 & 840 & 845 & $5+/-1$ & inner $3 \mathrm{~mm},<0.05$ & $1.75+/-3 \%$ \\
\hline
\end{tabular}

Notes

1. Note that Capsule-to-capsule density variations are limited

by another requirement to $+/-1.5 \%$ 


\section{Surface roughness and thickness power spectra for graded doped Be capsule - Rev 0}

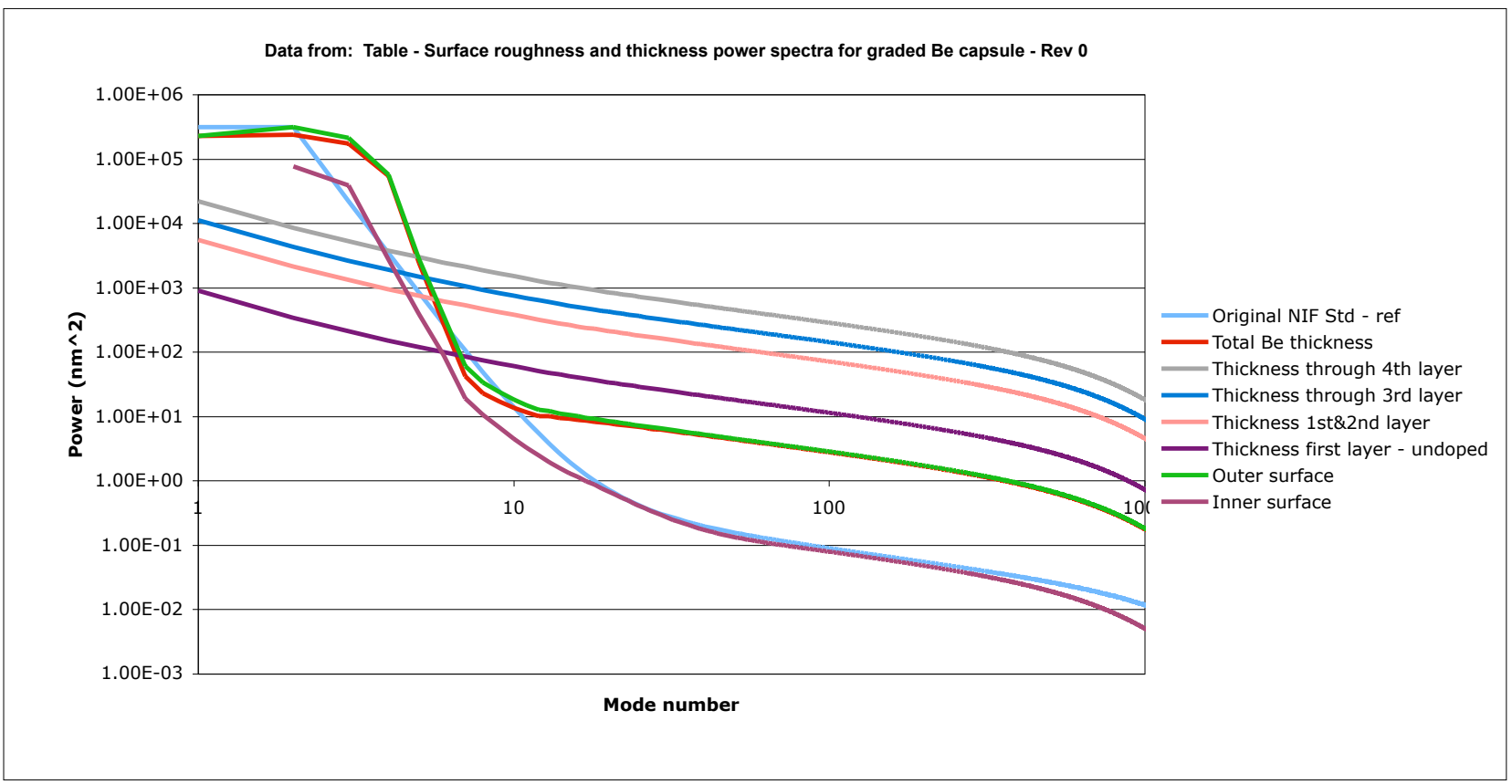

Notes:

1. First table is the $2 \mathrm{D}$ power in the low modes, in $\mathrm{nm}^{\wedge} 2$. Requirement for modes $1-12$.

2. Second table is the $1 D$ power for each surface in a trace, in $n m^{\wedge} 2$. Required for modes $>12$, included for information purposes for low modes

3. Inner surface roughness is defined relative to an ideal sphere of the same average radius.

Other surfaces are all defined relative to inner surfaces, i.e.they are actually the variation in the thickness

4. Coding that generates trace powers is in surfaces093005.i

5. Outer surface relative to inner surface

6. At low modes, assumes inner surface and thickness are randomly phased.

At high modes, assumes inner \& outer surfaces randomly phased.

7. Second dopant surface, relative to inside of Be shell

8. Third dopant surface, relative to inside of Be shell

9. Fourth dopant surface, relative to inside of $\mathrm{Be}$

\begin{tabular}{|c|c|c|c|c|c|c|c|c|}
\hline Mode number & $\begin{array}{l}\text { Original NIF } \\
\text { standard, } \\
\text { reference, } \mathrm{nm}^{\wedge} 2\end{array}$ & $\begin{array}{l}\text { Inner surface } \\
\text { (radius), } \mathrm{nm}^{\wedge} 2\end{array}$ & $\begin{array}{c}\text { Total shell } \\
\text { thickness, } \\
\text { nm^2 (note 5) }\end{array}$ & $\begin{array}{l}\text { Outer surface, } \\
\left.\mathrm{nm}^{\wedge} 2 \text { (note } 6\right)\end{array}$ & $\begin{array}{l}\text { Thickness of } \\
\text { first doped } \\
\text { layer, } \mathrm{nm}^{\wedge} 2\end{array}$ & $\begin{array}{c}\text { Thickness of } \\
\text { 1st+2nd doped } \\
\text { layer, } \mathrm{nm}^{\wedge} 2 \\
\text { (note } 7)\end{array}$ & $\begin{array}{l}\text { Thickness of } \\
1 \mathrm{st}+2 \mathrm{nd}+3 \mathrm{rd} \\
\text { doped layer, } \\
\mathrm{nm} \mathrm{m}^{\wedge} 2 \text { (note } 8 \text { ) }\end{array}$ & $\begin{array}{c}\text { Thickness of } \\
\text { 1st+2nd+3rd+4th } \\
\text { doped layer, } \mathrm{nm}^{\wedge} 2 \\
\text { (note 9) }\end{array}$ \\
\hline \multicolumn{9}{|l|}{ 2D power } \\
\hline 1 & \multirow[t]{12}{*}{ not applicable } & & 122500 & 122500 & 739.383 & 4621.15 & 9242.29 & 18484.6 \\
\hline 2 & & 101700 & 275625 & 377325 & 308.076 & 1925.48 & 3850.96 & 7701.91 \\
\hline 3 & & 61530 & 275625 & 337155 & 191.692 & 1198.08 & 2396.15 & 4792.3 \\
\hline 4 & & 4900 & 99225 & 104125 & 138.634 & 866.465 & 1732.93 & 3465.86 \\
\hline 5 & & 780 & 4900 & 5680 & 108.443 & 677.768 & 1355.54 & 2711.07 \\
\hline 6 & & 185 & 566.44 & 751.44 & 88.9999 & 556.249 & 1112.5 & 2225 \\
\hline 7 & & 36 & 70 & 106 & 75.4473 & 471.546 & 943.091 & 1886.18 \\
\hline 8 & & 19.8 & 31.36 & 51.16 & 65.4662 & 409.164 & 818.328 & 1636.66 \\
\hline 9 & & 12.4 & 20 & 32.4 & 57.8119 & 361.324 & 722.648 & 1445.3 \\
\hline 10 & & 8 & 14 & 22 & 51.7568 & 323.48 & 646.961 & 1293.92 \\
\hline 11 & & 5.8 & 9 & 14.8 & 46.848 & 292.8 & 585.6 & 1171.2 \\
\hline 12 & & 4.3 & 6 & 10.3 & 42.7884 & 267.427 & 534.855 & 1069.71 \\
\hline \multicolumn{9}{|l|}{ 1D trace power } \\
\hline 1 & $3.13 E+05$ & & 227031 & $2.27 \mathrm{E}+05$ & 896.646 & 5604.04 & 11208.1 & 22416.1 \\
\hline 2 & 313000 & 77828.8 & 237857 & 315685.8 & 345.657 & 2160.35 & 4320.71 & 8641.42 \\
\hline 3 & 22000 & 38678.5 & 173630 & 212308.5 & 211.227 & 1320.17 & 2640.33 & 5280.67 \\
\hline 4 & 3440 & 2737.4 & 54418.1 & 57155.5 & 152.616 & 953.848 & 1907.7 & 3815.39 \\
\hline 5 & 840 & 394.719 & 2439.48 & 2834.199 & 120.062 & 750.391 & 1500.78 & 3001.56 \\
\hline 6 & 272 & 90.2859 & 272.268 & 362.5539 & 99.4294 & 621.434 & 1242.87 & 2485.73 \\
\hline 7 & 107 & 19.2375 & 42.4041 & 61.6416 & 85.1895 & 532.434 & 1064.87 & 2129.74 \\
\hline 8 & 48.8 & 10.7192 & 23.2549 & 33.9741 & 74.7962 & 467.476 & 934.953 & 1869.91 \\
\hline 9 & 24.9 & 6.79828 & 17.1867 & 23.98498 & 66.8646 & 417.904 & 835.808 & 1671.62 \\
\hline 10 & 14 & 4.52319 & 13.7607 & 18.28389 & 60.6361 & 378.976 & 757.951 & 1515.9 \\
\hline
\end{tabular}




\begin{tabular}{|c|c|c|c|c|c|c|c|c|}
\hline 11 & 8.48 & 3.29369 & 11.7253 & 15.01899 & 55.5985 & 347.491 & 694.981 & 1389.96 \\
\hline 12 & 5.48 & 2.51608 & 10.2307 & 12.74678 & 51.4656 & 321.66 & 643.32 & 1286.64 \\
\hline 13 & 3.74 & 1.92477 & 10.1633 & 12.08807 & 47.9933 & 299.958 & 599.917 & 1199.83 \\
\hline 14 & 2.68 & 1.57638 & 9.6009 & 11.17728 & 45.0653 & 281.658 & 563.316 & 1126.63 \\
\hline 15 & 2 & 1.32717 & 9.25686 & 10.58403 & 42.5377 & 265.861 & 531.722 & 1063.44 \\
\hline 16 & 1.55 & 1.14487 & 8.92521 & 10.07008 & 40.2803 & 251.752 & 503.504 & 1007.01 \\
\hline 17 & 1.24 & 0.995459 & 8.62121 & 9.616669 & 38.4667 & 240.417 & 480.834 & 961.667 \\
\hline 18 & 1.01 & 0.871942 & 8.34124 & 9.213182 & 36.8527 & 230.329 & 460.659 & 921.318 \\
\hline 19 & 0.851 & 0.769054 & 8.08231 & 8.851364 & 35.4054 & 221.284 & 442.568 & 885.136 \\
\hline 20 & 0.729 & 0.682765 & 7.84196 & 8.524725 & 34.0989 & 213.118 & 426.236 & 852.472 \\
\hline 21 & 0.635 & 0.609953 & 7.61812 & 8.228073 & 32.9123 & 205.702 & 411.404 & 822.808 \\
\hline 22 & 0.561 & 0.548169 & 7.40905 & 7.957219 & 31.8289 & 198.93 & 397.861 & 795.722 \\
\hline 23 & 0.503 & 0.495473 & 7.21324 & 7.708713 & 30.8349 & 192.718 & 385.436 & 770.871 \\
\hline 24 & 0.455 & 0.450315 & 7.02941 & 7.479725 & 29.9189 & 186.993 & 373.986 & 747.972 \\
\hline 25 & 0.416 & 0.411445 & 6.85643 & 7.267875 & 29.0715 & 181.697 & 363.394 & 726.788 \\
\hline 26 & 0.384 & 0.377848 & 6.69334 & 7.071188 & 28.2847 & 176.78 & 353.559 & 707.119 \\
\hline 27 & 0.357 & 0.348694 & 6.53927 & 6.887964 & 27.5518 & 172.199 & 344.398 & 688.796 \\
\hline 28 & 0.333 & 0.323299 & 6.39347 & 6.716769 & 26.8671 & 167.919 & 335.838 & 671.677 \\
\hline 29 & 0.313 & 0.301098 & 6.25526 & 6.556358 & 26.2254 & 163.909 & 327.818 & 655.636 \\
\hline 30 & 0.296 & 0.281623 & 6.12405 & 6.405673 & 25.6227 & 160.142 & 320.284 & 640.567 \\
\hline 31 & 0.281 & 0.26448 & 5.9993 & 6.26378 & 25.0551 & 156.594 & 313.189 & 626.378 \\
\hline 32 & 0.268 & 0.249339 & 5.88053 & 6.129869 & 24.5195 & 153.247 & 306.493 & 612.987 \\
\hline 33 & 0.256 & 0.235925 & 5.76731 & 6.003235 & 24.0129 & 150.081 & 300.162 & 600.323 \\
\hline 34 & 0.245 & 0.224001 & 5.65924 & 5.883241 & 23.533 & 147.081 & 294.162 & 588.324 \\
\hline 35 & 0.236 & 0.213368 & 5.55597 & 5.769338 & 23.0773 & 144.233 & 288.467 & 576.934 \\
\hline 36 & 0.227 & 0.203857 & 5.45718 & 5.661037 & 22.6441 & 141.526 & 283.052 & 566.103 \\
\hline 37 & 0.219 & 0.195324 & 5.36256 & 5.557884 & 22.2316 & 138.947 & 277.894 & 555.789 \\
\hline 38 & 0.212 & 0.187643 & 5.27186 & 5.459503 & 21.838 & 136.488 & 272.975 & 545.951 \\
\hline 39 & 0.205 & 0.180709 & 5.18483 & 5.365539 & 21.4622 & 134.139 & 268.277 & 536.554 \\
\hline 40 & 0.199 & 0.17443 & 5.10124 & 5.27567 & 21.1027 & 131.892 & 263.783 & 527.567 \\
\hline 41 & 0.194 & 0.168726 & 5.02088 & 5.189606 & 20.7584 & 129.74 & 259.48 & 518.961 \\
\hline 42 & 0.189 & 0.16353 & 4.94356 & 5.10709 & 20.4284 & 127.677 & 255.355 & 510.709 \\
\hline 43 & 0.184 & 0.158782 & 4.86911 & 5.027892 & 20.1116 & 125.697 & 251.394 & 502.789 \\
\hline 44 & 0.179 & 0.15443 & 4.79735 & 4.95178 & 19.8071 & 123.795 & 247.589 & 495.178 \\
\hline 45 & 0.175 & 0.15043 & 4.72815 & 4.87858 & 19.5143 & 121.964 & 243.929 & 487.858 \\
\hline 46 & 0.171 & 0.146742 & 4.66136 & 4.808102 & 19.2324 & 120.202 & 240.405 & 480.81 \\
\hline 47 & 0.168 & 0.143332 & 4.59684 & 4.740172 & 18.9607 & 118.504 & 237.009 & 474.018 \\
\hline 48 & 0.164 & 0.14017 & 4.53449 & 4.67466 & 18.6986 & 116.867 & 233.733 & 467.466 \\
\hline 49 & 0.161 & 0.137231 & 4.47418 & 4.611411 & 18.4457 & 115.285 & 230.571 & 461.142 \\
\hline 50 & 0.158 & 0.13449 & 4.41582 & 4.55031 & 18.2012 & 113.758 & 227.516 & 455.031 \\
\hline 51 & 0.155 & 0.131928 & 4.3593 & 4.491228 & 17.9649 & 112.281 & 224.561 & 449.123 \\
\hline 52 & 0.152 & 0.129526 & 4.30454 & 4.434066 & 17.7362 & 110.852 & 221.703 & 443.406 \\
\hline 53 & 0.15 & 0.12727 & 4.25144 & 4.37871 & 17.5148 & 109.468 & 218.935 & 437.871 \\
\hline 54 & 0.147 & 0.125145 & 4.19993 & 4.325075 & 17.3003 & 108.127 & 216.254 & 432.507 \\
\hline 55 & 0.145 & 0.123138 & 4.14993 & 4.273068 & 17.0923 & 106.827 & 213.653 & 427.307 \\
\hline 56 & 0.142 & 0.121239 & 4.10137 & 4.222609 & 16.8904 & 105.565 & 211.131 & 422.261 \\
\hline 57 & 0.14 & 0.119438 & 4.05419 & 4.173628 & 16.6945 & 104.341 & 208.682 & 417.363 \\
\hline 58 & 0.138 & 0.117726 & 4.00833 & 4.126056 & 16.5042 & 103.151 & 206.303 & 412.605 \\
\hline 59 & 0.136 & 0.116096 & 3.96372 & 4.079816 & 16.3193 & 101.995 & 203.991 & 407.982 \\
\hline 60 & 0.134 & 0.11454 & 3.92031 & 4.03485 & 16.1394 & 100.871 & 201.743 & 403.485 \\
\hline 61 & 0.132 & 0.113053 & 3.87806 & 3.991113 & 15.9644 & 99.7777 & 199.555 & 399.111 \\
\hline 62 & 0.13 & 0.111629 & 3.8369 & 3.948529 & 15.7941 & 98.7132 & 197.426 & 394.853 \\
\hline 63 & 0.129 & 0.110263 & 3.7968 & 3.907063 & 15.6282 & 97.6765 & 195.353 & 390.706 \\
\hline 64 & 0.127 & 0.10895 & 3.75771 & 3.86666 & 15.4666 & 96.6664 & 193.333 & 386.666 \\
\hline 65 & 0.125 & 0.107687 & 3.71959 & 3.827277 & 15.3091 & 95.6819 & 191.364 & 382.727 \\
\hline 66 & 0.124 & 0.10647 & 3.6824 & 3.78887 & 15.1555 & 94.7217 & 189.443 & 378.887 \\
\hline 67 & 0.122 & 0.105296 & 3.6461 & 3.751396 & 15.0056 & 93.785 & 187.57 & 375.14 \\
\hline 68 & 0.121 & 0.104162 & 3.61067 & 3.714832 & 14.8593 & 92.8708 & 185.742 & 371.483 \\
\hline 69 & 0.12 & 0.103064 & 3.57606 & 3.679124 & 14.7165 & 91.9781 & 183.956 & 367.913 \\
\hline 70 & 0.118 & 0.102002 & 3.54225 & 3.644252 & 14.577 & 91.1063 & 182.213 & 364.425 \\
\hline 71 & 0.117 & 0.100971 & 3.5092 & 3.610171 & 14.4407 & 90.2543 & 180.509 & 361.017 \\
\hline 72 & 0.116 & 0.0999717 & 3.47689 & 3.5768617 & 14.3075 & 89.4216 & 178.843 & 357.686 \\
\hline 73 & 0.114 & 0.0990008 & 3.44529 & 3.5442908 & 14.1772 & 88.6073 & 177.215 & 354.429 \\
\hline 74 & 0.113 & 0.0980568 & 3.41438 & 3.5124368 & 14.0497 & 87.8109 & 175.622 & 351.244 \\
\hline 75 & 0.112 & 0.0971383 & 3.38412 & 3.4812583 & 13.9251 & 87.0316 & 174.063 & 348.126 \\
\hline 76 & 0.111 & 0.096244 & 3.35451 & 3.450754 & 13.803 & 86.2688 & 172.538 & 345.075 \\
\hline 77 & 0.11 & 0.0953724 & 3.32551 & 3.4208824 & 13.6835 & 85.522 & 171.044 & 342.088 \\
\hline 78 & 0.109 & 0.0945225 & 3.2971 & 3.3916225 & 13.5665 & 84.7906 & 169.581 & 339.162 \\
\hline 79 & 0.108 & 0.0936932 & 3.26927 & 3.3629632 & 13.4518 & 84.074 & 168.148 & 336.296 \\
\hline 80 & 0.107 & 0.0928834 & 3.24199 & 3.3348734 & 13.3395 & 83.3719 & 166.744 & 333.487 \\
\hline 81 & 0.106 & 0.0920922 & 3.21525 & 3.3073422 & 13.2294 & 82.6836 & 165.367 & 330.734 \\
\hline 82 & 0.105 & 0.0913188 & 3.18903 & 3.2803488 & 13.1214 & 82.0087 & 164.017 & 328.035 \\
\hline 83 & 0.104 & 0.0905624 & 3.16331 & 3.2538724 & 13.0155 & 81.3469 & 162.694 & 325.387 \\
\hline
\end{tabular}




\begin{tabular}{|c|c|c|}
\hline 84 & 0.103 & 0.0898222 \\
\hline 85 & 0.102 & 0.0890975 \\
\hline 86 & 0.101 & 0.0883878 \\
\hline 87 & 0.1 & 0.0876923 \\
\hline 88 & 0.0992 & 0.0870106 \\
\hline 89 & 0.0984 & 0.0863421 \\
\hline 90 & 0.0976 & 0.0856863 \\
\hline 91 & 0.0968 & 0.0850428 \\
\hline 92 & 0.096 & 0.0844111 \\
\hline 93 & 0.0953 & 0.0837908 \\
\hline 94 & 0.0945 & 0.0831815 \\
\hline 95 & 0.0938 & 0.0825829 \\
\hline 96 & 0.0931 & 0.0819946 \\
\hline 97 & 0.0924 & 0.0814163 \\
\hline 98 & 0.0917 & 0.0808476 \\
\hline 99 & 0.091 & 0.0802883 \\
\hline 100 & 0.0904 & 0.079738 \\
\hline 101 & 0.0897 & 0.0791966 \\
\hline 102 & 0.0891 & 0.0786638 \\
\hline 103 & 0.0885 & 0.0781392 \\
\hline 104 & 0.0879 & 0.0776228 \\
\hline 105 & 0.0873 & 0.0771142 \\
\hline 106 & 0.0867 & 0.0766133 \\
\hline 107 & 0.0861 & 0.0761197 \\
\hline 108 & 0.0855 & 0.0756335 \\
\hline 109 & 0.085 & 0.0751543 \\
\hline 110 & 0.0844 & 0.0746819 \\
\hline 111 & 0.0839 & 0.0742163 \\
\hline 112 & 0.0833 & 0.0737572 \\
\hline 113 & 0.0828 & 0.0733044 \\
\hline 114 & 0.0823 & 0.0728579 \\
\hline 115 & 0.0818 & 0.0724175 \\
\hline 116 & 0.0813 & 0.071983 \\
\hline 117 & 0.0808 & 0.0715542 \\
\hline 118 & 0.0803 & 0.0711311 \\
\hline 119 & 0.0798 & 0.0707136 \\
\hline 120 & 0.0793 & 0.0703014 \\
\hline 121 & 0.0789 & 0.0698945 \\
\hline 122 & 0.0784 & 0.0694928 \\
\hline 123 & 0.078 & 0.0690961 \\
\hline 124 & 0.0775 & 0.0687044 \\
\hline 125 & 0.0771 & 0.0683175 \\
\hline 126 & 0.0766 & 0.0679353 \\
\hline 127 & 0.0762 & 0.0675578 \\
\hline 128 & 0.0758 & 0.0671847 \\
\hline 129 & 0.0754 & 0.0668162 \\
\hline 130 & 0.075 & 0.0664519 \\
\hline 131 & 0.0746 & 0.066092 \\
\hline 132 & 0.0742 & 0.0657362 \\
\hline 133 & 0.0738 & 0.0653845 \\
\hline 134 & 0.0734 & 0.0650368 \\
\hline 135 & 0.073 & 0.064693 \\
\hline 136 & 0.0726 & 0.0643531 \\
\hline 137 & 0.0723 & 0.064017 \\
\hline 138 & 0.0719 & 0.0636846 \\
\hline 139 & 0.0715 & 0.0633558 \\
\hline 140 & 0.0712 & 0.0630306 \\
\hline 141 & 0.0708 & 0.0627089 \\
\hline 142 & 0.0705 & 0.0623906 \\
\hline 143 & 0.0701 & 0.0620758 \\
\hline 144 & 0.0698 & 0.0617642 \\
\hline 145 & 0.0694 & 0.0614559 \\
\hline 146 & 0.0691 & 0.0611508 \\
\hline 147 & 0.0688 & 0.0608488 \\
\hline 148 & 0.0684 & 0.0605499 \\
\hline 149 & 0.0681 & 0.060254 \\
\hline 150 & 0.0678 & 0.0599611 \\
\hline 151 & 0.0675 & 0.0596712 \\
\hline 152 & 0.0672 & 0.0593841 \\
\hline 153 & 0.0668 & 0.0590999 \\
\hline 154 & 0.0665 & 0.0588184 \\
\hline 155 & 0.0662 & 0.0585396 \\
\hline 156 & 0.0659 & 0.0582636 \\
\hline
\end{tabular}

\begin{tabular}{|c|c|}
\hline 3.13808 & 3.2279022 \\
\hline 3.11332 & 3.2024175 \\
\hline 3.08902 & 3.1774078 \\
\hline 3.06516 & 3.1528523 \\
\hline 3.04173 & 3.1287406 \\
\hline 3.01872 & 3.1050621 \\
\hline 2.99611 & 3.0817963 \\
\hline 2.97389 & 3.0589328 \\
\hline 2.95205 & 3.0364611 \\
\hline 2.93059 & 3.0143808 \\
\hline 2.90948 & 2.9926615 \\
\hline 2.88873 & 2.9713129 \\
\hline 2.86831 & 2.9503046 \\
\hline 2.84823 & 2.9296463 \\
\hline 2.82846 & 2.9093076 \\
\hline 2.80901 & 2.8892983 \\
\hline 2.78987 & 2.869608 \\
\hline 2.77102 & 2.8502166 \\
\hline 2.75246 & 2.8311238 \\
\hline 2.73418 & 2.8123192 \\
\hline 2.71618 & 2.7938028 \\
\hline 2.69844 & 2.7755542 \\
\hline 2.68096 & 2.7575733 \\
\hline 2.66374 & 2.7398597 \\
\hline 2.64677 & 2.7224035 \\
\hline 2.63004 & 2.7051943 \\
\hline 2.61354 & 2.6882219 \\
\hline 2.59728 & 2.6714963 \\
\hline 2.58124 & 2.6549972 \\
\hline 2.56542 & 2.6387244 \\
\hline 2.54982 & 2.6226779 \\
\hline 2.53442 & 2.6068375 \\
\hline 2.51924 & 2.591223 \\
\hline 2.50425 & 2.5758042 \\
\hline 2.48945 & 2.5605811 \\
\hline 2.47485 & 2.5455636 \\
\hline 2.46044 & 2.5307414 \\
\hline 2.44621 & 2.5161045 \\
\hline 2.43216 & 2.5016528 \\
\hline 2.41829 & 2.4873861 \\
\hline 2.40458 & 2.4732844 \\
\hline 2.39105 & 2.4593675 \\
\hline 2.37768 & 2.4456153 \\
\hline 2.36447 & 2.4320278 \\
\hline 2.35142 & 2.4186047 \\
\hline 2.33852 & 2.4053362 \\
\hline 2.32578 & 2.3922319 \\
\hline 2.31319 & 2.379282 \\
\hline 2.30074 & 2.3664762 \\
\hline 2.28843 & 2.3538145 \\
\hline 2.27626 & 2.3412968 \\
\hline 2.26423 & 2.328923 \\
\hline 2.25234 & 2.3166931 \\
\hline 2.24058 & 2.304597 \\
\hline 2.22894 & 2.2926246 \\
\hline 2.21744 & 2.2807958 \\
\hline 2.20606 & 2.2690906 \\
\hline 2.1948 & 2.2575089 \\
\hline 2.18366 & 2.2460506 \\
\hline 2.17264 & 2.2347158 \\
\hline 2.16174 & 2.2235042 \\
\hline 2.15095 & 2.2124059 \\
\hline 2.14027 & 2.2014208 \\
\hline 2.1297 & 2.1905488 \\
\hline 2.11924 & 2.1797899 \\
\hline 2.10889 & 2.169144 \\
\hline 2.09864 & 2.1586011 \\
\hline 2.08849 & 2.1481612 \\
\hline 2.07844 & 2.1378241 \\
\hline 2.06849 & 2.1275899 \\
\hline 2.05864 & 2.1174584 \\
\hline 2.04888 & 2.1074196 \\
\hline 2.03922 & 2.0974836 \\
\hline
\end{tabular}

\begin{tabular}{|c|c|}
\hline 12.9116 & 80.6976 \\
\hline 12.8097 & 80.0605 \\
\hline 12.7096 & 79.4352 \\
\hline 12.6114 & 78.8213 \\
\hline 12.515 & 78.2185 \\
\hline 12.4202 & 77.6264 \\
\hline 12.3272 & 77.0448 \\
\hline 12.2357 & 76.4733 \\
\hline 12.1459 & 75.9116 \\
\hline 12.0575 & 75.3595 \\
\hline 11.9707 & 74.8166 \\
\hline 11.8852 & 74.2828 \\
\hline 11.8012 & 73.7576 \\
\hline 11.7186 & 73.2411 \\
\hline 11.6372 & 72.7328 \\
\hline 11.5572 & 72.2325 \\
\hline 11.4784 & 71.7401 \\
\hline 11.4009 & 71.2554 \\
\hline 11.3245 & 70.778 \\
\hline 11.2493 & 70.308 \\
\hline 11.1752 & 69.845 \\
\hline 11.1022 & 69.3888 \\
\hline 11.0303 & 68.9394 \\
\hline 10.9595 & 68.4966 \\
\hline 10.8896 & 68.0601 \\
\hline 10.8208 & 67.6298 \\
\hline 10.7529 & 67.2057 \\
\hline 10.686 & 66.7874 \\
\hline 10.62 & 66.375 \\
\hline 10.5549 & 65.9682 \\
\hline 10.4907 & 65.5669 \\
\hline 10.4274 & 65.1711 \\
\hline 10.3649 & 64.7805 \\
\hline 10.3032 & 64.395 \\
\hline 10.2423 & 64.0147 \\
\hline 10.1823 & 63.6392 \\
\hline 10.123 & 63.2686 \\
\hline 10.0644 & 62.9026 \\
\hline 10.0066 & 62.5413 \\
\hline 9.94953 & 62.1846 \\
\hline 9.89315 & 61.8322 \\
\hline 9.83746 & 61.4842 \\
\hline 9.78246 & 61.1404 \\
\hline 9.72811 & 60.8007 \\
\hline 9.67442 & 60.4651 \\
\hline 9.62136 & 60.1335 \\
\hline 9.56893 & 59.8058 \\
\hline 9.51711 & 59.4819 \\
\hline 9.46589 & 59.1618 \\
\hline 9.41526 & 58.8453 \\
\hline 9.3652 & 58.5325 \\
\hline 9.31571 & 58.2232 \\
\hline 9.26677 & 57.9173 \\
\hline 9.21838 & 57.6148 \\
\hline 9.17052 & 57.3157 \\
\hline 9.12318 & 57.0199 \\
\hline 9.07635 & 56.7272 \\
\hline 9.03004 & 56.4377 \\
\hline 8.98421 & 56.1513 \\
\hline 8.93887 & 55.8679 \\
\hline 8.89401 & 55.5876 \\
\hline 8.84962 & 55.3101 \\
\hline 8.80568 & 55.0355 \\
\hline 8.7622 & 54.7638 \\
\hline 8.71916 & 54.4948 \\
\hline 8.67656 & 54.2285 \\
\hline 8.63439 & 53.9649 \\
\hline 8.59264 & 53.704 \\
\hline 8.5513 & 53.4456 \\
\hline 8.51037 & 53.1898 \\
\hline 8.46984 & 52.9365 \\
\hline 8.4297 & 52.6856 \\
\hline 8.38995 & 52.4372 \\
\hline
\end{tabular}

\begin{tabular}{|c|c|}
\hline 161.395 & 322.79 \\
\hline 160.121 & 320.242 \\
\hline 158.87 & 317.741 \\
\hline 157.643 & 315.285 \\
\hline 156.437 & 312.874 \\
\hline 155.253 & 310.506 \\
\hline 154.09 & 308.179 \\
\hline 152.947 & 305.893 \\
\hline 151.823 & 303.647 \\
\hline 150.719 & 301.438 \\
\hline 149.633 & 299.266 \\
\hline 148.566 & 297.131 \\
\hline 147.515 & 295.031 \\
\hline 146.482 & 292.964 \\
\hline 145.466 & 290.931 \\
\hline 144.465 & 288.93 \\
\hline 143.48 & 286.96 \\
\hline 142.511 & 285.021 \\
\hline 141.556 & 283.112 \\
\hline 140.616 & 281.232 \\
\hline 139.69 & 279.38 \\
\hline 138.778 & 277.555 \\
\hline 137.879 & 275.758 \\
\hline 136.993 & 273.986 \\
\hline 136.12 & 272.24 \\
\hline 135.26 & 270.519 \\
\hline 134.411 & 268.823 \\
\hline 133.575 & 267.15 \\
\hline 132.75 & 265.5 \\
\hline 131.936 & 263.873 \\
\hline 131.134 & 262.268 \\
\hline 130.342 & 260.684 \\
\hline 129.561 & 259.122 \\
\hline 128.79 & 257.58 \\
\hline 128.029 & 256.059 \\
\hline 127.278 & 254.557 \\
\hline 126.537 & 253.074 \\
\hline 125.805 & 251.611 \\
\hline 125.083 & 250.165 \\
\hline 124.369 & 248.738 \\
\hline 123.664 & 247.329 \\
\hline 122.968 & 245.937 \\
\hline 122.281 & 244.561 \\
\hline 121.601 & 243.203 \\
\hline 120.93 & 241.86 \\
\hline 120.267 & 240.534 \\
\hline 119.612 & 239.223 \\
\hline 118.964 & 237.928 \\
\hline 118.324 & 236.647 \\
\hline 117.691 & 235.381 \\
\hline 117.065 & 234.13 \\
\hline 116.446 & 232.893 \\
\hline 115.835 & 231.669 \\
\hline 115.23 & 230.459 \\
\hline 114.631 & 229.263 \\
\hline 114.04 & 228.079 \\
\hline 113.454 & 226.909 \\
\hline 112.875 & 225.751 \\
\hline 112.303 & 224.605 \\
\hline 111.736 & 223.472 \\
\hline 111.175 & 222.35 \\
\hline 110.62 & 221.24 \\
\hline 110.071 & 220.142 \\
\hline 109.528 & 219.055 \\
\hline 108.99 & 217.979 \\
\hline 108.457 & 216.914 \\
\hline 107.93 & 215.86 \\
\hline 107.408 & 214.816 \\
\hline 106.891 & 213.782 \\
\hline 106.38 & 212.759 \\
\hline 105.873 & 211.746 \\
\hline 105.371 & 210.742 \\
\hline 104.874 & 209.749 \\
\hline
\end{tabular}




\begin{tabular}{|c|c|c|}
\hline 157 & 0.0656 & 0.0579902 \\
\hline 158 & 0.0653 & 0.0577194 \\
\hline 159 & 0.0651 & 0.0574511 \\
\hline 160 & 0.0648 & 0.0571853 \\
\hline 161 & 0.0645 & 0.0569221 \\
\hline 162 & 0.0642 & 0.0566612 \\
\hline 163 & 0.0639 & 0.0564028 \\
\hline 164 & 0.0636 & 0.0561467 \\
\hline 165 & 0.0634 & 0.055893 \\
\hline 166 & 0.0631 & 0.0556415 \\
\hline 167 & 0.0628 & 0.0553923 \\
\hline 168 & 0.0626 & 0.0551453 \\
\hline 169 & 0.0623 & 0.0549004 \\
\hline 170 & 0.0621 & 0.0546577 \\
\hline 171 & 0.0618 & 0.0544172 \\
\hline 172 & 0.0615 & 0.0541787 \\
\hline 173 & 0.0613 & 0.0539422 \\
\hline 174 & 0.061 & 0.0537078 \\
\hline 175 & 0.0608 & 0.0534754 \\
\hline 176 & 0.0605 & 0.053245 \\
\hline 177 & 0.0603 & 0.0530164 \\
\hline 178 & 0.0601 & 0.0527898 \\
\hline 179 & 0.0598 & 0.0525651 \\
\hline 180 & 0.0596 & 0.0523422 \\
\hline 181 & 0.0594 & 0.0521211 \\
\hline 182 & 0.0591 & 0.0519018 \\
\hline 183 & 0.0589 & 0.0516844 \\
\hline 184 & 0.0587 & 0.0514686 \\
\hline 185 & 0.0584 & 0.0512546 \\
\hline 186 & 0.0582 & 0.0510423 \\
\hline 187 & 0.058 & 0.0508316 \\
\hline 188 & 0.0578 & 0.0506226 \\
\hline 189 & 0.0576 & 0.0504153 \\
\hline 190 & 0.0573 & 0.0502095 \\
\hline 191 & 0.0571 & 0.0500054 \\
\hline 192 & 0.0569 & 0.0498028 \\
\hline 193 & 0.0567 & 0.0496017 \\
\hline 194 & 0.0565 & 0.0494022 \\
\hline 195 & 0.0563 & 0.0492042 \\
\hline 196 & 0.0561 & 0.0490077 \\
\hline 197 & 0.0559 & 0.0488126 \\
\hline 198 & 0.0557 & 0.048619 \\
\hline 199 & 0.0555 & 0.0484268 \\
\hline 200 & 0.0553 & 0.048236 \\
\hline 201 & 0.0551 & 0.0480466 \\
\hline 202 & 0.0549 & 0.0478586 \\
\hline 203 & 0.0547 & 0.047672 \\
\hline 204 & 0.0545 & 0.0474867 \\
\hline 205 & 0.0543 & 0.0473027 \\
\hline 206 & 0.0541 & 0.04712 \\
\hline 207 & 0.0539 & 0.0469386 \\
\hline 208 & 0.0537 & 0.0467585 \\
\hline 209 & 0.0536 & 0.0465796 \\
\hline 210 & 0.0534 & 0.046402 \\
\hline 211 & 0.0532 & 0.0462256 \\
\hline 212 & 0.053 & 0.0460504 \\
\hline 213 & 0.0528 & 0.0458765 \\
\hline 214 & 0.0527 & 0.0457037 \\
\hline 215 & 0.0525 & 0.045532 \\
\hline 216 & 0.0523 & 0.0453616 \\
\hline 217 & 0.0521 & 0.0451923 \\
\hline 218 & 0.052 & 0.0450241 \\
\hline 219 & 0.0518 & 0.044857 \\
\hline 220 & 0.0516 & 0.044691 \\
\hline 221 & 0.0514 & 0.0445262 \\
\hline 222 & 0.0513 & 0.0443624 \\
\hline 223 & 0.0511 & 0.0441996 \\
\hline 224 & 0.0509 & 0.044038 \\
\hline 225 & 0.0508 & 0.0438773 \\
\hline 226 & 0.0506 & 0.0437177 \\
\hline 227 & 0.0504 & 0.0435591 \\
\hline 228 & 0.0503 & 0.0434015 \\
\hline 229 & 0.0501 & 0.043245 \\
\hline
\end{tabular}

\begin{tabular}{|c|c|c|c|}
\hline 2.02965 & 2.0876402 & 8.35058 & 52.1911 \\
\hline 2.02018 & 2.0778994 & 8.31158 & 51.9474 \\
\hline 2.01079 & 2.0682411 & 8.27295 & 51.7059 \\
\hline 2.00149 & 2.0586753 & 8.23468 & 51.4668 \\
\hline 1.99227 & 2.0491921 & 8.19677 & 51.2298 \\
\hline 1.98314 & 2.0398012 & 8.15921 & 50.9951 \\
\hline 1.9741 & 2.0305028 & 8.122 & 50.7625 \\
\hline 1.96513 & 2.0212767 & 8.08512 & 50.532 \\
\hline 1.95625 & 2.012143 & 8.04858 & 50.3036 \\
\hline 1.94745 & 2.0030915 & 8.01237 & 50.0773 \\
\hline 1.93873 & 1.9941223 & 7.97648 & 49.853 \\
\hline 1.93008 & 1.9852253 & 7.94091 & 49.6307 \\
\hline 1.92151 & 1.9764104 & 7.90566 & 49.4104 \\
\hline 1.91302 & 1.9676777 & 7.87071 & 49.1919 \\
\hline 1.9046 & 1.9590172 & 7.83607 & 48.9754 \\
\hline 1.89625 & 1.9504287 & 7.80173 & 48.7608 \\
\hline 1.88798 & 1.9419222 & 7.76768 & 48.548 \\
\hline 1.87977 & 1.9334778 & 7.73393 & 48.337 \\
\hline 1.87164 & 1.9251154 & 7.70046 & 48.1279 \\
\hline 1.86357 & 1.916815 & 7.66727 & 47.9205 \\
\hline 1.85557 & 1.9085864 & 7.63436 & 47.7148 \\
\hline 1.84764 & 1.9004298 & 7.60173 & 47.5108 \\
\hline 1.83978 & 1.8923451 & 7.56937 & 47.3086 \\
\hline 1.83198 & 1.8843222 & 7.53727 & 47.108 \\
\hline 1.82424 & 1.8763611 & 7.50544 & 46.909 \\
\hline 1.81656 & 1.8684618 & 7.47387 & 46.7117 \\
\hline 1.80895 & 1.8606344 & 7.44255 & 46.5159 \\
\hline 1.8014 & 1.8528686 & 7.41148 & 46.3217 \\
\hline 1.79391 & 1.8451646 & 7.38066 & 46.1291 \\
\hline 1.78648 & 1.8375223 & 7.35009 & 45.938 \\
\hline 1.77911 & 1.8299416 & 7.31975 & 45.7485 \\
\hline 1.77179 & 1.8224126 & 7.28966 & 45.5604 \\
\hline 1.76454 & 1.8149553 & 7.2598 & 45.3738 \\
\hline 1.75733 & 1.8075395 & 7.23017 & 45.1886 \\
\hline 1.75019 & 1.8001954 & 7.20078 & 45.0048 \\
\hline 1.7431 & 1.7929028 & 7.1716 & 44.8225 \\
\hline 1.73606 & 1.7856617 & 7.14265 & 44.6416 \\
\hline 1.72908 & 1.7784822 & 7.11392 & 44.462 \\
\hline 1.72215 & 1.7713542 & 7.08541 & 44.2838 \\
\hline 1.71527 & 1.7642777 & 7.05711 & 44.1069 \\
\hline 1.70844 & 1.7572526 & 7.02902 & 43.9314 \\
\hline 1.70166 & 1.750279 & 7.00114 & 43.7571 \\
\hline 1.69494 & 1.7433668 & 6.97346 & 43.5841 \\
\hline 1.68826 & 1.736496 & 6.94599 & 43.4124 \\
\hline 1.68163 & 1.7296766 & 6.91872 & 43.242 \\
\hline 1.67505 & 1.7229086 & 6.89164 & 43.0728 \\
\hline 1.66852 & 1.716192 & 6.86477 & 42.9048 \\
\hline 1.66203 & 1.7095167 & 6.83808 & 42.738 \\
\hline 1.65559 & 1.7028927 & 6.81158 & 42.5724 \\
\hline 1.6492 & 1.69632 & 6.78528 & 42.408 \\
\hline 1.64285 & 1.6897886 & 6.75916 & 42.2447 \\
\hline 1.63655 & 1.6833085 & 6.73322 & 42.0826 \\
\hline 1.63029 & 1.6768696 & 6.70746 & 41.9217 \\
\hline 1.62407 & 1.670472 & 6.68189 & 41.7618 \\
\hline 1.6179 & 1.6641256 & 6.65649 & 41.603 \\
\hline 1.61177 & 1.6578204 & 6.63126 & 41.4454 \\
\hline 1.60568 & 1.6515565 & 6.60621 & 41.2888 \\
\hline 1.59963 & 1.6453337 & 6.58133 & 41.1333 \\
\hline 1.59362 & 1.639152 & 6.55662 & 40.9788 \\
\hline 1.58766 & 1.6330216 & 6.53207 & 40.8254 \\
\hline 1.58173 & 1.6269223 & 6.50769 & 40.673 \\
\hline 1.57584 & 1.6208641 & 6.48347 & 40.5217 \\
\hline 1.57 & 1.614857 & 6.45941 & 40.3713 \\
\hline 1.56419 & 1.608881 & 6.43551 & 40.2219 \\
\hline 1.55842 & 1.6029462 & 6.41177 & 40.0735 \\
\hline 1.55268 & 1.5970424 & 6.38818 & 39.9261 \\
\hline 1.54699 & 1.5911896 & 6.36475 & 39.7797 \\
\hline 1.54133 & 1.585368 & 6.34146 & 39.6342 \\
\hline 1.53571 & 1.5795873 & 6.31833 & 39.4896 \\
\hline 1.53012 & 1.5738377 & 6.29535 & 39.3459 \\
\hline 1.52457 & 1.5681291 & 6.27251 & 39.2032 \\
\hline 1.51905 & 1.5624515 & 6.24982 & 39.0614 \\
\hline 1.51357 & 1.556815 & 6.22727 & 38.9205 \\
\hline
\end{tabular}

\begin{tabular}{|c|c|}
\hline 104.382 & 208.764 \\
\hline 103.895 & 207.789 \\
\hline 103.412 & 206.824 \\
\hline 102.934 & 205.867 \\
\hline 102.46 & 204.919 \\
\hline 101.99 & 203.98 \\
\hline 101.525 & 203.05 \\
\hline 101.064 & 202.128 \\
\hline 100.607 & 201.215 \\
\hline 100.155 & 200.309 \\
\hline 99.706 & 199.412 \\
\hline 99.2614 & 198.523 \\
\hline 98.8207 & 197.641 \\
\hline 98.3839 & 196.768 \\
\hline 97.9509 & 195.902 \\
\hline 97.5216 & 195.043 \\
\hline 97.096 & 194.192 \\
\hline 96.6741 & 193.348 \\
\hline 96.2557 & 192.511 \\
\hline 95.8409 & 191.682 \\
\hline 95.4296 & 190.859 \\
\hline 95.0216 & 190.043 \\
\hline 94.6171 & 189.234 \\
\hline 94.2159 & 188.432 \\
\hline 93.818 & 187.636 \\
\hline 93.4233 & 186.847 \\
\hline 93.0318 & 186.064 \\
\hline 92.6435 & 185.287 \\
\hline 92.2583 & 184.517 \\
\hline 91.8761 & 183.752 \\
\hline 91.4969 & 182.994 \\
\hline 91.1208 & 182.242 \\
\hline 90.7475 & 181.495 \\
\hline 90.3772 & 180.754 \\
\hline 90.0097 & 180.019 \\
\hline 89.645 & 179.29 \\
\hline 89.2831 & 178.566 \\
\hline 88.924 & 177.848 \\
\hline 88.5676 & 177.135 \\
\hline 88.2138 & 176.428 \\
\hline 87.8627 & 175.725 \\
\hline 87.5142 & 175.028 \\
\hline 87.1683 & 174.337 \\
\hline 86.8249 & 173.65 \\
\hline 86.484 & 172.968 \\
\hline 86.1455 & 172.291 \\
\hline 85.8096 & 171.619 \\
\hline 85.476 & 170.952 \\
\hline 85.1448 & 170.29 \\
\hline 84.816 & 169.632 \\
\hline 84.4895 & 168.979 \\
\hline 84.1653 & 168.331 \\
\hline 83.8433 & 167.687 \\
\hline 83.5236 & 167.047 \\
\hline 83.2061 & 166.412 \\
\hline 82.8908 & 165.782 \\
\hline 82.5776 & 165.155 \\
\hline 82.2666 & 164.533 \\
\hline 81.9577 & 163.915 \\
\hline 81.6509 & 163.302 \\
\hline 81.3461 & 162.692 \\
\hline 81.0433 & 162.087 \\
\hline 80.7426 & 161.485 \\
\hline 80.4439 & 160.888 \\
\hline 80.1471 & 160.294 \\
\hline 79.8523 & 159.705 \\
\hline 79.5593 & 159.119 \\
\hline 79.2683 & 158.537 \\
\hline 78.9792 & 157.958 \\
\hline 78.6919 & 157.384 \\
\hline 78.4064 & 156.813 \\
\hline 78.1228 & 156.246 \\
\hline 77.8409 & 155.682 \\
\hline
\end{tabular}




\begin{tabular}{|c|c|}
\hline 1.50813 & 1.5512194 \\
\hline 1.50272 & 1.5456547 \\
\hline 1.49734 & 1.5401211 \\
\hline 1.49199 & 1.5346184 \\
\hline 1.48668 & 1.5291566 \\
\hline 1.4814 & 1.5237258 \\
\hline 1.47616 & 1.5183359 \\
\hline 1.47094 & 1.5129669 \\
\hline 1.46576 & 1.5076388 \\
\hline 1.4606 & 1.5023316 \\
\hline 1.45548 & 1.4970653 \\
\hline 1.45039 & 1.4918298 \\
\hline 1.44533 & 1.4866252 \\
\hline 1.4403 & 1.4814515 \\
\hline 1.4353 & 1.4763086 \\
\hline 1.43033 & 1.4711966 \\
\hline 1.42539 & 1.4661154 \\
\hline 1.42048 & 1.461065 \\
\hline 1.41559 & 1.4560354 \\
\hline 1.41073 & 1.4510367 \\
\hline 1.4059 & 1.4460687 \\
\hline 1.4011 & 1.4411315 \\
\hline 1.39633 & 1.4362251 \\
\hline 1.39158 & 1.4313395 \\
\hline 1.38686 & 1.4264846 \\
\hline 1.38217 & 1.4216605 \\
\hline 1.3775 & 1.4168572 \\
\hline 1.37286 & 1.4120846 \\
\hline 1.36824 & 1.4073327 \\
\hline 1.36365 & 1.4026116 \\
\hline 1.35909 & 1.3979211 \\
\hline 1.35455 & 1.3932514 \\
\hline 1.35003 & 1.3886024 \\
\hline 1.34554 & 1.3839841 \\
\hline 1.34108 & 1.3793965 \\
\hline 1.33664 & 1.3748296 \\
\hline 1.33222 & 1.3702833 \\
\hline 1.32782 & 1.3657578 \\
\hline 1.32345 & 1.3612629 \\
\hline 1.3191 & 1.3567886 \\
\hline 1.31478 & 1.352345 \\
\hline 1.31047 & 1.3479121 \\
\hline 1.30619 & 1.3435098 \\
\hline 1.30194 & 1.3391382 \\
\hline 1.2977 & 1.3347772 \\
\hline 1.29349 & 1.3304468 \\
\hline 1.28929 & 1.326127 \\
\hline 1.28512 & 1.3218378 \\
\hline 1.28097 & 1.3175693 \\
\hline 1.27685 & 1.3133313 \\
\hline 1.27274 & 1.309104 \\
\hline 1.26865 & 1.3048972 \\
\hline 1.26459 & 1.3007211 \\
\hline 1.26054 & 1.2965555 \\
\hline 1.25652 & 1.2924205 \\
\hline 1.25251 & 1.288296 \\
\hline 1.24853 & 1.2842022 \\
\hline 1.24456 & 1.2801189 \\
\hline 1.24061 & 1.2760561 \\
\hline 1.23669 & 1.2720239 \\
\hline 1.23278 & 1.2680023 \\
\hline 1.22889 & 1.2640012 \\
\hline 1.22502 & 1.2600206 \\
\hline 1.22117 & 1.2560606 \\
\hline 1.21734 & 1.2521211 \\
\hline 1.21352 & 1.2481921 \\
\hline 1.20973 & 1.2442936 \\
\hline 1.20595 & 1.2404057 \\
\hline 1.20219 & 1.2365383 \\
\hline 1.19845 & 1.2326913 \\
\hline 1.19472 & $1.228854 \mathrm{~S}$ \\
\hline 1.19101 & 1.225039 \\
\hline 1.18732 & 1.2212436 \\
\hline
\end{tabular}

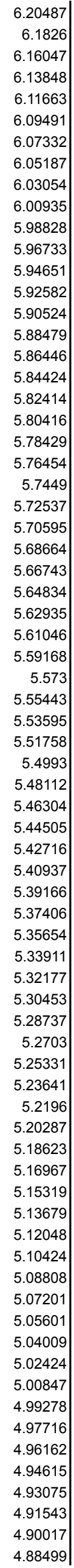

38.7804

38.6413

38.503

38.3655

38.2289

38.0932

37.9583

37.8242

37.6909

37.5584

37.4267

37.2958

37.1657

37.0364

36.9078

36.7799

36.6529

36.5265

36.4009

36.276

36.1518

36.0284

35.9056

35.7836

35.6622

35.5415

35.4215

35.3021

35.1834

35.0654

34.948

34.8313

34.7152

34.5997

34.4848

34.3706

34.257

34.144

34.0316

33.9198

33.8085

33.6979

33.5878

33.4784

33.3694

33.2611

33.1533

33.046

32.9394

32.8332

32.7276

32.6225

32.518

32.4139

32.3104

32.2074

32.105

32.003

31.9015

31.8005

31.7

31.6

31.5005

31.4015

31.303

31.2049

31.1073

31.0101

30.9134

30.8172

30.7214

30.6261

30.5312

\begin{tabular}{|c|c|}
\hline 77.5608 & 155.122 \\
\hline 77.2825 & 154.565 \\
\hline 77.0059 & 154.012 \\
\hline 76.7311 & 153.462 \\
\hline 76.4579 & 152.916 \\
\hline 76.1864 & 152.373 \\
\hline 75.9165 & 151.833 \\
\hline 75.6484 & 151.297 \\
\hline 75.3818 & 150.764 \\
\hline 75.1168 & 150.234 \\
\hline 74.8535 & 149.707 \\
\hline 74.5917 & 149.183 \\
\hline 74.3314 & 148.663 \\
\hline 74.0727 & 148.145 \\
\hline 73.8155 & 147.631 \\
\hline 73.5599 & 147.12 \\
\hline 73.3057 & 146.611 \\
\hline 73.053 & 146.106 \\
\hline 72.8018 & 145.604 \\
\hline 72.552 & 145.104 \\
\hline 72.3037 & 144.607 \\
\hline 72.0568 & 144.114 \\
\hline 71.8112 & 143.622 \\
\hline 71.5671 & 143.134 \\
\hline 71.3244 & 142.649 \\
\hline 71.083 & 142.166 \\
\hline 70.8429 & 141.686 \\
\hline 70.6042 & 141.208 \\
\hline 70.3669 & 140.734 \\
\hline 70.1308 & 140.262 \\
\hline 69.896 & 139.792 \\
\hline 69.6625 & 139.325 \\
\hline 69.4303 & 138.861 \\
\hline 69.1994 & 138.399 \\
\hline 68.9697 & 137.939 \\
\hline 68.7412 & 137.482 \\
\hline 68.514 & 137.028 \\
\hline 68.288 & 136.576 \\
\hline 68.0632 & 136.126 \\
\hline 67.8395 & 135.679 \\
\hline 67.6171 & 135.234 \\
\hline 67.3958 & 134.792 \\
\hline 67.1757 & 134.351 \\
\hline 66.9567 & 133.913 \\
\hline 66.7389 & 133.478 \\
\hline 66.5222 & 133.044 \\
\hline 66.3066 & 132.613 \\
\hline 66.0921 & 132.184 \\
\hline 65.8787 & 131.757 \\
\hline 65.6664 & 131.333 \\
\hline 65.4552 & 130.91 \\
\hline 65.245 & 130.49 \\
\hline 65.0359 & 130.072 \\
\hline 64.8279 & 129.656 \\
\hline 64.6209 & 129.242 \\
\hline 64.4149 & 128.83 \\
\hline 64.2099 & 128.42 \\
\hline 64.006 & 128.012 \\
\hline 63.803 & 127.606 \\
\hline 63.6011 & 127.202 \\
\hline 63.4001 & 126.8 \\
\hline 63.2001 & 126.4 \\
\hline 63.0011 & 126.002 \\
\hline 62.803 & 125.606 \\
\hline 62.6059 & 125.212 \\
\hline 62.4097 & 124.819 \\
\hline 62.2145 & 124.429 \\
\hline 62.0202 & 124.04 \\
\hline 61.8269 & 123.654 \\
\hline 61.6344 & 123.269 \\
\hline 61.4428 & 122.886 \\
\hline 61.2522 & 122.504 \\
\hline 61.0624 & 122.125 \\
\hline
\end{tabular}




\begin{tabular}{|c|c|c|}
\hline 303 & 0.0407 & 0.0338186 \\
\hline 304 & 0.0406 & 0.0337142 \\
\hline 305 & 0.0405 & 0.0336102 \\
\hline 306 & 0.0404 & 0.0335067 \\
\hline 307 & 0.0403 & 0.0334037 \\
\hline 308 & 0.0402 & 0.0333012 \\
\hline 309 & 0.04 & 0.0331991 \\
\hline 310 & 0.04 & 0.0330975 \\
\hline 311 & 0.0398 & 0.0329963 \\
\hline 312 & 0.0398 & 0.0328956 \\
\hline 313 & 0.0397 & 0.0327954 \\
\hline 314 & 0.0396 & 0.0326956 \\
\hline 315 & 0.0395 & 0.0325963 \\
\hline 316 & 0.0394 & 0.0324974 \\
\hline 317 & 0.0393 & 0.0323989 \\
\hline 318 & 0.0392 & 0.0323009 \\
\hline 319 & 0.0391 & 0.0322033 \\
\hline 320 & 0.039 & 0.0321061 \\
\hline 321 & 0.0389 & 0.0320094 \\
\hline 322 & 0.0388 & 0.031913 \\
\hline 323 & 0.0387 & 0.0318171 \\
\hline 324 & 0.0386 & 0.0317217 \\
\hline 325 & 0.0385 & 0.0316266 \\
\hline 326 & 0.0384 & 0.0315319 \\
\hline 327 & 0.0383 & 0.0314377 \\
\hline 328 & 0.0382 & 0.0313438 \\
\hline 329 & 0.0381 & 0.0312504 \\
\hline 330 & 0.038 & 0.0311573 \\
\hline 331 & 0.038 & 0.0310646 \\
\hline 332 & 0.0379 & 0.0309724 \\
\hline 333 & 0.0378 & 0.0308805 \\
\hline 334 & 0.0377 & 0.030789 \\
\hline 335 & 0.0376 & 0.0306979 \\
\hline 336 & 0.0375 & 0.0306071 \\
\hline 337 & 0.0374 & 0.0305168 \\
\hline 338 & 0.0373 & 0.0304268 \\
\hline 339 & 0.0372 & 0.0303372 \\
\hline 340 & 0.0372 & 0.0302479 \\
\hline 341 & 0.0371 & 0.0301591 \\
\hline 342 & 0.037 & 0.0300706 \\
\hline 343 & 0.0369 & 0.0299824 \\
\hline 344 & 0.0368 & 0.0298946 \\
\hline 345 & 0.0367 & 0.0298072 \\
\hline 346 & 0.0366 & 0.0297201 \\
\hline 347 & 0.0366 & 0.0296334 \\
\hline 348 & 0.0365 & 0.029547 \\
\hline 349 & 0.0364 & 0.029461 \\
\hline 350 & 0.0363 & 0.0293753 \\
\hline 351 & 0.0362 & 0.0292899 \\
\hline 352 & 0.0361 & 0.0292049 \\
\hline 353 & 0.0361 & 0.0291202 \\
\hline 354 & 0.036 & 0.0290359 \\
\hline 355 & 0.0359 & 0.0289519 \\
\hline 356 & 0.0358 & 0.0288682 \\
\hline 357 & 0.0357 & 0.0287849 \\
\hline 358 & 0.0357 & 0.0287018 \\
\hline 359 & 0.0356 & 0.0286191 \\
\hline 360 & 0.0355 & 0.0285367 \\
\hline 361 & 0.0354 & 0.0284547 \\
\hline 362 & 0.0353 & 0.0283729 \\
\hline 363 & 0.0353 & 0.0282915 \\
\hline 364 & 0.0352 & 0.0282104 \\
\hline 365 & 0.0351 & 0.0281296 \\
\hline 366 & 0.035 & 0.0280491 \\
\hline 367 & 0.035 & 0.0279689 \\
\hline 368 & 0.0349 & 0.027889 \\
\hline 369 & 0.0348 & 0.0278094 \\
\hline 370 & 0.0347 & 0.0277302 \\
\hline 371 & 0.0346 & 0.0276512 \\
\hline 372 & 0.0346 & 0.0275725 \\
\hline 373 & 0.0345 & 0.0274941 \\
\hline 374 & 0.0344 & 0.027416 \\
\hline 375 & 0.0343 & 0.0273382 \\
\hline
\end{tabular}

\begin{tabular}{|c|c|}
\hline 1.18365 & 1.2174686 \\
\hline 1.18 & 1.2137142 \\
\hline 1.17636 & 1.2099702 \\
\hline 1.17274 & 1.2062467 \\
\hline 1.16913 & 1.2025337 \\
\hline 1.16554 & 1.1988412 \\
\hline 1.16197 & 1.1951691 \\
\hline 1.15841 & 1.1915075 \\
\hline 1.15487 & 1.1878663 \\
\hline 1.15135 & 1.1842456 \\
\hline 1.14784 & 1.1806354 \\
\hline 1.14435 & 1.1770456 \\
\hline 1.14087 & 1.1734663 \\
\hline 1.13741 & 1.1699074 \\
\hline 1.13396 & 1.1663589 \\
\hline 1.13053 & 1.1628309 \\
\hline 1.12711 & 1.1593133 \\
\hline 1.12371 & 1.1558161 \\
\hline 1.12033 & 1.1523394 \\
\hline 1.11696 & 1.148873 \\
\hline 1.1136 & 1.1454171 \\
\hline 1.11026 & 1.1419817 \\
\hline 1.10693 & 1.1385566 \\
\hline 1.10362 & 1.1351519 \\
\hline 1.10032 & 1.1317577 \\
\hline 1.09703 & 1.1283738 \\
\hline 1.09376 & 1.1250104 \\
\hline 1.09051 & 1.1216673 \\
\hline 1.08726 & 1.1183246 \\
\hline 1.08403 & 1.1150024 \\
\hline 1.08082 & 1.1117005 \\
\hline 1.07761 & 1.108399 \\
\hline 1.07443 & 1.1051279 \\
\hline 1.07125 & 1.1018571 \\
\hline 1.06809 & 1.0986068 \\
\hline 1.06494 & 1.0953668 \\
\hline 1.0618 & 1.0921372 \\
\hline 1.05868 & 1.0889279 \\
\hline 1.05557 & 1.0857291 \\
\hline 1.05247 & 1.0825406 \\
\hline 1.04938 & 1.0793624 \\
\hline 1.04631 & 1.0762046 \\
\hline 1.04325 & 1.0730572 \\
\hline 1.0402 & 1.0699201 \\
\hline 1.03717 & 1.0668034 \\
\hline 1.03414 & 1.063687 \\
\hline 1.03113 & 1.060591 \\
\hline 1.02813 & 1.0575053 \\
\hline 1.02515 & 1.0544399 \\
\hline 1.02217 & 1.0513749 \\
\hline 1.01921 & 1.0483302 \\
\hline 1.01626 & 1.0452959 \\
\hline 1.01332 & 1.0422719 \\
\hline 1.01039 & 1.0392582 \\
\hline 1.00747 & 1.0362549 \\
\hline 1.00456 & 1.0332618 \\
\hline 1.00167 & 1.0302891 \\
\hline 0.998786 & 1.0273227 \\
\hline 0.995914 & 1.0243687 \\
\hline 0.993053 & 1.0214259 \\
\hline 0.990203 & 1.0184945 \\
\hline 0.987364 & 1.0155744 \\
\hline 0.984536 & 1.0126656 \\
\hline 0.981718 & 1.0097671 \\
\hline 0.978912 & 1.0068809 \\
\hline 0.976116 & 1.004005 \\
\hline 0.97333 & 1.0011394 \\
\hline 0.970555 & 0.9982852 \\
\hline 0.967791 & 0.9954422 \\
\hline 0.965037 & 0.9926095 \\
\hline 0.962293 & 0.9897871 \\
\hline 0.95956 & 0.986976 \\
\hline 0.956836 & 0.9841742 \\
\hline
\end{tabular}

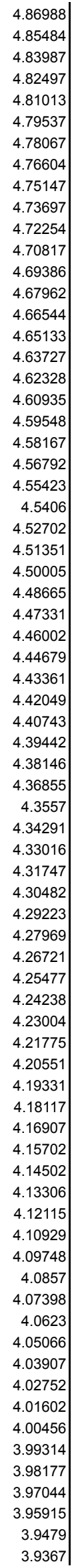

30.4368

30.3428

30.2492

30.1561

30.0633

29.9711

29.8792

29.7877

29.6967

29.6061

29.5159

29.4261

29.3366

29.2476

29.159

29.0708

28.983

28.8955

28.8084

28.7217

28.6354

28.5495

28.4639

28.3787

28.2939

28.2094

28.1253

28.0416

27.9582

27.8751

27.7924

27.7101

27.6281

27.5464

27.4651

27.3841

27.3035

27.2231

27.1432

27.0635

26.9842

26.9052

26.8265

26.7481

26.67

26.5923

26.5149

26.4377

26.3609

26.2844

26.2082

26.1323

26.0567

25.9814

25.9064

25.8316

25.7572

25.6831

25.6092

25.5357

25.4624

25.3894

25.3166

25.2442

25.172

25.1001

25.0285

24.9571

24.8861

24.8152

24.7447

24.6744

24.6044

\begin{tabular}{|c|c|}
\hline 60.8735 & 121.747 \\
\hline 60.6855 & 121.371 \\
\hline 60.4984 & 120.997 \\
\hline 60.3121 & 120.624 \\
\hline 60.1267 & 120.253 \\
\hline 59.9421 & 119.884 \\
\hline 59.7584 & 119.517 \\
\hline 59.5755 & 119.151 \\
\hline 59.3934 & 118.787 \\
\hline 59.2122 & 118.424 \\
\hline 59.0317 & 118.063 \\
\hline 58.8521 & 117.704 \\
\hline 58.6733 & 117.347 \\
\hline 58.4953 & 116.991 \\
\hline 58.318 & 116.636 \\
\hline 58.1416 & 116.283 \\
\hline 57.9659 & 115.932 \\
\hline 57.791 & 115.582 \\
\hline 57.6169 & 115.234 \\
\hline 57.4435 & 114.887 \\
\hline 57.2709 & 114.542 \\
\hline 57.099 & 114.198 \\
\hline 56.9279 & 113.856 \\
\hline 56.7575 & 113.515 \\
\hline 56.5878 & 113.176 \\
\hline 56.4189 & 112.838 \\
\hline 56.2506 & 112.501 \\
\hline 56.0831 & 112.166 \\
\hline 55.9163 & 111.833 \\
\hline 55.7503 & 111.501 \\
\hline 55.5849 & 111.17 \\
\hline 55.4202 & 110.84 \\
\hline 55.2562 & 110.512 \\
\hline 55.0928 & 110.186 \\
\hline 54.9302 & 109.86 \\
\hline 54.7682 & 109.536 \\
\hline 54.6069 & 109.214 \\
\hline 54.4463 & 108.893 \\
\hline 54.2863 & 108.573 \\
\hline 54.127 & 108.254 \\
\hline 53.9683 & 107.937 \\
\hline 53.8103 & 107.621 \\
\hline 53.6529 & 107.306 \\
\hline 53.4962 & 106.992 \\
\hline 53.3401 & 106.68 \\
\hline 53.1846 & 106.369 \\
\hline 53.0297 & 106.059 \\
\hline 52.8755 & 105.751 \\
\hline 52.7218 & 105.444 \\
\hline 52.5688 & 105.138 \\
\hline 52.4164 & 104.833 \\
\hline 52.2646 & 104.529 \\
\hline 52.1134 & 104.227 \\
\hline 51.9628 & 103.926 \\
\hline 51.8127 & 103.625 \\
\hline 51.6633 & 103.327 \\
\hline 51.5144 & 103.029 \\
\hline 51.3661 & 102.732 \\
\hline 51.2184 & 102.437 \\
\hline 51.0713 & 102.143 \\
\hline 50.9247 & 101.849 \\
\hline 50.7787 & 101.557 \\
\hline 50.6333 & 101.267 \\
\hline 50.4884 & 100.977 \\
\hline 50.344 & 100.688 \\
\hline 50.2002 & 100.4 \\
\hline 50.057 & 100.114 \\
\hline 49.9143 & 99.8286 \\
\hline 49.7721 & 99.5442 \\
\hline 49.6305 & 99.2609 \\
\hline 49.4894 & 98.9787 \\
\hline 49.3488 & 98.6976 \\
\hline 49.2087 & 98.4175 \\
\hline
\end{tabular}




\begin{tabular}{|c|c|}
\hline 376 & 0.0343 \\
\hline 377 & 0.0342 \\
\hline 378 & 0.0341 \\
\hline 379 & 0.034 \\
\hline 380 & 0.034 \\
\hline 381 & 0.0339 \\
\hline 382 & 0.0338 \\
\hline 383 & 0.0338 \\
\hline 384 & 0.0337 \\
\hline 385 & 0.0336 \\
\hline 386 & 0.0335 \\
\hline 387 & 0.0335 \\
\hline 388 & 0.0334 \\
\hline 389 & 0.0333 \\
\hline 390 & 0.0333 \\
\hline 391 & 0.0332 \\
\hline 392 & 0.0331 \\
\hline 393 & 0.033 \\
\hline 394 & 0.033 \\
\hline 395 & 0.0329 \\
\hline 396 & 0.0328 \\
\hline 397 & 0.0328 \\
\hline 398 & 0.0327 \\
\hline 399 & 0.0326 \\
\hline 400 & 0.0326 \\
\hline 401 & 0.0325 \\
\hline 402 & 0.0324 \\
\hline 403 & 0.0324 \\
\hline 404 & 0.0323 \\
\hline 405 & 0.0322 \\
\hline 406 & 0.0322 \\
\hline 407 & 0.0321 \\
\hline 408 & 0.032 \\
\hline 409 & 0.032 \\
\hline 410 & 0.0319 \\
\hline 411 & 0.0318 \\
\hline 412 & 0.0318 \\
\hline 413 & 0.0317 \\
\hline 414 & 0.0316 \\
\hline 415 & 0.0316 \\
\hline 416 & 0.0315 \\
\hline 417 & 0.0314 \\
\hline 418 & 0.0314 \\
\hline 419 & 0.0313 \\
\hline 420 & 0.0312 \\
\hline 421 & 0.0312 \\
\hline 422 & 0.0311 \\
\hline 423 & 0.0311 \\
\hline 424 & 0.031 \\
\hline 425 & 0.0309 \\
\hline 426 & 0.0309 \\
\hline 427 & 0.0308 \\
\hline 428 & 0.0307 \\
\hline 429 & 0.0307 \\
\hline 430 & 0.0306 \\
\hline 431 & 0.0306 \\
\hline 432 & 0.0305 \\
\hline 433 & 0.0304 \\
\hline 434 & 0.0304 \\
\hline 435 & 0.0303 \\
\hline 436 & 0.0303 \\
\hline 437 & 0.0302 \\
\hline 438 & 0.0301 \\
\hline 439 & 0.0301 \\
\hline 440 & 0.03 \\
\hline 441 & 0.03 \\
\hline 442 & 0.0299 \\
\hline 443 & 0.0298 \\
\hline 444 & 0.0298 \\
\hline 445 & 0.0297 \\
\hline 446 & 0.0297 \\
\hline 447 & 0.0296 \\
\hline 448 & 0.0296 \\
\hline
\end{tabular}

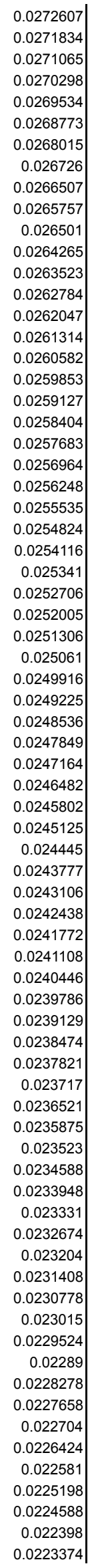

0.954123

0.95142

0.948727

0.946044

0.94337

0.940707

0.938053

0.935409

0.932774

0.930149

0.927534

0.924928

0.922331

0.919744

0.917166

0.914597

0.912038

0.909487

0.906946

0.904413

0.90189

0.899375

0.896869

0.894372

0.891884

0.889404

0.886934

0.884471

0.882017

0.879572

0.877135

0.874706

0.872286

0.869874

0.867471

0.865075

0.862688

0.860309

0.857937

0.855574

0.853219

0.850872

0.848532

0.846201

0.843877

0.841561

0.839253

0.836952

0.834659

0.832373

0.830095

0.827825

0.825562

0.823306

0.821058

0.818817

0.816584

0.814357

0.812138

0.809926

0.807722

0.805524

0.803333

0.80115

0.798973

0.796804

0.794641

0.792485

0.790336

0.788194

0.786059

0.78393

0.781809
0.9813837

0.9786034

0.9758335

0.9730738

0.9703234

0.9675843

0.9648545

0.962135

0.9594247

0.9567247

0.954035

0.9513545

0.9486833

0.9460224

0.9433707

0.9407284

0.9380962

0.9354723

0.9328587

0.9302534

0.9276583

0.9250714

0.9224938

0.9199255

0.9173664

0.9148156

0.912275

0.9097416

0.9072175

0.9047026

0.902196

0.8996976

0.8972085

0.8947276

0.8922559

0.8897914

0.8873362

0.8848892

0.8824495

0.880019

0.8775967

0.8751826

0.8727758

0.8703782

0.8679878

0.8656056

0.8632316

0.8608649

0.8585064

0.8561551

0.853812

0.8514771

0.8491495

0.846829

0.8445168

0.8422118

0.839915

0.8376244

0.835342

0.8330668

0.8307998

0.828539

0.8262854

0.82404

0.8218008

0.8195698

0.817345

0.8151274

0.812917

0.8107138

0.8085178

0.806328

0.8041464
3.92554

3.91441

3.90333

3.89229

3.88129

3.87034

3.85942

3.84854

3.8377

3.8269

3.81614

3.80542

3.79473

3.78409

3.77348

3.76291

3.75238

3.74189

3.73143

3.72101

3.71063

3.70029

3.68998

3.6797

3.66947

3.65926

3.6491

3.63897

3.62887

3.61881

3.60878

3.59879

3.58883

3.57891

3.56902

3.55917

3.54934

3.53956

3.5298

3.52008

3.51039

3.50073

3.4911

3.48151

3.47195

3.46242

3.45292

3.44346

3.43402

3.42462

3.41525

3.40591

3.3966

3.38732

3.37807

3.36885

3.35966

3.3505

3.34137

3.33227

3.3232

3.31416

3.30514

3.29616

3.2872

3.27828

3.26938

3.26051

3.25167

3.24286

3.23407

3.22531

3.21658

24.5346

24.4651

24.3958

24.3268

24.2581

24.1896

24.1214

24.0534

23.9856

23.9181

23.8509

23.7839

23.7171

23.6506

23.5843

23.5182

23.4524

23.3868

23.3215

23.2563

23.1915

23.1268

23.0624

22.9981

22.9342

22.8704

22.8069

22.7435

22.6804

22.6176

22.5549

22.4925

22.4302

22.3682

22.3064

22.2448

22.1834

22.1222

22.0612

22.0005

21.9399

21.8796

21.8194

21.7594

21.6997

21.6401

21.5808

21.5216

21.4627

21.4039

21.3453

21.2869

21.2287

21.1707

21.1129

21.0553

20.9979

20.9406

20.8836

20.8267

20.77

20.7135

20.6571

20.601

20.545

20.4892

20.4336

20.3782

20.3229 


\begin{tabular}{|c|c|c|}
\hline 449 & 0.0295 & 0.022277 \\
\hline 450 & 0.0294 & 0.0222167 \\
\hline 451 & 0.0294 & 0.0221567 \\
\hline 452 & 0.0293 & 0.0220968 \\
\hline 453 & 0.0293 & 0.0220371 \\
\hline 454 & 0.0292 & 0.0219776 \\
\hline 455 & 0.0292 & 0.0219183 \\
\hline 456 & 0.0291 & 0.0218592 \\
\hline 457 & 0.029 & 0.0218002 \\
\hline 458 & 0.029 & 0.0217415 \\
\hline 459 & 0.0289 & 0.0216829 \\
\hline 460 & 0.0289 & 0.0216245 \\
\hline 461 & 0.0288 & 0.0215663 \\
\hline 462 & 0.0288 & 0.0215083 \\
\hline 463 & 0.0287 & 0.0214504 \\
\hline 464 & 0.0287 & 0.0213927 \\
\hline 465 & 0.0286 & 0.0213352 \\
\hline 466 & 0.0285 & 0.0212779 \\
\hline 467 & 0.0285 & 0.0212207 \\
\hline 468 & 0.0284 & 0.0211638 \\
\hline 469 & 0.0284 & 0.021107 \\
\hline 470 & 0.0283 & 0.0210503 \\
\hline 471 & 0.0283 & 0.0209939 \\
\hline 472 & 0.0282 & 0.0209376 \\
\hline 473 & 0.0282 & 0.0208814 \\
\hline 474 & 0.0281 & 0.0208255 \\
\hline 475 & 0.0281 & 0.0207697 \\
\hline 476 & 0.028 & 0.0207141 \\
\hline 477 & 0.028 & 0.0206586 \\
\hline 478 & 0.0279 & 0.0206033 \\
\hline 479 & 0.0278 & 0.0205482 \\
\hline 480 & 0.0278 & 0.0204933 \\
\hline 481 & 0.0277 & 0.0204385 \\
\hline 482 & 0.0277 & 0.0203838 \\
\hline 483 & 0.0276 & 0.0203294 \\
\hline 484 & 0.0276 & 0.0202751 \\
\hline 485 & 0.0275 & 0.0202209 \\
\hline 486 & 0.0275 & 0.0201669 \\
\hline 487 & 0.0274 & 0.0201131 \\
\hline 488 & 0.0274 & 0.0200594 \\
\hline 489 & 0.0273 & 0.0200059 \\
\hline 490 & 0.0273 & 0.0199526 \\
\hline 491 & 0.0272 & 0.0198994 \\
\hline 492 & 0.0272 & 0.0198463 \\
\hline 493 & 0.0271 & 0.0197935 \\
\hline 494 & 0.0271 & 0.0197407 \\
\hline 495 & 0.027 & 0.0196882 \\
\hline 496 & 0.027 & 0.0196357 \\
\hline 497 & 0.0269 & 0.0195835 \\
\hline 498 & 0.0269 & 0.0195313 \\
\hline 499 & 0.0268 & 0.0194794 \\
\hline 500 & 0.0268 & 0.0194276 \\
\hline 501 & 0.0267 & 0.0193759 \\
\hline 502 & 0.0267 & 0.0193244 \\
\hline 503 & 0.0266 & 0.019273 \\
\hline 504 & 0.0266 & 0.0192218 \\
\hline 505 & 0.0265 & 0.0191707 \\
\hline 506 & 0.0265 & 0.0191198 \\
\hline 507 & 0.0264 & 0.019069 \\
\hline 508 & 0.0264 & 0.0190183 \\
\hline 509 & 0.0263 & 0.0189678 \\
\hline 510 & 0.0263 & 0.0189175 \\
\hline 511 & 0.0262 & 0.0188673 \\
\hline 512 & 0.0262 & 0.0188172 \\
\hline 513 & 0.0262 & 0.0187673 \\
\hline 514 & 0.0261 & 0.0187175 \\
\hline 515 & 0.0261 & 0.0186679 \\
\hline 516 & 0.026 & 0.0186184 \\
\hline 517 & 0.026 & 0.018569 \\
\hline 518 & 0.0259 & 0.0185198 \\
\hline 519 & 0.0259 & 0.0184707 \\
\hline 520 & 0.0258 & 0.0184218 \\
\hline 521 & 0.0258 & 0.018373 \\
\hline
\end{tabular}

\begin{tabular}{|c|c|}
\hline 0.779693 & 0.80197 \\
\hline 0.777585 & 0.7998017 \\
\hline 0.775483 & 0.7976397 \\
\hline 0.773388 & 0.7954848 \\
\hline 0.771299 & 0.7933361 \\
\hline 0.769217 & 0.7911946 \\
\hline 0.767141 & 0.7890593 \\
\hline 0.765072 & 0.7869312 \\
\hline 0.763009 & 0.7848092 \\
\hline 0.760952 & 0.7826935 \\
\hline 0.758902 & 0.7805849 \\
\hline 0.756858 & 0.7784825 \\
\hline 0.754821 & 0.7763873 \\
\hline 0.752789 & 0.7742973 \\
\hline 0.750764 & 0.7722144 \\
\hline 0.748745 & 0.7701377 \\
\hline 0.746733 & 0.7680682 \\
\hline 0.744726 & 0.7660039 \\
\hline 0.742726 & 0.7639467 \\
\hline 0.740732 & 0.7618958 \\
\hline 0.738743 & 0.75985 \\
\hline 0.736761 & 0.7578113 \\
\hline 0.734785 & 0.7557789 \\
\hline 0.732815 & 0.7537526 \\
\hline 0.73085 & 0.7517314 \\
\hline 0.728892 & 0.7497175 \\
\hline 0.726939 & 0.7477087 \\
\hline 0.724993 & 0.7457071 \\
\hline 0.723052 & 0.7437106 \\
\hline 0.721117 & 0.7417203 \\
\hline 0.719188 & 0.7397362 \\
\hline 0.717264 & 0.7377573 \\
\hline 0.715347 & 0.7357855 \\
\hline 0.713435 & 0.7338188 \\
\hline 0.711528 & 0.7318574 \\
\hline 0.709627 & 0.7299021 \\
\hline 0.707732 & 0.7279529 \\
\hline 0.705843 & 0.7260099 \\
\hline 0.703959 & 0.7240721 \\
\hline 0.702081 & 0.7221404 \\
\hline 0.700208 & 0.7202139 \\
\hline 0.69834 & 0.7182926 \\
\hline 0.696479 & 0.7163784 \\
\hline 0.694622 & 0.7144683 \\
\hline 0.692771 & 0.7125645 \\
\hline 0.690926 & 0.7106667 \\
\hline 0.689085 & 0.7087732 \\
\hline 0.687251 & 0.7068867 \\
\hline 0.685421 & 0.7050045 \\
\hline 0.683597 & 0.7031283 \\
\hline 0.681778 & 0.7012574 \\
\hline 0.679964 & 0.6993916 \\
\hline 0.678156 & 0.6975319 \\
\hline 0.676353 & 0.6956774 \\
\hline 0.674555 & 0.693828 \\
\hline 0.672762 & 0.6919838 \\
\hline 0.670974 & 0.6901447 \\
\hline 0.669192 & 0.6883118 \\
\hline 0.667414 & 0.686483 \\
\hline 0.665642 & 0.6846603 \\
\hline 0.663875 & 0.6828428 \\
\hline 0.662112 & 0.6810295 \\
\hline 0.660355 & 0.6792223 \\
\hline 0.658603 & 0.6774202 \\
\hline 0.656856 & 0.6756233 \\
\hline 0.655113 & 0.6738305 \\
\hline 0.653376 & 0.6720439 \\
\hline 0.651644 & 0.6702624 \\
\hline 0.649916 & 0.668485 \\
\hline 0.648193 & 0.6667128 \\
\hline 0.646476 & 0.6649467 \\
\hline 0.644763 & 0.6631848 \\
\hline 0.643055 & 0.661428 \\
\hline
\end{tabular}

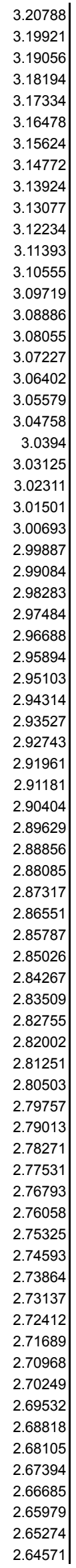

20.0493

19.995

19.941

19.8871

19.8334

19.7799

19.7265

19.6733

19.6202

19.5673

19.5146

19.4621

19.4097

19.3574

19.3054

19.2535

19.2017

19.1501

19.0987

19.0474

18.9963

18.9453

18.8945

18.8438

18.7933

18.7429

18.6927

18.6427

18.5928

18.543

18.4934

18.4439

18.3946

18.3455

18.2964

18.2476

18.1988

18.1502

18.1018

18.0535

18.0053

17.9573

17.9094

17.8617

17.8141

17.7667

17.7193

17.6722

17.6251

17.5782

17.5314

17.4848

17.4383

17.3919

17.3457

17.2996

17.2536

17.2078

17.1621

17.1165

17.0711

17.0257

16.9806

16.9355

16.8906

16.8458

16.8011

16.7565

16.7121

16.6678

16.6237

16.5796

16.5357

\begin{tabular}{|c|c|}
\hline 40.0985 & 80.197 \\
\hline 39.9901 & 79.9802 \\
\hline 39.882 & 79.764 \\
\hline 39.7742 & 79.5484 \\
\hline 39.6668 & 79.3336 \\
\hline 39.5597 & 79.1194 \\
\hline 39.453 & 78.9059 \\
\hline 39.3465 & 78.6931 \\
\hline 39.2404 & 78.4809 \\
\hline 39.1347 & 78.2694 \\
\hline 39.0292 & 78.0585 \\
\hline 38.9241 & 77.8483 \\
\hline 38.8193 & 77.6387 \\
\hline 38.7149 & 77.4298 \\
\hline 38.6107 & 77.2215 \\
\hline 38.5069 & 77.0138 \\
\hline 38.4034 & 76.8068 \\
\hline 38.3002 & 76.6004 \\
\hline 38.1973 & 76.3947 \\
\hline 38.0948 & 76.1895 \\
\hline 37.9925 & 75.985 \\
\hline 37.8906 & 75.7811 \\
\hline 37.7889 & 75.5779 \\
\hline 37.6876 & 75.3752 \\
\hline 37.5866 & 75.1732 \\
\hline 37.4859 & 74.9717 \\
\hline 37.3855 & 74.7709 \\
\hline 37.2853 & 74.5707 \\
\hline 37.1855 & 74.3711 \\
\hline 37.086 & 74.172 \\
\hline 36.9868 & 73.9736 \\
\hline 36.8879 & 73.7758 \\
\hline 36.7893 & 73.5785 \\
\hline 36.6909 & 73.3818 \\
\hline 36.5929 & 73.1858 \\
\hline 36.4951 & 72.9903 \\
\hline 36.3977 & 72.7953 \\
\hline 36.3005 & 72.601 \\
\hline 36.2036 & 72.4072 \\
\hline 36.107 & 72.214 \\
\hline 36.0107 & 72.0214 \\
\hline 35.9147 & 71.8293 \\
\hline 35.8189 & 71.6378 \\
\hline 35.7234 & 71.4469 \\
\hline 35.6282 & 71.2565 \\
\hline 35.5333 & 71.0666 \\
\hline 35.4387 & 70.8774 \\
\hline 35.3443 & 70.6886 \\
\hline 35.2502 & 70.5004 \\
\hline 35.1564 & 70.3128 \\
\hline 35.0629 & 70.1257 \\
\hline 34.9696 & 69.9392 \\
\hline 34.8766 & 69.7532 \\
\hline 34.7839 & 69.5677 \\
\hline 34.6914 & 69.3828 \\
\hline 34.5992 & 69.1984 \\
\hline 34.5072 & 69.0145 \\
\hline 34.4156 & 68.8311 \\
\hline 34.3242 & 68.6483 \\
\hline 34.233 & 68.466 \\
\hline 34.1421 & 68.2842 \\
\hline 34.0515 & 68.103 \\
\hline 33.9611 & 67.9222 \\
\hline 33.871 & 67.742 \\
\hline 33.7811 & 67.5623 \\
\hline 33.6915 & 67.3831 \\
\hline 33.6022 & 67.2044 \\
\hline 33.5131 & 67.0262 \\
\hline 33.4243 & 66.8485 \\
\hline 33.3357 & 66.6713 \\
\hline 33.2473 & 66.4946 \\
\hline 33.1592 & 66.3185 \\
\hline 33.0714 & 66.1428 \\
\hline
\end{tabular}




\begin{tabular}{|c|c|c|}
\hline 522 & 0.0257 & 0.0183243 \\
\hline 523 & 0.0257 & 0.0182758 \\
\hline 524 & 0.0256 & 0.0182274 \\
\hline 525 & 0.0256 & 0.0181791 \\
\hline 526 & 0.0255 & 0.018131 \\
\hline 527 & 0.0255 & 0.018083 \\
\hline 528 & 0.0255 & 0.0180352 \\
\hline 529 & 0.0254 & 0.0179875 \\
\hline 530 & 0.0254 & 0.0179399 \\
\hline 531 & 0.0253 & 0.0178924 \\
\hline 532 & 0.0253 & 0.0178451 \\
\hline 533 & 0.0252 & 0.0177979 \\
\hline 534 & 0.0252 & 0.0177508 \\
\hline 535 & 0.0251 & 0.0177039 \\
\hline 536 & 0.0251 & 0.0176571 \\
\hline 537 & 0.025 & 0.0176104 \\
\hline 538 & 0.025 & 0.0175639 \\
\hline 539 & 0.025 & 0.0175175 \\
\hline 540 & 0.0249 & 0.0174712 \\
\hline 541 & 0.0249 & 0.017425 \\
\hline 542 & 0.0248 & 0.017379 \\
\hline 543 & 0.0248 & 0.0173331 \\
\hline 544 & 0.0247 & 0.0172873 \\
\hline 545 & 0.0247 & 0.0172416 \\
\hline 546 & 0.0247 & 0.0171961 \\
\hline 547 & 0.0246 & 0.0171507 \\
\hline 548 & 0.0246 & 0.0171054 \\
\hline 549 & 0.0245 & 0.0170602 \\
\hline 550 & 0.0245 & 0.0170152 \\
\hline 551 & 0.0244 & 0.0169703 \\
\hline 552 & 0.0244 & 0.0169255 \\
\hline 553 & 0.0243 & 0.0168808 \\
\hline 554 & 0.0243 & 0.0168363 \\
\hline 555 & 0.0243 & 0.0167918 \\
\hline 556 & 0.0242 & 0.0167475 \\
\hline 557 & 0.0242 & 0.0167033 \\
\hline 558 & 0.0241 & 0.0166593 \\
\hline 559 & 0.0241 & 0.0166153 \\
\hline 560 & 0.0241 & 0.0165715 \\
\hline 561 & 0.024 & 0.0165278 \\
\hline 562 & 0.024 & 0.0164842 \\
\hline 563 & 0.0239 & 0.0164407 \\
\hline 564 & 0.0239 & 0.0163973 \\
\hline 565 & 0.0238 & 0.0163541 \\
\hline 566 & 0.0238 & 0.016311 \\
\hline 567 & 0.0238 & 0.016268 \\
\hline 568 & 0.0237 & 0.0162251 \\
\hline 569 & 0.0237 & 0.0161823 \\
\hline 570 & 0.0236 & 0.0161396 \\
\hline 571 & 0.0236 & 0.016097 \\
\hline 572 & 0.0236 & 0.0160546 \\
\hline 573 & 0.0235 & 0.0160123 \\
\hline 574 & 0.0235 & 0.0159701 \\
\hline 575 & 0.0234 & 0.015928 \\
\hline 576 & 0.0234 & 0.015886 \\
\hline 577 & 0.0234 & 0.0158441 \\
\hline 578 & 0.0233 & 0.0158023 \\
\hline 579 & 0.0233 & 0.0157607 \\
\hline 580 & 0.0232 & 0.0157191 \\
\hline 581 & 0.0232 & 0.0156777 \\
\hline 582 & 0.0232 & 0.0156363 \\
\hline 583 & 0.0231 & 0.0155951 \\
\hline 584 & 0.0231 & 0.015554 \\
\hline 585 & 0.023 & 0.015513 \\
\hline 586 & 0.023 & 0.0154721 \\
\hline 587 & 0.023 & 0.0154313 \\
\hline 588 & 0.0229 & 0.0153906 \\
\hline 589 & 0.0229 & 0.0153501 \\
\hline 590 & 0.0228 & 0.0153096 \\
\hline 591 & 0.0228 & 0.0152693 \\
\hline 592 & 0.0228 & 0.015229 \\
\hline 593 & 0.0227 & 0.0151889 \\
\hline 594 & 0.0227 & 0.0151488 \\
\hline
\end{tabular}

\begin{tabular}{|c|c|c|}
\hline 0.641351 & 0.6596753 & 2.6387 \\
\hline 0.639653 & 0.6579288 & 2.63171 \\
\hline 0.637959 & 0.6561864 & 2.62475 \\
\hline 0.63627 & 0.6544491 & 2.6178 \\
\hline 0.634586 & 0.652717 & 2.61087 \\
\hline 0.632906 & 0.650989 & 2.60396 \\
\hline 0.631231 & 0.6492662 & 2.59707 \\
\hline 0.629561 & 0.6475485 & 2.59019 \\
\hline 0.627895 & 0.6458349 & 2.58334 \\
\hline 0.626234 & 0.6441264 & 2.57651 \\
\hline 0.624578 & 0.6424231 & 2.56969 \\
\hline 0.622926 & 0.6407239 & 2.5629 \\
\hline 0.621279 & 0.6390298 & 2.55612 \\
\hline 0.619636 & 0.6373399 & 2.54936 \\
\hline 0.617998 & 0.6356551 & 2.54262 \\
\hline 0.616365 & 0.6339754 & 2.5359 \\
\hline 0.614736 & 0.6322999 & 2.5292 \\
\hline 0.613111 & 0.6306285 & 2.52251 \\
\hline 0.611491 & 0.6289622 & 2.51585 \\
\hline 0.609875 & 0.6273 & 2.5092 \\
\hline 0.608264 & 0.625643 & 2.50257 \\
\hline 0.606657 & 0.6239901 & 2.49596 \\
\hline 0.605055 & 0.6223423 & 2.48937 \\
\hline 0.603457 & 0.6206986 & 2.48279 \\
\hline 0.601863 & 0.6190591 & 2.47624 \\
\hline 0.600274 & 0.6174247 & 2.4697 \\
\hline 0.598689 & 0.6157944 & 2.46318 \\
\hline 0.597108 & 0.6141682 & 2.45667 \\
\hline 0.595532 & 0.6125472 & 2.45019 \\
\hline 0.59396 & 0.6109303 & 2.44372 \\
\hline 0.592392 & 0.6093175 & 2.43727 \\
\hline 0.590829 & 0.6077098 & 2.43084 \\
\hline 0.58927 & 0.6061063 & 2.42442 \\
\hline 0.587715 & 0.6045068 & 2.41803 \\
\hline 0.586164 & 0.6029115 & 2.41165 \\
\hline 0.584617 & 0.6013203 & 2.40528 \\
\hline 0.583075 & 0.5997343 & 2.39894 \\
\hline 0.581536 & 0.5981513 & 2.39261 \\
\hline 0.580002 & 0.5965735 & 2.3863 \\
\hline 0.578472 & 0.5949998 & 2.38 \\
\hline 0.576946 & 0.5934302 & 2.37372 \\
\hline 0.575425 & 0.5918657 & 2.36746 \\
\hline 0.573907 & 0.5903043 & 2.36122 \\
\hline 0.572393 & 0.5887471 & 2.35499 \\
\hline 0.570884 & 0.587195 & 2.34878 \\
\hline 0.569378 & 0.585646 & 2.34259 \\
\hline 0.567877 & 0.5841021 & 2.33641 \\
\hline 0.566379 & 0.5825613 & 2.33025 \\
\hline 0.564886 & 0.5810256 & 2.3241 \\
\hline 0.563397 & 0.579494 & 2.31797 \\
\hline 0.561911 & 0.5779656 & 2.31186 \\
\hline 0.56043 & 0.5764423 & 2.30577 \\
\hline 0.558952 & 0.5749221 & 2.29969 \\
\hline 0.557478 & 0.573406 & 2.29362 \\
\hline 0.556009 & 0.571895 & 2.28758 \\
\hline 0.554543 & 0.5703871 & 2.28155 \\
\hline 0.553081 & 0.5688833 & 2.27553 \\
\hline 0.551623 & 0.5673837 & 2.26953 \\
\hline 0.550169 & 0.5658881 & 2.26355 \\
\hline 0.548718 & 0.5643957 & 2.25758 \\
\hline 0.547272 & 0.5629083 & 2.25163 \\
\hline 0.545829 & 0.5614241 & 2.2457 \\
\hline 0.54439 & 0.559944 & 2.23978 \\
\hline 0.542955 & 0.558468 & 2.23387 \\
\hline 0.541524 & 0.5569961 & 2.22798 \\
\hline 0.540096 & 0.5555273 & 2.22211 \\
\hline 0.538673 & 0.5540636 & 2.21625 \\
\hline 0.537253 & 0.5526031 & 2.21041 \\
\hline 0.535836 & 0.5511456 & 2.20458 \\
\hline 0.534424 & 0.5496933 & 2.19877 \\
\hline 0.533015 & 0.548244 & 2.19298 \\
\hline 0.53161 & 0.5467989 & 2.1872 \\
\hline 0.530209 & 0.5453578 & 2.18143 \\
\hline
\end{tabular}

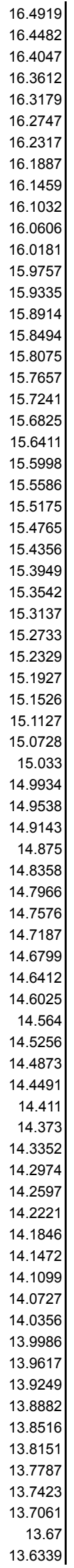

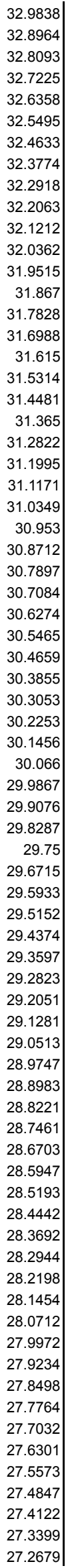

65.9676 65.7929 65.6186 65.4449 65.2717 65.0989 64.9266 64.7548 64.5835 64.4127 64.2423 64.0724 63.903 63.734 63.5655 63.3975 63.23 63.0629 62.8962 62.73 62.5643 62.399 62.2342 62.0699 61.9059 61.7425 61.5795 61.4169 61.2547 61.093 60.9318 60.771 60.6106 60.4506 60.2911 60.1321 59.9734 59.8152 59.6574 59.5 59.3431 59.1865 59.0304 58.8748 58.7195 58.5646 58.4102 58.2562 58.1026 57.9494 57.7966 57.6442 57.4922 57.3406 57.1895 57.0387 56.8883 56.7383 56.5888 56.4396 56.2908 56.1424 55.9944 55.8468 55.6996 55.5528 55.4063 55.2603 55.1146 54.9693 54.8244 54.6799 54.5357 


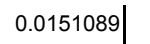

0.0150691

0.0150293
0.0149897

0.0149502

0.0149108

0.0148715

0.0148322

0.0147931

0.0147541

0.0147152

0.0146764

0.0146377

0.0145991

0.0145606

0.0145222

0.0144457

0.0144076

0.0143696

0.0143317

0.0142939

0.0142562

0.0142186

0.0141811

0.0141436
0.0141063

0.0140691

0.014032

0.0139949

0.013958

0.0139211

0.0138844

0.0138477

0.0138112

0.0137747

0.0137383

0.0137021

0.0136659

0.0136298

0.0135938

0.0135579

0.0135221

0.0134863

0.0134507
0.0134152
0.013397

0.0133797

0.0133444

0.0133091

0.0132739

0.0132388

0.0132038

0.0131689

0.0131341

0.0130993
0.0130647

0.0130301

0.0129957
0.0129613

0.0129613

0.012927
0.0128928

0.0128587

0.0128247

0.0127907

0.0127568

0.0127231

0.0126894

0.0126558

0.0126223

0.0125888

0.0125555

0.0125222

0.0124891

\begin{tabular}{|c|c|c|}
\hline 0.528811 & 0.5439199 & 2.17568 \\
\hline 0.527417 & 0.5424861 & 2.16994 \\
\hline 0.526026 & 0.5410553 & 2.16422 \\
\hline 0.52464 & 0.5396297 & 2.15852 \\
\hline 0.523256 & 0.5382062 & 2.15283 \\
\hline 0.521877 & 0.5367878 & 2.14715 \\
\hline 0.520501 & 0.5353725 & 2.14149 \\
\hline 0.519129 & 0.5339612 & 2.13584 \\
\hline 0.51776 & 0.5325531 & 2.13021 \\
\hline 0.516395 & 0.5311491 & 2.12459 \\
\hline 0.515033 & 0.5297482 & 2.11899 \\
\hline 0.513675 & 0.5283514 & 2.1134 \\
\hline 0.51232 & 0.5269577 & 2.10783 \\
\hline 0.510969 & 0.5255681 & 2.10227 \\
\hline 0.509622 & 0.5241826 & 2.09673 \\
\hline 0.508278 & 0.5228002 & 2.0912 \\
\hline 0.506937 & 0.5214209 & 2.08568 \\
\hline 0.5056 & 0.5200457 & 2.08018 \\
\hline 0.504266 & 0.5186736 & 2.0747 \\
\hline 0.502936 & 0.5173056 & 2.06922 \\
\hline 0.501609 & 0.5159407 & 2.06376 \\
\hline 0.500286 & 0.5145799 & 2.05832 \\
\hline 0.498966 & 0.5132222 & 2.05289 \\
\hline 0.49765 & 0.5118686 & 2.04747 \\
\hline 0.496337 & 0.5105181 & 2.04207 \\
\hline 0.495027 & 0.5091706 & 2.03668 \\
\hline 0.493721 & 0.5078273 & 2.03131 \\
\hline 0.492418 & 0.5064871 & 2.02595 \\
\hline 0.491119 & 0.505151 & 2.0206 \\
\hline 0.489822 & 0.5038169 & 2.01527 \\
\hline 0.488529 & 0.502487 & 2.00995 \\
\hline 0.48724 & 0.5011611 & 2.00464 \\
\hline 0.485954 & 0.4998384 & 1.99935 \\
\hline 0.484671 & 0.4985187 & 1.99407 \\
\hline 0.483391 & 0.4972022 & 1.98881 \\
\hline 0.482115 & 0.4958897 & 1.98356 \\
\hline 0.480842 & 0.4945803 & 1.97832 \\
\hline 0.479572 & 0.4932741 & 1.9731 \\
\hline 0.478306 & 0.4919719 & 1.96789 \\
\hline 0.477042 & 0.4906718 & 1.96269 \\
\hline 0.475782 & 0.4893758 & 1.95751 \\
\hline 0.474526 & 0.4880839 & 1.95233 \\
\hline 0.473272 & 0.4867941 & 1.94718 \\
\hline 0.472022 & 0.4855083 & 1.94203 \\
\hline 0.470775 & 0.4842257 & 1.9369 \\
\hline 0.469531 & 0.4829462 & 1.93178 \\
\hline 0.46829 & 0.4816697 & 1.92668 \\
\hline 0.467052 & 0.4803964 & 1.92159 \\
\hline 0.465818 & 0.4791271 & 1.91651 \\
\hline 0.464587 & 0.4778609 & 1.91144 \\
\hline 0.463359 & 0.4765978 & 1.90639 \\
\hline 0.462134 & 0.4753378 & 1.90135 \\
\hline 0.460912 & 0.4740809 & 1.89632 \\
\hline 0.459693 & 0.4728271 & 1.89131 \\
\hline 0.458477 & 0.4715763 & 1.88631 \\
\hline 0.457265 & 0.4703297 & 1.88132 \\
\hline 0.456055 & 0.4690851 & 1.87634 \\
\hline 0.454849 & 0.4678447 & 1.87138 \\
\hline 0.453646 & 0.4666073 & 1.86643 \\
\hline 0.452445 & 0.465372 & 1.86149 \\
\hline 0.451248 & 0.4641408 & 1.85656 \\
\hline 0.450054 & 0.4629127 & 1.85165 \\
\hline 0.448863 & 0.4616877 & 1.84675 \\
\hline 0.447675 & 0.4604657 & 1.84186 \\
\hline 0.44649 & 0.4592468 & 1.83699 \\
\hline 0.445308 & 0.4580311 & 1.83212 \\
\hline 0.444129 & 0.4568184 & 1.82727 \\
\hline 0.442953 & 0.4556088 & 1.82243 \\
\hline 0.44178 & 0.4544023 & 1.81761 \\
\hline 0.440609 & 0.4531978 & 1.81279 \\
\hline 0.439442 & 0.4519975 & 1.80799 \\
\hline 0.438278 & 0.4508002 & 1.8032 \\
\hline 0.437117 & 0.4496061 & 1.79842 \\
\hline
\end{tabular}

13.598

13.5621

13.5264

13.4907

13.4552

13.4197

13.3843

13.349

13.3138

13.2787

13.2437

13.2088

13.1739

13.1392

13.1046

13.07

13.0355

13.0011

12.9668

12.9326

12.8985

12.8645

12.8306

12.7967

12.7629

12.7293

12.6957

12.6622

12.6288

12.5954

12.5622

12.529

12.496

12.463

12.4301

12.3972

12.3645

12.3319

12.2993
12.2668

12.2668
12.2344

12.2344
12.2021

12.1699

12.1377

12.1056

12.0736

12.0417

12.0099

11.9782

11.9465

11.9149

11.8834

11.852

11.8207

11.7894

11.7582

11.7271

11.6961

11.6652

11.6343

11.6035

11.5728

11.5422

11.5116

11.4812

11.4508

11.4205

11.3902

11.36

11.33

11.2999

11.27

11.2402

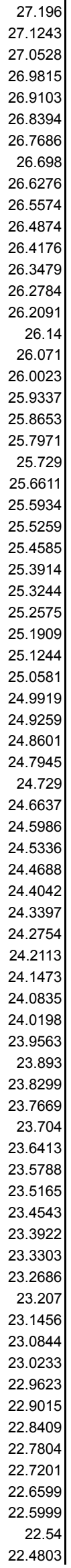

54.392

54.2486

54.1056

53.9629

53.8207

53.6788

53.5372

53.3961

53.2553

53.1149

52.9748

52.8351

52.6958

52.5568

52.4182

52.28

52.1421

52.0046

51.8674

51.7306

51.5941

51.458

51.3222

51.1868

51.0518

50.9171

50.7827

50.6487

50.515

50.3817

50.2487

50.1161

49.9838

49.8519

49.7202

49.589

49.458

49.3274

49.1972

49.0672

48.9376

48.8084

48.6794

48.5508

48.4225

48.2946

48.167

48.0397

47.9127

47.7861

47.6597

47.5337

47.4081

47.2827

47.1577

47.0329

46.9085

46.7845

46.6607

46.5372

46.4141

46.2913

46.1688

46.0465

45.9247

45.8031

45.6818

45.5608

45.4402

45.3198

45.1998

45.08

44.9606 
0.435959

0.434803

0.433651

0.432502

0.431355

0.430211

0.429071

0.427933

0.426798

0.425666

0.424536

0.42341

0.422286

0.421166

0.420048

0.418933

0.41782

0.416711

0.415604

0.4145

0.413399

0.412301

0.411206

0.410113

0.409023

0.407936

0.406851

0.405769

0.40469

0.403614

0.40254

0.40147

0.400401

0.399336

0.398273

0.397213

0.396155

0.395101

0.394048

0.392999

0.391952

0.390908

0.389866

0.388827

0.387791

0.386757

0.385726

0.384698

0.383672

0.382648

0.381627

0.380609

0.379594

0.37858

0.37757

0.376562

0.375556

0.374553

0.373553

0.372555

0.37156

0.370567

0.369576

0.368588

0.367603

0.36662

0.36564

0.364661

0.363686

0.362713

0.361742

0.360774

0.359808

0.448415
0.447226
0.446041
0.4448592
0.4436794
0.4425028
0.4413302
0.4401596
0.4389922
0.4378279
0.4366656
0.4355074
0.4343513
0.4331993
0.4320494
0.4309025

1.79366

1.78891

1.78416

1.77943

1.77472

1.77001

1.76532

1.76064

1.75597

1.75131

1.74666

1.74203

1.73741

1.7328

1.7282

1.72361

1.71903

1.71447

1.70991

1.70537

1.70084

1.69632

1.69182

1.68732

1.68284

1.67836

1.6739

1.66945

1.66501

1.66058

1.65617

1.65176

1.64737

1.64298

1.63861

1.63425

1.6299

1.62556

1.62123

1.61691

1.6126

1.60831

1.60402

1.59975

1.59548

1.59123

1.58699

1.58276

1.57853

1.57432

1.57012

1.56593

1.56176

1.55759

1.55343

1.54928

1.54515

1.54102

1.5369

1.5328

1.5287

1.52462

1.52054

1.51648

1.51242

1.50838

1.50435

1.50032

1.49631

1.4923

1.48831

1.48433

1.48035
11.2104

11.1807

11.151

11.1215

11.092

11.0626

11.0332

11.004

10.9748

10.9457

10.9166

10.8877

10.8588

10.83

10.8012

10.7726

10.744

10.7154

10.687

10.6586

10.6303

10.602

10.5739

10.5458

10.5177

10.4898

10.4619

10.4341

10.4063

10.3786

10.351

10.3235

10.296

10.2686

10.2413

10.214

10.1869

10.1597

10.1327

10.1057

10.0788

10.0519

10.0251

9.99842

9.97177

9.94519

9.91867

9.89222

9.86584

9.83953

9.81328

9.78709

9.76098

9.73492

9.70894

9.68302

9.65716

9.63137

9.60565

9.57999

9.55439

9.52886

9.50339

9.47799

9.45265

9.42737

9.40216

9.37701

9.35192

9.3269

9.30194

9.27705

9.25221

22.4207

22.3613

22.3021

22.2429

22.184

22.1252

22.0665

22.008

21.9496

21.8914

21.8333

21.7754

21.7176

21.6599

21.6025

21.5451

21.4879

21.4308

21.3739

21.3172

21.2605

21.2041

21.1477

21.0915

21.0355

20.9795

20.9238

20.8681

20.8126

20.7573

20.7021

20.647

20.5921

20.5373

20.4826

20.4281

20.3737

20.3195

20.2654

20.2114

20.1575

20.1038

20.0503

19.9968

19.9435

19.8904

19.8373

19.7844

19.7317

19.6791

19.6266

19.5742

19.522

19.4698

19.4179

19.366

19.3143

19.2627

19.2113

19.16

19.1088

19.0577

19.0068

18.956

18.9053

18.8547

18.8043

18.754 
0.358845

0.357884

0.356925

0.355969

0.355016

0.354064

0.353116

0.352169

0.351225

0.350283

0.349344

0.348407

0.347472

0.34654

0.34561

0.344682

0.343757

0.342834

0.341913

0.340995

0.340079

0.339165

0.338254

0.337345

0.336438

0.335534

0.334631

0.333732

0.332834

0.331938

0.331045

0.330154

0.329266

0.328379

0.327495

0.326613

0.325733

0.324856

0.323981

0.323108

0.322237

0.321368

0.320502

0.319637

0.318775

0.317915

0.317057

0.316202

0.315348

0.314497

0.313648

0.312801

0.311956

0.311113

0.310273

0.309434

0.308598

0.307764

0.306932

0.306102

0.305274

0.304448

0.303625

0.302803

0.301983

0.301166

0.300351

0.299537

0.298726

0.297917

0.29711

0.296305

0.295502
0.3690977

0.3681093

0.3671229

0.3661396

0.3651593

0.3641801

0.363205

0.362231

0.36126

0.3602911

0.35932525

0.35836148

0.35739977

0.35644114

0.35548457

0.35453006

0.35357863

0.35262926

0.35168195

0.35073772

0.34979555

0.34885544

0.3479184

0.34698343

0.34605052

0.34512068

0.3441919

0.34326719

0.34234354

0.34142196

0.34050344

0.33958698

0.33867359

0.33776127

0.336852

0.3359448

0.33503967

0.3341376

0.33323759

0.33233964

0.33144376

0.33054994

0.32965919

0.32876949

0.32788286

0.32699829

0.32611578

0.32523634

0.32435795

0.32348263

0.32260937

0.32173817

0.32086903

0.32000196

0.31913794

0.31827499

0.31741509

0.31655726

0.31570148

0.31484777

0.31399612

0.31314652

0.31229999

0.31145451

0.3106111

0.30977074

0.30893245

0.30809521

0.30726103

0.30642891

0.30559885

0.30477085

0.30394491
1.47639

1.47244

1.46849

1.46456

1.46064

1.45672

1.45282

1.44892

1.44504

1.44116

1.4373

1.43344

1.4296

1.42576

1.42194

1.41812

1.41431

1.41052

1.40673

1.40295

1.39918

1.39542

1.39167

1.38793

1.3842

1.38048

1.37677

1.37307

1.36937

1.36569

1.36201

1.35835

1.35469

1.35105

1.34741

1.34378

1.34016

1.33655

1.33295

1.32936

1.32577

1.3222

1.31863

1.31508

1.31153

1.30799

1.30446

1.30094

1.29743

1.29393

1.29044

1.28695

1.28348

1.28001

1.27655

1.2731

1.26966

1.26623

1.26281

1.25939

1.25598

1.25259

1.2492

1.24582

1.24245

1.23908

1.23573

1.23238

1.22904

1.22572

1.22239

1.21908

1.21578

9.22744

9.20273

9.17808

9.1535

9.12897

9.10451

9.08011

9.05577

9.0315

9.00728

8.98313

8.95903

8.935

8.91102

8.88711

8.86326

8.83947

8.81573

8.79206

8.76845

8.74489

8.7214

8.69796

8.67459

8.65127

8.62801

8.60481

8.58167

8.55858

8.53556

8.51259

8.48968

8.46683

8.44404

8.4213

8.39862

8.376

8.35344

8.33093

8.30848

8.28609

8.26375

8.24147

8.21924

8.19707

8.17496

8.1529

8.1309

8.10896

8.08707

8.06523

8.04345

8.02173

8.00006

7.97845

7.95689

7.93538

7.91393

7.89253

7.87119

7.8499

7.82867

7.80749

7.78636

7.76529

7.74427

7.7233

7.70239

7.68153 


\begin{tabular}{|c|c|c|c|c|c|c|c|c|}
\hline 814 & 0.0158 & 0.00842002 & 0.294701 & 0.30312102 & 1.21248 & 7.57802 & 15.156 & 30.3121 \\
\hline 815 & 0.0158 & 0.0083972 & 0.293902 & 0.3022992 & 1.2092 & 7.55748 & 15.115 & 30.2299 \\
\hline 816 & 0.0158 & 0.00837443 & 0.293105 & 0.30147943 & 1.20592 & 7.53698 & 15.074 & 30.1479 \\
\hline 817 & 0.0158 & 0.00835171 & 0.29231 & 0.30066171 & 1.20265 & 7.51654 & 15.0331 & 30.0662 \\
\hline 818 & 0.0157 & 0.00832906 & 0.291517 & 0.29984606 & 1.19938 & 7.49615 & 14.9923 & 29.9846 \\
\hline 819 & 0.0157 & 0.00830646 & 0.290726 & 0.29903246 & 1.19613 & 7.47582 & 14.9516 & 29.9033 \\
\hline 820 & 0.0157 & 0.00828393 & 0.289937 & 0.29822093 & 1.19289 & 7.45553 & 14.9111 & 29.8221 \\
\hline 821 & 0.0157 & 0.00826144 & 0.289151 & 0.29741244 & 1.18965 & 7.4353 & 14.8706 & 29.7412 \\
\hline 822 & 0.0156 & 0.00823902 & 0.288366 & 0.29660502 & 1.18642 & 7.41512 & 14.8302 & 29.6605 \\
\hline 823 & 0.0156 & 0.00821665 & 0.287583 & 0.29579965 & 1.1832 & 7.39499 & 14.79 & 29.58 \\
\hline 824 & 0.0156 & 0.00819434 & 0.286802 & 0.29499634 & 1.17999 & 7.37491 & 14.7498 & 29.4996 \\
\hline 825 & 0.0156 & 0.00817209 & 0.286023 & 0.29419509 & 1.17678 & 7.35488 & 14.7098 & 29.4195 \\
\hline 826 & 0.0156 & 0.00814989 & 0.285246 & 0.29339589 & 1.17358 & 7.3349 & 14.6698 & 29.3396 \\
\hline 827 & 0.0155 & 0.00812775 & 0.284471 & 0.29259875 & 1.1704 & 7.31498 & 14.63 & 29.2599 \\
\hline 828 & 0.0155 & 0.00810567 & 0.283698 & 0.29180367 & 1.16722 & 7.2951 & 14.5902 & 29.1804 \\
\hline 829 & 0.0155 & 0.00808364 & 0.282927 & 0.29101064 & 1.16404 & 7.27528 & 14.5506 & 29.1011 \\
\hline 830 & 0.0155 & 0.00806167 & 0.282158 & 0.29021967 & 1.16088 & 7.2555 & 14.511 & 29.022 \\
\hline 831 & 0.0154 & 0.00803976 & 0.281391 & 0.28943076 & 1.15772 & 7.23578 & 14.4716 & 28.9431 \\
\hline 832 & 0.0154 & 0.0080179 & 0.280626 & 0.2886439 & 1.15458 & 7.21611 & 14.4322 & 28.8644 \\
\hline 833 & 0.0154 & 0.00799609 & 0.279863 & 0.28785909 & 1.15144 & 7.19648 & 14.393 & 28.7859 \\
\hline 834 & 0.0154 & 0.00797434 & 0.279102 & 0.28707634 & 1.14831 & 7.17691 & 14.3538 & 28.7076 \\
\hline 835 & 0.0153 & 0.00795265 & 0.278343 & 0.28629565 & 1.14518 & 7.15739 & 14.3148 & 28.6295 \\
\hline 836 & 0.0153 & 0.00793101 & 0.277585 & 0.28551601 & 1.14207 & 7.13791 & 14.2758 & 28.5517 \\
\hline 837 & 0.0153 & 0.00790943 & 0.27683 & 0.28473943 & 1.13896 & 7.11849 & 14.237 & 28.474 \\
\hline 838 & 0.0153 & 0.00788791 & 0.276077 & 0.28396491 & 1.13586 & 7.09911 & 14.1982 & 28.3965 \\
\hline 839 & 0.0152 & 0.00786643 & 0.275325 & 0.28319143 & 1.13277 & 7.07979 & 14.1596 & 28.3192 \\
\hline 840 & 0.0152 & 0.00784502 & 0.274576 & 0.28242102 & 1.12968 & 7.06051 & 14.121 & 28.2421 \\
\hline 841 & 0.0152 & 0.00782365 & 0.273828 & 0.28165165 & 1.12661 & 7.04129 & 14.0826 & 28.1652 \\
\hline 842 & 0.0152 & 0.00780235 & 0.273082 & 0.28088435 & 1.12354 & 7.02211 & 14.0442 & 28.0884 \\
\hline 843 & 0.0151 & 0.00778109 & 0.272338 & 0.28011909 & 1.12048 & 7.00298 & 14.006 & 28.0119 \\
\hline 844 & 0.0151 & 0.00775989 & 0.271596 & 0.27935589 & 1.11742 & 6.9839 & 13.9678 & 27.9356 \\
\hline 845 & 0.0151 & 0.00773875 & 0.270856 & 0.27859475 & 1.11438 & 6.96487 & 13.9297 & 27.8595 \\
\hline 846 & 0.0151 & 0.00771766 & 0.270118 & 0.27783566 & 1.11134 & 6.94589 & 13.8918 & 27.7836 \\
\hline 847 & 0.015 & 0.00769662 & 0.269382 & 0.27707862 & 1.10831 & 6.92696 & 13.8539 & 27.7078 \\
\hline 848 & 0.015 & 0.00767564 & 0.268647 & 0.27632264 & 1.10529 & 6.90807 & 13.8161 & 27.6323 \\
\hline 849 & 0.015 & 0.00765471 & 0.267915 & 0.27556971 & 1.10228 & 6.88924 & 13.7785 & 27.557 \\
\hline 850 & 0.015 & 0.00763383 & 0.267184 & 0.27481783 & 1.09927 & 6.87045 & 13.7409 & 27.4818 \\
\hline 851 & 0.0149 & 0.00761301 & 0.266455 & 0.27406801 & 1.09627 & 6.85171 & 13.7034 & 27.4068 \\
\hline 852 & 0.0149 & 0.00759224 & 0.265729 & 0.27332124 & 1.09328 & 6.83302 & 13.666 & 27.3321 \\
\hline 853 & 0.0149 & 0.00757153 & 0.265003 & 0.27257453 & 1.0903 & 6.81438 & 13.6288 & 27.2575 \\
\hline 854 & 0.0149 & 0.00755087 & 0.26428 & 0.27183087 & 1.08732 & 6.79578 & 13.5916 & 27.1831 \\
\hline 855 & 0.0148 & 0.00753026 & 0.263559 & 0.27108926 & 1.08436 & 6.77723 & 13.5545 & 27.1089 \\
\hline 856 & 0.0148 & 0.0075097 & 0.262839 & 0.2703487 & 1.0814 & 6.75873 & 13.5175 & 27.0349 \\
\hline 857 & 0.0148 & 0.0074892 & 0.262122 & 0.2696112 & 1.07844 & 6.74028 & 13.4806 & 26.9611 \\
\hline 858 & 0.0148 & 0.00746874 & 0.261406 & 0.26887474 & 1.0755 & 6.72187 & 13.4437 & 26.8875 \\
\hline 859 & 0.0148 & 0.00744835 & 0.260692 & 0.26814035 & 1.07256 & 6.70351 & 13.407 & 26.814 \\
\hline 860 & 0.0147 & 0.007428 & 0.25998 & 0.267408 & 1.06963 & 6.6852 & 13.3704 & 26.7408 \\
\hline 861 & 0.0147 & 0.00740771 & 0.25927 & 0.26667771 & 1.06671 & 6.66694 & 13.3339 & 26.6677 \\
\hline 862 & 0.0147 & 0.00738746 & 0.258561 & 0.26594846 & 1.06379 & 6.64872 & 13.2974 & 26.5949 \\
\hline 863 & 0.0147 & 0.00736727 & 0.257855 & 0.26522227 & 1.06089 & 6.63055 & 13.2611 & 26.5222 \\
\hline 864 & 0.0146 & 0.00734714 & 0.25715 & 0.26449714 & 1.05799 & 6.61242 & 13.2248 & 26.4497 \\
\hline 865 & 0.0146 & 0.00732705 & 0.256447 & 0.26377405 & 1.0551 & 6.59435 & 13.1887 & 26.3774 \\
\hline 866 & 0.0146 & 0.00730702 & 0.255746 & 0.26305302 & 1.05221 & 6.57631 & 13.1526 & 26.3053 \\
\hline 867 & 0.0146 & 0.00728703 & 0.255046 & 0.26233303 & 1.04933 & 6.55833 & 13.1167 & 26.2333 \\
\hline 868 & 0.0145 & 0.0072671 & 0.254349 & 0.2616161 & 1.04646 & 6.54039 & 13.0808 & 26.1616 \\
\hline 869 & 0.0145 & 0.00724722 & 0.253653 & 0.26090022 & 1.0436 & 6.5225 & 13.045 & 26.09 \\
\hline 870 & 0.0145 & 0.00722739 & 0.252959 & 0.26018639 & 1.04074 & 6.50465 & 13.0093 & 26.0186 \\
\hline 871 & 0.0145 & 0.00720761 & 0.252266 & 0.25947361 & 1.0379 & 6.48685 & 12.9737 & 25.9474 \\
\hline 872 & 0.0145 & 0.00718789 & 0.251576 & 0.25876389 & 1.03506 & 6.4691 & 12.9382 & 25.8764 \\
\hline 873 & 0.0144 & 0.00716821 & 0.250887 & 0.25805521 & 1.03222 & 6.45139 & 12.9028 & 25.8056 \\
\hline 874 & 0.0144 & 0.00714859 & 0.2502 & 0.25734859 & 1.0294 & 6.43373 & 12.8675 & 25.7349 \\
\hline 875 & 0.0144 & 0.00712901 & 0.249515 & 0.25664401 & 1.02658 & 6.41611 & 12.8322 & 25.6644 \\
\hline 876 & 0.0144 & 0.00710949 & 0.248832 & 0.25594149 & 1.02377 & 6.39854 & 12.7971 & 25.5942 \\
\hline 877 & 0.0143 & 0.00709001 & 0.24815 & 0.25524001 & 1.02096 & 6.38101 & 12.762 & 25.524 \\
\hline 878 & 0.0143 & 0.00707059 & 0.247471 & 0.25454159 & 1.01816 & 6.36353 & 12.7271 & 25.4541 \\
\hline 879 & 0.0143 & 0.00705122 & 0.246793 & 0.25384422 & 1.01537 & 6.34609 & 12.6922 & 25.3844 \\
\hline 880 & 0.0143 & 0.00703189 & 0.246116 & 0.25314789 & 1.01259 & 6.3287 & 12.6574 & 25.3148 \\
\hline 881 & 0.0142 & 0.00701262 & 0.245442 & 0.25245462 & 1.00982 & 6.31136 & 12.6227 & 25.2454 \\
\hline 882 & 0.0142 & 0.00699339 & 0.244769 & 0.25176239 & 1.00705 & 6.29405 & 12.5881 & 25.1762 \\
\hline 883 & 0.0142 & 0.00697422 & 0.244098 & 0.25107222 & 1.00429 & 6.2768 & 12.5536 & 25.1072 \\
\hline 884 & 0.0142 & 0.0069551 & 0.243428 & 0.2503831 & 1.00153 & 6.25959 & 12.5192 & 25.0383 \\
\hline 885 & 0.0142 & 0.00693602 & 0.242761 & 0.24969702 & 0.998787 & 6.24242 & 12.4848 & 24.9697 \\
\hline 886 & 0.0141 & 0.00691699 & 0.242095 & 0.24901199 & 0.996047 & 6.2253 & 12.4506 & 24.9012 \\
\hline
\end{tabular}




\begin{tabular}{|c|c|c|c|c|c|c|c|c|}
\hline 887 & 0.0141 & 0.00689802 & 0.241431 & 0.24832902 & 0.993315 & 6.20822 & 12.4164 & 24.8329 \\
\hline 888 & 0.0141 & 0.00687909 & 0.240768 & 0.24764709 & 0.990589 & 6.19118 & 12.3824 & 24.7647 \\
\hline 889 & 0.0141 & 0.00686021 & 0.240107 & 0.24696721 & 0.987871 & 6.17419 & 12.3484 & 24.6968 \\
\hline 890 & 0.014 & 0.00684138 & 0.239448 & 0.24628938 & 0.985159 & 6.15724 & 12.3145 & 24.629 \\
\hline 891 & 0.014 & 0.0068226 & 0.238791 & 0.2456136 & 0.982455 & 6.14034 & 12.2807 & 24.5614 \\
\hline 892 & 0.014 & 0.00680387 & 0.238135 & 0.24493887 & 0.979757 & 6.12348 & 12.247 & 24.4939 \\
\hline 893 & 0.014 & 0.00678519 & 0.237482 & 0.24426719 & 0.977067 & 6.10667 & 12.2133 & 24.4267 \\
\hline 894 & 0.014 & 0.00676655 & 0.236829 & 0.24359555 & 0.974383 & 6.0899 & 12.1798 & 24.3596 \\
\hline 895 & 0.0139 & 0.00674796 & 0.236179 & 0.24292696 & 0.971707 & 6.07317 & 12.1463 & 24.2927 \\
\hline 896 & 0.0139 & 0.00672943 & 0.23553 & 0.24225943 & 0.969037 & 6.05648 & 12.113 & 24.2259 \\
\hline 897 & 0.0139 & 0.00671094 & 0.234883 & 0.24159394 & 0.966375 & 6.03984 & 12.0797 & 24.1594 \\
\hline 898 & 0.0139 & 0.00669249 & 0.234237 & 0.24092949 & 0.963719 & 6.02324 & 12.0465 & 24.093 \\
\hline 899 & 0.0138 & 0.0066741 & 0.233593 & 0.2402671 & 0.96107 & 6.00669 & 12.0134 & 24.0268 \\
\hline 900 & 0.0138 & 0.00665575 & 0.232951 & 0.23960675 & 0.958428 & 5.99018 & 11.9804 & 23.9607 \\
\hline 901 & 0.0138 & 0.00663745 & 0.232311 & 0.23894845 & 0.955793 & 5.97371 & 11.9474 & 23.8948 \\
\hline 902 & 0.0138 & 0.0066192 & 0.231672 & 0.2382912 & 0.953165 & 5.95728 & 11.9146 & 23.8291 \\
\hline 903 & 0.0138 & 0.006601 & 0.231035 & 0.237636 & 0.950544 & 5.9409 & 11.8818 & 23.7636 \\
\hline 904 & 0.0137 & 0.00658284 & 0.230399 & 0.23698184 & 0.947929 & 5.92456 & 11.8491 & 23.6982 \\
\hline 905 & 0.0137 & 0.00656473 & 0.229766 & 0.23633073 & 0.945321 & 5.90826 & 11.8165 & 23.633 \\
\hline 906 & 0.0137 & 0.00654667 & 0.229133 & 0.23567967 & 0.94272 & 5.892 & 11.784 & 23.568 \\
\hline 907 & 0.0137 & 0.00652865 & 0.228503 & 0.23503165 & 0.940126 & 5.87579 & 11.7516 & 23.5032 \\
\hline 908 & 0.0136 & 0.00651069 & 0.227874 & 0.23438469 & 0.937539 & 5.85962 & 11.7192 & 23.4385 \\
\hline 909 & 0.0136 & 0.00649276 & 0.227247 & 0.23373976 & 0.934958 & 5.84349 & 11.687 & 23.374 \\
\hline 910 & 0.0136 & 0.00647489 & 0.226621 & 0.23309589 & 0.932384 & 5.8274 & 11.6548 & 23.3096 \\
\hline 911 & 0.0136 & 0.00645706 & 0.225997 & 0.23245406 & 0.929817 & 5.81136 & 11.6227 & 23.2454 \\
\hline 912 & 0.0136 & 0.00643928 & 0.225375 & 0.23181428 & 0.927256 & 5.79535 & 11.5907 & 23.1814 \\
\hline 913 & 0.0135 & 0.00642154 & 0.224754 & 0.23117554 & 0.924702 & 5.77939 & 11.5588 & 23.1176 \\
\hline 914 & 0.0135 & 0.00640386 & 0.224135 & 0.23053886 & 0.922155 & 5.76347 & 11.5269 & 23.0539 \\
\hline 915 & 0.0135 & 0.00638621 & 0.223517 & 0.22990321 & 0.919615 & 5.74759 & 11.4952 & 22.9904 \\
\hline 916 & 0.0135 & 0.00636862 & 0.222902 & 0.22927062 & 0.917081 & 5.73175 & 11.4635 & 22.927 \\
\hline 917 & 0.0135 & 0.00635106 & 0.222287 & 0.22863806 & 0.914553 & 5.71596 & 11.4319 & 22.8638 \\
\hline 918 & 0.0134 & 0.00633356 & 0.221675 & 0.22800856 & 0.912033 & 5.7002 & 11.4004 & 22.8008 \\
\hline 919 & 0.0134 & 0.0063161 & 0.221063 & 0.2273791 & 0.909518 & 5.68449 & 11.369 & 22.738 \\
\hline 920 & 0.0134 & 0.00629869 & 0.220454 & 0.22675269 & 0.907011 & 5.66882 & 11.3376 & 22.6753 \\
\hline 921 & 0.0134 & 0.00628132 & 0.219846 & 0.22612732 & 0.90451 & 5.65319 & 11.3064 & 22.6127 \\
\hline 922 & 0.0133 & 0.00626399 & 0.21924 & 0.22550399 & 0.902015 & 5.6376 & 11.2752 & 22.5504 \\
\hline 923 & 0.0133 & 0.00624672 & 0.218635 & 0.22488172 & 0.899527 & 5.62205 & 11.2441 & 22.4882 \\
\hline 924 & 0.0133 & 0.00622949 & 0.218032 & 0.22426149 & 0.897046 & 5.60654 & 11.2131 & 22.4261 \\
\hline 925 & 0.0133 & 0.0062123 & 0.21743 & 0.2236423 & 0.894571 & 5.59107 & 11.1821 & 22.3643 \\
\hline 926 & 0.0133 & 0.00619516 & 0.21683 & 0.22302516 & 0.892102 & 5.57564 & 11.1513 & 22.3026 \\
\hline 927 & 0.0132 & 0.00617806 & 0.216232 & 0.22241006 & 0.88964 & 5.56025 & 11.1205 & 22.241 \\
\hline 928 & 0.0132 & 0.00616101 & 0.215635 & 0.22179601 & 0.887185 & 5.54491 & 11.0898 & 22.1796 \\
\hline 929 & 0.0132 & 0.006144 & 0.21504 & 0.221184 & 0.884736 & 5.5296 & 11.0592 & 22.1184 \\
\hline 930 & 0.0132 & 0.00612704 & 0.214446 & 0.22057304 & 0.882293 & 5.51433 & 11.0287 & 22.0573 \\
\hline 931 & 0.0132 & 0.00611012 & 0.213854 & 0.21996412 & 0.879857 & 5.49911 & 10.9982 & 21.9964 \\
\hline 932 & 0.0131 & 0.00609324 & 0.213263 & 0.21935624 & 0.877427 & 5.48392 & 10.9678 & 21.9357 \\
\hline 933 & 0.0131 & 0.00607641 & 0.212674 & 0.21875041 & 0.875003 & 5.46877 & 10.9375 & 21.8751 \\
\hline 934 & 0.0131 & 0.00605963 & 0.212087 & 0.21814663 & 0.872586 & 5.45366 & 10.9073 & 21.8147 \\
\hline 935 & 0.0131 & 0.00604289 & 0.211501 & 0.21754389 & 0.870175 & 5.4386 & 10.8772 & 21.7544 \\
\hline 936 & 0.0131 & 0.00602619 & 0.210917 & 0.21694319 & 0.867771 & 5.42357 & 10.8471 & 21.6943 \\
\hline 937 & 0.013 & 0.00600953 & 0.210334 & 0.21634353 & 0.865373 & 5.40858 & 10.8172 & 21.6343 \\
\hline 938 & 0.013 & 0.00599292 & 0.209752 & 0.21574492 & 0.862981 & 5.39363 & 10.7873 & 21.5745 \\
\hline 939 & 0.013 & 0.00597636 & 0.209172 & 0.21514836 & 0.860595 & 5.37872 & 10.7574 & 21.5149 \\
\hline 940 & 0.013 & 0.00595983 & 0.208594 & 0.21455383 & 0.858216 & 5.36385 & 10.7277 & 21.4554 \\
\hline 941 & 0.0129 & 0.00594335 & 0.208017 & 0.21396035 & 0.855843 & 5.34902 & 10.698 & 21.3961 \\
\hline 942 & 0.0129 & 0.00592692 & 0.207442 & 0.21336892 & 0.853476 & 5.33423 & 10.6685 & 21.3369 \\
\hline 943 & 0.0129 & 0.00591053 & 0.206868 & 0.21277853 & 0.851116 & 5.31947 & 10.6389 & 21.2779 \\
\hline 944 & 0.0129 & 0.00589418 & 0.206296 & 0.21219018 & 0.848761 & 5.30476 & 10.6095 & 21.219 \\
\hline 945 & 0.0129 & 0.00587787 & 0.205725 & 0.21160287 & 0.846413 & 5.29008 & 10.5802 & 21.1603 \\
\hline 946 & 0.0128 & 0.00586161 & 0.205156 & 0.21101761 & 0.844071 & 5.27545 & 10.5509 & 21.1018 \\
\hline 947 & 0.0128 & 0.00584539 & 0.204589 & 0.21043439 & 0.841736 & 5.26085 & 10.5217 & 21.0434 \\
\hline 948 & 0.0128 & 0.00582921 & 0.204022 & 0.20985121 & 0.839406 & 5.24629 & 10.4926 & 20.9852 \\
\hline 949 & 0.0128 & 0.00581307 & 0.203458 & 0.20927107 & 0.837083 & 5.23177 & 10.4635 & 20.9271 \\
\hline 950 & 0.0128 & 0.00579698 & 0.202894 & 0.20869098 & 0.834765 & 5.21728 & 10.4346 & 20.8691 \\
\hline 951 & 0.0127 & 0.00578093 & 0.202333 & 0.20811393 & 0.832454 & 5.20284 & 10.4057 & 20.8114 \\
\hline 952 & 0.0127 & 0.00576492 & 0.201772 & 0.20753692 & 0.830149 & 5.18843 & 10.3769 & 20.7537 \\
\hline 953 & 0.0127 & 0.00574896 & 0.201214 & 0.20696296 & 0.82785 & 5.17406 & 10.3481 & 20.6962 \\
\hline 954 & 0.0127 & 0.00573303 & 0.200656 & 0.20638903 & 0.825557 & 5.15973 & 10.3195 & 20.6389 \\
\hline 955 & 0.0127 & 0.00571715 & 0.2001 & 0.20581715 & 0.82327 & 5.14544 & 10.2909 & 20.5818 \\
\hline 956 & 0.0126 & 0.00570131 & 0.199546 & 0.20524731 & 0.820989 & 5.13118 & 10.2624 & 20.5247 \\
\hline 957 & 0.0126 & 0.00568552 & 0.198993 & 0.20467852 & 0.818714 & 5.11697 & 10.2339 & 20.4679 \\
\hline 958 & 0.0126 & 0.00566976 & 0.198442 & 0.20411176 & 0.816446 & 5.10279 & 10.2056 & 20.4111 \\
\hline 959 & 0.0126 & 0.00565405 & 0.197892 & 0.20354605 & 0.814183 & 5.08864 & 10.1773 & 20.3546 \\
\hline
\end{tabular}


0.197343

0.196796

0.19625

0.195706

0.195164

0.194622

0.194082

0.193544

0.193007

0.192471

0.191937

0.191404

0.190873

0.190343

0.189815

0.189288

0.188762

0.188238

0.187715

0.187193

0.186673

0.186154

0.185637

0.185121

0.184607

0.184093

0.183581

0.183071

0.182562

0.182054

0.181548

0.181043

0.180539

0.180037

0.179536

0.179036

0.178538

0.178041

0.177545

0.177051

0.176558

0.176066

0.175576

0.175087

0.174599

0.174113

0.173627

0.173144

0.172661

0.17218

0.1717

0.171221

0.170744

0.170268

0.169793

0.16932

0.168847

0.168377

0.167907

0.167438

0.166971

0.166505

0.166041

0.165577

0.165115

0.164654

0.164195

0.163736

0.163279

0.162823

0.162369

0.161915

0.161463
0.20298138

0.20241874

0.20185716

0.20129761

0.2007401

0.20018263

0.19962721

0.19907383

0.19852148

0.19797018

0.19742092

0.1968727

0.19632652

0.19578138

0.19523828

0.19469622

0.1941552

0.19361622

0.19307828

0.19254138

0.19200652

0.1914727

0.19094092

0.19041018

0.18988147

0.18935281

0.18882619

0.1883016

0.18777805

0.18725555

0.18673508

0.18621565

0.18569726

0.18518091

0.18466559

0.18415131

0.18363908

0.18312788

0.18261772

0.18210959

0.18160251

0.18109646

0.18059245

0.18008948

0.17958754

0.17908764

0.17858778

0.17809096

0.17759417

0.17709942

0.17660571

0.17611304

0.1756224

0.1751328

0.17464423

0.1741577

0.17367121

0.17318776

0.17270434

0.17222195

0.17174161

0.1712623

0.17078502

0.17030778

0.16983258

0.16935841

0.16888628

0.16841418

0.16794412

0.1674751

0.16700811

0.16654115

0.16607623
0.811926

0.809675

0.80743

0.805191

0.802958

0.800731

0.79851

0.796295

0.794086

0.791882

0.789684

0.787493

0.785307

0.783126

0.780952

0.778783

0.776621

0.774464

0.772312

0.770167

0.768027

0.765893

0.763764

0.761641

0.759524

0.757413

0.755307

0.753206

0.751112

0.749023

0.746939

0.744861

0.742789

0.740722

0.738661

0.736605

0.734555

0.73251

0.730471

0.728437

0.726409

0.724386

0.722369

0.720357

0.71835

0.716349

0.714353

0.712362

0.710377

0.708397

0.706423

0.704453

0.70249

0.700531

0.698578

0.696629

0.694687

0.692749

0.690817

0.688889

0.686968

0.685051

0.683139

0.681233

0.679331

0.677435

0.675544

0.673658

0.671778

0.669902

0.668031

0.666166

0.664305

5.07454

5.06047

5.04644

5.03245

5.01849

5.00457

4.99069

4.97684

4.96304

4.94926

4.93553

4.92183

4.90817

4.89454

4.88095

4.8674

4.85388

4.8404

4.82695

4.81354

4.80017

4.78683

4.77353

4.76026

4.74703

4.73383

4.72067

4.70754

4.69445

4.68139

4.66837

4.65538

4.64243

4.62951

4.61663

4.60378

4.59097

4.57819

4.56544

4.55273

4.54006

4.52741

4.5148

4.50223

4.48969

4.47718

4.4647

4.45226

4.43986

4.42748

4.41514

4.40283

4.39056

4.37832

4.36611

4.35393

4.34179

4.32968

4.3176

4.30556

4.29355

4.28157

4.26962

4.2577

4.24582

4.23397

4.22215

4.21036

4.19861 


\begin{tabular}{|c|c|c|c|c|c|c|c|c|}
\hline 1033 & 0.0112 & 0.00460034 & 0.161012 & 0.16561234 & 0.66245 & 4.14031 & 8.28062 & 16.5612 \\
\hline 1034 & 0.0111 & 0.00458749 & 0.160562 & 0.16514949 & 0.660599 & 4.12874 & 8.25749 & 16.515 \\
\hline 1035 & 0.0111 & 0.00457468 & 0.160114 & 0.16468868 & 0.658754 & 4.11721 & 8.23442 & 16.4688 \\
\hline 1036 & 0.0111 & 0.0045619 & 0.159666 & 0.1642279 & 0.656913 & 4.10571 & 8.21142 & 16.4228 \\
\hline 1037 & 0.0111 & 0.00454915 & 0.15922 & 0.16376915 & 0.655078 & 4.09424 & 8.18847 & 16.3769 \\
\hline 1038 & 0.0111 & 0.00453644 & 0.158775 & 0.16331144 & 0.653247 & 4.0828 & 8.16559 & 16.3312 \\
\hline 1039 & 0.011 & 0.00452376 & 0.158332 & 0.16285576 & 0.651422 & 4.07139 & 8.14277 & 16.2855 \\
\hline 1040 & 0.011 & 0.00451112 & 0.157889 & 0.16240012 & 0.649601 & 4.06001 & 8.12002 & 16.24 \\
\hline 1041 & 0.011 & 0.00449851 & 0.157448 & 0.16194651 & 0.647786 & 4.04866 & 8.09732 & 16.1946 \\
\hline 1042 & 0.011 & 0.00448594 & 0.157008 & 0.16149394 & 0.645975 & 4.03734 & 8.07469 & 16.1494 \\
\hline 1043 & 0.011 & 0.0044734 & 0.156569 & 0.1610424 & 0.644169 & 4.02606 & 8.05211 & 16.1042 \\
\hline 1044 & 0.011 & 0.00446089 & 0.156131 & 0.16059189 & 0.642368 & 4.0148 & 8.0296 & 16.0592 \\
\hline 1045 & 0.0109 & 0.00444842 & 0.155695 & 0.16014342 & 0.640572 & 4.00358 & 8.00715 & 16.0143 \\
\hline 1046 & 0.0109 & 0.00443598 & 0.155259 & 0.15969498 & 0.638781 & 3.99238 & 7.98477 & 15.9695 \\
\hline 1047 & 0.0109 & 0.00442358 & 0.154825 & 0.15924858 & 0.636995 & 3.98122 & 7.96244 & 15.9249 \\
\hline 1048 & 0.0109 & 0.00441121 & 0.154392 & 0.15880321 & 0.635214 & 3.97009 & 7.94017 & 15.8803 \\
\hline 1049 & 0.0109 & 0.00439887 & 0.15396 & 0.15835887 & 0.633437 & 3.95898 & 7.91797 & 15.8359 \\
\hline 1050 & 0.0108 & 0.00438657 & 0.15353 & 0.15791657 & 0.631666 & 3.94791 & 7.89582 & 15.7916 \\
\hline 1051 & 0.0108 & 0.0043743 & 0.1531 & 0.1574743 & 0.629899 & 3.93687 & 7.87373 & 15.7475 \\
\hline 1052 & 0.0108 & 0.00436206 & 0.152672 & 0.15703406 & 0.628137 & 3.92585 & 7.85171 & 15.7034 \\
\hline 1053 & 0.0108 & 0.00434986 & 0.152245 & 0.15659486 & 0.62638 & 3.91487 & 7.82974 & 15.6595 \\
\hline 1054 & 0.0108 & 0.00433769 & 0.151819 & 0.15615669 & 0.624627 & 3.90392 & 7.80784 & 15.6157 \\
\hline 1055 & 0.0108 & 0.00432555 & 0.151394 & 0.15571955 & 0.622879 & 3.893 & 7.78599 & 15.572 \\
\hline 1056 & 0.0107 & 0.00431345 & 0.150971 & 0.15528445 & 0.621137 & 3.8821 & 7.76421 & 15.5284 \\
\hline 1057 & 0.0107 & 0.00430138 & 0.150548 & 0.15484938 & 0.619398 & 3.87124 & 7.74248 & 15.485 \\
\hline 1058 & 0.0107 & 0.00428934 & 0.150127 & 0.15441634 & 0.617665 & 3.86041 & 7.72081 & 15.4416 \\
\hline 1059 & 0.0107 & 0.00427734 & 0.149707 & 0.15398434 & 0.615936 & 3.8496 & 7.69921 & 15.3984 \\
\hline 1060 & 0.0107 & 0.00426536 & 0.149288 & 0.15355336 & 0.614213 & 3.83883 & 7.67766 & 15.3553 \\
\hline 1061 & 0.0107 & 0.00425343 & 0.14887 & 0.15312343 & 0.612493 & 3.82808 & 7.65617 & 15.3123 \\
\hline 1062 & 0.0106 & 0.00424152 & 0.148453 & 0.15269452 & 0.610779 & 3.81737 & 7.63474 & 15.2695 \\
\hline 1063 & 0.0106 & 0.00422965 & 0.148038 & 0.15226765 & 0.609069 & 3.80668 & 7.61336 & 15.2267 \\
\hline 1064 & 0.0106 & 0.0042178 & 0.147623 & 0.1518408 & 0.607364 & 3.79602 & 7.59205 & 15.1841 \\
\hline 1065 & 0.0106 & 0.004206 & 0.14721 & 0.151416 & 0.605663 & 3.7854 & 7.57079 & 15.1416 \\
\hline 1066 & 0.0106 & 0.00419422 & 0.146798 & 0.15099222 & 0.603967 & 3.7748 & 7.54959 & 15.0992 \\
\hline 1067 & 0.0105 & 0.00418247 & 0.146387 & 0.15056947 & 0.602276 & 3.76423 & 7.52845 & 15.0569 \\
\hline 1068 & 0.0105 & 0.00417076 & 0.145977 & 0.15014776 & 0.60059 & 3.75369 & 7.50737 & 15.0147 \\
\hline 1069 & 0.0105 & 0.00415908 & 0.145568 & 0.14972708 & 0.598908 & 3.74317 & 7.48635 & 14.9727 \\
\hline 1070 & 0.0105 & 0.00414743 & 0.14516 & 0.14930743 & 0.59723 & 3.73269 & 7.46538 & 14.9308 \\
\hline 1071 & 0.0105 & 0.00413582 & 0.144754 & 0.14888982 & 0.595558 & 3.72223 & 7.44447 & 14.8889 \\
\hline 1072 & 0.0105 & 0.00412423 & 0.144348 & 0.14847223 & 0.593889 & 3.71181 & 7.42362 & 14.8472 \\
\hline 1073 & 0.0104 & 0.00411268 & 0.143944 & 0.14805668 & 0.592226 & 3.70141 & 7.40282 & 14.8056 \\
\hline 1074 & 0.0104 & 0.00410116 & 0.143541 & 0.14764216 & 0.590567 & 3.69104 & 7.38208 & 14.7642 \\
\hline 1075 & 0.0104 & 0.00408967 & 0.143138 & 0.14722767 & 0.588912 & 3.6807 & 7.3614 & 14.7228 \\
\hline 1076 & 0.0104 & 0.00407821 & 0.142737 & 0.14681521 & 0.587262 & 3.67039 & 7.34078 & 14.6816 \\
\hline 1077 & 0.0104 & 0.00406678 & 0.142337 & 0.14640378 & 0.585617 & 3.66011 & 7.32021 & 14.6404 \\
\hline 1078 & 0.0104 & 0.00405539 & 0.141939 & 0.14599439 & 0.583976 & 3.64985 & 7.2997 & 14.5994 \\
\hline 1079 & 0.0103 & 0.00404402 & 0.141541 & 0.14558502 & 0.58234 & 3.63962 & 7.27924 & 14.5585 \\
\hline 1080 & 0.0103 & 0.00403269 & 0.141144 & 0.14517669 & 0.580708 & 3.62942 & 7.25885 & 14.5177 \\
\hline 1081 & 0.0103 & 0.00402139 & 0.140749 & 0.14477039 & 0.57908 & 3.61925 & 7.2385 & 14.477 \\
\hline 1082 & 0.0103 & 0.00401012 & 0.140354 & 0.14436412 & 0.577457 & 3.60911 & 7.21822 & 14.4364 \\
\hline 1083 & 0.0103 & 0.00399888 & 0.139961 & 0.14395988 & 0.575839 & 3.59899 & 7.19798 & 14.396 \\
\hline 1084 & 0.0103 & 0.00398767 & 0.139569 & 0.14355667 & 0.574225 & 3.5889 & 7.17781 & 14.3556 \\
\hline 1085 & 0.0102 & 0.00397649 & 0.139177 & 0.14315349 & 0.572615 & 3.57884 & 7.15769 & 14.3154 \\
\hline 1086 & 0.0102 & 0.00396535 & 0.138787 & 0.14275235 & 0.57101 & 3.56881 & 7.13762 & 14.2752 \\
\hline 1087 & 0.0102 & 0.00395423 & 0.138398 & 0.14235223 & 0.569409 & 3.55881 & 7.11762 & 14.2352 \\
\hline 1088 & 0.0102 & 0.00394314 & 0.13801 & 0.14195314 & 0.567813 & 3.54883 & 7.09766 & 14.1953 \\
\hline 1089 & 0.0102 & 0.00393209 & 0.137623 & 0.14155509 & 0.566221 & 3.53888 & 7.07776 & 14.1555 \\
\hline 1090 & 0.0102 & 0.00392106 & 0.137237 & 0.14115806 & 0.564633 & 3.52896 & 7.05792 & 14.1158 \\
\hline 1091 & 0.0101 & 0.00391007 & 0.136852 & 0.14076207 & 0.56305 & 3.51906 & 7.03813 & 14.0763 \\
\hline 1092 & 0.0101 & 0.00389911 & 0.136469 & 0.14036811 & 0.561471 & 3.5092 & 7.01839 & 14.0368 \\
\hline 1093 & 0.0101 & 0.00388817 & 0.136086 & 0.13997417 & 0.559897 & 3.49935 & 6.99871 & 13.9974 \\
\hline 1094 & 0.0101 & 0.00387727 & 0.135704 & 0.13958127 & 0.558327 & 3.48954 & 6.97908 & 13.9582 \\
\hline 1095 & 0.0101 & 0.00386639 & 0.135324 & 0.13919039 & 0.556761 & 3.47976 & 6.95951 & 13.919 \\
\hline 1096 & 0.0101 & 0.00385555 & 0.134944 & 0.13879955 & 0.555199 & 3.47 & 6.93999 & 13.88 \\
\hline 1097 & 0.01 & 0.00384474 & 0.134566 & 0.13841074 & 0.553642 & 3.46026 & 6.92053 & 13.8411 \\
\hline 1098 & 0.01 & 0.00383395 & 0.134188 & 0.13802195 & 0.552089 & 3.45056 & 6.90112 & 13.8022 \\
\hline 1099 & 0.01 & 0.0038232 & 0.133812 & 0.1376352 & 0.550541 & 3.44088 & 6.88176 & 13.7635 \\
\hline 1100 & 0.00998 & 0.00381247 & 0.133437 & 0.13724947 & 0.548996 & 3.43123 & 6.86245 & 13.7249 \\
\hline 1101 & 0.00996 & 0.00380178 & 0.133062 & 0.13686378 & 0.547456 & 3.4216 & 6.8432 & 13.6864 \\
\hline 1102 & 0.00995 & 0.00379111 & 0.132689 & 0.13648011 & 0.54592 & 3.412 & 6.82401 & 13.648 \\
\hline 1103 & 0.00993 & 0.00378048 & 0.132317 & 0.13609748 & 0.544389 & 3.40243 & 6.80486 & 13.6097 \\
\hline 1104 & 0.00992 & 0.00376987 & 0.131946 & 0.13571587 & 0.542862 & 3.39288 & 6.78577 & 13.5715 \\
\hline 1105 & 0.0099 & 0.00375929 & 0.131575 & 0.13533429 & 0.541338 & 3.38337 & 6.76673 & 13.5335 \\
\hline
\end{tabular}




\begin{tabular}{|c|c|c|c|c|c|c|c|c|}
\hline 1106 & 0.00988 & 0.00374875 & 0.131206 & 0.13495475 & 0.53982 & 3.37387 & 6.74774 & 13.4955 \\
\hline 1107 & 0.00986 & 0.00373823 & 0.130838 & 0.13457623 & 0.538305 & 3.36441 & 6.72881 & 13.4576 \\
\hline 1108 & 0.00985 & 0.00372774 & 0.130471 & 0.13419874 & 0.536794 & 3.35496 & 6.70993 & 13.4199 \\
\hline 1109 & 0.00983 & 0.00371728 & 0.130105 & 0.13382228 & 0.535288 & 3.34555 & 6.6911 & 13.3822 \\
\hline 1110 & 0.00982 & 0.00370685 & 0.12974 & 0.13344685 & 0.533786 & 3.33616 & 6.67232 & 13.3446 \\
\hline 1111 & 0.0098 & 0.00369644 & 0.129376 & 0.13307244 & 0.532288 & 3.3268 & 6.6536 & 13.3072 \\
\hline 1112 & 0.00978 & 0.00368607 & 0.129012 & 0.13269807 & 0.530794 & 3.31746 & 6.63493 & 13.2699 \\
\hline 1113 & 0.00977 & 0.00367572 & 0.12865 & 0.13232572 & 0.529304 & 3.30815 & 6.6163 & 13.2326 \\
\hline 1114 & 0.00975 & 0.00366541 & 0.128289 & 0.13195441 & 0.527819 & 3.29887 & 6.59773 & 13.1955 \\
\hline 1115 & 0.00973 & 0.00365512 & 0.127929 & 0.13158412 & 0.526337 & 3.28961 & 6.57922 & 13.1584 \\
\hline 1116 & 0.00972 & 0.00364486 & 0.12757 & 0.13121486 & 0.52486 & 3.28037 & 6.56075 & 13.1215 \\
\hline 1117 & 0.0097 & 0.00363463 & 0.127212 & 0.13084663 & 0.523387 & 3.27117 & 6.54233 & 13.0847 \\
\hline 1118 & 0.00969 & 0.00362443 & 0.126855 & 0.13047943 & 0.521918 & 3.26198 & 6.52397 & 13.0479 \\
\hline 1119 & 0.00967 & 0.00361425 & 0.126499 & 0.13011325 & 0.520452 & 3.25283 & 6.50566 & 13.0113 \\
\hline 1120 & 0.00965 & 0.00360411 & 0.126144 & 0.12974811 & 0.518991 & 3.2437 & 6.48739 & 12.9748 \\
\hline 1121 & 0.00963 & 0.00359399 & 0.12579 & 0.12938399 & 0.517534 & 3.23459 & 6.46918 & 12.9384 \\
\hline 1122 & 0.00962 & 0.0035839 & 0.125436 & 0.1290199 & 0.516082 & 3.22551 & 6.45102 & 12.902 \\
\hline 1123 & 0.0096 & 0.00357384 & 0.125084 & 0.12865784 & 0.514633 & 3.21645 & 6.43291 & 12.8658 \\
\hline 1124 & 0.00959 & 0.0035638 & 0.124733 & 0.1282968 & 0.513188 & 3.20742 & 6.41485 & 12.8297 \\
\hline 1125 & 0.00957 & 0.0035538 & 0.124383 & 0.1279368 & 0.511747 & 3.19842 & 6.39684 & 12.7937 \\
\hline 1126 & 0.00956 & 0.00354382 & 0.124034 & 0.12757782 & 0.51031 & 3.18944 & 6.37888 & 12.7578 \\
\hline 1127 & 0.00954 & 0.00353387 & 0.123685 & 0.12721887 & 0.508877 & 3.18048 & 6.36097 & 12.7219 \\
\hline 1128 & 0.00952 & 0.00352395 & 0.123338 & 0.12686195 & 0.507449 & 3.17155 & 6.34311 & 12.6862 \\
\hline 1129 & 0.0095 & 0.00351405 & 0.122992 & 0.12650605 & 0.506024 & 3.16265 & 6.3253 & 12.6506 \\
\hline 1130 & 0.00949 & 0.00350419 & 0.122647 & 0.12615119 & 0.504603 & 3.15377 & 6.30753 & 12.6151 \\
\hline 1131 & 0.00947 & 0.00349435 & 0.122302 & 0.12579635 & 0.503186 & 3.14491 & 6.28982 & 12.5796 \\
\hline 1132 & 0.00946 & 0.00348453 & 0.121959 & 0.12544353 & 0.501773 & 3.13608 & 6.27216 & 12.5443 \\
\hline 1133 & 0.00944 & 0.00347475 & 0.121616 & 0.12509075 & 0.500364 & 3.12727 & 6.25455 & 12.5091 \\
\hline 1134 & 0.00943 & 0.00346499 & 0.121275 & 0.12473999 & 0.498959 & 3.11849 & 6.23698 & 12.474 \\
\hline 1135 & 0.00941 & 0.00345526 & 0.120934 & 0.12438926 & 0.497557 & 3.10973 & 6.21947 & 12.4389 \\
\hline 1136 & 0.00939 & 0.00344556 & 0.120594 & 0.12403956 & 0.49616 & 3.101 & 6.202 & 12.404 \\
\hline 1137 & 0.00938 & 0.00343588 & 0.120256 & 0.12369188 & 0.494767 & 3.09229 & 6.18458 & 12.3692 \\
\hline 1138 & 0.00936 & 0.00342623 & 0.119918 & 0.12334423 & 0.493377 & 3.08361 & 6.16721 & 12.3344 \\
\hline 1139 & 0.00934 & 0.00341661 & 0.119581 & 0.12299761 & 0.491991 & 3.07495 & 6.14989 & 12.2998 \\
\hline 1140 & 0.00933 & 0.00340701 & 0.119245 & 0.12265201 & 0.49061 & 3.06631 & 6.13262 & 12.2652 \\
\hline 1141 & 0.00931 & 0.00339744 & 0.11891 & 0.12230744 & 0.489232 & 3.0577 & 6.1154 & 12.2308 \\
\hline 1142 & 0.0093 & 0.0033879 & 0.118576 & 0.1219639 & 0.487858 & 3.04911 & 6.09822 & 12.1964 \\
\hline 1143 & 0.00928 & 0.00337838 & 0.118243 & 0.12162138 & 0.486487 & 3.04055 & 6.08109 & 12.1622 \\
\hline 1144 & 0.00927 & 0.00336889 & 0.117911 & 0.12127989 & 0.485121 & 3.03201 & 6.06401 & 12.128 \\
\hline 1145 & 0.00925 & 0.00335943 & 0.11758 & 0.12093943 & 0.483758 & 3.02349 & 6.04698 & 12.094 \\
\hline 1146 & 0.00924 & 0.00335 & 0.11725 & 0.1206 & 0.482399 & 3.015 & 6.02999 & 12.06 \\
\hline 1147 & 0.00922 & 0.00334059 & 0.11692 & 0.12026059 & 0.481044 & 3.00653 & 6.01305 & 12.0261 \\
\hline 1148 & 0.0092 & 0.0033312 & 0.116592 & 0.1199232 & 0.479693 & 2.99808 & 5.99616 & 11.9923 \\
\hline 1149 & 0.00919 & 0.00332184 & 0.116265 & 0.11958684 & 0.478346 & 2.98966 & 5.97932 & 11.9586 \\
\hline 1150 & 0.00917 & 0.00331251 & 0.115938 & 0.11925051 & 0.477002 & 2.98126 & 5.96252 & 11.925 \\
\hline 1151 & 0.00915 & 0.00330321 & 0.115612 & 0.11891521 & 0.475662 & 2.97289 & 5.94578 & 11.8916 \\
\hline 1152 & 0.00914 & 0.00329393 & 0.115288 & 0.11858193 & 0.474326 & 2.96454 & 5.92907 & 11.8581 \\
\hline 1153 & 0.00912 & 0.00328468 & 0.114964 & 0.11824868 & 0.472993 & 2.95621 & 5.91242 & 11.8248 \\
\hline 1154 & 0.00911 & 0.00327545 & 0.114641 & 0.11791645 & 0.471665 & 2.9479 & 5.89581 & 11.7916 \\
\hline 1155 & 0.00909 & 0.00326625 & 0.114319 & 0.11758525 & 0.47034 & 2.93962 & 5.87925 & 11.7585 \\
\hline 1156 & 0.00908 & 0.00325707 & 0.113998 & 0.11725507 & 0.469018 & 2.93137 & 5.86273 & 11.7255 \\
\hline 1157 & 0.00906 & 0.00324792 & 0.113677 & 0.11692492 & 0.467701 & 2.92313 & 5.84626 & 11.6925 \\
\hline 1158 & 0.00905 & 0.0032388 & 0.113358 & 0.1165968 & 0.466387 & 2.91492 & 5.82984 & 11.6597 \\
\hline 1159 & 0.00903 & 0.0032297 & 0.113039 & 0.1162687 & 0.465077 & 2.90673 & 5.81346 & 11.6269 \\
\hline 1160 & 0.00902 & 0.00322063 & 0.112722 & 0.11594263 & 0.46377 & 2.89856 & 5.79713 & 11.5943 \\
\hline 1161 & 0.009 & 0.00321158 & 0.112405 & 0.11561658 & 0.462467 & 2.89042 & 5.78084 & 11.5617 \\
\hline 1162 & 0.00899 & 0.00320256 & 0.112089 & 0.11529156 & 0.461168 & 2.8823 & 5.7646 & 11.5292 \\
\hline 1163 & 0.00897 & 0.00319356 & 0.111775 & 0.11496856 & 0.459873 & 2.8742 & 5.74841 & 11.4968 \\
\hline 1164 & 0.00895 & 0.00318459 & 0.111461 & 0.11464559 & 0.458581 & 2.86613 & 5.73226 & 11.4645 \\
\hline 1165 & 0.00894 & 0.00317564 & 0.111147 & 0.11432264 & 0.457292 & 2.85808 & 5.71615 & 11.4323 \\
\hline 1166 & 0.00892 & 0.00316672 & 0.110835 & 0.11400172 & 0.456008 & 2.85005 & 5.70009 & 11.4002 \\
\hline 1167 & 0.00891 & 0.00315782 & 0.110524 & 0.11368182 & 0.454726 & 2.84204 & 5.68408 & 11.3682 \\
\hline 1168 & 0.00889 & 0.00314895 & 0.110213 & 0.11336195 & 0.453449 & 2.83406 & 5.66811 & 11.3362 \\
\hline 1169 & 0.00888 & 0.0031401 & 0.109904 & 0.1130441 & 0.452175 & 2.82609 & 5.65219 & 11.3044 \\
\hline 1170 & 0.00886 & 0.00313128 & 0.109595 & 0.11272628 & 0.450905 & 2.81815 & 5.63631 & 11.2726 \\
\hline 1171 & 0.00885 & 0.00312249 & 0.109287 & 0.11240949 & 0.449638 & 2.81024 & 5.62047 & 11.2409 \\
\hline 1172 & 0.00883 & 0.00311371 & 0.10898 & 0.11209371 & 0.448375 & 2.80234 & 5.60468 & 11.2094 \\
\hline 1173 & 0.00881 & 0.00310497 & 0.108674 & 0.11177897 & 0.447115 & 2.79447 & 5.58894 & 11.1779 \\
\hline 1174 & 0.0088 & 0.00309624 & 0.108369 & 0.11146524 & 0.445859 & 2.78662 & 5.57324 & 11.1465 \\
\hline 1175 & 0.00878 & 0.00308754 & 0.108064 & 0.11115154 & 0.444606 & 2.77879 & 5.55758 & 11.1152 \\
\hline 1176 & 0.00877 & 0.00307887 & 0.10776 & 0.11083887 & 0.443357 & 2.77098 & 5.54197 & 11.0839 \\
\hline 1177 & 0.00875 & 0.00307022 & 0.107458 & 0.11052822 & 0.442112 & 2.7632 & 5.5264 & 11.0528 \\
\hline 1178 & 0.00874 & 0.0030616 & 0.107156 & 0.1102176 & 0.44087 & 2.75544 & 5.51087 & 11.0217 \\
\hline
\end{tabular}




\begin{tabular}{|c|c|}
\hline 1179 & 0.00872 \\
\hline 1180 & 0.00871 \\
\hline 1181 & 0.00869 \\
\hline 1182 & 0.00868 \\
\hline 1183 & 0.00866 \\
\hline 1184 & 0.00865 \\
\hline 1185 & 0.00863 \\
\hline 1186 & 0.00862 \\
\hline 1187 & 0.0086 \\
\hline 1188 & 0.00859 \\
\hline 1189 & 0.00857 \\
\hline 1190 & 0.00856 \\
\hline 1191 & 0.00854 \\
\hline 1192 & 0.00853 \\
\hline 1193 & 0.00851 \\
\hline 1194 & 0.0085 \\
\hline 1195 & 0.00848 \\
\hline 1196 & 0.00847 \\
\hline 1197 & 0.00845 \\
\hline 1198 & 0.00844 \\
\hline 1199 & 0.00842 \\
\hline 1200 & 0.00841 \\
\hline 1201 & 0.0084 \\
\hline 1202 & 0.00838 \\
\hline 1203 & 0.00837 \\
\hline 1204 & 0.00835 \\
\hline 1205 & 0.00834 \\
\hline 1206 & 0.00832 \\
\hline 1207 & 0.00831 \\
\hline 1208 & 0.00829 \\
\hline 1209 & 0.00828 \\
\hline 1210 & 0.00827 \\
\hline 1211 & 0.00825 \\
\hline 1212 & 0.00824 \\
\hline 1213 & 0.00822 \\
\hline 1214 & 0.00821 \\
\hline 1215 & 0.00819 \\
\hline 1216 & 0.00818 \\
\hline 1217 & 0.00816 \\
\hline 1218 & 0.00815 \\
\hline 1219 & 0.00813 \\
\hline 1220 & 0.00812 \\
\hline 1221 & 0.0081 \\
\hline 1222 & 0.00809 \\
\hline 1223 & 0.00808 \\
\hline 1224 & 0.00806 \\
\hline 1225 & 0.00805 \\
\hline 1226 & 0.00803 \\
\hline 1227 & 0.00802 \\
\hline 1228 & 0.00801 \\
\hline 1229 & 0.00799 \\
\hline 1230 & 0.00798 \\
\hline 1231 & 0.00796 \\
\hline 1232 & 0.00795 \\
\hline 1233 & 0.00793 \\
\hline 1234 & 0.00792 \\
\hline 1235 & 0.0079 \\
\hline 1236 & 0.00789 \\
\hline 1237 & 0.00788 \\
\hline 1238 & 0.00786 \\
\hline 1239 & 0.00785 \\
\hline 1240 & 0.00784 \\
\hline 1241 & 0.00782 \\
\hline 1242 & 0.00781 \\
\hline 1243 & 0.00779 \\
\hline 1244 & 0.00778 \\
\hline 1245 & 0.00776 \\
\hline 1246 & 0.00775 \\
\hline 1247 & 0.00774 \\
\hline 1248 & 0.00773 \\
\hline 1249 & 0.00771 \\
\hline 1250 & 0.0077 \\
\hline 1251 & 0.00768 \\
\hline
\end{tabular}

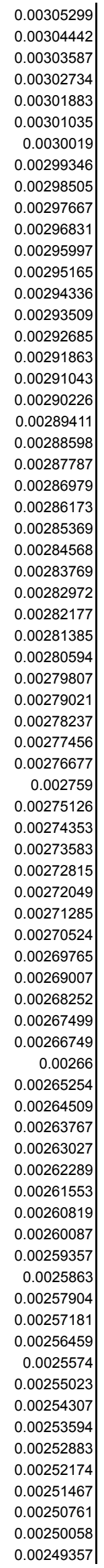

0.106855

0.106555

0.106255

0.105957

0.105659

0.105362

0.105066

0.104771

0.104477

0.104183

0.103891

0.103599

0.103308

0.103018

0.102728

0.10244

0.102152

0.101865

0.101579

0.101294

0.101009

0.100725

0.100443

0.100161

0.0998792

0.0995987

0.099319

0.0990401

0.098762

0.0984846

0.0982081

0.0979323

0.0976573

0.0973831

0.0971097

0.096837

0.0965651

0.096294

0.0960237

0.0957541

0.0954853

0.0952172

0.0949499

0.0946834

0.0944176

0.0941526

0.0938883

0.0936248

0.093362

0.0931

0.0928387

0.0925782

0.0923184

0.0920593

0.091801

0.0915434

0.0912866

0.0910305

0.0907751

0.0905204

0.0902665

0.0900132

0.0897607

0.089509

0.0892579

0.0890076

0.0887579

0.088509

0.0882608

0.0880133

0.0877665

0.0875204

0.087275
0.10990799

0.10959942

0.10929087

0.10898434

0.10867783

0.10837235

0.1080679

0.10776446

0.10746205

0.10715967

0.10685931

0.10655897

0.10625965

0.10596136

0.10566309

0.10536685

0.10507063

0.10477543

0.10448126

0.10418811

0.10389498

0.10360287

0.10331279

0.10302273

0.10273289

0.10244438

0.10215669

0.10186982

0.10158377

0.10129845

0.10101404

0.10073037

0.10044751

0.10016547

0.09988426

0.09960377

0.0993241

0.09904526

0.09876723

0.09848993

0.09821345

0.09793769

0.09766275

0.09738864

0.09711525

0.09684267

0.09657082

0.09629979

0.09602949

0.09576

0.09549124

0.09522329

0.09495607

0.09468957

0.09442389

0.09415893

0.09389479

0.09363137

0.09336867

0.0931067

0.09284554

0.09258501

0.09232529

0.0920664

0.09180813

0.09155067

0.09129384

0.09103783

0.09078254

0.09052797

0.09027411

0.09002098

0.08976857
0.439631

0.438396

0.437165

0.435937

0.434712

0.433491

0.432273

0.431059

0.429848

0.42864

0.427436

0.426235

0.425038

0.423844

0.422654

0.421466

0.420283

0.419102

0.417925

0.416751

0.415581

0.414413

0.41325

0.412089

0.410932

0.409778

0.408627

0.407479

0.406335

0.405194

0.404056

0.402921

0.40179

0.400662

0.399537

0.398415

0.397296

0.396181

0.395069

0.39396

0.392854

0.391751

0.390651

0.389555

0.388461

0.387371

0.386283

0.385199

0.384118

0.38304

0.381965

0.380893

0.379824

0.378758

0.377696

0.376636

0.375579

0.374525

0.373475

0.372427

0.371382

0.37034

0.369301

0.368265

0.367233

0.366203

0.365175

0.364151

0.36313

0.362112

0.361096

0.360084

0.359074

2.7477

2.73998

2.73228

2.7246

2.71695

2.70932

2.70171

2.69412

2.68655

2.679

2.67148

2.66397

2.65649

2.64903

2.64159

2.63417

2.62677

2.61939

2.61203

2.60469

2.59738

2.59008

2.58281

2.57556

2.56832

2.56111

2.55392

2.54674

2.53959

2.53246

2.52535

2.51826

2.51119

2.50414

2.49711

2.49009

2.4831

2.47613

2.46918

2.46225

2.45534

2.44844

2.44157

2.43472

2.42788

2.42107

2.41427

2.40749

2.40074

2.394

2.38728

2.38058

2.3739

2.36724

2.3606

2.35397

2.34737

2.34078

2.33422

2.32767

2.32114

2.31463

2.30813

2.30166

2.2952

2.28877

2.28235

2.27595

2.26956 


\begin{tabular}{|c|c|c|}
\hline 1252 & 0.00767 & 0.00248658 \\
\hline 1253 & 0.00765 & 0.00247961 \\
\hline 1254 & 0.00764 & 0.00247266 \\
\hline 1255 & 0.00763 & 0.00246573 \\
\hline 1256 & 0.00761 & 0.00245881 \\
\hline 1257 & 0.0076 & 0.00245192 \\
\hline 1258 & 0.00759 & 0.00244505 \\
\hline 1259 & 0.00757 & 0.0024382 \\
\hline 1260 & 0.00756 & 0.00243136 \\
\hline 1261 & 0.00754 & 0.00242455 \\
\hline 1262 & 0.00753 & 0.00241776 \\
\hline 1263 & 0.00752 & 0.00241098 \\
\hline 1264 & 0.00751 & 0.00240423 \\
\hline 1265 & 0.00749 & 0.00239749 \\
\hline 1266 & 0.00748 & 0.00239077 \\
\hline 1267 & 0.00746 & 0.00238407 \\
\hline 1268 & 0.00745 & 0.0023774 \\
\hline 1269 & 0.00744 & 0.00237074 \\
\hline 1270 & 0.00742 & 0.0023641 \\
\hline 1271 & 0.00741 & 0.00235748 \\
\hline 1272 & 0.0074 & 0.00235087 \\
\hline 1273 & 0.00738 & 0.00234429 \\
\hline 1274 & 0.00737 & 0.00233772 \\
\hline 1275 & 0.00735 & 0.00233118 \\
\hline 1276 & 0.00734 & 0.00232465 \\
\hline 1277 & 0.00733 & 0.00231814 \\
\hline 1278 & 0.00732 & 0.00231165 \\
\hline 1279 & 0.0073 & 0.00230518 \\
\hline 1280 & 0.00729 & 0.00229873 \\
\hline 1281 & 0.00727 & 0.00229229 \\
\hline 1282 & 0.00726 & 0.00228588 \\
\hline 1283 & 0.00725 & 0.00227948 \\
\hline 1284 & 0.00724 & 0.0022731 \\
\hline 1285 & 0.00722 & 0.00226674 \\
\hline 1286 & 0.00721 & 0.0022604 \\
\hline 1287 & 0.00719 & 0.00225407 \\
\hline 1288 & 0.00718 & 0.00224777 \\
\hline 1289 & 0.00717 & 0.00224148 \\
\hline 1290 & 0.00716 & 0.00223521 \\
\hline 1291 & 0.00714 & 0.00222896 \\
\hline 1292 & 0.00713 & 0.00222272 \\
\hline 1293 & 0.00712 & 0.00221651 \\
\hline 1294 & 0.0071 & 0.00221031 \\
\hline 1295 & 0.00709 & 0.00220413 \\
\hline 1296 & 0.00708 & 0.00219796 \\
\hline 1297 & 0.00706 & 0.00219182 \\
\hline 1298 & 0.00705 & 0.00218569 \\
\hline 1299 & 0.00704 & 0.00217958 \\
\hline 1300 & 0.00703 & 0.00217349 \\
\hline 1301 & 0.00701 & 0.00216741 \\
\hline 1302 & 0.007 & 0.00216136 \\
\hline 1303 & 0.00698 & 0.00215532 \\
\hline 1304 & 0.00697 & 0.00214929 \\
\hline 1305 & 0.00696 & 0.00214329 \\
\hline 1306 & 0.00695 & 0.0021373 \\
\hline 1307 & 0.00693 & 0.00213133 \\
\hline 1308 & 0.00692 & 0.00212537 \\
\hline 1309 & 0.00691 & 0.00211944 \\
\hline 1310 & 0.0069 & 0.00211352 \\
\hline 1311 & 0.00688 & 0.00210761 \\
\hline 1312 & 0.00687 & 0.00210173 \\
\hline 1313 & 0.00686 & 0.00209586 \\
\hline 1314 & 0.00685 & 0.00209001 \\
\hline 1315 & 0.00683 & 0.00208417 \\
\hline 1316 & 0.00682 & 0.00207835 \\
\hline 1317 & 0.0068 & 0.00207255 \\
\hline 1318 & 0.00679 & 0.00206677 \\
\hline 1319 & 0.00678 & 0.002061 \\
\hline 1320 & 0.00677 & 0.00205525 \\
\hline 1321 & 0.00675 & 0.00204951 \\
\hline 1322 & 0.00674 & 0.00204379 \\
\hline 1323 & 0.00673 & 0.00203809 \\
\hline 1324 & 0.00672 & 0.00203241 \\
\hline
\end{tabular}

\begin{tabular}{|c|c|c|c|}
\hline 0.0870303 & 0.08951688 & 0.358068 & 2.23792 \\
\hline 0.0867863 & 0.08926591 & 0.357064 & 2.23165 \\
\hline 0.086543 & 0.08901566 & 0.356063 & 2.22539 \\
\hline 0.0863004 & 0.08876613 & 0.355065 & 2.21915 \\
\hline 0.0860585 & 0.08851731 & 0.354069 & 2.21293 \\
\hline 0.0858173 & 0.08826922 & 0.353077 & 2.20673 \\
\hline 0.0855767 & 0.08802175 & 0.352087 & 2.20054 \\
\hline 0.0853369 & 0.0877751 & 0.3511 & 2.19438 \\
\hline 0.0850977 & 0.08752906 & 0.350116 & 2.18823 \\
\hline 0.0848593 & 0.08728385 & 0.349135 & 2.1821 \\
\hline 0.0846215 & 0.08703926 & 0.348157 & 2.17598 \\
\hline 0.0843843 & 0.08679528 & 0.347181 & 2.16988 \\
\hline 0.0841479 & 0.08655213 & 0.346208 & 2.1638 \\
\hline 0.0839121 & 0.08630959 & 0.345238 & 2.15774 \\
\hline 0.083677 & 0.08606777 & 0.344271 & 2.1517 \\
\hline 0.0834426 & 0.08582667 & 0.343307 & 2.14567 \\
\hline 0.0832089 & 0.0855863 & 0.342345 & 2.13966 \\
\hline 0.0829758 & 0.08534654 & 0.341386 & 2.13366 \\
\hline 0.0827434 & 0.0851075 & 0.34043 & 2.12769 \\
\hline 0.0825116 & 0.08486908 & 0.339476 & 2.12173 \\
\hline 0.0822806 & 0.08463147 & 0.338526 & 2.11579 \\
\hline 0.0820501 & 0.08439439 & 0.337578 & 2.10986 \\
\hline 0.0818204 & 0.08415812 & 0.336632 & 2.10395 \\
\hline 0.0815913 & 0.08392248 & 0.33569 & 2.09806 \\
\hline 0.0813628 & 0.08368745 & 0.33475 & 2.09219 \\
\hline 0.081135 & 0.08345314 & 0.333813 & 2.08633 \\
\hline 0.0809079 & 0.08321955 & 0.332878 & 2.08049 \\
\hline 0.0806814 & 0.08298658 & 0.331946 & 2.07466 \\
\hline 0.0804555 & 0.08275423 & 0.331017 & 2.06886 \\
\hline 0.0802303 & 0.08252259 & 0.33009 & 2.06307 \\
\hline 0.0800058 & 0.08229168 & 0.329167 & 2.05729 \\
\hline 0.0797819 & 0.08206138 & 0.328245 & 2.05153 \\
\hline 0.0795586 & 0.0818317 & 0.327327 & 2.04579 \\
\hline 0.079336 & 0.08160274 & 0.326411 & 2.04007 \\
\hline 0.079114 & 0.0813744 & 0.325497 & 2.03436 \\
\hline 0.0788926 & 0.08114667 & 0.324587 & 2.02867 \\
\hline 0.0786719 & 0.08091967 & 0.323679 & 2.02299 \\
\hline 0.0784518 & 0.08069328 & 0.322773 & 2.01733 \\
\hline 0.0782323 & 0.08046751 & 0.32187 & 2.01169 \\
\hline 0.0780135 & 0.08024246 & 0.32097 & 2.00606 \\
\hline 0.0777953 & 0.08001802 & 0.320072 & 2.00045 \\
\hline 0.0775777 & 0.07979421 & 0.319177 & 1.99486 \\
\hline 0.0773608 & 0.07957111 & 0.318284 & 1.98928 \\
\hline 0.0771445 & 0.07934863 & 0.317394 & 1.98371 \\
\hline 0.0769288 & 0.07912676 & 0.316507 & 1.97817 \\
\hline 0.0767137 & 0.07890552 & 0.315622 & 1.97264 \\
\hline 0.0764992 & 0.07868489 & 0.31474 & 1.96712 \\
\hline 0.0762853 & 0.07846488 & 0.31386 & 1.96162 \\
\hline 0.0760721 & 0.07824559 & 0.312982 & 1.95614 \\
\hline 0.0758595 & 0.07802691 & 0.312108 & 1.95067 \\
\hline 0.0756475 & 0.07780886 & 0.311235 & 1.94522 \\
\hline 0.075436 & 0.07759132 & 0.310365 & 1.93978 \\
\hline 0.0752252 & 0.07737449 & 0.309498 & 1.93436 \\
\hline 0.0750151 & 0.07715839 & 0.308633 & 1.92896 \\
\hline 0.0748055 & 0.0769428 & 0.307771 & 1.92357 \\
\hline 0.0745965 & 0.07672783 & 0.306911 & 1.91819 \\
\hline 0.0743881 & 0.07651347 & 0.306054 & 1.91284 \\
\hline 0.0741803 & 0.07629974 & 0.305199 & 1.90749 \\
\hline 0.0739731 & 0.07608662 & 0.304346 & 1.90217 \\
\hline 0.0737665 & 0.07587411 & 0.303496 & 1.89685 \\
\hline 0.0735605 & 0.07566223 & 0.302649 & 1.89156 \\
\hline 0.0733551 & 0.07545096 & 0.301804 & 1.88627 \\
\hline 0.0731503 & 0.07524031 & 0.300961 & 1.88101 \\
\hline 0.072946 & 0.07503017 & 0.300121 & 1.87576 \\
\hline 0.0727424 & 0.07482075 & 0.299283 & 1.87052 \\
\hline 0.0725393 & 0.07461185 & 0.298448 & 1.8653 \\
\hline 0.0723369 & 0.07440367 & 0.297615 & 1.86009 \\
\hline 0.072135 & 0.074196 & 0.296784 & 1.8549 \\
\hline 0.0719337 & 0.07398895 & 0.295956 & 1.84972 \\
\hline 0.0717329 & 0.07378241 & 0.29513 & 1.84456 \\
\hline 0.0715328 & 0.07357659 & 0.294306 & 1.83941 \\
\hline 0.0713332 & 0.07337129 & 0.293485 & 1.83428 \\
\hline 0.0711342 & 0.07316661 & 0.292666 & 1.82916 \\
\hline
\end{tabular}

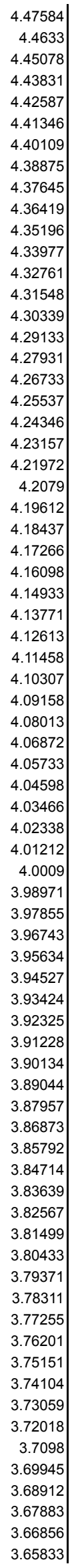

8.95169 8.92659 8.90157 8.87661 8.85173 8.82692 8.80218 8.77751 8.75291 8.72838 8.70392 8.67953 8.65521 8.63096 8.60678 8.58267 8.55863 8.53465 8.51075 8.48691 8.46314 8.43944 8.41581 8.39224 8.36875 8.34531 8.32195 8.29865 8.27542 8.25226 8.22916 8.20613 8.18317 8.16027 8.13744 8.11467 8.09197 8.06933 8.04676 8.02425 8.0018 7.97942 7.95711 7.93486 7.91267 7.89055 7.86849 7.84649 7.82456 7.80269 7.78088 7.75914 7.73745 7.71583 7.69428 7.67278 7.65135 7.62997 7.60866 7.58741 7.56622 7.54509 7.52403 7.50302 7.48207 7.46119 7.44036 7.4196 7.39889 7.37824 7.35766 7.33713 7.31666 


\begin{tabular}{|c|c|c|}
\hline 1325 & 0.0067 & 0.00202674 \\
\hline 1326 & 0.00669 & 0.00202108 \\
\hline 1327 & 0.00668 & 0.00201545 \\
\hline 1328 & 0.00667 & 0.00200983 \\
\hline 1329 & 0.00665 & 0.00200422 \\
\hline 1330 & 0.00664 & 0.00199863 \\
\hline 1331 & 0.00663 & 0.00199306 \\
\hline 1332 & 0.00662 & 0.0019875 \\
\hline 1333 & 0.0066 & 0.00198196 \\
\hline 1334 & 0.00659 & 0.00197644 \\
\hline 1335 & 0.00658 & 0.00197093 \\
\hline 1336 & 0.00657 & 0.00196544 \\
\hline 1337 & 0.00655 & 0.00195996 \\
\hline 1338 & 0.00654 & 0.0019545 \\
\hline 1339 & 0.00653 & 0.00194906 \\
\hline 1340 & 0.00652 & 0.00194363 \\
\hline 1341 & 0.0065 & 0.00193821 \\
\hline 1342 & 0.00649 & 0.00193282 \\
\hline 1343 & 0.00648 & 0.00192743 \\
\hline 1344 & 0.00647 & 0.00192207 \\
\hline 1345 & 0.00645 & 0.00191671 \\
\hline 1346 & 0.00644 & 0.00191138 \\
\hline 1347 & 0.00643 & 0.00190606 \\
\hline 1348 & 0.00642 & 0.00190075 \\
\hline 1349 & 0.0064 & 0.00189546 \\
\hline 1350 & 0.00639 & 0.00189019 \\
\hline 1351 & 0.00638 & 0.00188493 \\
\hline 1352 & 0.00637 & 0.00187968 \\
\hline 1353 & 0.00636 & 0.00187445 \\
\hline 1354 & 0.00635 & 0.00186924 \\
\hline 1355 & 0.00633 & 0.00186404 \\
\hline 1356 & 0.00632 & 0.00185886 \\
\hline 1357 & 0.00631 & 0.00185369 \\
\hline 1358 & 0.0063 & 0.00184853 \\
\hline 1359 & 0.00628 & 0.0018434 \\
\hline 1360 & 0.00627 & 0.00183827 \\
\hline 1361 & 0.00626 & 0.00183316 \\
\hline 1362 & 0.00625 & 0.00182807 \\
\hline 1363 & 0.00623 & 0.00182299 \\
\hline 1364 & 0.00622 & 0.00181792 \\
\hline 1365 & 0.00621 & 0.00181287 \\
\hline 1366 & 0.0062 & 0.00180783 \\
\hline 1367 & 0.00619 & 0.00180281 \\
\hline 1368 & 0.00618 & 0.0017978 \\
\hline 1369 & 0.00616 & 0.00179281 \\
\hline 1370 & 0.00615 & 0.00178783 \\
\hline 1371 & 0.00614 & 0.00178287 \\
\hline 1372 & 0.00613 & 0.00177792 \\
\hline 1373 & 0.00611 & 0.00177298 \\
\hline 1374 & 0.0061 & 0.00176806 \\
\hline 1375 & 0.00609 & 0.00176316 \\
\hline 1376 & 0.00608 & 0.00175826 \\
\hline 1377 & 0.00607 & 0.00175338 \\
\hline 1378 & 0.00606 & 0.00174852 \\
\hline 1379 & 0.00604 & 0.00174367 \\
\hline 1380 & 0.00603 & 0.00173883 \\
\hline 1381 & 0.00602 & 0.00173401 \\
\hline 1382 & 0.00601 & 0.0017292 \\
\hline 1383 & 0.006 & 0.00172441 \\
\hline 1384 & 0.00599 & 0.00171963 \\
\hline 1385 & 0.00597 & 0.00171486 \\
\hline 1386 & 0.00596 & 0.00171011 \\
\hline 1387 & 0.00595 & 0.00170537 \\
\hline 1388 & 0.00594 & 0.00170064 \\
\hline 1389 & 0.00592 & 0.00169593 \\
\hline 1390 & 0.00592 & 0.00169123 \\
\hline 1391 & 0.0059 & 0.00168655 \\
\hline 1392 & 0.00589 & 0.00168188 \\
\hline 1393 & 0.00588 & 0.00167722 \\
\hline 1394 & 0.00587 & 0.00167257 \\
\hline 1395 & 0.00586 & 0.00166794 \\
\hline 1396 & 0.00585 & 0.00166333 \\
\hline $1397 \mid$ & 0.00583 & 0.00165872 \\
\hline
\end{tabular}

\begin{tabular}{|c|c|c|c|}
\hline 0.0709358 & 0.07296254 & 0.29185 & 1.82406 \\
\hline 0.0707379 & 0.07275898 & 0.291036 & 1.81897 \\
\hline 0.0705406 & 0.07255605 & 0.290224 & 1.8139 \\
\hline 0.0703439 & 0.07235373 & 0.289415 & 1.80884 \\
\hline 0.0701477 & 0.07215192 & 0.288608 & 1.8038 \\
\hline 0.0699521 & 0.07195073 & 0.287803 & 1.79877 \\
\hline 0.0697571 & 0.07175016 & 0.287001 & 1.79375 \\
\hline 0.0695627 & 0.0715502 & 0.286201 & 1.78875 \\
\hline 0.0693687 & 0.07135066 & 0.285403 & 1.78377 \\
\hline 0.0691754 & 0.07115184 & 0.284607 & 1.7788 \\
\hline 0.0689826 & 0.07095353 & 0.283814 & 1.77384 \\
\hline 0.0687904 & 0.07075584 & 0.283023 & 1.7689 \\
\hline 0.0685987 & 0.07055866 & 0.282235 & 1.76397 \\
\hline 0.0684076 & 0.0703621 & 0.281448 & 1.75905 \\
\hline 0.068217 & 0.07016606 & 0.280664 & 1.75415 \\
\hline 0.068027 & 0.06997063 & 0.279882 & 1.74927 \\
\hline 0.0678375 & 0.06977571 & 0.279103 & 1.74439 \\
\hline 0.0676486 & 0.06958142 & 0.278326 & 1.73953 \\
\hline 0.0674602 & 0.06938763 & 0.27755 & 1.73469 \\
\hline 0.0672723 & 0.06919437 & 0.276778 & 1.72986 \\
\hline 0.067085 & 0.06900171 & 0.276007 & 1.72504 \\
\hline 0.0668983 & 0.06880968 & 0.275239 & 1.72024 \\
\hline 0.066712 & 0.06861806 & 0.274472 & 1.71545 \\
\hline 0.0665264 & 0.06842715 & 0.273708 & 1.71068 \\
\hline 0.0663412 & 0.06823666 & 0.272947 & 1.70592 \\
\hline 0.0661566 & 0.06804679 & 0.272187 & 1.70117 \\
\hline 0.0659725 & 0.06785743 & 0.27143 & 1.69644 \\
\hline 0.0657889 & 0.06766858 & 0.270675 & 1.69172 \\
\hline 0.0656059 & 0.06748035 & 0.269922 & 1.68701 \\
\hline 0.0654234 & 0.06729264 & 0.269171 & 1.68232 \\
\hline 0.0652415 & 0.06710554 & 0.268422 & 1.67764 \\
\hline 0.06506 & 0.06691886 & 0.267676 & 1.67297 \\
\hline 0.0648791 & 0.06673279 & 0.266931 & 1.66832 \\
\hline 0.0646987 & 0.06654723 & 0.266189 & 1.66368 \\
\hline 0.0645188 & 0.0663622 & 0.265449 & 1.65906 \\
\hline 0.0643395 & 0.06617777 & 0.264711 & 1.65444 \\
\hline 0.0641606 & 0.06599376 & 0.263975 & 1.64984 \\
\hline 0.0639823 & 0.06581037 & 0.263241 & 1.64526 \\
\hline 0.0638045 & 0.06562749 & 0.26251 & 1.64069 \\
\hline 0.0636272 & 0.06544512 & 0.261781 & 1.63613 \\
\hline 0.0634504 & 0.06526327 & 0.261053 & 1.63158 \\
\hline 0.0632742 & 0.06508203 & 0.260328 & 1.62705 \\
\hline 0.0630984 & 0.06490121 & 0.259605 & 1.62253 \\
\hline 0.0629231 & 0.0647209 & 0.258884 & 1.61802 \\
\hline 0.0627484 & 0.06454121 & 0.258165 & 1.61353 \\
\hline 0.0625741 & 0.06436193 & 0.257448 & 1.60905 \\
\hline 0.0624004 & 0.06418327 & 0.256733 & 1.60458 \\
\hline 0.0622272 & 0.06400512 & 0.25602 & 1.60013 \\
\hline 0.0620544 & 0.06382738 & 0.25531 & 1.59569 \\
\hline 0.0618822 & 0.06365026 & 0.254601 & 1.59126 \\
\hline 0.0617104 & 0.06347356 & 0.253894 & 1.58684 \\
\hline 0.0615392 & 0.06329746 & 0.25319 & 1.58244 \\
\hline 0.0613685 & 0.06312188 & 0.252487 & 1.57805 \\
\hline 0.0611982 & 0.06294672 & 0.251787 & 1.57367 \\
\hline 0.0610284 & 0.06277207 & 0.251088 & 1.5693 \\
\hline 0.0608592 & 0.06259803 & 0.250392 & 1.56495 \\
\hline 0.0606904 & 0.06242441 & 0.249698 & 1.56061 \\
\hline 0.0605221 & 0.0622513 & 0.249005 & 1.55628 \\
\hline 0.0603543 & 0.06207871 & 0.248315 & 1.55197 \\
\hline 0.060187 & 0.06190663 & 0.247626 & 1.54766 \\
\hline 0.0600201 & 0.06173496 & 0.24694 & 1.54337 \\
\hline 0.0598538 & 0.06156391 & 0.246255 & 1.5391 \\
\hline 0.0596879 & 0.06139327 & 0.245573 & 1.53483 \\
\hline 0.0595225 & 0.06122314 & 0.244893 & 1.53058 \\
\hline 0.0593576 & 0.06105353 & 0.244214 & 1.52634 \\
\hline 0.0591931 & 0.06088433 & 0.243537 & 1.52211 \\
\hline 0.0590292 & 0.06071575 & 0.242863 & 1.51789 \\
\hline 0.0588657 & 0.06054758 & 0.24219 & 1.51369 \\
\hline 0.0587027 & 0.06037992 & 0.241519 & 1.5095 \\
\hline 0.0585401 & 0.06021267 & 0.240851 & 1.50532 \\
\hline 0.058378 & 0.06004594 & 0.240184 & 1.50115 \\
\hline 0.0582164 & 0.05987973 & 0.239519 & 1.49699 \\
\hline 0.0580553 & 0.05971402 & 0.238856 & 1.49285 \\
\hline
\end{tabular}

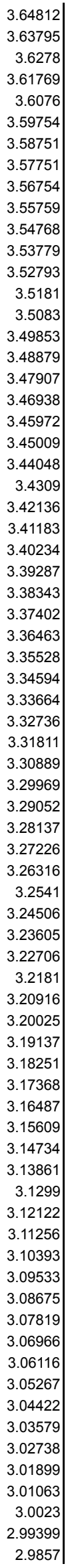

7.29625

7.2759

7.25561

7.23537

7.2152

7.19508

7.17502

7.15502

7.13507

7.11518

7.09535

7.07558

7.05587

7.03621

7.01661

6.99706

6.97757

6.95814

6.93876

6.91944

6.90017

6.88096

6.86181

6.84271

6.82367

6.80468

6.78574

6.76686

6.74804

6.72927

6.71055

6.69189

6.67328

6.65472

6.63622

6.61777

6.59938

6.58104

6.56275

6.54451

6.52633

6.5082

6.49012

6.47209

6.45412

6.4362

6.41833

6.40051

6.38274

6.36503

6.34736

6.32975

6.31218

6.29467

6.27721

6.2598

6.24244

6.22513

6.20787

6.19066

6.1735

6.15639

6.13933

6.12231

6.10535

6.08844

6.07157

6.05476

6.03799

6.02127

6.0046

5.98798

5.9714 


\begin{tabular}{|c|c|c|}
\hline 1398 & 0.00582 & 0.00165413 \\
\hline 1399 & 0.00581 & 0.00164955 \\
\hline 1400 & 0.0058 & 0.00164499 \\
\hline 1401 & 0.00579 & 0.00164044 \\
\hline 1402 & 0.00578 & 0.0016359 \\
\hline 1403 & 0.00576 & 0.00163138 \\
\hline 1404 & 0.00575 & 0.00162687 \\
\hline 1405 & 0.00574 & 0.00162237 \\
\hline 1406 & 0.00573 & 0.00161788 \\
\hline 1407 & 0.00572 & 0.00161341 \\
\hline 1408 & 0.00571 & 0.00160895 \\
\hline 1409 & 0.00569 & 0.0016045 \\
\hline 1410 & 0.00569 & 0.00160007 \\
\hline 1411 & 0.00567 & 0.00159565 \\
\hline 1412 & 0.00566 & 0.00159124 \\
\hline 1413 & 0.00565 & 0.00158684 \\
\hline 1414 & 0.00564 & 0.00158246 \\
\hline 1415 & 0.00563 & 0.00157809 \\
\hline 1416 & 0.00562 & 0.00157374 \\
\hline 1417 & 0.0056 & 0.00156939 \\
\hline 1418 & 0.00559 & 0.00156506 \\
\hline 1419 & 0.00558 & 0.00156074 \\
\hline 1420 & 0.00557 & 0.00155643 \\
\hline 1421 & 0.00556 & 0.00155214 \\
\hline 1422 & 0.00555 & 0.00154786 \\
\hline 1423 & 0.00554 & 0.00154359 \\
\hline 1424 & 0.00553 & 0.00153933 \\
\hline 1425 & 0.00551 & 0.00153508 \\
\hline 1426 & 0.0055 & 0.00153085 \\
\hline 1427 & 0.00549 & 0.00152663 \\
\hline 1428 & 0.00548 & 0.00152242 \\
\hline 1429 & 0.00547 & 0.00151823 \\
\hline 1430 & 0.00546 & 0.00151404 \\
\hline 1431 & 0.00545 & 0.00150987 \\
\hline 1432 & 0.00544 & 0.00150571 \\
\hline 1433 & 0.00542 & 0.00150156 \\
\hline 1434 & 0.00542 & 0.00149743 \\
\hline 1435 & 0.0054 & 0.0014933 \\
\hline 1436 & 0.00539 & 0.00148919 \\
\hline 1437 & 0.00538 & 0.00148509 \\
\hline 1438 & 0.00537 & 0.001481 \\
\hline 1439 & 0.00536 & 0.00147693 \\
\hline 1440 & 0.00535 & 0.00147286 \\
\hline 1441 & 0.00534 & 0.00146881 \\
\hline 1442 & 0.00533 & 0.00146477 \\
\hline 1443 & 0.00531 & 0.00146074 \\
\hline 1444 & 0.00531 & 0.00145672 \\
\hline 1445 & 0.00529 & 0.00145272 \\
\hline 1446 & 0.00528 & 0.00144872 \\
\hline 1447 & 0.00527 & 0.00144474 \\
\hline 1448 & 0.00526 & 0.00144077 \\
\hline 1449 & 0.00525 & 0.00143681 \\
\hline 1450 & 0.00524 & 0.00143286 \\
\hline 1451 & 0.00523 & 0.00142893 \\
\hline 1452 & 0.00522 & 0.001425 \\
\hline 1453 & 0.00521 & 0.00142109 \\
\hline 1454 & 0.0052 & 0.00141719 \\
\hline 1455 & 0.00518 & 0.00141329 \\
\hline 1456 & 0.00518 & 0.00140941 \\
\hline 1457 & 0.00516 & 0.00140555 \\
\hline 1458 & 0.00515 & 0.00140169 \\
\hline 1459 & 0.00514 & 0.00139784 \\
\hline 1460 & 0.00513 & 0.00139401 \\
\hline 1461 & 0.00512 & 0.00139018 \\
\hline 1462 & 0.00511 & 0.00138637 \\
\hline 1463 & 0.0051 & 0.00138257 \\
\hline 1464 & 0.00509 & 0.00137878 \\
\hline 1465 & 0.00508 & 0.001375 \\
\hline 1466 & 0.00507 & 0.00137123 \\
\hline 1467 & 0.00505 & 0.00136747 \\
\hline 1468 & 0.00505 & 0.00136372 \\
\hline 1469 & 0.00503 & 0.00135999 \\
\hline 1470 & 0.00503 & 0.00135626 \\
\hline
\end{tabular}

\begin{tabular}{|c|c|c|c|}
\hline 0.0578946 & 0.05954873 & 0.238195 & 1.48872 \\
\hline 0.0577344 & 0.05938395 & 0.237536 & 1.4846 \\
\hline 0.0575747 & 0.05921969 & 0.236879 & 1.48049 \\
\hline 0.0574154 & 0.05905584 & 0.236223 & 1.4764 \\
\hline 0.0572566 & 0.0588925 & 0.23557 & 1.47231 \\
\hline 0.0570982 & 0.05872958 & 0.234918 & 1.46824 \\
\hline 0.0569403 & 0.05856717 & 0.234269 & 1.46418 \\
\hline 0.0567828 & 0.05840517 & 0.233621 & 1.46013 \\
\hline 0.0566259 & 0.05824378 & 0.232975 & 1.45609 \\
\hline 0.0564693 & 0.05808271 & 0.232331 & 1.45207 \\
\hline 0.0563132 & 0.05792215 & 0.231689 & 1.44805 \\
\hline 0.0561576 & 0.0577621 & 0.231048 & 1.44405 \\
\hline 0.0560024 & 0.05760247 & 0.23041 & 1.44006 \\
\hline 0.0558477 & 0.05744335 & 0.229773 & 1.43608 \\
\hline 0.0556934 & 0.05728464 & 0.229139 & 1.43212 \\
\hline 0.0555396 & 0.05712644 & 0.228506 & 1.42816 \\
\hline 0.0553862 & 0.05696866 & 0.227875 & 1.42422 \\
\hline 0.0552332 & 0.05681129 & 0.227245 & 1.42028 \\
\hline 0.0550807 & 0.05665444 & 0.226618 & 1.41636 \\
\hline 0.0549287 & 0.05649809 & 0.225992 & 1.41245 \\
\hline 0.054777 & 0.05634206 & 0.225368 & 1.40855 \\
\hline 0.0546259 & 0.05618664 & 0.224746 & 1.40466 \\
\hline 0.0544751 & 0.05603153 & 0.224126 & 1.40079 \\
\hline 0.0543248 & 0.05587694 & 0.223508 & 1.39692 \\
\hline 0.0541749 & 0.05572276 & 0.222891 & 1.39307 \\
\hline 0.0540255 & 0.05556909 & 0.222276 & 1.38923 \\
\hline 0.0538765 & 0.05541583 & 0.221663 & 1.3854 \\
\hline 0.0537279 & 0.05526298 & 0.221052 & 1.38157 \\
\hline 0.0535798 & 0.05511065 & 0.220442 & 1.37777 \\
\hline 0.053432 & 0.05495863 & 0.219835 & 1.37397 \\
\hline 0.0532848 & 0.05480722 & 0.219229 & 1.37018 \\
\hline 0.0531379 & 0.05465613 & 0.218624 & 1.3664 \\
\hline 0.0529915 & 0.05450554 & 0.218022 & 1.36264 \\
\hline 0.0528455 & 0.05435537 & 0.217421 & 1.35888 \\
\hline 0.0526999 & 0.05420561 & 0.216822 & 1.35514 \\
\hline 0.0525547 & 0.05405626 & 0.216225 & 1.35141 \\
\hline 0.0524099 & 0.05390733 & 0.21563 & 1.34768 \\
\hline 0.0522656 & 0.0537589 & 0.215036 & 1.34397 \\
\hline 0.0521217 & 0.05361089 & 0.214444 & 1.34027 \\
\hline 0.0519782 & 0.05346329 & 0.213853 & 1.33658 \\
\hline 0.0518351 & 0.0533161 & 0.213265 & 1.3329 \\
\hline 0.0516925 & 0.05316943 & 0.212678 & 1.32924 \\
\hline 0.0515502 & 0.05302306 & 0.212092 & 1.32558 \\
\hline 0.0514084 & 0.05287721 & 0.211509 & 1.32193 \\
\hline 0.051267 & 0.05273177 & 0.210927 & 1.31829 \\
\hline 0.051126 & 0.05258674 & 0.210347 & 1.31467 \\
\hline 0.0509853 & 0.05244202 & 0.209768 & 1.31105 \\
\hline 0.0508451 & 0.05229782 & 0.209191 & 1.30745 \\
\hline 0.0507054 & 0.05215412 & 0.208616 & 1.30385 \\
\hline 0.050566 & 0.05201074 & 0.208043 & 1.30027 \\
\hline 0.050427 & 0.05186777 & 0.207471 & 1.29669 \\
\hline 0.0502884 & 0.05172521 & 0.206901 & 1.29313 \\
\hline 0.0501502 & 0.05158306 & 0.206332 & 1.28958 \\
\hline 0.0500124 & 0.05144133 & 0.205765 & 1.28603 \\
\hline 0.0498751 & 0.0513001 & 0.2052 & 1.2825 \\
\hline 0.0497381 & 0.05115919 & 0.204637 & 1.27898 \\
\hline 0.0496015 & 0.05101869 & 0.204075 & 1.27547 \\
\hline 0.0494653 & 0.05087859 & 0.203514 & 1.27196 \\
\hline 0.0493295 & 0.05073891 & 0.202956 & 1.26847 \\
\hline 0.0491941 & 0.05059965 & 0.202399 & 1.26499 \\
\hline 0.0490591 & 0.05046079 & 0.201843 & 1.26152 \\
\hline 0.0489245 & 0.05032234 & 0.201289 & 1.25806 \\
\hline 0.0487902 & 0.05018421 & 0.200737 & 1.25461 \\
\hline 0.0486564 & 0.05004658 & 0.200186 & 1.25116 \\
\hline 0.0485229 & 0.04990927 & 0.199637 & 1.24773 \\
\hline 0.0483899 & 0.04977247 & 0.19909 & 1.24431 \\
\hline 0.0482572 & 0.04963598 & 0.198544 & 1.2409 \\
\hline 0.0481249 & 0.0494999 & 0.198 & 1.2375 \\
\hline 0.047993 & 0.04936423 & 0.197457 & 1.23411 \\
\hline 0.0478615 & 0.04922897 & 0.196916 & 1.23072 \\
\hline 0.0477303 & 0.04909402 & 0.196376 & 1.22735 \\
\hline 0.0475995 & 0.04895949 & 0.195838 & 1.22399 \\
\hline 0.0474692 & 0.04882546 & 0.195302 & 1.22064 \\
\hline
\end{tabular}

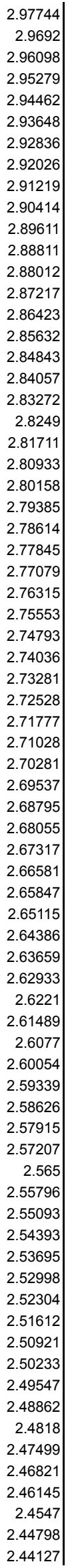

5.95487

5.9384

5.92197

5.90558

5.88925

5.87296

5.85672

5.84052

5.82437

5.80827

5.79222

5.77621

5.76025

5.74433

5.72847

5.71264

5.69686

5.68113

5.66545

5.64981

5.63421

5.61866

5.60315

5.58769

5.57228

5.55691

5.54158

5.5263

5.51106

5.49587

5.48072

5.46561

5.45055

5.43553

5.42056

5.40563

5.39074

5.37589

5.36109

5.34633

5.33161

5.31694

5.30231

5.28772

5.27317

5.25867

5.24421

5.22979

5.21541

5.20107

5.18677

5.17252

5.15831

5.14414

5.13001

5.11592

5.10187

5.08786

5.07389

5.05996

5.04608

5.03223

5.01842

5.00466

4.99093

4.97724

4.9636

4.94999

4.93642

4.92289

4.9094

4.89595

4.88254 


\begin{tabular}{|c|c|c|c|c|c|c|c|c|}
\hline 1471 & 0.00501 & 0.00135255 & 0.0473391 & 0.04869165 & 0.194767 & 1.21729 & 2.43458 & 4.86917 \\
\hline 1472 & 0.005 & 0.00134884 & 0.0472095 & 0.04855834 & 0.194233 & 1.21396 & 2.42792 & 4.85583 \\
\hline 1473 & 0.00499 & 0.00134515 & 0.0470802 & 0.04842535 & 0.193702 & 1.21063 & 2.42127 & 4.84254 \\
\hline 1474 & 0.00498 & 0.00134147 & 0.0469514 & 0.04829287 & 0.193171 & 1.20732 & 2.41464 & 4.82928 \\
\hline 1475 & 0.00497 & 0.0013378 & 0.0468228 & 0.0481606 & 0.192643 & 1.20402 & 2.40803 & 4.81606 \\
\hline 1476 & 0.00496 & 0.00133413 & 0.0466947 & 0.04802883 & 0.192115 & 1.20072 & 2.40144 & 4.80288 \\
\hline 1477 & 0.00495 & 0.00133048 & 0.0465669 & 0.04789738 & 0.19159 & 1.19744 & 2.39487 & 4.78974 \\
\hline 1478 & 0.00494 & 0.00132684 & 0.0464395 & 0.04776634 & 0.191066 & 1.19416 & 2.38832 & 4.77664 \\
\hline 1479 & 0.00493 & 0.00132321 & 0.0463125 & 0.04763571 & 0.190543 & 1.19089 & 2.38179 & 4.76357 \\
\hline 1480 & 0.00492 & 0.0013196 & 0.0461859 & 0.0475055 & 0.190022 & 1.18764 & 2.37527 & 4.75054 \\
\hline 1481 & 0.00491 & 0.00131599 & 0.0460596 & 0.04737559 & 0.189502 & 1.18439 & 2.36878 & 4.73755 \\
\hline 1482 & 0.0049 & 0.00131239 & 0.0459336 & 0.04724599 & 0.188984 & 1.18115 & 2.3623 & 4.7246 \\
\hline 1483 & 0.00489 & 0.0013088 & 0.0458081 & 0.0471169 & 0.188467 & 1.17792 & 2.35584 & 4.71169 \\
\hline 1484 & 0.00488 & 0.00130522 & 0.0456829 & 0.04698812 & 0.187952 & 1.1747 & 2.3494 & 4.69881 \\
\hline 1485 & 0.00487 & 0.00130166 & 0.045558 & 0.04685966 & 0.187439 & 1.17149 & 2.34298 & 4.68597 \\
\hline 1486 & 0.00486 & 0.0012981 & 0.0454336 & 0.0467317 & 0.186927 & 1.16829 & 2.33658 & 4.67317 \\
\hline 1487 & 0.00484 & 0.00129456 & 0.0453094 & 0.04660396 & 0.186416 & 1.1651 & 2.3302 & 4.6604 \\
\hline 1488 & 0.00484 & 0.00129102 & 0.0451857 & 0.04647672 & 0.185907 & 1.16192 & 2.32384 & 4.64767 \\
\hline 1489 & 0.00482 & 0.00128749 & 0.0450623 & 0.04634979 & 0.185399 & 1.15874 & 2.31749 & 4.63498 \\
\hline 1490 & 0.00482 & 0.00128398 & 0.0449393 & 0.04622328 & 0.184893 & 1.15558 & 2.31116 & 4.62232 \\
\hline 1491 & 0.0048 & 0.00128047 & 0.0448166 & 0.04609707 & 0.184388 & 1.15243 & 2.30485 & 4.6097 \\
\hline 1492 & 0.0048 & 0.00127698 & 0.0446942 & 0.04597118 & 0.183885 & 1.14928 & 2.29856 & 4.59712 \\
\hline 1493 & 0.00478 & 0.00127349 & 0.0445723 & 0.04584579 & 0.183383 & 1.14614 & 2.29229 & 4.58458 \\
\hline 1494 & 0.00477 & 0.00127002 & 0.0444506 & 0.04572062 & 0.182883 & 1.14302 & 2.28603 & 4.57207 \\
\hline 1495 & 0.00476 & 0.00126655 & 0.0443294 & 0.04559595 & 0.182384 & 1.1399 & 2.2798 & 4.55959 \\
\hline 1496 & 0.00475 & 0.0012631 & 0.0442085 & 0.0454716 & 0.181886 & 1.13679 & 2.27358 & 4.54716 \\
\hline 1497 & 0.00474 & 0.00125965 & 0.0440879 & 0.04534755 & 0.18139 & 1.13369 & 2.26738 & 4.53476 \\
\hline 1498 & 0.00473 & 0.00125622 & 0.0439677 & 0.04522392 & 0.180896 & 1.1306 & 2.2612 & 4.52239 \\
\hline 1499 & 0.00472 & 0.00125279 & 0.0438478 & 0.04510059 & 0.180402 & 1.12752 & 2.25503 & 4.51006 \\
\hline 1500 & 0.00471 & 0.00124938 & 0.0437283 & 0.04497768 & 0.179911 & 1.12444 & 2.24888 & 4.49777 \\
\hline 1501 & 0.0047 & 0.00124597 & 0.0436091 & 0.04485507 & 0.17942 & 1.12138 & 2.24275 & 4.48551 \\
\hline 1502 & 0.00469 & 0.00124258 & 0.0434903 & 0.04473288 & 0.178931 & 1.11832 & 2.23664 & 4.47329 \\
\hline 1503 & 0.00468 & 0.00123919 & 0.0433718 & 0.04461099 & 0.178444 & 1.11527 & 2.23055 & 4.4611 \\
\hline 1504 & 0.00467 & 0.00123582 & 0.0432537 & 0.04448952 & 0.177958 & 1.11224 & 2.22447 & 4.44895 \\
\hline 1505 & 0.00466 & 0.00123245 & 0.0431359 & 0.04436835 & 0.177473 & 1.10921 & 2.21842 & 4.43683 \\
\hline 1506 & 0.00465 & 0.0012291 & 0.0430184 & 0.0442475 & 0.17699 & 1.10619 & 2.21237 & 4.42475 \\
\hline 1507 & 0.00464 & 0.00122575 & 0.0429013 & 0.04412705 & 0.176508 & 1.10318 & 2.20635 & 4.4127 \\
\hline 1508 & 0.00463 & 0.00122241 & 0.0427845 & 0.04400691 & 0.176028 & 1.10017 & 2.20035 & 4.40069 \\
\hline 1509 & 0.00462 & 0.00121909 & 0.0426681 & 0.04388719 & 0.175549 & 1.09718 & 2.19436 & 4.38871 \\
\hline 1510 & 0.00461 & 0.00121577 & 0.042552 & 0.04376777 & 0.175071 & 1.09419 & 2.18839 & 4.37677 \\
\hline 1511 & 0.0046 & 0.00121246 & 0.0424362 & 0.04364866 & 0.174595 & 1.09122 & 2.18243 & 4.36486 \\
\hline 1512 & 0.00459 & 0.00120916 & 0.0423208 & 0.04352996 & 0.17412 & 1.08825 & 2.1765 & 4.35299 \\
\hline 1513 & 0.00458 & 0.00120588 & 0.0422057 & 0.04341158 & 0.173646 & 1.08529 & 2.17058 & 4.34115 \\
\hline 1514 & 0.00457 & 0.0012026 & 0.0420909 & 0.0432935 & 0.173174 & 1.08234 & 2.16467 & 4.32935 \\
\hline 1515 & 0.00456 & 0.00119933 & 0.0419765 & 0.04317583 & 0.172703 & 1.07939 & 2.15879 & 4.31758 \\
\hline 1516 & 0.00455 & 0.00119607 & 0.0418624 & 0.04305847 & 0.172234 & 1.07646 & 2.15292 & 4.30584 \\
\hline 1517 & 0.00454 & 0.00119282 & 0.0417486 & 0.04294142 & 0.171766 & 1.07353 & 2.14707 & 4.29414 \\
\hline 1518 & 0.00453 & 0.00118958 & 0.0416351 & 0.04282468 & 0.171299 & 1.07062 & 2.14124 & 4.28247 \\
\hline 1519 & 0.00452 & 0.00118634 & 0.041522 & 0.04270834 & 0.170833 & 1.06771 & 2.13542 & 4.27084 \\
\hline 1520 & 0.00451 & 0.00118312 & 0.0414092 & 0.04259232 & 0.170369 & 1.06481 & 2.12962 & 4.25924 \\
\hline 1521 & 0.0045 & 0.00117991 & 0.0412968 & 0.04247671 & 0.169907 & 1.06192 & 2.12383 & 4.24767 \\
\hline 1522 & 0.00449 & 0.0011767 & 0.0411846 & 0.0423613 & 0.169445 & 1.05903 & 2.11807 & 4.23613 \\
\hline 1523 & 0.00448 & 0.00117351 & 0.0410728 & 0.04224631 & 0.168985 & 1.05616 & 2.11232 & 4.22463 \\
\hline 1524 & 0.00447 & 0.00117032 & 0.0409613 & 0.04213162 & 0.168527 & 1.05329 & 2.10658 & 4.21317 \\
\hline 1525 & 0.00446 & 0.00116715 & 0.0408502 & 0.04201735 & 0.168069 & 1.05043 & 2.10087 & 4.20173 \\
\hline 1526 & 0.00445 & 0.00116398 & 0.0407393 & 0.04190328 & 0.167613 & 1.04758 & 2.09517 & 4.19033 \\
\hline 1527 & 0.00444 & 0.00116082 & 0.0406288 & 0.04178962 & 0.167159 & 1.04474 & 2.08948 & 4.17896 \\
\hline 1528 & 0.00443 & 0.00115767 & 0.0405186 & 0.04167627 & 0.166705 & 1.04191 & 2.08381 & 4.16763 \\
\hline 1529 & 0.00442 & 0.00115454 & 0.0404087 & 0.04156324 & 0.166253 & 1.03908 & 2.07816 & 4.15633 \\
\hline 1530 & 0.00441 & 0.0011514 & 0.0402992 & 0.0414506 & 0.165802 & 1.03626 & 2.07253 & 4.14506 \\
\hline 1531 & 0.0044 & 0.00114828 & 0.0401899 & 0.04133818 & 0.165353 & 1.03345 & 2.06691 & 4.13382 \\
\hline 1532 & 0.00439 & 0.00114517 & 0.040081 & 0.04122617 & 0.164905 & 1.03065 & 2.06131 & 4.12262 \\
\hline 1533 & 0.00438 & 0.00114207 & 0.0399724 & 0.04111447 & 0.164458 & 1.02786 & 2.05572 & 4.11144 \\
\hline 1534 & 0.00437 & 0.00113897 & 0.0398641 & 0.04100307 & 0.164012 & 1.02508 & 2.05015 & 4.1003 \\
\hline 1535 & 0.00436 & 0.00113589 & 0.0397561 & 0.04089199 & 0.163568 & 1.0223 & 2.0446 & 4.0892 \\
\hline 1536 & 0.00435 & 0.00113281 & 0.0396484 & 0.04078121 & 0.163125 & 1.01953 & 2.03906 & 4.07812 \\
\hline 1537 & 0.00434 & 0.00112974 & 0.039541 & 0.04067074 & 0.162683 & 1.01677 & 2.03354 & 4.06708 \\
\hline 1538 & 0.00433 & 0.00112669 & 0.039434 & 0.04056069 & 0.162243 & 1.01402 & 2.02803 & 4.05607 \\
\hline 1539 & 0.00432 & 0.00112364 & 0.0393272 & 0.04045084 & 0.161803 & 1.01127 & 2.02254 & 4.04509 \\
\hline 1540 & 0.00431 & 0.00112059 & 0.0392208 & 0.04034139 & 0.161366 & 1.00853 & 2.01707 & 4.03414 \\
\hline 1541 & 0.0043 & 0.00111756 & 0.0391147 & 0.04023226 & 0.160929 & 1.00581 & 2.01161 & 4.02322 \\
\hline 1542 & 0.00429 & 0.00111454 & 0.0390088 & 0.04012334 & 0.160494 & 1.00308 & 2.00617 & 4.01234 \\
\hline 1543 & 0.00428 & 0.00111152 & 0.0389033 & 0.04001482 & 0.160059 & 1.00037 & 2.00074 & 4.00149 \\
\hline
\end{tabular}




\begin{tabular}{|c|c|c|c|c|c|c|c|c|}
\hline 1544 & 0.00427 & 0.00110852 & 0.0387981 & 0.03990662 & 0.159627 & 0.997666 & 1.99533 & 3.99066 \\
\hline 1545 & 0.00426 & 0.00110552 & 0.0386932 & 0.03979872 & 0.159195 & 0.994968 & 1.98994 & 3.97987 \\
\hline 1546 & 0.00426 & 0.00110253 & 0.0385886 & 0.03969113 & 0.158765 & 0.992279 & 1.98456 & 3.96911 \\
\hline 1547 & 0.00424 & 0.00109955 & 0.0384843 & 0.03958385 & 0.158335 & 0.989596 & 1.97919 & 3.95839 \\
\hline 1548 & 0.00424 & 0.00109658 & 0.0383803 & 0.03947688 & 0.157908 & 0.986922 & 1.97384 & 3.94769 \\
\hline 1549 & 0.00422 & 0.00109362 & 0.0382766 & 0.03937022 & 0.157481 & 0.984256 & 1.96851 & 3.93702 \\
\hline 1550 & 0.00422 & 0.00109066 & 0.0381732 & 0.03926386 & 0.157056 & 0.981597 & 1.96319 & 3.92639 \\
\hline 1551 & 0.0042 & 0.00108772 & 0.0380701 & 0.03915782 & 0.156631 & 0.978946 & 1.95789 & 3.91578 \\
\hline 1552 & 0.0042 & 0.00108478 & 0.0379673 & 0.03905208 & 0.156208 & 0.976302 & 1.9526 & 3.90521 \\
\hline 1553 & 0.00418 & 0.00108185 & 0.0378648 & 0.03894665 & 0.155787 & 0.973666 & 1.94733 & 3.89467 \\
\hline 1554 & 0.00418 & 0.00107893 & 0.0377626 & 0.03884153 & 0.155366 & 0.971038 & 1.94208 & 3.88415 \\
\hline 1555 & 0.00417 & 0.00107602 & 0.0376607 & 0.03873672 & 0.154947 & 0.968418 & 1.93684 & 3.87367 \\
\hline 1556 & 0.00416 & 0.00107312 & 0.0375591 & 0.03863222 & 0.154529 & 0.965805 & 1.93161 & 3.86322 \\
\hline 1557 & 0.00415 & 0.00107022 & 0.0374577 & 0.03852792 & 0.154112 & 0.963199 & 1.9264 & 3.8528 \\
\hline 1558 & 0.00414 & 0.00106733 & 0.0373567 & 0.03842403 & 0.153696 & 0.960601 & 1.9212 & 3.8424 \\
\hline 1559 & 0.00413 & 0.00106446 & 0.037256 & 0.03832046 & 0.153282 & 0.958011 & 1.91602 & 3.83204 \\
\hline 1560 & 0.00412 & 0.00106159 & 0.0371555 & 0.03821709 & 0.152868 & 0.955428 & 1.91086 & 3.82171 \\
\hline 1561 & 0.00411 & 0.00105872 & 0.0370554 & 0.03811412 & 0.152456 & 0.952852 & 1.9057 & 3.81141 \\
\hline 1562 & 0.0041 & 0.00105587 & 0.0369555 & 0.03801137 & 0.152045 & 0.950284 & 1.90057 & 3.80114 \\
\hline 1563 & 0.00409 & 0.00105303 & 0.0368559 & 0.03790893 & 0.151636 & 0.947723 & 1.89545 & 3.79089 \\
\hline 1564 & 0.00408 & 0.00105019 & 0.0367566 & 0.03780679 & 0.151227 & 0.94517 & 1.89034 & 3.78068 \\
\hline 1565 & 0.00407 & 0.00104736 & 0.0366576 & 0.03770496 & 0.15082 & 0.942624 & 1.88525 & 3.7705 \\
\hline 1566 & 0.00406 & 0.00104454 & 0.0365589 & 0.03760344 & 0.150414 & 0.940086 & 1.88017 & 3.76034 \\
\hline 1567 & 0.00405 & 0.00104173 & 0.0364604 & 0.03750213 & 0.150009 & 0.937554 & 1.87511 & 3.75022 \\
\hline 1568 & 0.00404 & 0.00103892 & 0.0363623 & 0.03740122 & 0.149605 & 0.935031 & 1.87006 & 3.74012 \\
\hline 1569 & 0.00403 & 0.00103613 & 0.0362644 & 0.03730053 & 0.149202 & 0.932514 & 1.86503 & 3.73006 \\
\hline 1570 & 0.00403 & 0.00103334 & 0.0361668 & 0.03720014 & 0.148801 & 0.930005 & 1.86001 & 3.72002 \\
\hline 1571 & 0.00401 & 0.00103056 & 0.0360695 & 0.03710006 & 0.1484 & 0.927502 & 1.855 & 3.71001 \\
\hline 1572 & 0.00401 & 0.00102779 & 0.0359725 & 0.03700029 & 0.148001 & 0.925008 & 1.85002 & 3.70003 \\
\hline 1573 & 0.00399 & 0.00102502 & 0.0358758 & 0.03690082 & 0.147603 & 0.92252 & 1.84504 & 3.69008 \\
\hline 1574 & 0.00399 & 0.00102227 & 0.0357793 & 0.03680157 & 0.147206 & 0.920039 & 1.84008 & 3.68016 \\
\hline 1575 & 0.00398 & 0.00101952 & 0.0356831 & 0.03670262 & 0.146811 & 0.917566 & 1.83513 & 3.67026 \\
\hline 1576 & 0.00397 & 0.00101678 & 0.0355872 & 0.03660398 & 0.146416 & 0.9151 & 1.8302 & 3.6604 \\
\hline 1577 & 0.00396 & 0.00101405 & 0.0354916 & 0.03650565 & 0.146023 & 0.912641 & 1.82528 & 3.65056 \\
\hline 1578 & 0.00395 & 0.00101132 & 0.0353962 & 0.03640752 & 0.14563 & 0.910189 & 1.82038 & 3.64076 \\
\hline 1579 & 0.00394 & 0.0010086 & 0.0353011 & 0.0363097 & 0.145239 & 0.907744 & 1.81549 & 3.63098 \\
\hline 1580 & 0.00393 & 0.0010059 & 0.0352063 & 0.0362122 & 0.144849 & 0.905306 & 1.81061 & 3.62122 \\
\hline 1581 & 0.00392 & 0.00100319 & 0.0351118 & 0.03611499 & 0.14446 & 0.902875 & 1.80575 & 3.6115 \\
\hline 1582 & 0.00391 & 0.0010005 & 0.0350176 & 0.0360181 & 0.144072 & 0.900451 & 1.8009 & 3.60181 \\
\hline 1583 & 0.0039 & 0.000997816 & 0.0349236 & 0.035921416 & 0.143686 & 0.898035 & 1.79607 & 3.59214 \\
\hline 1584 & 0.00389 & 0.000995139 & 0.0348299 & 0.035825039 & 0.1433 & 0.895625 & 1.79125 & 3.5825 \\
\hline 1585 & 0.00388 & 0.000992469 & 0.0347364 & 0.035728869 & 0.142915 & 0.893222 & 1.78644 & 3.57289 \\
\hline 1586 & 0.00388 & 0.000989807 & 0.0346432 & 0.035633007 & 0.142532 & 0.890826 & 1.78165 & 3.5633 \\
\hline 1587 & 0.00386 & 0.000987152 & 0.0345503 & 0.035537452 & 0.14215 & 0.888437 & 1.77687 & 3.55375 \\
\hline 1588 & 0.00386 & 0.000984505 & 0.0344577 & 0.035442205 & 0.141769 & 0.886055 & 1.77211 & 3.54422 \\
\hline 1589 & 0.00385 & 0.000981866 & 0.0343653 & 0.035347166 & 0.141389 & 0.883679 & 1.76736 & 3.53472 \\
\hline 1590 & 0.00384 & 0.000979234 & 0.0342732 & 0.035252434 & 0.14101 & 0.881311 & 1.76262 & 3.52524 \\
\hline 1591 & 0.00383 & 0.00097661 & 0.0341814 & 0.03515801 & 0.140632 & 0.878949 & 1.7579 & 3.5158 \\
\hline 1592 & 0.00382 & 0.000973994 & 0.0340898 & 0.035063794 & 0.140255 & 0.876595 & 1.75319 & 3.50638 \\
\hline 1593 & 0.00381 & 0.000971385 & 0.0339985 & 0.034969885 & 0.139879 & 0.874246 & 1.74849 & 3.49699 \\
\hline 1594 & 0.0038 & 0.000968784 & 0.0339074 & 0.034876184 & 0.139505 & 0.871905 & 1.74381 & 3.48762 \\
\hline 1595 & 0.00379 & 0.00096619 & 0.0338166 & 0.03478279 & 0.139131 & 0.869571 & 1.73914 & 3.47828 \\
\hline 1596 & 0.00378 & 0.000963603 & 0.0337261 & 0.034689703 & 0.138759 & 0.867243 & 1.73449 & 3.46897 \\
\hline 1597 & 0.00377 & 0.000961024 & 0.0336359 & 0.034596924 & 0.138387 & 0.864922 & 1.72984 & 3.45969 \\
\hline 1598 & 0.00377 & 0.000958453 & 0.0335458 & 0.034504253 & 0.138017 & 0.862608 & 1.72522 & 3.45043 \\
\hline 1599 & 0.00375 & 0.000955889 & 0.0334561 & 0.034411989 & 0.137648 & 0.8603 & 1.7206 & 3.4412 \\
\hline 1600 & 0.00375 & 0.000953332 & 0.0333666 & 0.034319932 & 0.13728 & 0.857999 & 1.716 & 3.432 \\
\hline 1601 & 0.00374 & 0.000950783 & 0.0332774 & 0.034228183 & 0.136913 & 0.855704 & 1.71141 & 3.42282 \\
\hline 1602 & 0.00373 & 0.000948241 & 0.0331884 & 0.034136641 & 0.136547 & 0.853416 & 1.70683 & 3.41367 \\
\hline 1603 & 0.00372 & 0.000945706 & 0.0330997 & 0.034045406 & 0.136182 & 0.851135 & 1.70227 & 3.40454 \\
\hline 1604 & 0.00371 & 0.000943178 & 0.0330112 & 0.033954378 & 0.135818 & 0.848861 & 1.69772 & 3.39544 \\
\hline 1605 & 0.0037 & 0.000940658 & 0.032923 & 0.033863658 & 0.135455 & 0.846592 & 1.69318 & 3.38637 \\
\hline 1606 & 0.00369 & 0.000938145 & 0.0328351 & 0.033773245 & 0.135093 & 0.844331 & 1.68866 & 3.37732 \\
\hline 1607 & 0.00368 & 0.00093564 & 0.0327474 & 0.03368304 & 0.134732 & 0.842076 & 1.68415 & 3.3683 \\
\hline 1608 & 0.00367 & 0.000933141 & 0.0326599 & 0.033593041 & 0.134372 & 0.839827 & 1.67965 & 3.35931 \\
\hline 1609 & 0.00366 & 0.00093065 & 0.0325728 & 0.03350345 & 0.134014 & 0.837585 & 1.67517 & 3.35034 \\
\hline 1610 & 0.00366 & 0.000928166 & 0.0324858 & 0.033413966 & 0.133656 & 0.83535 & 1.6707 & 3.3414 \\
\hline 1611 & 0.00364 & 0.000925689 & 0.0323991 & 0.033324789 & 0.133299 & 0.83312 & 1.66624 & 3.33248 \\
\hline 1612 & 0.00364 & 0.000923219 & 0.0323127 & 0.033235919 & 0.132944 & 0.830898 & 1.6618 & 3.32359 \\
\hline 1613 & 0.00363 & 0.000920757 & 0.0322265 & 0.033147257 & 0.132589 & 0.828681 & 1.65736 & 3.31472 \\
\hline 1614 & 0.00362 & 0.000918301 & 0.0321405 & 0.033058801 & 0.132235 & 0.826471 & 1.65294 & 3.30588 \\
\hline 1615 & 0.00361 & 0.000915853 & 0.0320548 & 0.032970653 & 0.131883 & 0.824267 & 1.64853 & 3.29707 \\
\hline 1616 & 0.0036 & 0.000913411 & 0.0319694 & 0.032882811 & 0.131531 & 0.82207 & 1.64414 & 3.28828 \\
\hline
\end{tabular}




\begin{tabular}{|c|c|c|}
\hline 1617 & 0.00359 & 0.000910977 \\
\hline 1618 & 0.00359 & 0.000908549 \\
\hline 1619 & 0.00357 & 0.000906129 \\
\hline 1620 & 0.00357 & 0.000903715 \\
\hline 1621 & 0.00356 & 0.000901309 \\
\hline 1622 & 0.00355 & 0.000898909 \\
\hline 1623 & 0.00354 & 0.000896516 \\
\hline 1624 & 0.00353 & 0.000894131 \\
\hline 1625 & 0.00352 & 0.000891752 \\
\hline 1626 & 0.00351 & 0.000889379 \\
\hline 1627 & 0.0035 & 0.000887014 \\
\hline 1628 & 0.0035 & 0.000884656 \\
\hline 1629 & 0.00348 & 0.000882304 \\
\hline 1630 & 0.00348 & 0.000879959 \\
\hline 1631 & 0.00347 & 0.00087762 \\
\hline 1632 & 0.00346 & 0.000875289 \\
\hline 1633 & 0.00345 & 0.000872964 \\
\hline 1634 & 0.00344 & 0.000870646 \\
\hline 1635 & 0.00343 & 0.000868334 \\
\hline 1636 & 0.00343 & 0.00086603 \\
\hline 1637 & 0.00341 & 0.000863731 \\
\hline 1638 & 0.00341 & 0.00086144 \\
\hline 1639 & 0.0034 & 0.000859155 \\
\hline 1640 & 0.00339 & 0.000856876 \\
\hline 1641 & 0.00338 & 0.000854604 \\
\hline 1642 & 0.00337 & 0.000852339 \\
\hline 1643 & 0.00336 & 0.00085008 \\
\hline 1644 & 0.00336 & 0.000847827 \\
\hline 1645 & 0.00334 & 0.000845581 \\
\hline 1646 & 0.00334 & 0.000843342 \\
\hline 1647 & 0.00333 & 0.000841108 \\
\hline 1648 & 0.00332 & 0.000838882 \\
\hline 1649 & 0.00331 & 0.000836661 \\
\hline 1650 & 0.0033 & 0.000834447 \\
\hline 1651 & 0.00329 & 0.00083224 \\
\hline 1652 & 0.00329 & 0.000830038 \\
\hline 1653 & 0.00327 & 0.000827843 \\
\hline 1654 & 0.00327 & 0.000825655 \\
\hline 1655 & 0.00326 & 0.000823472 \\
\hline 1656 & 0.00325 & 0.000821296 \\
\hline 1657 & 0.00324 & 0.000819126 \\
\hline 1658 & 0.00323 & 0.000816963 \\
\hline 1659 & 0.00322 & 0.000814805 \\
\hline 1660 & 0.00322 & 0.000812654 \\
\hline 1661 & 0.00321 & 0.000810508 \\
\hline 1662 & 0.0032 & 0.000808369 \\
\hline 1663 & 0.00319 & 0.000806237 \\
\hline 1664 & 0.00318 & 0.00080411 \\
\hline 1665 & 0.00317 & 0.000801989 \\
\hline 1666 & 0.00317 & 0.000799874 \\
\hline 1667 & 0.00315 & 0.000797766 \\
\hline 1668 & 0.00315 & 0.000795663 \\
\hline 1669 & 0.00314 & 0.000793567 \\
\hline 1670 & 0.00313 & 0.000791476 \\
\hline 1671 & 0.00312 & 0.000789392 \\
\hline 1672 & 0.00312 & 0.000787313 \\
\hline 1673 & 0.0031 & 0.00078524 \\
\hline 1674 & 0.0031 & 0.000783174 \\
\hline 1675 & 0.00309 & 0.000781113 \\
\hline 1676 & 0.00308 & 0.000779058 \\
\hline 1677 & 0.00307 & 0.000777009 \\
\hline 1678 & 0.00306 & 0.000774966 \\
\hline 1679 & 0.00305 & 0.000772929 \\
\hline 1680 & 0.00305 & 0.000770897 \\
\hline 1681 & 0.00304 & 0.000768871 \\
\hline 1682 & 0.00303 & 0.000766851 \\
\hline 1683 & 0.00302 & 0.000764837 \\
\hline 1684 & 0.00301 & 0.000762829 \\
\hline 1685 & 0.003 & 0.000760826 \\
\hline 1686 & 0.003 & 0.000758829 \\
\hline 1687 & 0.00299 & 0.000756838 \\
\hline 1688 & 0.00298 & 0.000754853 \\
\hline 1689 & 0.00297 & 0.000752873 \\
\hline
\end{tabular}

\begin{tabular}{|c|c|c|c|}
\hline 0.0318842 & 0.032795177 & 0.131181 & 0.819879 \\
\hline 0.0317992 & 0.032707749 & 0.130831 & 0.817694 \\
\hline 0.0317145 & 0.032620629 & 0.130483 & 0.815516 \\
\hline 0.03163 & 0.032533715 & 0.130135 & 0.813344 \\
\hline 0.0315458 & 0.032447109 & 0.129788 & 0.811178 \\
\hline 0.0314618 & 0.032360709 & 0.129443 & 0.809018 \\
\hline 0.0313781 & 0.032274616 & 0.129098 & 0.806865 \\
\hline 0.0312946 & 0.032188731 & 0.128755 & 0.804718 \\
\hline 0.0312113 & 0.032103052 & 0.128412 & 0.802576 \\
\hline 0.0311283 & 0.032017679 & 0.128071 & 0.800442 \\
\hline 0.0310455 & 0.031932514 & 0.12773 & 0.798313 \\
\hline 0.0309629 & 0.031847556 & 0.12739 & 0.79619 \\
\hline 0.0308806 & 0.031762904 & 0.127052 & 0.794073 \\
\hline 0.0307986 & 0.031678559 & 0.126714 & 0.791963 \\
\hline 0.0307167 & 0.03159432 & 0.126377 & 0.789858 \\
\hline 0.0306351 & 0.031510389 & 0.126042 & 0.78776 \\
\hline 0.0305537 & 0.031426664 & 0.125707 & 0.785668 \\
\hline 0.0304726 & 0.031343246 & 0.125373 & 0.783581 \\
\hline 0.0303917 & 0.031260034 & 0.12504 & 0.781501 \\
\hline 0.030311 & 0.03117703 & 0.124708 & 0.779427 \\
\hline 0.0302306 & 0.031094331 & 0.124377 & 0.777358 \\
\hline 0.0301504 & 0.03101184 & 0.124047 & 0.775296 \\
\hline 0.0300704 & 0.030929555 & 0.123718 & 0.773239 \\
\hline 0.0299907 & 0.030847576 & 0.12339 & 0.771188 \\
\hline 0.0299111 & 0.030765704 & 0.123063 & 0.769144 \\
\hline 0.0298319 & 0.030684239 & 0.122737 & 0.767105 \\
\hline 0.0297528 & 0.03060288 & 0.122411 & 0.765072 \\
\hline 0.029674 & 0.030521827 & 0.122087 & 0.763044 \\
\hline 0.0295953 & 0.030440881 & 0.121764 & 0.761023 \\
\hline 0.029517 & 0.030360342 & 0.121441 & 0.759007 \\
\hline 0.0294388 & 0.030279908 & 0.12112 & 0.756998 \\
\hline 0.0293609 & 0.030199782 & 0.120799 & 0.754994 \\
\hline 0.0292831 & 0.030119761 & 0.120479 & 0.752995 \\
\hline 0.0292057 & 0.030040147 & 0.12016 & 0.751003 \\
\hline 0.0291284 & 0.02996064 & 0.119843 & 0.749016 \\
\hline 0.0290513 & 0.029881338 & 0.119526 & 0.747035 \\
\hline 0.0289745 & 0.029802343 & 0.119209 & 0.745059 \\
\hline 0.0288979 & 0.029723555 & 0.118894 & 0.743089 \\
\hline 0.0288215 & 0.029644972 & 0.11858 & 0.741125 \\
\hline 0.0287454 & 0.029566696 & 0.118267 & 0.739167 \\
\hline 0.0286694 & 0.029488526 & 0.117954 & 0.737214 \\
\hline 0.0285937 & 0.029410663 & 0.117643 & 0.735266 \\
\hline 0.0285182 & 0.029333005 & 0.117332 & 0.733324 \\
\hline 0.0284429 & 0.029255554 & 0.117022 & 0.731388 \\
\hline 0.0283678 & 0.029178308 & 0.116713 & 0.729458 \\
\hline 0.0282929 & 0.029101269 & 0.116405 & 0.727533 \\
\hline 0.0282183 & 0.029024537 & 0.116098 & 0.725613 \\
\hline 0.0281438 & 0.02894791 & 0.115792 & 0.723699 \\
\hline 0.0280696 & 0.028871589 & 0.115486 & 0.72179 \\
\hline 0.0279956 & 0.028795474 & 0.115182 & 0.719887 \\
\hline 0.0279218 & 0.028719566 & 0.114878 & 0.717989 \\
\hline 0.0278482 & 0.028643863 & 0.114576 & 0.716097 \\
\hline 0.0277748 & 0.028568367 & 0.114274 & 0.71421 \\
\hline 0.0277017 & 0.028493176 & 0.113973 & 0.712329 \\
\hline 0.0276287 & 0.028418092 & 0.113672 & 0.710453 \\
\hline 0.027556 & 0.028343313 & 0.113373 & 0.708582 \\
\hline 0.0274834 & 0.02826864 & 0.113075 & 0.706716 \\
\hline 0.0274111 & 0.028194274 & 0.112777 & 0.704856 \\
\hline 0.027339 & 0.028120113 & 0.11248 & 0.703002 \\
\hline 0.027267 & 0.028046058 & 0.112184 & 0.701152 \\
\hline 0.0271953 & 0.027972309 & 0.111889 & 0.699308 \\
\hline 0.0271238 & 0.027898766 & 0.111595 & 0.697469 \\
\hline 0.0270525 & 0.027825429 & 0.111302 & 0.695636 \\
\hline 0.0269814 & 0.027752297 & 0.111009 & 0.693807 \\
\hline 0.0269105 & 0.027679371 & 0.110717 & 0.691984 \\
\hline 0.0268398 & 0.027606651 & 0.110427 & 0.690166 \\
\hline 0.0267693 & 0.027534137 & 0.110137 & 0.688354 \\
\hline 0.026699 & 0.027461829 & 0.109847 & 0.686546 \\
\hline 0.0266289 & 0.027389726 & 0.109559 & 0.684744 \\
\hline 0.026559 & 0.027317829 & 0.109271 & 0.682946 \\
\hline 0.0264893 & 0.027246138 & 0.108985 & 0.681154 \\
\hline 0.0264198 & 0.027174653 & 0.108699 & 0.679367 \\
\hline 0.0263505 & 0.027103373 & 0.108414 & 0.677585 \\
\hline
\end{tabular}

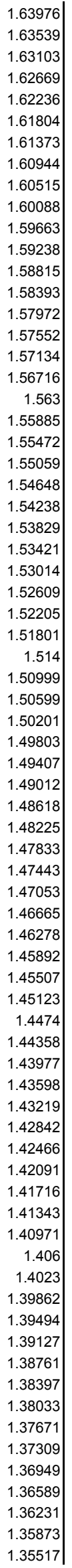

3.27952

3.27078

3.26206

3.25338

3.24471

3.23607

3.22746

3.21887

3.21031

3.20177

3.19325

3.18476

3.17629

3.16785

3.15943

3.15104

3.14267

3.13433

3.126

3.11771

3.10943

3.10118

3.09296

3.08475

3.07657

3.06842

3.06029

3.05218

3.04409

3.03603

3.02799

3.01997

3.01198

3.00401

2.99606

2.98814

2.98024

2.97236

2.9645

2.95667

2.94885

2.94107

2.9333

2.92555

2.91783

2.91013

2.90245

2.8948

2.88716

2.87955

2.87196

2.86439

2.85684

2.84931

2.84181

2.83433

2.82687

2.81943

2.81201

2.80461

2.79723

2.78988

2.78254

2.77523

2.76794

2.76067

2.75341

2.74618

2.73897

2.73179

2.72462

2.71747

2.71034 


\begin{tabular}{|c|c|c|}
\hline 1690 & 0.00296 & 0.000750898 \\
\hline 1691 & 0.00295 & 0.00074893 \\
\hline 1692 & 0.00295 & 0.000746967 \\
\hline 1693 & 0.00294 & 0.00074501 \\
\hline 1694 & 0.00293 & 0.000743058 \\
\hline 1695 & 0.00292 & 0.000741112 \\
\hline 1696 & 0.00291 & 0.000739171 \\
\hline 1697 & 0.0029 & 0.000737236 \\
\hline 1698 & 0.0029 & 0.000735306 \\
\hline 1699 & 0.00289 & 0.000733382 \\
\hline 1700 & 0.00288 & 0.000731464 \\
\hline 1701 & 0.00287 & 0.00072955 \\
\hline 1702 & 0.00287 & 0.000727643 \\
\hline 1703 & 0.00285 & 0.00072574 \\
\hline 1704 & 0.00285 & 0.000723844 \\
\hline 1705 & 0.00284 & 0.000721952 \\
\hline 1706 & 0.00283 & 0.000720066 \\
\hline 1707 & 0.00282 & 0.000718185 \\
\hline 1708 & 0.00282 & 0.00071631 \\
\hline 1709 & 0.0028 & 0.00071444 \\
\hline 1710 & 0.0028 & 0.000712576 \\
\hline 1711 & 0.00279 & 0.000710716 \\
\hline 1712 & 0.00278 & 0.000708862 \\
\hline 1713 & 0.00277 & 0.000707013 \\
\hline 1714 & 0.00277 & 0.00070517 \\
\hline 1715 & 0.00276 & 0.000703332 \\
\hline 1716 & 0.00275 & 0.000701499 \\
\hline 1717 & 0.00274 & 0.000699671 \\
\hline 1718 & 0.00273 & 0.000697848 \\
\hline 1719 & 0.00272 & 0.000696031 \\
\hline 1720 & 0.00272 & 0.000694219 \\
\hline 1721 & 0.00271 & 0.000692411 \\
\hline 1722 & 0.0027 & 0.00069061 \\
\hline 1723 & 0.00269 & 0.000688813 \\
\hline 1724 & 0.00269 & 0.000687021 \\
\hline 1725 & 0.00267 & 0.000685234 \\
\hline 1726 & 0.00267 & 0.000683453 \\
\hline 1727 & 0.00266 & 0.000681676 \\
\hline 1728 & 0.00265 & 0.000679905 \\
\hline 1729 & 0.00264 & 0.000678138 \\
\hline 1730 & 0.00264 & 0.000676377 \\
\hline 1731 & 0.00263 & 0.000674621 \\
\hline 1732 & 0.00262 & 0.000672869 \\
\hline 1733 & 0.00261 & 0.000671123 \\
\hline 1734 & 0.00261 & 0.000669381 \\
\hline 1735 & 0.00259 & 0.000667645 \\
\hline 1736 & 0.00259 & 0.000665913 \\
\hline 1737 & 0.00258 & 0.000664186 \\
\hline 1738 & 0.00257 & 0.000662465 \\
\hline 1739 & 0.00256 & 0.000660748 \\
\hline 1740 & 0.00256 & 0.000659036 \\
\hline 1741 & 0.00255 & 0.000657328 \\
\hline 1742 & 0.00254 & 0.000655626 \\
\hline 1743 & 0.00253 & 0.000653928 \\
\hline 1744 & 0.00253 & 0.000652236 \\
\hline 1745 & 0.00251 & 0.000650548 \\
\hline 1746 & 0.00251 & 0.000648865 \\
\hline 1747 & 0.0025 & 0.000647186 \\
\hline 1748 & 0.00249 & 0.000645513 \\
\hline 1749 & 0.00248 & 0.000643844 \\
\hline 1750 & 0.00248 & 0.00064218 \\
\hline 1751 & 0.00247 & 0.00064052 \\
\hline 1752 & 0.00246 & 0.000638866 \\
\hline 1753 & 0.00245 & 0.000637216 \\
\hline 1754 & 0.00245 & 0.00063557 \\
\hline 1755 & 0.00244 & 0.000633929 \\
\hline 1756 & 0.00243 & 0.000632293 \\
\hline 1757 & 0.00242 & 0.000630662 \\
\hline 1758 & 0.00242 & 0.000629035 \\
\hline 1759 & 0.0024 & 0.000627413 \\
\hline 1760 & 0.0024 & 0.000625795 \\
\hline 1761 & 0.00239 & 0.000624182 \\
\hline 1762 & 0.00238 & 0.000622574 \\
\hline
\end{tabular}

\begin{tabular}{|c|c|c|c|}
\hline 0.0262814 & 0.027032298 & 0.108129 & 0.675809 \\
\hline 0.0262125 & 0.02696143 & 0.107846 & 0.674037 \\
\hline 0.0261438 & 0.026890767 & 0.107563 & 0.67227 \\
\hline 0.0260753 & 0.02682031 & 0.107281 & 0.670509 \\
\hline 0.026007 & 0.026750058 & 0.107 & 0.668752 \\
\hline 0.0259389 & 0.026680012 & 0.10672 & 0.667 \\
\hline 0.025871 & 0.026610171 & 0.106441 & 0.665254 \\
\hline 0.0258033 & 0.026540536 & 0.106162 & 0.663512 \\
\hline 0.0257357 & 0.026471006 & 0.105884 & 0.661776 \\
\hline 0.0256684 & 0.026401782 & 0.105607 & 0.660044 \\
\hline 0.0256012 & 0.026332664 & 0.105331 & 0.658317 \\
\hline 0.0255343 & 0.02626385 & 0.105055 & 0.656595 \\
\hline 0.0254675 & 0.026195143 & 0.104781 & 0.654878 \\
\hline 0.0254009 & 0.02612664 & 0.104507 & 0.653166 \\
\hline 0.0253345 & 0.026058344 & 0.104233 & 0.651459 \\
\hline 0.0252683 & 0.025990252 & 0.103961 & 0.649757 \\
\hline 0.0252023 & 0.025922366 & 0.10369 & 0.64806 \\
\hline 0.0251365 & 0.025854685 & 0.103419 & 0.646367 \\
\hline 0.0250709 & 0.02578721 & 0.103149 & 0.644679 \\
\hline 0.0250054 & 0.02571984 & 0.102879 & 0.642996 \\
\hline 0.0249401 & 0.025652676 & 0.102611 & 0.641318 \\
\hline 0.0248751 & 0.025585816 & 0.102343 & 0.639645 \\
\hline 0.0248102 & 0.025519062 & 0.102076 & 0.637976 \\
\hline 0.0247455 & 0.025452513 & 0.10181 & 0.636312 \\
\hline 0.0246809 & 0.02538607 & 0.101544 & 0.634653 \\
\hline 0.0246166 & 0.025319932 & 0.10128 & 0.632998 \\
\hline 0.0245525 & 0.025253999 & 0.101016 & 0.631349 \\
\hline 0.0244885 & 0.025188171 & 0.100753 & 0.629704 \\
\hline 0.0244247 & 0.025122548 & 0.10049 & 0.628063 \\
\hline 0.0243611 & 0.025057131 & 0.100228 & 0.626428 \\
\hline 0.0242977 & 0.024991919 & 0.0999675 & 0.624797 \\
\hline 0.0242344 & 0.024926811 & 0.0997072 & 0.62317 \\
\hline 0.0241713 & 0.02486191 & 0.0994478 & 0.621549 \\
\hline 0.0241084 & 0.024797213 & 0.099189 & 0.619931 \\
\hline 0.0240457 & 0.024732721 & 0.098931 & 0.618319 \\
\hline 0.0239832 & 0.024668434 & 0.0986737 & 0.616711 \\
\hline 0.0239208 & 0.024604253 & 0.0984172 & 0.615108 \\
\hline 0.0238587 & 0.024540376 & 0.0981614 & 0.613509 \\
\hline 0.0237967 & 0.024476605 & 0.0979063 & 0.611914 \\
\hline 0.0237348 & 0.024412938 & 0.0976519 & 0.610325 \\
\hline 0.0236732 & 0.024349577 & 0.0973983 & 0.608739 \\
\hline 0.0236117 & 0.024286321 & 0.0971454 & 0.607158 \\
\hline 0.0235504 & 0.024223269 & 0.0968932 & 0.605582 \\
\hline 0.0234893 & 0.024160423 & 0.0966417 & 0.60401 \\
\hline 0.0234283 & 0.024097681 & 0.0963909 & 0.602443 \\
\hline 0.0233676 & 0.024035245 & 0.0961408 & 0.60088 \\
\hline 0.023307 & 0.023972913 & 0.0958915 & 0.599322 \\
\hline 0.0232465 & 0.023910686 & 0.0956428 & 0.597768 \\
\hline 0.0231863 & 0.023848765 & 0.0953949 & 0.596218 \\
\hline 0.0231262 & 0.023786948 & 0.0951477 & 0.594673 \\
\hline 0.0230662 & 0.023725236 & 0.0949011 & 0.593132 \\
\hline 0.0230065 & 0.023663828 & 0.0946553 & 0.591596 \\
\hline 0.0229469 & 0.023602526 & 0.0944102 & 0.590063 \\
\hline 0.0228875 & 0.023541428 & 0.0941657 & 0.588536 \\
\hline 0.0228283 & 0.023480536 & 0.093922 & 0.587012 \\
\hline 0.0227692 & 0.023419748 & 0.0936789 & 0.585493 \\
\hline 0.0227103 & 0.023359165 & 0.0934365 & 0.583978 \\
\hline 0.0226515 & 0.023298686 & 0.0931948 & 0.582468 \\
\hline 0.0225929 & 0.023238413 & 0.0929538 & 0.580962 \\
\hline 0.0225345 & 0.023178344 & 0.0927135 & 0.579459 \\
\hline 0.0224763 & 0.02311848 & 0.0924739 & 0.577962 \\
\hline 0.0224182 & 0.02305872 & 0.0922349 & 0.576468 \\
\hline 0.0223603 & 0.022999166 & 0.0919966 & 0.574979 \\
\hline 0.0223025 & 0.022939716 & 0.091759 & 0.573494 \\
\hline 0.022245 & 0.02288057 & 0.0915221 & 0.572013 \\
\hline 0.0221875 & 0.022821429 & 0.0912858 & 0.570536 \\
\hline 0.0221303 & 0.022762593 & 0.0910502 & 0.569064 \\
\hline 0.0220732 & 0.022703862 & 0.0908153 & 0.567596 \\
\hline 0.0220162 & 0.022645235 & 0.090581 & 0.566132 \\
\hline 0.0219594 & 0.022586813 & 0.0903474 & 0.564672 \\
\hline 0.0219028 & 0.022528595 & 0.0901145 & 0.563216 \\
\hline 0.0218464 & 0.022470582 & 0.0898822 & 0.561764 \\
\hline 0.0217901 & 0.022412674 & 0.0896506 & 0.560316 \\
\hline
\end{tabular}

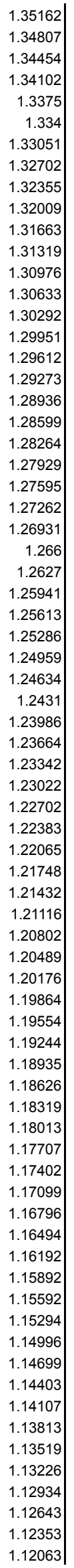

2.70323 2.69615 2.68908 2.68203

Page 25 of 42 


\begin{tabular}{|c|c|c|}
\hline 1763 & 0.00237 & 0.00062097 \\
\hline 1764 & 0.00237 & 0.00061937 \\
\hline 1765 & 0.00236 & 0.000617775 \\
\hline 1766 & 0.00235 & 0.000616185 \\
\hline 1767 & 0.00234 & 0.000614599 \\
\hline 1768 & 0.00234 & 0.000613017 \\
\hline 1769 & 0.00232 & 0.00061144 \\
\hline 1770 & 0.00232 & 0.000609868 \\
\hline 1771 & 0.00231 & 0.0006083 \\
\hline 1772 & 0.00231 & 0.000606736 \\
\hline 1773 & 0.00229 & 0.000605177 \\
\hline 1774 & 0.00229 & 0.000603622 \\
\hline 1775 & 0.00228 & 0.000602072 \\
\hline 1776 & 0.00227 & 0.000600526 \\
\hline 1777 & 0.00226 & 0.000598984 \\
\hline 1778 & 0.00226 & 0.000597447 \\
\hline 1779 & 0.00225 & 0.000595913 \\
\hline 1780 & 0.00224 & 0.000594385 \\
\hline 1781 & 0.00223 & 0.00059286 \\
\hline 1782 & 0.00223 & 0.00059134 \\
\hline 1783 & 0.00222 & 0.000589824 \\
\hline 1784 & 0.00221 & 0.000588313 \\
\hline 1785 & 0.0022 & 0.000586806 \\
\hline 1786 & 0.0022 & 0.000585303 \\
\hline 1787 & 0.00218 & 0.000583804 \\
\hline 1788 & 0.00218 & 0.000582309 \\
\hline 1789 & 0.00217 & 0.000580819 \\
\hline 1790 & 0.00217 & 0.000579333 \\
\hline 1791 & 0.00215 & 0.00057785 \\
\hline 1792 & 0.00215 & 0.000576373 \\
\hline 1793 & 0.00214 & 0.000574899 \\
\hline 1794 & 0.00213 & 0.000573429 \\
\hline 1795 & 0.00212 & 0.000571964 \\
\hline 1796 & 0.00212 & 0.000570503 \\
\hline 1797 & 0.00211 & 0.000569046 \\
\hline 1798 & 0.0021 & 0.000567592 \\
\hline 1799 & 0.00209 & 0.000566143 \\
\hline 1800 & 0.00209 & 0.000564699 \\
\hline 1801 & 0.00208 & 0.000563258 \\
\hline 1802 & 0.00207 & 0.000561821 \\
\hline 1803 & 0.00206 & 0.000560388 \\
\hline 1804 & 0.00206 & 0.000558959 \\
\hline 1805 & 0.00205 & 0.000557535 \\
\hline 1806 & 0.00204 & 0.000556114 \\
\hline 1807 & 0.00203 & 0.000554697 \\
\hline 1808 & 0.00203 & 0.000553284 \\
\hline 1809 & 0.00201 & 0.000551875 \\
\hline 1810 & 0.00201 & 0.000550471 \\
\hline 1811 & 0.002 & 0.00054907 \\
\hline 1812 & 0.002 & 0.000547673 \\
\hline 1813 & 0.00198 & 0.00054628 \\
\hline 1814 & 0.00198 & 0.00054489 \\
\hline 1815 & 0.00197 & 0.000543505 \\
\hline 1816 & 0.00197 & 0.000542124 \\
\hline 1817 & 0.00195 & 0.000540746 \\
\hline 1818 & 0.00195 & 0.000539373 \\
\hline 1819 & 0.00194 & 0.000538003 \\
\hline 1820 & 0.00193 & 0.000536637 \\
\hline 1821 & 0.00192 & 0.000535275 \\
\hline 1822 & 0.00192 & 0.000533916 \\
\hline 1823 & 0.00191 & 0.000532562 \\
\hline 1824 & 0.0019 & 0.000531211 \\
\hline 1825 & 0.00189 & 0.000529864 \\
\hline 1826 & 0.00189 & 0.000528521 \\
\hline 1827 & 0.00188 & 0.000527181 \\
\hline 1828 & 0.00187 & 0.000525846 \\
\hline 1829 & 0.00186 & 0.000524514 \\
\hline 1830 & 0.00186 & 0.000523185 \\
\hline 1831 & 0.00185 & 0.000521861 \\
\hline 1832 & 0.00184 & 0.00052054 \\
\hline 1833 & 0.00183 & 0.000519223 \\
\hline 1834 & 0.00183 & 0.000517909 \\
\hline 1835 & 0.00182 & 0.0005166 \\
\hline
\end{tabular}

\begin{tabular}{|c|c|c|c|}
\hline 0.0217339 & 0.02235487 & 0.0894196 & 0.558873 \\
\hline 0.021678 & 0.02229737 & 0.0891893 & 0.557433 \\
\hline 0.0216221 & 0.022239875 & 0.0889596 & 0.555998 \\
\hline 0.0215665 & 0.022182685 & 0.0887306 & 0.554566 \\
\hline 0.021511 & 0.022125599 & 0.0885022 & 0.553139 \\
\hline 0.0214556 & 0.022068617 & 0.0882745 & 0.551716 \\
\hline 0.0214004 & 0.02201184 & 0.0880474 & 0.550296 \\
\hline 0.0213454 & 0.021955268 & 0.087821 & 0.548881 \\
\hline 0.0212905 & 0.0218988 & 0.0875952 & 0.54747 \\
\hline 0.0212358 & 0.021842536 & 0.08737 & 0.546063 \\
\hline 0.0211812 & 0.021786377 & 0.0871455 & 0.544659 \\
\hline 0.0211268 & 0.021730422 & 0.0869216 & 0.54326 \\
\hline 0.0210725 & 0.021674572 & 0.0866983 & 0.541865 \\
\hline 0.0210184 & 0.021618926 & 0.0864757 & 0.540473 \\
\hline 0.0209644 & 0.021563384 & 0.0862537 & 0.539085 \\
\hline 0.0209106 & 0.021508047 & 0.0860323 & 0.537702 \\
\hline 0.020857 & 0.021452913 & 0.0858115 & 0.536322 \\
\hline 0.0208035 & 0.021397885 & 0.0855914 & 0.534946 \\
\hline 0.0207501 & 0.02134296 & 0.0853719 & 0.533574 \\
\hline 0.0206969 & 0.02128824 & 0.085153 & 0.532206 \\
\hline 0.0206439 & 0.021233724 & 0.0849347 & 0.530842 \\
\hline 0.020591 & 0.021179313 & 0.0847171 & 0.529482 \\
\hline 0.0205382 & 0.021125006 & 0.0845 & 0.528125 \\
\hline 0.0204856 & 0.021070903 & 0.0842836 & 0.526772 \\
\hline 0.0204331 & 0.021016904 & 0.0840677 & 0.525423 \\
\hline 0.0203808 & 0.020963109 & 0.0838525 & 0.524078 \\
\hline 0.0203287 & 0.020909519 & 0.0836379 & 0.522737 \\
\hline 0.0202766 & 0.020855933 & 0.0834239 & 0.521399 \\
\hline 0.0202248 & 0.02080265 & 0.0832105 & 0.520065 \\
\hline 0.020173 & 0.020749373 & 0.0829977 & 0.518735 \\
\hline 0.0201215 & 0.020696399 & 0.0827854 & 0.517409 \\
\hline 0.02007 & 0.020643429 & 0.0825738 & 0.516086 \\
\hline 0.0200187 & 0.020590664 & 0.0823628 & 0.514768 \\
\hline 0.0199676 & 0.020538103 & 0.0821524 & 0.513452 \\
\hline 0.0199166 & 0.020485646 & 0.0819426 & 0.512141 \\
\hline 0.0198657 & 0.020433292 & 0.0817333 & 0.510833 \\
\hline 0.019815 & 0.020381143 & 0.0815247 & 0.509529 \\
\hline 0.0197644 & 0.020329099 & 0.0813166 & 0.508229 \\
\hline 0.019714 & 0.020277258 & 0.0811091 & 0.506932 \\
\hline 0.0196637 & 0.020225521 & 0.0809022 & 0.505639 \\
\hline 0.0196136 & 0.020173988 & 0.0806959 & 0.504349 \\
\hline 0.0195636 & 0.020122559 & 0.0804901 & 0.503063 \\
\hline 0.0195137 & 0.020071235 & 0.080285 & 0.501781 \\
\hline 0.019464 & 0.020020114 & 0.0800804 & 0.500502 \\
\hline 0.0194144 & 0.019969097 & 0.0798764 & 0.499227 \\
\hline 0.0193649 & 0.019918184 & 0.0796729 & 0.497956 \\
\hline 0.0193156 & 0.019867475 & 0.0794701 & 0.496688 \\
\hline 0.0192665 & 0.019816971 & 0.0792678 & 0.495424 \\
\hline 0.0192174 & 0.01976647 & 0.079066 & 0.494163 \\
\hline 0.0191685 & 0.019716173 & 0.0788649 & 0.492905 \\
\hline 0.0191198 & 0.01966608 & 0.0786643 & 0.491652 \\
\hline 0.0190712 & 0.01961609 & 0.0784642 & 0.490401 \\
\hline 0.0190227 & 0.019566205 & 0.0782647 & 0.489155 \\
\hline 0.0189743 & 0.019516424 & 0.0780658 & 0.487911 \\
\hline 0.0189261 & 0.019466846 & 0.0778674 & 0.486672 \\
\hline 0.018878 & 0.019417373 & 0.0776696 & 0.485435 \\
\hline 0.0188301 & 0.019368103 & 0.0774724 & 0.484202 \\
\hline 0.0187823 & 0.019318937 & 0.0772757 & 0.482973 \\
\hline 0.0187346 & 0.019269875 & 0.0770795 & 0.481747 \\
\hline 0.0186871 & 0.019221016 & 0.0768839 & 0.480525 \\
\hline 0.0186397 & 0.019172262 & 0.0766889 & 0.479305 \\
\hline 0.0185924 & 0.019123611 & 0.0764944 & 0.47809 \\
\hline 0.0185452 & 0.019075064 & 0.0763004 & 0.476878 \\
\hline 0.0184982 & 0.019026721 & 0.076107 & 0.475669 \\
\hline 0.0184513 & 0.018978481 & 0.0759141 & 0.474463 \\
\hline 0.0184046 & 0.018930446 & 0.0757218 & 0.473261 \\
\hline 0.018358 & 0.018882514 & 0.07553 & 0.472062 \\
\hline 0.0183115 & 0.018834685 & 0.0753387 & 0.470867 \\
\hline 0.0182651 & 0.018786961 & 0.075148 & 0.469675 \\
\hline 0.0182189 & 0.01873944 & 0.0749578 & 0.468486 \\
\hline 0.0181728 & 0.018692023 & 0.0747681 & 0.467301 \\
\hline 0.0181268 & 0.018644709 & 0.074579 & 0.466119 \\
\hline 0.018081 & 0.0185976 & 0.0743903 & 0.46494 \\
\hline
\end{tabular}

\begin{tabular}{|c|c|}
\hline 1.11775 & 2.23549 \\
\hline 1.11487 & 2.22973 \\
\hline 1.112 & 2.22399 \\
\hline 1.10913 & 2.21827 \\
\hline 1.10628 & 2.21256 \\
\hline 1.10343 & 2.20686 \\
\hline 1.10059 & 2.20119 \\
\hline 1.09776 & 2.19552 \\
\hline 1.09494 & 2.18988 \\
\hline 1.09213 & 2.18425 \\
\hline 1.08932 & 2.17864 \\
\hline 1.08652 & 2.17304 \\
\hline 1.08373 & 2.16746 \\
\hline 1.08095 & 2.16189 \\
\hline 1.07817 & 2.15634 \\
\hline 1.0754 & 2.15081 \\
\hline 1.07264 & 2.14529 \\
\hline 1.06989 & 2.13979 \\
\hline 1.06715 & 2.1343 \\
\hline 1.06441 & 2.12882 \\
\hline 1.06168 & 2.12337 \\
\hline 1.05896 & 2.11793 \\
\hline 1.05625 & 2.1125 \\
\hline 1.05354 & 2.10709 \\
\hline 1.05085 & 2.10169 \\
\hline 1.04816 & 2.09631 \\
\hline 1.04547 & 2.09095 \\
\hline 1.0428 & 2.0856 \\
\hline 1.04013 & 2.08026 \\
\hline 1.03747 & 2.07494 \\
\hline 1.03482 & 2.06964 \\
\hline 1.03217 & 2.06435 \\
\hline 1.02954 & 2.05907 \\
\hline 1.0269 & 2.05381 \\
\hline 1.02428 & 2.04856 \\
\hline 1.02167 & 2.04333 \\
\hline 1.01906 & 2.03812 \\
\hline 1.01646 & 2.03291 \\
\hline 1.01386 & 2.02773 \\
\hline 1.01128 & 2.02256 \\
\hline 1.0087 & 2.0174 \\
\hline 1.00613 & 2.01225 \\
\hline 1.00356 & 2.00712 \\
\hline 1.001 & 2.00201 \\
\hline 0.998455 & 1.99691 \\
\hline 0.995912 & 1.99182 \\
\hline 0.993376 & 1.98675 \\
\hline 0.990847 & 1.98169 \\
\hline 0.988325 & 1.97665 \\
\hline 0.985811 & 1.97162 \\
\hline 0.983303 & 1.96661 \\
\hline 0.980803 & 1.96161 \\
\hline 0.978309 & 1.95662 \\
\hline 0.975823 & 1.95165 \\
\hline 0.973343 & 1.94669 \\
\hline 0.970871 & 1.94174 \\
\hline 0.968405 & 1.93681 \\
\hline 0.965946 & 1.93189 \\
\hline 0.963494 & 1.92699 \\
\hline 0.961049 & 1.9221 \\
\hline 0.958611 & 1.91722 \\
\hline 0.95618 & 1.91236 \\
\hline 0.953755 & 1.90751 \\
\hline 0.951337 & 1.90267 \\
\hline 0.948926 & 1.89785 \\
\hline 0.946522 & 1.89304 \\
\hline 0.944124 & 1.88825 \\
\hline 0.941734 & 1.88347 \\
\hline 0.93935 & 1.8787 \\
\hline 0.936972 & 1.87394 \\
\hline 0.934601 & 1.8692 \\
\hline 0.932237 & 1.86447 \\
\hline 0.929879 & 1.85976 \\
\hline
\end{tabular}




\begin{tabular}{|c|c|c|}
\hline 1836 & 0.00181 & 0.000515294 \\
\hline 1837 & 0.0018 & 0.000513991 \\
\hline 1838 & 0.0018 & 0.000512692 \\
\hline 1839 & 0.00178 & 0.000511397 \\
\hline 1840 & 0.00178 & 0.000510105 \\
\hline 1841 & 0.00177 & 0.000508817 \\
\hline 1842 & 0.00177 & 0.000507533 \\
\hline 1843 & 0.00175 & 0.000506252 \\
\hline 1844 & 0.00175 & 0.000504975 \\
\hline 1845 & 0.00174 & 0.000503701 \\
\hline 1846 & 0.00174 & 0.000502431 \\
\hline 1847 & 0.00172 & 0.000501165 \\
\hline 1848 & 0.00172 & 0.000499902 \\
\hline 1849 & 0.00171 & 0.000498642 \\
\hline 1850 & 0.0017 & 0.000497386 \\
\hline 1851 & 0.00169 & 0.000496133 \\
\hline 1852 & 0.00169 & 0.000494884 \\
\hline 1853 & 0.00168 & 0.000493639 \\
\hline 1854 & 0.00167 & 0.000492397 \\
\hline 1855 & 0.00166 & 0.000491158 \\
\hline 1856 & 0.00166 & 0.000489923 \\
\hline 1857 & 0.00165 & 0.000488691 \\
\hline 1858 & 0.00164 & 0.000487463 \\
\hline 1859 & 0.00163 & 0.000486238 \\
\hline 1860 & 0.00163 & 0.000485016 \\
\hline 1861 & 0.00161 & 0.000483798 \\
\hline 1862 & 0.00161 & 0.000482584 \\
\hline 1863 & 0.0016 & 0.000481372 \\
\hline 1864 & 0.0016 & 0.000480164 \\
\hline 1865 & 0.00158 & 0.000478959 \\
\hline 1866 & 0.00158 & 0.000477758 \\
\hline 1867 & 0.00157 & 0.00047656 \\
\hline 1868 & 0.00157 & 0.000475366 \\
\hline 1869 & 0.00155 & 0.000474174 \\
\hline 1870 & 0.00155 & 0.000472986 \\
\hline 1871 & 0.00154 & 0.000471802 \\
\hline 1872 & 0.00153 & 0.00047062 \\
\hline 1873 & 0.00152 & 0.000469442 \\
\hline 1874 & 0.00152 & 0.000468267 \\
\hline 1875 & 0.00151 & 0.000467096 \\
\hline 1876 & 0.0015 & 0.000465927 \\
\hline 1877 & 0.00149 & 0.000464762 \\
\hline 1878 & 0.00149 & 0.0004636 \\
\hline 1879 & 0.00147 & 0.000462442 \\
\hline 1880 & 0.00147 & 0.000461286 \\
\hline 1881 & 0.00146 & 0.000460134 \\
\hline 1882 & 0.00146 & 0.000458985 \\
\hline 1883 & 0.00144 & 0.000457839 \\
\hline 1884 & 0.00144 & 0.000456696 \\
\hline 1885 & 0.00143 & 0.000455557 \\
\hline 1886 & 0.00142 & 0.00045442 \\
\hline 1887 & 0.00141 & 0.000453287 \\
\hline 1888 & 0.00141 & 0.000452157 \\
\hline 1889 & 0.0014 & 0.00045103 \\
\hline 1890 & 0.00139 & 0.000449906 \\
\hline 1891 & 0.00138 & 0.000448785 \\
\hline 1892 & 0.00138 & 0.000447668 \\
\hline 1893 & 0.00136 & 0.000446553 \\
\hline 1894 & 0.00136 & 0.000445442 \\
\hline 1895 & 0.00135 & 0.000444333 \\
\hline 1896 & 0.00135 & 0.000443228 \\
\hline 1897 & 0.00133 & 0.000442126 \\
\hline 1898 & 0.00133 & 0.000441026 \\
\hline 1899 & 0.00132 & 0.00043993 \\
\hline 1900 & 0.00131 & 0.000438837 \\
\hline 1901 & 0.0013 & 0.000437747 \\
\hline 1902 & 0.0013 & 0.00043666 \\
\hline 1903 & 0.00128 & 0.000435575 \\
\hline 1904 & 0.00128 & 0.000434494 \\
\hline 1905 & 0.00127 & 0.000433416 \\
\hline 1906 & 0.00126 & 0.000432341 \\
\hline 1907 & 0.00125 & 0.000431268 \\
\hline 1908 & 0.00125 & 0.00043019 \\
\hline
\end{tabular}

\begin{tabular}{|c|c|c|c|}
\hline 0.0180353 & 0.018550594 & 0.0742023 & 0.463764 \\
\hline 0.0179897 & 0.018503691 & 0.0740147 & 0.462592 \\
\hline 0.0179442 & 0.018456892 & 0.0738277 & 0.461423 \\
\hline 0.0178989 & 0.018410297 & 0.0736412 & 0.460257 \\
\hline 0.0178537 & 0.018363805 & 0.0734552 & 0.459095 \\
\hline 0.0178086 & 0.018317417 & 0.0732697 & 0.457936 \\
\hline 0.0177637 & 0.018271233 & 0.0730848 & 0.45678 \\
\hline 0.0177188 & 0.018225052 & 0.0729003 & 0.455627 \\
\hline 0.0176741 & 0.018179075 & 0.0727164 & 0.454477 \\
\hline 0.0176295 & 0.018133201 & 0.072533 & 0.453331 \\
\hline 0.0175851 & 0.018087531 & 0.0723501 & 0.452188 \\
\hline 0.0175408 & 0.018041965 & 0.0721677 & 0.451048 \\
\hline 0.0174966 & 0.017996502 & 0.0719858 & 0.449911 \\
\hline 0.0174525 & 0.017951142 & 0.0718044 & 0.448778 \\
\hline 0.0174085 & 0.017905886 & 0.0716236 & 0.447647 \\
\hline 0.0173647 & 0.017860833 & 0.0714432 & 0.44652 \\
\hline 0.017321 & 0.017815884 & 0.0712633 & 0.445396 \\
\hline 0.0172774 & 0.017771039 & 0.071084 & 0.444275 \\
\hline 0.0172339 & 0.017726297 & 0.0709051 & 0.443157 \\
\hline 0.0171905 & 0.017681658 & 0.0707268 & 0.442042 \\
\hline 0.0171473 & 0.017637223 & 0.0705489 & 0.440931 \\
\hline 0.0171042 & 0.017592891 & 0.0703715 & 0.439822 \\
\hline 0.0170612 & 0.017548663 & 0.0701946 & 0.438716 \\
\hline 0.0170183 & 0.017504538 & 0.0700182 & 0.437614 \\
\hline 0.0169756 & 0.017460616 & 0.0698424 & 0.436515 \\
\hline 0.0169329 & 0.017416698 & 0.0696669 & 0.435418 \\
\hline 0.0168904 & 0.017372984 & 0.069492 & 0.434325 \\
\hline 0.016848 & 0.017329372 & 0.0693176 & 0.433235 \\
\hline 0.0168057 & 0.017285864 & 0.0691436 & 0.432148 \\
\hline 0.0167636 & 0.017242559 & 0.0689702 & 0.431064 \\
\hline 0.0167215 & 0.017199258 & 0.0687972 & 0.429982 \\
\hline 0.0166796 & 0.01715616 & 0.0686247 & 0.428904 \\
\hline 0.0166378 & 0.017113166 & 0.0684527 & 0.427829 \\
\hline 0.0165961 & 0.017070274 & 0.0682811 & 0.426757 \\
\hline 0.0165545 & 0.017027486 & 0.06811 & 0.425688 \\
\hline 0.0165131 & 0.016984902 & 0.0679394 & 0.424622 \\
\hline 0.0164717 & 0.01694232 & 0.0677693 & 0.423558 \\
\hline 0.0164305 & 0.016899942 & 0.0675997 & 0.422498 \\
\hline 0.0163894 & 0.016857667 & 0.0674305 & 0.421441 \\
\hline 0.0163483 & 0.016815396 & 0.0672618 & 0.420386 \\
\hline 0.0163075 & 0.016773427 & 0.0670935 & 0.419335 \\
\hline 0.0162667 & 0.016731462 & 0.0669258 & 0.418286 \\
\hline 0.016226 & 0.0166896 & 0.0667585 & 0.41724 \\
\hline 0.0161855 & 0.016647942 & 0.0665916 & 0.416197 \\
\hline 0.016145 & 0.016606286 & 0.0664252 & 0.415158 \\
\hline 0.0161047 & 0.016564834 & 0.0662593 & 0.414121 \\
\hline 0.0160645 & 0.016523485 & 0.0660938 & 0.413086 \\
\hline 0.0160244 & 0.016482239 & 0.0659288 & 0.412055 \\
\hline 0.0159844 & 0.016441096 & 0.0657643 & 0.411027 \\
\hline 0.0159445 & 0.016400057 & 0.0656002 & 0.410001 \\
\hline 0.0159047 & 0.01635912 & 0.0654365 & 0.408978 \\
\hline 0.015865 & 0.016318287 & 0.0652733 & 0.407958 \\
\hline 0.0158255 & 0.016277657 & 0.0651106 & 0.406941 \\
\hline 0.0157861 & 0.01623713 & 0.0649483 & 0.405927 \\
\hline 0.0157467 & 0.016196606 & 0.0647865 & 0.404916 \\
\hline 0.0157075 & 0.016156285 & 0.0646251 & 0.403907 \\
\hline 0.0156684 & 0.016116068 & 0.0644642 & 0.402901 \\
\hline 0.0156294 & 0.016075953 & 0.0643037 & 0.401898 \\
\hline 0.0155905 & 0.016035942 & 0.0641436 & 0.400898 \\
\hline 0.0155517 & 0.015996033 & 0.063984 & 0.3999 \\
\hline 0.015513 & 0.015956228 & 0.0638248 & 0.398905 \\
\hline 0.0154744 & 0.015916526 & 0.0636661 & 0.397913 \\
\hline 0.0154359 & 0.015876926 & 0.0635078 & 0.396924 \\
\hline 0.0153976 & 0.01583753 & 0.0633499 & 0.395937 \\
\hline 0.0153593 & 0.015798137 & 0.0631925 & 0.394953 \\
\hline 0.0153211 & 0.015758847 & 0.0630355 & 0.393972 \\
\hline 0.0152831 & 0.01571976 & 0.062879 & 0.392994 \\
\hline 0.0152451 & 0.015680675 & 0.0627229 & 0.392018 \\
\hline 0.0152073 & 0.015641794 & 0.0625672 & 0.391045 \\
\hline 0.0151696 & 0.015603016 & 0.0624119 & 0.390074 \\
\hline 0.0151319 & 0.015564241 & 0.0622571 & 0.389107 \\
\hline 0.0150944 & 0.015525668 & 0.0621027 & 0.388142 \\
\hline 0.015057 & 0.015487199 & 0.0619487 & 0.387179 \\
\hline
\end{tabular}

\begin{tabular}{|c|c|}
\hline 0.927528 & 1.85506 \\
\hline 0.925184 & 1.85037 \\
\hline 0.922846 & 1.84569 \\
\hline 0.920515 & 1.84103 \\
\hline 0.91819 & 1.83638 \\
\hline 0.915871 & 1.83174 \\
\hline 0.913559 & 1.82712 \\
\hline 0.911254 & 1.82251 \\
\hline 0.908955 & 1.81791 \\
\hline 0.906662 & 1.81332 \\
\hline 0.904376 & 1.80875 \\
\hline 0.902096 & 1.80419 \\
\hline 0.899823 & 1.79965 \\
\hline 0.897555 & 1.79511 \\
\hline 0.895295 & 1.79059 \\
\hline 0.89304 & 1.78608 \\
\hline 0.890792 & 1.78158 \\
\hline 0.88855 & 1.7771 \\
\hline 0.886314 & 1.77263 \\
\hline 0.884084 & 1.76817 \\
\hline 0.881861 & 1.76372 \\
\hline 0.879644 & 1.75929 \\
\hline 0.877433 & 1.75487 \\
\hline 0.875228 & 1.75046 \\
\hline 0.873029 & 1.74606 \\
\hline 0.870837 & 1.74167 \\
\hline 0.86865 & 1.7373 \\
\hline 0.86647 & 1.73294 \\
\hline 0.864296 & 1.72859 \\
\hline 0.862127 & 1.72425 \\
\hline 0.859965 & 1.71993 \\
\hline 0.857809 & 1.71562 \\
\hline 0.855658 & 1.71132 \\
\hline 0.853514 & 1.70703 \\
\hline 0.851376 & 1.70275 \\
\hline 0.849243 & 1.69849 \\
\hline 0.847117 & 1.69423 \\
\hline 0.844996 & 1.68999 \\
\hline 0.842881 & 1.68576 \\
\hline 0.840772 & 1.68154 \\
\hline 0.838669 & 1.67734 \\
\hline 0.836572 & 1.67314 \\
\hline 0.834481 & 1.66896 \\
\hline 0.832395 & 1.66479 \\
\hline 0.830315 & 1.66063 \\
\hline 0.828241 & 1.65648 \\
\hline 0.826173 & 1.65235 \\
\hline 0.82411 & 1.64822 \\
\hline 0.822053 & 1.64411 \\
\hline 0.820002 & 1.64 \\
\hline 0.817957 & 1.63591 \\
\hline 0.815917 & 1.63183 \\
\hline 0.813883 & 1.62777 \\
\hline 0.811854 & 1.62371 \\
\hline 0.809831 & 1.61966 \\
\hline 0.807814 & 1.61563 \\
\hline 0.805802 & 1.6116 \\
\hline 0.803796 & 1.60759 \\
\hline 0.801795 & 1.60359 \\
\hline 0.7998 & 1.5996 \\
\hline 0.79781 & 1.59562 \\
\hline 0.795826 & 1.59165 \\
\hline 0.793847 & 1.58769 \\
\hline 0.791874 & 1.58375 \\
\hline 0.789906 & 1.57981 \\
\hline 0.787944 & 1.57589 \\
\hline 0.785987 & 1.57197 \\
\hline 0.784036 & 1.56807 \\
\hline 0.78209 & 1.56418 \\
\hline 0.780149 & 1.5603 \\
\hline 0.778213 & 1.55643 \\
\hline 0.776283 & 1.55257 \\
\hline 0.774359 & 1.54872 \\
\hline
\end{tabular}




\begin{tabular}{|c|c|c|}
\hline 1909| & 0.00123 & 0.000429133 \\
\hline 1910 & 0.00123 & 0.000428069 \\
\hline 1911 & 0.00122 & 0.000427009 \\
\hline 1912 & 0.00122 & 0.000425951 \\
\hline 1913 & 0.0012 & 0.000424897 \\
\hline 1914 & 0.0012 & 0.000423845 \\
\hline 1915 & 0.00118 & 0.000422796 \\
\hline 1916 & 0.00118 & 0.00042175 \\
\hline $1917 \mid$ & 0.00117 & 0.000420707 \\
\hline 1918 & 0.00117 & 0.000419667 \\
\hline 1919 & 0.00115 & 0.000418629 \\
\hline 1920 & 0.00115 & 0.000417595 \\
\hline 1921 & 0.00113 & 0.000416563 \\
\hline 1922 & 0.00113 & 0.000415534 \\
\hline 1923 & 0.00112 & 0.000414508 \\
\hline 1924 & 0.00111 & 0.000413485 \\
\hline 1925 & 0.0011 & 0.000412465 \\
\hline 1926 & 0.0011 & 0.000411447 \\
\hline 1927 & 0.00108 & 0.000410432 \\
\hline 1928 & 0.00108 & 0.00040942 \\
\hline 1929 & 0.00106 & 0.000408411 \\
\hline 1930 & 0.00106 & 0.000407405 \\
\hline 1931 & 0.00105 & 0.000406401 \\
\hline 1932 & 0.00105 & 0.0004054 \\
\hline 1933 & 0.00103 & 0.000404402 \\
\hline 1934 & 0.00103 & 0.000403406 \\
\hline 1935 & 0.00101 & 0.000402413 \\
\hline 1936 & 0.00101 & 0.000401423 \\
\hline $1937 \mid$ & 0.000994 & 0.000400436 \\
\hline 1938 & 0.000992 & 0.000399452 \\
\hline 1939 & 0.000976 & 0.00039847 \\
\hline 1940 & 0.000974 & 0.00039749 \\
\hline 1941 & 0.000957 & 0.000396514 \\
\hline 1942 & 0.000956 & 0.00039554 \\
\hline 1943 & 0.000939 & 0.000394569 \\
\hline 1944 & 0.000937 & 0.0003936 \\
\hline 1945 & 0.00092 & 0.000392634 \\
\hline 1946 & 0.000918 & 0.000391671 \\
\hline 1947 & 0.000901 & 0.000390711 \\
\hline 1948 & 0.0009 & 0.000389753 \\
\hline 1949 & 0.000882 & 0.000388797 \\
\hline 1950 & 0.00088 & 0.000387845 \\
\hline 1951 & 0.000863 & 0.000386894 \\
\hline 1952 & 0.000861 & 0.000385947 \\
\hline 1953 & 0.000843 & 0.000385002 \\
\hline 1954 & 0.000841 & 0.000384059 \\
\hline 1955 & 0.000823 & 0.00038312 \\
\hline 1956 & 0.000822 & 0.000382182 \\
\hline 1957 & 0.000803 & 0.000381248 \\
\hline 1958 & 0.000801 & 0.000380316 \\
\hline 1959 & 0.000782 & 0.000379386 \\
\hline 1960 & 0.000781 & 0.000378459 \\
\hline 1961 & 0.000761 & 0.000377534 \\
\hline 1962 & 0.00076 & 0.000376612 \\
\hline 1963 & 0.00074 & 0.000375693 \\
\hline 1964 & 0.000739 & 0.000374776 \\
\hline 1965 & 0.000718 & 0.000373861 \\
\hline 1966 & 0.000717 & 0.000372949 \\
\hline 1967 & 0.000696 & 0.00037204 \\
\hline 1968 & 0.000695 & 0.000371133 \\
\hline 1969 & 0.000673 & 0.000370228 \\
\hline 1970 & 0.000672 & 0.000369326 \\
\hline 1971 & 0.00065 & 0.000368426 \\
\hline 1972 & 0.000649 & 0.000367529 \\
\hline 1973 & 0.000626 & 0.000366634 \\
\hline 1974 & 0.000625 & 0.000365742 \\
\hline 1975 & 0.000601 & 0.000364852 \\
\hline 1976 & 0.0006 & 0.000363965 \\
\hline 1977 & 0.000576 & 0.000363079 \\
\hline 1978 & 0.000575 & 0.000362197 \\
\hline 1979 & 0.00055 & 0.000361317 \\
\hline 1980 & 0.000549 & 0.000360439 \\
\hline 1981 & 0.000522 & 0.000359563 \\
\hline
\end{tabular}

\begin{tabular}{|c|c|c|c|}
\hline 0.0150196 & 0.015448733 & 0.0617951 & 0.386219 \\
\hline 0.0149824 & 0.015410469 & 0.061642 & 0.385262 \\
\hline 0.0149453 & 0.015372309 & 0.0614893 & 0.384308 \\
\hline 0.0149083 & 0.015334251 & 0.061337 & 0.383356 \\
\hline 0.0148714 & 0.015296297 & 0.0611851 & 0.382407 \\
\hline 0.0148346 & 0.015258445 & 0.0610337 & 0.38146 \\
\hline 0.0147979 & 0.015220696 & 0.0608826 & 0.380516 \\
\hline 0.0147613 & 0.01518305 & 0.060732 & 0.379575 \\
\hline 0.0147247 & 0.015145407 & 0.0605818 & 0.378636 \\
\hline 0.0146883 & 0.015107967 & 0.060432 & 0.3777 \\
\hline 0.014652 & 0.015070629 & 0.0602826 & 0.376766 \\
\hline 0.0146158 & 0.015033395 & 0.0601336 & 0.375835 \\
\hline 0.0145797 & 0.014996263 & 0.0599851 & 0.374907 \\
\hline 0.0145437 & 0.014959234 & 0.0598369 & 0.373981 \\
\hline 0.0145078 & 0.014922308 & 0.0596892 & 0.373057 \\
\hline 0.014472 & 0.014885485 & 0.0595418 & 0.372137 \\
\hline 0.0144363 & 0.014848765 & 0.0593949 & 0.371218 \\
\hline 0.0144006 & 0.014812047 & 0.0592484 & 0.370302 \\
\hline 0.0143651 & 0.014775532 & 0.0591022 & 0.369389 \\
\hline 0.0143297 & 0.01473912 & 0.0589565 & 0.368478 \\
\hline 0.0142944 & 0.014702811 & 0.0588112 & 0.36757 \\
\hline 0.0142592 & 0.014666605 & 0.0586663 & 0.366664 \\
\hline 0.014224 & 0.014630401 & 0.0585217 & 0.365761 \\
\hline 0.014189 & 0.0145944 & 0.0583776 & 0.36486 \\
\hline 0.0141541 & 0.014558502 & 0.0582338 & 0.363961 \\
\hline 0.0141192 & 0.014522606 & 0.0580905 & 0.363066 \\
\hline 0.0140845 & 0.014486913 & 0.0579475 & 0.362172 \\
\hline 0.0140498 & 0.014451223 & 0.057805 & 0.361281 \\
\hline 0.0140153 & 0.014415736 & 0.0576628 & 0.360393 \\
\hline 0.0139808 & 0.014380252 & 0.057521 & 0.359506 \\
\hline 0.0139464 & 0.01434487 & 0.0573796 & 0.358623 \\
\hline 0.0139122 & 0.01430969 & 0.0572386 & 0.357741 \\
\hline 0.013878 & 0.014274514 & 0.057098 & 0.356863 \\
\hline 0.0138439 & 0.01423944 & 0.0569578 & 0.355986 \\
\hline 0.0138099 & 0.014204469 & 0.0568179 & 0.355112 \\
\hline 0.013776 & 0.0141696 & 0.0566785 & 0.35424 \\
\hline 0.0137422 & 0.014134834 & 0.0565394 & 0.353371 \\
\hline 0.0137085 & 0.014100171 & 0.0564007 & 0.352504 \\
\hline 0.0136749 & 0.014065611 & 0.0562623 & 0.35164 \\
\hline 0.0136413 & 0.014031053 & 0.0561244 & 0.350777 \\
\hline 0.0136079 & 0.013996697 & 0.0559868 & 0.349917 \\
\hline 0.0135746 & 0.013962445 & 0.0558496 & 0.34906 \\
\hline 0.0135413 & 0.013928194 & 0.0557128 & 0.348205 \\
\hline 0.0135081 & 0.013894047 & 0.0555763 & 0.347352 \\
\hline 0.0134751 & 0.013860102 & 0.0554403 & 0.346502 \\
\hline 0.0134421 & 0.013826159 & 0.0553046 & 0.345653 \\
\hline 0.0134092 & 0.01379232 & 0.0551692 & 0.344808 \\
\hline 0.0133764 & 0.013758582 & 0.0550343 & 0.343964 \\
\hline 0.0133437 & 0.013724948 & 0.0548997 & 0.343123 \\
\hline 0.013311 & 0.013691316 & 0.0547654 & 0.342284 \\
\hline 0.0132785 & 0.013657886 & 0.0546316 & 0.341447 \\
\hline 0.0132461 & 0.013624559 & 0.0544981 & 0.340613 \\
\hline 0.0132137 & 0.013591234 & 0.0543649 & 0.339781 \\
\hline 0.0131814 & 0.013558012 & 0.0542322 & 0.338951 \\
\hline 0.0131492 & 0.013524893 & 0.0540998 & 0.338123 \\
\hline 0.0131172 & 0.013491976 & 0.0539677 & 0.337298 \\
\hline 0.0130851 & 0.013458961 & 0.053836 & 0.336475 \\
\hline 0.0130532 & 0.013426149 & 0.0537047 & 0.335654 \\
\hline 0.0130214 & 0.01339344 & 0.0535737 & 0.334836 \\
\hline 0.0129896 & 0.013360733 & 0.0534431 & 0.334019 \\
\hline 0.012958 & 0.013328228 & 0.0533128 & 0.333205 \\
\hline 0.0129264 & 0.013295726 & 0.0531829 & 0.332393 \\
\hline 0.0128949 & 0.013263326 & 0.0530534 & 0.331584 \\
\hline 0.0128635 & 0.013231029 & 0.0529242 & 0.330776 \\
\hline 0.0128322 & 0.013198834 & 0.0527953 & 0.329971 \\
\hline 0.012801 & 0.013166742 & 0.0526669 & 0.329168 \\
\hline 0.0127698 & 0.013134652 & 0.0525387 & 0.328367 \\
\hline 0.0127388 & 0.013102765 & 0.0524109 & 0.327568 \\
\hline 0.0127078 & 0.013070879 & 0.0522834 & 0.326772 \\
\hline 0.0126769 & 0.013039097 & 0.0521563 & 0.325977 \\
\hline 0.0126461 & 0.013007417 & 0.0520296 & 0.325185 \\
\hline 0.0126154 & 0.012975839 & 0.0519032 & 0.324395 \\
\hline 0.0125847 & 0.012944263 & 0.0517771 & 0.323607 \\
\hline
\end{tabular}

\begin{tabular}{|c|c|}
\hline 0.772439 & 1.54488 \\
\hline 0.770525 & 1.54105 \\
\hline 0.768616 & 1.53723 \\
\hline 0.766712 & 1.53342 \\
\hline 0.764814 & 1.52963 \\
\hline 0.762921 & 1.52584 \\
\hline 0.761033 & 1.52207 \\
\hline 0.75915 & 1.5183 \\
\hline 0.757272 & 1.51454 \\
\hline 0.7554 & 1.5108 \\
\hline 0.753533 & 1.50707 \\
\hline 0.751671 & 1.50334 \\
\hline 0.749813 & 1.49963 \\
\hline 0.747962 & 1.49592 \\
\hline 0.746115 & 1.49223 \\
\hline 0.744273 & 1.48855 \\
\hline 0.742436 & 1.48487 \\
\hline 0.740605 & 1.48121 \\
\hline 0.738778 & 1.47756 \\
\hline 0.736956 & 1.47391 \\
\hline 0.73514 & 1.47028 \\
\hline 0.733328 & 1.46666 \\
\hline 0.731521 & 1.46304 \\
\hline 0.72972 & 1.45944 \\
\hline 0.727923 & 1.45585 \\
\hline 0.726131 & 1.45226 \\
\hline 0.724344 & 1.44869 \\
\hline 0.722562 & 1.44512 \\
\hline 0.720785 & 1.44157 \\
\hline 0.719013 & 1.43803 \\
\hline 0.717245 & 1.43449 \\
\hline 0.715483 & 1.43097 \\
\hline 0.713725 & 1.42745 \\
\hline 0.711972 & 1.42394 \\
\hline 0.710224 & 1.42045 \\
\hline 0.708481 & 1.41696 \\
\hline 0.706742 & 1.41348 \\
\hline 0.705008 & 1.41002 \\
\hline 0.703279 & 1.40656 \\
\hline 0.701555 & 1.40311 \\
\hline 0.699835 & 1.39967 \\
\hline 0.69812 & 1.39624 \\
\hline 0.69641 & 1.39282 \\
\hline 0.694704 & 1.38941 \\
\hline 0.693003 & 1.38601 \\
\hline 0.691307 & 1.38261 \\
\hline 0.689615 & 1.37923 \\
\hline 0.687928 & 1.37586 \\
\hline 0.686246 & 1.37249 \\
\hline 0.684568 & 1.36914 \\
\hline 0.682895 & 1.36579 \\
\hline 0.681226 & 1.36245 \\
\hline 0.679562 & 1.35912 \\
\hline 0.677902 & 1.3558 \\
\hline 0.676247 & 1.35249 \\
\hline 0.674596 & 1.34919 \\
\hline 0.67295 & 1.3459 \\
\hline 0.671309 & 1.34262 \\
\hline 0.669672 & 1.33934 \\
\hline 0.668039 & 1.33608 \\
\hline 0.666411 & 1.33282 \\
\hline 0.664787 & 1.32957 \\
\hline 0.663167 & 1.32633 \\
\hline 0.661552 & 1.3231 \\
\hline 0.659942 & 1.31988 \\
\hline 0.658336 & 1.31667 \\
\hline 0.656734 & 1.31347 \\
\hline 0.655136 & 1.31027 \\
\hline 0.653543 & 1.30709 \\
\hline 0.651954 & 1.30391 \\
\hline 0.65037 & 1.30074 \\
\hline 0.64879 & 1.29758 \\
\hline 0.647214 & 1.29443 \\
\hline
\end{tabular}




\begin{tabular}{|c|c|c|}
\hline 1982 & 0.000521 & 0.00035869 \\
\hline 1983 & 0.000493 & 0.000357819 \\
\hline 1984 & 0.000493 & 0.000356951 \\
\hline 1985 & 0.000463 & 0.000356085 \\
\hline 1986 & 0.000463 & 0.000355221 \\
\hline 1987 & 0.000431 & 0.00035436 \\
\hline 1988 & 0.000431 & 0.000353501 \\
\hline 1989 & 0.000397 & 0.000352644 \\
\hline 1990 & 0.000397 & 0.00035179 \\
\hline 1991 & 0.00036 & 0.000350938 \\
\hline 1992 & 0.00036 & 0.000350088 \\
\hline 1993 & 0.000319 & 0.00034924 \\
\hline 1994 & 0.000319 & 0.000348395 \\
\hline 1995 & 0.000273 & 0.000347552 \\
\hline 1996 & 0.000273 & 0.000346712 \\
\hline 1997 & 0.000218 & 0.000345873 \\
\hline 1998 & 0.000218 & 0.000345037 \\
\hline 1999 & 0.000145 & 0.000344204 \\
\hline 2000 & & 0.000343372 \\
\hline 2001 & & 0.000342543 \\
\hline 2002 & & 0.000341716 \\
\hline 2003 & & 0.000340891 \\
\hline 2004 & & 0.000340069 \\
\hline 2005 & & 0.000339248 \\
\hline 2006 & & 0.00033843 \\
\hline 2007 & & 0.000337614 \\
\hline 2008 & & 0.0003368 \\
\hline 2009 & & 0.000335989 \\
\hline 2010 & & 0.00033518 \\
\hline 2011 & & 0.000334372 \\
\hline 2012 & & 0.000333567 \\
\hline 2013 & & 0.000332765 \\
\hline 2014 & & 0.000331964 \\
\hline 2015 & & 0.000331165 \\
\hline 2016 & & 0.000330369 \\
\hline 2017 & & 0.000329575 \\
\hline 2018 & & 0.000328783 \\
\hline 2019 & & 0.000327993 \\
\hline 2020 & & 0.000327205 \\
\hline 2021 & & 0.000326419 \\
\hline 2022 & & 0.000325636 \\
\hline 2023 & & 0.000324854 \\
\hline 2024 & & 0.000324075 \\
\hline 2025 & & 0.000323298 \\
\hline 2026 & & 0.000322523 \\
\hline 2027 & & 0.00032175 \\
\hline 2028 & & 0.000320979 \\
\hline 2029 & & 0.00032021 \\
\hline 2030 & & 0.000319443 \\
\hline 2031 & & 0.000318678 \\
\hline 2032 & & 0.000317915 \\
\hline 2033 & & 0.000317155 \\
\hline 2034 & & 0.000316396 \\
\hline 2035 & & 0.000315639 \\
\hline 2036 & & 0.000314885 \\
\hline 2037 & & 0.000314132 \\
\hline 2038 & & 0.000313382 \\
\hline 2039 & & 0.000312633 \\
\hline 2040 & & 0.000311887 \\
\hline 2041 & & 0.000311142 \\
\hline 2042 & & 0.0003104 \\
\hline 2043 & & 0.000309659 \\
\hline 2044 & & 0.00030892 \\
\hline 2045 & & 0.000308184 \\
\hline 2046 & & 0.000307449 \\
\hline 2047 & & 0.000306717 \\
\hline 2048 & & 0.000305986 \\
\hline 2049 & & 0.000305257 \\
\hline 2050 & & 0.00030453 \\
\hline 2051 & & 0.000303805 \\
\hline 2052 & & 0.000303083 \\
\hline 2053 & & 0.000302362 \\
\hline 2054 & & 0.000301643 \\
\hline
\end{tabular}

\begin{tabular}{|c|c|c|c|}
\hline 0.0125542 & 0.01291289 & 0.0516514 & 0.322821 \\
\hline 0.0125237 & 0.012881519 & 0.051526 & 0.322037 \\
\hline 0.0124933 & 0.012850251 & 0.0514009 & 0.321256 \\
\hline 0.012463 & 0.012819085 & 0.0512762 & 0.320476 \\
\hline 0.0124327 & 0.012787921 & 0.0511518 & 0.319699 \\
\hline 0.0124026 & 0.01275696 & 0.0510278 & 0.318924 \\
\hline 0.0123725 & 0.012726001 & 0.0509041 & 0.318151 \\
\hline 0.0123425 & 0.012695144 & 0.0507807 & 0.31738 \\
\hline 0.0123126 & 0.01266439 & 0.0506577 & 0.316611 \\
\hline 0.0122828 & 0.012633738 & 0.050535 & 0.315844 \\
\hline 0.0122531 & 0.012603188 & 0.0504127 & 0.315079 \\
\hline 0.0122234 & 0.01257264 & 0.0502906 & 0.314316 \\
\hline 0.0121938 & 0.012542195 & 0.0501689 & 0.313556 \\
\hline 0.0121643 & 0.012511852 & 0.0500475 & 0.312797 \\
\hline 0.0121349 & 0.012481612 & 0.0499265 & 0.312041 \\
\hline 0.0121056 & 0.012451473 & 0.0498058 & 0.311286 \\
\hline 0.0120763 & 0.012421337 & 0.0496854 & 0.310534 \\
\hline 0.0120471 & 0.012391304 & 0.0495653 & 0.309783 \\
\hline 0.012018 & 0.012361372 & 0.0494456 & 0.309035 \\
\hline 0.011989 & 0.012331543 & 0.0493262 & 0.308289 \\
\hline 0.0119601 & 0.012301816 & 0.0492071 & 0.307544 \\
\hline 0.0119312 & 0.012272091 & 0.0490883 & 0.306802 \\
\hline 0.0119024 & 0.012242469 & 0.0489699 & 0.306062 \\
\hline 0.0118737 & 0.012212948 & 0.0488517 & 0.305323 \\
\hline 0.0118451 & 0.01218353 & 0.0487339 & 0.304587 \\
\hline 0.0118165 & 0.012154114 & 0.0486164 & 0.303853 \\
\hline 0.011788 & 0.0121248 & 0.0484993 & 0.30312 \\
\hline 0.0117596 & 0.012095589 & 0.0483824 & 0.30239 \\
\hline 0.0117313 & 0.01206648 & 0.0482658 & 0.301662 \\
\hline 0.011703 & 0.012037372 & 0.0481496 & 0.300935 \\
\hline 0.0116749 & 0.012008467 & 0.0480337 & 0.300211 \\
\hline 0.0116468 & 0.011979565 & 0.0479181 & 0.299488 \\
\hline 0.0116187 & 0.011950664 & 0.0478028 & 0.298768 \\
\hline 0.0115908 & 0.011921965 & 0.0476878 & 0.298049 \\
\hline 0.0115629 & 0.011893269 & 0.0475732 & 0.297332 \\
\hline 0.0115351 & 0.011864675 & 0.0474588 & 0.296617 \\
\hline 0.0115074 & 0.011836183 & 0.0473447 & 0.295905 \\
\hline 0.0114798 & 0.011807793 & 0.047231 & 0.295194 \\
\hline 0.0114522 & 0.011779405 & 0.0471175 & 0.294485 \\
\hline 0.0114247 & 0.011751119 & 0.0470044 & 0.293778 \\
\hline 0.0113973 & 0.011722936 & 0.0468916 & 0.293072 \\
\hline 0.0113699 & 0.011694754 & 0.046779 & 0.292369 \\
\hline 0.0113426 & 0.011666675 & 0.0466668 & 0.291668 \\
\hline 0.0113154 & 0.011638698 & 0.0465549 & 0.290968 \\
\hline 0.0112883 & 0.011610823 & 0.0464433 & 0.29027 \\
\hline 0.0112612 & 0.01158295 & 0.0463319 & 0.289575 \\
\hline 0.0112343 & 0.011555279 & 0.0462209 & 0.288881 \\
\hline 0.0112073 & 0.01152751 & 0.0461102 & 0.288189 \\
\hline 0.0111805 & 0.011499943 & 0.0459998 & 0.287499 \\
\hline 0.0111537 & 0.011472378 & 0.0458896 & 0.28681 \\
\hline 0.011127 & 0.011444915 & 0.0457798 & 0.286124 \\
\hline 0.0111004 & 0.011417555 & 0.0456703 & 0.285439 \\
\hline 0.0110739 & 0.011390296 & 0.045561 & 0.284756 \\
\hline 0.0110474 & 0.011363039 & 0.0454521 & 0.284075 \\
\hline 0.011021 & 0.011335885 & 0.0453434 & 0.283396 \\
\hline 0.0109946 & 0.011308732 & 0.045235 & 0.282719 \\
\hline 0.0109684 & 0.011281782 & 0.045127 & 0.282043 \\
\hline 0.0109422 & 0.011254833 & 0.0450192 & 0.28137 \\
\hline 0.010916 & 0.011227887 & 0.0449117 & 0.280698 \\
\hline 0.01089 & 0.011201142 & 0.0448045 & 0.280028 \\
\hline 0.010864 & 0.0111744 & 0.0446975 & 0.27936 \\
\hline 0.0108381 & 0.011147759 & 0.0445909 & 0.278693 \\
\hline 0.0108122 & 0.01112112 & 0.0444845 & 0.278028 \\
\hline 0.0107864 & 0.011094584 & 0.0443785 & 0.277365 \\
\hline 0.0107607 & 0.011068149 & 0.0442727 & 0.276704 \\
\hline 0.0107351 & 0.011041817 & 0.0441672 & 0.276045 \\
\hline 0.0107095 & 0.011015486 & 0.044062 & 0.275387 \\
\hline 0.010684 & 0.010989257 & 0.043957 & 0.274731 \\
\hline 0.0106586 & 0.01096313 & 0.0438524 & 0.274077 \\
\hline 0.0106332 & 0.010937005 & 0.043748 & 0.273425 \\
\hline 0.0106079 & 0.010910983 & 0.0436439 & 0.272774 \\
\hline 0.0105827 & 0.010885062 & 0.0435401 & 0.272125 \\
\hline 0.0105575 & 0.010859143 & 0.0434365 & 0.271478 \\
\hline
\end{tabular}

\begin{tabular}{|c|c|}
\hline 0.645642 & 1.29128 \\
\hline 0.644075 & 1.28815 \\
\hline 0.642512 & 1.28502 \\
\hline 0.640953 & 1.28191 \\
\hline 0.639398 & 1.2788 \\
\hline 0.637847 & 1.27569 \\
\hline 0.636301 & 1.2726 \\
\hline 0.634759 & 1.26952 \\
\hline 0.633221 & 1.26644 \\
\hline 0.631688 & 1.26338 \\
\hline 0.630158 & 1.26032 \\
\hline 0.628633 & 1.25727 \\
\hline 0.627112 & 1.25422 \\
\hline 0.625594 & 1.25119 \\
\hline 0.624081 & 1.24816 \\
\hline 0.622572 & 1.24514 \\
\hline 0.621067 & 1.24213 \\
\hline 0.619567 & 1.23913 \\
\hline 0.61807 & 1.23614 \\
\hline 0.616577 & 1.23315 \\
\hline 0.615089 & 1.23018 \\
\hline 0.613604 & 1.22721 \\
\hline 0.612123 & 1.22425 \\
\hline 0.610647 & 1.22129 \\
\hline 0.609174 & 1.21835 \\
\hline 0.607705 & 1.21541 \\
\hline 0.606241 & 1.21248 \\
\hline 0.60478 & 1.20956 \\
\hline 0.603323 & 1.20665 \\
\hline 0.60187 & 1.20374 \\
\hline 0.600421 & 1.20084 \\
\hline 0.598976 & 1.19795 \\
\hline 0.597535 & 1.19507 \\
\hline 0.596098 & 1.1922 \\
\hline 0.594664 & 1.18933 \\
\hline 0.593235 & 1.18647 \\
\hline 0.591809 & 1.18362 \\
\hline 0.590387 & 1.18077 \\
\hline 0.588969 & 1.17794 \\
\hline 0.587555 & 1.17511 \\
\hline 0.586145 & 1.17229 \\
\hline 0.584738 & 1.16948 \\
\hline 0.583335 & 1.16667 \\
\hline 0.581936 & 1.16387 \\
\hline 0.580541 & 1.16108 \\
\hline 0.579149 & 1.1583 \\
\hline 0.577762 & 1.15552 \\
\hline 0.576377 & 1.15275 \\
\hline 0.574997 & 1.14999 \\
\hline 0.57362 & 1.14724 \\
\hline 0.572248 & 1.1445 \\
\hline 0.570878 & 1.14176 \\
\hline 0.569513 & 1.13903 \\
\hline 0.568151 & 1.1363 \\
\hline 0.566793 & 1.13359 \\
\hline 0.565438 & 1.13088 \\
\hline 0.564087 & 1.12817 \\
\hline 0.56274 & 1.12548 \\
\hline 0.561396 & 1.12279 \\
\hline 0.560056 & 1.12011 \\
\hline 0.558719 & 1.11744 \\
\hline 0.557386 & 1.11477 \\
\hline 0.556057 & 1.11211 \\
\hline 0.554731 & 1.10946 \\
\hline 0.553409 & 1.10682 \\
\hline 0.55209 & 1.10418 \\
\hline 0.550775 & 1.10155 \\
\hline 0.549463 & 1.09893 \\
\hline 0.548155 & 1.09631 \\
\hline 0.54685 & 1.0937 \\
\hline 0.545549 & 1.0911 \\
\hline 0.544251 & 1.0885 \\
\hline 0.542957 & 1.08591 \\
\hline
\end{tabular}


\begin{tabular}{l|r|}
0.0105324 & 0.010833325 \\
\hline
\end{tabular}

0.0105074

0.0104824

0.0104575

0.0104327

0.0104079

0.0103832

0.0103586

0.010334

0.0103095

0.010285

0.0102607

0.0102364

0.0102121

0.0101879

0.0101638

0.0101398

0.0101158

0.0100919

0.010068

0.0100442

0.0100205

0.00999682

0.00997322

0.00994967

0.00992619

0.00990277

0.00987942

0.00985612

0.00983289

0.00980972

0.00978661

0.00976356

0.00974058

0.00971765

0.00969479

0.00967198

0.00964924

0.00962655

0.00960393

0.00958136

0.00955886

0.00953642

0.00951403

0.0094917

0.00946944

0.00944723

0.00942508

0.00940299

0.00938096

0.00935898

0.00933706

0.0093152

0.0092934

0.00927166

0.00924997

0.00922834

0.00920677

0.00918526

0.0091638

0.0091424

0.00912105

0.00909976

0.00907853

0.00905735

0.00903623

0.00901516

0.00899415

0.00897319

0.0089523

0.00893145

0.00891066

0.00888992
0.01080761

0.010781897

0.010756286

0.010730776

0.010705268

0.010679863

0.010654559

0.010629257

0.010604057

0.010578859

0.010553862

0.010528868

0.010503875

0.010478984

0.010454195

0.010429508

0.010404823

0.010380239

0.010355658

0.010331178

0.0103068

0.010282443

0.010258169

0.010233946

0.010209796

0.010185706

0.010161689

0.010137724

0.01011383

0.010089998

0.010066228

0.010042519

0.010018882

0.009995297

0.009971784

0.009948322

0.009924933

0.009901594

0.009878328

0.009855113

0.00983197

0.009808889

0.009785859

0.009762892

0.009739995

0.009717151

0.009694368

0.009671647

0.009648987

0.009626379

0.009603833

0.009581349

0.009558926

0.009536565

0.009514255

0.009492007

0.009469821

0.009447696

0.009425623

0.009403611

0.009381651

0.009359753

0.009337917

0.009316131

0.009294408

0.009272736

0.009251126

0.009229567

0.00920808

0.009186634

0.00916525

0.009143918

\begin{tabular}{|c|c|}
\hline 0.0433333 & 0.270833 \\
\hline 0.0432303 & 0.270189 \\
\hline 0.0431276 & 0.269547 \\
\hline 0.0430251 & 0.268907 \\
\hline 0.0429229 & 0.268268 \\
\hline 0.0428211 & 0.267632 \\
\hline 0.0427194 & 0.266996 \\
\hline 0.0426181 & 0.266363 \\
\hline 0.042517 & 0.265731 \\
\hline 0.0424162 & 0.265101 \\
\hline 0.0423156 & 0.264473 \\
\hline 0.0422154 & 0.263846 \\
\hline 0.0421153 & 0.263221 \\
\hline 0.0420156 & 0.262598 \\
\hline 0.0419161 & 0.261976 \\
\hline 0.0418169 & 0.261356 \\
\hline 0.041718 & 0.260737 \\
\hline 0.0416193 & 0.260121 \\
\hline 0.0415209 & 0.259505 \\
\hline 0.0414227 & 0.258892 \\
\hline 0.0413248 & 0.25828 \\
\hline 0.0412272 & 0.25767 \\
\hline 0.0411298 & 0.257061 \\
\hline 0.0410327 & 0.256454 \\
\hline 0.0409358 & 0.255849 \\
\hline 0.0408392 & 0.255245 \\
\hline 0.0407428 & 0.254643 \\
\hline 0.0406468 & 0.254042 \\
\hline 0.0405509 & 0.253443 \\
\hline 0.0404553 & 0.252846 \\
\hline 0.04036 & 0.25225 \\
\hline 0.0402649 & 0.251656 \\
\hline 0.0401701 & 0.251063 \\
\hline 0.0400755 & 0.250472 \\
\hline 0.0399812 & 0.249882 \\
\hline 0.0398871 & 0.249295 \\
\hline 0.0397933 & 0.248708 \\
\hline 0.0396997 & 0.248123 \\
\hline 0.0396064 & 0.24754 \\
\hline 0.0395133 & 0.246958 \\
\hline 0.0394205 & 0.246378 \\
\hline 0.0393279 & 0.245799 \\
\hline 0.0392355 & 0.245222 \\
\hline 0.0391434 & 0.244647 \\
\hline 0.0390516 & 0.244072 \\
\hline 0.03896 & 0.2435 \\
\hline 0.0388686 & 0.242929 \\
\hline 0.0387775 & 0.242359 \\
\hline 0.0386866 & 0.241791 \\
\hline 0.0385959 & 0.241225 \\
\hline 0.0385055 & 0.240659 \\
\hline 0.0384154 & 0.240096 \\
\hline 0.0383254 & 0.239534 \\
\hline 0.0382357 & 0.238973 \\
\hline 0.0381463 & 0.238414 \\
\hline 0.038057 & 0.237856 \\
\hline 0.037968 & 0.2373 \\
\hline 0.0378793 & 0.236746 \\
\hline 0.0377908 & 0.236192 \\
\hline 0.0377025 & 0.235641 \\
\hline 0.0376144 & 0.23509 \\
\hline 0.0375266 & 0.234541 \\
\hline 0.037439 & 0.233994 \\
\hline 0.0373517 & 0.233448 \\
\hline 0.0372645 & 0.232903 \\
\hline 0.0371776 & 0.23236 \\
\hline 0.0370909 & 0.231818 \\
\hline 0.0370045 & 0.231278 \\
\hline 0.0369183 & 0.230739 \\
\hline 0.0368323 & 0.230202 \\
\hline 0.0367465 & 0.229666 \\
\hline 0.036661 & 0.229131 \\
\hline 0.0365757 & 0.228598 \\
\hline
\end{tabular}

0.541666

0.540378

0.539094

0.537814

0.536537

0.535263

0.533993

0.532726

0.531462

0.530202

0.528945

0.527692

0.526442

0.525195

0.523952

0.522712

0.521475

0.520241

0.519011

0.517784

0.51656

0.51534

0.514122

0.512908

0.511697

0.51049

0.509285

0.508084

0.506886

0.505692

0.5045

0.503312

0.502126

0.500944

0.499765

0.498589

0.497416

0.496247

0.49508

0.493916

0.492756

0.491599

0.490444

0.489293

0.488145

0.487

0.485857

0.484718

0.483582

0.482449

0.481319

0.480192

0.479068

0.477947

0.476828

0.475713

0.474601

0.473491

0.472385

0.471281

0.47018

0.469083

0.467988

0.466896

0.465807

0.46472

0.463637

0.462556

0.461479

0.460404

0.459332

0.458262

0.457196

1.08333

1.08076

1.07819

1.07563

1.07307

1.07053

1.06799

1.06545

1.06292

1.0604

1.05789

1.05538

1.05288

1.05039

1.0479

1.04542

1.04295

1.04048

1.03802

1.03557

1.03312

1.03068

1.02824

1.02582

1.02339

1.02098

1.01857

1.01617

1.01377

1.01138

1.009

1.00662

1.00425

1.00189

0.99953

0.997178

0.994832

0.992493

0.99016

0.987833

0.985512

0.983197

0.980888

0.978586

0.976289

0.973999

0.971715

0.969437

0.967164

0.964898

0.962638

0.960384

0.958135

0.955893

0.953656

0.951426

0.949201

0.946982

0.944769

0.942562

0.940361

0.938165

0.935975

0.933792

0.931613

0.929441

0.927274

0.925113

0.922957 
0.00886924 0.00884861 0.00882804

0.00880752

0.00878706

0.00876664

0.00874629

0.00872598

0.00870573

0.00868553

0.00866538

0.00864529

0.00862525

0.00860526

0.00858533

0.00856544

0.00854561

0.00852583

0.00850611

0.00848643

0.0084668

0.00844723

0.00842771

0.00840824

0.00838882

0.00836944

0.00835013

0.00833086

0.00831164

0.00829247

0.00827335

0.00825428

0.00823526

0.00821629

0.00819737

0.0081785

0.00815968

0.00814091

0.00812219

0.00810351

0.00808488

0.0080663

0.00804778

0.00802929

0.00801086

0.00799247

0.00797414

0.00795584

0.0079376

0.0079194

0.00790126

0.00788316

0.0078651

0.00784709

0.00782913

0.00781122

0.00779335

0.00777553

0.00775775

0.00774002

0.00772234

0.0077047

0.00768711

0.00766956

0.00765206

0.0076346

0.00761719

0.00759983

0.00758251

0.00756523

0.007548

0.00753081

0.00751367 0.009101427 0.00908027 0.009059163 0.009038119

0.009017115

0.008996184

0.008975294

0.008954465

0.008933688

0.008912962

0.008892298

0.008871686

0.008851125

0.008830625

0.008810167

0.00878977

0.008769425

0.008749142

0.008728899

0.008708709

0.008688579

0.008668502

0.008648475

0.0086285

0.008608567

0.008588705

0.008568884

0.008549115

0.008529398

0.008509731

0.008490117

0.008470553

0.008451041

0.008431581

0.008412171

0.008392814

0.008373507

0.008354252

0.008335039

0.008315877

0.008296766

0.008277716

0.008258698

0.008239742

0.008220826

0.008201972

0.00818315

0.008164389

0.008145669

0.00812701

0.008108393

0.008089817

0.008071293

0.00805282

0.008034398

0.008016017

0.007997688

0.0079794

0.007961163

0.007942978

0.007924834

0.007906742

0.00788869

0.00787069

0.007852732

0.007834824

0.007816968

0.007799153

0.007781379

0.007763657

0.007745976

0.007728346
0.0364906

0.0364057

0.0363211

0.0362367

0.0361525

0.0360685

0.0359847

0.0359012

0.0358179

0.0357347

0.0356519

0.0355692

0.0354867

0.0354045

0.0353225

0.0352407

0.0351591

0.0350777

0.0349965

0.0349156

0.0348349

0.0347543

0.034674

0.0345939

0.034514

0.0344343

0.0343548

0.0342755

0.0341965

0.0341176

0.0340389

0.0339605

0.0338822

0.0338042

0.0337263

0.0336487

0.0335713

0.033494

0.033417

0.0333401

0.0332635

0.0331871

0.0331108

0.0330348

0.032959

0.0328833

0.0328079

0.0327326

0.0326576

0.0325827

0.032508

0.0324336

0.0323593

0.0322852

0.0322113

0.0321376

0.0320641

0.0319907

0.0319176

0.0318447

0.0317719

0.0316993

0.031627

0.0315548

0.0314828

0.0314109

0.0313393

0.0312679

0.0311966

0.0311255

0.0310546

0.0309839

0.0309134

0.228066

0.227536

0.227007

0.226479

0.225953

0.225428

0.224904

0.224382

0.223862

0.223342

0.222824

0.222307

0.221792

0.221278

0.220766

0.220254

0.219744

0.219236

0.218728

0.218222

0.217718

0.217214

0.216713

0.216212

0.215712

0.215214

0.214718

0.214222

0.213728

0.213235

0.212743

0.212253

0.211764

0.211276

0.21079

0.210304

0.20982

0.209338

0.208856

0.208376

0.207897

0.207419

0.206943

0.206468

0.205994

0.205521

0.205049

0.204579

0.20411

0.203642

0.203175

0.20271

0.202245

0.201782

0.201321

0.20086

0.2004

0.199942

0.199485

0.199029

0.198574

0.198121

0.197669

0.197217

0.196767

0.196318

0.195871

0.195424

0.194979 
0.000214188 0.0002137

0.000213214

0.00021273

0.000212246

0.000211764

0.000211283

0.000210803

0.000210324

0.000209847

0.000209371

0.000208896

0.000208422

0.00020795

0.000207479

0.000207009

0.00020654

0.000206072

0.000205606

0.000205141

0.000204677

0.000204214

0.000203752

0.000203292

0.000202833

0.000202375

0.000201918

0.000201462

0.000201007

0.000200554

0.000200102

0.000199651

0.000199201

0.000198752

0.000198304

0.000197858

0.000197412

0.000196968

0.000196525

0.000196083

0.000195642

0.000195203

0.000194764

0.000194327

0.00019389

0.000193455

0.000193021

0.000192588

0.000192156

0.000191725

0.000191295

0.000190867

0.000190439

0.000190013

0.000189588

0.000189163

0.00018874

0.000188318

0.000187897

0.000187477

0.000187058

0.00018664

0.000186223

0.000185808

0.000185393

0.00018498

0.000184567

0.000184155

0.000183745

0.000183336

0.000182927

0.00018252

0.000182113
0.00749657

0.0074795

0.0074625

0.00744554

0.00742861

0.00741173

0.0073949

0.0073781

0.00736135

0.00734465

0.00732798

0.00731136

0.00729478

0.00727825

0.00726176

0.00724531

0.0072289

0.00721253

0.00719621

0.00717993

0.00716369

0.00714749

0.00713133

0.00711522

0.00709914

0.00708311

0.00706712

0.00705117

0.00703525

0.00701939

0.00700356

0.00698777

0.00697202

0.00695631

0.00694065

0.00692502

0.00690943

0.00689389

0.00687838

0.00686291

0.00684748

0.00683209

0.00681674

0.00680143

0.00678616

0.00677093

0.00675573

0.00674058

0.00672546

0.00671038

0.00669534

0.00668034

0.00666538

0.00665045

0.00663556

0.00662072

0.0066059

0.00659113

0.00657639

0.0065617

0.00654703

0.00653241

0.00651782

0.00650327

0.00648876

0.00647428

0.00645984

0.00644544

0.00643107

0.00641675

0.00640245

0.0063882

0.00637397
0.007710758 0.00769321 0.007675714

0.00765827

0.007640856

0.007623494

0.007606183

0.007588903

0.007571674

0.007554497

0.007537351

0.007520256

0.007503202

0.0074862

0.007469239

0.007452319

0.00743544

0.007418602

0.007401816

0.007385071

0.007368367

0.007351704

0.007335082

0.007318512

0.007301973

0.007285485

0.007269038

0.007252632

0.007236257

0.007219944

0.007203662

0.007187421

0.007171221

0.007155062

0.007138954

0.007122878

0.007106842

0.007090858

0.007074905

0.007058993

0.007043122

0.007027293

0.007011504

0.006995757

0.00698005

0.006964385

0.006948751

0.006933168

0.006917616

0.006902105

0.006886635

0.006871207

0.006855819

0.006840463

0.006825148

0.006809883

0.00679464

0.006779448

0.006764287

0.006749177

0.006734088

0.00671905

0.006704043

0.006689078

0.006674153

0.00665926

0.006644407

0.006629595

0.006614815

0.006600086

0.006585377

0.00657072

0.006556083

0.0307729

0.0307029

0.0306331

0.0305634

0.030494

0.0304247

0.0303556

0.0302867

0.030218

0.0301494

0.030081

0.0300128

0.0299448

0.0298769

0.0298093

0.0297418

0.0296744

0.0296073

0.0295403

0.0294734

0.0294068

0.0293403

0.029274

0.0292079

0.0291419

0.0290761

0.0290105

0.028945

0.0288798

0.0288146

0.0287497

0.0286849

0.0286203

0.0285558

0.0284915

0.0284274

0.0283634

0.0282996

0.028236

0.0281725

0.0281092

0.028046

0.027983

0.0279202

0.0278575

0.027795

0.0277327

0.0276705

0.0276084

0.0275465

0.0274848

0.0274233

0.0273619

0.0273006

0.0272395

0.0271786

0.0271178

0.0270572

0.0269967

0.0269364

0.0268762

0.0268162

0.0267563

0.0266966

0.0266371

0.0265776

0.0265184

0.0264593

0.0264003 
0.000181708

0.000181304

0.000180901

0.000180498

0.000180097

0.000179697

0.000179298

0.0001789

0.000178503

0.000178106

0.000177711

0.000177317

0.000176924

0.000176532

0.000176141

0.00017575

0.000175361

0.000174973

0.000174586

0.000174199

0.000173814

0.00017343

0.000173046

0.000172664

0.000172283

0.000171902

0.000171523

0.000171144

0.000170766

0.00017039

0.000170014

0.000169639

0.000169265

0.000168892

0.000168521

0.000168149

0.000167779

0.00016741

0.000167042

0.000166675

0.000166308

0.000165943

0.000165578

0.000165215

0.000164852

0.00016449

0.000164129

0.000163769

0.00016341

0.000163052

0.000162694

0.000162338

0.000161982

0.000161628

0.000161274

0.000160921

0.000160569

0.000160218

0.000159868

0.000159518

0.00015917

0.000158822

0.000158475

0.000158129

0.000157784

0.00015744

0.000157097

0.000156754

0.000156413

0.000156072

0.000155732

0.000155393

0.000155055 $0.00635979|\quad 0.006541498|$ 0.00634564

0.00633153

0.00631745

0.00630341

0.0062894

0.00627543

0.00626149

0.00624759

0.00623372

0.00621989

0.0062061

0.00619234

0.00617861

0.00616492

0.00615126

0.00613764

0.00612405

0.0061105

0.00609698

0.00608349

0.00607004

0.00605662

0.00604324

0.00602989

0.00601657

0.00600329

0.00599004

0.00597682

0.00596364

0.00595049

0.00593737

0.00592429

0.00591124

0.00589822

0.00588523

0.00587228

0.00585936

0.00584647

0.00583361

0.00582079

0.005808

0.00579524

0.00578251

0.00576982

0.00575715

0.00574452

0.00573192

0.00571935

0.00570681

0.0056943

0.00568183

0.00566938

0.00565697

0.00564459

0.00563224

0.00561992

0.00560763

0.00559537

0.00558314

0.00557094

0.00555877

0.00554664

0.00553453

0.00552246

0.00551041

0.00549839

0.0054864

0.00547445

0.00546252

0.00545062

0.00543876

0.00542692 0.006526944

0.006512431

0.006497948

0.006483507

0.006454728

0.00644039

0.006426093

0.006411826

0.006397601

0.006383417

0.006369264

0.006355142

0.006341061

0.00632701

0.006313001

0.006299023

0.006285086

0.006271179

0.006257304

0.00624347

0.006229666

0.006215904

0.006202173

0.006188472

0.006174813

0.006161184

0.006147586

0.00613403

0.006120504

0.006107009

0.006093555

0.006080132

0.006066741

0.006053379

0.006040059

0.00602677

0.006013512

0.006000285

0.005987098

0.005973943

0.005960818

0.005947725

0.005934672

0.00592164

0.005908649

0.005895689

0.00588276

0.005869862

0.005856994

0.005844168

0.005831362

0.005818598

0.005805864

0.005793161

0.005780489

0.005767848

0.005755238

0.005742658

0.00573011

0.005717592

0.005705115

0.005692659

0.005680244

0.00566785

0.005655487

0.005643154

0.005630863

0.005618592

0.005606352

0.005594153

0.005581975
0.006469097

0.0261078

0.0260497

0.0259918

0.025934

0.0258764

0.0258189

0.0257616

0.0257044

0.0256473

0.0255904

0.0255337

0.025477

0.0254206

0.0253642

0.025308

0.025252

0.0251961

0.0251403

0.0250847

0.0250292

0.0249739

0.0249187

0.0248636

0.0248087

0.0247539

0.0246993

0.0246447

0.0245904

0.0245361

0.024482

0.024428

0.0243742

0.0243205

0.024267

0.0242135

0.0241602

0.0241071

0.024054

0.0240011

0.0239484

0.0238958

0.0238433

0.0237909

0.0237387

0.0236866

0.0236346

0.0235827

0.023531

0.0234794

0.023428

0.0233767

0.0233255

0.0232744

0.0232235

0.0231726

0.023122

0.0230714

0.023021

0.0229706

0.0229205

0.0228704

0.0228205

0.0227706

0.022721

0.0226714

0.022622

0.0225726

0.0225234

0.0224744 
0.000154717

0.000154381

0.000154045

0.00015371

0.000153376

0.000153043

0.00015271

0.000152379

0.000152048

0.000151718

0.000151389

0.000151061

0.000150733

0.000150407

0.000150081

0.000149756

0.000149431

0.000149108

0.000148785

0.000148463

0.000148142

0.000147822

0.000147503

0.000147184

0.000146866

0.000146549

0.000146233

0.000145917

0.000145602

0.000145288

0.000144975

0.000144663

0.000144351

0.00014404

0.00014373

0.00014342

0.000143112

0.000142804

0.000142497

0.000142191

0.000141885

0.00014158

0.000141276

0.000140973

0.00014067

0.000140368

0.000140067

0.000139767

0.000139467

0.000139168

0.00013887

0.000138572

0.000138276

0.00013798

0.000137684

0.00013739

0.000137096

0.000136803

0.000136511

0.000136219

0.000135928

0.000135638

0.000135348

0.000135059

0.000134771

0.000134484

0.000134197

0.000133911

0.000133626

0.000133341

0.000133057

0.000132774

0.000132491
0.00541511

0.00540333

0.00539158

0.00537986

0.00536816

0.0053565

0.00534487

0.00533326

0.00532169

0.00531014

0.00529862

0.00528713

0.00527567

0.00526423

0.00525283

0.00524145

0.0052301

0.00521878

0.00520749

0.00519622

0.00518498

0.00517378

0.00516259

0.00515144

0.00514031

0.00512921

0.00511814

0.0051071

0.00509608

0.00508509

0.00507413

0.00506319

0.00505228

0.0050414

0.00503054

0.00501972

0.00500891

0.00499814

4.99E-03

$4.98 \mathrm{E}-03$

4. $97 \mathrm{E}-03$

4.96E-03

4.94E-03

4.93E-03

4.92E-03

$4.91 \mathrm{E}-03$

$4.90 \mathrm{E}-03$

4.89E-03

$4.88 \mathrm{E}-03$

4.87E-03

4.86E-03

4.85E-03

4.84E-03

4.83E-03

4.82E-03

$4.81 \mathrm{E}-03$

4.80E-03

4.79E-03

4.78E-03

4.77E-03

4.76E-03

4.75E-03

4.74E-03

4.73E-03

4.72E-03

4.71E-03

4.70E-03

4.69E-03

4.68E-03

4.67E-03

4.66E-03

4.65E-03

4.64E-03
0.005569827

0.005557711

0.005545625

0.00553357

0.005521536

0.005509543

0.00549758

0.005485639

0.005473738

0.005461858

0.005450009

0.005438191

0.005426403

0.005414637

0.005402911

0.005391206

0.005379531

0.005367888

0.005356275

0.005344683

0.005333122

0.005321602

0.005310093

0.005298624

0.005287176

0.005275759

0.005264373

0.005253017

0.005241682

0.005230378

0.005219105

0.005207853

0.005196631

0.00518544

0.00517427

0.00516314

0.005152022

0.005140944

5.13E-03

5.12E-03

$5.11 \mathrm{E}-03$

5.10E-03

$5.09 \mathrm{E}-03$

$5.08 \mathrm{E}-03$

5.06E-03

5.05E-03

5.04E-03

5.03E-03

5.02E-03

$5.01 \mathrm{E}-03$

5.00E-03

4. $99 \mathrm{E}-03$

4.98E-03

4.97E-03

4.96E-03

4.95E-03

4.94E-03

4.92E-03

4.91E-03

4.90E-03

$4.89 \mathrm{E}-03$

4.88E-03

4.87E-03

4.86E-03

4.85E-03

4.84E-03

4.83E-03

4.82E-03

$4.81 \mathrm{E}-03$

4.80E-03

4.79E-03

4.78E-03

4.77E-03
0.022279
0.022230
0.022182
0.022134
0.0220862
0.022038
0.021990
0.021942
0.021894
0.021847
0.021
0.0217528

0.0217528

0.0217056

0.0216586

0.0216116

0.0215648

0.0215181

0.0214716

0.0214251

0.0213787

0.0213325

0.0212864

0.0212404

0.0211945

0.0211487

0.0211031

0.0210575

0.0210121

0.0209667

0.0209215

0.0208764

0.0208314

0.0207865

0.0207418

0.0206971

0.0206525

0.0206081

0.0205638

0.0205195

0.0204754

0.0204314

0.0203875

0.0203437

0.0203001

0.0202565

0.020213

0.0201696

0.0201264

0.0200832

0.0200402

0.0199973

0.0199544

0.0199117

0.0198691

0.0198266

0.0197841

0.0197418

0.0196996

0.0196575

0.0196155

0.0195736

0.0195318

0.0194901

0.0194485

0.019407

0.0193657

0.0193244

0.0192832

0.0192421

0.0192011

0.0191602

0.0191194

0.0190788

0.139246

0.138943

0.138641

0.138339

0.138039 
4.63E-03

4.62E-03

4.61E-03

$4.60 \mathrm{E}-03$

4.59E-03

$4.58 \mathrm{E}-03$

4.57E-03

$4.56 \mathrm{E}-03$

4.55E-03

4.54E-03

$4.53 \mathrm{E}-03$

$4.52 \mathrm{E}-03$

$4.51 \mathrm{E}-03$

$4.50 \mathrm{E}-03$

4.49E-03

$4.48 \mathrm{E}-03$

4.47E-03

4. $46 \mathrm{E}-03$

4.45E-03

4.44E-03

4. $44 \mathrm{E}-03$

4.43E-03

4.42E-03

$4.41 \mathrm{E}-03$

4. $40 \mathrm{E}-03$

4. $39 \mathrm{E}-03$

4. $38 \mathrm{E}-03$

4. $37 \mathrm{E}-03$

4. $36 \mathrm{E}-03$

4.35E-03

4.34E-03

4.33E-03

4.32E-03

4.31E-03

4.31E-03

4. $30 \mathrm{E}-03$

4.29E-03

4.28E-03

4.27E-03

4. $26 \mathrm{E}-03$

4.25E-03

4.24E-03

4.23E-03

4.23E-03

4.22E-03

$4.21 \mathrm{E}-03$

4.20E-03

4.19E-03

4.18E-03

4.17E-03

4.16E-03

4.15E-03

4.15E-03

4.14E-03

4.13E-03

4.12E-03

4.11E-03

4.10E-03

4.09E-03

4.09E-03

4.08E-03

4.07E-03

4.06E-03

4.05E-03

4.04E-03

4.03E-03

4.03E-03

4.02E-03

4.01E-03

4.00E-03

$3.99 \mathrm{E}-03$

3.98E-03

3.98E-03
4.76E-03

4.75E-03

$4.74 \mathrm{E}-03$

4.73E-03

$4.72 \mathrm{E}-03$

4.71E-03

4.70E-03

4.69E-03

$4.68 \mathrm{E}-03$

4.67E-03

4.66E-03

4.65E-03

4.64E-03

4.63E-03

4.62E-03

$4.61 \mathrm{E}-03$

4.60E-03

4. $.59 \mathrm{E}-03$

$4.58 \mathrm{E}-03$

4.57E-03

4.56E-03

$4.55 \mathrm{E}-03$

4.54E-03

4.53E-03

4.52E- 03

4.51E-03

4.50E-03

4. $49 \mathrm{E}-03$

$4.49 \mathrm{E}-03$

$4.48 \mathrm{E}-03$

4.47E-03

$4.46 \mathrm{E}-03$

4. $45 \mathrm{E}-03$

4.44E-03

4.43E-03

4.42E-03

$4.41 \mathrm{E}-03$

$4.40 \mathrm{E}-03$

4. 39E-03

4.38E-03

4.37E-03

4. $36 \mathrm{E}-03$

4.35E-03

$4.35 \mathrm{E}-03$

4.34E-03

4.33E-03

4.32E-03

4. $31 \mathrm{E}-03$

4.30E-03

4.29E-03

$4.28 \mathrm{E}-03$

$4.27 \mathrm{E}-03$

4.26E-03

$4.26 \mathrm{E}-03$

4.25E-03

4.24E-03

$4.23 \mathrm{E}-03$

$4.22 \mathrm{E}-03$

4.21E-03

$4.20 \mathrm{E}-03$

4.19E-03

4.18E-03

4.18E-03

4.17E-03

4.16E-03

4.15E-03

4.14E-03

4.13E-03

4.12E-03

4.12E-03

4.11E-03

4.10E-03

4.09E-03

\begin{tabular}{|c|c|}
\hline 0.0190382 & 0.118989 \\
\hline 0.0189977 & 0.118735 \\
\hline 0.0189573 & 0.118483 \\
\hline 0.018917 & 0.118231 \\
\hline 0.0188768 & 0.11798 \\
\hline 0.0188367 & 0.117729 \\
\hline 0.0187967 & 0.117479 \\
\hline 0.0187568 & 0.11723 \\
\hline 0.018717 & 0.116981 \\
\hline 0.0186773 & 0.116733 \\
\hline 0.0186377 & 0.116486 \\
\hline 0.0185982 & 0.116239 \\
\hline 0.0185588 & 0.115992 \\
\hline 0.0185194 & 0.115746 \\
\hline 0.0184802 & 0.115501 \\
\hline 0.0184411 & 0.115257 \\
\hline 0.018402 & 0.115013 \\
\hline 0.0183631 & 0.114769 \\
\hline 0.0183243 & 0.114527 \\
\hline 0.0182855 & 0.114284 \\
\hline 0.0182468 & 0.114043 \\
\hline 0.0182083 & 0.113802 \\
\hline 0.0181698 & 0.113561 \\
\hline 0.0181314 & 0.113321 \\
\hline 0.0180931 & 0.113082 \\
\hline 0.0180549 & 0.112843 \\
\hline 0.0180168 & 0.112605 \\
\hline 0.0179788 & 0.112368 \\
\hline 0.0179409 & 0.112131 \\
\hline 0.0179031 & 0.111894 \\
\hline 0.0178654 & 0.111658 \\
\hline 0.0178277 & 0.111423 \\
\hline 0.0177902 & 0.111189 \\
\hline 0.0177527 & 0.110954 \\
\hline 0.0177153 & 0.110721 \\
\hline 0.017678 & 0.110488 \\
\hline 0.0176409 & 0.110255 \\
\hline 0.0176038 & 0.110023 \\
\hline 0.0175668 & 0.109792 \\
\hline 0.0175298 & 0.109561 \\
\hline 0.017493 & 0.109331 \\
\hline 0.0174562 & 0.109102 \\
\hline 0.0174196 & 0.108872 \\
\hline 0.017383 & 0.108644 \\
\hline 0.0173466 & 0.108416 \\
\hline 0.0173102 & 0.108188 \\
\hline 0.0172739 & 0.107962 \\
\hline 0.0172376 & 0.107735 \\
\hline 0.0172015 & 0.107509 \\
\hline 0.0171655 & 0.107284 \\
\hline 0.0171295 & 0.107059 \\
\hline 0.0170936 & 0.106835 \\
\hline 0.0170579 & 0.106612 \\
\hline 0.0170222 & 0.106389 \\
\hline 0.0169866 & 0.106166 \\
\hline 0.016951 & 0.105944 \\
\hline 0.0169156 & 0.105722 \\
\hline 0.0168802 & 0.105501 \\
\hline 0.016845 & 0.105281 \\
\hline 0.0168098 & 0.105061 \\
\hline 0.0167747 & 0.104842 \\
\hline 0.0167397 & 0.104623 \\
\hline 0.0167047 & 0.104405 \\
\hline 0.0166699 & 0.104187 \\
\hline 0.0166351 & 0.103969 \\
\hline 0.0166004 & 0.103753 \\
\hline 0.0165658 & 0.103536 \\
\hline 0.0165313 & 0.103321 \\
\hline 0.0164969 & 0.103106 \\
\hline 0.0164625 & 0.102891 \\
\hline 0.0164283 & 0.102677 \\
\hline 0.0163941 & 0.102463 \\
\hline 0.01636 & 0.10225 \\
\hline
\end{tabular}

0.237977

0.237471

0.236966

0.236462

0.23596

0.235459

0.234959

0.23446

0.233963

0.233466

0.232971

0.232477

0.231985

0.231493

0.231003

0.230513

0.230026

0.229539

0.229053

0.228569

0.228086

0.227603

0.227123

0.226643

0.226164

0.225687

0.225211

0.224735

0.224261

0.223789

0.223317

0.222846

0.222377

0.221909

0.221442

0.220976

0.220511

0.220047

0.219584

0.219123

0.218662

0.218203

0.217745

0.217288

0.216832

0.216377

0.215923

0.21547

0.215019

0.214568

0.214119

0.213671

0.213223

0.212777

0.212332

0.211888

0.211445

0.211003

0.210562

0.210122

0.209684

0.209246

0.208809

0.208373

0.207939

0.207505

0.207073

0.206641

0.206211

0.205782

0.205353

0.204926

0.2045

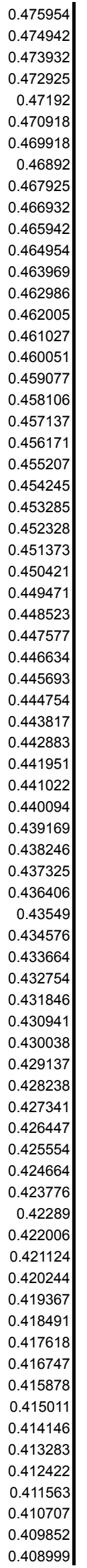


$3.97 \mathrm{E}-03$

3.96E-03

3.95E-03

$3.94 \mathrm{E}-03$

$3.94 \mathrm{E}-03$

3.93E- 03

$3.92 \mathrm{E}-03$

$3.91 \mathrm{E}-03$

$3.90 \mathrm{E}-03$

$3.89 \mathrm{E}-03$

$3.89 \mathrm{E}-03$

$3.88 \mathrm{E}-03$

3.87E-03

$3.86 \mathrm{E}-03$

3.85E-03

$3.85 \mathrm{E}-03$

3.84E-03

3.83E-03

3. $82 \mathrm{E}-03$

3.81E-03

$3.81 \mathrm{E}-03$

3.80E-03

3.79E-03

$3.78 \mathrm{E}-03$

$3.78 \mathrm{E}-03$

3.77E-03

3.76E-03

3.75E-03

3.74E-03

3.74E- 03

3.73E-03

3.72E-03

3.71E-03

3.71E-03

$3.70 \mathrm{E}-03$

3.69E-03

3.68E-03

3.68E-03

3.67E-03

3.66E-03

3.65E-03

3.65E-03

3.64E-03

3.63E-03

3.62E-03

$3.62 \mathrm{E}-03$

3.61E-03

3.60E-03

3.59E-03

3.59E-03

3.58E-03

$3.57 \mathrm{E}-03$

3.56E-03

3.56E-03

3.55E-03

3.54E-03

3.54E-03

3.53E-03

3.52E- 03

3.51E-03

3.51E-03

$3.50 \mathrm{E}-03$

$3.49 \mathrm{E}-03$

3. $48 \mathrm{E}-03$

$3.48 \mathrm{E}-03$

3. $47 \mathrm{E}-03$

3.46E-03

3. $46 \mathrm{E}-03$

3. $45 \mathrm{E}-03$

3. $44 \mathrm{E}-03$

3.44E-03

3.43E-03

$3.42 \mathrm{E}-03$
4.08E-03

4.07E-03

$4.06 \mathrm{E}-03$

4.06E-03

4.05E-03

4.04E-03

4.03E-03

4.02E-03

4.01E-03

4.01E-03

4.00E-03

3.99E-03

$3.98 \mathrm{E}-03$

3.97E-03

3.96E-03

3.96E-03

3.95E-03

3.94E-03

3.93E-03

3.92E-03

3.92E-03

$3.91 \mathrm{E}-03$

$3.90 \mathrm{E}-03$

3.89E-03

3. $88 \mathrm{E}-03$

3.88E-03

3. $87 \mathrm{E}-03$

3.86E-03

3. $85 \mathrm{E}-03$

3.84E-03

$3.84 \mathrm{E}-03$

3.83E-03

3.82E-03

$3.81 \mathrm{E}-03$

$3.80 \mathrm{E}-03$

$3.80 \mathrm{E}-03$

$3.79 \mathrm{E}-03$

$3.78 \mathrm{E}-03$

$3.77 \mathrm{E}-03$

3.77E-03

$3.76 \mathrm{E}-03$

3.75E-03

3.74E-03

3.73E-03

3.73E-03

3.72E-03

$3.71 \mathrm{E}-03$

3.70E-03

3.70E-03

3.69E-03

$3.68 \mathrm{E}-03$

3.67E-03

3.67E-03

3.66E-03

3.65E-03

3.64E-03

3.64E-03

3.63E-03

3.62E-03

$3.61 \mathrm{E}-03$

3.61E-03

3. $60 \mathrm{E}-03$

3.59E-03

$3.58 \mathrm{E}-03$

$3.58 \mathrm{E}-03$

3.57E-03

$3.56 \mathrm{E}-03$

3.56E-03

$3.55 \mathrm{E}-03$

3.54E-03

3.53E-03

3.53E-03

3.52E-03
0.0163259

0.016292

0.0162581

0.0162244

0.0161907

0.016157

0.0161235

0.01609

0.0160567

0.0160234

0.0159901

0.015957

0.0159239

0.0158909

0.015858

0.0158252

0.0157925

0.0157598

0.0157272

0.0156947

0.0156622

0.0156299

0.0155976

0.0155654

0.0155333

0.0155012

0.0154692

0.0154373

0.0154055

0.0153737

0.0153421

0.0153105

0.0152789

0.0152475

0.0152161

0.0151848

0.0151536

0.0151224

0.0150913

0.0150603

0.0150294

0.0149985

0.0149677

0.014937

0.0149064

0.0148758

0.0148453

0.0148149

0.0147845

0.0147542

0.014724

0.0146939

0.0146638

0.0146338

0.0146039

0.014574

0.0145442

0.0145145

0.0144849

0.0144553

0.0144258

0.0143964

0.014367

0.0143377

0.0143084

0.0142793

0.0142502

0.0142212

0.0141922

0.0141633

0.0141345

0.0141057

0.014077

0.102037

0.101825

0.101613

0.101402

0.101192

0.100981

0.100772

0.100563

0.100354

0.100146

0.0999383

0.0997312

0.0995245

0.0993184

0.0991127

0.0989076

0.0987029

0.0984987

0.098295

0.0980918

0.097889

0.0976868

0.097485

0.0972837

0.0970828

0.0968825

0.0966826

0.0964833

0.0962843

0.0960859

0.0958879

0.0956904

0.0954933

0.0952968

0.0951006

0.094905

0.0947098

0.0945151

0.0943208

0.094127

0.0939337

0.0937408

0.0935483

0.0933564

0.0931648

0.0929738

0.0927831

0.092593

0.0924032

0.092214

0.0920251

0.0918368

0.0916488

0.0914614

0.0912743

0.0910877

0.0909015

0.0907158

0.0905304

0.0903456

0.0901612

0.0899772

0.0897936

0.0896105

0.0894278

0.0892455

0.0890636

0.0888823

0.0887012 
Table_of_specs_Rev0.xls,Layered Be surface roughness Printed 10/2/05,4:16 PM
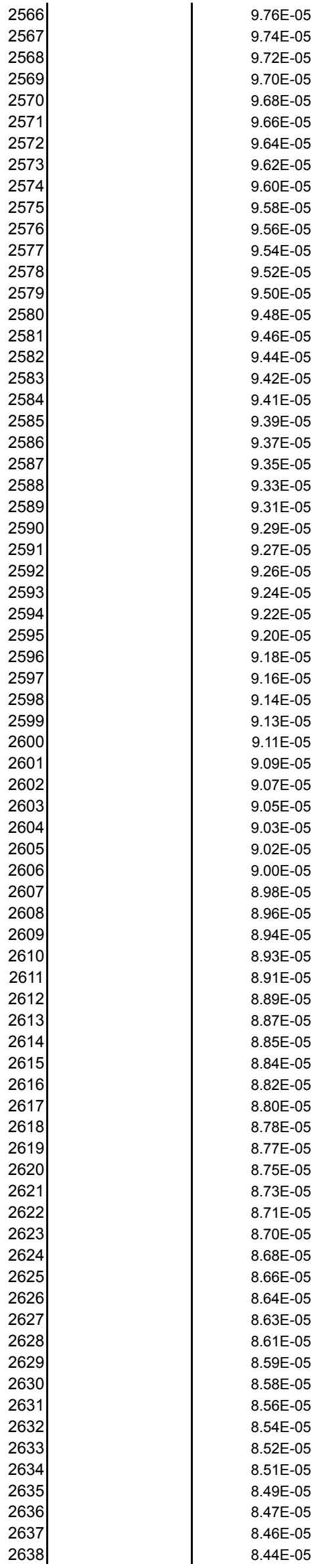

$3.41 \mathrm{E}-03$

$3.41 \mathrm{E}-03$

$3.40 \mathrm{E}-03$

$3.39 \mathrm{E}-03$

3.39E-03

$3.38 \mathrm{E}-03$

$3.37 \mathrm{E}-03$

3.37E-03

$3.36 \mathrm{E}-03$

3.35E-03

3.35E-03

$3.34 \mathrm{E}-03$

3.33E-03

$3.33 \mathrm{E}-03$

$3.32 \mathrm{E}-03$

$3.31 \mathrm{E}-03$

3. $31 \mathrm{E}-03$

3.30E-03

3. $29 \mathrm{E}-03$

3.29E-03

3.28E-03

3.27E-03

3.27E-03

3. $26 \mathrm{E}-03$

3.25E-03

3.25E-03

3.24E-03

3.23E-03

3.23E-03

3.22E-03

3.21E-03

3. $21 \mathrm{E}-03$

3.20E-03

3.19E-03

3.19E-03

3.18E-03

3.17E-03

3.17E-03

3.16E-03

3.16E-03

3.15E-03

3.14E-03

3.14E-03

3.13E-03

3.12E-03

3.12E-03

3.11E-03

3.11E-03

3.10E-03

3.09E-03

3.09E-03

3.08E-03

3.07E-03

3.07E-03

3.06E-03

3.06E-03

3.05E-03

3.04E-03

3.04E-03

3.03E-03

3.03E-03

3.02E-03

3.01E-03

3.01E-03

3.00E-03

3.00E-03

2.99E-03

2.98E-03

$2.98 \mathrm{E}-03$

2.97E-03

2.97E-03

2.96E-03

2.95E-03
3.50E-03

$3.50 \mathrm{E}-03$

3.49E-03

3. $48 \mathrm{E}-03$

3. $48 \mathrm{E}-03$

3. $47 \mathrm{E}-03$

3.46E-03

$3.46 \mathrm{E}-03$

3.45E-03

3. $44 \mathrm{E}-03$

3.43E-03

3.43E-03

3. $42 \mathrm{E}-03$

3. $41 \mathrm{E}-03$

$3.41 \mathrm{E}-03$

3.40E-03

$3.39 \mathrm{E}-03$

3.39E-03

3.38E-03

3.37E-03

3.37E-03

3. $36 \mathrm{E}-03$

3.35E-03

3. $35 \mathrm{E}-03$

3. $34 \mathrm{E}-03$

3.33E-03

3.33E-03

3.32E-03

3.31E-03

$3.31 \mathrm{E}-03$

3. 30E-03

3.29E-03

3.29E-03

$3.28 \mathrm{E}-03$

3.27E-03

$3.27 \mathrm{E}-03$

3.26E-03

3.25E-03

3. $25 \mathrm{E}-03$

3.24E-03

3.23E-03

3.23E-03

3.22E-03

3.21E-03

3.21E-03

3.20E-03

3.19E-03

3.19E-03

3.18E-03

3.17E-03

3.17E-03

3.16E-03

3.16E-03

3.15E-03

3.14E-03

3.14E-03

3.13E-03

3.12E-03

3.12E-03

3.11E-03

3.11E-03

3.10E-03

3.09E-03

3.09E-03

$3.08 \mathrm{E}-03$

3.07E-03

3.07E-03

3.06E-03

3.06E-03

3.05E-03

3.04E-03

3.04E-03

\begin{tabular}{|c|c|}
\hline 0.0140484 & 0.0878027 \\
\hline 0.0140199 & 0.0876241 \\
\hline 0.0139914 & 0.0874461 \\
\hline 0.013963 & 0.0872685 \\
\hline 0.0139346 & 0.0870913 \\
\hline 0.0139063 & 0.0869145 \\
\hline 0.0138781 & 0.0867381 \\
\hline 0.0138499 & 0.0865621 \\
\hline 0.0138219 & 0.0863866 \\
\hline 0.0137938 & 0.0862114 \\
\hline 0.0137659 & 0.0860367 \\
\hline 0.013738 & 0.0858623 \\
\hline 0.0137102 & 0.0856885 \\
\hline 0.0136824 & 0.0855149 \\
\hline 0.0136547 & 0.0853418 \\
\hline 0.0136271 & 0.0851691 \\
\hline 0.0135995 & 0.0849968 \\
\hline 0.013572 & 0.0848249 \\
\hline 0.0135445 & 0.0846534 \\
\hline 0.0135172 & 0.0844822 \\
\hline 0.0134899 & 0.0843116 \\
\hline 0.0134626 & 0.0841412 \\
\hline 0.0134354 & 0.0839714 \\
\hline 0.0134083 & 0.0838018 \\
\hline 0.0133812 & 0.0836327 \\
\hline 0.0133542 & 0.083464 \\
\hline 0.0133273 & 0.0832957 \\
\hline 0.0133004 & 0.0831277 \\
\hline 0.0132736 & 0.0829602 \\
\hline 0.0132469 & 0.082793 \\
\hline 0.0132202 & 0.0826262 \\
\hline 0.0131936 & 0.0824598 \\
\hline 0.013167 & 0.0822938 \\
\hline 0.0131405 & 0.0821282 \\
\hline 0.0131141 & 0.081963 \\
\hline 0.0130877 & 0.0817981 \\
\hline 0.0130614 & 0.0816337 \\
\hline 0.0130351 & 0.0814696 \\
\hline 0.0130089 & 0.0813059 \\
\hline 0.0129828 & 0.0811425 \\
\hline 0.0129567 & 0.0809796 \\
\hline 0.0129307 & 0.080817 \\
\hline 0.0129048 & 0.0806549 \\
\hline 0.0128789 & 0.080493 \\
\hline 0.0128531 & 0.0803316 \\
\hline 0.0128273 & 0.0801705 \\
\hline 0.0128016 & 0.0800098 \\
\hline 0.0127759 & 0.0798495 \\
\hline 0.0127503 & 0.0796896 \\
\hline 0.0127248 & 0.0795299 \\
\hline 0.0126993 & 0.0793708 \\
\hline 0.0126739 & 0.0792119 \\
\hline 0.0126486 & 0.0790534 \\
\hline 0.0126232 & 0.0788953 \\
\hline 0.012598 & 0.0787376 \\
\hline 0.0125728 & 0.0785802 \\
\hline 0.0125477 & 0.0784232 \\
\hline 0.0125226 & 0.0782665 \\
\hline 0.0124976 & 0.0781102 \\
\hline 0.0124727 & 0.0779542 \\
\hline 0.0124478 & 0.0777987 \\
\hline 0.012423 & 0.0776435 \\
\hline 0.0123982 & 0.0774886 \\
\hline 0.0123735 & 0.0773341 \\
\hline 0.0123488 & 0.07718 \\
\hline 0.0123242 & 0.0770261 \\
\hline 0.0122996 & 0.0768727 \\
\hline 0.0122751 & 0.0767196 \\
\hline 0.0122507 & 0.0765669 \\
\hline 0.0122263 & 0.0764145 \\
\hline 0.012202 & 0.0762625 \\
\hline 0.0121777 & 0.0761108 \\
\hline 0.0121535 & 0.0759595 \\
\hline
\end{tabular}

0.175605

0.175248

0.174892

0.174537

0.174183

0.173829

0.173476

0.173124

0.172773

0.172423

0.172073

0.171725

0.171377

0.17103

0.170684

0.170338

0.169994

0.16965

0.169307

0.168964

0.168623

0.168282

0.167943

0.167604

0.167265

0.166928

0.166591

0.166255

0.16592

0.165586

0.165252

0.16492

0.164588

0.164256

0.163926

0.163596

0.163267

0.162939

0.162612

0.162285

0.161959

0.161634

0.16131

0.160986

0.160663

0.160341

0.16002

0.159699

0.159379

0.15906

0.158742

0.158424

0.158107

0.157791

0.157475

0.15716

0.156846

0.156533

0.15622

0.155908

0.155597

0.155287

0.154977

0.154668

0.15436

0.154052

0.153745

0.153439

0.153134

0.152829

0.152525

0.152222

0.151919

0.351211

0.350497

0.349785

0.349074

0.348365

0.347658

0.346953

0.346248

0.345546

0.344846

0.344147

0.343449

0.342754

0.34206

0.341367

0.340676

0.339987

0.339299

0.338614

0.337929

0.337246

0.336565

0.335885

0.335207

0.334531

0.333856

0.333183

0.332511

0.331841

0.331172

0.330505

0.329839

0.329175

0.328513

0.327852

0.327192

0.326535

0.325878

0.325224

0.32457

0.323918

0.323268

0.322619

0.321972

0.321326

0.320682

0.320039

0.319398

0.318758

0.31812

0.317483

0.316848

0.316214

0.315581

0.31495

0.314321

0.313693

0.313066

0.312441

0.311817

0.311195

0.310574

0.309955

0.309336

0.30872

0.308105

0.307491 
Table_of_specs_Rev0.xls,Layered Be surface roughness Printed 10/2/05,4:16 PM
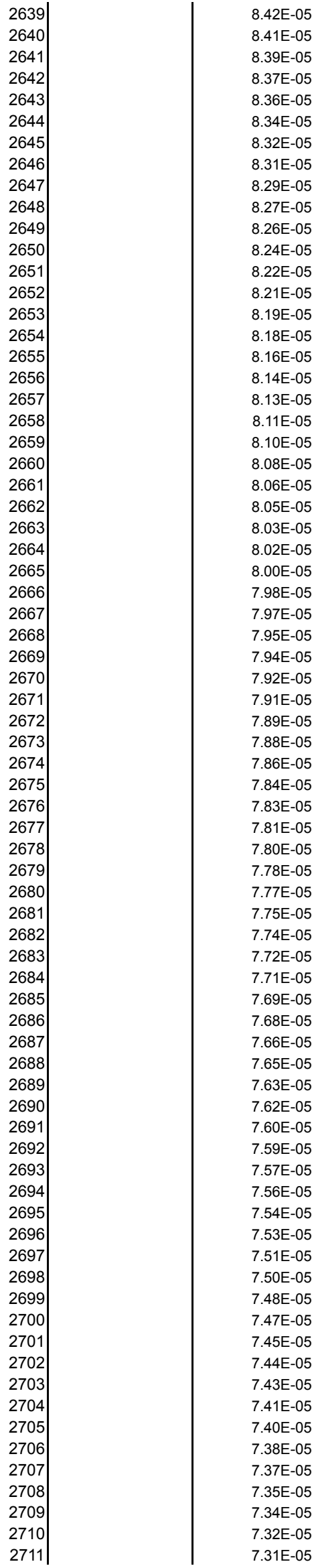

2.95E-03

$2.94 \mathrm{E}-03$

2.94E-03

2.93E-03

$2.92 \mathrm{E}-03$

2.92E-03

$2.91 \mathrm{E}-03$

$2.91 \mathrm{E}-03$

$2.90 \mathrm{E}-03$

$2.90 \mathrm{E}-03$

$2.89 \mathrm{E}-03$

$2.88 \mathrm{E}-03$

$2.88 \mathrm{E}-03$

2.87E-03

$2.87 \mathrm{E}-03$

$2.86 \mathrm{E}-03$

$2.86 \mathrm{E}-03$

$2.85 \mathrm{E}-03$

2.84E-03

2.84E-03

2.83E-03

2.83E-03

2.82E-03

2.82E-03

2.81E-03

$2.81 \mathrm{E}-03$

2.80E-03

2.79E-03

2.79E-03

$2.78 \mathrm{E}-03$

2.78E-03

2.77E-03

2.77E-03

2.76E-03

2.76E-03

2.75E-03

2.75E-03

2.74E-03

2.73E-03

2.73E-03

2.72E-03

2.72E-03

$2.71 \mathrm{E}-03$

2.71E-03

2.70E-03

2.70E-03

2.69E-03

2.69E-03

2.68E-03

2.68E-03

2.67E-03

2.67E-03

2.66E-03

2.66E-03

2.65E-03

2.65E-03

2.64E-03

2.63E-03

2.63E-03

2.62E-03

2.62E-03

$2.61 \mathrm{E}-03$

2.61E-03

2.60E-03

$2.60 \mathrm{E}-03$

2.59E-03

2.59E-03

$2.58 \mathrm{E}-03$

$2.58 \mathrm{E}-03$

2.57E-03

2.57E-03

2.56E-03

2.56E-03
3.03E-03

3.02E-03

3.01E-03

3.01E-03

3.00E-03

3.00E-03

2.99E-03

2.98E-03

2.98E-03

2.97E-03

2.97E-03

2.96E-03

2.96E-03

2.95E-03

2.94E-03

2.94E-03

2.93E-03

2.93E-03

2.92E-03

2.91E-03

2.91E-03

$2.90 \mathrm{E}-03$

$2.90 \mathrm{E}-03$

2.89E-03

2.89E-03

2.88E-03

2.87E-03

2.87E-03

2.86E-03

2.86E-03

2.85E-03

$2.85 \mathrm{E}-03$

$2.84 \mathrm{E}-03$

2.84E-03

2.83E-03

2.82E-03

2.82E-03

2.81E-03

2.81E-03

$2.80 \mathrm{E}-03$

2.80E-03

$2.79 \mathrm{E}-03$

2.79E-03

2.78E-03

2.77E-03

2.77E-03

2.76E-03

2.76E-03

2.75E-03

2.75E-03

2.74E-03

2.74E-03

2.73E-03

2.73E-03

2.72E-03

2.72E-03

$2.71 \mathrm{E}-03$

2.70E-03

$2.70 \mathrm{E}-03$

2.69E-03

2.69E-03

2.68E-03

2.68E-03

2.67E-03

2.67E-03

2.66E-03

2.66E-03

2.65E-03

2.65E-03

2.64E-03

2.64E-03

2.63E-03

\begin{tabular}{|c|c|}
\hline 0.0121294 & 0.0758085 \\
\hline 0.0121053 & 0.0756579 \\
\hline 0.0120812 & 0.0755075 \\
\hline 0.0120572 & 0.0753576 \\
\hline 0.0120333 & 0.075208 \\
\hline 0.0120094 & 0.0750587 \\
\hline 0.0119856 & 0.0749098 \\
\hline 0.0119618 & 0.0747612 \\
\hline 0.0119381 & 0.074613 \\
\hline 0.0119144 & 0.0744651 \\
\hline 0.0118908 & 0.0743175 \\
\hline 0.0118673 & 0.0741703 \\
\hline 0.0118437 & 0.0740234 \\
\hline 0.0118203 & 0.0738769 \\
\hline 0.0117969 & 0.0737306 \\
\hline 0.0117736 & 0.0735848 \\
\hline 0.0117503 & 0.0734392 \\
\hline 0.011727 & 0.073294 \\
\hline 0.0117039 & 0.0731491 \\
\hline 0.0116807 & 0.0730046 \\
\hline 0.0116577 & 0.0728603 \\
\hline 0.0116346 & 0.0727165 \\
\hline 0.0116117 & 0.0725729 \\
\hline 0.0115887 & 0.0724297 \\
\hline 0.0115659 & 0.0722867 \\
\hline 0.0115431 & 0.0721442 \\
\hline 0.0115203 & 0.0720019 \\
\hline 0.0114976 & 0.07186 \\
\hline 0.0114749 & 0.0717183 \\
\hline 0.0114523 & 0.0715771 \\
\hline 0.0114298 & 0.0714361 \\
\hline 0.0114073 & 0.0712955 \\
\hline 0.0113848 & 0.0711551 \\
\hline 0.0113624 & 0.0710152 \\
\hline 0.0113401 & 0.0708754 \\
\hline 0.0113178 & 0.0707361 \\
\hline 0.0112955 & 0.070597 \\
\hline 0.0112733 & 0.0704583 \\
\hline 0.0112512 & 0.0703198 \\
\hline 0.0112291 & 0.0701818 \\
\hline 0.011207 & 0.0700439 \\
\hline 0.011185 & 0.0699065 \\
\hline 0.0111631 & 0.0697693 \\
\hline 0.0111412 & 0.0696325 \\
\hline 0.0111193 & 0.0694959 \\
\hline 0.0110976 & 0.0693597 \\
\hline 0.0110758 & 0.0692237 \\
\hline 0.0110541 & 0.0690882 \\
\hline 0.0110324 & 0.0689528 \\
\hline 0.0110109 & 0.0688178 \\
\hline 0.0109893 & 0.0686831 \\
\hline 0.0109678 & 0.0685488 \\
\hline 0.0109463 & 0.0684146 \\
\hline 0.0109249 & 0.0682809 \\
\hline 0.0109036 & 0.0681474 \\
\hline 0.0108823 & 0.0680142 \\
\hline 0.010861 & 0.0678813 \\
\hline 0.0108398 & 0.0677488 \\
\hline 0.0108186 & 0.0676164 \\
\hline 0.0107975 & 0.0674845 \\
\hline 0.0107764 & 0.0673528 \\
\hline 0.0107554 & 0.0672214 \\
\hline 0.0107344 & 0.0670903 \\
\hline 0.0107135 & 0.0669595 \\
\hline 0.0106926 & 0.066829 \\
\hline 0.0106718 & 0.0666988 \\
\hline 0.010651 & 0.0665689 \\
\hline 0.0106303 & 0.0664393 \\
\hline 0.0106096 & 0.06631 \\
\hline 0.010589 & 0.066181 \\
\hline 0.0105683 & 0.0660522 \\
\hline 0.0105478 & 0.0659238 \\
\hline 0.0105273 & 0.0657956 \\
\hline
\end{tabular}

0.151316

0.151015

0.150715

0.150416

0.150117

0.14982

0.149522

0.149226

0.14893

0.148635

0.148341

0.148047

0.147754

0.147461

0.14717

0.146878

0.146588

0.146298

0.146009

0.145721

0.145433

0.145146

0.144859

0.144573

0.144288

0.144004

0.14372

0.143437

0.143154

0.142872

0.142591

0.14231

0.14203

0.141751

0.141472

0.141194

0.140917

0.14064

0.140364

0.140088

0.139813

0.139539

0.139265

0.138992

0.138719

0.138447

0.138176

0.137906

0.137636

0.137366

0.137098

0.136829

0.136562

0.136295

0.136028

0.135763

0.135498

0.135233

0.134969

0.134706

0.134443

0.134181

0.133919

0.133658

0.133398

0.133138

0.132879

0.13262

0.132362

0.132104

0.131848

0.131591

0.303234

0.302631

0.30203

0.30143

0.300832

0.300235

0.299639

0.299045

0.298452

0.29786

0.29727

0.296681

0.296094

0.295508

0.294922

0.294339

0.293757

0.293176

0.292596

0.292018

0.291441

0.290866

0.290291

0.289719

0.289147

0.288577

0.288008

0.28744

0.286873

0.286308

0.285744

0.285182

0.28462

0.284061

0.283502

0.282944

0.282388

0.281833

0.281279

0.280727

0.280176

0.279626

0.279077

0.27853

0.277984

0.277439

0.276895

0.276353

0.275811

0.275271

0.274732

0.274195

0.273658

0.273124

0.272589

0.272057

0.271525

0.270995

0.270466

0.269938

0.269411

0.268886

0.268361

0.267838

0.267316

0.266795

0.266276 
Table_of_specs_Rev0.xls,Layered Be surface roughness Printed 10/2/05,4:16 PM

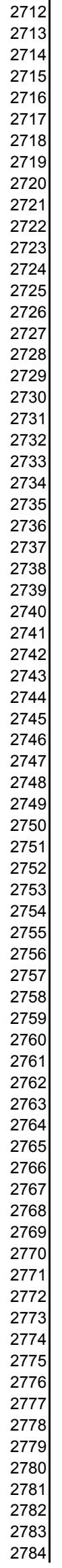

\begin{tabular}{|c|c|}
\hline 7.30E-05 & $2.55 \mathrm{E}-03$ \\
\hline 7.28E-05 & 2.55E-03 \\
\hline 7.27E-05 & $2.54 \mathrm{E}-03$ \\
\hline 7.25E-05 & $2.54 \mathrm{E}-03$ \\
\hline 7.24E-05 & 2.53E-03 \\
\hline 7.23E-05 & 2.53E-03 \\
\hline 7.21E-05 & $2.52 \mathrm{E}-03$ \\
\hline 7.20E-05 & $2.52 \mathrm{E}-03$ \\
\hline 7.18E-05 & $2.51 \mathrm{E}-03$ \\
\hline 7.17E-05 & $2.51 \mathrm{E}-03$ \\
\hline 7.16E-05 & $2.50 \mathrm{E}-03$ \\
\hline 7.14E-05 & $2.50 \mathrm{E}-03$ \\
\hline 7.13E-05 & $2.49 \mathrm{E}-03$ \\
\hline 7.11E-05 & $2.49 \mathrm{E}-03$ \\
\hline $7.10 \mathrm{E}-05$ & $2.49 \mathrm{E}-03$ \\
\hline 7.09E-05 & $2.48 \mathrm{E}-03$ \\
\hline 7.07E-05 & $2.48 \mathrm{E}-03$ \\
\hline 7.06E-05 & 2.47E-03 \\
\hline 7.05E-05 & 2.47E-03 \\
\hline 7.03E-05 & $2.46 \mathrm{E}-03$ \\
\hline $7.02 \mathrm{E}-05$ & $2.46 \mathrm{E}-03$ \\
\hline 7.01E-05 & $2.45 E-03$ \\
\hline $6.99 \mathrm{E}-05$ & $2.45 \mathrm{E}-03$ \\
\hline $6.98 \mathrm{E}-05$ & $2.44 \mathrm{E}-03$ \\
\hline $6.96 \mathrm{E}-05$ & 2.44E-03 \\
\hline $6.95 \mathrm{E}-05$ & $2.43 E-03$ \\
\hline $6.94 \mathrm{E}-05$ & $2.43 \mathrm{E}-03$ \\
\hline $6.92 \mathrm{E}-05$ & $2.42 \mathrm{E}-03$ \\
\hline $6.91 \mathrm{E}-05$ & $2.42 \mathrm{E}-03$ \\
\hline $6.90 \mathrm{E}-05$ & $2.41 \mathrm{E}-03$ \\
\hline $6.88 \mathrm{E}-05$ & $2.41 \mathrm{E}-03$ \\
\hline $6.87 \mathrm{E}-05$ & $2.41 \mathrm{E}-03$ \\
\hline $6.86 \mathrm{E}-05$ & $2.40 \mathrm{E}-03$ \\
\hline $6.85 E-05$ & $2.40 \mathrm{E}-03$ \\
\hline $6.83 \mathrm{E}-05$ & $2.39 \mathrm{E}-03$ \\
\hline $6.82 \mathrm{E}-05$ & $2.39 E-03$ \\
\hline $6.81 \mathrm{E}-05$ & $2.38 \mathrm{E}-03$ \\
\hline $6.79 \mathrm{E}-05$ & $2.38 \mathrm{E}-03$ \\
\hline $6.78 \mathrm{E}-05$ & 2.37E-03 \\
\hline $6.77 \mathrm{E}-05$ & 2.37E-03 \\
\hline $6.75 \mathrm{E}-05$ & 2.36E-03 \\
\hline $6.74 \mathrm{E}-05$ & 2.36E-03 \\
\hline 6.73E-05 & 2.35E-03 \\
\hline $6.71 \mathrm{E}-05$ & $2.35 \mathrm{E}-03$ \\
\hline $6.70 \mathrm{E}-05$ & $2.35 \mathrm{E}-03$ \\
\hline $6.69 \mathrm{E}-05$ & $2.34 \mathrm{E}-03$ \\
\hline $6.68 \mathrm{E}-05$ & $2.34 \mathrm{E}-03$ \\
\hline $6.66 \mathrm{E}-05$ & $2.33 \mathrm{E}-03$ \\
\hline $6.65 \mathrm{E}-05$ & 2.33E-03 \\
\hline $6.64 \mathrm{E}-05$ & $2.32 \mathrm{E}-03$ \\
\hline $6.63 \mathrm{E}-05$ & $2.32 \mathrm{E}-03$ \\
\hline $6.61 \mathrm{E}-05$ & $2.31 \mathrm{E}-03$ \\
\hline $6.60 \mathrm{E}-05$ & $2.31 \mathrm{E}-03$ \\
\hline $6.59 \mathrm{E}-05$ & $2.31 \mathrm{E}-03$ \\
\hline $6.57 \mathrm{E}-05$ & $2.30 \mathrm{E}-03$ \\
\hline $6.56 \mathrm{E}-05$ & $2.30 \mathrm{E}-03$ \\
\hline $6.55 \mathrm{E}-05$ & $2.29 \mathrm{E}-03$ \\
\hline $6.54 \mathrm{E}-05$ & $2.29 \mathrm{E}-03$ \\
\hline $6.52 \mathrm{E}-05$ & $2.28 \mathrm{E}-03$ \\
\hline $6.51 \mathrm{E}-05$ & $2.28 \mathrm{E}-03$ \\
\hline $6.50 \mathrm{E}-05$ & 2.27E-03 \\
\hline $6.49 \mathrm{E}-05$ & $2.27 \mathrm{E}-03$ \\
\hline $6.47 \mathrm{E}-05$ & 2.27E-03 \\
\hline 6.46E-05 & $2.26 \mathrm{E}-03$ \\
\hline $6.45 \mathrm{E}-05$ & $2.26 \mathrm{E}-03$ \\
\hline $6.44 \mathrm{E}-05$ & $2.25 \mathrm{E}-03$ \\
\hline $6.43 \mathrm{E}-05$ & $2.25 \mathrm{E}-03$ \\
\hline $6.41 \mathrm{E}-05$ & $2.24 \mathrm{E}-03$ \\
\hline $6.40 \mathrm{E}-05$ & $2.24 \mathrm{E}-03$ \\
\hline $6.39 \mathrm{E}-05$ & $2.24 \mathrm{E}-03$ \\
\hline $6.38 \mathrm{E}-05$ & $2.23 \mathrm{E}-03$ \\
\hline $6.36 \mathrm{E}-05$ & $2.23 \mathrm{E}-03$ \\
\hline $6.35 \mathrm{E}-05$ & $2.22 \mathrm{E}-03$ \\
\hline
\end{tabular}

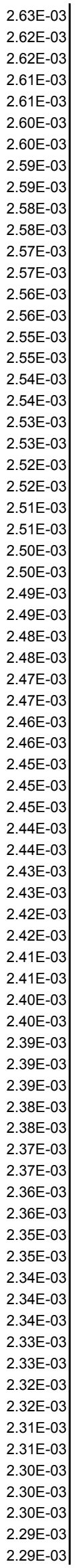

0.0105068 0.0104864 0.0104661 0.0104457 0.0104255 0.0104052 0.010385

0.0103649

0.0103448

0.0103247

0.0103048

0.0102848

0.0102649

0.010245

0.0102252

0.0102054

0.0101857

0.010166

0.0101463

0.0101267

0.0101072

0.0100876

0.0100682

0.0100487

0.0100293

0.01001

0.00999071

0.00997144

0.00995224

0.00993306

0.00991394

0.00989484

0.00987581

0.0098568

0.00983785

0.00981893

0.00980006

0.00978122

0.00976244

0.00974369

0.00972499

0.00970632

0.0096877

0.00966911

0.00965058

0.00963207

0.00961362

0.0095952

0.00957683

0.00955849

0.0095402

0.00952194

0.00950374

0.00948556

0.00946743

0.00944933

0.00943129

0.00941327

0.0093953

0.00937736

0.00935948

0.00934162

0.00932381

0.00930603

0.0092883

0.0092706

0.00925295

0.00923533

0.00921775

0.00920021

0.00918271

0.00916524

0.00914783

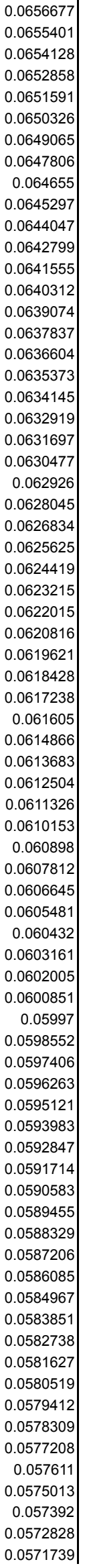

0.131335

0.13108

0.130826

0.130572

0.130318

0.130065

0.129813

0.129561

0.12931

0.129059

0.128809

0.12856

0.128311

0.128062

0.127815

0.127567

0.127321

0.127075

0.126829

0.126584

0.126339

0.126095

0.125852

0.125609

0.125367

0.125125

0.124884

0.124643

0.124403

0.124163

0.123924

0.123686

0.123448

0.12321

0.122973

0.122737

0.122501

0.122265

0.122031

0.121796

0.121562

0.121329

0.121096

0.120864

0.120632

0.120401

0.12017

0.11994

0.11971

0.119481

0.119253

0.119024

0.118797

0.118569

0.118343

0.118117

0.117891

0.117666

0.117441

0.117217

0.116993

0.11677

0.116548

0.116325

0.116104

0.115882

0.115662

0.115442

0.115222

0.115003

0.114784

0.114566

0.114348

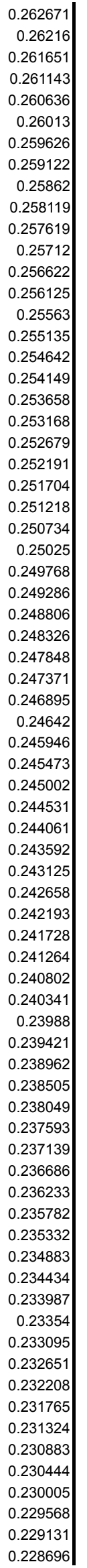


Table_of_specs_Rev0.xls,Layered Be surface roughness Printed 10/2/05,4:16 PM
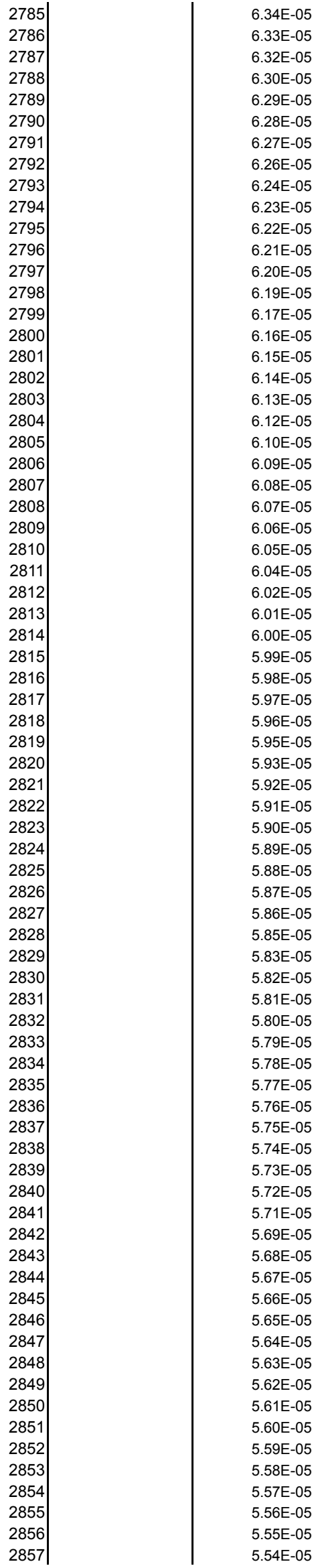

$2.22 \mathrm{E}-03$ $2.21 \mathrm{E}-03$

$2.21 \mathrm{E}-03$

$2.21 \mathrm{E}-03$

2.20E-03

$2.20 \mathrm{E}-03$

2.19E-03

2.19E-03

2.19E-03

2.18E-03

$2.18 \mathrm{E}-03$

2.17E-03

2.17E-03

2.17E-03

2.16E-03

2.16E-03

2.15E-03

2.15E-03

2.14E-03

2.14E-03

2.14E-03

2.13E-03

2.13E-03

2.12E-03

2.12E-03

2.12E-03

2.11E-03

2.11E-03

2.10E-03

2.10E-03

2.10E-03

2.09E-03

2.09E-03

$2.08 \mathrm{E}-03$

$2.08 \mathrm{E}-03$

$2.08 \mathrm{E}-03$

2.07E-03

2.07E-03

2.07E-03

2.06E-03

2.06E-03

2.05E-03

2.05E-03

2.05E-03

2.04E-03

$2.04 \mathrm{E}-03$

2.03E-03

2.03E-03

2.03E-03

2.02E-03

2.02E-03

2.02E-03

2.01E-03

2.01E-03

2.00E-03

2.00E-03

2.00E-03

$1.99 \mathrm{E}-03$

1.99E-03

1.99E-03

$1.98 \mathrm{E}-03$

$1.98 \mathrm{E}-03$

1.97E-03

1.97E-03

1.97E-03

$1.96 \mathrm{E}-03$

$1.96 \mathrm{E}-03$

1.96E-03

1.95E-03

1.95E-03

1.95E-03

$1.94 \mathrm{E}-03$

$1.94 \mathrm{E}-03$
$2.28 \mathrm{E}-03$

2.28E-03

2.27E-03

2.27E-03

2.27E-03

2.26E-03

2.26E-03

2.25E-03

2.25E-03

2.24E- 03

2.24E-03

2.24E-03

2.23E-03

2.23E-03

2.22E-03

2.22E-03

2.21E-03

$2.21 \mathrm{E}-03$

2.21E-03

2.20E-03

2.20E-03

2.19E-03

2.19E-03

2.19E-03

$2.18 \mathrm{E}-03$

2.18E-03

2.17E-03

2.17E-03

2.16E-03

2.16E-03

2.16E-03

2.15E-03

2.15E-03

2.14E-03

2.14E-03

2.14E-03

2.13E-03

2.13E-03

2.12E-03

2.12E-03

2.12E-03

2.11E-03

$2.11 \mathrm{E}-03$

2.10E-03

2.10E-03

2.10E-03

2.09E-03

2.09E-03

$2.08 \mathrm{E}-03$

2.08E-03

2.08E-03

2.07E-03

2.07E-03

2.07E-03

2.06E-03

2.06E-03

2.05E-03

$2.05 \mathrm{E}-03$

2.05E-03

2.04E-03

2.04E-03

2.03E-03

2.03E-03

2.03E-03

2.02E-03

2.02E-03

2.02E-03

2.01E-03

2.01E-03

2.00E-03

2.00E-03

$2.00 \mathrm{E}-03$

$1.99 \mathrm{E}-03$
0.00913044

0.0091131

0.00909578

0.00907852

0.00906128

0.00904409

0.00902693

0.00900981

0.00899273

0.00897569

0.00895868

0.00894171

0.00892478

0.00890789

0.00889102

0.00887421

0.00885742

0.00884068

0.00882397

0.0088073

0.00879066

0.00877406

0.00875749

0.00874097

0.00872448

0.00870803

0.0086916

0.00867523

0.00865887

0.00864257

0.00862629

0.00861005

0.00859384

0.00857768

0.00856154

0.00854545

0.00852938

0.00851336

0.00849736

0.00848141

0.00846548

0.0084496

0.00843373

0.00841792

0.00840213

0.00838639

0.00837066

0.00835499

0.00833933

0.00832373

0.00830814

0.0082926

0.00827708

0.00826161

0.00824616

0.00823076

0.00821537

0.00820003

0.00818472

0.00816945

0.00815419

0.00813899

0.0081238

0.00810867

0.00809355

0.00807847

0.00806342

0.00804841

0.00803342

0.00801848

0.00800356

0.00798868

0.00797382

0.0569568

0.0568486

0.0567407

0.056633

0.0565256

0.0564183

0.0563113

0.0562045

0.0560981

0.0559917

0.0558857

0.0557798

0.0556743

0.0555689

0.0554638

0.0553589

0.0552543

0.0551498

0.0550456

0.0549416

0.0548379

0.0547343

0.0546311

0.054528

0.0544252

0.0543225

0.0542202

0.054118

0.0540161

0.0539143

0.0538128

0.0537115

0.0536105

0.0535096

0.0534091

0.0533086

0.0532085

0.0531085

0.0530088

0.0529092

0.05281

0.0527108

0.052612

0.0525133

0.0524149

0.0523166

0.0522187

0.0521208

0.0520233

0.0519259

0.0518288

0.0517318

0.0516351

0.0515385

0.0514422

0.0513461

0.0512502

0.0511545

0.051059

0.0509637

0.0508687

0.0507738

0.0506792

0.0505847

0.0504905

0.0503964

0.0503026

0.0502089 
Table_of_specs_Rev0.xls,Layered Be surface roughness Printed 10/2/05,4:16 PM
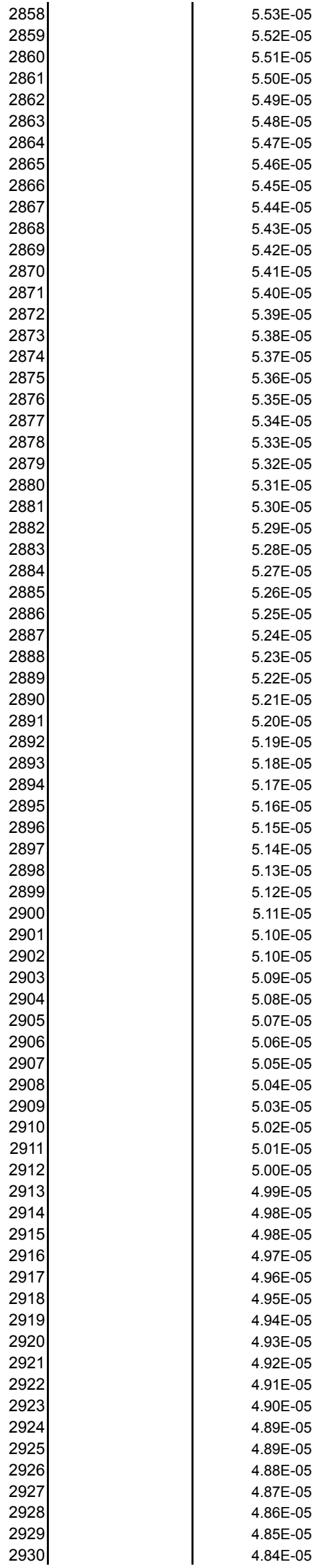

$1.93 \mathrm{E}-03$

$1.93 \mathrm{E}-03$

$1.93 \mathrm{E}-03$

$1.92 \mathrm{E}-03$

$1.92 \mathrm{E}-03$

$1.92 \mathrm{E}-03$

$1.91 \mathrm{E}-03$

$1.91 \mathrm{E}-03$

$1.91 \mathrm{E}-03$

$1.90 \mathrm{E}-03$

$1.90 \mathrm{E}-03$

$1.90 \mathrm{E}-03$

$1.89 \mathrm{E}-03$

$1.89 \mathrm{E}-03$

$1.88 \mathrm{E}-03$

$1.88 \mathrm{E}-03$

$1.88 \mathrm{E}-03$

1.87E-03

1.87E-03

1.87E-03

1.86E-03

$1.86 \mathrm{E}-03$

$1.86 \mathrm{E}-03$

1.85E-03

1.85E-03

1.85E-03

$1.84 \mathrm{E}-03$

$1.84 \mathrm{E}-03$

$1.84 \mathrm{E}-03$

1.83E-03

$1.83 \mathrm{E}-03$

1.83E-03

$1.82 \mathrm{E}-03$

$1.82 \mathrm{E}-03$

1.82E-03

1.81E-03

$1.81 \mathrm{E}-03$

1.81E-03

$1.80 \mathrm{E}-03$

$1.80 \mathrm{E}-03$

1.80E-03

1.79E-03

1.79E-03

1.79E-03

$1.78 \mathrm{E}-03$

$1.78 \mathrm{E}-03$

$1.78 \mathrm{E}-03$

1.77E-03

1.77E-03

$1.77 \mathrm{E}-03$

1.76E-03

$1.76 \mathrm{E}-03$

1.76E-03

1.75E-03

1.75E-03

1.75E-03

1.74E-03

1.74E-03

1.74E-03

1.74E-03

1.73E-03

1.73E-03

1.73E-03

1.72E-03

$1.72 \mathrm{E}-03$

1.72E-03

$1.71 \mathrm{E}-03$

$1.71 \mathrm{E}-03$

$1.71 \mathrm{E}-03$

1.70E-03

$1.70 \mathrm{E}-03$

1.70E-03

1.69E-03
1.99E-03

1.99E-03

$1.98 \mathrm{E}-03$

$1.98 \mathrm{E}-03$

$1.98 \mathrm{E}-03$

$1.97 \mathrm{E}-03$

1.97E-03

$1.96 \mathrm{E}-03$

$1.96 \mathrm{E}-03$

$1.96 \mathrm{E}-03$

$1.95 \mathrm{E}-03$

1.95E-03

$1.95 \mathrm{E}-03$

$1.94 \mathrm{E}-03$

1.94E-03

$1.94 \mathrm{E}-03$

1.93E-03

1.93E-03

1.92E-03

$1.92 \mathrm{E}-03$

$1.92 \mathrm{E}-03$

$1.91 \mathrm{E}-03$

$1.91 \mathrm{E}-03$

$1.91 \mathrm{E}-03$

$1.90 \mathrm{E}-03$

$1.90 \mathrm{E}-03$

$1.90 \mathrm{E}-03$

$1.89 \mathrm{E}-03$

1.89E-03

1.89E-03

$1.88 \mathrm{E}-03$

$1.88 \mathrm{E}-03$

$1.88 \mathrm{E}-03$

1.87E-03

$1.87 \mathrm{E}-03$

$1.86 \mathrm{E}-03$

$1.86 \mathrm{E}-03$

$1.86 \mathrm{E}-03$

$1.85 \mathrm{E}-03$

1.85E-03

$1.85 \mathrm{E}-03$

1.84E-03

$1.84 \mathrm{E}-03$

$1.84 \mathrm{E}-03$

1.83E-03

1.83E-03

1.83E-03

$1.82 \mathrm{E}-03$

$1.82 \mathrm{E}-03$

$1.82 \mathrm{E}-03$

$1.81 \mathrm{E}-03$

$1.81 \mathrm{E}-03$

$1.81 \mathrm{E}-03$

$1.80 \mathrm{E}-03$

$1.80 \mathrm{E}-03$

1.80E-03

1.79E-03

1.79E-03

1.79E-03

$1.78 \mathrm{E}-03$

$1.78 \mathrm{E}-03$

$1.78 \mathrm{E}-03$

1.77E-03

1.77E-03

1.77E-03

$1.77 \mathrm{E}-03$

1.76E-03

1.76E-03

1.76E-03

1.75E-03

1.75E-03

1.75E-03

$1.74 \mathrm{E}-03$
0.007959

0.00794421

0.00792946

0.00791473

0.00790004

0.00788537

0.00787075

0.00785614

0.00784158

0.00782704

0.00781254

0.00779806

0.00778363

0.00776921

0.00775484

0.00774049

0.00772618

0.00771188

0.00769763

0.0076834

0.00766921

0.00765504

0.00764091

0.0076268

0.00761274

0.00759869

0.00758468

0.00757069

0.00755675

0.00754282

0.00752893

0.00751506

0.00750124

0.00748742

0.00747366

0.00745991

0.0074462

0.00743251

0.00741886

0.00740522

0.00739163

0.00737805

0.00736452

0.007351

0.00733753

0.00732407

0.00731065

0.00729725

0.00728389

0.00727054

0.00725724

0.00724395

0.0072307

0.00721747

0.00720428

0.0071911

0.00717797

0.00716485

0.00715177

0.0071387

0.00712568

0.00711267

0.0070997

0.00708675

0.00707384

0.00706094

0.00704808

0.00703524

0.00702243

0.00700964

0.00699689

0.00698416

0.00697146

0.0496513

0.0495591

0.049467

0.0493753

0.0492836

0.0491922

0.0491009

0.0490099

0.048919

0.0488284

0.0487379

0.0486477

0.0485576

0.0484678

0.048378

0.0482886

0.0481993

0.0481102

0.0480212

0.0479326

0.047844

0.0477557

0.0476675

0.0475796

0.0474918

0.0474043

0.0473168

0.0472297

0.0471426

0.0470558

0.0469691

0.0468827

0.0467964

0.0467104

0.0466244

0.0465387

0.0464532

0.0463679

0.0462826

0.0461977

0.0461128

0.0460283

0.0459438

0.0458595

0.0457754

0.0456916

0.0456078

0.0455243

0.0454409

0.0453577

0.0452747

0.0451919

0.0451092

0.0450267

0.0449444

0.0448623

0.0447803

0.0446985

0.0446169

0.0445355

0.0444542

0.0443731

0.0442922

0.0442115

0.0441309

0.0440505

0.0439702

0.0438902 
Table_of_specs_Rev0.xls,Layered Be surface roughness Printed 10/2/05,4:16 PM

\begin{tabular}{|c|c|c|c|c|c|c|c|}
\hline 2931 & 4.83E-05 & $1.69 \mathrm{E}-03$ & $1.74 \mathrm{E}-03$ & 0.00695878 & 0.0434924 & 0.0869848 & 0.17397 \\
\hline 2932 & 4.82E-05 & $1.69 \mathrm{E}-03$ & $1.74 \mathrm{E}-03$ & 0.00694614 & 0.0434134 & 0.0868268 & 0.173654 \\
\hline 2933 & $4.81 \mathrm{E}-05$ & $1.69 \mathrm{E}-03$ & $1.73 \mathrm{E}-03$ & 0.00693351 & 0.0433345 & 0.0866689 & 0.173338 \\
\hline 2934 & $4.81 \mathrm{E}-05$ & $1.68 \mathrm{E}-03$ & $1.73 \mathrm{E}-03$ & 0.00692093 & 0.0432558 & 0.0865116 & 0.173023 \\
\hline 2935 & $4.80 \mathrm{E}-05$ & $1.68 \mathrm{E}-03$ & $1.73 \mathrm{E}-03$ & 0.00690835 & 0.0431772 & 0.0863544 & 0.172709 \\
\hline 2936 & 4.79E-05 & $1.68 \mathrm{E}-03$ & $1.72 \mathrm{E}-03$ & 0.00689582 & 0.0430989 & 0.0861977 & 0.172395 \\
\hline 2937 & $4.78 \mathrm{E}-05$ & $1.67 \mathrm{E}-03$ & $1.72 \mathrm{E}-03$ & 0.00688329 & 0.0430206 & 0.0860412 & 0.172082 \\
\hline 2938 & 4.77E-05 & $1.67 \mathrm{E}-03$ & $1.72 \mathrm{E}-03$ & 0.00687081 & 0.0429426 & 0.0858852 & 0.17177 \\
\hline 2939 & 4.76E-05 & $1.67 \mathrm{E}-03$ & $1.71 \mathrm{E}-03$ & 0.00685834 & 0.0428646 & 0.0857293 & 0.171459 \\
\hline 2940 & 4.75E-05 & $1.66 \mathrm{E}-03$ & $1.71 \mathrm{E}-03$ & 0.00684592 & 0.042787 & 0.0855739 & 0.171148 \\
\hline 2941 & 4.75E-05 & $1.66 \mathrm{E}-03$ & $1.71 \mathrm{E}-03$ & 0.0068335 & 0.0427094 & 0.0854187 & 0.170837 \\
\hline 2942 & 4.74E-05 & $1.66 \mathrm{E}-03$ & $1.71 \mathrm{E}-03$ & 0.00682112 & 0.042632 & 0.085264 & 0.170528 \\
\hline 2943 & 4.73E-05 & $1.65 \mathrm{E}-03$ & $1.70 \mathrm{E}-03$ & 0.00680876 & 0.0425547 & 0.0851095 & 0.170219 \\
\hline 2944 & 4.72E-05 & $1.65 \mathrm{E}-03$ & $1.70 \mathrm{E}-03$ & 0.00679644 & 0.0424777 & 0.0849555 & 0.169911 \\
\hline 2945 & 4.71E-05 & $1.65 \mathrm{E}-03$ & $1.70 \mathrm{E}-03$ & 0.00678412 & 0.0424008 & 0.0848016 & 0.169603 \\
\hline 2946 & 4.70E-05 & $1.65 \mathrm{E}-03$ & $1.69 \mathrm{E}-03$ & 0.00677185 & 0.0423241 & 0.0846482 & 0.169296 \\
\hline 2947 & 4.69E-05 & $1.64 \mathrm{E}-03$ & $1.69 \mathrm{E}-03$ & 0.00675959 & 0.0422475 & 0.0844949 & 0.16899 \\
\hline 2948 & 4.69E-05 & $1.64 \mathrm{E}-03$ & $1.69 \mathrm{E}-03$ & 0.00674737 & 0.0421711 & 0.0843422 & 0.168684 \\
\hline 2949 & $4.68 \mathrm{E}-05$ & $1.64 \mathrm{E}-03$ & $1.68 \mathrm{E}-03$ & 0.00673516 & 0.0420948 & 0.0841896 & 0.168379 \\
\hline 2950 & 4.67E-05 & $1.63 \mathrm{E}-03$ & $1.68 \mathrm{E}-03$ & 0.006723 & 0.0420187 & 0.0840375 & 0.168075 \\
\hline 2951 & $4.66 \mathrm{E}-05$ & $1.63 \mathrm{E}-03$ & $1.68 \mathrm{E}-03$ & 0.00671084 & 0.0419427 & 0.0838855 & 0.167771 \\
\hline 2952 & 4.65E-05 & $1.63 \mathrm{E}-03$ & 1.67E-03 & 0.00669872 & 0.041867 & 0.083734 & 0.167468 \\
\hline 2953 & 4.64E-05 & 1.63E-03 & $1.67 \mathrm{E}-03$ & 0.00668662 & 0.0417914 & 0.0835827 & 0.167165 \\
\hline 2954 & 4.64E-05 & $1.62 \mathrm{E}-03$ & $1.67 \mathrm{E}-03$ & 0.00667455 & 0.0417159 & 0.0834319 & 0.166864 \\
\hline 2955 & 4.63E-05 & $1.62 \mathrm{E}-03$ & $1.67 \mathrm{E}-03$ & 0.0066625 & 0.0416406 & 0.0832812 & 0.166562 \\
\hline 2956 & 4.62E-05 & $1.62 \mathrm{E}-03$ & $1.66 \mathrm{E}-03$ & 0.00665048 & 0.0415655 & 0.083131 & 0.166262 \\
\hline 2957 & 4.61E-05 & $1.61 \mathrm{E}-03$ & $1.66 \mathrm{E}-03$ & 0.00663848 & 0.0414905 & 0.082981 & 0.165962 \\
\hline 2958 & $4.60 \mathrm{E}-05$ & $1.61 \mathrm{E}-03$ & $1.66 \mathrm{E}-03$ & 0.00662651 & 0.0414157 & 0.0828314 & 0.165663 \\
\hline 2959 & 4.59E-05 & $1.61 \mathrm{E}-03$ & $1.65 \mathrm{E}-03$ & 0.00661456 & 0.041341 & 0.082682 & 0.165364 \\
\hline 2960 & 4.59E-05 & $1.60 \mathrm{E}-03$ & $1.65 \mathrm{E}-03$ & 0.00660264 & 0.0412665 & 0.082533 & 0.165066 \\
\hline 2961 & 4.58E-05 & $1.60 \mathrm{E}-03$ & $1.65 \mathrm{E}-03$ & 0.00659074 & 0.0411921 & 0.0823842 & 0.164768 \\
\hline 2962 & 4.57E-05 & $1.60 \mathrm{E}-03$ & $1.64 \mathrm{E}-03$ & 0.00657888 & 0.041118 & 0.0822359 & 0.164472 \\
\hline 2963 & 4.56E-05 & $1.60 \mathrm{E}-03$ & $1.64 \mathrm{E}-03$ & 0.00656702 & 0.0410439 & 0.0820878 & 0.164176 \\
\hline 2964 & 4.55E-05 & $1.59 \mathrm{E}-03$ & $1.64 \mathrm{E}-03$ & 0.00655521 & 0.04097 & 0.0819401 & 0.16388 \\
\hline 2965 & 4.54E-05 & $1.59 \mathrm{E}-03$ & $1.64 \mathrm{E}-03$ & 0.0065434 & 0.0408963 & 0.0817925 & 0.163585 \\
\hline 2966 & 4.54E-05 & $1.59 \mathrm{E}-03$ & $1.63 \mathrm{E}-03$ & 0.00653164 & 0.0408227 & 0.0816455 & 0.163291 \\
\hline 2967 & 4.53E-05 & $1.58 \mathrm{E}-03$ & $1.63 \mathrm{E}-03$ & 0.00651988 & 0.0407493 & 0.0814985 & 0.162997 \\
\hline 2968 & 4.52E-05 & $1.58 \mathrm{E}-03$ & $1.63 \mathrm{E}-03$ & 0.00650817 & 0.0406761 & 0.0813521 & 0.162704 \\
\hline 2969 & 4.51E-05 & $1.58 \mathrm{E}-03$ & $1.62 \mathrm{E}-03$ & 0.00649646 & 0.0406029 & 0.0812058 & 0.162412 \\
\hline 2970 & $4.50 \mathrm{E}-05$ & $1.58 \mathrm{E}-03$ & $1.62 \mathrm{E}-03$ & 0.0064848 & 0.04053 & 0.08106 & 0.16212 \\
\hline 2971 & $4.50 \mathrm{E}-05$ & $1.57 \mathrm{E}-03$ & $1.62 \mathrm{E}-03$ & 0.00647314 & 0.0404571 & 0.0809142 & 0.161828 \\
\hline 2972 & 4.49E-05 & $1.57 \mathrm{E}-03$ & $1.62 \mathrm{E}-03$ & 0.00646152 & 0.0403845 & 0.080769 & 0.161538 \\
\hline 2973 & 4.48E-05 & $1.57 \mathrm{E}-03$ & $1.61 \mathrm{E}-03$ & 0.00644991 & 0.040312 & 0.0806239 & 0.161248 \\
\hline 2974 & 4.47E-05 & $1.56 \mathrm{E}-03$ & $1.61 \mathrm{E}-03$ & 0.00643835 & 0.0402397 & 0.0804793 & 0.160959 \\
\hline 2975 & 4.46E-05 & $1.56 \mathrm{E}-03$ & $1.61 \mathrm{E}-03$ & 0.00642679 & 0.0401674 & 0.0803348 & 0.16067 \\
\hline 2976 & 4.46E-05 & $1.56 \mathrm{E}-03$ & $1.60 \mathrm{E}-03$ & 0.00641527 & 0.0400954 & 0.0801908 & 0.160382 \\
\hline 2977 & 4.45E-05 & $1.56 \mathrm{E}-03$ & $1.60 \mathrm{E}-03$ & 0.00640376 & 0.0400235 & 0.0800469 & 0.160094 \\
\hline 2978 & 4.44E-05 & $1.55 \mathrm{E}-03$ & $1.60 \mathrm{E}-03$ & 0.00639228 & 0.0399518 & 0.0799035 & 0.159807 \\
\hline 2979 & 4.43E-05 & $1.55 \mathrm{E}-03$ & $1.60 \mathrm{E}-03$ & 0.00638082 & 0.0398801 & 0.0797603 & 0.159521 \\
\hline 2980 & $4.42 \mathrm{E}-05$ & $1.55 \mathrm{E}-03$ & $1.59 \mathrm{E}-03$ & 0.0063694 & 0.0398087 & 0.0796175 & 0.159235 \\
\hline 2981 & $4.42 \mathrm{E}-05$ & $1.55 \mathrm{E}-03$ & $1.59 \mathrm{E}-03$ & 0.00635798 & 0.0397374 & 0.0794748 & 0.15895 \\
\hline 2982 & 4.41E-05 & $1.54 \mathrm{E}-03$ & $1.59 \mathrm{E}-03$ & 0.0063466 & 0.0396663 & 0.0793326 & 0.158665 \\
\hline 2983 & $4.40 \mathrm{E}-05$ & $1.54 \mathrm{E}-03$ & $1.58 \mathrm{E}-03$ & 0.00633524 & 0.0395952 & 0.0791905 & 0.158381 \\
\hline 2984 & 4.39E-05 & $1.54 \mathrm{E}-03$ & $1.58 \mathrm{E}-03$ & 0.00632391 & 0.0395244 & 0.0790489 & 0.158098 \\
\hline 2985 & 4.38E-05 & $1.53 \mathrm{E}-03$ & $1.58 \mathrm{E}-03$ & 0.00631259 & 0.0394537 & 0.0789074 & 0.157815 \\
\hline 2986 & 4.38E-05 & $1.53 \mathrm{E}-03$ & $1.58 \mathrm{E}-03$ & 0.00630131 & 0.0393832 & 0.0787663 & 0.157533 \\
\hline 2987 & 4.37E-05 & $1.53 \mathrm{E}-03$ & 1.57E-03 & 0.00629003 & 0.0393127 & 0.0786254 & 0.157251 \\
\hline 2988 & 4.36E-05 & $1.53 \mathrm{E}-03$ & 1.57E-03 & 0.0062788 & 0.0392425 & 0.078485 & 0.15697 \\
\hline 2989 & 4.35E-05 & $1.52 \mathrm{E}-03$ & $1.57 \mathrm{E}-03$ & 0.00626757 & 0.0391723 & 0.0783447 & 0.156689 \\
\hline 2990 & 4.34E-05 & $1.52 \mathrm{E}-03$ & $1.56 \mathrm{E}-03$ & 0.00625639 & 0.0391024 & 0.0782048 & 0.15641 \\
\hline 2991 & 4.34E-05 & $1.52 \mathrm{E}-03$ & $1.56 \mathrm{E}-03$ & 0.00624521 & 0.0390325 & 0.0780651 & 0.15613 \\
\hline 2992 & 4.33E-05 & $1.52 \mathrm{E}-03$ & $1.56 \mathrm{E}-03$ & 0.00623407 & 0.0389629 & 0.0779258 & 0.155852 \\
\hline 2993 & 4.32E-05 & $1.51 \mathrm{E}-03$ & $1.56 \mathrm{E}-03$ & 0.00622293 & 0.0388933 & 0.0777867 & 0.155573 \\
\hline 2994 & 4.31E-05 & $1.51 \mathrm{E}-03$ & $1.55 \mathrm{E}-03$ & 0.00621184 & 0.038824 & 0.077648 & 0.155296 \\
\hline 2995 & 4.31E-05 & $1.51 \mathrm{E}-03$ & $1.55 \mathrm{E}-03$ & 0.00620075 & 0.0387547 & 0.0775094 & 0.155019 \\
\hline 2996 & 4.30E-05 & $1.50 \mathrm{E}-03$ & $1.55 \mathrm{E}-03$ & 0.0061897 & 0.0386856 & 0.0773713 & 0.154743 \\
\hline 2997 & 4.29E-05 & $1.50 \mathrm{E}-03$ & $1.54 \mathrm{E}-03$ & 0.00617866 & 0.0386166 & 0.0772333 & 0.154467 \\
\hline 2998 & 4.28E-05 & $1.50 \mathrm{E}-03$ & $1.54 \mathrm{E}-03$ & 0.00616766 & 0.0385479 & 0.0770958 & 0.154192 \\
\hline 2999 & $4.28 \mathrm{E}-05$ & $1.50 \mathrm{E}-03$ & $1.54 \mathrm{E}-03$ & 0.00615667 & 0.0384792 & 0.0769583 & 0.153917 \\
\hline 3000 & 4.27E-05 & $1.49 \mathrm{E}-03$ & $1.54 \mathrm{E}-03$ & 0.00614571 & 0.0384107 & 0.0768214 & 0.153643 \\
\hline
\end{tabular}




\section{Isolated surface defects - Rev 0}

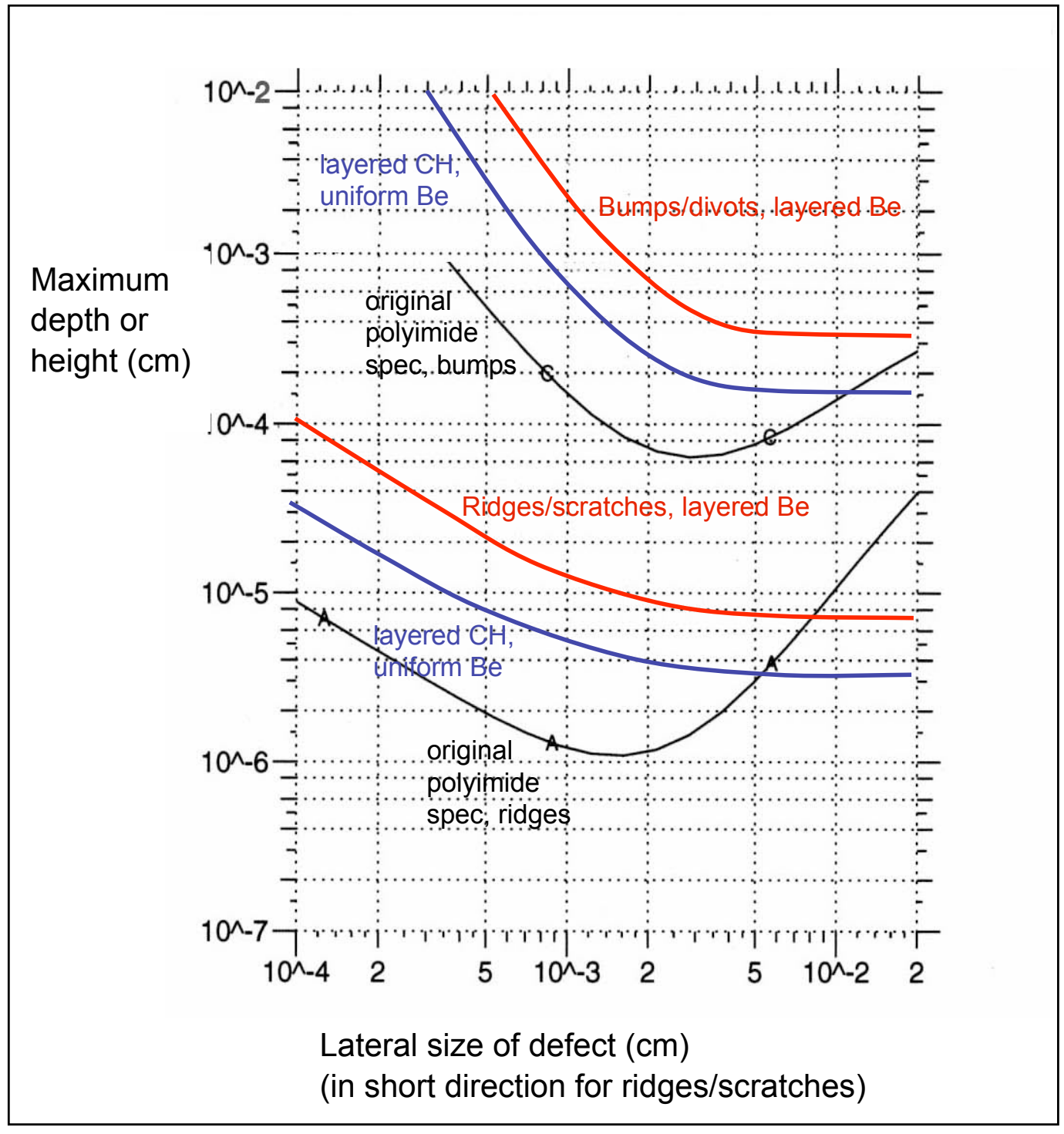

Notes:

Horizontal axis: characteristic FWHM size of defect, in $\mathrm{cm}$, either width if scratch/ridge or diameter if divot/bump

Upper curves: maximum depth of long defect (scratch or ridge) that has length >> width.

Horizontal axis in plot is FWHM.

Lower curves: maximum depth of defect that has length $\sim$ width $=$ horizontal axis (bump or divot) FWHM

Up to 20 defects allowed whose size is between the indicated limits and that limit divided by 3 . Up to 100 defects allowed whose size is between the indicated limit/3 and limit/10. Defects smaller and/or more numerous than that are limited by surface roughness specification. 
DT layer requirements - Rev 0

\begin{tabular}{|c|c|c|c|}
\hline Capsule type & $\begin{array}{c}\text { Range of possible requested DT } \\
\text { layer thickness, } \mu \mathrm{m}\end{array}$ & $\begin{array}{c}\text { Point Design } \\
\text { thickness, } \mu \mathrm{m}\end{array}$ & $\begin{array}{c}\text { Thickness tolerance, based on } \\
\text { specified thickness, } \mu \mathrm{m}\end{array}$ \\
\hline $\mathrm{Be}$ (graded doped) & $65-85$ & 75 & $\pm 3 \mu \mathrm{m}$ \\
\hline $\mathrm{CH}$ & $65-85$ & 75 & $\pm 3 \mu \mathrm{m}$ \\
\hline $\mathrm{Be}$ (uniform) & $65-85$ & 72 & $\pm 3 \mu \mathrm{m}$ \\
\hline
\end{tabular}




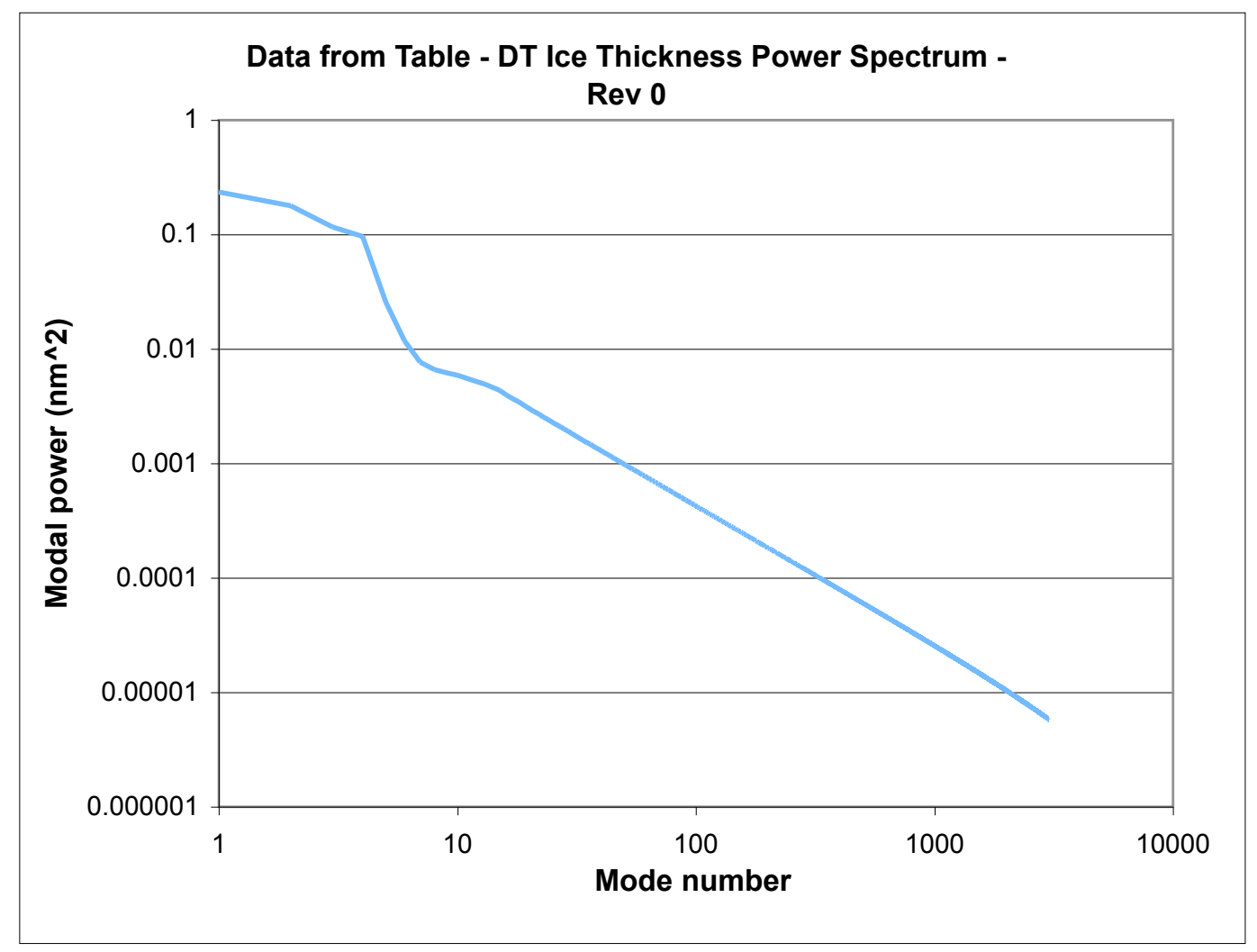

DT Ice Thickenss Power Spectrum - Rev 0

\begin{tabular}{|c|c|c|}
\hline Mode & $\begin{array}{l}\text { 1D trace modal } \\
\text { power, } \mu \mathrm{m}^{\wedge} 2\end{array}$ & $\begin{array}{l}\text { Two-D modal } \\
\text { power, } \mu \mathrm{m} \wedge 2\end{array}$ \\
\hline 1 & 0.234829 & 0.16 \\
\hline 2 & 0.177731 & 0.16 \\
\hline 3 & 0.116558 & 0.16 \\
\hline 4 & 0.0959921 & 0.16 \\
\hline 5 & 0.0257543 & 0.04 \\
\hline 6 & 0.0118326 & 0.014 \\
\hline 7 & 0.00768883 & 0.0064 \\
\hline 8 & 0.00661045 & 0.0049 \\
\hline 9 & 0.00623101 & 0.0049 \\
\hline 10 & 0.00585909 & 0.0049 \\
\hline 11 & 0.00554174 & 0.0049 \\
\hline 12 & 0.00521337 & 0.0049 \\
\hline 13 & 0.00493286 & 0.0049 \\
\hline 14 & 0.0046212 & 0.0049 \\
\hline 15 & 0.00435737 & 0.0049 \\
\hline 16 & 0.00400084 & 0.0045 \\
\hline 17 & 0.00370978 & 0.00413 \\
\hline 18 & 0.00345529 & 0.00386 \\
\hline 19 & 0.00323105 & \\
\hline 20 & 0.0030321 & \\
\hline 21 & 0.0028545 & \\
\hline 22 & 0.00269506 & \\
\hline 23 & 0.00255122 & \\
\hline 24 & 0.00242084 & \\
\hline 25 & 0.00230217 & \\
\hline 26 & 0.00219373 & \\
\hline 27 & 0.0020943 & \\
\hline 28 & 0.00200283 & \\
\hline 29 & 0.00191841 & \\
\hline 30 & 0.0018403 & \\
\hline 31 & 0.00176781 & \\
\hline
\end{tabular}




\begin{tabular}{|c|c|}
\hline 97 & 0.000441423 \\
\hline 98 & 0.000435973 \\
\hline 99 & 0.000430645 \\
\hline 100 & 0.000425435 \\
\hline 101 & 0.000420338 \\
\hline 102 & 0.000415353 \\
\hline 103 & 0.000410474 \\
\hline 104 & 0.000405699 \\
\hline 105 & 0.000401025 \\
\hline 106 & 0.000396448 \\
\hline 107 & 0.000391965 \\
\hline 108 & 0.000387575 \\
\hline 109 & 0.000383273 \\
\hline 110 & 0.000379058 \\
\hline 111 & 0.000374927 \\
\hline 112 & 0.000370877 \\
\hline 113 & 0.000366907 \\
\hline 114 & 0.000363014 \\
\hline 115 & 0.000359195 \\
\hline 116 & 0.000355449 \\
\hline 117 & 0.000351774 \\
\hline 118 & 0.000348167 \\
\hline 119 & 0.000344628 \\
\hline 120 & 0.000341154 \\
\hline 121 & 0.000337743 \\
\hline 122 & 0.000334394 \\
\hline 123 & 0.000331105 \\
\hline 124 & 0.000327875 \\
\hline 125 & 0.000324702 \\
\hline 126 & 0.000321584 \\
\hline 127 & 0.000318521 \\
\hline 128 & 0.00031551 \\
\hline 129 & 0.000312551 \\
\hline 130 & 0.000309643 \\
\hline 131 & 0.000306783 \\
\hline 132 & 0.000303972 \\
\hline 133 & 0.000301206 \\
\hline 134 & 0.000298487 \\
\hline 135 & 0.000295812 \\
\hline 136 & 0.000293181 \\
\hline 137 & 0.000290591 \\
\hline 138 & 0.000288044 \\
\hline 139 & 0.000285536 \\
\hline 140 & 0.000283069 \\
\hline 141 & 0.00028064 \\
\hline 142 & 0.000278249 \\
\hline 143 & 0.000275894 \\
\hline 144 & 0.000273576 \\
\hline 145 & 0.000271293 \\
\hline 146 & 0.000269045 \\
\hline 147 & 0.00026683 \\
\hline 148 & 0.000264649 \\
\hline 149 & 0.000262499 \\
\hline 150 & 0.000260382 \\
\hline 151 & 0.000258295 \\
\hline 152 & 0.000256239 \\
\hline 153 & 0.000254212 \\
\hline 154 & 0.000252214 \\
\hline 155 & 0.000250245 \\
\hline 156 & 0.000248304 \\
\hline 157 & 0.000246389 \\
\hline 158 & 0.000244502 \\
\hline 159 & 0.000242641 \\
\hline 160 & 0.000240806 \\
\hline 161 & 0.000238995 \\
\hline
\end{tabular}




\begin{tabular}{|c|c|}
\hline 162 & 0.00023721 \\
\hline 163 & 0.000235448 \\
\hline 164 & 0.000233711 \\
\hline 165 & 0.000231996 \\
\hline 166 & 0.000230305 \\
\hline 167 & 0.000228635 \\
\hline 168 & 0.000226988 \\
\hline 169 & 0.000225362 \\
\hline 170 & 0.000223757 \\
\hline 171 & 0.000222173 \\
\hline 172 & 0.000220609 \\
\hline 173 & 0.000219065 \\
\hline 174 & 0.000217541 \\
\hline 175 & 0.000216036 \\
\hline 176 & 0.00021455 \\
\hline 177 & 0.000213082 \\
\hline 178 & 0.000211633 \\
\hline 179 & 0.000210201 \\
\hline 180 & 0.000208787 \\
\hline 181 & 0.00020739 \\
\hline 182 & 0.000206011 \\
\hline 183 & 0.000204647 \\
\hline 184 & 0.000203301 \\
\hline 185 & 0.00020197 \\
\hline 186 & 0.000200655 \\
\hline 187 & 0.000199355 \\
\hline 188 & 0.000198071 \\
\hline 189 & 0.000196802 \\
\hline 190 & 0.000195547 \\
\hline 191 & 0.000194307 \\
\hline 192 & 0.000193081 \\
\hline 193 & 0.000191869 \\
\hline 194 & 0.000190672 \\
\hline 195 & 0.000189487 \\
\hline 196 & 0.000188316 \\
\hline 197 & 0.000187158 \\
\hline 198 & 0.000186013 \\
\hline 199 & 0.00018488 \\
\hline 200 & 0.000183761 \\
\hline 201 & 0.000182653 \\
\hline 202 & 0.000181557 \\
\hline 203 & 0.000180474 \\
\hline 204 & 0.000179402 \\
\hline 205 & 0.000178341 \\
\hline 206 & 0.000177292 \\
\hline 207 & 0.000176254 \\
\hline 208 & 0.000175228 \\
\hline 209 & 0.000174212 \\
\hline 210 & 0.000173206 \\
\hline 211 & 0.000172211 \\
\hline 212 & 0.000171227 \\
\hline 213 & 0.000170253 \\
\hline 214 & 0.000169289 \\
\hline 215 & 0.000168334 \\
\hline 216 & 0.00016739 \\
\hline 217 & 0.000166454 \\
\hline 218 & 0.000165529 \\
\hline 219 & 0.000164613 \\
\hline 220 & 0.000163706 \\
\hline 221 & 0.000162807 \\
\hline 222 & 0.000161919 \\
\hline 223 & 0.000161038 \\
\hline 224 & 0.000160167 \\
\hline 225 & 0.000159303 \\
\hline 226 & 0.000158449 \\
\hline
\end{tabular}




\begin{tabular}{|c|c|}
\hline 227 & 0.000157602 \\
\hline 228 & 0.000156764 \\
\hline 229 & 0.000155934 \\
\hline 230 & 0.000155112 \\
\hline 231 & 0.000154298 \\
\hline 232 & 0.000153491 \\
\hline 233 & 0.000152692 \\
\hline 234 & 0.000151901 \\
\hline 235 & 0.000151117 \\
\hline 236 & 0.00015034 \\
\hline 237 & 0.000149571 \\
\hline 238 & 0.000148809 \\
\hline 239 & 0.000148053 \\
\hline 240 & 0.000147305 \\
\hline 241 & 0.000146563 \\
\hline 242 & 0.000145829 \\
\hline 243 & 0.0001451 \\
\hline 244 & 0.000144379 \\
\hline 245 & 0.000143664 \\
\hline 246 & 0.000142956 \\
\hline 247 & 0.000142253 \\
\hline 248 & 0.000141557 \\
\hline 249 & 0.000140867 \\
\hline 250 & 0.000140184 \\
\hline 251 & 0.000139506 \\
\hline 252 & 0.000138834 \\
\hline 253 & 0.000138168 \\
\hline 254 & 0.000137508 \\
\hline 255 & 0.000136853 \\
\hline 256 & 0.000136205 \\
\hline 257 & 0.000135561 \\
\hline 258 & 0.000134923 \\
\hline 259 & 0.000134291 \\
\hline 260 & 0.000133664 \\
\hline 261 & 0.000133042 \\
\hline 262 & 0.000132426 \\
\hline 263 & 0.000131814 \\
\hline 264 & 0.000131208 \\
\hline 265 & 0.000130607 \\
\hline 266 & 0.000130011 \\
\hline 267 & 0.00012942 \\
\hline 268 & 0.000128833 \\
\hline 269 & 0.000128252 \\
\hline 270 & 0.000127675 \\
\hline 271 & 0.000127103 \\
\hline 272 & 0.000126535 \\
\hline 273 & 0.000125972 \\
\hline 274 & 0.000125414 \\
\hline 275 & 0.00012486 \\
\hline 276 & 0.000124311 \\
\hline 277 & 0.000123765 \\
\hline 278 & 0.000123225 \\
\hline 279 & 0.000122688 \\
\hline 280 & 0.000122156 \\
\hline 281 & 0.000121627 \\
\hline 282 & 0.000121104 \\
\hline 283 & 0.000120583 \\
\hline 284 & 0.000120068 \\
\hline 285 & 0.000119556 \\
\hline 286 & 0.000119048 \\
\hline 287 & 0.000118544 \\
\hline 288 & 0.000118044 \\
\hline 289 & 0.000117547 \\
\hline 290 & 0.000117055 \\
\hline 291 & 0.000116566 \\
\hline
\end{tabular}




\begin{tabular}{|c|c|}
\hline 292 & 0.000116081 \\
\hline 293 & 0.000115599 \\
\hline 294 & 0.000115121 \\
\hline 295 & 0.000114646 \\
\hline 296 & 0.000114176 \\
\hline 297 & 0.000113708 \\
\hline 298 & 0.000113245 \\
\hline 299 & 0.000112784 \\
\hline 300 & 0.000112327 \\
\hline 301 & 0.000111873 \\
\hline 302 & 0.000111423 \\
\hline 303 & 0.000110976 \\
\hline 304 & 0.000110532 \\
\hline 305 & 0.000110091 \\
\hline 306 & 0.000109654 \\
\hline 307 & 0.000109219 \\
\hline 308 & 0.000108788 \\
\hline 309 & 0.00010836 \\
\hline 310 & 0.000107935 \\
\hline 311 & 0.000107512 \\
\hline 312 & 0.000107093 \\
\hline 313 & 0.000106677 \\
\hline 314 & 0.000106264 \\
\hline 315 & 0.000105853 \\
\hline 316 & 0.000105446 \\
\hline 317 & 0.000105041 \\
\hline 318 & 0.000104639 \\
\hline 319 & 0.00010424 \\
\hline 320 & 0.000103844 \\
\hline 321 & 0.00010345 \\
\hline 322 & 0.000103059 \\
\hline 323 & 0.000102671 \\
\hline 324 & 0.000102285 \\
\hline 325 & 0.000101902 \\
\hline 326 & 0.000101522 \\
\hline 327 & 0.000101144 \\
\hline 328 & 0.000100768 \\
\hline 329 & 0.000100395 \\
\hline 330 & 0.000100025 \\
\hline 331 & $9.96572 \mathrm{E}-05$ \\
\hline 332 & 0.000099292 \\
\hline 333 & $9.89288 \mathrm{E}-05$ \\
\hline 334 & 9.85683E-05 \\
\hline 335 & 9.82099E-05 \\
\hline 336 & $9.78542 \mathrm{E}-05$ \\
\hline 337 & $9.75004 \mathrm{E}-05$ \\
\hline 338 & $9.71493 \mathrm{E}-05$ \\
\hline 339 & 9.68002E-05 \\
\hline 340 & $9.64536 \mathrm{E}-05$ \\
\hline 341 & 9.61089E-05 \\
\hline 342 & 9.57669E-05 \\
\hline 343 & $9.54266 \mathrm{E}-05$ \\
\hline 344 & $9.50889 \mathrm{E}-05$ \\
\hline 345 & 0.000094753 \\
\hline 346 & 9.44196E-05 \\
\hline 347 & 9.40879E-05 \\
\hline 348 & 9.37587E-05 \\
\hline 349 & $9.34312 \mathrm{E}-05$ \\
\hline 350 & $9.31062 \mathrm{E}-05$ \\
\hline 351 & $9.27828 \mathrm{E}-05$ \\
\hline 352 & $9.24618 \mathrm{E}-05$ \\
\hline 353 & $9.21425 \mathrm{E}-05$ \\
\hline 354 & $9.18255 \mathrm{E}-05$ \\
\hline 355 & $9.15101 \mathrm{E}-05$ \\
\hline 356 & 0.000091197 \\
\hline
\end{tabular}




\begin{tabular}{|c|c|}
\hline 357 & $9.08855 \mathrm{E}-05$ \\
\hline 358 & $9.05763 \mathrm{E}-05$ \\
\hline 359 & $9.02686 \mathrm{E}-05$ \\
\hline 360 & $8.99631 \mathrm{E}-05$ \\
\hline 361 & 8.96592E-05 \\
\hline 362 & $8.93574 \mathrm{E}-05$ \\
\hline 363 & $8.90572 \mathrm{E}-05$ \\
\hline 364 & 8.87591E-05 \\
\hline 365 & $8.84624 \mathrm{E}-05$ \\
\hline 366 & 8.81679E-05 \\
\hline 367 & 8.78748E-05 \\
\hline 368 & $8.75838 \mathrm{E}-05$ \\
\hline 369 & $8.72942 \mathrm{E}-05$ \\
\hline 370 & 8.70067E-05 \\
\hline 371 & 8.67205E-05 \\
\hline 372 & $8.64364 \mathrm{E}-05$ \\
\hline 373 & 8.61536E-05 \\
\hline 374 & 8.58729E-05 \\
\hline 375 & 8.55934E-05 \\
\hline 376 & 8.53159E-05 \\
\hline 377 & 8.50397E-05 \\
\hline 378 & $8.47654 \mathrm{E}-05$ \\
\hline 379 & 8.44924E-05 \\
\hline 380 & $8.42213 \mathrm{E}-05$ \\
\hline 381 & $8.39514 \mathrm{E}-05$ \\
\hline 382 & 8.36835E-05 \\
\hline 383 & 8.34167E-05 \\
\hline 384 & $8.31518 \mathrm{E}-05$ \\
\hline 385 & $8.28881 \mathrm{E}-05$ \\
\hline 386 & 8.26262E-05 \\
\hline 387 & $8.23655 \mathrm{E}-05$ \\
\hline 388 & $8.21066 \mathrm{E}-05$ \\
\hline 389 & $8.18488 \mathrm{E}-05$ \\
\hline 390 & 8.15929E-05 \\
\hline 391 & 0.000081338 \\
\hline 392 & $8.10849 \mathrm{E}-05$ \\
\hline 393 & $8.08328 \mathrm{E}-05$ \\
\hline 394 & $8.05826 \mathrm{E}-05$ \\
\hline 395 & $8.03333 \mathrm{E}-05$ \\
\hline 396 & $8.00859 \mathrm{E}-05$ \\
\hline 397 & 7.98394E-05 \\
\hline 398 & 7.95946E-05 \\
\hline 399 & 7.93509E-05 \\
\hline 400 & $7.91088 \mathrm{E}-05$ \\
\hline 401 & 7.88677E-05 \\
\hline 402 & 7.86284E-05 \\
\hline 403 & 7.83899E-05 \\
\hline 404 & $7.81531 \mathrm{E}-05$ \\
\hline 405 & 7.79172E-05 \\
\hline 406 & 0.000077683 \\
\hline 407 & 7.74497E-05 \\
\hline 408 & 0.000077218 \\
\hline 409 & 7.69872E-05 \\
\hline 410 & 0.000076758 \\
\hline 411 & 7.65296E-05 \\
\hline 412 & 7.63029E-05 \\
\hline 413 & $7.60769 \mathrm{E}-05$ \\
\hline 414 & 7.58526E-05 \\
\hline 415 & $7.56291 \mathrm{E}-05$ \\
\hline 416 & 7.54071E-05 \\
\hline 417 & 7.51859E-05 \\
\hline 418 & 7.49663E-05 \\
\hline 419 & $7.47475 \mathrm{E}-05$ \\
\hline 420 & $7.45301 \mathrm{E}-05$ \\
\hline 421 & $7.43136 \mathrm{E}-05$ \\
\hline
\end{tabular}




\begin{tabular}{|c|c|}
\hline 422 & 7.40985E-05 \\
\hline 423 & 7.38842E-05 \\
\hline 424 & 7.36714E-05 \\
\hline 425 & 7.34593E-05 \\
\hline 426 & 7.32486E-05 \\
\hline 427 & 7.30387E-05 \\
\hline 428 & 7.28303E-05 \\
\hline 429 & 7.26225E-05 \\
\hline 430 & 7.24162E-05 \\
\hline 431 & $7.22105 \mathrm{E}-05$ \\
\hline 432 & 7.20063E-05 \\
\hline 433 & 7.18027E-05 \\
\hline 434 & $7.16006 \mathrm{E}-05$ \\
\hline 435 & $7.13991 \mathrm{E}-05$ \\
\hline 436 & 0.000071199 \\
\hline 437 & 7.09995E-05 \\
\hline 438 & 7.08014E-05 \\
\hline 439 & 7.06039E-05 \\
\hline 440 & 7.04078E-05 \\
\hline 441 & 7.02123E-05 \\
\hline 442 & 7.00181E-05 \\
\hline 443 & 6.98246E-05 \\
\hline 444 & $6.96323 \mathrm{E}-05$ \\
\hline 445 & 6.94407E-05 \\
\hline 446 & 6.92503E-05 \\
\hline 447 & $6.90605 \mathrm{E}-05$ \\
\hline 448 & $6.88721 \mathrm{E}-05$ \\
\hline 449 & $6.86842 \mathrm{E}-05$ \\
\hline 450 & $6.84975 \mathrm{E}-05$ \\
\hline 451 & $6.83115 \mathrm{E}-05$ \\
\hline 452 & 6.81266E-05 \\
\hline 453 & 6.79424E-05 \\
\hline 454 & 6.77594E-05 \\
\hline 455 & $6.75769 \mathrm{E}-05$ \\
\hline 456 & $6.73956 \mathrm{E}-05$ \\
\hline 457 & $6.72149 \mathrm{E}-05$ \\
\hline 458 & $6.70354 \mathrm{E}-05$ \\
\hline 459 & $6.68563 \mathrm{E}-05$ \\
\hline 460 & 6.66786E-05 \\
\hline 461 & $6.65012 \mathrm{E}-05$ \\
\hline 462 & 6.63252E-05 \\
\hline 463 & 6.61495E-05 \\
\hline 464 & $6.59751 \mathrm{E}-05$ \\
\hline 465 & $6.58011 \mathrm{E}-05$ \\
\hline 466 & 6.56284E-05 \\
\hline 467 & 0.000065456 \\
\hline 468 & $6.52849 \mathrm{E}-05$ \\
\hline 469 & $6.51142 \mathrm{E}-05$ \\
\hline 470 & 6.49446E-05 \\
\hline 471 & $6.47755 \mathrm{E}-05$ \\
\hline 472 & $6.46076 \mathrm{E}-05$ \\
\hline 473 & 0.00006444 \\
\hline 474 & $6.42736 \mathrm{E}-05$ \\
\hline 475 & $6.41076 \mathrm{E}-05$ \\
\hline 476 & 6.39428E-05 \\
\hline 477 & 6.37783E-05 \\
\hline 478 & $6.36149 \mathrm{E}-05$ \\
\hline 479 & 0.000063452 \\
\hline 480 & $6.32901 \mathrm{E}-05$ \\
\hline 481 & 6.31287E-05 \\
\hline 482 & 6.29683E-05 \\
\hline 483 & $6.28083 \mathrm{E}-05$ \\
\hline 484 & 6.26494E-05 \\
\hline 485 & $6.24908 \mathrm{E}-05$ \\
\hline 486 & 6.23334E-05 \\
\hline
\end{tabular}




\begin{tabular}{|c|c|}
\hline 487 & $6.21762 \mathrm{E}-05$ \\
\hline 488 & $6.20202 \mathrm{E}-05$ \\
\hline 489 & $6.18645 \mathrm{E}-05$ \\
\hline 490 & 6.17099E-05 \\
\hline 491 & $6.15555 \mathrm{E}-05$ \\
\hline 492 & 6.14023E-05 \\
\hline 493 & $6.12493 \mathrm{E}-05$ \\
\hline 494 & 6.10974E-05 \\
\hline 495 & $6.09459 \mathrm{E}-05$ \\
\hline 496 & $6.07953 \mathrm{E}-05$ \\
\hline 497 & $6.06451 \mathrm{E}-05$ \\
\hline 498 & $6.04959 \mathrm{E}-05$ \\
\hline 499 & 6.03469E-05 \\
\hline 500 & 0.000060199 \\
\hline 501 & $6.00514 \mathrm{E}-05$ \\
\hline 502 & $5.99048 \mathrm{E}-05$ \\
\hline 503 & 5.97585E-05 \\
\hline 504 & $5.96132 \mathrm{E}-05$ \\
\hline 505 & $5.94681 \mathrm{E}-05$ \\
\hline 506 & $5.93241 \mathrm{E}-05$ \\
\hline 507 & 5.91803E-05 \\
\hline 508 & $5.90375 \mathrm{E}-05$ \\
\hline 509 & 5.88949E-05 \\
\hline 510 & 5.87534E-05 \\
\hline 511 & 0.000058612 \\
\hline 512 & $5.84717 \mathrm{E}-05$ \\
\hline 513 & $5.83316 \mathrm{E}-05$ \\
\hline 514 & $5.81924 \mathrm{E}-05$ \\
\hline 515 & $5.80535 \mathrm{E}-05$ \\
\hline 516 & 5.79155E-05 \\
\hline 517 & $5.77778 \mathrm{E}-05$ \\
\hline 518 & 0.000057641 \\
\hline 519 & 5.75044E-05 \\
\hline 520 & $5.73688 \mathrm{E}-05$ \\
\hline 521 & $5.72334 \mathrm{E}-05$ \\
\hline 522 & 5.70989E-05 \\
\hline 523 & 5.69646E-05 \\
\hline 524 & $5.68312 \mathrm{E}-05$ \\
\hline 525 & $5.66981 \mathrm{E}-05$ \\
\hline 526 & $5.65658 \mathrm{E}-05$ \\
\hline 527 & $5.64338 \mathrm{E}-05$ \\
\hline 528 & $5.63026 \mathrm{E}-05$ \\
\hline 529 & 5.61717E-05 \\
\hline 530 & $5.60416 \mathrm{E}-05$ \\
\hline 531 & $5.59118 \mathrm{E}-05$ \\
\hline 532 & $5.57828 \mathrm{E}-05$ \\
\hline 533 & 0.000055654 \\
\hline 534 & 5.55261E-05 \\
\hline 535 & 5.53983E-05 \\
\hline 536 & $5.52715 \mathrm{E}-05$ \\
\hline 537 & $5.51448 \mathrm{E}-05$ \\
\hline 538 & 0.000055019 \\
\hline 539 & 5.48933E-05 \\
\hline 540 & $5.47685 \mathrm{E}-05$ \\
\hline 541 & 5.46439E-05 \\
\hline 542 & $5.45201 \mathrm{E}-05$ \\
\hline 543 & $5.43965 \mathrm{E}-05$ \\
\hline 544 & $5.42737 \mathrm{E}-05$ \\
\hline 545 & 0.000054151 \\
\hline 546 & $5.40293 \mathrm{E}-05$ \\
\hline 547 & 5.39076E-05 \\
\hline 548 & $5.37868 \mathrm{E}-05$ \\
\hline 549 & $5.36661 \mathrm{E}-05$ \\
\hline 550 & $5.35463 \mathrm{E}-05$ \\
\hline 551 & $5.34266 \mathrm{E}-05$ \\
\hline
\end{tabular}




\begin{tabular}{|c|c|}
\hline 552 & 5.33077E-05 \\
\hline 553 & 5.31889E-05 \\
\hline 554 & 0.000053071 \\
\hline 555 & 5.29532E-05 \\
\hline 556 & $5.28362 \mathrm{E}-05$ \\
\hline 557 & 5.27193E-05 \\
\hline 558 & $5.26032 \mathrm{E}-05$ \\
\hline 559 & $5.24872 \mathrm{E}-05$ \\
\hline 560 & 0.000052372 \\
\hline 561 & 0.000052257 \\
\hline 562 & $5.21427 \mathrm{E}-05$ \\
\hline 563 & $5.20285 \mathrm{E}-05$ \\
\hline 564 & 5.19152E-05 \\
\hline 565 & $5.18019 \mathrm{E}-05$ \\
\hline 566 & 5.16894E-05 \\
\hline 567 & 0.000051577 \\
\hline 568 & $5.14654 \mathrm{E}-05$ \\
\hline 569 & $5.13538 \mathrm{E}-05$ \\
\hline 570 & $5.12431 \mathrm{E}-05$ \\
\hline 571 & $5.11324 \mathrm{E}-05$ \\
\hline 572 & $5.10225 \mathrm{E}-05$ \\
\hline 573 & $5.09127 \mathrm{E}-05$ \\
\hline 574 & $5.08036 \mathrm{E}-05$ \\
\hline 575 & 5.06946E-05 \\
\hline 576 & $5.05864 \mathrm{E}-05$ \\
\hline 577 & $5.04782 \mathrm{E}-05$ \\
\hline 578 & $5.03708 \mathrm{E}-05$ \\
\hline 579 & $5.02635 \mathrm{E}-05$ \\
\hline 580 & 5.01569E-05 \\
\hline 581 & $5.00504 \mathrm{E}-05$ \\
\hline 582 & 4.99446E-05 \\
\hline 583 & 4.98389E-05 \\
\hline 584 & 4.97339E-05 \\
\hline 585 & 0.000049629 \\
\hline 586 & 4.95248E-05 \\
\hline 587 & 4.94206E-05 \\
\hline 588 & $4.93172 \mathrm{E}-05$ \\
\hline 589 & 4.92138E-05 \\
\hline 590 & $4.91112 \mathrm{E}-05$ \\
\hline 591 & $4.90086 \mathrm{E}-05$ \\
\hline 592 & 4.89068E-05 \\
\hline 593 & 4.88049E-05 \\
\hline 594 & 4.87038E-05 \\
\hline 595 & 4.86027E-05 \\
\hline 596 & 4.85023E-05 \\
\hline 597 & 0.000048402 \\
\hline 598 & 4.83024E-05 \\
\hline 599 & 4.82027E-05 \\
\hline 600 & 4.81038E-05 \\
\hline 601 & 4.80049E-05 \\
\hline 602 & $4.79068 \mathrm{E}-05$ \\
\hline 603 & 4.78086E-05 \\
\hline 604 & 4.77112E-05 \\
\hline 605 & 4.76137E-05 \\
\hline 606 & 0.000047517 \\
\hline 607 & 4.74202E-05 \\
\hline 608 & $4.73242 \mathrm{E}-05$ \\
\hline 609 & $4.72281 \mathrm{E}-05$ \\
\hline 610 & $4.71328 \mathrm{E}-05$ \\
\hline 611 & 4.70374E-05 \\
\hline 612 & 4.69427E-05 \\
\hline 613 & $4.68481 \mathrm{E}-05$ \\
\hline 614 & $4.67541 \mathrm{E}-05$ \\
\hline 615 & $4.66601 \mathrm{E}-05$ \\
\hline 616 & $4.65668 \mathrm{E}-05$ \\
\hline
\end{tabular}




\begin{tabular}{|c|c|}
\hline 617 & $4.64734 \mathrm{E}-05$ \\
\hline 618 & 4.63808E-05 \\
\hline 619 & $4.62881 \mathrm{E}-05$ \\
\hline 620 & 4.61961E-05 \\
\hline 621 & $4.61041 \mathrm{E}-05$ \\
\hline 622 & 4.60128E-05 \\
\hline 623 & 4.59214E-05 \\
\hline 624 & 4.58307E-05 \\
\hline 625 & 0.00004574 \\
\hline 626 & 4.56499E-05 \\
\hline 627 & $4.55598 \mathrm{E}-05$ \\
\hline 628 & 4.54704E-05 \\
\hline 629 & 0.000045381 \\
\hline 630 & 4.52922E-05 \\
\hline 631 & 4.52033E-05 \\
\hline 632 & $4.51152 \mathrm{E}-05$ \\
\hline 633 & 4.50269E-05 \\
\hline 634 & 4.49394E-05 \\
\hline 635 & $4.48518 \mathrm{E}-05$ \\
\hline 636 & 4.47648E-05 \\
\hline 637 & 4.46778E-05 \\
\hline 638 & $4.45914 \mathrm{E}-05$ \\
\hline 639 & 0.000044505 \\
\hline 640 & 4.44193E-05 \\
\hline 641 & 4.43334E-05 \\
\hline 642 & 4.42483E-05 \\
\hline 643 & 0.000044163 \\
\hline 644 & $4.40785 \mathrm{E}-05$ \\
\hline 645 & 4.39938E-05 \\
\hline 646 & 4.39098E-05 \\
\hline 647 & 4.38257E-05 \\
\hline 648 & 4.37423E-05 \\
\hline 649 & $4.36588 \mathrm{E}-05$ \\
\hline 650 & 4.35759E-05 \\
\hline 651 & 4.34929E-05 \\
\hline 652 & 4.34106E-05 \\
\hline 653 & 4.33282E-05 \\
\hline 654 & 4.32465E-05 \\
\hline 655 & $4.31646 \mathrm{E}-05$ \\
\hline 656 & 4.30834E-05 \\
\hline 657 & 4.30021E-05 \\
\hline 658 & 4.29215E-05 \\
\hline 659 & 4.28407E-05 \\
\hline 660 & 4.27606E-05 \\
\hline 661 & 4.26804E-05 \\
\hline 662 & 4.26008E-05 \\
\hline 663 & $4.25211 \mathrm{E}-05$ \\
\hline 664 & $4.24421 \mathrm{E}-05$ \\
\hline 665 & 4.23629E-05 \\
\hline 666 & $4.22844 \mathrm{E}-05$ \\
\hline 667 & 4.22057E-05 \\
\hline 668 & $4.21277 \mathrm{E}-05$ \\
\hline 669 & 4.20496E-05 \\
\hline 670 & 4.19721E-05 \\
\hline 671 & 4.18945E-05 \\
\hline 672 & 4.18175E-05 \\
\hline 673 & $4.17404 \mathrm{E}-05$ \\
\hline 674 & 4.16639E-05 \\
\hline 675 & 4.15873E-05 \\
\hline 676 & $4.15113 \mathrm{E}-05$ \\
\hline 677 & 4.14352E-05 \\
\hline 678 & 4.13597E-05 \\
\hline 679 & 4.12841E-05 \\
\hline 680 & $4.12091 \mathrm{E}-05$ \\
\hline 681 & $4.11339 \mathrm{E}-05$ \\
\hline
\end{tabular}




\begin{tabular}{|c|c|}
\hline 682 & 4.10594E-05 \\
\hline 683 & 4.09847E-05 \\
\hline 684 & 4.09107E-05 \\
\hline 685 & $4.08365 \mathrm{E}-05$ \\
\hline 686 & 0.000040763 \\
\hline 687 & 4.06893E-05 \\
\hline 688 & $4.06162 \mathrm{E}-05$ \\
\hline 689 & 4.05429E-05 \\
\hline 690 & 4.04703E-05 \\
\hline 691 & 4.03976E-05 \\
\hline 692 & $4.03254 \mathrm{E}-05$ \\
\hline 693 & $4.02531 \mathrm{E}-05$ \\
\hline 694 & $4.01814 \mathrm{E}-05$ \\
\hline 695 & $4.01095 \mathrm{E}-05$ \\
\hline 696 & $4.00383 \mathrm{E}-05$ \\
\hline 697 & 3.99669E-05 \\
\hline 698 & $3.98961 \mathrm{E}-05$ \\
\hline 699 & $3.98252 \mathrm{E}-05$ \\
\hline 700 & $3.97548 \mathrm{E}-05$ \\
\hline 701 & $3.96843 \mathrm{E}-05$ \\
\hline 702 & $3.96144 \mathrm{E}-05$ \\
\hline 703 & 3.95443E-05 \\
\hline 704 & $3.94749 \mathrm{E}-05$ \\
\hline 705 & $3.94052 \mathrm{E}-05$ \\
\hline 706 & $3.93362 \mathrm{E}-05$ \\
\hline 707 & 0.000039267 \\
\hline 708 & $3.91984 \mathrm{E}-05$ \\
\hline 709 & $3.91296 \mathrm{E}-05$ \\
\hline 710 & $3.90614 \mathrm{E}-05$ \\
\hline 711 & $3.89931 \mathrm{E}-05$ \\
\hline 712 & $3.89253 \mathrm{E}-05$ \\
\hline 713 & $3.88574 \mathrm{E}-05$ \\
\hline 714 & 3.87901E-05 \\
\hline 715 & $3.87226 \mathrm{E}-05$ \\
\hline 716 & $3.86556 \mathrm{E}-05$ \\
\hline 717 & $3.85886 \mathrm{E}-05$ \\
\hline 718 & 0.000038522 \\
\hline 719 & $3.84554 \mathrm{E}-05$ \\
\hline 720 & $3.83892 \mathrm{E}-05$ \\
\hline 721 & 0.000038323 \\
\hline 722 & $3.82573 \mathrm{E}-05$ \\
\hline 723 & $3.81914 \mathrm{E}-05$ \\
\hline 724 & $3.81261 \mathrm{E}-05$ \\
\hline 725 & $3.80606 \mathrm{E}-05$ \\
\hline 726 & 3.79957E-05 \\
\hline 727 & $3.79306 \mathrm{E}-05$ \\
\hline 728 & $3.78661 \mathrm{E}-05$ \\
\hline 729 & $3.78014 \mathrm{E}-05$ \\
\hline 730 & $3.77373 \mathrm{E}-05$ \\
\hline 731 & 0.000037673 \\
\hline 732 & 3.76093E-05 \\
\hline 733 & $3.75453 \mathrm{E}-05$ \\
\hline 734 & 0.000037482 \\
\hline 735 & $3.74185 \mathrm{E}-05$ \\
\hline 736 & $3.73555 \mathrm{E}-05$ \\
\hline 737 & 3.72923E-05 \\
\hline 738 & 3.72297E-05 \\
\hline 739 & $3.71669 \mathrm{E}-05$ \\
\hline 740 & 3.71047E-05 \\
\hline 741 & 3.70423E-05 \\
\hline 742 & $3.69805 E-05$ \\
\hline 743 & 3.69184E-05 \\
\hline 744 & $3.68569 \mathrm{E}-05$ \\
\hline 745 & $3.67953 \mathrm{E}-05$ \\
\hline 746 & $3.67341 \mathrm{E}-05$ \\
\hline
\end{tabular}




\begin{tabular}{|c|c|}
\hline 747 & $3.66728 \mathrm{E}-05$ \\
\hline 748 & $3.66121 \mathrm{E}-05$ \\
\hline 749 & $3.65511 \mathrm{E}-05$ \\
\hline 750 & $3.64907 \mathrm{E}-05$ \\
\hline 751 & $3.64301 \mathrm{E}-05$ \\
\hline 752 & 0.00003637 \\
\hline 753 & $3.63098 \mathrm{E}-05$ \\
\hline 754 & $3.62501 \mathrm{E}-05$ \\
\hline 755 & $3.61902 \mathrm{E}-05$ \\
\hline 756 & $3.61308 \mathrm{E}-05$ \\
\hline 757 & $3.60713 \mathrm{E}-05$ \\
\hline 758 & $3.60123 \mathrm{E}-05$ \\
\hline 759 & $3.59531 \mathrm{E}-05$ \\
\hline 760 & $3.58944 \mathrm{E}-05$ \\
\hline 761 & $3.58356 \mathrm{E}-05$ \\
\hline 762 & $3.57772 \mathrm{E}-05$ \\
\hline 763 & $3.57187 \mathrm{E}-05$ \\
\hline 764 & $3.56607 \mathrm{E}-05$ \\
\hline 765 & $3.56025 \mathrm{E}-05$ \\
\hline 766 & $3.55449 \mathrm{E}-05$ \\
\hline 767 & 0.000035487 \\
\hline 768 & $3.54297 \mathrm{E}-05$ \\
\hline 769 & $3.53722 \mathrm{E}-05$ \\
\hline 770 & $3.53152 \mathrm{E}-05$ \\
\hline 771 & 0.000035258 \\
\hline 772 & $3.52013 \mathrm{E}-05$ \\
\hline 773 & $3.51444 \mathrm{E}-05$ \\
\hline 774 & $3.50881 \mathrm{E}-05$ \\
\hline 775 & $3.50315 \mathrm{E}-05$ \\
\hline 776 & $3.49755 \mathrm{E}-05$ \\
\hline 777 & $3.49193 \mathrm{E}-05$ \\
\hline 778 & $3.48636 \mathrm{E}-05$ \\
\hline 779 & $3.48076 \mathrm{E}-05$ \\
\hline 780 & $3.47523 \mathrm{E}-05$ \\
\hline 781 & $3.46967 \mathrm{E}-05$ \\
\hline 782 & $3.46416 \mathrm{E}-05$ \\
\hline 783 & $3.45863 \mathrm{E}-05$ \\
\hline 784 & $3.45315 \mathrm{E}-05$ \\
\hline 785 & $3.44765 \mathrm{E}-05$ \\
\hline 786 & $3.44221 \mathrm{E}-05$ \\
\hline 787 & $3.43674 \mathrm{E}-05$ \\
\hline 788 & $3.43132 \mathrm{E}-05$ \\
\hline 789 & 3.42589E-05 \\
\hline 790 & 0.000034205 \\
\hline 791 & $3.41509 \mathrm{E}-05$ \\
\hline 792 & $3.40974 \mathrm{E}-05$ \\
\hline 793 & $3.40436 \mathrm{E}-05$ \\
\hline 794 & $3.39904 \mathrm{E}-05$ \\
\hline 795 & $3.39369 \mathrm{E}-05$ \\
\hline 796 & $3.38839 \mathrm{E}-05$ \\
\hline 797 & 3.38307E-05 \\
\hline 798 & $3.37781 \mathrm{E}-05$ \\
\hline 799 & 3.37252E-05 \\
\hline 800 & $3.36728 \mathrm{E}-05$ \\
\hline 801 & $3.36202 \mathrm{E}-05$ \\
\hline 802 & $3.35681 \mathrm{E}-05$ \\
\hline 803 & $3.35158 \mathrm{E}-05$ \\
\hline 804 & 0.000033464 \\
\hline 805 & 0.000033412 \\
\hline 806 & $3.33604 \mathrm{E}-05$ \\
\hline 807 & $3.33087 \mathrm{E}-05$ \\
\hline 808 & $3.32575 \mathrm{E}-05$ \\
\hline 809 & 0.000033206 \\
\hline 810 & 0.000033155 \\
\hline 811 & $3.31038 \mathrm{E}-05$ \\
\hline
\end{tabular}




\begin{tabular}{|c|c|}
\hline 812 & $3.30532 \mathrm{E}-05$ \\
\hline 813 & $3.30022 \mathrm{E}-05$ \\
\hline 814 & $3.29518 \mathrm{E}-05$ \\
\hline 815 & $3.29012 \mathrm{E}-05$ \\
\hline 816 & $3.28511 \mathrm{E}-05$ \\
\hline 817 & 3.28007E-05 \\
\hline 818 & $3.27508 \mathrm{E}-05$ \\
\hline 819 & $3.27007 \mathrm{E}-05$ \\
\hline 820 & $3.26512 \mathrm{E}-05$ \\
\hline 821 & $3.26013 \mathrm{E}-05$ \\
\hline 822 & 0.000032552 \\
\hline 823 & $3.25024 \mathrm{E}-05$ \\
\hline 824 & $3.24534 \mathrm{E}-05$ \\
\hline 825 & $3.24041 \mathrm{E}-05$ \\
\hline 826 & $3.23553 \mathrm{E}-05$ \\
\hline 827 & $3.23062 \mathrm{E}-05$ \\
\hline 828 & $3.22577 \mathrm{E}-05$ \\
\hline 829 & $3.22089 E-05$ \\
\hline 830 & $3.21606 \mathrm{E}-05$ \\
\hline 831 & $3.21121 \mathrm{E}-05$ \\
\hline 832 & $3.20641 \mathrm{E}-05$ \\
\hline 833 & $3.20158 \mathrm{E}-05$ \\
\hline 834 & 0.000031968 \\
\hline 835 & 0.00003192 \\
\hline 836 & $3.18725 \mathrm{E}-05$ \\
\hline 837 & $3.18247 \mathrm{E}-05$ \\
\hline 838 & $3.17775 \mathrm{E}-05$ \\
\hline 839 & $3.17299 \mathrm{E}-05$ \\
\hline 840 & $3.16829 \mathrm{E}-05$ \\
\hline 841 & $3.16356 \mathrm{E}-05$ \\
\hline 842 & $3.15889 E-05$ \\
\hline 843 & $3.15419 \mathrm{E}-05$ \\
\hline 844 & $3.14953 \mathrm{E}-05$ \\
\hline 845 & $3.14485 \mathrm{E}-05$ \\
\hline 846 & $3.14023 \mathrm{E}-05$ \\
\hline 847 & $3.13557 \mathrm{E}-05$ \\
\hline 848 & $3.13097 \mathrm{E}-05$ \\
\hline 849 & $3.12634 \mathrm{E}-05$ \\
\hline 850 & $3.12176 \mathrm{E}-05$ \\
\hline 851 & $3.11715 \mathrm{E}-05$ \\
\hline 852 & 0.000031126 \\
\hline 853 & $3.10801 \mathrm{E}-05$ \\
\hline 854 & $3.10348 \mathrm{E}-05$ \\
\hline 855 & $3.09892 \mathrm{E}-05$ \\
\hline 856 & $3.09441 \mathrm{E}-05$ \\
\hline 857 & $3.08988 \mathrm{E}-05$ \\
\hline 858 & $3.08539 \mathrm{E}-05$ \\
\hline 859 & $3.08088 \mathrm{E}-05$ \\
\hline 860 & $3.07641 \mathrm{E}-05$ \\
\hline 861 & $3.07192 \mathrm{E}-05$ \\
\hline 862 & $3.06748 \mathrm{E}-05$ \\
\hline 863 & $3.06302 \mathrm{E}-05$ \\
\hline 864 & 0.000030586 \\
\hline 865 & $3.05415 \mathrm{E}-05$ \\
\hline 866 & $3.04976 \mathrm{E}-05$ \\
\hline 867 & $3.04534 \mathrm{E}-05$ \\
\hline 868 & $3.04097 \mathrm{E}-05$ \\
\hline 869 & $3.03657 \mathrm{E}-05$ \\
\hline 870 & $3.03222 \mathrm{E}-05$ \\
\hline 871 & $3.02784 \mathrm{E}-05$ \\
\hline 872 & $3.02351 \mathrm{E}-05$ \\
\hline 873 & $3.01915 \mathrm{E}-05$ \\
\hline 874 & $3.01485 \mathrm{E}-05$ \\
\hline 875 & $3.01051 \mathrm{E}-05$ \\
\hline 876 & 3.00623E-05 \\
\hline
\end{tabular}




\begin{tabular}{|c|c|}
\hline 877 & 3.00192E-05 \\
\hline 878 & $2.99765 \mathrm{E}-05$ \\
\hline 879 & $2.99336 \mathrm{E}-05$ \\
\hline 880 & $2.98912 \mathrm{E}-05$ \\
\hline 881 & $2.98485 \mathrm{E}-05$ \\
\hline 882 & $2.98063 \mathrm{E}-05$ \\
\hline 883 & $2.97638 \mathrm{E}-05$ \\
\hline 884 & $2.97218 \mathrm{E}-05$ \\
\hline 885 & $2.96796 \mathrm{E}-05$ \\
\hline 886 & $2.96378 \mathrm{E}-05$ \\
\hline 887 & 2.95957E-05 \\
\hline 888 & $2.95541 \mathrm{E}-05$ \\
\hline 889 & 2.95123E-05 \\
\hline 890 & $2.94709 \mathrm{E}-05$ \\
\hline 891 & 2.94293E-05 \\
\hline 892 & 2.93881E-05 \\
\hline 893 & 2.93467E-05 \\
\hline 894 & $2.93057 \mathrm{E}-05$ \\
\hline 895 & 2.92644E-05 \\
\hline 896 & 2.92237E-05 \\
\hline 897 & $2.91826 \mathrm{E}-05$ \\
\hline 898 & $2.91421 \mathrm{E}-05$ \\
\hline 899 & $2.91012 \mathrm{E}-05$ \\
\hline 900 & $2.90609 \mathrm{E}-05$ \\
\hline 901 & 2.90202E-05 \\
\hline 902 & $2.89801 \mathrm{E}-05$ \\
\hline 903 & $2.89396 \mathrm{E}-05$ \\
\hline 904 & 2.88997E-05 \\
\hline 905 & $2.88594 \mathrm{E}-05$ \\
\hline 906 & 2.88196E-05 \\
\hline 907 & 2.87796E-05 \\
\hline 908 & 0.00002874 \\
\hline 909 & $2.87001 \mathrm{E}-05$ \\
\hline 910 & $2.86608 \mathrm{E}-05$ \\
\hline 911 & $2.86211 \mathrm{E}-05$ \\
\hline 912 & $2.85819 \mathrm{E}-05$ \\
\hline 913 & $2.85424 \mathrm{E}-05$ \\
\hline 914 & $2.85034 \mathrm{E}-05$ \\
\hline 915 & $2.84641 \mathrm{E}-05$ \\
\hline 916 & $2.84253 \mathrm{E}-05$ \\
\hline 917 & $2.83862 E-05$ \\
\hline 918 & $2.83476 \mathrm{E}-05$ \\
\hline 919 & $2.83086 \mathrm{E}-05$ \\
\hline 920 & $2.82702 \mathrm{E}-05$ \\
\hline 921 & $2.82315 \mathrm{E}-05$ \\
\hline 922 & $2.81932 \mathrm{E}-05$ \\
\hline 923 & $2.81547 \mathrm{E}-05$ \\
\hline 924 & $2.81166 \mathrm{E}-05$ \\
\hline 925 & $2.80782 \mathrm{E}-05$ \\
\hline 926 & 2.80403E-05 \\
\hline 927 & $2.80021 \mathrm{E}-05$ \\
\hline 928 & $2.79644 \mathrm{E}-05$ \\
\hline 929 & $2.79264 \mathrm{E}-05$ \\
\hline 930 & $2.78889 \mathrm{E}-05$ \\
\hline 931 & 0.000027851 \\
\hline 932 & $2.78137 \mathrm{E}-05$ \\
\hline 933 & 0.000027776 \\
\hline 934 & 2.77389E-05 \\
\hline 935 & $2.77014 \mathrm{E}-05$ \\
\hline 936 & $2.76644 \mathrm{E}-05$ \\
\hline 937 & $2.76271 \mathrm{E}-05$ \\
\hline 938 & $2.75902 \mathrm{E}-05$ \\
\hline 939 & $2.75531 \mathrm{E}-05$ \\
\hline 940 & $2.75165 \mathrm{E}-05$ \\
\hline 941 & $2.74795 \mathrm{E}-05$ \\
\hline
\end{tabular}




\begin{tabular}{|c|c|}
\hline 942 & 0.000027443 \\
\hline 943 & $2.74062 \mathrm{E}-05$ \\
\hline 944 & $2.73699 \mathrm{E}-05$ \\
\hline 945 & 2.73333E-05 \\
\hline 946 & 2.72972E-05 \\
\hline 947 & 2.72607E-05 \\
\hline 948 & $2.72248 \mathrm{E}-05$ \\
\hline 949 & $2.71885 \mathrm{E}-05$ \\
\hline 950 & 2.71527E-05 \\
\hline 951 & $2.71166 \mathrm{E}-05$ \\
\hline 952 & 2.70809E-05 \\
\hline 953 & 0.000027045 \\
\hline 954 & 2.70095E-05 \\
\hline 955 & 2.69737E-05 \\
\hline 956 & 2.69384E-05 \\
\hline 957 & $2.69028 \mathrm{E}-05$ \\
\hline 958 & 2.68677E-05 \\
\hline 959 & 2.68322E-05 \\
\hline 960 & 2.67972E-05 \\
\hline 961 & 2.67619E-05 \\
\hline 962 & $2.67271 \mathrm{E}-05$ \\
\hline 963 & 0.000026692 \\
\hline 964 & $2.66573 \mathrm{E}-05$ \\
\hline 965 & 2.66223E-05 \\
\hline 966 & $2.65878 \mathrm{E}-05$ \\
\hline 967 & 0.000026553 \\
\hline 968 & $2.65187 \mathrm{E}-05$ \\
\hline 969 & 0.000026484 \\
\hline 970 & $2.64498 \mathrm{E}-05$ \\
\hline 971 & $2.64153 \mathrm{E}-05$ \\
\hline 972 & 2.63813E-05 \\
\hline 973 & $2.63469 \mathrm{E}-05$ \\
\hline 974 & 0.000026313 \\
\hline 975 & 2.62789E-05 \\
\hline 976 & $2.62451 \mathrm{E}-05$ \\
\hline 977 & $2.62111 \mathrm{E}-05$ \\
\hline 978 & $2.61775 \mathrm{E}-05$ \\
\hline 979 & $2.61436 \mathrm{E}-05$ \\
\hline 980 & $2.61102 \mathrm{E}-05$ \\
\hline 981 & 2.60765E-05 \\
\hline 982 & 2.60432E-05 \\
\hline 983 & 2.60096E-05 \\
\hline 984 & $2.59765 \mathrm{E}-05$ \\
\hline 985 & 0.000025943 \\
\hline 986 & $2.59101 \mathrm{E}-05$ \\
\hline 987 & $2.58768 \mathrm{E}-05$ \\
\hline 988 & 0.000025844 \\
\hline 989 & $2.58108 \mathrm{E}-05$ \\
\hline 990 & $2.57781 \mathrm{E}-05$ \\
\hline 991 & $2.57451 \mathrm{E}-05$ \\
\hline 992 & 2.57126E-05 \\
\hline 993 & $2.56798 \mathrm{E}-05$ \\
\hline 994 & $2.56474 \mathrm{E}-05$ \\
\hline 995 & $2.56147 \mathrm{E}-05$ \\
\hline 996 & $2.55824 \mathrm{E}-05$ \\
\hline 997 & 2.55499E-05 \\
\hline 998 & 2.55177E-05 \\
\hline 999 & $2.54853 \mathrm{E}-05$ \\
\hline 1000 & $2.54534 \mathrm{E}-05$ \\
\hline 1001 & $2.54211 \mathrm{E}-05$ \\
\hline 1002 & $2.53893 \mathrm{E}-05$ \\
\hline 1003 & $2.53571 \mathrm{E}-05$ \\
\hline 1004 & 2.53254E-05 \\
\hline 1005 & $2.52935 \mathrm{E}-05$ \\
\hline 1006 & $2.52619 \mathrm{E}-05$ \\
\hline
\end{tabular}




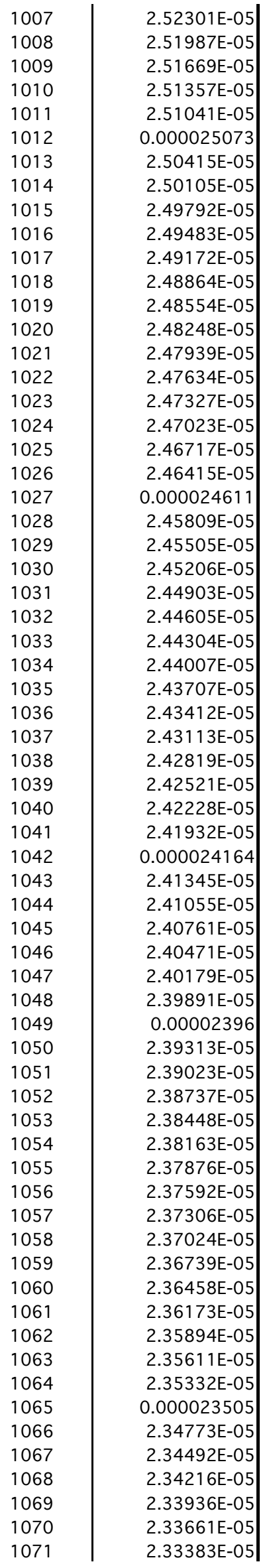




\begin{tabular}{|c|c|}
\hline 1072 & $2.33109 \mathrm{E}-05$ \\
\hline 1073 & $2.32832 \mathrm{E}-05$ \\
\hline 1074 & $2.32559 \mathrm{E}-05$ \\
\hline 1075 & $2.32283 \mathrm{E}-05$ \\
\hline 1076 & $2.32011 \mathrm{E}-05$ \\
\hline 1077 & $2.31736 \mathrm{E}-05$ \\
\hline 1078 & $2.31465 \mathrm{E}-05$ \\
\hline 1079 & $2.31191 \mathrm{E}-05$ \\
\hline 1080 & $2.30922 \mathrm{E}-05$ \\
\hline 1081 & $2.30649 \mathrm{E}-05$ \\
\hline 1082 & $2.30381 \mathrm{E}-05$ \\
\hline 1083 & $2.30109 \mathrm{E}-05$ \\
\hline 1084 & $2.29841 \mathrm{E}-05$ \\
\hline 1085 & $2.29571 \mathrm{E}-05$ \\
\hline 1086 & $2.29305 \mathrm{E}-05$ \\
\hline 1087 & $2.29035 \mathrm{E}-05$ \\
\hline 1088 & 0.000022877 \\
\hline 1089 & $2.28502 \mathrm{E}-05$ \\
\hline 1090 & $2.28238 \mathrm{E}-05$ \\
\hline 1091 & 0.000022797 \\
\hline 1092 & $2.27707 \mathrm{E}-05$ \\
\hline 1093 & $2.27441 \mathrm{E}-05$ \\
\hline 1094 & $2.27179 \mathrm{E}-05$ \\
\hline 1095 & $2.26914 \mathrm{E}-05$ \\
\hline 1096 & $2.26653 \mathrm{E}-05$ \\
\hline 1097 & $2.26389 \mathrm{E}-05$ \\
\hline 1098 & $2.26129 \mathrm{E}-05$ \\
\hline 1099 & $2.25866 \mathrm{E}-05$ \\
\hline 1100 & $2.25607 \mathrm{E}-05$ \\
\hline 1101 & $2.25345 \mathrm{E}-05$ \\
\hline 1102 & $2.25087 \mathrm{E}-05$ \\
\hline 1103 & $2.24826 \mathrm{E}-05$ \\
\hline 1104 & $2.24569 \mathrm{E}-05$ \\
\hline 1105 & $2.24309 \mathrm{E}-05$ \\
\hline 1106 & 2.24054E-05 \\
\hline 1107 & $2.23795 \mathrm{E}-05$ \\
\hline 1108 & 0.000022354 \\
\hline 1109 & $2.23282 E-05$ \\
\hline 1110 & $2.23028 \mathrm{E}-05$ \\
\hline 1111 & $2.22772 \mathrm{E}-05$ \\
\hline 1112 & $2.22519 \mathrm{E}-05$ \\
\hline 1113 & 2.22263E-05 \\
\hline 1114 & $2.22011 \mathrm{E}-05$ \\
\hline 1115 & $2.21756 \mathrm{E}-05$ \\
\hline 1116 & $2.21506 \mathrm{E}-05$ \\
\hline 1117 & $2.21252 \mathrm{E}-05$ \\
\hline 1118 & $2.21002 E-05$ \\
\hline 1119 & $2.20749 \mathrm{E}-05$ \\
\hline 1120 & $2.20501 \mathrm{E}-05$ \\
\hline 1121 & $2.20249 \mathrm{E}-05$ \\
\hline 1122 & $2.20001 \mathrm{E}-05$ \\
\hline 1123 & 0.000021975 \\
\hline 1124 & $2.19503 \mathrm{E}-05$ \\
\hline 1125 & $2.19253 \mathrm{E}-05$ \\
\hline 1126 & $2.19008 \mathrm{E}-05$ \\
\hline 1127 & $2.18759 \mathrm{E}-05$ \\
\hline 1128 & $2.18514 \mathrm{E}-05$ \\
\hline 1129 & $2.18266 \mathrm{E}-05$ \\
\hline 1130 & $2.18022 \mathrm{E}-05$ \\
\hline 1131 & $2.17775 \mathrm{E}-05$ \\
\hline 1132 & $2.17532 \mathrm{E}-05$ \\
\hline 1133 & $2.17286 \mathrm{E}-05$ \\
\hline 1134 & $2.17044 \mathrm{E}-05$ \\
\hline 1135 & $2.16799 \mathrm{E}-05$ \\
\hline 1136 & $2.16558 \mathrm{E}-05$ \\
\hline
\end{tabular}




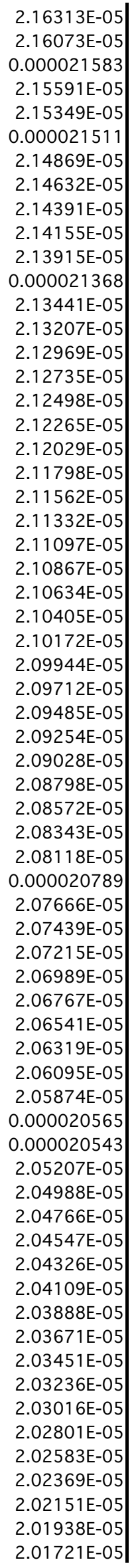




\begin{tabular}{|c|c|}
\hline 1202 & $2.01509 \mathrm{E}-05$ \\
\hline 1203 & $2.01293 \mathrm{E}-05$ \\
\hline 1204 & $2.01081 \mathrm{E}-05$ \\
\hline 1205 & $2.00866 \mathrm{E}-05$ \\
\hline 1206 & $2.00655 \mathrm{E}-05$ \\
\hline 1207 & 0.000020044 \\
\hline 1208 & 0.000020023 \\
\hline 1209 & $2.00016 \mathrm{E}-05$ \\
\hline 1210 & 1.99807E-05 \\
\hline 1211 & $1.99594 \mathrm{E}-05$ \\
\hline 1212 & $1.99385 \mathrm{E}-05$ \\
\hline 1213 & $1.99173 \mathrm{E}-05$ \\
\hline 1214 & $1.98965 \mathrm{E}-05$ \\
\hline 1215 & $1.98754 \mathrm{E}-05$ \\
\hline 1216 & $1.98547 \mathrm{E}-05$ \\
\hline 1217 & $1.98336 \mathrm{E}-05$ \\
\hline 1218 & 0.000019813 \\
\hline 1219 & 0.000019792 \\
\hline 1220 & $1.97714 \mathrm{E}-05$ \\
\hline 1221 & $1.97505 \mathrm{E}-05$ \\
\hline 1222 & 0.00001973 \\
\hline 1223 & $1.97092 \mathrm{E}-05$ \\
\hline 1224 & $1.96888 \mathrm{E}-05$ \\
\hline 1225 & 0.000019668 \\
\hline 1226 & $1.96477 \mathrm{E}-05$ \\
\hline 1227 & 0.000019627 \\
\hline 1228 & $1.96067 \mathrm{E}-05$ \\
\hline 1229 & $1.95861 \mathrm{E}-05$ \\
\hline 1230 & $1.95659 \mathrm{E}-05$ \\
\hline 1231 & $1.95454 \mathrm{E}-05$ \\
\hline 1232 & $1.95253 \mathrm{E}-05$ \\
\hline 1233 & $1.95048 \mathrm{E}-05$ \\
\hline 1234 & $1.94847 \mathrm{E}-05$ \\
\hline 1235 & $1.94643 \mathrm{E}-05$ \\
\hline 1236 & $1.94444 \mathrm{E}-05$ \\
\hline 1237 & 0.000019424 \\
\hline 1238 & $1.94041 \mathrm{E}-05$ \\
\hline 1239 & $1.93839 \mathrm{E}-05$ \\
\hline 1240 & 0.000019364 \\
\hline 1241 & $1.93439 \mathrm{E}-05$ \\
\hline 1242 & $1.93241 \mathrm{E}-05$ \\
\hline 1243 & 0.000019304 \\
\hline 1244 & $1.92843 \mathrm{E}-05$ \\
\hline 1245 & $1.92642 \mathrm{E}-05$ \\
\hline 1246 & $1.92446 \mathrm{E}-05$ \\
\hline 1247 & $1.92246 \mathrm{E}-05$ \\
\hline 1248 & $1.92051 \mathrm{E}-05$ \\
\hline 1249 & $1.91852 \mathrm{E}-05$ \\
\hline 1250 & $1.91657 \mathrm{E}-05$ \\
\hline 1251 & $1.91459 \mathrm{E}-05$ \\
\hline 1252 & $1.91265 \mathrm{E}-05$ \\
\hline 1253 & 1.91067E-05 \\
\hline 1254 & $1.90874 \mathrm{E}-05$ \\
\hline 1255 & 1.90677E-05 \\
\hline 1256 & $1.90484 \mathrm{E}-05$ \\
\hline 1257 & $1.90288 \mathrm{E}-05$ \\
\hline 1258 & $1.90095 \mathrm{E}-05$ \\
\hline 1259 & 0.00001899 \\
\hline 1260 & $1.89708 \mathrm{E}-05$ \\
\hline 1261 & $1.89513 \mathrm{E}-05$ \\
\hline 1262 & 1.89323E-05 \\
\hline 1263 & $1.89128 \mathrm{E}-05$ \\
\hline 1264 & $1.88938 \mathrm{E}-05$ \\
\hline 1265 & $1.88745 \mathrm{E}-05$ \\
\hline 1266 & $1.88555 \mathrm{E}-05$ \\
\hline
\end{tabular}




\begin{tabular}{|c|c|}
\hline 1267 & $1.88362 \mathrm{E}-05$ \\
\hline 1268 & $1.88174 \mathrm{E}-05$ \\
\hline 1269 & $1.87981 \mathrm{E}-05$ \\
\hline 1270 & $1.87793 \mathrm{E}-05$ \\
\hline 1271 & $1.87602 \mathrm{E}-05$ \\
\hline 1272 & $1.87414 \mathrm{E}-05$ \\
\hline 1273 & $1.87223 \mathrm{E}-05$ \\
\hline 1274 & 1.87037E-05 \\
\hline 1275 & $1.86846 \mathrm{E}-05$ \\
\hline 1276 & 0.000018666 \\
\hline 1277 & $1.86471 \mathrm{E}-05$ \\
\hline 1278 & $1.86285 \mathrm{E}-05$ \\
\hline 1279 & $1.86096 \mathrm{E}-05$ \\
\hline 1280 & $1.85911 \mathrm{E}-05$ \\
\hline 1281 & $1.85723 \mathrm{E}-05$ \\
\hline 1282 & 1.85539E-05 \\
\hline 1283 & $1.85351 \mathrm{E}-05$ \\
\hline 1284 & $1.85167 \mathrm{E}-05$ \\
\hline 1285 & 0.000018498 \\
\hline 1286 & $1.84797 \mathrm{E}-05$ \\
\hline 1287 & $1.84611 \mathrm{E}-05$ \\
\hline 1288 & $1.84429 \mathrm{E}-05$ \\
\hline 1289 & $1.84243 \mathrm{E}-05$ \\
\hline 1290 & $1.84061 \mathrm{E}-05$ \\
\hline 1291 & $1.83876 \mathrm{E}-05$ \\
\hline 1292 & $1.83695 \mathrm{E}-05$ \\
\hline 1293 & 0.000018351 \\
\hline 1294 & 0.000018333 \\
\hline 1295 & $1.83146 \mathrm{E}-05$ \\
\hline 1296 & $1.82966 \mathrm{E}-05$ \\
\hline 1297 & $1.82783 \mathrm{E}-05$ \\
\hline 1298 & $1.82604 \mathrm{E}-05$ \\
\hline 1299 & $1.82421 \mathrm{E}-05$ \\
\hline 1300 & $1.82242 \mathrm{E}-05$ \\
\hline 1301 & 0.000018206 \\
\hline 1302 & $1.81882 \mathrm{E}-05$ \\
\hline 1303 & $1.81701 \mathrm{E}-05$ \\
\hline 1304 & $1.81524 \mathrm{E}-05$ \\
\hline 1305 & $1.81343 \mathrm{E}-05$ \\
\hline 1306 & $1.81166 \mathrm{E}-05$ \\
\hline 1307 & $1.80986 \mathrm{E}-05$ \\
\hline 1308 & $1.80809 \mathrm{E}-05$ \\
\hline 1309 & 0.000018063 \\
\hline 1310 & $1.80454 \mathrm{E}-05$ \\
\hline 1311 & $1.80275 \mathrm{E}-05$ \\
\hline 1312 & 0.00001801 \\
\hline 1313 & $1.79922 \mathrm{E}-05$ \\
\hline 1314 & $1.79747 \mathrm{E}-05$ \\
\hline 1315 & 1.79569E-05 \\
\hline 1316 & $1.79396 \mathrm{E}-05$ \\
\hline 1317 & $1.79218 \mathrm{E}-05$ \\
\hline 1318 & $1.79045 \mathrm{E}-05$ \\
\hline 1319 & $1.78868 \mathrm{E}-05$ \\
\hline 1320 & $1.78696 \mathrm{E}-05$ \\
\hline 1321 & 0.000017852 \\
\hline 1322 & $1.78348 \mathrm{E}-05$ \\
\hline 1323 & $1.78172 \mathrm{E}-05$ \\
\hline 1324 & $1.78001 \mathrm{E}-05$ \\
\hline 1325 & $1.77826 \mathrm{E}-05$ \\
\hline 1326 & $1.77655 \mathrm{E}-05$ \\
\hline 1327 & 0.000017748 \\
\hline 1328 & 0.000017731 \\
\hline 1329 & $1.77136 \mathrm{E}-05$ \\
\hline 1330 & $1.76966 \mathrm{E}-05$ \\
\hline 1331 & $1.76793 \mathrm{E}-05$ \\
\hline
\end{tabular}




\begin{tabular}{|c|c|}
\hline 1332 & $1.76624 \mathrm{E}-05$ \\
\hline 1333 & $1.76451 \mathrm{E}-05$ \\
\hline 1334 & $1.76283 \mathrm{E}-05$ \\
\hline 1335 & 0.000017611 \\
\hline 1336 & $1.75942 \mathrm{E}-05$ \\
\hline 1337 & $1.75771 \mathrm{E}-05$ \\
\hline 1338 & $1.75603 \mathrm{E}-05$ \\
\hline 1339 & $1.75432 \mathrm{E}-05$ \\
\hline 1340 & $1.75265 \mathrm{E}-05$ \\
\hline 1341 & $1.75095 \mathrm{E}-05$ \\
\hline 1342 & $1.74928 \mathrm{E}-05$ \\
\hline 1343 & $1.74758 \mathrm{E}-05$ \\
\hline 1344 & $1.74593 \mathrm{E}-05$ \\
\hline 1345 & $1.74423 \mathrm{E}-05$ \\
\hline 1346 & $1.74258 \mathrm{E}-05$ \\
\hline 1347 & $1.74089 \mathrm{E}-05$ \\
\hline 1348 & $1.73924 \mathrm{E}-05$ \\
\hline 1349 & $1.73756 \mathrm{E}-05$ \\
\hline 1350 & $1.73592 \mathrm{E}-05$ \\
\hline 1351 & $1.73424 \mathrm{E}-05$ \\
\hline 1352 & 0.000017326 \\
\hline 1353 & $1.73093 \mathrm{E}-05$ \\
\hline 1354 & 0.000017293 \\
\hline 1355 & $1.72763 \mathrm{E}-05$ \\
\hline 1356 & $1.72601 \mathrm{E}-05$ \\
\hline 1357 & $1.72435 \mathrm{E}-05$ \\
\hline 1358 & $1.72273 \mathrm{E}-05$ \\
\hline 1359 & $1.72107 \mathrm{E}-05$ \\
\hline 1360 & $1.71946 \mathrm{E}-05$ \\
\hline 1361 & 0.000017178 \\
\hline 1362 & $1.71619 \mathrm{E}-05$ \\
\hline 1363 & $1.71455 \mathrm{E}-05$ \\
\hline 1364 & $1.71294 \mathrm{E}-05$ \\
\hline 1365 & 0.000017113 \\
\hline 1366 & 0.000017097 \\
\hline 1367 & $1.70807 \mathrm{E}-05$ \\
\hline 1368 & $1.70648 \mathrm{E}-05$ \\
\hline 1369 & $1.70485 \mathrm{E}-05$ \\
\hline 1370 & $1.70326 \mathrm{E}-05$ \\
\hline 1371 & $1.70163 \mathrm{E}-05$ \\
\hline 1372 & $1.70005 \mathrm{E}-05$ \\
\hline 1373 & $1.69843 \mathrm{E}-05$ \\
\hline 1374 & $1.69685 \mathrm{E}-05$ \\
\hline 1375 & $1.69523 \mathrm{E}-05$ \\
\hline 1376 & $1.69366 \mathrm{E}-05$ \\
\hline 1377 & $1.69205 \mathrm{E}-05$ \\
\hline 1378 & $1.69048 \mathrm{E}-05$ \\
\hline 1379 & $1.68888 \mathrm{E}-05$ \\
\hline 1380 & 1.68731E-05 \\
\hline 1381 & $1.68572 \mathrm{E}-05$ \\
\hline 1382 & $1.68416 \mathrm{E}-05$ \\
\hline 1383 & $1.68256 \mathrm{E}-05$ \\
\hline 1384 & $1.68101 \mathrm{E}-05$ \\
\hline 1385 & $1.67942 \mathrm{E}-05$ \\
\hline 1386 & 1.67787E-05 \\
\hline 1387 & $1.67629 \mathrm{E}-05$ \\
\hline 1388 & $1.67474 \mathrm{E}-05$ \\
\hline 1389 & 1.67316E-05 \\
\hline 1390 & $1.67163 \mathrm{E}-05$ \\
\hline 1391 & $1.67005 \mathrm{E}-05$ \\
\hline 1392 & $1.66852 \mathrm{E}-05$ \\
\hline 1393 & $1.66695 \mathrm{E}-05$ \\
\hline 1394 & $1.66542 \mathrm{E}-05$ \\
\hline 1395 & $1.66385 \mathrm{E}-05$ \\
\hline 1396 & $1.66233 \mathrm{E}-05$ \\
\hline
\end{tabular}




\begin{tabular}{|c|c|}
\hline 1397 & 1.66077E-05 \\
\hline 1398 & $1.65925 \mathrm{E}-05$ \\
\hline 1399 & 0.000016577 \\
\hline 1400 & $1.65618 \mathrm{E}-05$ \\
\hline 1401 & $1.65463 \mathrm{E}-05$ \\
\hline 1402 & $1.65312 \mathrm{E}-05$ \\
\hline 1403 & $1.65158 \mathrm{E}-05$ \\
\hline 1404 & 1.65007E-05 \\
\hline 1405 & $1.64853 \mathrm{E}-05$ \\
\hline 1406 & $1.64703 \mathrm{E}-05$ \\
\hline 1407 & 0.000016455 \\
\hline 1408 & 0.00001644 \\
\hline 1409 & 1.64247E-05 \\
\hline 1410 & $1.64098 \mathrm{E}-05$ \\
\hline 1411 & $1.63945 \mathrm{E}-05$ \\
\hline 1412 & 1.63797E-05 \\
\hline 1413 & $1.63645 \mathrm{E}-05$ \\
\hline 1414 & 1.63496E-05 \\
\hline 1415 & $1.63345 \mathrm{E}-05$ \\
\hline 1416 & $1.63197 \mathrm{E}-05$ \\
\hline 1417 & $1.63046 \mathrm{E}-05$ \\
\hline 1418 & 1.62899E-05 \\
\hline 1419 & $1.62748 \mathrm{E}-05$ \\
\hline 1420 & $1.62601 \mathrm{E}-05$ \\
\hline 1421 & $1.62451 \mathrm{E}-05$ \\
\hline 1422 & $1.62305 \mathrm{E}-05$ \\
\hline 1423 & $1.62155 \mathrm{E}-05$ \\
\hline 1424 & $1.62009 \mathrm{E}-05$ \\
\hline 1425 & 0.000016186 \\
\hline 1426 & $1.61714 \mathrm{E}-05$ \\
\hline 1427 & $1.61565 \mathrm{E}-05$ \\
\hline 1428 & 0.000016142 \\
\hline 1429 & $1.61272 \mathrm{E}-05$ \\
\hline 1430 & $1.61128 \mathrm{E}-05$ \\
\hline 1431 & 1.60979E-05 \\
\hline 1432 & $1.60835 \mathrm{E}-05$ \\
\hline 1433 & $1.60688 \mathrm{E}-05$ \\
\hline 1434 & $1.60544 \mathrm{E}-05$ \\
\hline 1435 & 1.60397E-05 \\
\hline 1436 & $1.60254 \mathrm{E}-05$ \\
\hline 1437 & $1.60107 \mathrm{E}-05$ \\
\hline 1438 & $1.59965 \mathrm{E}-05$ \\
\hline 1439 & $1.59818 \mathrm{E}-05$ \\
\hline 1440 & $1.59676 \mathrm{E}-05$ \\
\hline 1441 & 0.000015953 \\
\hline 1442 & $1.59389 \mathrm{E}-05$ \\
\hline 1443 & $1.59243 \mathrm{E}-05$ \\
\hline 1444 & $1.59102 \mathrm{E}-05$ \\
\hline 1445 & 1.58957E-05 \\
\hline 1446 & $1.58816 \mathrm{E}-05$ \\
\hline 1447 & $1.58672 \mathrm{E}-05$ \\
\hline 1448 & $1.58531 \mathrm{E}-05$ \\
\hline 1449 & $1.58387 \mathrm{E}-05$ \\
\hline 1450 & $1.58247 \mathrm{E}-05$ \\
\hline 1451 & $1.58103 \mathrm{E}-05$ \\
\hline 1452 & $1.57964 \mathrm{E}-05$ \\
\hline 1453 & $1.57821 \mathrm{E}-05$ \\
\hline 1454 & $1.57681 \mathrm{E}-05$ \\
\hline 1455 & $1.57539 \mathrm{E}-05$ \\
\hline 1456 & 0.00001574 \\
\hline 1457 & $1.57257 \mathrm{E}-05$ \\
\hline 1458 & $1.57119 \mathrm{E}-05$ \\
\hline 1459 & 1.56977E-05 \\
\hline 1460 & $1.56839 \mathrm{E}-05$ \\
\hline 1461 & $1.56698 \mathrm{E}-05$ \\
\hline
\end{tabular}




\begin{tabular}{|c|c|}
\hline 1462 & 0.000015656 \\
\hline 1463 & $1.56419 \mathrm{E}-05$ \\
\hline 1464 & $1.56282 \mathrm{E}-05$ \\
\hline 1465 & $1.56141 \mathrm{E}-05$ \\
\hline 1466 & $1.56005 \mathrm{E}-05$ \\
\hline 1467 & $1.55864 \mathrm{E}-05$ \\
\hline 1468 & $1.55728 \mathrm{E}-05$ \\
\hline 1469 & $1.55588 \mathrm{E}-05$ \\
\hline 1470 & $1.55453 \mathrm{E}-05$ \\
\hline 1471 & $1.55313 \mathrm{E}-05$ \\
\hline 1472 & $1.55178 \mathrm{E}-05$ \\
\hline 1473 & $1.55039 \mathrm{E}-05$ \\
\hline 1474 & $1.54904 \mathrm{E}-05$ \\
\hline 1475 & $1.54765 \mathrm{E}-05$ \\
\hline 1476 & 0.000015463 \\
\hline 1477 & $1.54492 \mathrm{E}-05$ \\
\hline 1478 & $1.54358 \mathrm{E}-05$ \\
\hline 1479 & 0.000015422 \\
\hline 1480 & $1.54086 \mathrm{E}-05$ \\
\hline 1481 & $1.53949 \mathrm{E}-05$ \\
\hline 1482 & $1.53816 \mathrm{E}-05$ \\
\hline 1483 & $1.53679 \mathrm{E}-05$ \\
\hline 1484 & $1.53546 \mathrm{E}-05$ \\
\hline 1485 & $1.53409 \mathrm{E}-05$ \\
\hline 1486 & $1.53276 \mathrm{E}-05$ \\
\hline 1487 & 0.000015314 \\
\hline 1488 & $1.53008 \mathrm{E}-05$ \\
\hline 1489 & $1.52872 \mathrm{E}-05$ \\
\hline 1490 & 0.000015274 \\
\hline 1491 & $1.52605 \mathrm{E}-05$ \\
\hline 1492 & $1.52474 \mathrm{E}-05$ \\
\hline 1493 & $1.52339 \mathrm{E}-05$ \\
\hline 1494 & $1.52208 \mathrm{E}-05$ \\
\hline 1495 & 1.52073E-05 \\
\hline 1496 & 1.51942E-05 \\
\hline 1497 & 1.51808E-05 \\
\hline 1498 & $1.51678 \mathrm{E}-05$ \\
\hline 1499 & $1.51544 \mathrm{E}-05$ \\
\hline 1500 & $1.51414 \mathrm{E}-05$ \\
\hline 1501 & $1.51281 \mathrm{E}-05$ \\
\hline 1502 & $1.51151 \mathrm{E}-05$ \\
\hline 1503 & $1.51018 \mathrm{E}-05$ \\
\hline 1504 & $1.50889 \mathrm{E}-05$ \\
\hline 1505 & $1.50756 \mathrm{E}-05$ \\
\hline 1506 & $1.50628 \mathrm{E}-05$ \\
\hline 1507 & $1.50495 \mathrm{E}-05$ \\
\hline 1508 & 1.50367E-05 \\
\hline 1509 & $1.50235 \mathrm{E}-05$ \\
\hline 1510 & 1.50107E-05 \\
\hline 1511 & 1.49976E-05 \\
\hline 1512 & $1.49848 \mathrm{E}-05$ \\
\hline 1513 & $1.49717 \mathrm{E}-05$ \\
\hline 1514 & 0.000014959 \\
\hline 1515 & $1.49459 \mathrm{E}-05$ \\
\hline 1516 & $1.49332 \mathrm{E}-05$ \\
\hline 1517 & $1.49202 \mathrm{E}-05$ \\
\hline 1518 & $1.49075 \mathrm{E}-05$ \\
\hline 1519 & $1.48945 \mathrm{E}-05$ \\
\hline 1520 & 1.48819E-05 \\
\hline 1521 & $1.48689 \mathrm{E}-05$ \\
\hline 1522 & $1.48564 \mathrm{E}-05$ \\
\hline 1523 & $1.48434 \mathrm{E}-05$ \\
\hline 1524 & $1.48309 \mathrm{E}-05$ \\
\hline 1525 & 0.000014818 \\
\hline 1526 & $1.48055 \mathrm{E}-05$ \\
\hline
\end{tabular}




\begin{tabular}{|c|c|}
\hline 1527 & $1.47927 \mathrm{E}-05$ \\
\hline 1528 & $1.47802 \mathrm{E}-05$ \\
\hline 1529 & $1.47674 \mathrm{E}-05$ \\
\hline 1530 & 0.000014755 \\
\hline 1531 & $1.47422 \mathrm{E}-05$ \\
\hline 1532 & $1.47298 \mathrm{E}-05$ \\
\hline 1533 & 0.000014717 \\
\hline 1534 & $1.47047 \mathrm{E}-05$ \\
\hline 1535 & 0.000014692 \\
\hline 1536 & $1.46797 \mathrm{E}-05$ \\
\hline 1537 & 0.000014667 \\
\hline 1538 & $1.46547 \mathrm{E}-05$ \\
\hline 1539 & 0.000014642 \\
\hline 1540 & $1.46298 \mathrm{E}-05$ \\
\hline 1541 & $1.46172 \mathrm{E}-05$ \\
\hline 1542 & 0.000014605 \\
\hline 1543 & $1.45924 \mathrm{E}-05$ \\
\hline 1544 & $1.45802 \mathrm{E}-05$ \\
\hline 1545 & 1.45677E-05 \\
\hline 1546 & $1.45556 \mathrm{E}-05$ \\
\hline 1547 & $1.45431 \mathrm{E}-05$ \\
\hline 1548 & 0.000014531 \\
\hline 1549 & $1.45185 \mathrm{E}-05$ \\
\hline 1550 & $1.45064 \mathrm{E}-05$ \\
\hline 1551 & 0.000014494 \\
\hline 1552 & 0.000014482 \\
\hline 1553 & $1.44696 \mathrm{E}-05$ \\
\hline 1554 & $1.44576 \mathrm{E}-05$ \\
\hline 1555 & $1.44452 \mathrm{E}-05$ \\
\hline 1556 & $1.44332 \mathrm{E}-05$ \\
\hline 1557 & $1.44209 \mathrm{E}-05$ \\
\hline 1558 & 0.000014409 \\
\hline 1559 & $1.43967 \mathrm{E}-05$ \\
\hline 1560 & $1.43848 \mathrm{E}-05$ \\
\hline 1561 & $1.43725 \mathrm{E}-05$ \\
\hline 1562 & 1.43607E-05 \\
\hline 1563 & $1.43484 \mathrm{E}-05$ \\
\hline 1564 & $1.43366 \mathrm{E}-05$ \\
\hline 1565 & $1.43244 \mathrm{E}-05$ \\
\hline 1566 & $1.43126 \mathrm{E}-05$ \\
\hline 1567 & $1.43005 \mathrm{E}-05$ \\
\hline 1568 & 1.42887E-05 \\
\hline 1569 & 1.42766E-05 \\
\hline 1570 & $1.42649 \mathrm{E}-05$ \\
\hline 1571 & $1.42528 \mathrm{E}-05$ \\
\hline 1572 & $1.42411 \mathrm{E}-05$ \\
\hline 1573 & 0.000014229 \\
\hline 1574 & $1.42174 \mathrm{E}-05$ \\
\hline 1575 & 1.42053E-05 \\
\hline 1576 & 1.41937E-05 \\
\hline 1577 & 1.41817E-05 \\
\hline 1578 & $1.41701 \mathrm{E}-05$ \\
\hline 1579 & $1.41581 \mathrm{E}-05$ \\
\hline 1580 & $1.41466 \mathrm{E}-05$ \\
\hline 1581 & 1.41347E-05 \\
\hline 1582 & $1.41231 \mathrm{E}-05$ \\
\hline 1583 & $1.41112 \mathrm{E}-05$ \\
\hline 1584 & 1.40997E-05 \\
\hline 1585 & 1.40879E-05 \\
\hline 1586 & $1.40764 \mathrm{E}-05$ \\
\hline 1587 & $1.40646 \mathrm{E}-05$ \\
\hline 1588 & $1.40531 \mathrm{E}-05$ \\
\hline 1589 & $1.40414 \mathrm{E}-05$ \\
\hline 1590 & 0.00001403 \\
\hline 1591 & $1.40182 \mathrm{E}-05$ \\
\hline
\end{tabular}




\begin{tabular}{|c|c|}
\hline 1592 & $1.40068 \mathrm{E}-05$ \\
\hline 1593 & $1.39951 \mathrm{E}-05$ \\
\hline 1594 & $1.39837 \mathrm{E}-05$ \\
\hline 1595 & 0.000013972 \\
\hline 1596 & $1.39607 \mathrm{E}-05$ \\
\hline 1597 & $1.39491 \mathrm{E}-05$ \\
\hline 1598 & $1.39378 \mathrm{E}-05$ \\
\hline 1599 & $1.39262 \mathrm{E}-05$ \\
\hline 1600 & $1.39149 \mathrm{E}-05$ \\
\hline 1601 & $1.39033 \mathrm{E}-05$ \\
\hline 1602 & $1.38921 \mathrm{E}-05$ \\
\hline 1603 & $1.38805 \mathrm{E}-05$ \\
\hline 1604 & $1.38693 \mathrm{E}-05$ \\
\hline 1605 & $1.38578 \mathrm{E}-05$ \\
\hline 1606 & $1.38467 \mathrm{E}-05$ \\
\hline 1607 & $1.38351 \mathrm{E}-05$ \\
\hline 1608 & 0.000013824 \\
\hline 1609 & $1.38125 \mathrm{E}-05$ \\
\hline 1610 & $1.38015 \mathrm{E}-05$ \\
\hline 1611 & 0.00001379 \\
\hline 1612 & $1.37789 \mathrm{E}-05$ \\
\hline 1613 & $1.37675 \mathrm{E}-05$ \\
\hline 1614 & $1.37565 \mathrm{E}-05$ \\
\hline 1615 & $1.37451 \mathrm{E}-05$ \\
\hline 1616 & $1.37341 \mathrm{E}-05$ \\
\hline 1617 & $1.37228 \mathrm{E}-05$ \\
\hline 1618 & $1.37118 \mathrm{E}-05$ \\
\hline 1619 & $1.37005 \mathrm{E}-05$ \\
\hline 1620 & $1.36895 \mathrm{E}-05$ \\
\hline 1621 & $1.36782 \mathrm{E}-05$ \\
\hline 1622 & $1.36673 \mathrm{E}-05$ \\
\hline 1623 & 0.000013656 \\
\hline 1624 & $1.36452 \mathrm{E}-05$ \\
\hline 1625 & $1.36339 \mathrm{E}-05$ \\
\hline 1626 & $1.36231 \mathrm{E}-05$ \\
\hline 1627 & 1.36119E-05 \\
\hline 1628 & $1.36011 \mathrm{E}-05$ \\
\hline 1629 & $1.35899 \mathrm{E}-05$ \\
\hline 1630 & $1.35791 \mathrm{E}-05$ \\
\hline 1631 & 0.000013568 \\
\hline 1632 & $1.35572 \mathrm{E}-05$ \\
\hline 1633 & $1.35461 \mathrm{E}-05$ \\
\hline 1634 & $1.35354 \mathrm{E}-05$ \\
\hline 1635 & 1.35243E-05 \\
\hline 1636 & $1.35136 \mathrm{E}-05$ \\
\hline 1637 & $1.35025 \mathrm{E}-05$ \\
\hline 1638 & $1.34918 \mathrm{E}-05$ \\
\hline 1639 & $1.34808 \mathrm{E}-05$ \\
\hline 1640 & $1.34702 \mathrm{E}-05$ \\
\hline 1641 & 1.34592E-05 \\
\hline 1642 & $1.34486 \mathrm{E}-05$ \\
\hline 1643 & $1.34376 \mathrm{E}-05$ \\
\hline 1644 & 0.000013427 \\
\hline 1645 & 0.000013416 \\
\hline 1646 & $1.34055 \mathrm{E}-05$ \\
\hline 1647 & $1.33946 \mathrm{E}-05$ \\
\hline 1648 & $1.33841 \mathrm{E}-05$ \\
\hline 1649 & $1.33732 \mathrm{E}-05$ \\
\hline 1650 & 1.33627E-05 \\
\hline 1651 & $1.33518 \mathrm{E}-05$ \\
\hline 1652 & $1.33413 \mathrm{E}-05$ \\
\hline 1653 & $1.33305 \mathrm{E}-05$ \\
\hline 1654 & $1.33201 \mathrm{E}-05$ \\
\hline 1655 & $1.33093 \mathrm{E}-05$ \\
\hline 1656 & 1.32989E-05 \\
\hline
\end{tabular}




\begin{tabular}{|c|c|}
\hline 1657 & $1.32881 \mathrm{E}-05$ \\
\hline 1658 & 1.32777E-05 \\
\hline 1659 & 0.000013267 \\
\hline 1660 & $1.32566 \mathrm{E}-05$ \\
\hline 1661 & $1.32459 \mathrm{E}-05$ \\
\hline 1662 & $1.32356 \mathrm{E}-05$ \\
\hline 1663 & $1.32249 \mathrm{E}-05$ \\
\hline 1664 & $1.32146 \mathrm{E}-05$ \\
\hline 1665 & $1.32039 \mathrm{E}-05$ \\
\hline 1666 & $1.31936 \mathrm{E}-05$ \\
\hline 1667 & 0.000013183 \\
\hline 1668 & $1.31728 \mathrm{E}-05$ \\
\hline 1669 & $1.31621 \mathrm{E}-05$ \\
\hline 1670 & $1.31519 \mathrm{E}-05$ \\
\hline 1671 & $1.31413 \mathrm{E}-05$ \\
\hline 1672 & $1.31312 \mathrm{E}-05$ \\
\hline 1673 & $1.31206 \mathrm{E}-05$ \\
\hline 1674 & $1.31104 \mathrm{E}-05$ \\
\hline 1675 & $1.30999 \mathrm{E}-05$ \\
\hline 1676 & $1.30898 \mathrm{E}-05$ \\
\hline 1677 & $1.30793 \mathrm{E}-05$ \\
\hline 1678 & $1.30692 \mathrm{E}-05$ \\
\hline 1679 & $1.30587 \mathrm{E}-05$ \\
\hline 1680 & $1.30486 \mathrm{E}-05$ \\
\hline 1681 & $1.30382 \mathrm{E}-05$ \\
\hline 1682 & $1.30281 \mathrm{E}-05$ \\
\hline 1683 & $1.30177 \mathrm{E}-05$ \\
\hline 1684 & $1.30077 \mathrm{E}-05$ \\
\hline 1685 & $1.29973 \mathrm{E}-05$ \\
\hline 1686 & $1.29873 \mathrm{E}-05$ \\
\hline 1687 & $1.29769 \mathrm{E}-05$ \\
\hline 1688 & $1.29669 \mathrm{E}-05$ \\
\hline 1689 & $1.29566 \mathrm{E}-05$ \\
\hline 1690 & $1.29467 \mathrm{E}-05$ \\
\hline 1691 & $1.29363 \mathrm{E}-05$ \\
\hline 1692 & $1.29264 \mathrm{E}-05$ \\
\hline 1693 & $1.29161 \mathrm{E}-05$ \\
\hline 1694 & $1.29062 \mathrm{E}-05$ \\
\hline 1695 & 0.000012896 \\
\hline 1696 & $1.28861 \mathrm{E}-05$ \\
\hline 1697 & $1.28759 \mathrm{E}-05$ \\
\hline 1698 & 0.000012866 \\
\hline 1699 & $1.28558 \mathrm{E}-05$ \\
\hline 1700 & 0.000012846 \\
\hline 1701 & $1.28358 \mathrm{E}-05$ \\
\hline 1702 & 0.000012826 \\
\hline 1703 & 1.28159E-05 \\
\hline 1704 & $1.28061 \mathrm{E}-05$ \\
\hline 1705 & 0.000012796 \\
\hline 1706 & $1.27862 \mathrm{E}-05$ \\
\hline 1707 & $1.27761 \mathrm{E}-05$ \\
\hline 1708 & $1.27664 \mathrm{E}-05$ \\
\hline 1709 & $1.27563 \mathrm{E}-05$ \\
\hline 1710 & $1.27467 \mathrm{E}-05$ \\
\hline 1711 & 1.27366E-05 \\
\hline 1712 & 1.27269E-05 \\
\hline 1713 & $1.27169 \mathrm{E}-05$ \\
\hline 1714 & 1.27073E-05 \\
\hline 1715 & 1.26973E-05 \\
\hline 1716 & $1.26876 \mathrm{E}-05$ \\
\hline 1717 & 1.26777E-05 \\
\hline 1718 & $1.26681 \mathrm{E}-05$ \\
\hline 1719 & $1.26581 \mathrm{E}-05$ \\
\hline 1720 & $1.26486 \mathrm{E}-05$ \\
\hline 1721 & $1.26386 \mathrm{E}-05$ \\
\hline
\end{tabular}




\begin{tabular}{|c|c|}
\hline 1722 & $1.26291 \mathrm{E}-05$ \\
\hline 1723 & $1.26192 \mathrm{E}-05$ \\
\hline 1724 & $1.26097 \mathrm{E}-05$ \\
\hline 1725 & $1.25998 \mathrm{E}-05$ \\
\hline 1726 & $1.25903 \mathrm{E}-05$ \\
\hline 1727 & $1.25805 \mathrm{E}-05$ \\
\hline 1728 & 0.000012571 \\
\hline 1729 & $1.25612 \mathrm{E}-05$ \\
\hline 1730 & $1.25517 \mathrm{E}-05$ \\
\hline 1731 & $1.25419 \mathrm{E}-05$ \\
\hline 1732 & $1.25325 \mathrm{E}-05$ \\
\hline 1733 & 1.25227E-05 \\
\hline 1734 & $1.25133 \mathrm{E}-05$ \\
\hline 1735 & $1.25036 \mathrm{E}-05$ \\
\hline 1736 & $1.24942 \mathrm{E}-05$ \\
\hline 1737 & $1.24845 \mathrm{E}-05$ \\
\hline 1738 & $1.24751 \mathrm{E}-05$ \\
\hline 1739 & $1.24654 \mathrm{E}-05$ \\
\hline 1740 & $1.24561 \mathrm{E}-05$ \\
\hline 1741 & $1.24464 \mathrm{E}-05$ \\
\hline 1742 & $1.24371 \mathrm{E}-05$ \\
\hline 1743 & $1.24275 \mathrm{E}-05$ \\
\hline 1744 & $1.24182 \mathrm{E}-05$ \\
\hline 1745 & $1.24085 \mathrm{E}-05$ \\
\hline 1746 & $1.23993 \mathrm{E}-05$ \\
\hline 1747 & $1.23897 \mathrm{E}-05$ \\
\hline 1748 & $1.23805 \mathrm{E}-05$ \\
\hline 1749 & $1.23709 \mathrm{E}-05$ \\
\hline 1750 & 1.23617E-05 \\
\hline 1751 & $1.23521 \mathrm{E}-05$ \\
\hline 1752 & $1.23429 \mathrm{E}-05$ \\
\hline 1753 & $1.23334 \mathrm{E}-05$ \\
\hline 1754 & $1.23242 \mathrm{E}-05$ \\
\hline 1755 & $1.23147 \mathrm{E}-05$ \\
\hline 1756 & $1.23056 \mathrm{E}-05$ \\
\hline 1757 & $1.22961 \mathrm{E}-05$ \\
\hline 1758 & 0.000012287 \\
\hline 1759 & $1.22775 \mathrm{E}-05$ \\
\hline 1760 & $1.22684 \mathrm{E}-05$ \\
\hline 1761 & 0.000012259 \\
\hline 1762 & $1.22499 \mathrm{E}-05$ \\
\hline 1763 & $1.22405 \mathrm{E}-05$ \\
\hline 1764 & $1.22315 \mathrm{E}-05$ \\
\hline 1765 & $1.22221 \mathrm{E}-05$ \\
\hline 1766 & 0.000012213 \\
\hline 1767 & $1.22037 \mathrm{E}-05$ \\
\hline 1768 & 1.21947E-05 \\
\hline 1769 & $1.21853 \mathrm{E}-05$ \\
\hline 1770 & $1.21763 \mathrm{E}-05$ \\
\hline 1771 & 0.000012167 \\
\hline 1772 & $1.21581 \mathrm{E}-05$ \\
\hline 1773 & $1.21487 \mathrm{E}-05$ \\
\hline 1774 & $1.21398 \mathrm{E}-05$ \\
\hline 1775 & $1.21305 \mathrm{E}-05$ \\
\hline 1776 & $1.21216 \mathrm{E}-05$ \\
\hline 1777 & $1.21124 \mathrm{E}-05$ \\
\hline 1778 & $1.21035 \mathrm{E}-05$ \\
\hline 1779 & $1.20942 \mathrm{E}-05$ \\
\hline 1780 & $1.20854 \mathrm{E}-05$ \\
\hline 1781 & $1.20762 \mathrm{E}-05$ \\
\hline 1782 & $1.20673 \mathrm{E}-05$ \\
\hline 1783 & $1.20581 \mathrm{E}-05$ \\
\hline 1784 & $1.20493 \mathrm{E}-05$ \\
\hline 1785 & $1.20401 \mathrm{E}-05$ \\
\hline 1786 & $1.20314 \mathrm{E}-05$ \\
\hline
\end{tabular}




\begin{tabular}{|c|c|}
\hline 1787 & $1.20222 \mathrm{E}-05$ \\
\hline 1788 & $1.20134 \mathrm{E}-05$ \\
\hline 1789 & $1.20043 \mathrm{E}-05$ \\
\hline 1790 & $1.19956 \mathrm{E}-05$ \\
\hline 1791 & $1.19864 \mathrm{E}-05$ \\
\hline 1792 & 1.19777E-05 \\
\hline 1793 & $1.19686 \mathrm{E}-05$ \\
\hline 1794 & 1.19599E-05 \\
\hline 1795 & 1.19509E-05 \\
\hline 1796 & $1.19422 \mathrm{E}-05$ \\
\hline 1797 & $1.19331 \mathrm{E}-05$ \\
\hline 1798 & $1.19245 \mathrm{E}-05$ \\
\hline 1799 & $1.19154 \mathrm{E}-05$ \\
\hline 1800 & $1.19068 \mathrm{E}-05$ \\
\hline 1801 & $1.18978 \mathrm{E}-05$ \\
\hline 1802 & $1.18892 \mathrm{E}-05$ \\
\hline 1803 & $1.18802 \mathrm{E}-05$ \\
\hline 1804 & $1.18716 \mathrm{E}-05$ \\
\hline 1805 & $1.18627 \mathrm{E}-05$ \\
\hline 1806 & $1.18541 \mathrm{E}-05$ \\
\hline 1807 & $1.18451 \mathrm{E}-05$ \\
\hline 1808 & $1.18366 \mathrm{E}-05$ \\
\hline 1809 & $1.18277 \mathrm{E}-05$ \\
\hline 1810 & $1.18191 \mathrm{E}-05$ \\
\hline 1811 & $1.18102 \mathrm{E}-05$ \\
\hline 1812 & $1.18017 \mathrm{E}-05$ \\
\hline 1813 & $1.17929 \mathrm{E}-05$ \\
\hline 1814 & $1.17844 \mathrm{E}-05$ \\
\hline 1815 & $1.17755 \mathrm{E}-05$ \\
\hline 1816 & $1.17671 \mathrm{E}-05$ \\
\hline 1817 & $1.17582 \mathrm{E}-05$ \\
\hline 1818 & $1.17498 \mathrm{E}-05$ \\
\hline 1819 & 0.000011741 \\
\hline 1820 & $1.17325 \mathrm{E}-05$ \\
\hline 1821 & $1.17237 \mathrm{E}-05$ \\
\hline 1822 & $1.17153 \mathrm{E}-05$ \\
\hline 1823 & $1.17066 \mathrm{E}-05$ \\
\hline 1824 & $1.16982 \mathrm{E}-05$ \\
\hline 1825 & $1.16894 \mathrm{E}-05$ \\
\hline 1826 & $1.16811 \mathrm{E}-05$ \\
\hline 1827 & $1.16723 \mathrm{E}-05$ \\
\hline 1828 & 0.000011664 \\
\hline 1829 & $1.16553 \mathrm{E}-05$ \\
\hline 1830 & 0.000011647 \\
\hline 1831 & $1.16383 \mathrm{E}-05$ \\
\hline 1832 & 0.00001163 \\
\hline 1833 & $1.16213 \mathrm{E}-05$ \\
\hline 1834 & 0.000011613 \\
\hline 1835 & $1.16044 \mathrm{E}-05$ \\
\hline 1836 & $1.15961 \mathrm{E}-05$ \\
\hline 1837 & $1.15875 \mathrm{E}-05$ \\
\hline 1838 & $1.15792 \mathrm{E}-05$ \\
\hline 1839 & $1.15706 \mathrm{E}-05$ \\
\hline 1840 & $1.15624 \mathrm{E}-05$ \\
\hline 1841 & $1.15538 \mathrm{E}-05$ \\
\hline 1842 & $1.15456 \mathrm{E}-05$ \\
\hline 1843 & $1.15371 \mathrm{E}-05$ \\
\hline 1844 & $1.15289 \mathrm{E}-05$ \\
\hline 1845 & $1.15203 \mathrm{E}-05$ \\
\hline 1846 & $1.15122 \mathrm{E}-05$ \\
\hline 1847 & $1.15036 \mathrm{E}-05$ \\
\hline 1848 & $1.14955 \mathrm{E}-05$ \\
\hline 1849 & 0.000011487 \\
\hline 1850 & $1.14789 \mathrm{E}-05$ \\
\hline 1851 & $1.14704 \mathrm{E}-05$ \\
\hline
\end{tabular}




\begin{tabular}{|c|c|}
\hline 1917 & $1.09435 \mathrm{E}-05$ \\
\hline 1918 & 0.000010936 \\
\hline 1919 & $1.09282 \mathrm{E}-05$ \\
\hline 1920 & 1.09207E-05 \\
\hline 1921 & $1.09129 \mathrm{E}-05$ \\
\hline 1922 & $1.09054 \mathrm{E}-05$ \\
\hline 1923 & $1.08976 \mathrm{E}-05$ \\
\hline 1924 & $1.08902 \mathrm{E}-05$ \\
\hline 1925 & $1.08824 \mathrm{E}-05$ \\
\hline 1926 & $1.08749 \mathrm{E}-05$ \\
\hline 1927 & $1.08671 \mathrm{E}-05$ \\
\hline 1928 & $1.08598 \mathrm{E}-05$ \\
\hline 1929 & 0.000010852 \\
\hline 1930 & $1.08446 \mathrm{E}-05$ \\
\hline 1931 & $1.08368 \mathrm{E}-05$ \\
\hline 1932 & $1.08295 \mathrm{E}-05$ \\
\hline 1933 & $1.08217 \mathrm{E}-05$ \\
\hline 1934 & $1.08144 \mathrm{E}-05$ \\
\hline 1935 & $1.08067 \mathrm{E}-05$ \\
\hline 1936 & $1.07993 \mathrm{E}-05$ \\
\hline 1937 & $1.07916 \mathrm{E}-05$ \\
\hline 1938 & $1.07843 \mathrm{E}-05$ \\
\hline 1939 & $1.07766 \mathrm{E}-05$ \\
\hline 1940 & $1.07693 \mathrm{E}-05$ \\
\hline 1941 & $1.07617 \mathrm{E}-05$ \\
\hline 1942 & $1.07544 \mathrm{E}-05$ \\
\hline 1943 & $1.07467 \mathrm{E}-05$ \\
\hline 1944 & $1.07395 \mathrm{E}-05$ \\
\hline 1945 & $1.07318 \mathrm{E}-05$ \\
\hline 1946 & $1.07246 \mathrm{E}-05$ \\
\hline 1947 & 0.000010717 \\
\hline 1948 & $1.07097 \mathrm{E}-05$ \\
\hline 1949 & $1.07021 \mathrm{E}-05$ \\
\hline 1950 & $1.06949 \mathrm{E}-05$ \\
\hline 1951 & $1.06873 \mathrm{E}-05$ \\
\hline 1952 & $1.06802 \mathrm{E}-05$ \\
\hline 1953 & $1.06726 \mathrm{E}-05$ \\
\hline 1954 & $1.06654 \mathrm{E}-05$ \\
\hline 1955 & $1.06578 \mathrm{E}-05$ \\
\hline 1956 & $1.06507 \mathrm{E}-05$ \\
\hline 1957 & $1.06431 \mathrm{E}-05$ \\
\hline 1958 & 0.000010636 \\
\hline 1959 & $1.06285 \mathrm{E}-05$ \\
\hline 1960 & $1.06214 \mathrm{E}-05$ \\
\hline 1961 & $1.06139 \mathrm{E}-05$ \\
\hline 1962 & $1.06067 \mathrm{E}-05$ \\
\hline 1963 & $1.05993 \mathrm{E}-05$ \\
\hline 1964 & $1.05922 \mathrm{E}-05$ \\
\hline 1965 & $1.05847 \mathrm{E}-05$ \\
\hline 1966 & $1.05776 \mathrm{E}-05$ \\
\hline 1967 & $1.05701 \mathrm{E}-05$ \\
\hline 1968 & $1.05631 \mathrm{E}-05$ \\
\hline 1969 & $1.05556 \mathrm{E}-05$ \\
\hline 1970 & $1.05486 \mathrm{E}-05$ \\
\hline 1971 & $1.05412 \mathrm{E}-05$ \\
\hline 1972 & $1.05341 \mathrm{E}-05$ \\
\hline 1973 & $1.05267 \mathrm{E}-05$ \\
\hline 1974 & 1.05197E-05 \\
\hline 1975 & $1.05123 \mathrm{E}-05$ \\
\hline 1976 & $1.05053 \mathrm{E}-05$ \\
\hline 1977 & 0.000010498 \\
\hline 1978 & 0.000010491 \\
\hline 1979 & $1.04836 \mathrm{E}-05$ \\
\hline 1980 & $1.04766 \mathrm{E}-05$ \\
\hline 1981 & 1.04693E-05 \\
\hline
\end{tabular}




\begin{tabular}{|c|c|}
\hline 1982 & $1.04624 \mathrm{E}-05$ \\
\hline 1983 & 0.000010455 \\
\hline 1984 & $1.04481 \mathrm{E}-05$ \\
\hline 1985 & $1.04408 \mathrm{E}-05$ \\
\hline 1986 & $1.04339 \mathrm{E}-05$ \\
\hline 1987 & $1.04266 \mathrm{E}-05$ \\
\hline 1988 & $1.04197 \mathrm{E}-05$ \\
\hline 1989 & $1.04124 \mathrm{E}-05$ \\
\hline 1990 & $1.04055 \mathrm{E}-05$ \\
\hline 1991 & $1.03982 \mathrm{E}-05$ \\
\hline 1992 & $1.03914 \mathrm{E}-05$ \\
\hline 1993 & $1.03841 \mathrm{E}-05$ \\
\hline 1994 & $1.03772 \mathrm{E}-05$ \\
\hline 1995 & 0.00001037 \\
\hline 1996 & $1.03632 \mathrm{E}-05$ \\
\hline 1997 & 0.000010356 \\
\hline 1998 & $1.03491 \mathrm{E}-05$ \\
\hline 1999 & $1.03419 \mathrm{E}-05$ \\
\hline 2000 & $1.03351 \mathrm{E}-05$ \\
\hline 2001 & $1.03279 \mathrm{E}-05$ \\
\hline 2002 & $1.03211 \mathrm{E}-05$ \\
\hline 2003 & 0.000010314 \\
\hline 2004 & $1.03072 \mathrm{E}-05$ \\
\hline 2005 & 0.0000103 \\
\hline 2006 & $1.02933 \mathrm{E}-05$ \\
\hline 2007 & $1.02861 \mathrm{E}-05$ \\
\hline 2008 & $1.02794 \mathrm{E}-05$ \\
\hline 2009 & $1.02722 \mathrm{E}-05$ \\
\hline 2010 & $1.02655 \mathrm{E}-05$ \\
\hline 2011 & $1.02584 \mathrm{E}-05$ \\
\hline 2012 & $1.02517 \mathrm{E}-05$ \\
\hline 2013 & $1.02446 \mathrm{E}-05$ \\
\hline 2014 & $1.02379 \mathrm{E}-05$ \\
\hline 2015 & $1.02308 \mathrm{E}-05$ \\
\hline 2016 & $1.02241 \mathrm{E}-05$ \\
\hline 2017 & 0.000010217 \\
\hline 2018 & $1.02104 \mathrm{E}-05$ \\
\hline 2019 & $1.02033 \mathrm{E}-05$ \\
\hline 2020 & $1.01966 \mathrm{E}-05$ \\
\hline 2021 & $1.01896 \mathrm{E}-05$ \\
\hline 2022 & 0.000010183 \\
\hline 2023 & $1.01759 \mathrm{E}-05$ \\
\hline 2024 & $1.01693 \mathrm{E}-05$ \\
\hline 2025 & $1.01623 \mathrm{E}-05$ \\
\hline 2026 & $1.01557 \mathrm{E}-05$ \\
\hline 2027 & $1.01487 \mathrm{E}-05$ \\
\hline 2028 & $1.01421 \mathrm{E}-05$ \\
\hline 2029 & $1.01351 \mathrm{E}-05$ \\
\hline 2030 & $1.01285 \mathrm{E}-05$ \\
\hline 2031 & $1.01216 \mathrm{E}-05$ \\
\hline 2032 & 0.000010115 \\
\hline 2033 & 0.000010108 \\
\hline 2034 & $1.01015 \mathrm{E}-05$ \\
\hline 2035 & $1.00946 \mathrm{E}-05$ \\
\hline 2036 & 0.000010088 \\
\hline 2037 & $1.00811 \mathrm{E}-05$ \\
\hline 2038 & $1.00746 \mathrm{E}-05$ \\
\hline 2039 & $1.00677 \mathrm{E}-05$ \\
\hline 2040 & $1.00612 \mathrm{E}-05$ \\
\hline 2041 & $1.00543 \mathrm{E}-05$ \\
\hline 2042 & $1.00478 \mathrm{E}-05$ \\
\hline 2043 & $1.00409 \mathrm{E}-05$ \\
\hline 2044 & $1.00344 \mathrm{E}-05$ \\
\hline 2045 & $1.00275 \mathrm{E}-05$ \\
\hline 2046 & $1.00211 \mathrm{E}-05$ \\
\hline
\end{tabular}




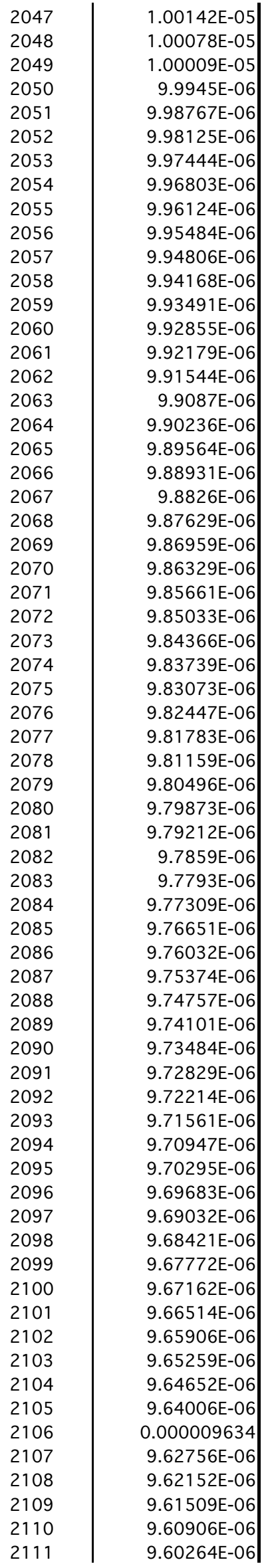


9.59662E-06

$9.58421 \mathrm{E}-06$

(57782E-06

$56545 \mathrm{E}-06$

(55947E-06

$54714 \mathrm{E}-06$

.54079E-06

$52849 \mathrm{E}-06$

.51029E-06

$50398 \mathrm{E}-06$

$9.49176 \mathrm{E}-06$

$8586 \mathrm{E}-06$

$47368 \mathrm{E}-06$

$.45526 \mathrm{E}-06$

44939E-06

.43729E-06

.43105E-06

$41898 \mathrm{E}-06$

$315 \mathrm{E}-06$

$40112 \mathrm{E}-06$

9492E-06

38292E-06

$7713 \mathrm{E}-06$

36517E-06

.000009359

9.35324E-06

$34133 E-06$

$.33519 \mathrm{E}-06$

32944E-06

$31758 \mathrm{E}-06$

.31146E-06

9.30574E-06

(2906

28784E-06

$.28214 \mathrm{E}-06$

27606E-06

$.27038 \mathrm{E}-06$

25863E-06

25258E-06

.

$.23522 \mathrm{E}-06$

$.22919 \mathrm{E}-06$

9.21753E-06

$9.2119 \mathrm{E}-06$ 


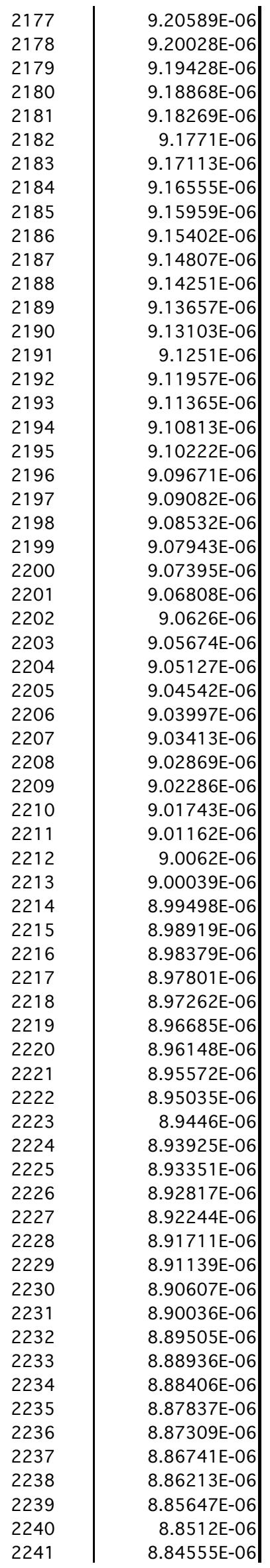

9.20589E-06 $9.20028 \mathrm{E}-06$ 9.19428E-06 9.18868E-06 9.18269E-06 9.1771E-06 9.17113E-06 9.16555E-06 9.15959E-06 9.15402E-06 9.14807E-06 9.14251E-06 9.13657E-06 9.13103E-06 9.1251E-06 9.11957E-06 $9.11365 \mathrm{E}-06$ 9.10813E-06 9.10222E-06 9.09671E-06 9.09082E-06 $9.08532 \mathrm{E}-06$ 9.07943E-06 9.07395E-06 9.06808E-06 9.0626E-06 9.05674E-06 9.05127E-06 9.04542E-06 9.03997E-06 $9.03413 \mathrm{E}-06$ 9.02869E-06 9.02286E-06 9.01743E-06 9.01162E-06 9.0062E-06 9.00039E-06 8.99498E-06 8.98919E-06 8.98379E-06 8.97801E-06 8.97262E-06 8.96685E-06 8.96148E-06 8.95572E-06 8.95035E-06 8.9446E-06 8.93925E-06 $8.93351 \mathrm{E}-06$ 8.92817E-06 8.92244E-06 $8.91711 \mathrm{E}-06$ 8.91139E-06 8.90607E-06 8.90036E-06 8.89505E-06 8.88936E-06 8.88406E-06 8.87837E-06 8.87309E-06 8.86741E-06 8.86213E-06 8.85647E-06 8.8512E-06 8.84555E-06 


\begin{tabular}{|c|c|}
\hline 2437 & $7.8701 \mathrm{E}-06$ \\
\hline 2438 & 7.86576E-06 \\
\hline 2439 & 7.86103E-06 \\
\hline 2440 & 7.8567E-06 \\
\hline 2441 & $7.85197 \mathrm{E}-06$ \\
\hline 2442 & $7.84765 \mathrm{E}-06$ \\
\hline 2443 & 7.84293E-06 \\
\hline 2444 & 7.83862E-06 \\
\hline 2445 & 7.83391E-06 \\
\hline 2446 & 7.8296E-06 \\
\hline 2447 & 7.8249E-06 \\
\hline 2448 & 7.8206E-06 \\
\hline 2449 & $7.81591 \mathrm{E}-06$ \\
\hline 2450 & $7.81162 \mathrm{E}-06$ \\
\hline 2451 & 7.80694E-06 \\
\hline 2452 & 7.80265E-06 \\
\hline 2453 & 7.79798E-06 \\
\hline 2454 & 7.7937E-06 \\
\hline 2455 & 7.78903E-06 \\
\hline 2456 & $7.78477 \mathrm{E}-06$ \\
\hline 2457 & $7.78011 \mathrm{E}-06$ \\
\hline 2458 & 7.77585E-06 \\
\hline 2459 & 7.7712E-06 \\
\hline 2460 & 7.76694E-06 \\
\hline 2461 & 7.7623E-06 \\
\hline 2462 & $7.75806 \mathrm{E}-06$ \\
\hline 2463 & 7.75342E-06 \\
\hline 2464 & 7.74918E-06 \\
\hline 2465 & $7.74456 \mathrm{E}-06$ \\
\hline 2466 & 7.74033E-06 \\
\hline 2467 & 7.73571E-06 \\
\hline 2468 & $7.73149 E-06$ \\
\hline 2469 & $7.72687 \mathrm{E}-06$ \\
\hline 2470 & 7.72266E-06 \\
\hline 2471 & 7.71806E-06 \\
\hline 2472 & 7.71385E-06 \\
\hline 2473 & 7.70926E-06 \\
\hline 2474 & $7.70506 \mathrm{E}-06$ \\
\hline 2475 & 7.70047E-06 \\
\hline 2476 & 7.69628E-06 \\
\hline 2477 & 7.6917E-06 \\
\hline 2478 & $7.68752 \mathrm{E}-06$ \\
\hline 2479 & 7.68294E-06 \\
\hline 2480 & 7.67877E-06 \\
\hline 2481 & 7.6742E-06 \\
\hline 2482 & 7.67004E-06 \\
\hline 2483 & $7.66548 \mathrm{E}-06$ \\
\hline 2484 & 7.66132E-06 \\
\hline 2485 & 7.65677E-06 \\
\hline 2486 & 7.65262E-06 \\
\hline 2487 & $7.64808 \mathrm{E}-06$ \\
\hline 2488 & 7.64393E-06 \\
\hline 2489 & 7.6394E-06 \\
\hline 2490 & 7.63526E-06 \\
\hline 2491 & 7.63074E-06 \\
\hline 2492 & $7.62661 \mathrm{E}-06$ \\
\hline 2493 & 7.62209E-06 \\
\hline 2494 & 7.61797E-06 \\
\hline 2495 & 7.61346E-06 \\
\hline 2496 & 7.60934E-06 \\
\hline 2497 & 7.60484E-06 \\
\hline 2498 & 7.60073E-06 \\
\hline 2499 & 7.59624E-06 \\
\hline 2500 & 7.59214E-06 \\
\hline 2501 & $7.58765 \mathrm{E}-06$ \\
\hline
\end{tabular}




\begin{tabular}{|c|c|}
\hline 2502 & 7.58356E-06 \\
\hline 2503 & 7.57908E-06 \\
\hline 2504 & 7.57499E-06 \\
\hline 2505 & 7.57052E-06 \\
\hline 2506 & 7.56644E-06 \\
\hline 2507 & 7.56198E-06 \\
\hline 2508 & $7.55791 \mathrm{E}-06$ \\
\hline 2509 & $7.55345 \mathrm{E}-06$ \\
\hline 2510 & 7.54939E-06 \\
\hline 2511 & 7.54493E-06 \\
\hline 2512 & $7.54088 \mathrm{E}-06$ \\
\hline 2513 & 7.53644E-06 \\
\hline 2514 & 7.53239E-06 \\
\hline 2515 & 7.52795E-06 \\
\hline 2516 & 7.52392E-06 \\
\hline 2517 & $7.51948 \mathrm{E}-06$ \\
\hline 2518 & $7.51545 \mathrm{E}-06$ \\
\hline 2519 & 7.51103E-06 \\
\hline 2520 & 7.50701E-06 \\
\hline 2521 & 7.50259E-06 \\
\hline 2522 & $7.49858 \mathrm{E}-06$ \\
\hline 2523 & 7.49417E-06 \\
\hline 2524 & $7.49016 \mathrm{E}-06$ \\
\hline 2525 & $7.48576 \mathrm{E}-06$ \\
\hline 2526 & 7.48176E-06 \\
\hline 2527 & 7.47736E-06 \\
\hline 2528 & 7.47337E-06 \\
\hline 2529 & $7.46898 \mathrm{E}-06$ \\
\hline 2530 & 7.46499E-06 \\
\hline 2531 & $7.46061 \mathrm{E}-06$ \\
\hline 2532 & $7.45663 \mathrm{E}-06$ \\
\hline 2533 & $7.45226 \mathrm{E}-06$ \\
\hline 2534 & $7.44829 \mathrm{E}-06$ \\
\hline 2535 & $7.44392 \mathrm{E}-06$ \\
\hline 2536 & 7.43996E-06 \\
\hline 2537 & 7.4356E-06 \\
\hline 2538 & $7.43164 \mathrm{E}-06$ \\
\hline 2539 & 7.42729E-06 \\
\hline 2540 & 7.42334E-06 \\
\hline 2541 & 0.000007419 \\
\hline 2542 & $7.41505 \mathrm{E}-06$ \\
\hline 2543 & $7.41072 \mathrm{E}-06$ \\
\hline 2544 & $7.40678 \mathrm{E}-06$ \\
\hline 2545 & $7.40245 \mathrm{E}-06$ \\
\hline 2546 & $7.39852 \mathrm{E}-06$ \\
\hline 2547 & 7.3942E-06 \\
\hline 2548 & 7.39027E-06 \\
\hline 2549 & 7.38596E-06 \\
\hline 2550 & 7.38204E-06 \\
\hline 2551 & 7.37773E-06 \\
\hline 2552 & 7.37383E-06 \\
\hline 2553 & 7.36952E-06 \\
\hline 2554 & 7.36562E-06 \\
\hline 2555 & 7.36133E-06 \\
\hline 2556 & $7.35743 \mathrm{E}-06$ \\
\hline 2557 & $7.35315 \mathrm{E}-06$ \\
\hline 2558 & 7.34926E-06 \\
\hline 2559 & $7.34498 \mathrm{E}-06$ \\
\hline 2560 & $7.3411 \mathrm{E}-06$ \\
\hline 2561 & 7.33682E-06 \\
\hline 2562 & 7.33295E-06 \\
\hline 2563 & $7.32868 \mathrm{E}-06$ \\
\hline 2564 & 7.32482E-06 \\
\hline 2565 & $7.32056 \mathrm{E}-06$ \\
\hline 2566 & 7.3167E-06 \\
\hline
\end{tabular}




\begin{tabular}{|c|c|}
\hline 2567 & $7.31245 \mathrm{E}-06$ \\
\hline 2568 & 7.30859E-06 \\
\hline 2569 & 7.30435E-06 \\
\hline 2570 & 7.3005E-06 \\
\hline 2571 & $7.29626 \mathrm{E}-06$ \\
\hline 2572 & $7.29242 \mathrm{E}-06$ \\
\hline 2573 & 7.28819E-06 \\
\hline 2574 & $7.28436 \mathrm{E}-06$ \\
\hline 2575 & $7.28013 \mathrm{E}-06$ \\
\hline 2576 & $7.27631 \mathrm{E}-06$ \\
\hline 2577 & 7.27209E-06 \\
\hline 2578 & 7.26827E-06 \\
\hline 2579 & $7.26406 \mathrm{E}-06$ \\
\hline 2580 & $7.26025 E-06$ \\
\hline 2581 & $7.25604 \mathrm{E}-06$ \\
\hline 2582 & $7.25224 \mathrm{E}-06$ \\
\hline 2583 & 7.24804E-06 \\
\hline 2584 & 7.24424E-06 \\
\hline 2585 & $7.24005 E-06$ \\
\hline 2586 & $7.23626 \mathrm{E}-06$ \\
\hline 2587 & 7.23207E-06 \\
\hline 2588 & 7.22829E-06 \\
\hline 2589 & $7.22411 \mathrm{E}-06$ \\
\hline 2590 & 7.22033E-06 \\
\hline 2591 & $7.21616 \mathrm{E}-06$ \\
\hline 2592 & $7.21239 E-06$ \\
\hline 2593 & 7.20823E-06 \\
\hline 2594 & $7.20446 \mathrm{E}-06$ \\
\hline 2595 & 7.2003E-06 \\
\hline 2596 & 7.19655E-06 \\
\hline 2597 & 7.1924E-06 \\
\hline 2598 & $7.18864 \mathrm{E}-06$ \\
\hline 2599 & 7.1845E-06 \\
\hline 2600 & $7.18076 \mathrm{E}-06$ \\
\hline 2601 & 7.17662E-06 \\
\hline 2602 & $7.17288 \mathrm{E}-06$ \\
\hline 2603 & $7.16875 \mathrm{E}-06$ \\
\hline 2604 & $7.16502 E-06$ \\
\hline 2605 & $7.16089 \mathrm{E}-06$ \\
\hline 2606 & 7.15717E-06 \\
\hline 2607 & 7.15305E-06 \\
\hline 2608 & $7.14933 \mathrm{E}-06$ \\
\hline 2609 & 7.14522E-06 \\
\hline 2610 & 7.14151E-06 \\
\hline 2611 & 7.1374E-06 \\
\hline 2612 & 7.1337E-06 \\
\hline 2613 & 7.1296E-06 \\
\hline 2614 & 7.1259E-06 \\
\hline 2615 & $7.12181 \mathrm{E}-06$ \\
\hline 2616 & $7.11812 \mathrm{E}-06$ \\
\hline 2617 & 7.11403E-06 \\
\hline 2618 & 7.11034E-06 \\
\hline 2619 & 7.10626E-06 \\
\hline 2620 & 7.10259E-06 \\
\hline 2621 & $7.09851 \mathrm{E}-06$ \\
\hline 2622 & 7.09484E-06 \\
\hline 2623 & 7.09077E-06 \\
\hline 2624 & $7.08711 \mathrm{E}-06$ \\
\hline 2625 & 7.08305E-06 \\
\hline 2626 & 7.07939E-06 \\
\hline 2627 & 7.07533E-06 \\
\hline 2628 & $7.07168 \mathrm{E}-06$ \\
\hline 2629 & 7.06763E-06 \\
\hline 2630 & 7.06399E-06 \\
\hline 2631 & 7.05995E-06 \\
\hline
\end{tabular}


6.58297E-06

$6.57606 \mathrm{E}-06$

$57241 \mathrm{E}-06$

$56553 \mathrm{E}-06$

(5)

$55542 \mathrm{E}-06$

$5178 \mathrm{E}-06$

$54493 \mathrm{E}-06$

$54171 \mathrm{E}-06$

$53487 \mathrm{E}-06$

$53125 \mathrm{E}-06$

$52443 \mathrm{E}-06$

$51442 \mathrm{E}-06$

$6.50762 \mathrm{E}-06$

$50402 \mathrm{E}-06$

$6.49724 \mathrm{E}-06$

$6.49406 \mathrm{E}-06$

$6.48729 \mathrm{E}-06$

.48372E-06

$6.4738 \mathrm{E}-06$

233E-06

$6.4635 \mathrm{E}-06$

-

$6.45363 \mathrm{E}-06$

$6.44338 \mathrm{E}-06$

$43356 \mathrm{E}-06$

.42336E-06

$.41358 \mathrm{E}-06$

$6 \mathrm{E}-06$

$6.40342 \mathrm{E}-06$

39369E-06

9019E-06

.38358E-06

6.3739E-06

$6.36732 \mathrm{E}-06$ 


\begin{tabular}{|l|r|}
2957 & $5.95728 \mathrm{E}-06$ \\
2958 & $5.9545 \mathrm{E}-06$ \\
2959 & $5.95132 \mathrm{E}-06$ \\
2960 & $5.94855 \mathrm{E}-06$ \\
2961 & $5.94538 \mathrm{E}-06$ \\
2962 & $5.94262 \mathrm{E}-06$ \\
2963 & $5.93945 \mathrm{E}-06$ \\
2964 & $5.93669 \mathrm{E}-06$ \\
2965 & $5.93352 \mathrm{E}-06$ \\
2966 & $5.93076 \mathrm{E}-06$ \\
2967 & $5.9276 \mathrm{E}-06$ \\
2968 & $5.92485 \mathrm{E}-06$ \\
2969 & $5.9217 \mathrm{E}-06$ \\
2970 & $5.91895 \mathrm{E}-06$ \\
2971 & $5.9158 \mathrm{E}-06$ \\
2972 & $5.91305 \mathrm{E}-06$ \\
2973 & $5.90991 \mathrm{E}-06$ \\
2974 & $5.90717 \mathrm{E}-06$ \\
2975 & $5.90402 \mathrm{E}-06$ \\
2976 & $5.90129 \mathrm{E}-06$ \\
2977 & $5.89815 \mathrm{E}-06$ \\
2978 & $5.89542 \mathrm{E}-06$ \\
2979 & $5.89228 \mathrm{E}-06$ \\
2980 & $5.88956 \mathrm{E}-06$ \\
2981 & $5.88643 \mathrm{E}-06$ \\
2982 & $5.8837 \mathrm{E}-06$ \\
2983 & $5.88058 \mathrm{E}-06$ \\
2984 & $5.87786 \mathrm{E}-06$ \\
2985 & $5.87474 \mathrm{E}-06$ \\
2986 & $5.87202 \mathrm{E}-06$ \\
2987 & $5.86891 \mathrm{E}-06$ \\
2988 & $5.8662 \mathrm{E}-06$ \\
2989 & $5.86308 \mathrm{E}-06$ \\
2990 & $5.86038 \mathrm{E}-06$ \\
2991 & $5.85727 \mathrm{E}-06$ \\
2992 & $5.85457 \mathrm{E}-06$ \\
2993 & $5.85146 \mathrm{E}-06$ \\
2994 & $5.84877 \mathrm{E}-06$ \\
2995 & $5.84567 \mathrm{E}-06$ \\
2996 & $5.84297 \mathrm{E}-06$ \\
2997 & $5.83988 \mathrm{E}-06$ \\
2998 & $5.83719 \mathrm{E}-06$ \\
2999 & $5.8341 \mathrm{E}-06$ \\
3000 & $5.83141 \mathrm{E}-06$ \\
\hline
\end{tabular}


Fill hole configuration (a)

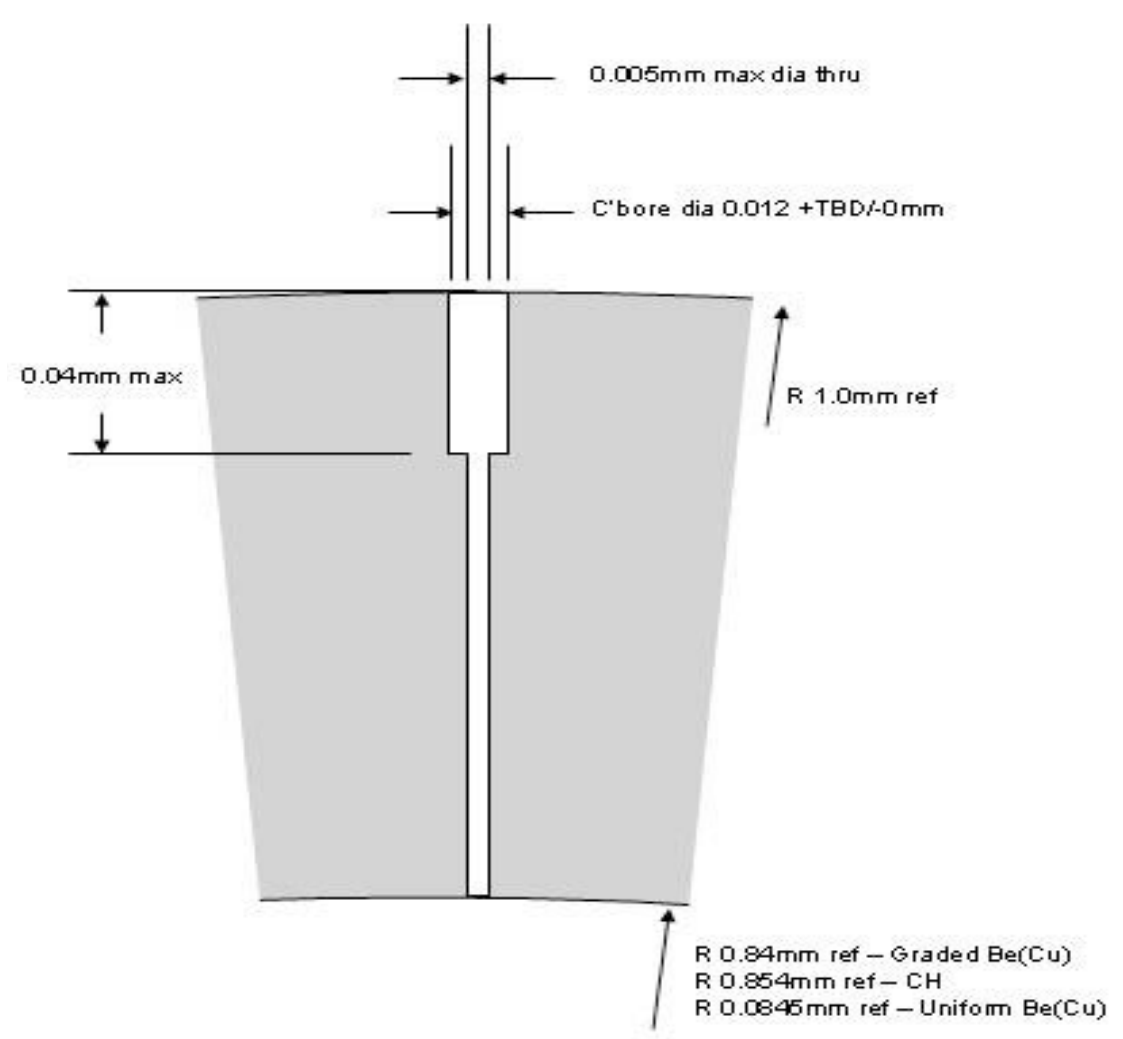

Fill tube installation (b)

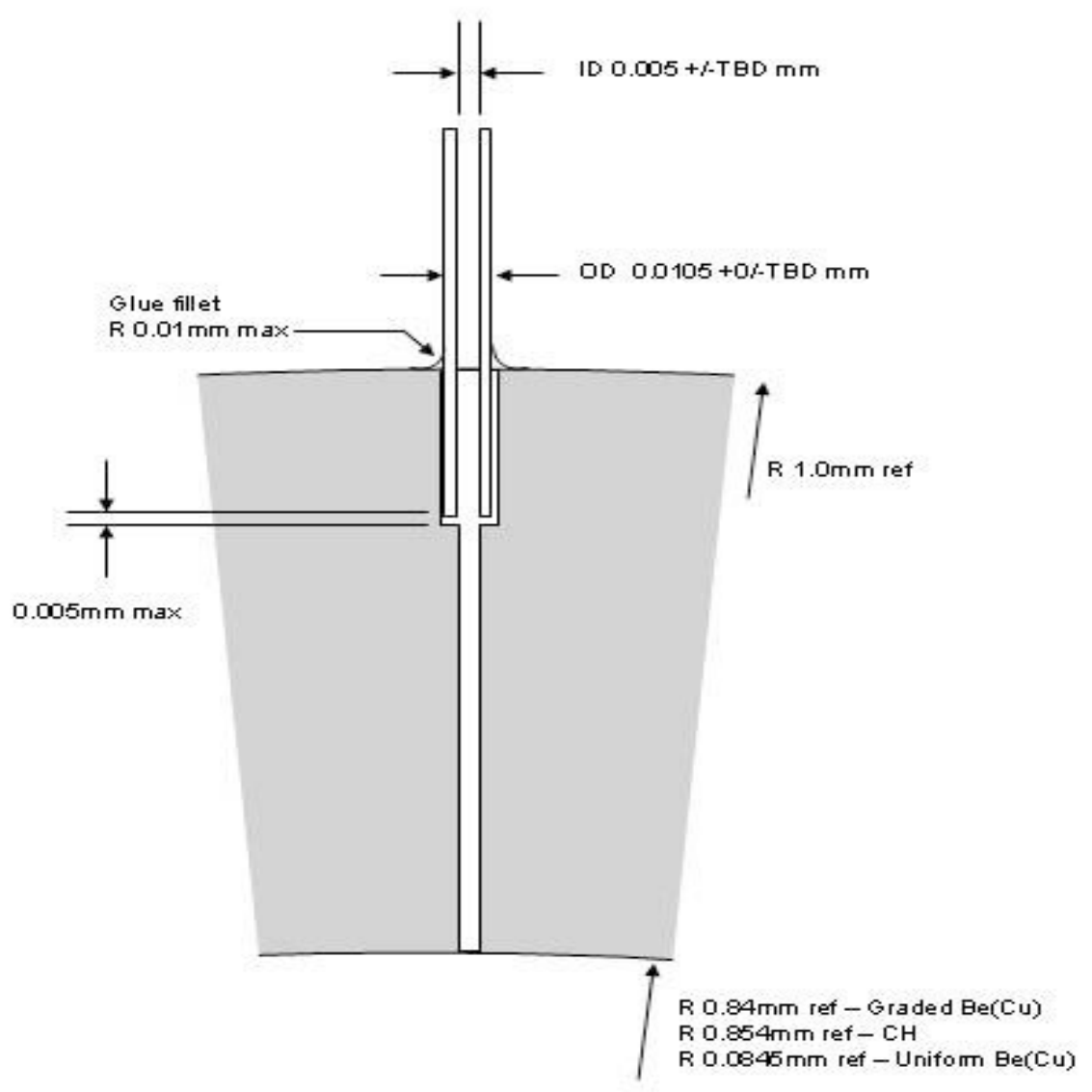


Range of possible hohlraum dimensions

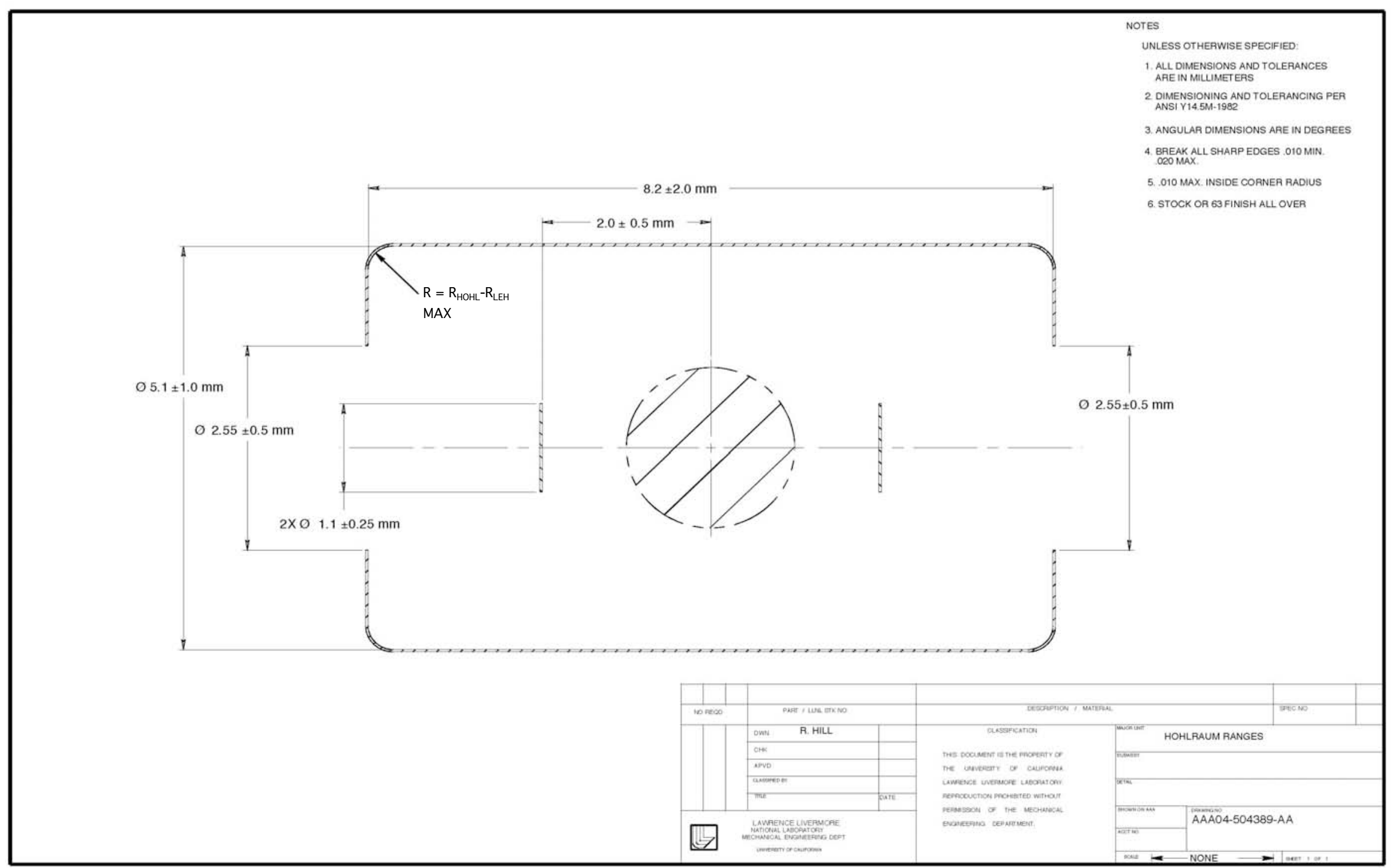




\section{Gas filled hohlraum design (a)}

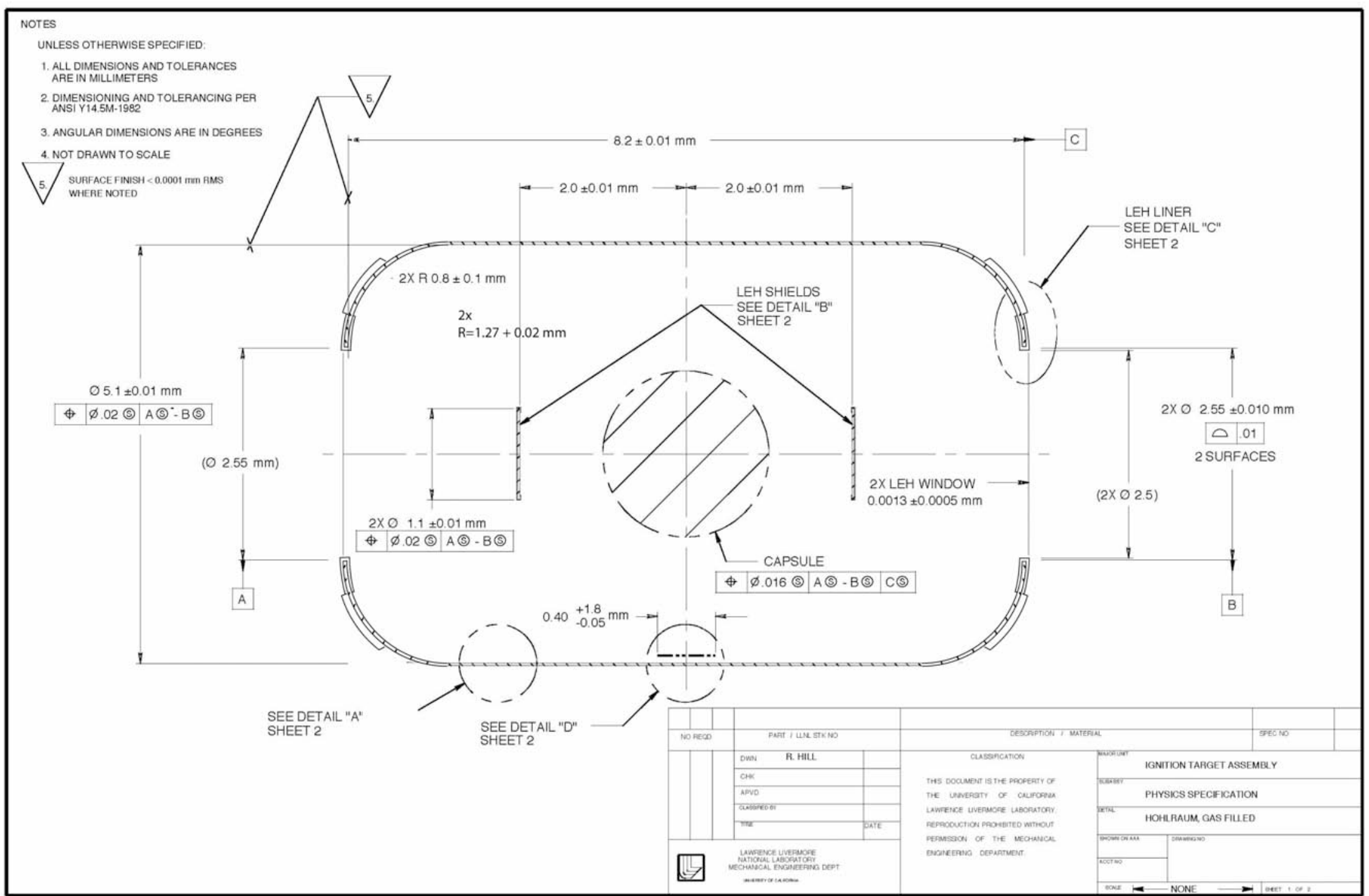




\section{Gas filled hohlraum design (b)}

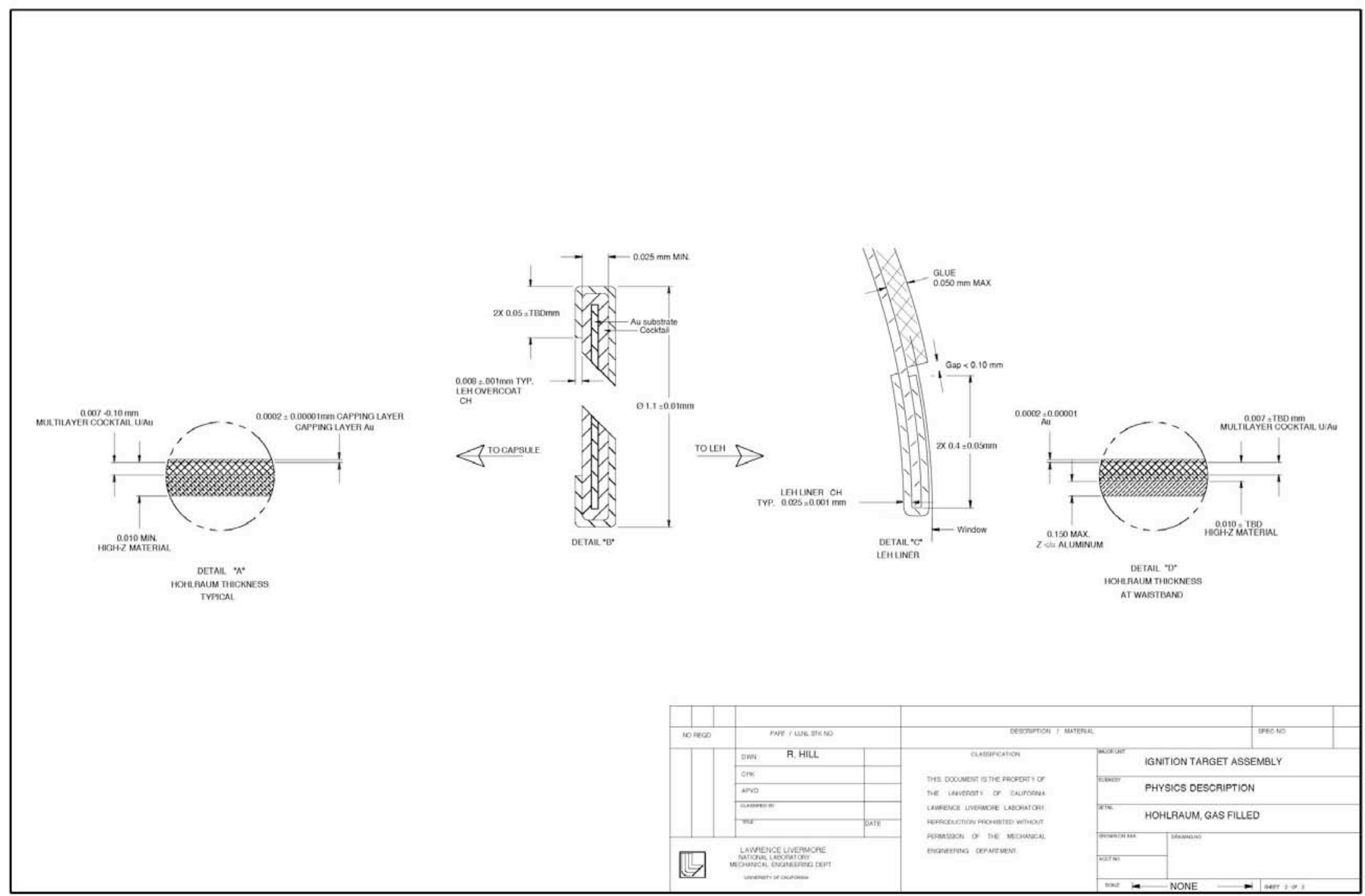




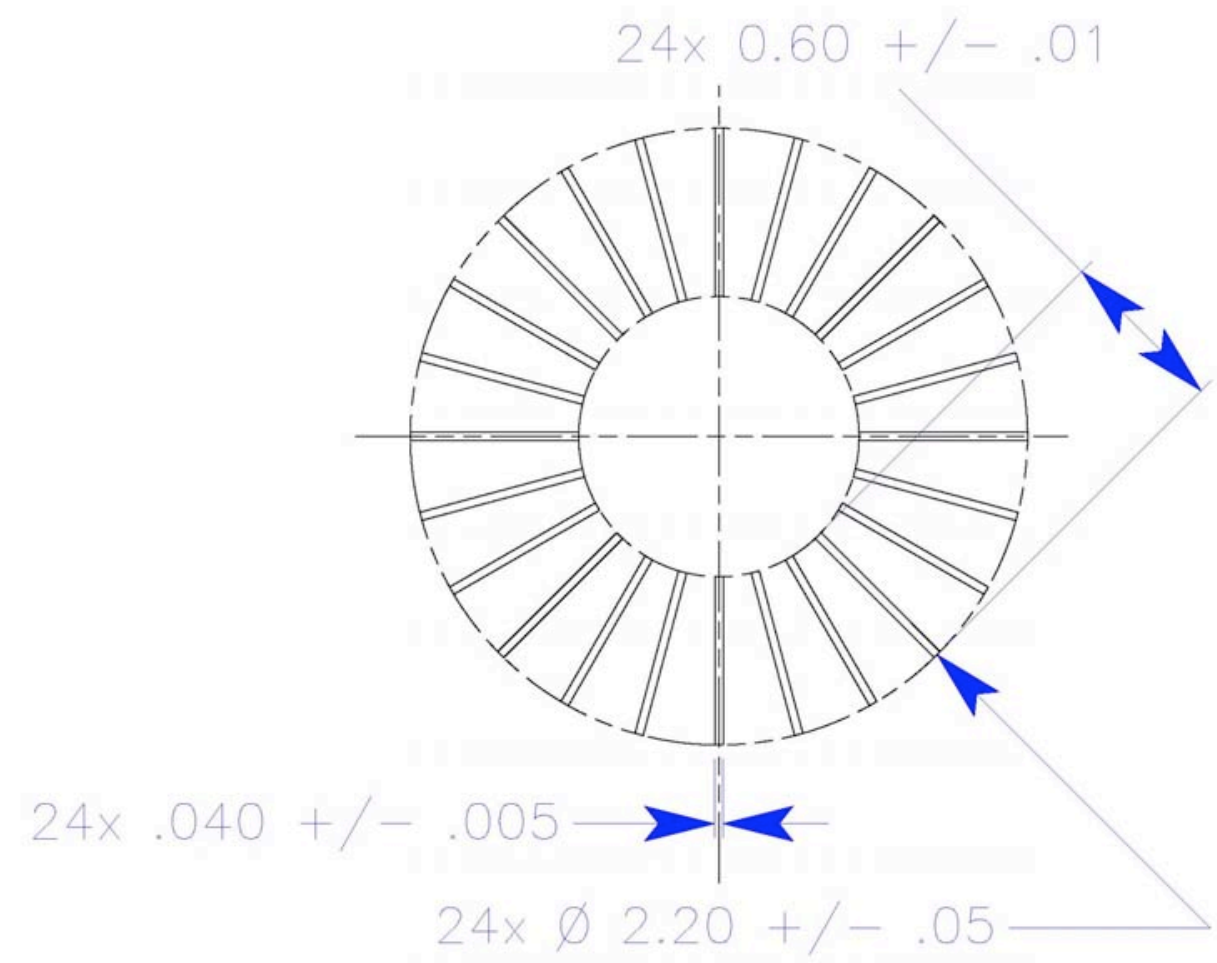




\section{LEH Stayout Zone - Rev 0}

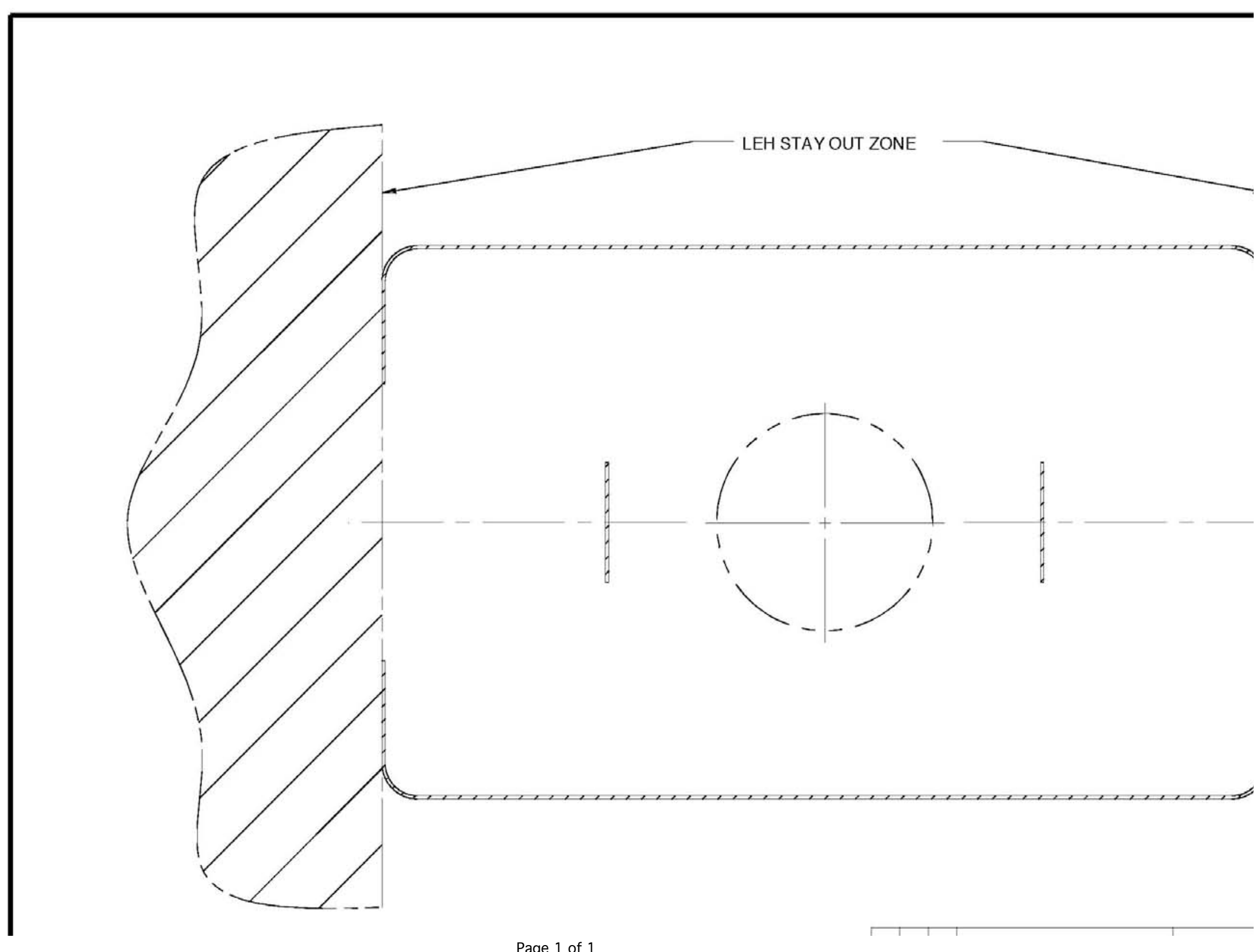




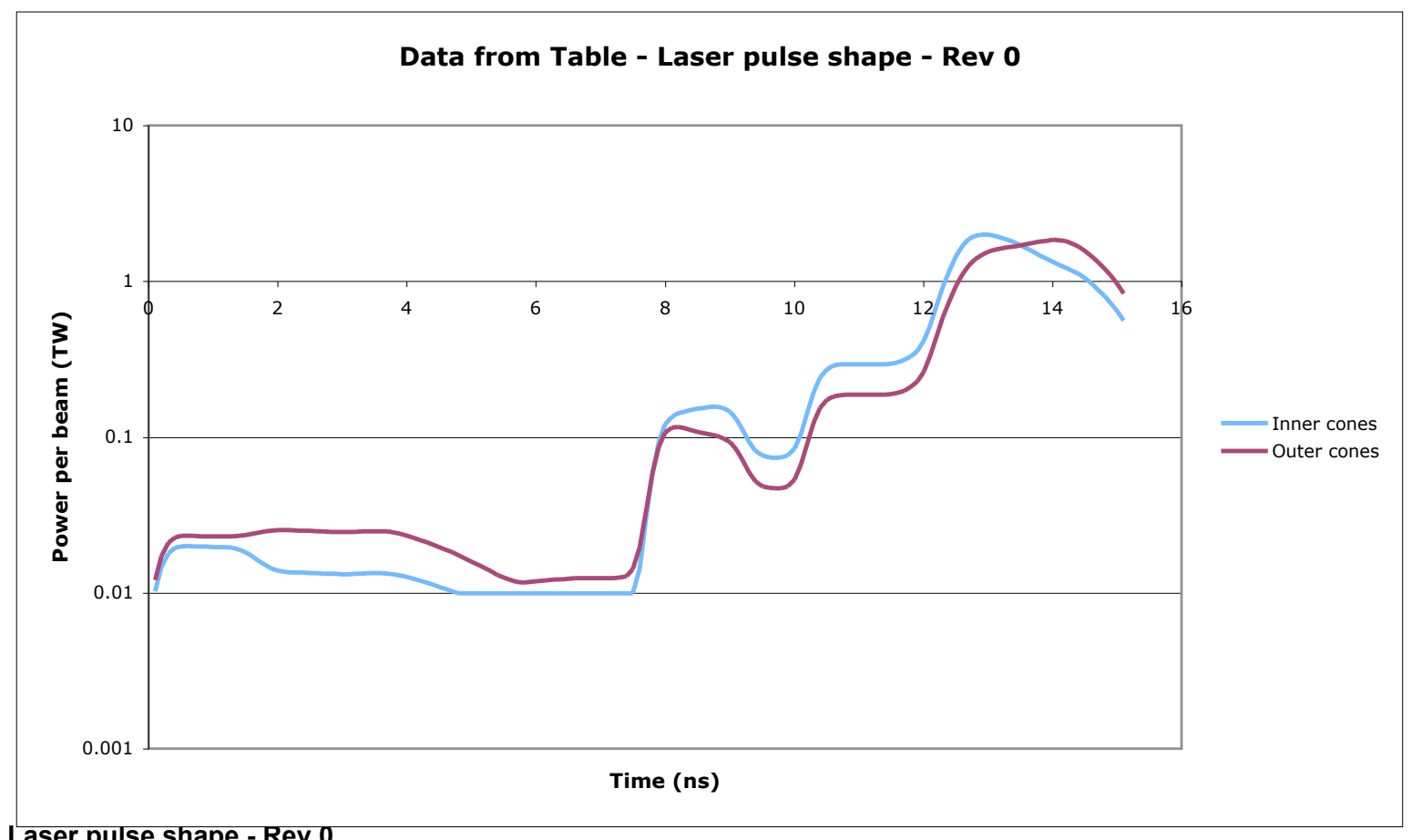

Laser pulse shape - Rev 0

\begin{tabular}{|c|c|c|}
\hline time (ns) & $\begin{array}{l}\text { Power per beam, } 23.5 \\
\& 30 \text { degree cones } \\
\text { (TW) }\end{array}$ & $\begin{array}{l}\text { Power per beam, } 44.5 \\
\& 50 \text { degree cones } \\
\text { (TW) }\end{array}$ \\
\hline 0 & $0.00 \mathrm{E}+00$ & $0.00 \mathrm{E}+00$ \\
\hline 0.1 & 0.010278 & 0.0121111 \\
\hline 0.2 & 0.0148291 & 0.0173658 \\
\hline 0.3 & 0.0180097 & 0.0210379 \\
\hline 0.4 & 0.0195137 & 0.0227739 \\
\hline 0.5 & 0.0199712 & 0.0233014 \\
\hline 0.6 & 0.0200267 & 0.0233647 \\
\hline 0.7 & 0.0199818 & 0.0233122 \\
\hline 0.8 & 0.0199206 & 0.0232408 \\
\hline 0.9 & 0.0198592 & 0.0231693 \\
\hline 1 & 0.0198022 & 0.0231047 \\
\hline 1.1 & 0.0197484 & 0.0230574 \\
\hline 1.2 & 0.0196697 & 0.023047 \\
\hline 1.3 & 0.0194778 & 0.0231099 \\
\hline 1.4 & 0.0190394 & 0.0232961 \\
\hline 1.5 & 0.0182764 & 0.0236312 \\
\hline 1.6 & 0.0172527 & 0.0240821 \\
\hline 1.7 & 0.0161377 & 0.0245691 \\
\hline 1.8 & 0.0151257 & 0.0249989 \\
\hline 1.9 & 0.0143746 & 0.0252927 \\
\hline 2 & 0.0139362 & 0.0254246 \\
\hline 2.1 & 0.0137341 & 0.0254328 \\
\hline 2.2 & 0.013646 & 0.025377 \\
\hline 2.3 & 0.0135901 & 0.0252932 \\
\hline 2.4 & 0.0135344 & 0.0251922 \\
\hline 2.5 & 0.0134729 & 0.025078 \\
\hline 2.6 & 0.0134087 & 0.0249585 \\
\hline 2.7 & 0.0133488 & 0.024847 \\
\hline 2.8 & 0.0133015 & 0.0247591 \\
\hline 2.9 & 0.0132723 & 0.0247047 \\
\hline 3 & 0.0132605 & 0.0246828 \\
\hline 3.1 & 0.013265 & 0.0246912 \\
\hline 3.2 & 0.0132907 & 0.024739 \\
\hline 3.3 & 0.013342 & 0.0248344 \\
\hline 3.4 & 0.0134064 & 0.0249539 \\
\hline 3.5 & 0.0134544 & 0.0250411 \\
\hline 3.6 & 0.0134566 & 0.0250368 \\
\hline 3.7 & 0.013389 & 0.0248883 \\
\hline 3.8 & 0.0132335 & 0.0245532 \\
\hline 3.9 & 0.0129919 & 0.024034 \\
\hline
\end{tabular}

Laser peak power and total energy for required pulse shapes - $\operatorname{Rev} 0$

\begin{tabular}{|c|r|r|}
\multicolumn{1}{c|}{} & Per Beam & For 192 beams \\
\hline Total Energy & & \\
inner cone & $5.068 \mathrm{~kJ}$ & $973.0 \mathrm{MJ}$ \\
outer cone & $4.967 \mathrm{~kJ}$ & $953.7 \mathrm{MJ}$ \\
\hline Peak power & $2.000 \mathrm{TW}$ & $384.1 \mathrm{TW}$ \\
inner cone & $1.851 \mathrm{TW}$ & $355.4 \mathrm{TW}$ \\
\hline outer cone &
\end{tabular}




\begin{tabular}{|c|c|c|}
\hline 4 & 0.012691 & 0.0233874 \\
\hline 4.1 & 0.0123626 & 0.0226819 \\
\hline 4.2 & 0.0120259 & 0.0219583 \\
\hline 4.3 & 0.0116874 & 0.0212311 \\
\hline 4.4 & 0.0113479 & 0.020503 \\
\hline 4.5 & 0.0110045 & 0.0197725 \\
\hline 4.6 & 0.0106495 & 0.0190349 \\
\hline 4.7 & 0.0102715 & 0.0182834 \\
\hline 4.8 & 0.01 & 0.017515 \\
\hline 4.9 & 0.01 & 0.0167358 \\
\hline 5 & 0.01 & 0.0159604 \\
\hline 5.1 & 0.01 & 0.0152058 \\
\hline 5.2 & 0.01 & 0.0144829 \\
\hline 5.3 & 0.01 & 0.0137923 \\
\hline 5.4 & 0.01 & 0.0131371 \\
\hline 5.5 & 0.01 & 0.0125489 \\
\hline 5.6 & 0.01 & 0.0120938 \\
\hline 5.7 & 0.01 & 0.0118291 \\
\hline 5.8 & 0.01 & 0.0117487 \\
\hline 5.9 & 0.01 & 0.011788 \\
\hline 6 & 0.01 & 0.0118795 \\
\hline 6.1 & 0.01 & 0.0119861 \\
\hline 6.2 & 0.01 & 0.0120953 \\
\hline 6.3 & 0.01 & 0.0122036 \\
\hline 6.4 & 0.01 & 0.0123067 \\
\hline 6.5 & 0.01 & 0.012396 \\
\hline 6.6 & 0.01 & 0.0124608 \\
\hline 6.7 & 0.01 & 0.0124971 \\
\hline 6.8 & 0.01 & 0.0125119 \\
\hline 6.9 & 0.01 & 0.0125161 \\
\hline 7 & 0.01 & 0.0125169 \\
\hline 7.1 & 0.01 & 0.0125172 \\
\hline 7.2 & 0.01 & 0.0125204 \\
\hline 7.3 & 0.01 & 0.0125548 \\
\hline 7.4 & 0.01 & 0.0128184 \\
\hline 7.5 & 0.01 & 0.01425 \\
\hline 7.6 & 0.0143978 & 0.0196799 \\
\hline 7.7 & 0.0299697 & 0.033784 \\
\hline 7.8 & 0.0584624 & 0.0583475 \\
\hline 7.9 & 0.0929264 & 0.0864126 \\
\hline 8 & 0.120611 & 0.106807 \\
\hline 8.1 & 0.135989 & 0.115398 \\
\hline 8.2 & 0.142918 & 0.116212 \\
\hline 8.3 & 0.146652 & 0.114139 \\
\hline 8.4 & 0.14965 & 0.111432 \\
\hline 8.5 & 0.15248 & 0.108696 \\
\hline 8.6 & 0.155064 & 0.10615 \\
\hline 8.7 & 0.157005 & 0.103963 \\
\hline 8.8 & 0.157431 & 0.101942 \\
\hline 8.9 & 0.154577 & 0.0989487 \\
\hline 9 & 0.145991 & 0.0930431 \\
\hline 9.1 & 0.130616 & 0.0831429 \\
\hline 9.2 & 0.111234 & 0.0707878 \\
\hline 9.3 & 0.0935306 & 0.0595197 \\
\hline 9.4 & 0.0818247 & 0.0520702 \\
\hline 9.5 & 0.076282 & 0.048543 \\
\hline 9.6 & 0.0744283 & 0.0473633 \\
\hline 9.7 & 0.0740382 & 0.0471151 \\
\hline 9.8 & 0.0743536 & 0.0473158 \\
\hline 9.9 & 0.0764887 & 0.0486745 \\
\hline 10 & 0.0845587 & 0.05381 \\
\hline 10.1 & 0.105625 & 0.0672162 \\
\hline 10.2 & 0.144401 & 0.091892 \\
\hline 10.3 & 0.195235 & 0.124241 \\
\hline 10.4 & 0.242713 & 0.154454 \\
\hline 10.5 & 0.274055 & 0.174399 \\
\hline 10.6 & 0.288511 & 0.183598 \\
\hline 10.7 & 0.293124 & 0.186533 \\
\hline 10.8 & 0.294134 & 0.187176 \\
\hline 10.9 & 0.294286 & 0.187272 \\
\hline 11 & 0.294301 & 0.187282 \\
\hline 11.1 & 0.294304 & 0.187284 \\
\hline
\end{tabular}




\begin{tabular}{|c|c|c|}
\hline 11.2 & 0.294321 & 0.187295 \\
\hline 11.3 & 0.29445 & 0.187377 \\
\hline 11.4 & 0.295104 & 0.187793 \\
\hline 11.5 & 0.297361 & 0.18923 \\
\hline 11.6 & 0.302883 & 0.192744 \\
\hline 11.7 & 0.313159 & 0.199283 \\
\hline 11.8 & 0.329866 & 0.209915 \\
\hline 11.9 & 0.358695 & 0.228261 \\
\hline 12 & 0.415004 & 0.264095 \\
\hline 12.1 & 0.5217 & 0.331995 \\
\hline 12.2 & 0.692903 & 0.440977 \\
\hline 12.3 & 0.921016 & 0.586441 \\
\hline 12.4 & 1.18385 & 0.755493 \\
\hline 12.5 & 1.4535 & 0.934464 \\
\hline 12.6 & 1.69433 & 1.10899 \\
\hline 12.7 & 1.87033 & 1.26485 \\
\hline 12.8 & 1.96727 & 1.3929 \\
\hline 12.9 & 2.00031 & 1.49049 \\
\hline 13 & 1.99439 & 1.55883 \\
\hline 13.1 & 1.96537 & 1.60296 \\
\hline 13.2 & 1.91869 & 1.63208 \\
\hline 13.3 & 1.85626 & 1.65542 \\
\hline 13.4 & 1.78219 & 1.67876 \\
\hline 13.5 & 1.7033 & 1.7049 \\
\hline 13.6 & 1.62531 & 1.73481 \\
\hline 13.7 & 1.55022 & 1.7678 \\
\hline 13.8 & 1.47755 & 1.80163 \\
\hline 13.9 & 1.40728 & 1.83197 \\
\hline 14 & 1.34135 & 1.85089 \\
\hline 14.1 & 1.28234 & 1.84835 \\
\hline 14.2 & 1.22967 & 1.81785 \\
\hline 14.3 & 1.17746 & 1.75938 \\
\hline 14.4 & 1.11828 & 1.67621 \\
\hline 14.5 & 1.0489 & 1.57321 \\
\hline 14.6 & 0.971628 & 1.45743 \\
\hline 14.7 & 0.890533 & 1.3358 \\
\hline 14.8 & 0.808258 & 1.21237 \\
\hline 14.9 & 0.725798 & 1.08859 \\
\hline 15 & 0.643177 & 0.964763 \\
\hline 15.1 & 0.560603 & 0.8409 \\
\hline 15.2 & 0.478029 & 0.717038 \\
\hline 15.3 & 0.403282 & 0.593173 \\
\hline
\end{tabular}


Laser Spot Shapes - Rev 0

NOTE: Spot shape requirement is TBD. The following data table is a sample intended only to show the form of the requirement

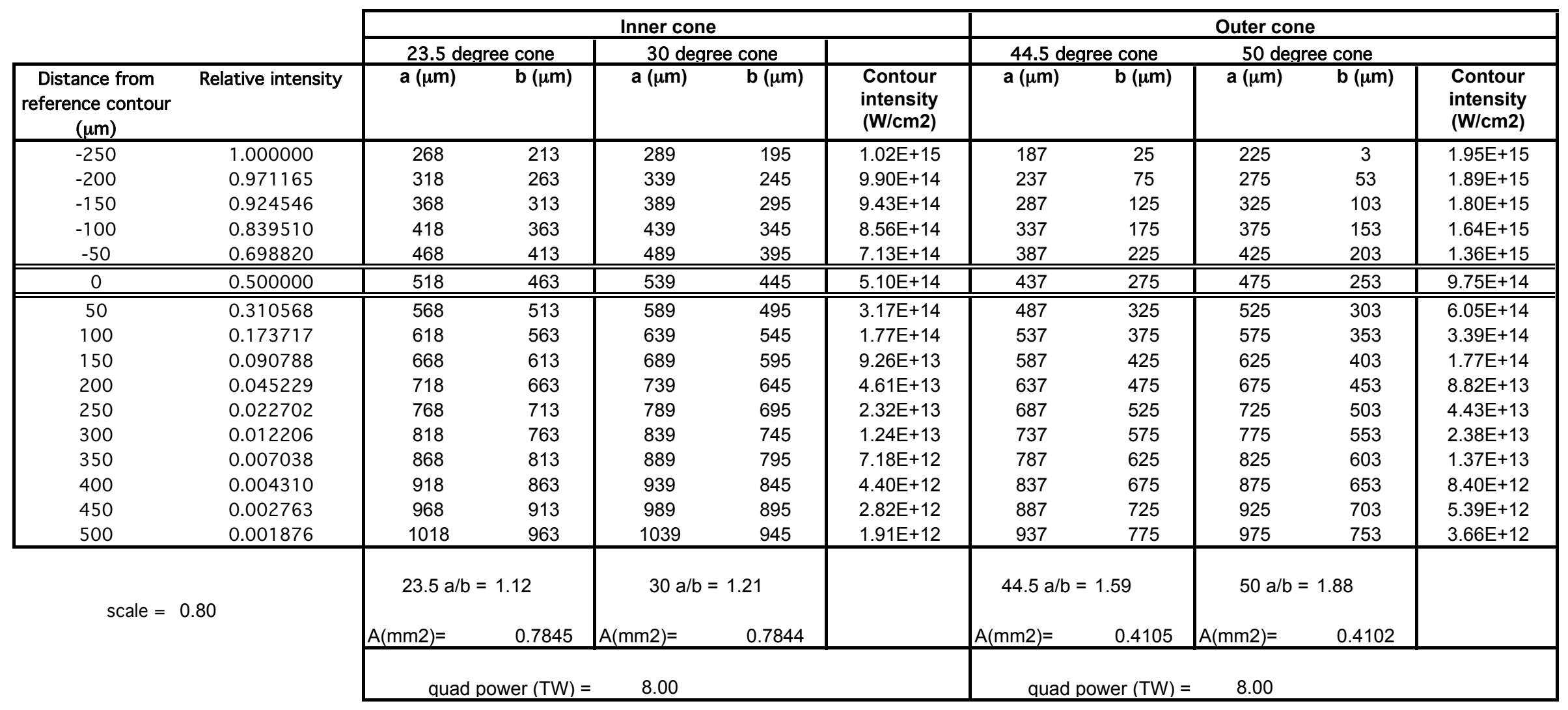

Notes:

1. Intensity contours are constant displacement $d$ from reference ellipse $d=0$.

2. The $a$ and $b$ values are orthogonal displacements relative to beam center.

3. Spot scale 0.8 has been used in all Lasnex calculations of $1.0 \mathrm{MJ}$ designs.

4. You can adjust spot scale (D1) or quad power (F24 and K24) to see effects.

5. From Munro's file D1spot20050815 


\section{Beam pointing - Rev 0}

\begin{tabular}{|c|c|c|}
\hline Cone, degrees & $\begin{array}{c}\text { Beam aim point radial distance from } \\
\text { hohlraum axis }(\mu \mathrm{m})\end{array}$ & $\begin{array}{c}\text { Beam aim point Z- } \\
\text { coordinate, from LEH plane } \\
(\mu \mathrm{m})\end{array}$ \\
\hline 23.5 & 600 & 0 \\
30 & 300 & 0 \\
44.5 & 0 & 0 \\
50 & 0 & 0 \\
\hline
\end{tabular}


Requirements for pre-ignition experimental programs. These are not requirements for fielding or fabrication, but are

included here to provide a common source for information on the requirements that drive the experimental programs leading up to ignition.

\begin{tabular}{|c|c|c|}
\hline Description & Remark & Requirement \\
\hline Peak temperature & & $300 \mathrm{eV}$ \\
\hline Maximum reproducible part of backscatter & Energy lost, reproducibly shot to shot & $15 \%$ \\
\hline Maximum irreproducible part of backscatter & Average $0 \%$ & $10 \% \mathrm{rms}$ \\
\hline Hot electrons, fraction of laser energy & $\begin{array}{l}\text { Maximum allowable fraction of energy is } \\
\text { function of Thot, log-lin interpolation of these } \\
\text { points } \\
\qquad \begin{array}{l}T_{\text {hot }}=10 \mathrm{keV} \\
\mathrm{T}_{\text {hot }}=30 \mathrm{keV} \\
\mathrm{T}_{\text {hot }}=70 \mathrm{keV}\end{array}\end{array}$ & $\begin{array}{l}<5 \% \\
<0.5 \% \\
<0.1 \%\end{array}$ \\
\hline M-band uncertainty & $\begin{array}{l}\text { Measure spectrum in } 1.5-4 \mathrm{keV} \text { range to } \pm 30 \% \text {. } \\
\text { Current model is assumed to be within } 50 \%\end{array}$ & $50 \%$ \\
\hline Cone-to-cone scatter, reproducible part & & $5 \%$ \\
\hline Cone-to-cone scatter, irreproducible part & & $2 \%$ \\
\hline Intrinsic asymmetry, mode 2 average & $\begin{array}{c}\text { All asymmetries are given as PI coefficients, in } \\
\text { flux. If non-zero } \mathrm{m} \text { values are present, they are } \\
\text { to included in an effective PI coefficient with } \\
\text { the same rms as the total. }\end{array}$ & $0.25 \%$ \\
\hline Intrinsic asymmetry, mode 4 average & & $0.25 \%$ \\
\hline Intrinsic asymmetry, mode 2 foot & & $0.50 \%$ \\
\hline Intrinsic asymmetry, mode 4 foot & & $0.50 \%$ \\
\hline Intrinsic asymmetry, time-average, rms in modes $>4$, foot & & $0.50 \%$ \\
\hline Intrinsic asymmetry, time average, rms sum in modes $>4$, peak & & $0.25 \%$ \\
\hline Intrinsic asymmetry, mode 2 swings during foot, 2 ns average & & $10 \%$ \\
\hline Intrinsic asymmetry, mode 4 swings during foot & & $5 \%$ \\
\hline Intrinsic asymmetry, mode 5 swings during foot & & $4 \%$ \\
\hline Intrinsic asymmetry, mode 6 swings during foot & & $3 \%$ \\
\hline Intrinsic asymmetry, mode 2 swings during peak & & $5 \%$ \\
\hline Intrinsic asymmetry, mode 4 swings during peak & & $3 \%$ \\
\hline Intrinsic asymmetry, mode 5 swings during peak & & $2 \%$ \\
\hline Intrinsic asymmetry, mode 6 swings during peak & & $2 \%$ \\
\hline Preheat asymmetry & & TBD \\
\hline Strength of first shock (reproducibility) & $\begin{array}{c}\begin{array}{c}\text { Shock strengths are given as \% variation in flux, } \\
\text { and are meant to constrain reproducibility not } \\
\text { absolute value. }\end{array} \\
\text { absolut }\end{array}$ & $3 \%$ \\
\hline Strength of second shock & & $3 \%$ \\
\hline Strength of 3rd shock & & $3 \%$ \\
\hline Strength of 4 th shock & & $5 \%$ \\
\hline Time of 1 st shock & $\begin{array}{l}\text { Times are relative to time outer cone power }= \\
1 \mathrm{TW} \text { per beam }\end{array}$ & $100 \mathrm{ps}$ \\
\hline Time of 2 nd shock & & 50 ps \\
\hline Time of 3rd shock & & $50 \mathrm{ps}$ \\
\hline Time of 4 th shock & & $100 \mathrm{ps}$ \\
\hline Shape of rise to peak & & TBD \\
\hline Ablation rate (total mass ablated until peak velocity) & & $1 \%$ \\
\hline Be microstructure as seed for $\mathrm{RT}$ & & $\begin{array}{c}\text { Velocity of ablator/DT } \\
\text { interface, } 100 \text { ps after } \\
\text { breakout of first shock, } \\
\text { uniform to } 2: 10^{\wedge} 4\end{array}$ \\
\hline RT growth confirmation & & TBD \\
\hline
\end{tabular}


Alternate Capsule Requirements - CH

\begin{tabular}{|c|c|c|c|c|c|c|}
\hline Number & Rev & WBS & Component & Title & short req't & Requirement Text \\
\hline $\mathrm{CH} 1$ & 0 & 1.4 .1 .1 & CH Capsule & Applicability of other requirements & & $\begin{array}{l}\text { All requirments in table "Point Design Req'ts" except those designated for component } \\
\text { "Layered Be capsule" shall apply to the CH capsule }\end{array}$ \\
\hline $\mathrm{CH} 2$ & 0 & I.4.1.1.4 & CH Capsule & Ablator composition & see table & $\begin{array}{l}\text { The ablator material shall be } \mathrm{C}: \mathrm{H}: \mathrm{O} \quad 0.43: 0.56: 0.01 \text {. C:H ratio shall be within } 10 \% \text { of } \\
\text { specified. O at\% shall be less than } 4 \% \text {. Dopant Ge fraction shall be as specified in Table } \\
\text { "Ablator layer requirements for } \mathrm{CH} \text { Capsule - Rev } 0 . " \text { Atomic fraction } \mathrm{x} \text { of Ge reduces } \mathrm{C} \\
\text { atomic fraction by } \mathrm{x} \text {, leaving } \mathrm{H} \text { and O fractions unchanged. }\end{array}$ \\
\hline $\mathrm{CH} 3$ & 0 & I.4.1.1.3 & CH Capsule & $\begin{array}{l}\text { Ablator total thickness - range of possible } \\
\text { requested values }\end{array}$ & $140-160 \mu \mathrm{m}$ & $\begin{array}{l}\text { Capsule ablator shall be producable with total ablator thickness within the range } 140-160 \mu \mathrm{m} \text {. } \\
\text { The ablator thickness for each capsule will be specified within that range. Current design } 146 \\
\mu \mathrm{m} \text {. }\end{array}$ \\
\hline $\mathrm{CH} 4$ & 0 & 1.4 .1 .1 .3 & CH Capsule & Ablator Dopant & Ge & The dopant material for all doped layers shall be Ge \\
\hline $\mathrm{CH} 5$ & 0 & 1.4.1.1.3 & CH Capsule & Ablator layer thicknesses & see table & $\begin{array}{l}\text { The thickness of the layers within the ablator shall be as listed in Table "Ablator layer } \\
\text { requirements for } \mathrm{CH} \text { Capsule - Rev 0" }\end{array}$ \\
\hline $\mathrm{CH} 6$ & 0 & I.4.1.1.3 & CH Capsule & Ablator layer dopant concentration & see table & $\begin{array}{l}\text { The dopant concentration in each of the layers within the ablator shall be as listed in Table } \\
\text { "Ablator layer requirements for } \mathrm{CH} \text { Capsule - Rev 0" }\end{array}$ \\
\hline $\mathrm{CH} 7$ & 0 & I.4.1.1.3 & CH Capsule & Ablator layer density & see table & $\begin{array}{l}\text { The density of each of the layers within the ablator shall be as listed in Table "Ablator layer } \\
\text { requirements for CH Capsule - Rev 0" }\end{array}$ \\
\hline $\mathrm{CH} 8$ & 0 & $\mid 1.4 .1 .1 .3$ & CH Capsule & Ablator inner surface figure & see table & $\begin{array}{l}\text { The power spectrum of the deviation of the inner surface of the Be ablator from a perfect } \\
\text { sphere of the same average radius shall not exceed the spectrum in Table "Surface } \\
\text { roughness and thickness power spectra for layered } \mathrm{CH} \text { capsule }- \text { Rev } 0 " \text { ". "Shall not exceed" } \\
\text { means that the 2D power in modes } 1-12 \text { shall be less than that tabulated, and the } 1 \mathrm{D} \text { power } \\
\text { in a trace, summed over any }+/-15 \% \text { bandwidth above mode } 12 \text { shall be less than that of the } \\
\text { same bandwidth for the reference power spectra. }\end{array}$ \\
\hline $\mathrm{CH} 9$ & 0 & I.4.1.1.3 & CH Capsule & Ablator thickness non-uniformity & see table & $\begin{array}{l}\text { The power spectra of the non-uniformity of the cumulative thickness of each layer shall not } \\
\text { exceed the spectrum in Table "Surface roughness and thickness power spectra for layered } \\
\mathrm{CH} \text { capsule - Rev 0". "Shall not exceed" means that the } 2 \mathrm{D} \text { power in modes } 1-12 \text { shall be } \\
\text { less than that tabulated, and the } 1 \mathrm{D} \text { power in a trace, summed over any }+/-15 \% \text { bandwidth } \\
\text { above mode } 12 \text { shall be less than that of the same bandwidth for the reference power } \\
\text { spectra. }\end{array}$ \\
\hline & & & & & & \\
\hline & & & & & & \\
\hline
\end{tabular}


Ablator layer requirements for $\mathrm{CH}$ Capsule - Rev 0

\begin{tabular}{|c|c|c|c|c|c|}
\hline Layer no & \begin{tabular}{|c|}
$\begin{array}{c}\text { Point Design } \\
\text { Inner Radius, } \mu \mathrm{m} \\
\text { (ref) }\end{array}$ \\
\end{tabular} & \begin{tabular}{|c} 
Point Design Outer \\
Radius, $\mu \mathrm{m}$ (ref)
\end{tabular} & $\begin{array}{c}\text { Required Thickness, } \\
\mu \mathrm{m}\end{array}$ & $\begin{array}{c}\text { Required Dopant } \\
\text { Concentration, at \% }\end{array}$ & $\begin{array}{c}\text { Required } \\
\text { Density, g/cc } \\
\text { (note 1) } \\
\end{array}$ \\
\hline Total, all layers & 854 & 1000 & $146+/-3$ & $n / a$ & $n / a$ \\
\hline 4 & 916 & 1000 & $\begin{array}{l}\text { as required (nominally } \\
84 \text { ) }\end{array}$ & $\begin{array}{l}\text { outer } 70 \mu \mathrm{m}<0.05 \\
\text { outer } 80 \mu \mathrm{m}<0.10\end{array}$ & $1.09+/-3 \%$ \\
\hline 3 & 906 & 916 & $10+/-2.5$ & $0.4+/-0.1$ & $1.13+/-3 \%$ \\
\hline 2 & 864 & 906 & $42+/-3$ & $0.8+/-0.1$ & $1.18+/-3 \%$ \\
\hline 1 & 854 & 864 & $10+/-1.5$ & inner $3 \mu \mathrm{m},<0.05$ & $1.09+/-3 \%$ \\
\hline Notes & $\begin{array}{r}1 . \text { Note that Cap } \\
\text { by anoth }\end{array}$ & $\begin{array}{l}\text { osule-to-capsule den } \\
\text { her requirement to }\end{array}$ & $\begin{array}{l}\text { sity variations are lim } \\
+/-1.5 \%\end{array}$ & ited & \\
\hline
\end{tabular}


Surface roughness and thickness power spectra for layered $\mathrm{CH}$ capsule - Rev 0

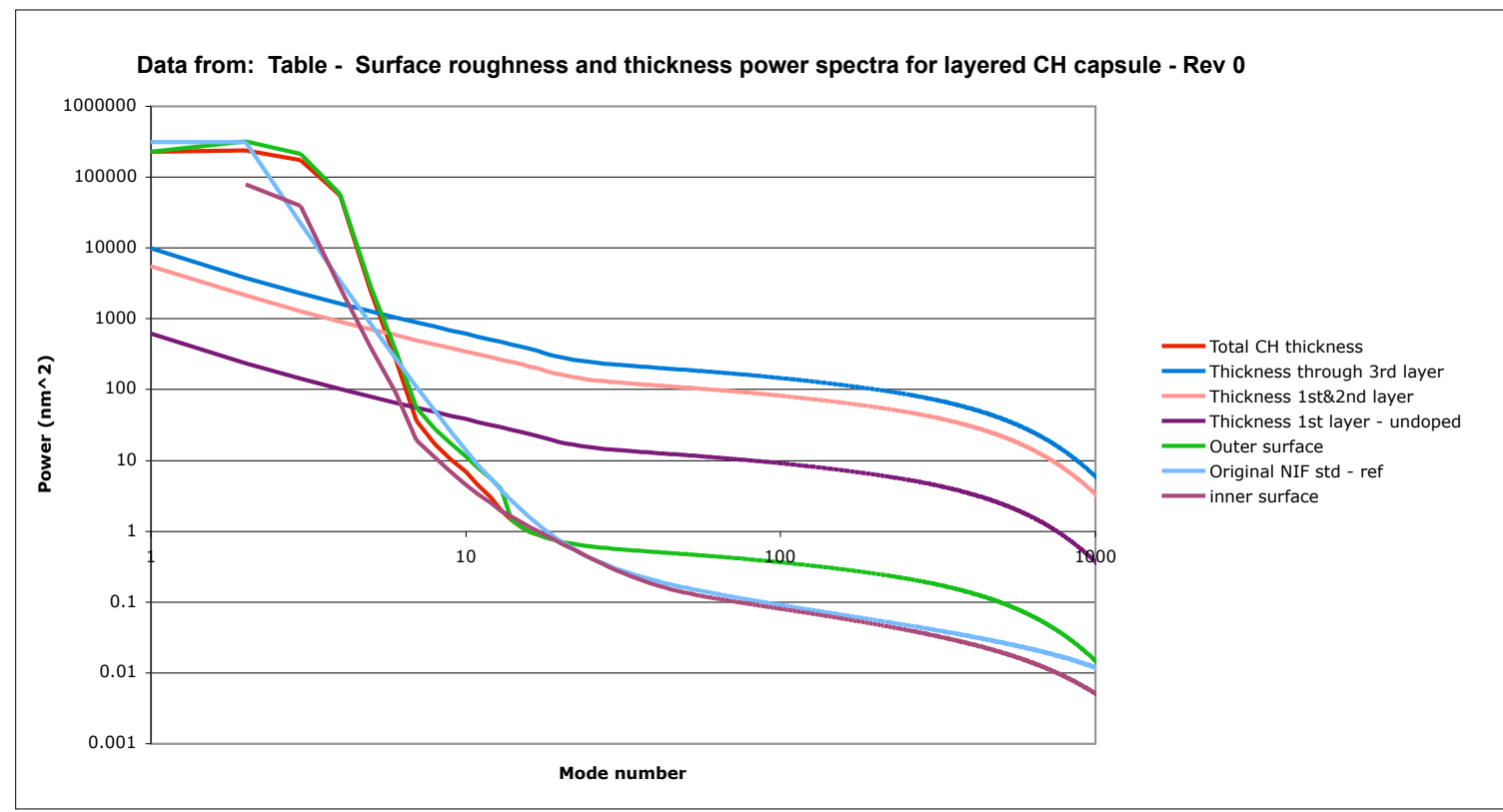

Notes:

1. First table is $2 \mathrm{D}$ power, in $n m^{\wedge} 2$, applies to modes $1-12$

2. Second table is the 1D power for each surface in a trace, in $\mathrm{nm}^{\wedge} 2$. Applies to modes $>12$, included for low modes for information only.

3. Inner surface roughness is defined relative to an ideal sphere of the same average radius.

Other surfaces are all defined relative to inner surfaces, i.e.they are actually the variation in the thickness

4. Coding that generates trace powers is in Haan's file surfacesCH093005.i

5. Outer surface relative to inner surface. Note does not apply at modes 20 and above.

6. At low modes, assumes inner surface and thickness are randomly phased.

At high modes, assumes inner \& outer surfaces randomly phased.

7. Second dopant surface, relative to inside of $\mathrm{CH}$ shell

8. Third dopant surface, relative to inside of $\mathrm{Ch}$ shell

\begin{tabular}{|c|c|c|c|c|c|c|c|}
\hline $\begin{array}{c}\text { Mode } \\
\text { number }\end{array}$ & $\begin{array}{l}\text { Original NIF } \\
\text { standard, for } \\
\text { reference, } \mathrm{nm}^{\wedge} \mathbf{2}\end{array}$ & $\begin{array}{l}\text { Inner surface } \\
\text { (radius), } \text { nm^2 }^{-}\end{array}$ & $\begin{array}{c}\text { Total shell } \\
\text { thickness, nm^^2 } \\
\text { (note 5) }\end{array}$ & $\begin{array}{l}\text { Outer surface, } \\
\mathrm{nm}^{\wedge} 2 \text { (note 6) }\end{array}$ & $\begin{array}{l}\text { Thickness of first } \\
\text { doped layer, } \mathrm{nm}^{\wedge} 2\end{array}$ & $\begin{array}{c}\text { Thickness of } \\
\text { 1st+2nd doped } \\
\text { layer, nm^2 (note } 7)\end{array}$ & $\begin{array}{c}\text { Thickness of } \\
\text { 1st }+2 \mathrm{nd}+3 \mathrm{rd} \text { doped } \\
\text { layer, nm^2 (note } 8)\end{array}$ \\
\hline \multicolumn{8}{|l|}{ 2D power } \\
\hline 1 & not applicable & & 122500 & 122500 & 505.509 & 4549.58 & 8088.14 \\
\hline 2 & & 101667 & 275625 & 377292 & 210.629 & 1895.66 & 3370.06 \\
\hline 3 & & 61529.2 & 275625 & 337154.2 & 131.058 & 1179.52 & 2096.93 \\
\hline 4 & & 4911.74 & 99225 & 104136.74 & 94.7829 & 853.046 & 1516.53 \\
\hline 5 & & 778.152 & 4900 & 5678.152 & 74.1413 & 667.272 & 1186.26 \\
\hline 6 & & 185.31 & 566.44 & 751.75 & 60.8483 & 547.634 & 973.572 \\
\hline 7 & & 35.5897 & 70 & 105.5897 & 51.5825 & 464.243 & 825.32 \\
\hline 8 & & 19.7554 & 31.36 & 51.1154 & 44.7586 & 402.827 & 716.137 \\
\hline 9 & & 12.4171 & 20 & 32.4171 & 39.5254 & 355.728 & 632.406 \\
\hline 10 & & 7.98983 & 14 & 21.98983 & 35.3856 & 318.471 & 566.17 \\
\hline 11 & & 5.80356 & 9 & 14.80356 & 32.0295 & 288.265 & 512.472 \\
\hline 12 & & 4.34232 & 6 & 10.34232 & 29.254 & 263.286 & 468.064 \\
\hline \multicolumn{8}{|l|}{$\begin{array}{l}\text { 1D trace } \\
\text { power }\end{array}$} \\
\hline 1 & $3.13 E+05$ & & 227025 & $2.27 \mathrm{E}+05$ & 609.848 & 5488.63 & 9757.56 \\
\hline 2 & 313000 & 77828.8 & 237851 & 315679.8 & 233.075 & 2097.68 & 3729.21 \\
\hline 3 & 22000 & 38678.5 & 173623 & 212301.5 & 141.214 & 1270.93 & 2259.43 \\
\hline 4 & 3440 & 2737.4 & 54411.7 & 57149.1 & 101.065 & 909.581 & 1617.03 \\
\hline 5 & 840 & 394.719 & 2432.86 & 2827.579 & 78.846 & 709.614 & 1261.54 \\
\hline 6 & 272 & 90.2859 & 265.775 & 356.0609 & 64.6481 & 581.833 & 1034.37 \\
\hline 7 & 107 & 19.2375 & 35.6521 & 54.8896 & 54.9403 & 494.463 & 879.045 \\
\hline 8 & 48.8 & 10.7192 & 16.6239 & 27.3431 & 47.7266 & 429.54 & 763.626 \\
\hline 9 & 24.9 & 6.79828 & 10.2291 & 17.02738 & 42.3206 & 380.886 & 677.13 \\
\hline 10 & 14 & 4.52319 & 6.91169 & 11.43488 & 37.9325 & 341.392 & 606.92 \\
\hline 11 & 8.48 & 3.29369 & 4.41024 & 7.70393 & 34.4913 & 310.421 & 551.86 \\
\hline 12 & 5.48 & 2.51608 & 3.00411 & 5.52019 & 31.5047 & 283.542 & 504.075 \\
\hline 13 & 3.74 & 1.92477 & 1.96865 & 3.89342 & 29.115 & 262.035 & 465.841 \\
\hline 14 & 2.68 & 1.57638 & 1.42527 & 1.42527 & 26.9023 & 242.121 & 430.437 \\
\hline 15 & 2 & 1.32717 & 1.1256 & 1.1256 & 25.1294 & 226.165 & 402.071 \\
\hline 16 & 1.55 & 1.14487 & 0.97817 & 0.97817 & 23.3403 & 210.062 & 373.444 \\
\hline 17 & 1.24 & 0.995459 & 0.87737 & 0.87737 & 21.9342 & 197.408 & 350.948 \\
\hline 18 & 1.01 & 0.871942 & 0.805799 & 0.805799 & 20.145 & 181.305 & 322.32 \\
\hline 19 & 0.851 & 0.769054 & 0.753231 & 0.753231 & 18.8308 & 169.477 & 301.292 \\
\hline 20 & 0.729 & 0.682765 & & 0.71343 & 17.8357 & 160.522 & 285.372 \\
\hline 21 & 0.635 & 0.609953 & & 0.68246 & 17.0615 & 153.553 & 272.984 \\
\hline 22 & 0.561 & 0.548169 & & 0.657763 & 16.4441 & 147.997 & 263.105 \\
\hline 23 & 0.503 & 0.495473 & & 0.637628 & 15.9407 & 143.466 & 255.051 \\
\hline 24 & 0.455 & 0.450315 & & 0.620881 & 15.522 & 139.698 & 248.352 \\
\hline 25 & 0.416 & 0.411445 & & 0.6067 & 15.1675 & 136.508 & 242.68 \\
\hline 26 & 0.384 & 0.377848 & & 0.594496 & 14.8624 & 133.762 & 237.798 \\
\hline 27 & 0.357 & 0.348694 & & Page 10.583837 & 14.5959 & 131.363 & 233.535 \\
\hline
\end{tabular}


Table_of_specs_Rev0.xls,CH Surface roughness

\begin{tabular}{|c|c|c|}
\hline 28 & 0.333 & 0.323299 \\
\hline 29 & 0.313 & 0.301098 \\
\hline 30 & 0.296 & 0.281623 \\
\hline 31 & 0.281 & 0.26448 \\
\hline 32 & 0.268 & 0.249339 \\
\hline 33 & 0.256 & 0.235925 \\
\hline 34 & 0.245 & 0.224001 \\
\hline 35 & 0.236 & 0.213368 \\
\hline 36 & 0.227 & 0.203857 \\
\hline 37 & 0.219 & 0.195324 \\
\hline 38 & 0.212 & 0.187643 \\
\hline 39 & 0.205 & 0.180709 \\
\hline 40 & 0.199 & 0.17443 \\
\hline 41 & 0.194 & 0.168726 \\
\hline 42 & 0.189 & 0.16353 \\
\hline 43 & 0.184 & 0.158782 \\
\hline 44 & 0.179 & 0.15443 \\
\hline 45 & 0.175 & 0.15043 \\
\hline 46 & 0.171 & 0.146742 \\
\hline 47 & 0.168 & 0.143332 \\
\hline 48 & 0.164 & 0.14017 \\
\hline 49 & 0.161 & 0.137231 \\
\hline 50 & 0.158 & 0.13449 \\
\hline 51 & 0.155 & 0.131928 \\
\hline 52 & 0.152 & 0.129526 \\
\hline 53 & 0.15 & 0.12727 \\
\hline 54 & 0.147 & 0.125145 \\
\hline 55 & 0.145 & 0.123138 \\
\hline 56 & 0.142 & 0.121239 \\
\hline 57 & 0.14 & 0.119438 \\
\hline 58 & 0.138 & 0.117726 \\
\hline 59 & 0.136 & 0.116096 \\
\hline 60 & 0.134 & 0.11454 \\
\hline 61 & 0.132 & 0.113053 \\
\hline 62 & 0.13 & 0.111629 \\
\hline 63 & 0.129 & 0.110263 \\
\hline 64 & 0.127 & 0.10895 \\
\hline 65 & 0.125 & 0.107687 \\
\hline 66 & 0.124 & 0.10647 \\
\hline 67 & 0.122 & 0.105296 \\
\hline 68 & 0.121 & 0.104162 \\
\hline 69 & 0.12 & 0.103064 \\
\hline 70 & 0.118 & 0.102002 \\
\hline 71 & 0.117 & 0.100971 \\
\hline 72 & 0.116 & 0.0999717 \\
\hline 73 & 0.114 & 0.0990008 \\
\hline 74 & 0.113 & 0.0980568 \\
\hline 75 & 0.112 & 0.0971383 \\
\hline 76 & 0.111 & 0.096244 \\
\hline 77 & 0.11 & 0.0953724 \\
\hline 78 & 0.109 & 0.0945225 \\
\hline 79 & 0.108 & 0.0936932 \\
\hline 80 & 0.107 & 0.0928834 \\
\hline 81 & 0.106 & 0.0920922 \\
\hline 82 & 0.105 & 0.0913188 \\
\hline 83 & 0.104 & 0.0905624 \\
\hline 84 & 0.103 & 0.0898222 \\
\hline 85 & 0.102 & 0.0890975 \\
\hline 86 & 0.101 & 0.0883878 \\
\hline 87 & 0.1 & 0.0876923 \\
\hline 88 & 0.0992 & 0.0870106 \\
\hline 89 & 0.0984 & 0.0863421 \\
\hline 90 & 0.0976 & 0.0856863 \\
\hline 91 & 0.0968 & 0.0850428 \\
\hline 92 & 0.096 & 0.0844111 \\
\hline 93 & 0.0953 & 0.0837908 \\
\hline 94 & 0.0945 & 0.0831815 \\
\hline 95 & 0.0938 & 0.0825829 \\
\hline 96 & 0.0931 & 0.0819946 \\
\hline 97 & 0.0924 & 0.0814163 \\
\hline 98 & 0.0917 & 0.0808476 \\
\hline 99 & 0.091 & 0.0802883 \\
\hline 100 & 0.0904 & 0.079738 \\
\hline 101 & 0.0897 & 0.0791966 \\
\hline 102 & 0.0891 & 0.0786638 \\
\hline 103 & 0.0885 & 0.0781392 \\
\hline 104 & 0.0879 & 0.0776228 \\
\hline 105 & 0.0873 & 0.0771142 \\
\hline 106 & 0.0867 & 0.0766133 \\
\hline 107 & 0.0861 & 0.0761197 \\
\hline 108 & 0.0855 & 0.0756335 \\
\hline 109 & 0.085 & 0.0751543 \\
\hline 110 & 0.0844 & 0.0746819 \\
\hline 111 & 0.0839 & 0.0742163 \\
\hline 112 & 0.0833 & 0.0737572 \\
\hline 113 & 0.0828 & 0.0733044 \\
\hline 114 & 0.0823 & 0.0728579 \\
\hline 115 & 0.0818 & 0.0724175 \\
\hline 116 & 0.0813 & 0.071983 \\
\hline 117 & 0.0808 & 0.0715542 \\
\hline 118 & 0.0803 & 0.0711311 \\
\hline 119 & 0.0798 & 0.0707136 \\
\hline 120 & 0.0793 & 0.0703014 \\
\hline 121 & 0.0789 & 0.0698945 \\
\hline 122 & 0.0784 & 0.0694928 \\
\hline
\end{tabular}

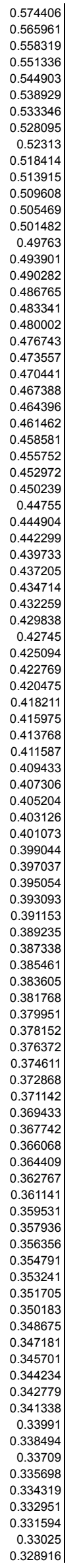

14.3602

14.149

13.958

13.7834
13.6226

13.6226
13.4732
13.3336

13.3336

13.2024

13.0783

12.9604
12.8479

12.7402

12.6367

12.5371

12.4408

12.3475

12.2571
12.1691

12.0835

12.0001

11.9186

11.8389

11.761
11.6847

11.6847
11.6099

11.5365

11.4645

11.3938

11.3243

11.256

11.1887

11.1226

10.9933

10.9301

10.8679
10.8065

10.7459

10.6862

10.6273

10.5692

10.5119

10.4553

10.3994
10.3442

10.2897

10.2358

10.1826

10.1301

10.0782

10.0268

9.92593

9.82731

9.77883

9.73088

9.68345

9.5442
9.49877

9.45381

9.40931

9.36528

9.32169

9.27855

9.23584

9.19355

9.15169

9.11023

9.06919

9.02854

8.98828

8.9484
8.9089

8.9089
8.86978

8.83102

8.79262

8.75458

8.71689

8.67954

8.64252

8.60584

8.53346
8.49774

8.46234

8.42725

8.39246

8.35797

8.28986

8.25624

8.2229
Printed 10/2/0

\begin{tabular}{l|l|}
129.241 & 229.762 \\
127.341 & 226.384 \\
125.622 & 223.328 \\
124.051 & 220.535
\end{tabular}

$124.051 \quad 220.535$

122.603
121.259

$\begin{array}{ll}121.259 & 215.572 \\ 120.003 & 213.338 \\ 118.821\end{array}$

$118.821 \quad 211.238$

$117.704 \quad 209.252$
116.643

$116.643 \quad 207.366$

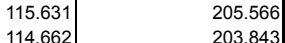

$113.731 \quad 202.188$

112.833

$111.967 \quad 199.052$

111.128

110.314

109.522
108.752 $\quad \begin{aligned} & 194.706 \\ & 108.001\end{aligned}$

$108.001 \quad 192.001$

$107.267 \quad 190.697$

106.55
189.423

$105.849 \quad 188.176$

\begin{tabular}{l|l}
105.162 & 186.955 \\
104.489 & 185.759
\end{tabular}

$103.829 \quad 184.585$

$103.181 \quad 183.433$

102.544

\begin{tabular}{l|l}
101.919 & 181.189 \\
101.304 & 180.096 \\
100.699 & 179.02
\end{tabular}

$100.699 \quad 179.02$

100.103
99.5172

98172

98.3712

\begin{tabular}{r|r|}
97.8107 & 173.886 \\
97.2582 & 172.904
\end{tabular}

$97.2582 \quad 172.904$

$96.7135 \quad 171.935$

96.1762

95.6461

\begin{tabular}{r|r|}
95.1231 & 169.108 \\
94.607 & 168.19 \\
94.0975
\end{tabular}

\begin{tabular}{r|r}
94.607 & 168.19 \\
94.0975 & 167.284 \\
93.594 & 166.39
\end{tabular}

$93.5944 \quad 166.39$

$93.0977 \quad 165.507$

$92.6071 \quad 164.635$

92.1225

91.6438
91.1708

\begin{tabular}{r|r}
91.1708 & 162.081 \\
90.7034 & 161.25 \\
90.2414
\end{tabular}

$90.2414 \quad 160.429$

$89.7848 \quad 159.617$

$89.3334 \quad 158.815$

$88.8871 \quad 158.022$

88.4458
88.0095

$\begin{array}{ll}88.0095 & 156.461 \\ 87.5779 & 155.694 \\ 87.151\end{array}$

$87.151 \quad 154.935$

$86.7288 \quad 154.185$

$86.3111 \quad 153.442$

$85.8978 \quad 152.707$

$85.4889 \quad 151.98$

\begin{tabular}{l|l}
85.0842 & 151.261 \\
84.6838 & 150.549 \\
84.2875
\end{tabular}

$84.2875 \quad 149.844$

$83.8952 \quad 149.147$

83.5069

\begin{tabular}{r|r|}
83.1225 & 147.773 \\
\hline & 147.097
\end{tabular}

$82.742 \quad 147.097$

82.3652

81.9921
81.6227

81.6227
81.2568
80.8945

81.2568
80.8945

$80.5356 \quad 143.174$

$80.1801 \quad 142.542$

79.828

\begin{tabular}{r|r|}
79.4792 & 141.296 \\
79.1336
\end{tabular}

78.7912

78.452

\begin{tabular}{r|r}
78.1158 & 138.473 \\
77.7827 & 138.28 \\
\hline
\end{tabular}

$77.7827 \quad 138.28$

$77.4526 \quad 137.693$

$77.1254 \quad 137.112$

$76.8011 \quad 136.535$

$76.4797 \quad 135.964$

$76.1611 \quad 135.397$

$75.8452 \quad 134.836$

\begin{tabular}{l|l}
75.5321 & 134.279 \\
75.2217 & 133.727 \\
74.9139
\end{tabular}

$74.9139+\quad 133.18$

$74.6088 \quad 132.638$

74.3062

74.0061

Page 2 of 33 
Table_of_specs_Rev0.xls,CH Surface roughness

\begin{tabular}{|c|c|c|}
\hline 123 & 0.078 & 0.0690961 \\
\hline 124 & 0.0775 & 0.0687044 \\
\hline 125 & 0.0771 & 0.0683175 \\
\hline 126 & 0.0766 & 0.0679353 \\
\hline 127 & 0.0762 & 0.0675578 \\
\hline 128 & 0.0758 & 0.0671847 \\
\hline 129 & 0.0754 & 0.0668162 \\
\hline 130 & 0.075 & 0.0664519 \\
\hline 131 & 0.0746 & 0.066092 \\
\hline 132 & 0.0742 & 0.0657362 \\
\hline 133 & 0.0738 & 0.0653845 \\
\hline 134 & 0.0734 & 0.0650368 \\
\hline 135 & 0.073 & 0.064693 \\
\hline 136 & 0.0726 & 0.0643531 \\
\hline 137 & 0.0723 & 0.064017 \\
\hline 138 & 0.0719 & 0.0636846 \\
\hline 139 & 0.0715 & 0.0633558 \\
\hline 140 & 0.0712 & 0.0630306 \\
\hline 141 & 0.0708 & 0.0627089 \\
\hline 142 & 0.0705 & 0.0623906 \\
\hline 143 & 0.0701 & 0.0620758 \\
\hline 144 & 0.0698 & 0.0617642 \\
\hline 145 & 0.0694 & 0.0614559 \\
\hline 146 & 0.0691 & 0.0611508 \\
\hline 147 & 0.0688 & 0.0608488 \\
\hline 148 & 0.0684 & 0.0605499 \\
\hline 149 & 0.0681 & 0.060254 \\
\hline 150 & 0.0678 & 0.0599611 \\
\hline 151 & 0.0675 & 0.0596712 \\
\hline 152 & 0.0672 & 0.0593841 \\
\hline 153 & 0.0668 & 0.0590999 \\
\hline 154 & 0.0665 & 0.0588184 \\
\hline 155 & 0.0662 & 0.0585396 \\
\hline 156 & 0.0659 & 0.0582636 \\
\hline 157 & 0.0656 & 0.0579902 \\
\hline 158 & 0.0653 & 0.0577194 \\
\hline 159 & 0.0651 & 0.0574511 \\
\hline 160 & 0.0648 & 0.0571853 \\
\hline 161 & 0.0645 & 0.0569221 \\
\hline 162 & 0.0642 & 0.0566612 \\
\hline 163 & 0.0639 & 0.0564028 \\
\hline 164 & 0.0636 & 0.0561467 \\
\hline 165 & 0.0634 & 0.055893 \\
\hline 166 & 0.0631 & 0.0556415 \\
\hline 167 & 0.0628 & 0.0553923 \\
\hline 168 & 0.0626 & 0.0551453 \\
\hline 169 & 0.0623 & 0.0549004 \\
\hline 170 & 0.0621 & 0.0546577 \\
\hline 171 & 0.0618 & 0.0544172 \\
\hline 172 & 0.0615 & 0.0541787 \\
\hline 173 & 0.0613 & 0.0539422 \\
\hline 174 & 0.061 & 0.0537078 \\
\hline 175 & 0.0608 & 0.0534754 \\
\hline 176 & 0.0605 & 0.053245 \\
\hline 177 & 0.0603 & 0.0530164 \\
\hline 178 & 0.0601 & 0.0527898 \\
\hline 179 & 0.0598 & 0.0525651 \\
\hline 180 & 0.0596 & 0.0523422 \\
\hline 181 & 0.0594 & 0.0521211 \\
\hline 182 & 0.0591 & 0.0519018 \\
\hline 183 & 0.0589 & 0.0516844 \\
\hline 184 & 0.0587 & 0.0514686 \\
\hline 185 & 0.0584 & 0.0512546 \\
\hline 186 & 0.0582 & 0.0510423 \\
\hline 187 & 0.058 & 0.0508316 \\
\hline 188 & 0.0578 & 0.0506226 \\
\hline 189 & 0.0576 & 0.0504153 \\
\hline 190 & 0.0573 & 0.0502095 \\
\hline 191 & 0.0571 & 0.0500054 \\
\hline 192 & 0.0569 & 0.0498028 \\
\hline 193 & 0.0567 & 0.0496017 \\
\hline 194 & 0.0565 & 0.0494022 \\
\hline 195 & 0.0563 & 0.0492042 \\
\hline 196 & 0.0561 & 0.0490077 \\
\hline 197 & 0.0559 & 0.0488126 \\
\hline 198 & 0.0557 & 0.048619 \\
\hline 199 & 0.0555 & 0.0484268 \\
\hline 200 & 0.0553 & 0.048236 \\
\hline 201 & 0.0551 & 0.0480466 \\
\hline 202 & 0.0549 & 0.0478586 \\
\hline 203 & 0.0547 & 0.047672 \\
\hline 204 & 0.0545 & 0.0474867 \\
\hline 205 & 0.0543 & 0.0473027 \\
\hline 206 & 0.0541 & 0.04712 \\
\hline 207 & 0.0539 & 0.0469386 \\
\hline 208 & 0.0537 & 0.0467585 \\
\hline 209 & 0.0536 & 0.0465796 \\
\hline 210 & 0.0534 & 0.046402 \\
\hline 211 & 0.0532 & 0.0462256 \\
\hline 212 & 0.053 & 0.0460504 \\
\hline 213 & 0.0528 & 0.0458765 \\
\hline 214 & 0.0527 & 0.0457037 \\
\hline 215 & 0.0525 & 0.045532 \\
\hline 216 & 0.0523 & 0.0453616 \\
\hline 217 & 0.0521 & 0.0451923 \\
\hline
\end{tabular}

\begin{tabular}{|c|c|}
\hline 0.327593 & 8.18984 \\
\hline 0.326282 & 8.15704 \\
\hline 0.324981 & 8.12452 \\
\hline 0.323691 & 8.09227 \\
\hline 0.322411 & 8.06027 \\
\hline 0.321141 & 8.02853 \\
\hline 0.319882 & 7.99705 \\
\hline 0.318633 & 7.96582 \\
\hline 0.317393 & 7.93483 \\
\hline 0.316163 & 7.90409 \\
\hline 0.314943 & 7.87358 \\
\hline 0.313733 & 7.84331 \\
\hline 0.312531 & 7.81328 \\
\hline 0.311339 & 7.78348 \\
\hline 0.310156 & 7.7539 \\
\hline 0.308982 & 7.72454 \\
\hline 0.307816 & 7.69541 \\
\hline 0.30666 & 7.66649 \\
\hline 0.305512 & 7.63779 \\
\hline 0.304372 & 7.6093 \\
\hline 0.30324 & 7.58101 \\
\hline 0.302117 & 7.55293 \\
\hline 0.301002 & 7.52506 \\
\hline 0.299895 & 7.49738 \\
\hline 0.298796 & 7.46991 \\
\hline 0.297705 & 7.44262 \\
\hline 0.296621 & 7.41553 \\
\hline 0.295545 & 7.38863 \\
\hline 0.294477 & 7.36191 \\
\hline 0.293415 & 7.33538 \\
\hline 0.292361 & 7.30903 \\
\hline 0.291315 & 7.28286 \\
\hline 0.290275 & 7.25687 \\
\hline 0.289242 & 7.23105 \\
\hline 0.288216 & 7.20541 \\
\hline 0.287197 & 7.17993 \\
\hline 0.286185 & 7.15462 \\
\hline 0.285179 & 7.12948 \\
\hline 0.28418 & 7.1045 \\
\hline 0.283187 & 7.07968 \\
\hline 0.282201 & 7.05502 \\
\hline 0.281221 & 7.03051 \\
\hline 0.280247 & 7.00617 \\
\hline 0.279279 & 6.98197 \\
\hline 0.278317 & 6.95793 \\
\hline 0.277361 & 6.93403 \\
\hline 0.276411 & 6.91028 \\
\hline 0.275467 & 6.88668 \\
\hline 0.274529 & 6.86321 \\
\hline 0.273596 & 6.83989 \\
\hline 0.272668 & 6.81671 \\
\hline 0.271747 & 6.79367 \\
\hline 0.27083 & 6.77076 \\
\hline 0.269919 & 6.74799 \\
\hline 0.269014 & 6.72534 \\
\hline 0.268113 & 6.70283 \\
\hline 0.267218 & 6.68045 \\
\hline 0.266328 & 6.65819 \\
\hline 0.265442 & 6.63606 \\
\hline 0.264562 & 6.61406 \\
\hline 0.263687 & 6.59217 \\
\hline 0.262816 & 6.57041 \\
\hline 0.26195 & 6.54876 \\
\hline 0.261089 & 6.52723 \\
\hline 0.260233 & 6.50582 \\
\hline 0.259381 & 6.48453 \\
\hline 0.258534 & 6.46334 \\
\hline 0.257691 & 6.44227 \\
\hline 0.256852 & 6.42131 \\
\hline 0.256018 & 6.40045 \\
\hline 0.255188 & 6.37971 \\
\hline 0.254363 & 6.35907 \\
\hline 0.253541 & 6.33853 \\
\hline 0.252724 & 6.3181 \\
\hline 0.251911 & 6.29777 \\
\hline 0.251102 & 6.27754 \\
\hline 0.250296 & 6.25741 \\
\hline 0.249495 & 6.23738 \\
\hline 0.248698 & 6.21744 \\
\hline 0.247904 & 6.19761 \\
\hline 0.247114 & 6.17786 \\
\hline 0.246328 & 6.15821 \\
\hline 0.245546 & 6.13865 \\
\hline 0.244767 & 6.11919 \\
\hline 0.243992 & 6.09981 \\
\hline 0.243221 & 6.08052 \\
\hline 0.242453 & 6.06132 \\
\hline 0.241688 & 6.04221 \\
\hline 0.240927 & 6.02318 \\
\hline 0.240169 & 6.00424 \\
\hline 0.239415 & 5.98538 \\
\hline 0.238664 & 5.9666 \\
\hline 0.237916 & 5.9479 \\
\hline 0.237172 & 5.92929 \\
\hline & \\
\hline
\end{tabular}

73.7085

73.4134

73.1207

72.8304
72.5424

72.2568

71.9735

71.6924

71.4135

71.1368
70.8622

70.5898

70.3195

70.0513

69.7851

69.5209

69.2587
68.9984
68.7401

68.7401

68.4837

68.2291

67.9764

67.725

67.4765

66.9836

66.7398

66.4977

66.2572

66.018

65.7813

65.5458

65.0795

64.8486

64.6194
64.3916

64.1653

63.9405

63.7171

63.4952

63.2746

63.0555

62.8377

62.4063

62.1925

61.9801

61.9801

61.7689
61.559

61.3504

61.143

60.9368

60.528

60.3255
60.124

60.124
59.9237
59.7246

59.7246

59.5265

59.5265

59.3295

59.1337
58.9389

58.9389
58.7451

58.5524

58.3607

58.1701

57.9804

57.9804
57.7918

57.7918
57.6041

57.4174

57.2316

57.0468

56.8629

56.6799

56.4979

56.3167

56.1364
55.957

55.957
55.7785

55.6008

55.4239

55.2479

55.0727

54.8983

54.7247

54.5519

54.3799

54.2086

54.0381

53.8684

53.5311

(53.5311

53.3636
53.1968
Printed 10/2/05,4.24 PM

131.037

129.513

129.476

128.964

28.457

127.453

126.957

126.465

125.977

125.012

124.536

124.062

123.593

123.127

122.205

121.749

121.296

120.847

119.958

119.519

119.082

118.218

117.791

117.366

116.945

116.526

116.11

115.697
115.286

114.879

114.474

114.072

113.672

11288

112.488

112.099

111.712

111.327
110.944

110.564

110.187

109.811

109.438

109.067

108.699

107.968

107.968

107.605

107.245
106.887

106.531

06.177

105.825
105.475

105.126

104.78

104.436

104.093

103.752

103.413

103.076

102.741

102.407

102.075

01.745

101.09

100.764

100.441

100.119

99.7981

99.1617

98.8458

98.5314

98.2185

97.907

97.2883

96.9811

96.6753

96.3709

96.0678

95.766

95.1665

95.1665

94.8686
94.5721

Page 3 of 33 
Table_of_specs_Rev0.xls,CH Surface roughness

\begin{tabular}{|c|c|}
\hline 0.052 & 0.0450241 \\
\hline 0.0518 & 0.044857 \\
\hline 0.0516 & 0.044691 \\
\hline 0.0514 & 0.0445262 \\
\hline 0.0513 & 0.0443624 \\
\hline 0.0511 & 0.0441996 \\
\hline 0.0509 & 0.044038 \\
\hline 0.0508 & 0.0438773 \\
\hline 0.0506 & 0.0437177 \\
\hline 0.0504 & 0.0435591 \\
\hline 0.0503 & 0.0434015 \\
\hline 0.0501 & 0.043245 \\
\hline 0.05 & 0.0430894 \\
\hline 0.0498 & 0.0429347 \\
\hline 0.0497 & 0.0427811 \\
\hline 0.0495 & 0.0426284 \\
\hline 0.0493 & 0.0424766 \\
\hline 0.0492 & 0.0423258 \\
\hline 0.049 & 0.0421759 \\
\hline 0.0489 & 0.0420269 \\
\hline 0.0487 & 0.0418788 \\
\hline 0.0486 & 0.0417316 \\
\hline 0.0484 & 0.0415853 \\
\hline 0.0483 & 0.0414398 \\
\hline 0.0481 & 0.0412952 \\
\hline 0.048 & 0.0411515 \\
\hline 0.0479 & 0.0410086 \\
\hline 0.0477 & 0.0408666 \\
\hline 0.0476 & 0.0407254 \\
\hline 0.0474 & 0.040585 \\
\hline 0.0473 & 0.0404454 \\
\hline 0.0471 & 0.0403067 \\
\hline 0.047 & 0.0401687 \\
\hline 0.0469 & 0.0400315 \\
\hline 0.0467 & 0.0398951 \\
\hline 0.0466 & 0.0397595 \\
\hline 0.0465 & 0.0396246 \\
\hline 0.0463 & 0.0394905 \\
\hline 0.0462 & 0.0393572 \\
\hline 0.046 & 0.0392246 \\
\hline 0.0459 & 0.0390927 \\
\hline 0.0458 & 0.0389616 \\
\hline 0.0457 & 0.0388311 \\
\hline 0.0455 & 0.0387014 \\
\hline 0.0454 & 0.0385724 \\
\hline 0.0453 & 0.0384441 \\
\hline 0.0451 & 0.0383165 \\
\hline 0.045 & 0.0381896 \\
\hline 0.0449 & 0.0380633 \\
\hline 0.0448 & 0.0379378 \\
\hline 0.0446 & 0.0378129 \\
\hline 0.0445 & 0.0376886 \\
\hline 0.0444 & 0.037565 \\
\hline 0.0443 & 0.0374421 \\
\hline 0.0441 & 0.0373198 \\
\hline 0.044 & 0.0371982 \\
\hline 0.0439 & 0.0370772 \\
\hline 0.0438 & 0.0369568 \\
\hline 0.0437 & 0.036837 \\
\hline 0.0435 & 0.0367178 \\
\hline 0.0434 & 0.0365993 \\
\hline 0.0433 & 0.0364813 \\
\hline 0.0432 & 0.036364 \\
\hline 0.0431 & 0.0362472 \\
\hline 0.043 & 0.0361311 \\
\hline 0.0428 & 0.0360155 \\
\hline 0.0427 & 0.0359005 \\
\hline 0.0426 & 0.035786 \\
\hline 0.0425 & 0.0356722 \\
\hline 0.0424 & 0.0355589 \\
\hline 0.0423 & 0.0354461 \\
\hline 0.0422 & 0.0353339 \\
\hline 0.042 & 0.0352223 \\
\hline 0.0419 & 0.0351112 \\
\hline 0.0418 & 0.0350006 \\
\hline 0.0417 & 0.0348906 \\
\hline 0.0416 & 0.0347811 \\
\hline 0.0415 & 0.0346721 \\
\hline 0.0414 & 0.0345636 \\
\hline 0.0413 & 0.0344557 \\
\hline 0.0412 & 0.0343483 \\
\hline 0.0411 & 0.0342413 \\
\hline 0.041 & 0.0341349 \\
\hline 0.0409 & 0.034029 \\
\hline 0.0408 & 0.0339236 \\
\hline 0.0407 & 0.0338186 \\
\hline 0.0406 & 0.0337142 \\
\hline 0.0405 & 0.0336102 \\
\hline 0.0404 & 0.0335067 \\
\hline 0.0403 & 0.0334037 \\
\hline 0.0402 & 0.0333012 \\
\hline 0.04 & 0.0331991 \\
\hline 0.04 & 0.0330975 \\
\hline 0.0398 & 0.0329963 \\
\hline 0.0398 & 0.0328956 \\
\hline
\end{tabular}

0.235692

0.234957

0.234225

0.233496

0.232047

0.231327

0.23061

0.229895

0.229184
0.228475

0.227769

0.227066

0.225668

0.224973

0.224281

0.223591
0.222904

0.222219

0.221537

0.220857

0.22018
0.219505

0.218833

0.218163

0.21683

0.216167

0.215507

0.214849
0.214193

0.213539

0.212887

0.212238

0.211591
0.210946

0.210303

0.209663

0.209024

0.208388
0.207753

0.207121

0.206491

0.205236

0.204612

0.20399

0.2033

0.202751
0.202135

0.201521

0.200908

0.200297

0.199689
0.199082

0.199082
0.198477

0.197874

0.197273

0.196673

0.196075

0.19548
0.194886

0.194886
0.194293

0.193703

0.193114

0.192527

0.191942
0.191359

0.191359
0.190777

0.190777
0.190197

0.189618

0.189042

0.188467

0.18789

0.187322
0.186752

0.186184

0.185617

0.185052

0.184489

0.183927

0.183367
0.182809

0.182252

0.181696

0.181143

0.180591

0.18004

0.179491
0.178944

0.178944

0.1783854
0.177311
0.17677

$\begin{array}{r}0.17677 \\ \hline\end{array}$
5.8923

5.87392

5.85562

5.83739
5.81924

5.80117

5.78317

5.76524

5.74738
5.72959

5.71188

5.69423
5.67665

5.65914

5.6417

5.62432

5.60701

5.58977
5.57259

5.55547

5.53842

5.52143

5.5045

5.48763
5.47082

5.45407

5.43738

5.42075

5.40418

5.38767

5.37121
5.35481

5.33847

5.32218

5.30595

5.28977

5.27365

5.25758

5.24156

5.2256

5.19383

5.17802

5.16227

5.14656

5.1153
5.09975
5.08424

5.08424

5.06878

5.05338

$\begin{array}{r}5.0227 \\ 5.00744 \\ \hline\end{array}$

5.00744
4.99222

4.97705

4.96192

4.94685

4.93181

4.91683

4.90189

4.88699

4.87214

4.85733

4.84257

4.82785

4.81318

4.79855

4.78396

4.76942

4.75492

4.74046

4.72604

4.71167

4.69734
4.68305

4.6688

4.6546

4.64043

4.62631

4.61222

4.59818

4.58418
4.57022

4.55629

4.54241

4.52857

4.51477

4.501

4.48728
4.4736

4.4736

4.44634

4.43278

4.41925
53.0307

52.8653

52.7006
52.5366

52.5366
52.3732

52.2105

52.0485

51.8871

51.7264

51.5663
51.4069

51.2481

51.0899

50.9323

50.7753

50.4631

50.3079

50.1533

49.9992

49.8458

49.6928

49.5405

49.3886
49.2374

49.0866

48.9365

48.7868

48.6376

48.489
48.3409

48.1933

48.0462

47.8997

47.7536

47.608
47.4629

47.3182

47.1741

47.0304

46.8872

46.7445

46.6022

46.4604

46.3178

46.0377

45.8977

45.7582

45.6191

45.4804

45.2043

45.0669

44.93
44.7934

44.7934

44.6573

44.5216

44.3863

44.2515

44.117

43.9829

43.8493
43.716

43.5832

43.4507

43.3186

43.187

43.0557

42.9248

42.7943

42.6641

42.5344

42.405

42.2761
42.1475

42.0192

41.8914

41.7639

41.6368

41.51

41.3836

41.2576

41.1319

41.0066

40.8817

40.7571

40.6329

40.509
40.3855

40.2624

40.1396

40.0171

40.0171

39.895
39.7732

94.2768

93.6899
93.3983

93.1079

92.8187

2.2438

91.6735

91.39
91.1077

90.8265

90.5463

90.2672

89.9892

89.7122

89.4363

89.1614
88.8875

88.6147

88.3428

88.0719

87.802

87.2652

86.7321

86.4669

86.2027

85.9394

85.677
85.4155

85.1549

84.8952

84.6364

84.3784

84.1213

83.865

83.355

83.1013

82.8484

82.5963

82.345
82.0945

81.8448

81.596

81.3479

81.1005

80.854 


\begin{tabular}{|c|c|c|}
\hline 313 & 0.0397 & 0.0327954 \\
\hline 314 & 0.0396 & 0.0326956 \\
\hline 315 & 0.0395 & 0.0325963 \\
\hline 316 & 0.0394 & 0.0324974 \\
\hline 317 & 0.0393 & 0.0323989 \\
\hline 318 & 0.0392 & 0.0323009 \\
\hline 319 & 0.0391 & 0.0322033 \\
\hline 320 & 0.039 & 0.0321061 \\
\hline 321 & 0.0389 & 0.0320094 \\
\hline 322 & 0.0388 & 0.031913 \\
\hline 323 & 0.0387 & 0.0318171 \\
\hline 324 & 0.0386 & 0.0317217 \\
\hline 325 & 0.0385 & 0.0316266 \\
\hline 326 & 0.0384 & 0.0315319 \\
\hline 327 & 0.0383 & 0.0314377 \\
\hline 328 & 0.0382 & 0.0313438 \\
\hline 329 & 0.0381 & 0.0312504 \\
\hline 330 & 0.038 & 0.0311573 \\
\hline 331 & 0.038 & 0.0310646 \\
\hline 332 & 0.0379 & 0.0309724 \\
\hline 333 & 0.0378 & 0.0308805 \\
\hline 334 & 0.0377 & 0.030789 \\
\hline 335 & 0.0376 & 0.0306979 \\
\hline 336 & 0.0375 & 0.0306071 \\
\hline 337 & 0.0374 & 0.0305168 \\
\hline 338 & 0.0373 & 0.0304268 \\
\hline 339 & 0.0372 & 0.0303372 \\
\hline 340 & 0.0372 & 0.0302479 \\
\hline 341 & 0.0371 & 0.0301591 \\
\hline 342 & 0.037 & 0.0300706 \\
\hline 343 & 0.0369 & 0.0299824 \\
\hline 344 & 0.0368 & 0.0298946 \\
\hline 345 & 0.0367 & 0.0298072 \\
\hline 346 & 0.0366 & 0.0297201 \\
\hline 347 & 0.0366 & 0.0296334 \\
\hline 348 & 0.0365 & 0.029547 \\
\hline 349 & 0.0364 & 0.029461 \\
\hline 350 & 0.0363 & 0.0293753 \\
\hline 351 & 0.0362 & 0.0292899 \\
\hline 352 & 0.0361 & 0.0292049 \\
\hline 353 & 0.0361 & 0.0291202 \\
\hline 354 & 0.036 & 0.0290359 \\
\hline 355 & 0.0359 & 0.0289519 \\
\hline 356 & 0.0358 & 0.0288682 \\
\hline 357 & 0.0357 & 0.0287849 \\
\hline 358 & 0.0357 & 0.0287018 \\
\hline 359 & 0.0356 & 0.0286191 \\
\hline 360 & 0.0355 & 0.0285367 \\
\hline 361 & 0.0354 & 0.0284547 \\
\hline 362 & 0.0353 & 0.0283729 \\
\hline 363 & 0.0353 & 0.0282915 \\
\hline 364 & 0.0352 & 0.0282104 \\
\hline 365 & 0.0351 & 0.0281296 \\
\hline 366 & 0.035 & 0.0280491 \\
\hline 367 & 0.035 & 0.0279689 \\
\hline 368 & 0.0349 & 0.027889 \\
\hline 369 & 0.0348 & 0.0278094 \\
\hline 370 & 0.0347 & 0.0277302 \\
\hline 371 & 0.0346 & 0.0276512 \\
\hline 372 & 0.0346 & 0.0275725 \\
\hline 373 & 0.0345 & 0.0274941 \\
\hline 374 & 0.0344 & 0.027416 \\
\hline 375 & 0.0343 & 0.0273382 \\
\hline 376 & 0.0343 & 0.0272607 \\
\hline 377 & 0.0342 & 0.0271834 \\
\hline 378 & 0.0341 & 0.0271065 \\
\hline 379 & 0.034 & 0.0270298 \\
\hline 380 & 0.034 & 0.0269534 \\
\hline 381 & 0.0339 & 0.0268773 \\
\hline 382 & 0.0338 & 0.0268015 \\
\hline 383 & 0.0338 & 0.026726 \\
\hline 384 & 0.0337 & 0.0266507 \\
\hline 385 & 0.0336 & 0.0265757 \\
\hline 386 & 0.0335 & 0.026501 \\
\hline 387 & 0.0335 & 0.0264265 \\
\hline 388 & 0.0334 & 0.0263523 \\
\hline 389 & 0.0333 & 0.0262784 \\
\hline 390 & 0.0333 & 0.0262047 \\
\hline 391 & 0.0332 & 0.0261314 \\
\hline 392 & 0.0331 & 0.0260582 \\
\hline 393 & 0.033 & 0.0259853 \\
\hline 394 & 0.033 & 0.0259127 \\
\hline 395 & 0.0329 & 0.0258404 \\
\hline 396 & 0.0328 & 0.0257683 \\
\hline 397 & 0.0328 & 0.0256964 \\
\hline 398 & 0.0327 & 0.0256248 \\
\hline 399 & 0.0326 & 0.0255535 \\
\hline 400 & 0.0326 & 0.0254824 \\
\hline 401 & 0.0325 & 0.0254116 \\
\hline 402 & 0.0324 & 0.025341 \\
\hline 403 & 0.0324 & 0.0252706 \\
\hline 404 & 0.0323 & 0.0252005 \\
\hline 405 & 0.0322 & 0.0251306 \\
\hline 406 & 0.0322 & 0.025061 \\
\hline 407 & 0.0321 & 0.0249916 \\
\hline
\end{tabular}

\begin{tabular}{r|}
0.17623 \\
0.175692 \\
0.175156 \\
0.17462 \\
0.174087 \\
0.173555 \\
0.173024 \\
0.172495 \\
0.171968 \\
0.171442 \\
0.170917 \\
0.170394 \\
0.169872 \\
0.169352 \\
0.168833 \\
0.168316 \\
0.1678 \\
0.167286 \\
0.166773 \\
0.166262 \\
0.165752 \\
0.165243
\end{tabular}

0.165243

0.164736
0.16423

0.163726

0.163223

0.162721

0.16222

0.161722
0.161225

0.160729

0.160234

0.15925
0.158759
0.15827

0.15827

0.157782

0.157296
0.156811

0.156811

0.155845

0.155364

0.154885

0.154406
0.153929

0.153454

0.152979

0.152506

0.152035

0.151564
0.151095

0.150628

0.150161

0.149696

0.149232
0.14877

0.14877
0.148309

0.147849

0.14739

0.146932

0.146476

0.14602

0.145568
0.145115

0.144664

0.144214

0.143766
0.143319

0.142872

0.142428

0.141984
0.141541

0.14154

0.14066

0.140222

0.139784

0.139348

0.138913

0.138046

0.137614

0.137184

0.136755
0.136327

0.135

0.135475

0.13505

0.134627

0.134205

0.133784
0.133364

0.132946

0.132528

0.132112

0.131697
4.40576

4.3923

4.37889

4.36551
4.35217

4.33887

4.32561

4.31238

4.29919
4.28604

4.27293

4.25985
4.24681

4.24681

4.2338

4.22084
4.2079

4.19501

4.18215
4.16933

4.15654

4.14379

4.14379
4.13107

4.1184

4.10575

4.09314
4.08057

4.06803

4.06803

4.04306

4.03063

4.01823

4.00586

3.98124

3.96898
3.95675

3.94456

3.9324

3.90818

3.90818

3.89613
3.8841

3.87211

3.86016

3.84823
3.83634

3.82449

3.81266
3.80087
3.78911

3.78911

3.77739

3.76569

3.75403

3.7424
3.73081

3.73081
3.71925

3.70771

3.69621
3.68475

3.67331

3.66191
3.65054

3.6392

3.62789

3.61661

3.60536

3.59415

3.58296

3.57181
3.56069

3.5496

3.53854

3.52751

3.51651

3.50554

3.4946
3.48369

3.47281

3.46197

3.45115

3.44036

3.4296

3.41887

3.40817
3.39751

3.39751

3.38687
3.37626

3.37626
3.36568
3.35512

3.35512

3.3446
3.33411

3.33411
3.32364

3.32364

3.31321
3.3028
3.29242

$\begin{array}{r}3.29242 \\ \hline\end{array}$
Table_of_specs_Rev0.xls,CH Surface roughness

Printed 10/2/05,4:24 PM

39.6518 $\quad 70.4921$

39.5307
39.41

\begin{tabular}{r|r}
39.2896 & 69.8482 \\
39.1696 & 69.6348
\end{tabular}

\begin{tabular}{r|r}
39.1696 & 69.6348 \\
39.0498 & 69.4219 \\
\hline
\end{tabular}

$38.9305 \quad 69.2097$

$38.8114 \quad 68.9981$

38.6927
38.5744

$38.5744 \quad 68.5767$

\begin{tabular}{l|l}
38.4563 & 68.3668 \\
38.3386 & 68.1576 \\
38.2213
\end{tabular}

$38.2213 \quad 67.9489$

$38.1042 \quad 67.7409$

$37.9875 \quad 67.5334$

$37.8711 \quad 67.3265$

37.7551
37.6394
37.524 $\quad \begin{aligned} & 67.1202 \\ & 66.9144\end{aligned}$

37.524
37.4089

$37.4089 \quad 66.5047$

$37.2941 \quad 66.3006$

37.1797
37.0656

$37.0656 \quad 65.8943$

\begin{tabular}{r|r}
36.9518 & 65.692 \\
36.8383 & 65.4903 \\
36.7251
\end{tabular}

\begin{tabular}{l|l}
36.7251 & 65.2891 \\
36.6123
\end{tabular}

$36.6123 \quad 65.0885$

36.4997

36.3875
36.2756

\begin{tabular}{r|r}
36.2756 & 64.49 \\
36.164 & 64.2916 \\
36.0528 & 64.0938
\end{tabular}

$36.0528 \quad 64.0938$

$35.9418 \quad 63.8965$

$35.8311 \quad 63.6998$

$35.7208 \quad 63.5036$

$35.6108 \quad 63.308$

$35.501 \quad 63.1129$

35.3916
35.2825
35.1737

35.1737

35.0651

34.9569
34.849

$34.849 \quad 61.9538$

$34.7414 \quad 61.7625$

34.6341
34.5271 $\quad \begin{aligned} & 61.5717 \\ & 34.4204\end{aligned}$

34.4204

$34.314 \quad 61.0026$

$34.2078 \quad 60.8139$

$34.102 \quad 60.6258$

\begin{tabular}{l|l}
33.9965 & 60.4382 \\
33.8912 & 60.2511 \\
33.7863 & 60.0645
\end{tabular}

$33.7863 \quad 60.0645$

33.6816

$33.5773 \quad 59.6929$

$33.4732 \quad 59.5079$

$33.3694 \quad 59.3234$

33.2659
33.1627

33.0598

33.0598
32.9572

\begin{tabular}{l|l}
32.8548 & 58.4086 \\
32.7528
\end{tabular}

$32.7528 \quad 58.227$

$32.651 \quad 58.0462$

32.5495
57.8657

\begin{tabular}{l|l}
32.4483 \\
32.3473
\end{tabular}

32.3473
32.2467

$32.1463 \quad 57.149$
32.0462

$32.0462 \quad 56.97$

$31.9464 \quad 56.7935$

$31.8468 \quad 56.6166$

31.7476

31.6486

31.5498
31.4514

$31.4514 \quad 55.9136$

$31.3532 \quad 55.739$

\begin{tabular}{r|r}
31.2553 & 55.565 \\
31.1577 & 55.3914
\end{tabular}

$31.0603-55.3914$

30.9632

$30.8664 \quad 54.8736$

$30.7699 \quad 54.702$

30.6736
30.5776

30.4818

30.3863

$30.2911 \quad 53.8508$

$30.1961 \quad 53.682$

30.1014
30.007 $\quad 53.5136$

$29.9128 \quad 53.1783$

$29.8189 \quad 53.0113$

$29.7252 \quad 52.8448$

\begin{tabular}{r|r|}
29.6318 & 52.6788
\end{tabular}
:24 PM

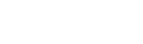


Table_of_specs_Rev0.xls,CH Surface roughness

\begin{tabular}{|c|c|c|}
\hline 408 & 0.032 & 0.0249225 \\
\hline 409 & 0.032 & 0.0248536 \\
\hline 410 & 0.0319 & 0.0247849 \\
\hline 411 & 0.0318 & 0.0247164 \\
\hline 412 & 0.0318 & 0.0246482 \\
\hline 413 & 0.0317 & 0.0245802 \\
\hline 414 & 0.0316 & 0.0245125 \\
\hline 415 & 0.0316 & 0.024445 \\
\hline 416 & 0.0315 & 0.0243777 \\
\hline 417 & 0.0314 & 0.0243106 \\
\hline 418 & 0.0314 & 0.0242438 \\
\hline 419 & 0.0313 & 0.0241772 \\
\hline 420 & 0.0312 & 0.0241108 \\
\hline 421 & 0.0312 & 0.0240446 \\
\hline 422 & 0.0311 & 0.0239786 \\
\hline 423 & 0.0311 & 0.0239129 \\
\hline 424 & 0.031 & 0.0238474 \\
\hline 425 & 0.0309 & 0.0237821 \\
\hline 426 & 0.0309 & 0.023717 \\
\hline 427 & 0.0308 & 0.0236521 \\
\hline 428 & 0.0307 & 0.0235875 \\
\hline 429 & 0.0307 & 0.023523 \\
\hline 430 & 0.0306 & 0.0234588 \\
\hline 431 & 0.0306 & 0.0233948 \\
\hline 432 & 0.0305 & 0.023331 \\
\hline 433 & 0.0304 & 0.0232674 \\
\hline 434 & 0.0304 & 0.023204 \\
\hline 435 & 0.0303 & 0.0231408 \\
\hline 436 & 0.0303 & 0.0230778 \\
\hline 437 & 0.0302 & 0.023015 \\
\hline 438 & 0.0301 & 0.0229524 \\
\hline 439 & 0.0301 & 0.02289 \\
\hline 440 & 0.03 & 0.0228278 \\
\hline 441 & 0.03 & 0.0227658 \\
\hline 442 & 0.0299 & 0.022704 \\
\hline 443 & 0.0298 & 0.0226424 \\
\hline 444 & 0.0298 & 0.022581 \\
\hline 445 & 0.0297 & 0.0225198 \\
\hline 446 & 0.0297 & 0.0224588 \\
\hline 447 & 0.0296 & 0.022398 \\
\hline 448 & 0.0296 & 0.0223374 \\
\hline 449 & 0.0295 & 0.022277 \\
\hline 450 & 0.0294 & 0.0222167 \\
\hline 451 & 0.0294 & 0.0221567 \\
\hline 452 & 0.0293 & 0.0220968 \\
\hline 453 & 0.0293 & 0.0220371 \\
\hline 454 & 0.0292 & 0.0219776 \\
\hline 455 & 0.0292 & 0.0219183 \\
\hline 456 & 0.0291 & 0.0218592 \\
\hline 457 & 0.029 & 0.0218002 \\
\hline 458 & 0.029 & 0.0217415 \\
\hline 459 & 0.0289 & 0.0216829 \\
\hline 460 & 0.0289 & 0.0216245 \\
\hline 461 & 0.0288 & 0.0215663 \\
\hline 462 & 0.0288 & 0.0215083 \\
\hline 463 & 0.0287 & 0.0214504 \\
\hline 464 & 0.0287 & 0.0213927 \\
\hline 465 & 0.0286 & 0.0213352 \\
\hline 466 & 0.0285 & 0.0212779 \\
\hline 467 & 0.0285 & 0.0212207 \\
\hline 468 & 0.0284 & 0.0211638 \\
\hline 469 & 0.0284 & 0.021107 \\
\hline 470 & 0.0283 & 0.0210503 \\
\hline 471 & 0.0283 & 0.0209939 \\
\hline 472 & 0.0282 & 0.0209376 \\
\hline 473 & 0.0282 & 0.0208814 \\
\hline 474 & 0.0281 & 0.0208255 \\
\hline 475 & 0.0281 & 0.0207697 \\
\hline 476 & 0.028 & 0.0207141 \\
\hline 477 & 0.028 & 0.0206586 \\
\hline 478 & 0.0279 & 0.0206033 \\
\hline 479 & 0.0278 & 0.0205482 \\
\hline 480 & 0.0278 & 0.0204933 \\
\hline 481 & 0.0277 & 0.0204385 \\
\hline 482 & 0.0277 & 0.0203838 \\
\hline 483 & 0.0276 & 0.0203294 \\
\hline 484 & 0.0276 & 0.0202751 \\
\hline 485 & 0.0275 & 0.0202209 \\
\hline 486 & 0.0275 & 0.0201669 \\
\hline 487 & 0.0274 & 0.0201131 \\
\hline 488 & 0.0274 & 0.0200594 \\
\hline 489 & 0.0273 & 0.0200059 \\
\hline 490 & 0.0273 & 0.0199526 \\
\hline 491 & 0.0272 & 0.0198994 \\
\hline 492 & 0.0272 & 0.0198463 \\
\hline 493 & 0.0271 & 0.0197935 \\
\hline 494 & 0.0271 & 0.0197407 \\
\hline 495 & 0.027 & 0.0196882 \\
\hline 496 & 0.027 & 0.0196357 \\
\hline 497 & 0.0269 & 0.0195835 \\
\hline 498 & 0.0269 & 0.0195313 \\
\hline 499 & 0.0268 & 0.0194794 \\
\hline 500 & 0.0268 & 0.0194276 \\
\hline 501 & 0.0267 & 0.0193759 \\
\hline 502 & 0.0267 & 0.0193244 \\
\hline
\end{tabular}

\begin{tabular}{|c|c|}
\hline 0.131283 & 3.28207 \\
\hline 0.13087 & 3.27175 \\
\hline 0.130458 & 3.26146 \\
\hline 0.130048 & 3.2512 \\
\hline 0.129638 & 3.24096 \\
\hline 0.12923 & 3.23075 \\
\hline 0.128823 & 3.22057 \\
\hline 0.128417 & 3.21042 \\
\hline 0.128012 & 3.2003 \\
\hline 0.127608 & 3.1902 \\
\hline 0.127205 & 3.18014 \\
\hline 0.126804 & 3.1701 \\
\hline 0.126403 & 3.16008 \\
\hline 0.126004 & 3.1501 \\
\hline 0.125606 & 3.14014 \\
\hline 0.125208 & 3.13021 \\
\hline 0.124812 & 3.12031 \\
\hline 0.124417 & 3.11043 \\
\hline 0.124023 & 3.10058 \\
\hline 0.12363 & 3.09076 \\
\hline 0.123239 & 3.08097 \\
\hline 0.122848 & 3.0712 \\
\hline 0.122458 & 3.06146 \\
\hline 0.12207 & 3.05175 \\
\hline 0.121682 & 3.04206 \\
\hline 0.121296 & 3.0324 \\
\hline 0.120911 & 3.02276 \\
\hline 0.120526 & 3.01316 \\
\hline 0.120143 & 3.00357 \\
\hline 0.119761 & 2.99402 \\
\hline 0.11938 & 2.98449 \\
\hline 0.119 & 2.97499 \\
\hline 0.11862 & 2.96551 \\
\hline 0.118242 & 2.95606 \\
\hline 0.117865 & 2.94664 \\
\hline 0.117489 & 2.93724 \\
\hline 0.117115 & 2.92786 \\
\hline 0.116741 & 2.91852 \\
\hline 0.116368 & 2.90919 \\
\hline 0.115996 & 2.8999 \\
\hline 0.115625 & 2.89063 \\
\hline 0.115255 & 2.88138 \\
\hline 0.114886 & 2.87216 \\
\hline 0.114519 & 2.86296 \\
\hline 0.114152 & 2.85379 \\
\hline 0.113786 & 2.84465 \\
\hline 0.113421 & 2.83553 \\
\hline 0.113057 & 2.82643 \\
\hline 0.112695 & 2.81736 \\
\hline 0.112333 & 2.80832 \\
\hline 0.111972 & 2.7993 \\
\hline 0.111612 & 2.7903 \\
\hline 0.111253 & 2.78133 \\
\hline 0.110895 & 2.77238 \\
\hline 0.110538 & 2.76346 \\
\hline 0.110182 & 2.75456 \\
\hline 0.109828 & 2.74569 \\
\hline 0.109474 & 2.73684 \\
\hline 0.109121 & 2.72801 \\
\hline 0.108768 & 2.71921 \\
\hline 0.108417 & 2.71043 \\
\hline 0.108067 & 2.70168 \\
\hline 0.107718 & 2.69295 \\
\hline 0.10737 & 2.68425 \\
\hline 0.107023 & 2.67556 \\
\hline 0.106676 & 2.6669 \\
\hline 0.106331 & 2.65827 \\
\hline 0.105986 & 2.64966 \\
\hline 0.105643 & 2.64107 \\
\hline 0.1053 & 2.63251 \\
\hline 0.104959 & 2.62397 \\
\hline 0.104618 & 2.61545 \\
\hline 0.104278 & 2.60696 \\
\hline 0.103939 & 2.59848 \\
\hline 0.103601 & 2.59004 \\
\hline 0.103264 & 2.58161 \\
\hline 0.102928 & 2.57321 \\
\hline 0.102593 & 2.56483 \\
\hline 0.102259 & 2.55647 \\
\hline 0.101926 & 2.54814 \\
\hline 0.101593 & 2.53983 \\
\hline 0.101262 & 2.53154 \\
\hline 0.100931 & 2.52328 \\
\hline 0.100601 & 2.51503 \\
\hline 0.100273 & 2.50681 \\
\hline 0.0999447 & 2.49862 \\
\hline 0.0996176 & 2.49044 \\
\hline 0.0992915 & 2.48229 \\
\hline 0.0989663 & 2.47416 \\
\hline 0.0986419 & 2.46605 \\
\hline 0.0983185 & 2.45796 \\
\hline 0.0979959 & 2.4499 \\
\hline 0.0976742 & 2.44186 \\
\hline 0.0973534 & 2.43383 \\
\hline & \\
\hline
\end{tabular}

29.5387

29.4458

29.3531

29.2608
29.1686

29.0768

28.9852

28.8938

28.8027

28.7118

28.6212

28.4407

28.3509

28.2613

28.1719

28.0828

27.9939

27.9053

(27.7.640

27.5531

27.4657

27.3785

27.2916

27.2049

27.0322

26.9462

26.8604

26.6045

26.5197

26.4351

26.3508

26.1827

26.0991

26.0156

25.9324

25.8494

25.7667

25.6018

25.5198

25.4379

25.3563

25.2749

25.1937

25.032

24.0514

24.8711

24.7911

24.7112

24.6315

24.5521

24.4729

24.3939

24.3151
24.2366

24.2366
24.1582

24.0801

24.0021

23.9244

23.8469

23.7696

23.6926

23.6157

23.539

23.4626

23.3864

23.3103
23.2345

23.2345
23.1589

23.0835

23.0083

22.9333

22.8585

22.7839

22.7095

22.6353

22.5613

22.4875

22.414

22.3406

22.2674

22.1944

22.0491

11.9767

(1.9767

21.9045
21.8325
Printed 10/2/0

52.5132

52.348

52.1834

52.0191

51.8554
51.692

51.692
51.5292

51.3668

51.3668
51.2048

51.0432

50.8822

50.7215
50.5613

50.4016

50.2422

50.0834

49.9249

49.7669

49.6093

4.4522

49.2955

49.1392
48.9834

48.8279

48.6729
48.5184

48.5184

48.3642

48.2105
48.0572

47.9043

47.7518

47.5998

47.4482

47.297

47.1462

46.9958

46.8458

46.6962

46.5471
46.3983

46.3983

46.1021

45.9545

45.8074

45.6607

(5.5144

45.3685

45.2229

45.0778
44.9331

44.7888

44.6448

44.5013

44.3581

44.2154

44.073

43.931

43.6482

43.5074

43.5074

43.2269

43.0872

42.9479

42.809

42.5323

42.3945

42.2571

42.1201

41.9835

41.7113

41.7113

41.4406

41.3058

41.1713

40.9036

40.7703

40.6373

40.5047

40.3724

40.2406

40.109

39.8471

39.7166

39.5865

39.4568

9.1984

39.0697

38.9414

38.9414
38.8134 
Table_of_specs_Rev0.xls,CH Surface roughness

\begin{tabular}{|c|c|c|}
\hline 503 & 0.0266 & 0.019273 \\
\hline 504 & 0.0266 & 0.0192218 \\
\hline 505 & 0.0265 & 0.0191707 \\
\hline 506 & 0.0265 & 0.0191198 \\
\hline 507 & 0.0264 & 0.019069 \\
\hline 508 & 0.0264 & 0.0190183 \\
\hline 509 & 0.0263 & 0.0189678 \\
\hline 510 & 0.0263 & 0.0189175 \\
\hline 511 & 0.0262 & 0.0188673 \\
\hline 512 & 0.0262 & 0.0188172 \\
\hline 513 & 0.0262 & 0.0187673 \\
\hline 514 & 0.0261 & 0.0187175 \\
\hline 515 & 0.0261 & 0.0186679 \\
\hline 516 & 0.026 & 0.0186184 \\
\hline 517 & 0.026 & 0.018569 \\
\hline 518 & 0.0259 & 0.0185198 \\
\hline 519 & 0.0259 & 0.0184707 \\
\hline 520 & 0.0258 & 0.0184218 \\
\hline 521 & 0.0258 & 0.018373 \\
\hline 522 & 0.0257 & 0.0183243 \\
\hline 523 & 0.0257 & 0.0182758 \\
\hline 524 & 0.0256 & 0.0182274 \\
\hline 525 & 0.0256 & 0.0181791 \\
\hline 526 & 0.0255 & 0.018131 \\
\hline 527 & 0.0255 & 0.018083 \\
\hline 528 & 0.0255 & 0.0180352 \\
\hline 529 & 0.0254 & 0.0179875 \\
\hline 530 & 0.0254 & 0.0179399 \\
\hline 531 & 0.0253 & 0.0178924 \\
\hline 532 & 0.0253 & 0.0178451 \\
\hline 533 & 0.0252 & 0.0177979 \\
\hline 534 & 0.0252 & 0.0177508 \\
\hline 535 & 0.0251 & 0.0177039 \\
\hline 536 & 0.0251 & 0.0176571 \\
\hline 537 & 0.025 & 0.0176104 \\
\hline 538 & 0.025 & 0.0175639 \\
\hline 539 & 0.025 & 0.0175175 \\
\hline 540 & 0.0249 & 0.0174712 \\
\hline 541 & 0.0249 & 0.017425 \\
\hline 542 & 0.0248 & 0.017379 \\
\hline 543 & 0.0248 & 0.0173331 \\
\hline 544 & 0.0247 & 0.0172873 \\
\hline 545 & 0.0247 & 0.0172416 \\
\hline 546 & 0.0247 & 0.0171961 \\
\hline 547 & 0.0246 & 0.0171507 \\
\hline 548 & 0.0246 & 0.0171054 \\
\hline 549 & 0.0245 & 0.0170602 \\
\hline 550 & 0.0245 & 0.0170152 \\
\hline 551 & 0.0244 & 0.0169703 \\
\hline 552 & 0.0244 & 0.0169255 \\
\hline 553 & 0.0243 & 0.0168808 \\
\hline 554 & 0.0243 & 0.0168363 \\
\hline 555 & 0.0243 & 0.0167918 \\
\hline 556 & 0.0242 & 0.0167475 \\
\hline 557 & 0.0242 & 0.0167033 \\
\hline 558 & 0.0241 & 0.0166593 \\
\hline 559 & 0.0241 & 0.0166153 \\
\hline 560 & 0.0241 & 0.0165715 \\
\hline 561 & 0.024 & 0.0165278 \\
\hline 562 & 0.024 & 0.0164842 \\
\hline 563 & 0.0239 & 0.0164407 \\
\hline 564 & 0.0239 & 0.0163973 \\
\hline 565 & 0.0238 & 0.0163541 \\
\hline 566 & 0.0238 & 0.016311 \\
\hline 567 & 0.0238 & 0.016268 \\
\hline 568 & 0.0237 & 0.0162251 \\
\hline 569 & 0.0237 & 0.0161823 \\
\hline 570 & 0.0236 & 0.0161396 \\
\hline 571 & 0.0236 & 0.016097 \\
\hline 572 & 0.0236 & 0.0160546 \\
\hline 573 & 0.0235 & 0.0160123 \\
\hline 574 & 0.0235 & 0.0159701 \\
\hline 575 & 0.0234 & 0.015928 \\
\hline 576 & 0.0234 & 0.015886 \\
\hline 577 & 0.0234 & 0.0158441 \\
\hline 578 & 0.0233 & 0.0158023 \\
\hline 579 & 0.0233 & 0.0157607 \\
\hline 580 & 0.0232 & 0.0157191 \\
\hline 581 & 0.0232 & 0.0156777 \\
\hline 582 & 0.0232 & 0.0156363 \\
\hline 583 & 0.0231 & 0.0155951 \\
\hline 584 & 0.0231 & 0.015554 \\
\hline 585 & 0.023 & 0.015513 \\
\hline 586 & 0.023 & 0.0154721 \\
\hline 587 & 0.023 & 0.0154313 \\
\hline 588 & 0.0229 & 0.0153906 \\
\hline 589 & 0.0229 & 0.0153501 \\
\hline 590 & 0.0228 & 0.0153096 \\
\hline 591 & 0.0228 & 0.0152693 \\
\hline 592 & 0.0228 & 0.015229 \\
\hline 593 & 0.0227 & 0.0151889 \\
\hline 594 & 0.0227 & 0.0151488 \\
\hline 595 & 0.0226 & 0.0151089 \\
\hline 596 & 0.0226 & 0.0150691 \\
\hline 597 & 0.0226 & 0.0150293 \\
\hline
\end{tabular}

\begin{tabular}{|c|c|}
\hline 0.0967144 & 2.41786 \\
\hline 0.0963962 & 2.4099 \\
\hline 0.0960789 & 2.40197 \\
\hline 0.0957624 & 2.39406 \\
\hline 0.0954468 & 2.38617 \\
\hline 0.0951321 & 2.3783 \\
\hline 0.0948182 & 2.37045 \\
\hline 0.0945052 & 2.36263 \\
\hline 0.094193 & 2.35483 \\
\hline 0.0938817 & 2.34704 \\
\hline 0.0935713 & 2.33928 \\
\hline 0.0932617 & 2.33154 \\
\hline 0.0929529 & 2.32382 \\
\hline 0.092645 & 2.31613 \\
\hline 0.0923379 & 2.30845 \\
\hline 0.0920317 & 2.30079 \\
\hline 0.0917263 & 2.29316 \\
\hline 0.0914218 & 2.28554 \\
\hline 0.0911181 & 2.27795 \\
\hline 0.0908152 & 2.27038 \\
\hline 0.0905131 & 2.26283 \\
\hline 0.0902119 & 2.2553 \\
\hline 0.0899115 & 2.24779 \\
\hline 0.0896119 & 2.2403 \\
\hline 0.0893132 & 2.23283 \\
\hline 0.0890153 & 2.22538 \\
\hline 0.0887182 & 2.21795 \\
\hline 0.0884219 & 2.21055 \\
\hline 0.0881264 & 2.20316 \\
\hline 0.0878317 & 2.19579 \\
\hline 0.0875379 & 2.18845 \\
\hline 0.0872448 & 2.18112 \\
\hline 0.0869526 & 2.17381 \\
\hline 0.0866611 & 2.16653 \\
\hline 0.0863705 & 2.15926 \\
\hline 0.0860807 & 2.15202 \\
\hline 0.0857916 & 2.14479 \\
\hline 0.0855034 & 2.13758 \\
\hline 0.0852159 & 2.1304 \\
\hline 0.0849293 & 2.12323 \\
\hline 0.0846434 & 2.11609 \\
\hline 0.0843584 & 2.10896 \\
\hline 0.0840741 & 2.10185 \\
\hline 0.0837906 & 2.09476 \\
\hline 0.0835079 & 2.0877 \\
\hline 0.0832259 & 2.08065 \\
\hline 0.0829448 & 2.07362 \\
\hline 0.0826644 & 2.06661 \\
\hline 0.0823848 & 2.05962 \\
\hline 0.082106 & 2.05265 \\
\hline 0.0818279 & 2.0457 \\
\hline 0.0815507 & 2.03877 \\
\hline 0.0812741 & 2.03185 \\
\hline 0.0809984 & 2.02496 \\
\hline 0.0807234 & 2.01809 \\
\hline 0.0804492 & 2.01123 \\
\hline 0.0801758 & 2.00439 \\
\hline 0.0799031 & 1.99758 \\
\hline 0.0796311 & 1.99078 \\
\hline 0.07936 & 1.984 \\
\hline 0.0790895 & 1.97724 \\
\hline 0.0788199 & 1.9705 \\
\hline 0.078551 & 1.96377 \\
\hline 0.0782828 & 1.95707 \\
\hline 0.0780154 & 1.95038 \\
\hline 0.0777487 & 1.94372 \\
\hline 0.0774828 & 1.93707 \\
\hline 0.0772176 & 1.93044 \\
\hline 0.0769532 & 1.92383 \\
\hline 0.0766895 & 1.91724 \\
\hline 0.0764265 & 1.91066 \\
\hline 0.0761643 & 1.90411 \\
\hline 0.0759028 & 1.89757 \\
\hline 0.0756421 & 1.89105 \\
\hline 0.075382 & 1.88455 \\
\hline 0.0751228 & 1.87807 \\
\hline 0.0748642 & 1.8716 \\
\hline 0.0746064 & 1.86516 \\
\hline 0.0743492 & 1.85873 \\
\hline 0.0740929 & 1.85232 \\
\hline 0.0738372 & 1.84593 \\
\hline 0.0735823 & 1.83956 \\
\hline 0.073328 & 1.8332 \\
\hline 0.0730745 & 1.82686 \\
\hline 0.0728217 & 1.82054 \\
\hline 0.0725697 & 1.81424 \\
\hline 0.0723183 & 1.80796 \\
\hline 0.0720677 & 1.80169 \\
\hline 0.0718177 & 1.79544 \\
\hline 0.0715685 & 1.78921 \\
\hline 0.07132 & 1.783 \\
\hline 0.0710721 & 1.7768 \\
\hline 0.070825 & 1.77063 \\
\hline 0.0705786 & 1.76447 \\
\hline & \\
\hline
\end{tabular}

21.7607

21.6891

21.6177

21.5465

21.4755
21.4047

21.3341

21.2637

21.1934

21.1234

21.0535

20.9839

20.9144

20.8451
20.776

20.7071

20.6384

20.5699
20.5016

20.4334

20.3655

20.2977

20.2301
20.1627

20.0955

20.0284

19.9616

19.8949

19.8284

19.7621

19.696
19.6301
19.5643

19.5643

19.4988

19.4334

19.3681

19.3031

19.1736

19.1091

19.0448

18.9806

18.9167

18.8529

18.7893

18.7258

18.6626

18.5995

18.5366

18.4738

18.413
18.3489

18.2867

18.2246

18.1628

18.1011

18.0395

17.9782
17.917

17.917

17.856

17.7951
17.7345

17.7345
17.674

17.6136

17.5535

17.4935

17.4336

17.374

17.3145

17.2551

17.196
17.137

17.137

17.0781

17.0195

16.961
16.9026

16.8444

16.7864

16.7286

16.6709

16.6134

16.556

16.4988

16.4418

16.3282

16.2716

16.2152

16.159
16.1029
16.047

16.047

15.9912

15.9356

15.8802

15.8249
Printed 10/2/0

38.6857

38.4315
38.305
38.1787

38.1787

38.0528

37.9273

37.8021

37.6772

37.4285

37.3047

37.1812

37.058
36.9352

36.8127

36.6905

36.5687

36.3261

36.2053

36.0848

35.9646

35.8448

35.7253

(5.6061

35.3687

35.2506

35.1327

35.0151

34.8979

34.6645

34.6482

34.5482

34.4323

34.3167

34.0864

33.9717

33.8574

33.7433

33.6296

33.5162

33.4031

(3.2904

33.1779

33.0658

32.9539

32.8424

32.7312

32.6203

32.5097

32.3994

32.2894

32.1797
32.0703

31.9612

31.8525

31.744

31.6358

31.528

31.4204

31.3131

31.2062

31.0995

30.9931

30.887
30.7813
30.6758

30.6758

30.5706

30.4657

30.3611

30.2568

30.1528
30.0491

29.9457

29.8425

29.7397

29.6371

29.5349

29.4329

29.3312

9.1287

29.0279

28.9273

28.8271

28.7271

28.6274

28.528

28.33

28.33
28.2314

28.1332 
Table_of_specs_Rev0.xls,CH Surface roughness

\begin{tabular}{|c|c|c|}
\hline 598 & 0.0225 & 0.0149897 \\
\hline 599 & 0.0225 & 0.0149502 \\
\hline 600 & 0.0225 & 0.0149108 \\
\hline 601 & 0.0224 & 0.0148715 \\
\hline 602 & 0.0224 & 0.0148322 \\
\hline 603 & 0.0223 & 0.0147931 \\
\hline 604 & 0.0223 & 0.0147541 \\
\hline 605 & 0.0223 & 0.0147152 \\
\hline 606 & 0.0222 & 0.0146764 \\
\hline 607 & 0.0222 & 0.0146377 \\
\hline 608 & 0.0222 & 0.0145991 \\
\hline 609 & 0.0221 & 0.0145606 \\
\hline 610 & 0.0221 & 0.0145222 \\
\hline 611 & 0.022 & 0.0144839 \\
\hline 612 & 0.022 & 0.0144457 \\
\hline 613 & 0.022 & 0.0144076 \\
\hline 614 & 0.0219 & 0.0143696 \\
\hline 615 & 0.0219 & 0.0143317 \\
\hline 616 & 0.0219 & 0.0142939 \\
\hline 617 & 0.0218 & 0.0142562 \\
\hline 618 & 0.0218 & 0.0142186 \\
\hline 619 & 0.0217 & 0.0141811 \\
\hline 620 & 0.0217 & 0.0141436 \\
\hline 621 & 0.0217 & 0.0141063 \\
\hline 622 & 0.0216 & 0.0140691 \\
\hline 623 & 0.0216 & 0.014032 \\
\hline 624 & 0.0216 & 0.0139949 \\
\hline 625 & 0.0215 & 0.013958 \\
\hline 626 & 0.0215 & 0.0139211 \\
\hline 627 & 0.0215 & 0.0138844 \\
\hline 628 & 0.0214 & 0.0138477 \\
\hline 629 & 0.0214 & 0.0138112 \\
\hline 630 & 0.0213 & 0.0137747 \\
\hline 631 & 0.0213 & 0.0137383 \\
\hline 632 & 0.0213 & 0.0137021 \\
\hline 633 & 0.0212 & 0.0136659 \\
\hline 634 & 0.0212 & 0.0136298 \\
\hline 635 & 0.0212 & 0.0135938 \\
\hline 636 & 0.0211 & 0.0135579 \\
\hline 637 & 0.0211 & 0.0135221 \\
\hline 638 & 0.0211 & 0.0134863 \\
\hline 639 & 0.021 & 0.0134507 \\
\hline 640 & 0.021 & 0.0134152 \\
\hline 641 & 0.021 & 0.0133797 \\
\hline 642 & 0.0209 & 0.0133444 \\
\hline 643 & 0.0209 & 0.0133091 \\
\hline 644 & 0.0209 & 0.0132739 \\
\hline 645 & 0.0208 & 0.0132388 \\
\hline 646 & 0.0208 & 0.0132038 \\
\hline 647 & 0.0208 & 0.0131689 \\
\hline 648 & 0.0207 & 0.0131341 \\
\hline 649 & 0.0207 & 0.0130993 \\
\hline 650 & 0.0207 & 0.0130647 \\
\hline 651 & 0.0206 & 0.0130301 \\
\hline 652 & 0.0206 & 0.0129957 \\
\hline 653 & 0.0205 & 0.0129613 \\
\hline 654 & 0.0205 & 0.012927 \\
\hline 655 & 0.0205 & 0.0128928 \\
\hline 656 & 0.0204 & 0.0128587 \\
\hline 657 & 0.0204 & 0.0128247 \\
\hline 658 & 0.0204 & 0.0127907 \\
\hline 659 & 0.0203 & 0.0127568 \\
\hline 660 & 0.0203 & 0.0127231 \\
\hline 661 & 0.0203 & 0.0126894 \\
\hline 662 & 0.0202 & 0.0126558 \\
\hline 663 & 0.0202 & 0.0126223 \\
\hline 664 & 0.0202 & 0.0125888 \\
\hline 665 & 0.0201 & 0.0125555 \\
\hline 666 & 0.0201 & 0.0125222 \\
\hline 667 & 0.0201 & 0.0124891 \\
\hline 668 & 0.02 & 0.012456 \\
\hline 669 & 0.02 & 0.012423 \\
\hline 670 & 0.02 & 0.01239 \\
\hline 671 & 0.0199 & 0.0123572 \\
\hline 672 & 0.0199 & 0.0123244 \\
\hline 673 & 0.0199 & 0.0122918 \\
\hline 674 & 0.0199 & 0.0122592 \\
\hline 675 & 0.0198 & 0.0122266 \\
\hline 676 & 0.0198 & 0.0121942 \\
\hline 677 & 0.0198 & 0.0121619 \\
\hline 678 & 0.0197 & 0.0121296 \\
\hline 679 & 0.0197 & 0.0120974 \\
\hline 680 & 0.0197 & 0.0120653 \\
\hline 681 & 0.0196 & 0.0120333 \\
\hline 682 & 0.0196 & 0.0120014 \\
\hline 683 & 0.0196 & 0.0119695 \\
\hline 684 & 0.0195 & 0.0119377 \\
\hline 685 & 0.0195 & 0.011906 \\
\hline 686 & 0.0195 & 0.0118744 \\
\hline 687 & 0.0194 & 0.0118429 \\
\hline 688 & 0.0194 & 0.0118114 \\
\hline 689 & 0.0194 & 0.01178 \\
\hline 690 & 0.0193 & 0.0117487 \\
\hline 691 & 0.0193 & 0.0117175 \\
\hline 692 & 0.0193 & 0.0116864 \\
\hline
\end{tabular}

\begin{tabular}{|c|c|}
\hline 0.0700879 & 1.7522 \\
\hline 0.0698436 & 1.74609 \\
\hline 0.0695999 & 1.74 \\
\hline 0.069357 & 1.73393 \\
\hline 0.0691148 & 1.72787 \\
\hline 0.0688733 & 1.72183 \\
\hline 0.0686324 & 1.71581 \\
\hline 0.0683923 & 1.70981 \\
\hline 0.0681528 & 1.70382 \\
\hline 0.067914 & 1.69785 \\
\hline 0.0676759 & 1.6919 \\
\hline 0.0674385 & 1.68596 \\
\hline 0.0672017 & 1.68004 \\
\hline 0.0669657 & 1.67414 \\
\hline 0.0667303 & 1.66826 \\
\hline 0.0664956 & 1.66239 \\
\hline 0.0662616 & 1.65654 \\
\hline 0.0660283 & 1.65071 \\
\hline 0.0657956 & 1.64489 \\
\hline 0.0655636 & 1.63909 \\
\hline 0.0653322 & 1.63331 \\
\hline 0.0651016 & 1.62754 \\
\hline 0.0648716 & 1.62179 \\
\hline 0.0646423 & 1.61606 \\
\hline 0.0644136 & 1.61034 \\
\hline 0.0641856 & 1.60464 \\
\hline 0.0639583 & 1.59896 \\
\hline 0.0637316 & 1.59329 \\
\hline 0.0635056 & 1.58764 \\
\hline 0.0632802 & 1.58201 \\
\hline 0.0630555 & 1.57639 \\
\hline 0.0628315 & 1.57079 \\
\hline 0.0626081 & 1.5652 \\
\hline 0.0623853 & 1.55963 \\
\hline 0.0621632 & 1.55408 \\
\hline 0.0619418 & 1.54855 \\
\hline 0.061721 & 1.54303 \\
\hline 0.0615009 & 1.53752 \\
\hline 0.0612814 & 1.53203 \\
\hline 0.0610625 & 1.52656 \\
\hline 0.0608443 & 1.52111 \\
\hline 0.0606268 & 1.51567 \\
\hline 0.0604098 & 1.51025 \\
\hline 0.0601935 & 1.50484 \\
\hline 0.0599779 & 1.49945 \\
\hline 0.0597629 & 1.49407 \\
\hline 0.0595485 & 1.48871 \\
\hline 0.0593348 & 1.48337 \\
\hline 0.0591217 & 1.47804 \\
\hline 0.0589092 & 1.47273 \\
\hline 0.0586973 & 1.46743 \\
\hline 0.0584861 & 1.46215 \\
\hline 0.0582755 & 1.45689 \\
\hline 0.0580656 & 1.45164 \\
\hline 0.0578562 & 1.44641 \\
\hline 0.0576475 & 1.44119 \\
\hline 0.0574394 & 1.43599 \\
\hline 0.0572319 & 1.4308 \\
\hline 0.0570251 & 1.42563 \\
\hline 0.0568189 & 1.42047 \\
\hline 0.0566133 & 1.41533 \\
\hline 0.0564083 & 1.41021 \\
\hline 0.0562039 & 1.4051 \\
\hline 0.0560001 & 1.4 \\
\hline 0.0557969 & 1.39492 \\
\hline 0.0555944 & 1.38986 \\
\hline 0.0553925 & 1.38481 \\
\hline 0.0551911 & 1.37978 \\
\hline 0.0549904 & 1.37476 \\
\hline 0.0547903 & 1.36976 \\
\hline 0.0545908 & 1.36477 \\
\hline 0.0543919 & 1.3598 \\
\hline 0.0541936 & 1.35484 \\
\hline 0.0539959 & 1.3499 \\
\hline 0.0537988 & 1.34497 \\
\hline 0.0536023 & 1.34006 \\
\hline 0.0534064 & 1.33516 \\
\hline 0.053211 & 1.33028 \\
\hline 0.0530163 & 1.32541 \\
\hline 0.0528222 & 1.32056 \\
\hline 0.0526287 & 1.31572 \\
\hline 0.0524357 & 1.31089 \\
\hline 0.0522434 & 1.30608 \\
\hline 0.0520516 & 1.30129 \\
\hline 0.0518605 & 1.29651 \\
\hline 0.0516699 & 1.29175 \\
\hline 0.0514799 & 1.287 \\
\hline 0.0512905 & 1.28226 \\
\hline 0.0511016 & 1.27754 \\
\hline 0.0509134 & 1.27283 \\
\hline 0.0507257 & 1.26814 \\
\hline 0.0505386 & 1.26347 \\
\hline 0.0503521 & 1.2588 \\
\hline 0.0501662 & 1.25415 \\
\hline 0.0499808 & \\
\hline
\end{tabular}

15.7698

15.7148

15.66

15.6053

15.5508

15.4965

15.4423

(15.383

15.2806

15.2271

15.1204

15.0673

15.0143

14.9615

14.9089

14.8564

14.7518

14.6998

14.6998

14.5961

14.5445

14.4931

14.4418
14.3906

14.3396

14.2888

14.238

14.1875

14.1371

14.0868

14.0367

13.9867

13.9369

13.8872
13.8377

13.7883

13.7391

13.69

13.641

13.5922

13.5435

\begin{tabular}{l}
13.495 \\
3.4466 \\
\hline 3.3984
\end{tabular}

13.3984

13.3503

13.3024

13.2546

13.2069

13.1594

13.112

13.0648

13.0177
12.9707

12.9239

12.8772

12.8306

12.7842

12.738

12.6919
12.6459

$\begin{array}{r}12.6 \\ \hline\end{array}$

12.5543

12.5087

12.4633

12.3728

12.3278

12.2829

12.2382

12.1936

12.1491

12.1047
12.0605

12.0605
12.0164

11.9725

11.9287

11.885

11.8415

11.798
11.7548

11.7548
11.7116

11.6686

11.6257

11.583

11.5404

11.4979

11.4555

11.4133

11.3712

11.3292

11.2874

11.2457
Printed 10/2/

28.0352

27.9374
27.8

27.7428

27.6459
27.5493

27.453

27.3569

27.2611

27.1656
27.0704

26.9754

26.8807

26.7863

26.6921

26.5982

26.5046

26.4113
26.3182

26.2254

26.1329

26.0406

25.9486

25.8569

25.7654

25.6742

25.4926

25.4022

25.3121

25.2222
25.1326

25.0432

24.9541

24.8653

24.7767

24.6884

24.6004
24.5126

24.425

24.425
24.3377

24.3377
24.2507

24.1639

24.0774
23.9912

23.9052

23.8194

23.7339

23.6487

23.5637

23.4789
23.3944

23.3944

23.2262

23.1425

23.059

22.9758
22.8928

22.891
22.81

22.7275

22.6453

22.5633
22.4815

22.4
2.3188

22.3188
22.2378

22.157

22.0765

21.9962

21.9161
21.8363

21.7568

21.6774

21.5983

21.5195

21.4409

21.3625

21.2844

21.2065

21.1289

21.0515

20.9743
20.8974

20.8207

20.7442

20.668

20.592
20.5162

20.5162
20.4407

20.3654

20.2903

(20.2154

20.1408

20.0665
19.9923

Page 8 of 33 
Table_of_specs_Rev0.xls, $\mathrm{CH}$ Surface roughness

\begin{tabular}{|c|c|c|}
\hline 693 & 0.0192 & 0.0116553 \\
\hline 694 & 0.0192 & 0.0116243 \\
\hline 695 & 0.0192 & 0.0115934 \\
\hline 696 & 0.0192 & 0.0115626 \\
\hline 697 & 0.0191 & 0.0115318 \\
\hline 698 & 0.0191 & 0.0115012 \\
\hline 699 & 0.0191 & 0.0114706 \\
\hline 700 & 0.019 & 0.01144 \\
\hline 701 & 0.019 & 0.0114096 \\
\hline 702 & 0.019 & 0.0113792 \\
\hline 703 & 0.0189 & 0.0113489 \\
\hline 704 & 0.0189 & 0.0113187 \\
\hline 705 & 0.0189 & 0.0112886 \\
\hline 706 & 0.0188 & 0.0112585 \\
\hline 707 & 0.0188 & 0.0112285 \\
\hline 708 & 0.0188 & 0.0111986 \\
\hline 709 & 0.0187 & 0.0111688 \\
\hline 710 & 0.0187 & 0.011139 \\
\hline 711 & 0.0187 & 0.0111094 \\
\hline 712 & 0.0187 & 0.0110797 \\
\hline 713 & 0.0186 & 0.0110502 \\
\hline 714 & 0.0186 & 0.0110207 \\
\hline 715 & 0.0186 & 0.0109914 \\
\hline 716 & 0.0185 & 0.010962 \\
\hline 717 & 0.0185 & 0.0109328 \\
\hline 718 & 0.0185 & 0.0109036 \\
\hline 719 & 0.0184 & 0.0108745 \\
\hline 720 & 0.0184 & 0.0108455 \\
\hline 721 & 0.0184 & 0.0108166 \\
\hline 722 & 0.0184 & 0.0107877 \\
\hline 723 & 0.0183 & 0.0107589 \\
\hline 724 & 0.0183 & 0.0107302 \\
\hline 725 & 0.0183 & 0.0107015 \\
\hline 726 & 0.0182 & 0.0106729 \\
\hline 727 & 0.0182 & 0.0106444 \\
\hline 728 & 0.0182 & 0.010616 \\
\hline 729 & 0.0182 & 0.0105876 \\
\hline 730 & 0.0181 & 0.0105593 \\
\hline 731 & 0.0181 & 0.0105311 \\
\hline 732 & 0.0181 & 0.0105029 \\
\hline 733 & 0.018 & 0.0104749 \\
\hline 734 & 0.018 & 0.0104468 \\
\hline 735 & 0.018 & 0.0104189 \\
\hline 736 & 0.018 & 0.010391 \\
\hline 737 & 0.0179 & 0.0103632 \\
\hline 738 & 0.0179 & 0.0103355 \\
\hline 739 & 0.0179 & 0.0103078 \\
\hline 740 & 0.0178 & 0.0102802 \\
\hline 741 & 0.0178 & 0.0102527 \\
\hline 742 & 0.0178 & 0.0102253 \\
\hline 743 & 0.0177 & 0.0101979 \\
\hline 744 & 0.0177 & 0.0101706 \\
\hline 745 & 0.0177 & 0.0101433 \\
\hline 746 & 0.0177 & 0.0101161 \\
\hline 747 & 0.0176 & 0.010089 \\
\hline 748 & 0.0176 & 0.010062 \\
\hline 749 & 0.0176 & 0.010035 \\
\hline 750 & 0.0176 & 0.0100081 \\
\hline 751 & 0.0175 & 0.00998125 \\
\hline 752 & 0.0175 & 0.00995448 \\
\hline 753 & 0.0175 & 0.00992777 \\
\hline 754 & 0.0174 & 0.00990114 \\
\hline 755 & 0.0174 & 0.00987457 \\
\hline 756 & 0.0174 & 0.00984806 \\
\hline 757 & 0.0174 & 0.00982163 \\
\hline 758 & 0.0173 & 0.00979526 \\
\hline 759 & 0.0173 & 0.00976895 \\
\hline 760 & 0.0173 & 0.00974272 \\
\hline 761 & 0.0172 & 0.00971655 \\
\hline 762 & 0.0172 & 0.00969044 \\
\hline 763 & 0.0172 & 0.0096644 \\
\hline 764 & 0.0172 & 0.00963843 \\
\hline 765 & 0.0171 & 0.00961252 \\
\hline 766 & 0.0171 & 0.00958668 \\
\hline 767 & 0.0171 & 0.0095609 \\
\hline 768 & 0.0171 & 0.00953519 \\
\hline 769 & 0.017 & 0.00950954 \\
\hline 770 & 0.017 & 0.00948396 \\
\hline 771 & 0.017 & 0.00945844 \\
\hline 772 & 0.0169 & 0.00943298 \\
\hline 773 & 0.0169 & 0.00940759 \\
\hline 774 & 0.0169 & 0.00938227 \\
\hline 775 & 0.0169 & 0.009357 \\
\hline 776 & 0.0168 & 0.0093318 \\
\hline 777 & 0.0168 & 0.00930667 \\
\hline 778 & 0.0168 & 0.0092816 \\
\hline 779 & 0.0168 & 0.00925659 \\
\hline 780 & 0.0167 & 0.00923164 \\
\hline 781 & 0.0167 & 0.00920676 \\
\hline 782 & 0.0167 & 0.00918194 \\
\hline 783 & 0.0166 & 0.00915719 \\
\hline 784 & 0.0166 & 0.00913249 \\
\hline 785 & 0.0166 & 0.00910786 \\
\hline 786 & 0.0166 & 0.00908329 \\
\hline 787 & 0.0165 & 0.00905878 \\
\hline
\end{tabular}

0.049796 0.0496118 0.0494282 0.0492451 0.0490626 0.0486993 0.0485185 0.0483382 0.0481586 0.0479795
0.0478009 0.0476229 0.0474455 0.0472686 0.0470923 0.0469165 0.0467413 0.0465667 0.0463926

0.046219 0.046046 0.0458736 0.0457016 0.0455303 0.0453595 0.0451892 0.0450195 0.0448503 0.0446816 0.0445135 0.0443459 0.0441789 0.0440124 0.0438464 0.04368 0.0435161 0.0433517 0.0431879 0.043024 0.0428617
0.0426995 0.0425377 0.0423765 0.0422158 0.0420557

0.041896 0.0417369 0.0415783 0.0414202 0.0412626 0.0411055

0.040949 0.0407929 0.0406374 0.0404824 0.0403279 0.0401739 0.0400204 0.0398674 0.0397149 0.0395629 0.0394114 0.0392604 0.0391099 0.0389599 0.0388105 0.0386615 0.038513 0.03836 0.0382174 0.0380704 0.0379239 0.0377778 0.0376323 0.0374872 0.0373426 0.0371985 0.0370549 0.0369118 0.0367691 0.036627 0.0364853 0.0363441 0.0362033 0.0360631 0.0359233 0.035784 0.0356451 0.0355068 0.0353689 0.0352314 0.0350945 0.034958
0.0348219 9 of 33
1.2449 1.24029 1.2357 1.23113 1.22656

1.21748

1.21296 1.20846

1.20396

1.19949
1.19502
1.19057

1.19057

1.18614

1.18172

1.17731

1.17291

1.16853

1.15981

1.15548

1.15115

1.14684

1.14254

1.13826

1.12973

1.12549

1.12126

1.11704

1.11284

1.10865

1.10031

1.09616
1.09202

1.0879

1.08379

1.0797

1.07561

1.07154

1.06749

1.06344

1.05941
1.0554

1.05139

1.04342

1.03946

1.0355
1.03156

1.02764

1.02372

1.01982

1.01593

1.01206
1.0082

1.0082
1.00435

1.00051

0.996684

0.992872

0.989072

0.985285

0.98151

0.977748

0.97026

0.966536

0.962824

0.959124

0.955436

0.95176

0.948097

0.944446

0.940807

0.937181

0.933566

0.929964

0.926373

0.922795

0.919229

0.915674

0.912132

0.908602

0.905083

0.901577

0.898082

0.894599

0.891128

0.887669

0.884222

0.880786

0.877362

0.873949
Printed 10/2/0

$11.2041 \mid \quad 19.9184$

\begin{tabular}{l|l}
11.1627 \\
11.1213
\end{tabular}$\quad 19.8447$

11.0801

10.9981

10.9573

10.9167

10.8761

10.8357

10.7954
10.7552

10.7152

10.6752

10.6354

10.5958

10.5562

10.5168
10.4775

10.4383

10.3993

10.3604

10.3215

10.2829

10.2443

10.1676

10.1294

10.0913

10.0534

10.0155

9.97783

9.90279

9.86544

9.82822

9.79112

9.75413

9.71727

9.64389

9.60738

9.57099

9.53472

9.49856

9.46253

9.4266

9.3908

9.35511
9.31954

9.28408

9.24874

9.21352

9.17841

9.14341

9.10853

9.07377

9.03912

8.97016

8.93585

8.93585

8.90165
8.86756

8.83359

8.79973

8.76599

8.73235

8.69883

8.66542

8.63211

8.59892

8.56584

8.53287

8.50001

8.46727
8.43462

8.40209

8.36967

8.33736

8.30515

8.27306

8.24107

8.20919

8.17742

8.14575

8.11419

8.08274

8.05139

8.02015
7.98902

7.95799

7.92707

7.89625

7.86554

7.86554
7.83494

19.698

19.625
19.5523

19.4797

19.4074

19.4074
19.3353

19.2634

19.1918

19.1204

19.0492

18.9074

18.9074
18.8369

18.7666

18.6965

18.6267
18.557

18.4876

18.4876
18.4184

18.3494

18.2807

18.2121

18.1438

18.0757

18.0078

17.9401
17.8726

17.8726
17.8054

17.7384 
Table_of_specs_Rev0.xls,CH Surface roughness

0.00903434 0.00900995

$788 \mid-0.0165$ 0.0165 0.0165

0.0164
0.0164

0.0164

0.0164

0.0163

0.0163

0.0163
0.0163

0.0162

0.0162

0.0162

0.0162
0.0161

0.0161

0.0161

0.0161

0.016
0.016
0.016
0.016

0.016

0.0159

0.015

0.0159

0.0158

0.0158

0.0158

0.0157

0.0157

0.0157

0.0157

0.0156
0.0156

0.0156

0.015

0.0156

0.0155

0.0155
0.0155

0.0155

0.0154

0.0154

0.0154

0.0154

0.0153

0.0153

0.015

0.0152

0.0152

0.0152
0.0152

0.0151

0.0151

0.0151

0.0151

0.015

0.015

0.015
0.015

0.0149

0.0149

0.0149

0.0149

0.0148
0.0148

0.0148
0.0148

0.0148

0.0148

0.0147

0.0147

0.0147

0.0147

0.0146

0.0146

0.0146

0.0146

0.0145
0.0145

0.0145

0.0145

0.0145

0.0144

0.0144

0.0144

0.0144

0.0143

0.0143

0.0143

0.0143
0.0142
0.0142

0.0142
0.0142
0.00898563

0.00896137

0.00893717

0.00888896

0.00886494

0.00884099

0.00881709

0.00879326

0.00876948
0.00874577

0.00872212

0.00869852

0.00867499

0.00865151

0.0086281
0.00860474

0.00858145

0.00855821

0.00853503

0.00851191

0.00848885

0.00844291

0.00842002

0.0083972

0.00837443

0.00835171

0.00832906

0.00828393

0.00826144

0.00823902

0.00821665

0.00819434

0.00817209

0.00812775

0.00810567

0.00808364

0.00806167

0.00803976

0.0080179
0.00799609

0.00797434

0.00795265

0.00793101

0.00790943
0.00788791

0.00786643

0.00784502

0.00782365

0.00780235

0.00778109
0.00775989

0.00773875

0.00771766

0.00769662

0.00767564

0.00765471

0.00763383
0.00761301

0.00759224

0.00755087

0.00753026
0.0075097

0.0074892

0.00746874

0.00744835

0.007428

0.00740771

0.00738746
0.00736727
0.00734714

0.00734714

0.00732705

0.00728703

0.0072671

0.00724722
0.00722739

0.00720761

0.00718789

0.00716821

0.00714859

0.00712901

0.00710949
0.00709001

0.00707059

0.00705122

0.00703189

0.00701262

0.00699339
0.0346864 0.0345513

0.0344166

0.0342824

0.0341487

0.0340154

0.0337503

0.0336184

0.033487

0.033356

0.0330953

0.0329657

0.0328365

0.0327078

0.0325795

0.0324516

0.0321973

0.0320707

0.0319447

0.031819

0.0316938

0.0315691
0.0314447

0.0313208

0.0311974

0.0310744

0.0309518

0.0308296

0.0307079

0.0304657

0.0303452

0.0302252

0.0301056

0.0299864

0.0298676

0.0297493

0.0296314
0.0295139

0.0293968

0.0292801

0.0291639

0.029048

0.0289326

0.0288176

0.028703
0.0285888

0.028475

0.0283617

0.0282487

0.0281361

0.028024

0.0279122

0.0278008

0.0276899

0.0275793

0.0274692

0.0273594

0.0272501

0.0271411

0.0270325

0.0269243

0.0267092

0.0266022

0.0264955

0.0263893

0.0262835

0.026178

0.026073

0.0259683

025864

0.02576

0.0256565

0.0255533

0.0254505

0.0253481

0.0252461

0.0251444

0.0250432

0.0249423

0.0248417

0.0247416

0.0245423

0.0244433

0.0243446

0.0242462

0.0241483

0.0240507

0.0239534

0.0238566

Page 10 of 33

0.867159

0.863782

0.860415

0.857061
0.853718

0.850386

0.847066

0.843757

0.84046

0.833899

0.830636

0.827384

0.824143

0.817694

0.814487

0.808106

0.804932

0.801769

0.798617

0.795476

0.792346

0.789226
0.786118

0.783021

0.779934

0.776859

0.773794

0.767696

0.764664

0.761642

0.75863

0.75563

0.75264
0.74966

0.746691

0.743733

0.740785

0.737847

0.73492

0.732003

0.729097

0.723316

0.72044

0.717575 
Table_of_specs_Rev0.xls,CH Surface roughness

\begin{tabular}{|c|c|c|}
\hline 883 & 0.0142 & 0.00697422 \\
\hline 884 & 0.0142 & 0.0069551 \\
\hline 885 & 0.0142 & 0.00693602 \\
\hline 886 & 0.0141 & 0.00691699 \\
\hline 887 & 0.0141 & 0.00689802 \\
\hline 888 & 0.0141 & 0.00687909 \\
\hline 889 & 0.0141 & 0.00686021 \\
\hline 890 & 0.014 & 0.00684138 \\
\hline 891 & 0.014 & 0.0068226 \\
\hline 892 & 0.014 & 0.00680387 \\
\hline 893 & 0.014 & 0.00678519 \\
\hline 894 & 0.014 & 0.00676655 \\
\hline 895 & 0.0139 & 0.00674796 \\
\hline 896 & 0.0139 & 0.00672943 \\
\hline 897 & 0.0139 & 0.00671094 \\
\hline 898 & 0.0139 & 0.00669249 \\
\hline 899 & 0.0138 & 0.0066741 \\
\hline 900 & 0.0138 & 0.00665575 \\
\hline 901 & 0.0138 & 0.00663745 \\
\hline 902 & 0.0138 & 0.0066192 \\
\hline 903 & 0.0138 & 0.006601 \\
\hline 904 & 0.0137 & 0.00658284 \\
\hline 905 & 0.0137 & 0.00656473 \\
\hline 906 & 0.0137 & 0.00654667 \\
\hline 907 & 0.0137 & 0.00652865 \\
\hline 908 & 0.0136 & 0.00651069 \\
\hline 909 & 0.0136 & 0.00649276 \\
\hline 910 & 0.0136 & 0.00647489 \\
\hline 911 & 0.0136 & 0.00645706 \\
\hline 912 & 0.0136 & 0.00643928 \\
\hline 913 & 0.0135 & 0.00642154 \\
\hline 914 & 0.0135 & 0.00640386 \\
\hline 915 & 0.0135 & 0.00638621 \\
\hline 916 & 0.0135 & 0.00636862 \\
\hline 917 & 0.0135 & 0.00635106 \\
\hline 918 & 0.0134 & 0.00633356 \\
\hline 919 & 0.0134 & 0.0063161 \\
\hline 920 & 0.0134 & 0.00629869 \\
\hline 921 & 0.0134 & 0.00628132 \\
\hline 922 & 0.0133 & 0.00626399 \\
\hline 923 & 0.0133 & 0.00624672 \\
\hline 924 & 0.0133 & 0.00622949 \\
\hline 925 & 0.0133 & 0.0062123 \\
\hline 926 & 0.0133 & 0.00619516 \\
\hline 927 & 0.0132 & 0.00617806 \\
\hline 928 & 0.0132 & 0.00616101 \\
\hline 929 & 0.0132 & 0.006144 \\
\hline 930 & 0.0132 & 0.00612704 \\
\hline 931 & 0.0132 & 0.00611012 \\
\hline 932 & 0.0131 & 0.00609324 \\
\hline 933 & 0.0131 & 0.00607641 \\
\hline 934 & 0.0131 & 0.00605963 \\
\hline 935 & 0.0131 & 0.00604289 \\
\hline 936 & 0.0131 & 0.00602619 \\
\hline 937 & 0.013 & 0.00600953 \\
\hline 938 & 0.013 & 0.00599292 \\
\hline 939 & 0.013 & 0.00597636 \\
\hline 940 & 0.013 & 0.00595983 \\
\hline 941 & 0.0129 & 0.00594335 \\
\hline 942 & 0.0129 & 0.00592692 \\
\hline 943 & 0.0129 & 0.00591053 \\
\hline 944 & 0.0129 & 0.00589418 \\
\hline 945 & 0.0129 & 0.00587787 \\
\hline 946 & 0.0128 & 0.00586161 \\
\hline 947 & 0.0128 & 0.00584539 \\
\hline 948 & 0.0128 & 0.00582921 \\
\hline 949 & 0.0128 & 0.00581307 \\
\hline 950 & 0.0128 & 0.00579698 \\
\hline 951 & 0.0127 & 0.00578093 \\
\hline 952 & 0.0127 & 0.00576492 \\
\hline 953 & 0.0127 & 0.00574896 \\
\hline 954 & 0.0127 & 0.00573303 \\
\hline 955 & 0.0127 & 0.00571715 \\
\hline 956 & 0.0126 & 0.00570131 \\
\hline 957 & 0.0126 & 0.00568552 \\
\hline 958 & 0.0126 & 0.00566976 \\
\hline 959 & 0.0126 & 0.00565405 \\
\hline 960 & 0.0126 & 0.00563838 \\
\hline 961 & 0.0125 & 0.00562274 \\
\hline 962 & 0.0125 & 0.00560716 \\
\hline 963 & 0.0125 & 0.00559161 \\
\hline 964 & 0.0125 & 0.0055761 \\
\hline 965 & 0.0125 & 0.00556063 \\
\hline 966 & 0.0124 & 0.00554521 \\
\hline 967 & 0.0124 & 0.00552983 \\
\hline 968 & 0.0124 & 0.00551448 \\
\hline 969 & 0.0124 & 0.00549918 \\
\hline 970 & 0.0124 & 0.00548392 \\
\hline 971 & 0.0123 & 0.0054687 \\
\hline 972 & 0.0123 & 0.00545352 \\
\hline 973 & 0.0123 & 0.00543838 \\
\hline 974 & 0.0123 & 0.00542328 \\
\hline 975 & 0.0123 & 0.00540822 \\
\hline 976 & 0.0122 & 0.0053932 \\
\hline 977 & 0.0122 & 0.00537822 \\
\hline
\end{tabular}

\begin{tabular}{|c|}
\hline 0.02376 \\
\hline 0.0236639 \\
\hline 0.0235681 \\
\hline 0.0234726 \\
\hline 0.0233776 \\
\hline 0.0232828 \\
\hline 0.0231884 \\
\hline 0.0230944 \\
\hline 0.0230007 \\
\hline 0.0229074 \\
\hline 0.0228144 \\
\hline 0.0227218 \\
\hline 0.0226295 \\
\hline 0.0225376 \\
\hline 0.022446 \\
\hline 0.0223548 \\
\hline 0.0222639 \\
\hline 0.0221733 \\
\hline 0.0220831 \\
\hline 0.0219933 \\
\hline 0.0219037 \\
\hline 0.0218145 \\
\hline 0.0217257 \\
\hline 0.0216372 \\
\hline 0.021549 \\
\hline 0.0214611 \\
\hline 0.0213736 \\
\hline 0.0212864 \\
\hline 0.0211996 \\
\hline 0.021113 \\
\hline 0.0210268 \\
\hline 0.020941 \\
\hline 0.0208554 \\
\hline 0.0207702 \\
\hline 0.0206853 \\
\hline 0.0206008 \\
\hline 0.0205165 \\
\hline 0.0204326 \\
\hline 0.020349 \\
\hline 0.0202658 \\
\hline 0.0201828 \\
\hline 0.0201002 \\
\hline 0.0200178 \\
\hline 0.0199358 \\
\hline 0.0198541 \\
\hline 0.0197728 \\
\hline 0.0196917 \\
\hline 0.0196109 \\
\hline 0.0195305 \\
\hline 0.0194504 \\
\hline 0.0193706 \\
\hline 0.019291 \\
\hline 0.0192118 \\
\hline 0.0191329 \\
\hline 0.0190544 \\
\hline 0.0189761 \\
\hline 0.0188981 \\
\hline 0.0188204 \\
\hline 0.018743 \\
\hline 0.0186659 \\
\hline 0.0185892 \\
\hline 0.0185127 \\
\hline 0.0184365 \\
\hline 0.0183606 \\
\hline 0.018285 \\
\hline 0.0182097 \\
\hline 0.0181347 \\
\hline 0.01806 \\
\hline 0.0179856 \\
\hline 0.0179114 \\
\hline 0.0178376 \\
\hline 0.0177641 \\
\hline 0.0176908 \\
\hline 0.0176178 \\
\hline 0.0175451 \\
\hline 0.0174727 \\
\hline 0.0174006 \\
\hline 0.0173288 \\
\hline 0.0172572 \\
\hline 0.0171859 \\
\hline 0.017115 \\
\hline 0.0170442 \\
\hline 0.0169738 \\
\hline 0.0169037 \\
\hline 0.0168338 \\
\hline 0.0167642 \\
\hline 0.0166948 \\
\hline 0.0166258 \\
\hline 0.016557 \\
\hline 0.0164885 \\
\hline 0.0164203 \\
\hline 0.0163523 \\
\hline 0.0162846 \\
\hline 0.0162172 \\
\hline \\
\hline
\end{tabular}

Page 11 of 33
0.594001

0.591597

0.589202

0.586816

0.584439

0.579711

0.57736

0.575019

0.572685
0.570361

0.568046

0.565739

0.56344

0.561151
0.55887

0.556597

0.554334

0.552078

0.549831

0.547593

0.545363

0.543142
0.540929

0.538724

0.536528

0.53434

0.53216

0.529989

0.527826

0.525671
0.523525

0.523525

0.519256

0.517134

0.51502

0.512914

0.510816

0.508726

0.506644
0.50457

0.502504

0.500446

0.498396

0.496353

0.492292

0.490274

0.488263
0.486259

0.484264

0.482276

0.480296

0.478324

0.476359

0.474402

0.472452

0.47051
0.468575

0.466648
0.464729

0.464729
0.462817

0.462817
0.460912

0.459015

0.457125

0.455243

0.453368

0.4515
0.449639

0.449639
0.447786

0.447786

0.44594
0.444101

0.44227

0.440445

0.438628
0.436818

0.436818

0.435015
0.433219

0.433219
0.43143

0.429649

0.427874
0.426106

0.424345

0.422591

0.420844

0.419104

0.417371

0.415645

0.413925
0.412212

0.410506

0.408807

0.407115

0.405429
0.40375
Printed 10/2/05,4.24 PM

5.34601

5.32437
5.30282

\begin{tabular}{l|l}
5.30282 & 9.42723 \\
5.28134 & 9.38906 \\
5.25995 & 9.3510
\end{tabular}

$5.25995 \quad 9.35102$

5.23863
5.2174

\begin{tabular}{r|r|}
5.19624 & 9.23777 \\
5.17557 & 9.2003
\end{tabular}

\begin{tabular}{r|r|}
5.17517 & 9.2003 \\
5.15417 & 9.16297 \\
\hline
\end{tabular}

\begin{tabular}{l|l}
5.15417 & 9.16297 \\
5.13325 & 9.12578 \\
5.1241 & 9.08873
\end{tabular}

$5.11241 \quad 9.08873$

5.09165
5.07096

$5.07096 \quad 9.01505$

$5.05036 \quad 8.97841$

\begin{tabular}{l|r|}
5.02983 & 8.94192 \\
5.00938 & 8.90556
\end{tabular}

5.90556
4.989 $\quad 8.86934$

4.9687

4.94848

4.92834

4.90827

4.88828

4.86836
4.84852

4.84852

4.80906

4.78944

4.7699

4.75043

4.73104

4.69247

4.6733

4.6542

4.63518

4.61622
4.59734

4.57853

4.55979

4.54113

4.52253

4.50401
4.48556

4.48556
4.46718

4.44887

4.43063

4.41246

4.39436

4.37634

4.35838

4.34049

4.32266

4.30491

4.28723
4.26961

4.25207

4.23459

4.21718

4.19984

4.18256

4.16535

4.14821

4.13113

4.11413

4.09718

4.08031

4.0635

4.04675

4.03007

4.01346

3.99691

3.98043

3.96401

3.94765
3.93136

3.91514

3.89897

3.88287

3.86684

3.85086

3.83495
3.81911

3.81911
3.80332

3.7876

3.77194

3.75634

3.7408

3.72533

3.70991
3.69456

3.69456

3.66403

3.66403

3.64886
3.63375

8.83325
8.7973

8.7973

8.76149
8.72581

8.69027

8.65486

8.61959

8.58445

8.51457

8.47983

8.44522

8.41074

8.37639
8.34218

30809

8.30809

8.24031

8.20662

8.17305

8.13961

8.1063
8.07312
8.04006

8.04006

8.00713

7.97433

7.94166
7.9091
7.87668

7.87668

7.84438

7.8122

7.78015

7.74822

7.71642

7.65318

7.62174

7.59043

7.55923

7.52816

7.46637

7.43566

7.40507

7.37459

7.34424
7.314
7.28388

7.28388

7.25388

7.224

7.19423

7.16458

7.10562

7.07632

7.04713

7.01805

6.98909

6.96024

6.93151

6.87438

6.84598

6.8177

6.78952

6.76146

6.73351

6.70567

6.67794

6.65031

6.5954

6.5681

6.54092

6.51384

6.48686 
Table_of_specs_Rev0.xls,CH Surface roughness

\begin{tabular}{|c|c|c|}
\hline 978 & 0.0122 & 0.00536328 \\
\hline 979 & 0.0122 & 0.00534838 \\
\hline 980 & 0.0122 & 0.00533352 \\
\hline 981 & 0.0121 & 0.0053187 \\
\hline 982 & 0.0121 & 0.00530392 \\
\hline 983 & 0.0121 & 0.00528918 \\
\hline 984 & 0.0121 & 0.00527447 \\
\hline 985 & 0.0121 & 0.00525981 \\
\hline 986 & 0.012 & 0.00524519 \\
\hline 987 & 0.012 & 0.0052306 \\
\hline 988 & 0.012 & 0.00521605 \\
\hline 989 & 0.012 & 0.00520155 \\
\hline 990 & 0.012 & 0.00518708 \\
\hline 991 & 0.0119 & 0.00517265 \\
\hline 992 & 0.0119 & 0.00515826 \\
\hline 993 & 0.0119 & 0.00514391 \\
\hline 994 & 0.0119 & 0.00512959 \\
\hline 995 & 0.0119 & 0.00511531 \\
\hline 996 & 0.0118 & 0.00510108 \\
\hline 997 & 0.0118 & 0.00508688 \\
\hline 998 & 0.0118 & 0.00507272 \\
\hline 999 & 0.0118 & 0.00505859 \\
\hline 000 & 0.0118 & 0.00504451 \\
\hline 001 & 0.0118 & 0.00503046 \\
\hline 002 & 0.0117 & 0.00501645 \\
\hline 003 & 0.0117 & 0.00500248 \\
\hline 004 & 0.0117 & 0.00498854 \\
\hline 005 & 0.0117 & 0.00497464 \\
\hline 006 & 0.0117 & 0.00496078 \\
\hline 007 & 0.0116 & 0.00494696 \\
\hline 008 & 0.0116 & 0.00493317 \\
\hline 009 & 0.0116 & 0.00491942 \\
\hline 010 & 0.0116 & 0.00490571 \\
\hline 011 & 0.0116 & 0.00489204 \\
\hline 012 & 0.0115 & 0.0048784 \\
\hline 013 & 0.0115 & 0.0048648 \\
\hline 014 & 0.0115 & 0.00485123 \\
\hline 015 & 0.0115 & 0.0048377 \\
\hline 016 & 0.0115 & 0.00482421 \\
\hline 017 & 0.0114 & 0.00481076 \\
\hline 018 & 0.0114 & 0.00479734 \\
\hline 019 & 0.0114 & 0.00478395 \\
\hline 020 & 0.0114 & 0.00477061 \\
\hline 021 & 0.0114 & 0.0047573 \\
\hline 022 & 0.0114 & 0.00474402 \\
\hline 023 & 0.0113 & 0.00473078 \\
\hline 024 & 0.0113 & 0.00471758 \\
\hline 025 & 0.0113 & 0.00470441 \\
\hline 026 & 0.0113 & 0.00469128 \\
\hline 027 & 0.0113 & 0.00467818 \\
\hline 02 & 0.0112 & 0.00466512 \\
\hline 029 & 0.0112 & 0.0046521 \\
\hline 030 & 0.0112 & 0.00463911 \\
\hline 031 & 0.0112 & 0.00462615 \\
\hline 032 & 0.0112 & 0.00461323 \\
\hline 033 & 0.0112 & 0.00460034 \\
\hline 0 & 0.0111 & 0.00458749 \\
\hline 0 & 0.0111 & 0.00457468 \\
\hline 036 & 0.0111 & 0.0045619 \\
\hline 03 & 0.0111 & 0.00454915 \\
\hline 0 & 0.0111 & 0.00453644 \\
\hline 03 & 0.011 & 0.00452376 \\
\hline 0 & 0.011 & 0.00451112 \\
\hline 0 & 0.011 & 0.00449851 \\
\hline 042 & 0.011 & 0.00448594 \\
\hline 0 & 0.011 & 0.0044734 \\
\hline 0 & 0.011 & 0.00446089 \\
\hline 04 & 0.0109 & 0.00444842 \\
\hline 0 & 0.0109 & 0.00443598 \\
\hline 04 & 0.0109 & 0.00442358 \\
\hline 048 & 0.0109 & 0.00441121 \\
\hline & 0.0109 & 0.00439887 \\
\hline & 0.0108 & 0.00438657 \\
\hline 0 & 0.0108 & 0.0043743 \\
\hline 05 & 0.0108 & 0.00436206 \\
\hline & 0.0108 & 0.00434986 \\
\hline 0 & 0.0108 & 0.00433769 \\
\hline & 0.0108 & 0.00432555 \\
\hline & 0.0107 & 0.00431345 \\
\hline 0 & 0.0107 & 0.00430138 \\
\hline & 0.0107 & 0.00428934 \\
\hline & 0.0107 & 0.00427734 \\
\hline Oe & 0.0107 & 0.00426536 \\
\hline & 0.0107 & 0.00425343 \\
\hline & 0.0106 & 0.00424152 \\
\hline & 0.0106 & 0.00422965 \\
\hline & 0.0106 & 0.0042178 \\
\hline & 0.0106 & 0.004206 \\
\hline & 0.0106 & 0.00419422 \\
\hline & 0.0105 & 0.00418247 \\
\hline & 0.0105 & 0.00417076 \\
\hline & 0.0105 & 0.00415908 \\
\hline & 0.0105 & 0.00414743 \\
\hline & 0.0105 & 0.00413582 \\
\hline & 0.0105 & 0.00412423 \\
\hline
\end{tabular}

\begin{tabular}{|c|c|}
\hline 0.0160831 & 0.402078 \\
\hline 0.0160165 & 0.400412 \\
\hline 0.0159501 & 0.398753 \\
\hline 0.015884 & 0.3971 \\
\hline 0.0158182 & 0.395454 \\
\hline 0.0157526 & 0.393815 \\
\hline 0.0156873 & 0.392182 \\
\hline 0.0156222 & 0.390556 \\
\hline 0.0155574 & 0.388936 \\
\hline 0.0154929 & 0.387322 \\
\hline 0.0154286 & 0.385715 \\
\hline 0.0153646 & 0.384115 \\
\hline 0.0153008 & 0.382521 \\
\hline 0.0152373 & 0.380933 \\
\hline 0.0151741 & 0.379352 \\
\hline 0.0151111 & 0.377777 \\
\hline 0.0150483 & 0.376208 \\
\hline 0.0149858 & 0.374645 \\
\hline 0.0149236 & 0.373089 \\
\hline 0.0148616 & 0.371539 \\
\hline 0.0147998 & 0.369995 \\
\hline 0.0147383 & 0.368458 \\
\hline 0.0146771 & 0.366927 \\
\hline 0.0146161 & 0.365401 \\
\hline 0.0145553 & 0.363882 \\
\hline 0.0144948 & 0.362369 \\
\hline 0.0144345 & 0.360862 \\
\hline 0.0143745 & 0.359362 \\
\hline 0.0143147 & 0.357867 \\
\hline 0.0142551 & 0.356378 \\
\hline 0.0141958 & 0.354896 \\
\hline 0.0141368 & 0.353419 \\
\hline 0.0140779 & 0.351948 \\
\hline 0.0140193 & 0.350483 \\
\hline 0.013961 & 0.349024 \\
\hline 0.0139029 & 0.347571 \\
\hline 0.013845 & 0.346124 \\
\hline 0.0137873 & 0.344683 \\
\hline 0.0137299 & 0.343248 \\
\hline 0.0136727 & 0.341818 \\
\hline 0.0136158 & 0.340394 \\
\hline 0.0135591 & 0.338976 \\
\hline 0.0135026 & 0.337564 \\
\hline 0.0134463 & 0.336158 \\
\hline 0.0133903 & 0.334757 \\
\hline 0.0133345 & 0.333362 \\
\hline 0.0132789 & 0.331972 \\
\hline 0.0132235 & 0.330588 \\
\hline 0.0131684 & 0.32921 \\
\hline 0.0131135 & 0.327838 \\
\hline 0.0130588 & 0.326471 \\
\hline 0.0130044 & 0.325109 \\
\hline 0.0129501 & 0.323754 \\
\hline 0.0128961 & 0.322403 \\
\hline 0.0128423 & 0.321059 \\
\hline 0.0127888 & 0.319719 \\
\hline 0.0127354 & 0.318385 \\
\hline 0.0126823 & 0.317057 \\
\hline 0.0126294 & 0.315734 \\
\hline 0.0125767 & 0.314417 \\
\hline 0.0125242 & 0.313105 \\
\hline 0.0124719 & 0.311798 \\
\hline 0.0124199 & 0.310496 \\
\hline 0.012368 & 0.3092 \\
\hline 0.0123164 & 0.30791 \\
\hline 0.012265 & 0.306624 \\
\hline 0.0122138 & 0.305344 \\
\hline 0.0121628 & 0.304069 \\
\hline 0.012112 & 0.302799 \\
\hline 0.0120614 & 0.301535 \\
\hline 0.012011 & 0.300276 \\
\hline 0.0119609 & 0.299022 \\
\hline 0.0119109 & 0.297773 \\
\hline 0.0118612 & 0.296529 \\
\hline 0.0118116 & 0.29529 \\
\hline 0.0117623 & 0.294057 \\
\hline 0.0117131 & 0.292828 \\
\hline 0.0116642 & 0.291605 \\
\hline 0.0116154 & 0.290386 \\
\hline 0.0115669 & 0.289173 \\
\hline 0.0115186 & 0.287965 \\
\hline 0.0114704 & 0.286761 \\
\hline 0.0114225 & 0.285563 \\
\hline 0.0113748 & 0.284369 \\
\hline 0.0113272 & 0.283181 \\
\hline 0.0112799 & 0.281997 \\
\hline 0.0112327 & 0.280818 \\
\hline 0.0111858 & 0.279645 \\
\hline 0.011139 & 0.278476 \\
\hline 0.0110925 & 0.277312 \\
\hline 0.0110461 & 0.276152 \\
\hline 0.0109999 & 0.274998 \\
\hline 0.0109539 & 0.273848 \\
\hline 0.0109081 & 0.272703 \\
\hline 0.0108625 & 0.271563 \\
\hline
\end{tabular}

3.6187 3.60371

3.58877

3.5739
3.55909

3.54433

3.52964

3.515

3.50042

3.4859
3.47144
3.45703

3.45703

3.44269

3.4284

3.41416

3.39999

3.38587

3.37181

3.3578

3.3299

3.32996

3.30234

3.28861

3.27494

3.26132

3.2477

3.23426
3.2208

3.2074

3.19406

3.18077

3.16753

3.15435

3.14122

3.12814
3.11512

3.10215

3.08923

3.07636

3.06355

3.05079

3.03808
3.02542

3.01281

3.00025

2.98775

2.9753

2.96289
2.95054

2.93824

2.92599

2.91378

2.90163

2.88953

2.87747

2.86547

2.85351

2.84161

2.82975

2.81794
2.80618

2.79447

2.7828
2.77119

2.77119

2.7481

2.73662

2.72519

2.71381

2.70248

2.69119

2.67995

2.66876

2.65761

2.64651

2.63545

2.62444

2.61348

2.60256

2.59168

2.58085
2.57006

2.55932

2.54863

2.53797

2.52737

2.5168

2.50628

2.4958
2.48537

2.47498

(.47498

2.45433
2.44407
Printed 10/2/05

6.43324

0.3800

6.38004
6.3536
6.32727

6.32727

6.30104

.27491

6.24889

6.22297

6.19716

6.17145

6.12033

6.09493

6.06963

6.04443

6.01933

5.99433

5.96943

5.91993

5.89533

5.87082

5.84642

5.82212

5.79791
5.7738

5.7738
5.74979
5.72587

5.72587

5.70205

5.67833

5.6547
.63117

5.60773

5.58439

5.56114

5.53799

5.51493

5.49196

5.46909

5.42362

5.40102

5.37852

5.33379

5.31156

5.28941

5.26736

5.2454
5.22353
5.2015

5.20175

5.18006

5.15845

5.13694

5.11551

5.09417

5.07291

5.03067

5.00967

4.98877

4.96794

4.94721

.92655

4.98555
4.8651

4.8651

4.84479

4.82456

4.80441

4.78434

4.76436

4.74446

4.72464

4.7049

4.66567

4.64618

4.62677

4.60743

4.58818

4.569

4.53089

4.51196

4.4931

4.47431

4.45561

4.41844

4.39996

4.38157

4.36325

Page 12 of 33 


\begin{tabular}{|c|c|c|}
\hline 1073 & 0.0104 & 0.00411268 \\
\hline 1074 & 0.0104 & 0.00410116 \\
\hline 1075 & 0.0104 & 0.00408967 \\
\hline 1076 & 0.0104 & 0.00407821 \\
\hline 1077 & 0.0104 & 0.00406678 \\
\hline 1078 & 0.0104 & 0.00405539 \\
\hline 1079 & 0.0103 & 0.00404402 \\
\hline 1080 & 0.0103 & 0.00403269 \\
\hline 1081 & 0.0103 & 0.00402139 \\
\hline 1082 & 0.0103 & 0.00401012 \\
\hline 1083 & 0.0103 & 0.00399888 \\
\hline 1084 & 0.0103 & 0.00398767 \\
\hline 1085 & 0.0102 & 0.00397649 \\
\hline 1086 & 0.0102 & 0.00396535 \\
\hline 1087 & 0.0102 & 0.00395423 \\
\hline 1088 & 0.0102 & 0.00394314 \\
\hline 1089 & 0.0102 & 0.00393209 \\
\hline 1090 & 0.0102 & 0.00392106 \\
\hline 1091 & 0.0101 & 0.00391007 \\
\hline 1092 & 0.0101 & 0.00389911 \\
\hline 1093 & 0.0101 & 0.00388817 \\
\hline 1094 & 0.0101 & 0.00387727 \\
\hline 1095 & 0.0101 & 0.00386639 \\
\hline 1096 & 0.0101 & 0.00385555 \\
\hline 1097 & 0.01 & 0.00384474 \\
\hline 1098 & 0.01 & 0.00383395 \\
\hline 1099 & 0.01 & 0.0038232 \\
\hline 1100 & 0.00998 & 0.00381247 \\
\hline 1101 & 0.00996 & 0.00380178 \\
\hline 1102 & 0.00995 & 0.00379111 \\
\hline 1103 & 0.00993 & 0.00378048 \\
\hline 1104 & 0.00992 & 0.00376987 \\
\hline 1105 & 0.0099 & 0.00375929 \\
\hline 1106 & 0.00988 & 0.00374875 \\
\hline 1107 & 0.00986 & 0.00373823 \\
\hline 1108 & 0.00985 & 0.00372774 \\
\hline 1109 & 0.00983 & 0.00371728 \\
\hline 1110 & 0.00982 & 0.00370685 \\
\hline 1111 & 0.0098 & 0.00369644 \\
\hline 1112 & 0.00978 & 0.00368607 \\
\hline 1113 & 0.00977 & 0.00367572 \\
\hline 1114 & 0.00975 & 0.00366541 \\
\hline 1115 & 0.00973 & 0.00365512 \\
\hline 1116 & 0.00972 & 0.00364486 \\
\hline 1117 & 0.0097 & 0.00363463 \\
\hline 1118 & 0.00969 & 0.00362443 \\
\hline 1119 & 0.00967 & 0.00361425 \\
\hline 1120 & 0.00965 & 0.00360411 \\
\hline 1121 & 0.00963 & 0.00359399 \\
\hline 1122 & 0.00962 & 0.0035839 \\
\hline 1123 & 0.0096 & 0.00357384 \\
\hline 1124 & 0.00959 & 0.0035638 \\
\hline 1125 & 0.00957 & 0.0035538 \\
\hline 1126 & 0.00956 & 0.00354382 \\
\hline 1127 & 0.00954 & 0.00353387 \\
\hline 1128 & 0.00952 & 0.00352395 \\
\hline 1129 & 0.0095 & 0.00351405 \\
\hline 1130 & 0.00949 & 0.00350419 \\
\hline 1131 & 0.00947 & 0.00349435 \\
\hline 1132 & 0.00946 & 0.00348453 \\
\hline 1133 & 0.00944 & 0.00347475 \\
\hline 1134 & 0.00943 & 0.00346499 \\
\hline 1135 & 0.00941 & 0.00345526 \\
\hline 1136 & 0.00939 & 0.00344556 \\
\hline 1137 & 0.00938 & 0.00343588 \\
\hline 1138 & 0.00936 & 0.00342623 \\
\hline 1139 & 0.00934 & 0.00341661 \\
\hline 1140 & 0.00933 & 0.00340701 \\
\hline 1141 & 0.00931 & 0.00339744 \\
\hline 1142 & 0.0093 & 0.0033879 \\
\hline 1143 & 0.00928 & 0.00337838 \\
\hline 1144 & 0.00927 & 0.00336889 \\
\hline 1145 & 0.00925 & 0.00335943 \\
\hline 1146 & 0.00924 & 0.00335 \\
\hline 1147 & 0.00922 & 0.00334059 \\
\hline 1148 & 0.0092 & 0.0033312 \\
\hline 1149 & 0.00919 & 0.00332184 \\
\hline 1150 & 0.00917 & 0.00331251 \\
\hline 1151 & 0.00915 & 0.00330321 \\
\hline 1152 & 0.00914 & 0.00329393 \\
\hline 1153 & 0.00912 & 0.00328468 \\
\hline 1154 & 0.00911 & 0.00327545 \\
\hline 1155 & 0.00909 & 0.00326625 \\
\hline 1156 & 0.00908 & 0.00325707 \\
\hline 1157 & 0.00906 & 0.00324792 \\
\hline 1158 & 0.00905 & 0.0032388 \\
\hline 1159 & 0.00903 & 0.0032297 \\
\hline 1160 & 0.00902 & 0.00322063 \\
\hline 1161 & 0.009 & 0.00321158 \\
\hline 1162 & 0.00899 & 0.00320256 \\
\hline 1163 & 0.00897 & 0.00319356 \\
\hline 1164 & 0.00895 & 0.00318459 \\
\hline 1165 & 0.00894 & 0.00317564 \\
\hline 1166 & 0.00892 & 0.00316672 \\
\hline 1167 & 0.00891 & 0.00315782 \\
\hline
\end{tabular}

\begin{tabular}{|c|c|}
\hline 0.0108171 & 0.270427 \\
\hline 0.0107719 & 0.269297 \\
\hline 0.0107268 & 0.268171 \\
\hline 0.010682 & 0.267049 \\
\hline 0.0106373 & 0.265933 \\
\hline 0.0105928 & 0.26482 \\
\hline 0.0105485 & 0.263713 \\
\hline 0.0105044 & 0.26261 \\
\hline 0.0104605 & 0.261512 \\
\hline 0.0104167 & 0.260418 \\
\hline 0.0103732 & 0.259329 \\
\hline 0.0103298 & 0.258245 \\
\hline 0.0102866 & 0.257164 \\
\hline 0.0102436 & 0.256089 \\
\hline 0.0102007 & 0.255018 \\
\hline 0.010158 & 0.253951 \\
\hline 0.0101156 & 0.252889 \\
\hline 0.0100733 & 0.251831 \\
\hline 0.0100311 & 0.250778 \\
\hline 0.00998916 & 0.249729 \\
\hline .00994738 & 0.248684 \\
\hline .00990577 & 0.247644 \\
\hline 0.00986434 & 0.246609 \\
\hline .00982308 & 0.245577 \\
\hline .00978199 & 0.24455 \\
\hline .00974108 & 0.243527 \\
\hline .00970033 & 0.242508 \\
\hline .00965976 & 0.241494 \\
\hline .00961936 & 0.240484 \\
\hline .00957912 & 0.239478 \\
\hline .00953906 & 0.238476 \\
\hline 0.00949916 & 0.237479 \\
\hline .00945943 & 0.236486 \\
\hline 0.00941987 & 0.235497 \\
\hline 0.00938047 & 0.234512 \\
\hline .00934124 & 0.233531 \\
\hline 0.00930217 & 0.232554 \\
\hline 0.00926327 & 0.231582 \\
\hline .00922453 & 0.230613 \\
\hline .00918595 & 0.229649 \\
\hline 0.00914753 & 0.228688 \\
\hline 0.00910928 & 0.227732 \\
\hline 0.00907119 & 0.22678 \\
\hline 0.00903325 & 0.225831 \\
\hline 0.00899548 & 0.224887 \\
\hline .00895787 & 0.223947 \\
\hline 0.00892041 & 0.22301 \\
\hline 0.00888311 & 0.222078 \\
\hline .00884597 & 0.221149 \\
\hline 0.00880899 & 0.220225 \\
\hline 0.00877216 & 0.219304 \\
\hline .00873549 & 0.218387 \\
\hline 0.00869897 & 0.217474 \\
\hline 0.0086626 & 0.216565 \\
\hline .00862639 & 0.21566 \\
\hline 0.00859033 & 0.214758 \\
\hline 0.00855442 & 0.213861 \\
\hline 0.00851867 & 0.212967 \\
\hline 0.00848307 & 0.212077 \\
\hline 0.00844761 & 0.21119 \\
\hline 0.00841231 & 0.210308 \\
\hline 0.00837715 & 0.209429 \\
\hline 0.00834215 & 0.208554 \\
\hline 0.00830729 & 0.207682 \\
\hline 0.00827258 & 0.206814 \\
\hline 0.00823801 & 0.20595 \\
\hline 0.0082036 & 0.20509 \\
\hline .00816932 & 0.204233 \\
\hline 0.0081352 & 0.20338 \\
\hline 0.00810121 & 0.20253 \\
\hline 0.00806738 & 0.201684 \\
\hline .00803368 & 0.200842 \\
\hline 0.00800013 & 0.200003 \\
\hline 0.00796672 & 0.199168 \\
\hline .00793345 & 0.198336 \\
\hline 0.00790032 & 0.197508 \\
\hline 0.00786733 & 0.196683 \\
\hline .00783448 & 0.195862 \\
\hline 0.00780178 & 0.195044 \\
\hline 0.00776921 & 0.19423 \\
\hline 0.00773677 & 0.193419 \\
\hline .00770448 & 0.192612 \\
\hline 0.00767232 & 0.191808 \\
\hline 0.0076403 & 0.191008 \\
\hline .00760842 & 0.19021 \\
\hline 0.00757667 & 0.189417 \\
\hline .00754506 & 0.188626 \\
\hline .00751358 & 0.187839 \\
\hline 0.00748223 & 0.187056 \\
\hline .00745102 & 0.186275 \\
\hline .00741994 & 0.185498 \\
\hline .00738899 & 0.184725 \\
\hline .00735817 & 0.183954 \\
\hline .00732749 & 0.183187 \\
\hline .00729693 & 0.182423 \\
\hline
\end{tabular}

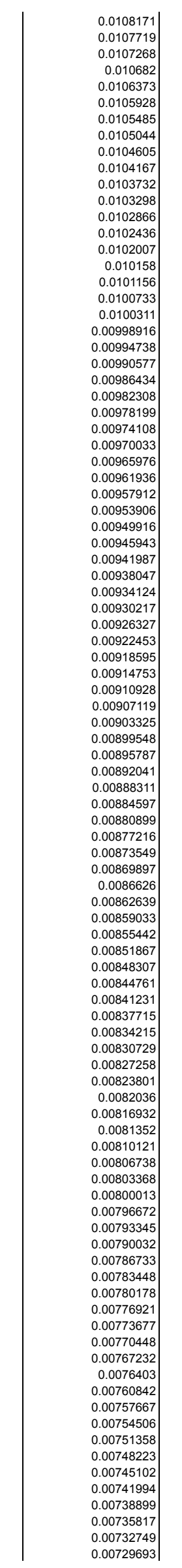

Page 13 of 33

$\begin{aligned} & 2.43385 \\
& 2.42367 \\
& 2.41354 \\
& 2.40344\end{aligned} \mid$\begin{tabular}{l}
4.32684 \\
2.30875 \\
\hline 39339
\end{tabular}

2.40344

\begin{tabular}{r|r|}
2.39339 & 4.25492 \\
2.38338 & 4.23713 \\
\hline
\end{tabular}

$2.37342 \quad 4.21941$

$2.36349 \quad 4.20176$

$2.35361 \quad 4.18419$

2.34376

\begin{tabular}{r|r}
2.33396 & 4.14927 \\
2.3242 & 4.13191 \\
\hline 2.31448
\end{tabular}

$2.31448 \quad 4.11463$

$2.3048 \quad 4.09742$

$2.29516 \quad 4.08028$

$2.28556 \quad 4.06322$

\begin{tabular}{r|r}
2.276 & 4.04622 \\
2.26648
\end{tabular}

2.26648
2.257

$2.24756 \quad 3.99566$

$2.23816 \quad 3.97895$

\begin{tabular}{r|r}
2.2288 & 3.96231 \\
2.21948 & 3.94574 \\
2.21019
\end{tabular}

$2.21019 \quad 3.92923$

\begin{tabular}{r|r}
2.20095 & 3.9128 \\
2.19174 & 3.89643
\end{tabular}

2.18258

\begin{tabular}{r|r|}
2.17258 \\
2.17345 & 3.8639 \\
2.16436
\end{tabular}

$2.16436 \quad 3.84774$

$2.1553 \quad 3.83165$

\begin{tabular}{l|l}
2.14629 & 3.81562 \\
2.13731 & 3.79966 \\
\hline
\end{tabular}

$2.12837 \quad 3.78377$

$2.11947 \quad 3.76795$

2.11061
2.10178

$2.10178 \quad 3.73649$

$2.09299 \quad 3.72087$

2.08423
2.07552

2.06684

2.0582

\begin{tabular}{r|r|}
2.04959 & 3.64371 \\
2.0402
\end{tabular}

$2.04102 \quad 3.62847$

\begin{tabular}{r|r}
2.03248 & 3.6133 \\
2.02398 & 3.59819 \\
\hline
\end{tabular}

$2.01552 \quad 3.58315$

$2.00709 \quad 3.56816$

$1.9987 \quad 3.55325$

$1.99034 \quad 3.53839$

1.98202

1.97374
1.96548 $\quad 3.50886$

1.95727

1.94909

$1.94094 \quad 3.45056$

$1.93282 \quad 3.43613$

1.92475

\begin{tabular}{r|r|}
1.9167 & 3.40747 \\
1.90869 & 3.39323 \\
1.90071
\end{tabular}

1.90071

$1.89277 \quad 3.36492$

$1.88486 \quad 3.35086$

1.87698

1.86914

\begin{tabular}{l|l}
1.86133 \\
1.85355
\end{tabular}

1.84581

$1.8381 \quad 3.26773$

1.83042

$1.82277 \quad 3.2404$

\begin{tabular}{l|l}
1.81516 & 3.22695 \\
1.80758 & 3.21347 \\
1.80003 & 3.20005
\end{tabular}

1.80003

1.79251

1.78503

$1.77757 \quad 3.16013$

$1.77015 \quad 3.14693$

\begin{tabular}{r|r|}
1.76276 & 3.13379 \\
1.7554 & 3.12071
\end{tabular}

1.74807

1.74077

1.73351
1.72627

1.72627

$1.71907 \quad 3.05612$

$1.71189 \quad 3.04337$

1.70475

$1.69764 \quad 3.01802$

1.69055

1.6835

$1.67648 \quad 2.9804$

1.66949
1.66252

1.66252
1.65559
1.64868

1.65559
1.64868 $\quad 2.94327$

2.93099
1.64181 $\quad 2.91877$ 
Table_of_specs_Rev0.xls,CH Surface roughness

\begin{tabular}{|c|c|c|}
\hline 1168 & 0.00889 & 0.00314895 \\
\hline 1169 & 0.00888 & 0.0031401 \\
\hline 1170 & 0.00886 & 0.00313128 \\
\hline 1171 & 0.00885 & 0.00312249 \\
\hline 1172 & 0.00883 & 0.00311371 \\
\hline 1173 & 0.00881 & 0.00310497 \\
\hline 1174 & 0.0088 & 0.00309624 \\
\hline 1175 & 0.00878 & 0.00308754 \\
\hline 1176 & 0.00877 & 0.00307887 \\
\hline 1177 & 0.00875 & 0.00307022 \\
\hline 1178 & 0.00874 & 0.0030616 \\
\hline 1179 & 0.00872 & 0.00305299 \\
\hline 1180 & 0.00871 & 0.00304442 \\
\hline 1181 & 0.00869 & 0.00303587 \\
\hline 1182 & 0.00868 & 0.00302734 \\
\hline 1183 & 0.00866 & 0.00301883 \\
\hline 1184 & 0.00865 & 0.00301035 \\
\hline 1185 & 0.00863 & 0.0030019 \\
\hline 1186 & 0.00862 & 0.00299346 \\
\hline 1187 & 0.0086 & 0.00298505 \\
\hline 1188 & 0.00859 & 0.00297667 \\
\hline 1189 & 0.00857 & 0.00296831 \\
\hline 1190 & 0.00856 & 0.00295997 \\
\hline 1191 & 0.00854 & 0.00295165 \\
\hline 1192 & 0.00853 & 0.00294336 \\
\hline 1193 & 0.00851 & 0.00293509 \\
\hline 1194 & 0.0085 & 0.00292685 \\
\hline 1195 & 0.00848 & 0.00291863 \\
\hline 1196 & 0.00847 & 0.00291043 \\
\hline 1197 & 0.00845 & 0.00290226 \\
\hline 1198 & 0.00844 & 0.00289411 \\
\hline 1199 & 0.00842 & 0.00288598 \\
\hline 1200 & 0.00841 & 0.00287787 \\
\hline 1201 & 0.0084 & 0.00286979 \\
\hline 1202 & 0.00838 & 0.00286173 \\
\hline 1203 & 0.00837 & 0.00285369 \\
\hline 1204 & 0.00835 & 0.00284568 \\
\hline 1205 & 0.00834 & 0.00283769 \\
\hline 1206 & 0.00832 & 0.00282972 \\
\hline 1207 & 0.00831 & 0.00282177 \\
\hline 1208 & 0.00829 & 0.00281385 \\
\hline 1209 & 0.00828 & 0.00280594 \\
\hline 1210 & 0.00827 & 0.00279807 \\
\hline 1211 & 0.00825 & 0.00279021 \\
\hline 1212 & 0.00824 & 0.00278237 \\
\hline 1213 & 0.00822 & 0.00277456 \\
\hline 1214 & 0.00821 & 0.00276677 \\
\hline 1215 & 0.00819 & 0.002759 \\
\hline 1216 & 0.00818 & 0.00275126 \\
\hline 1217 & 0.00816 & 0.00274353 \\
\hline 1218 & 0.00815 & 0.00273583 \\
\hline 1219 & 0.00813 & 0.00272815 \\
\hline 1220 & 0.00812 & 0.00272049 \\
\hline 1221 & 0.0081 & 0.00271285 \\
\hline 1222 & 0.00809 & 0.00270524 \\
\hline 1223 & 0.00808 & 0.00269765 \\
\hline 1224 & 0.00806 & 0.00269007 \\
\hline 1225 & 0.00805 & 0.00268252 \\
\hline 1226 & 0.00803 & 0.00267499 \\
\hline 1227 & 0.00802 & 0.00266749 \\
\hline 1228 & 0.00801 & 0.00266 \\
\hline 1229 & 0.00799 & 0.00265254 \\
\hline 1230 & 0.00798 & 0.00264509 \\
\hline 1231 & 0.00796 & 0.00263767 \\
\hline 1232 & 0.00795 & 0.00263027 \\
\hline 1233 & 0.00793 & 0.00262289 \\
\hline 1234 & 0.00792 & 0.00261553 \\
\hline 1235 & 0.0079 & 0.00260819 \\
\hline 1236 & 0.00789 & 0.00260087 \\
\hline 1237 & 0.00788 & 0.00259357 \\
\hline 1238 & 0.00786 & 0.0025863 \\
\hline 1239 & 0.00785 & 0.00257904 \\
\hline 1240 & 0.00784 & 0.00257181 \\
\hline 1241 & 0.00782 & 0.00256459 \\
\hline 1242 & 0.00781 & 0.0025574 \\
\hline 1243 & 0.00779 & 0.00255023 \\
\hline 1244 & 0.00778 & 0.00254307 \\
\hline 1245 & 0.00776 & 0.00253594 \\
\hline 1246 & 0.00775 & 0.00252883 \\
\hline 1247 & 0.00774 & 0.00252174 \\
\hline 1248 & 0.00773 & 0.00251467 \\
\hline 1249 & 0.00771 & 0.00250761 \\
\hline 1250 & 0.0077 & 0.00250058 \\
\hline 1251 & 0.00768 & 0.00249357 \\
\hline 1252 & 0.00767 & 0.00248658 \\
\hline 1253 & 0.00765 & 0.00247961 \\
\hline 1254 & 0.00764 & 0.00247266 \\
\hline 1255 & 0.00763 & 0.00246573 \\
\hline 1256 & 0.00761 & 0.00245881 \\
\hline 1257 & 0.0076 & 0.00245192 \\
\hline 1258 & 0.00759 & 0.00244505 \\
\hline 1259 & 0.00757 & 0.0024382 \\
\hline 1260 & 0.00756 & 0.00243136 \\
\hline 1261 & 0.00754 & 0.00242455 \\
\hline 1262 & 0.00753 & 0.00241776 \\
\hline
\end{tabular}

0.00726651 0.00723621 0.00720604 0.0071760 0.0071461 0.00708666 0.00705713 0.00702772 0.00699845 0.00696929
0.00694026 0.00691136 0.00688257 0.00685391 0.00682538 0.00679696 0.00676867 0.00671244

0.0066845 0.00665669 0.00662899 0.00660141
0.00657395 0.00654661

0.00651939

0.00649228

0.00646528

0.0064384 0.00641164

0.00635845

0.00633203

0.00630572

0.00627953

0.00625344

0.00622747

0.00617586

0.00615021

0.00612468

0.00609926

0.00607395

0.00604874

0.00602365

0.00599866

0.00597377

0.005949

0.00592433

0.00589976

0.0058753

0.0058267

0.00580255

0.00577851

0.00575457

0.00573073

0.00568336

0.00565983

0.0056364
0.00561307

.00558984

0.00556671

0.00554368

0.00552074

0.00549791

0.00547517

0.00545253

0.00542999

0.00538519
0.00536294

0.00534078

0.00531872

0.00529675

0.0052531

0.00523141

0.00520981

0.00518831

0.0051669

0.00514559

0.00512436

0.00510323

0.00508218

0.00506123

0.00504037

0.00501959

0.00497831

0.00495781

0.00493739

0.00491705

Page 14 of 33
0.181663

0.180905

0.180151
0.1794

0.178652

0.177908
0.177166

0.176428

0.175693

0.174961
0.174232

0.173507

0.172784

0.172064

0.170634

0.169924

0.169217
0.168512

0.167811

0.167113

0.166417

0.165725
0.165035

0.164349

0.163665

0.162985

0.162307

0.16096

0.160291

0.159625

0.158961

0.158301

0.157643

0.156988
0.156336

0.155687

0.15504

0.154396

0.153755

0.153117
0.152482

0.151849

0.151219

0.150591

0.149966

0.149344

0.148725

0.148108
0.147494

0.147494
0.146883

0.146274

0.145667

0.145064

0.144463

0.143864

0.143268

0.142675

0.142084

0.141496

0.14091
0.140327

0.140327
0.139746

0.139168

0.138592

0.138019

0.137448

0.136879

0.136313

0.13575
0.135189

0.13463

0.134073

0.13352
0.132968
0.132419

0.132968
0.132419

0.131872

0.131327

0.130785

0.130245

0.129708

0.129173

0.12864
0.128109

0.127581

0.127055

0.126531

0.126009
0.12549

0.124973

0.124458

0.123435

0.122926
Printed 10/2/0

1.63496

1.62815
1.62136
1.6146

1.6146
1.60787

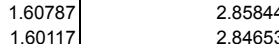

1.5945

$1.58785 \quad 2.82285$

1.58124

\begin{tabular}{l|l}
1.57465 & 2.79938 \\
1.56809 & 2.78772 \\
1.56156 & 2.7761
\end{tabular}

\begin{tabular}{r|r}
1.56156 & 2.7761 \\
1.55506 & 2.76454 \\
1.54858 &
\end{tabular}

1.54858

$1.54213 \quad 2.74157$

$1.53571 \quad 2.73015$

1.52932
1.52295

1.52932
1.51661
1.5103

$1.5103 \quad 2.68497$

$1.50401 \quad 2.6738$

$1.49775 \quad 2.66268$

1.49152
1.48532

1.48532

1.48532
1.47914
1.47299
1.46686

1.46686

$1.46076 \quad 2.59691$

1.45469

1.44864

1.44262
1.43662
1.43065

$1.43065 \quad 2.54338$

$1.42471 \quad 2.53281$

$1.41879 \quad 2.52229$

1.41289

$1.40702 \quad 2.50138$

\begin{tabular}{l|l}
1.40118 & 2.49099 \\
1.39536 & 2.48064 \\
1.38957 & 2.47034
\end{tabular}

$1.38957 \quad 2.47034$

1.3838

$\begin{array}{ll}1.37805 & 2.44987 \\ 1.37233\end{array}$

$1.37233 \quad 2.4397$

\begin{tabular}{r|r}
1.36664 & 2.42958 \\
1.36097 & 2.4195 \\
1.35532 & 2.40946
\end{tabular}

$1.35532 \quad 2.40946$

$1.3497 \quad 2.39946$

\begin{tabular}{r|r}
1.3441 & 2.3895 \\
1.33852
\end{tabular}

\begin{tabular}{r|r}
1.33852 & 2.3796 \\
1.33297 & 2.36973 \\
1.32745 & 2.3599
\end{tabular}

1.32745

1.32194

131101

$1.30557 \quad 2.32102$

$1.30016 \quad 2.3114$

$1.29478 \quad 2.30183$

1.28941

1.27876

$1.27346 \quad 2.26393$

1.26819

1.26294

1.25771
1.25251
1.24733

1.24733

1.24217
1.23783

$1.23703 \quad 2.19916$

1.23191

1.22682

1.22175
1.2167

1.21167

$1.20666 \quad 2.14518$

$1.20168 \quad 2.13631$

$1.19671 \quad 2.12749$

$1.19177 \quad 2.1187$

1.18685
1.18195

1.17707

$1.16737 \quad 2.07533$

1.16255

$1.15776 \quad 2.05824$

$1.15298 \quad 2.04974$

$1.14823 \quad 2.04129$

$1.14349 \quad 2.03287$

$\begin{array}{ll}1.13878 & 2.02449\end{array}$

$1.13408 \quad 2.01615$

1.12941
1.12475
1.12012

1.12012

1.11551

1.11091

1.11091
1.10634 


\begin{tabular}{|c|c|c|}
\hline 1263 & 0.00752 & 0.00241098 \\
\hline 1264 & 0.00751 & 0.00240423 \\
\hline 1265 & 0.00749 & 0.00239749 \\
\hline 1266 & 0.00748 & 0.00239077 \\
\hline 1267 & 0.00746 & 0.00238407 \\
\hline 1268 & 0.00745 & 0.0023774 \\
\hline 1269 & 0.00744 & 0.00237074 \\
\hline 1270 & 0.00742 & 0.0023641 \\
\hline 1271 & 0.00741 & 0.00235748 \\
\hline 1272 & 0.0074 & 0.00235087 \\
\hline 1273 & 0.00738 & 0.00234429 \\
\hline 1274 & 0.00737 & 0.00233772 \\
\hline 1275 & 0.00735 & 0.00233118 \\
\hline 1276 & 0.00734 & 0.00232465 \\
\hline 1277 & 0.00733 & 0.00231814 \\
\hline 1278 & 0.00732 & 0.00231165 \\
\hline 1279 & 0.0073 & 0.00230518 \\
\hline 1280 & 0.00729 & 0.00229873 \\
\hline 1281 & 0.00727 & 0.00229229 \\
\hline 1282 & 0.00726 & 0.00228588 \\
\hline 1283 & 0.00725 & 0.00227948 \\
\hline 1284 & 0.00724 & 0.0022731 \\
\hline 1285 & 0.00722 & 0.00226674 \\
\hline 1286 & 0.00721 & 0.0022604 \\
\hline 1287 & 0.00719 & 0.00225407 \\
\hline 1288 & 0.00718 & 0.00224777 \\
\hline 1289 & 0.00717 & 0.00224148 \\
\hline 1290 & 0.00716 & 0.00223521 \\
\hline 1291 & 0.00714 & 0.00222896 \\
\hline 1292 & 0.00713 & 0.00222272 \\
\hline 1293 & 0.00712 & 0.00221651 \\
\hline 1294 & 0.0071 & 0.00221031 \\
\hline 1295 & 0.00709 & 0.00220413 \\
\hline 1296 & 0.00708 & 0.00219796 \\
\hline 1297 & 0.00706 & 0.00219182 \\
\hline 1298 & 0.00705 & 0.00218569 \\
\hline 1299 & 0.00704 & 0.00217958 \\
\hline 1300 & 0.00703 & 0.00217349 \\
\hline 1301 & 0.00701 & 0.00216741 \\
\hline 1302 & 0.007 & 0.00216136 \\
\hline 1303 & 0.00698 & 0.00215532 \\
\hline 1304 & 0.00697 & 0.00214929 \\
\hline 1305 & 0.00696 & 0.00214329 \\
\hline 1306 & 0.00695 & 0.0021373 \\
\hline 1307 & 0.00693 & 0.00213133 \\
\hline 1308 & 0.00692 & 0.00212537 \\
\hline 1309 & 0.00691 & 0.00211944 \\
\hline 1310 & 0.0069 & 0.00211352 \\
\hline 1311 & 0.00688 & 0.00210761 \\
\hline 1312 & 0.00687 & 0.00210173 \\
\hline 1313 & 0.00686 & 0.00209586 \\
\hline 1314 & 0.00685 & 0.00209001 \\
\hline 1315 & 0.00683 & 0.00208417 \\
\hline 1316 & 0.00682 & 0.00207835 \\
\hline 1317 & 0.0068 & 0.00207255 \\
\hline 1318 & 0.00679 & 0.00206677 \\
\hline 1319 & 0.00678 & 0.002061 \\
\hline 1320 & 0.00677 & 0.00205525 \\
\hline 1321 & 0.00675 & 0.00204951 \\
\hline 1322 & 0.00674 & 0.00204379 \\
\hline 1323 & 0.00673 & 0.00203809 \\
\hline 1324 & 0.00672 & 0.00203241 \\
\hline 1325 & 0.0067 & 0.00202674 \\
\hline 1326 & 0.00669 & 0.00202108 \\
\hline 1327 & 0.00668 & 0.00201545 \\
\hline 1328 & 0.00667 & 0.00200983 \\
\hline 1329 & 0.00665 & 0.00200422 \\
\hline 1330 & 0.00664 & 0.00199863 \\
\hline 1331 & 0.00663 & 0.00199306 \\
\hline 1332 & 0.00662 & 0.0019875 \\
\hline 1333 & 0.0066 & 0.00198196 \\
\hline 1334 & 0.00659 & 0.00197644 \\
\hline 1335 & 0.00658 & 0.00197093 \\
\hline 1336 & 0.00657 & 0.00196544 \\
\hline 1337 & 0.00655 & 0.00195996 \\
\hline 1338 & 0.00654 & 0.0019545 \\
\hline 1339 & 0.00653 & 0.00194906 \\
\hline 1340 & 0.00652 & 0.00194363 \\
\hline 1341 & 0.0065 & 0.00193821 \\
\hline 1342 & 0.00649 & 0.00193282 \\
\hline 1343 & 0.00648 & 0.00192743 \\
\hline 1344 & 0.00647 & 0.00192207 \\
\hline 1345 & 0.00645 & 0.00191671 \\
\hline 1346 & 0.00644 & 0.00191138 \\
\hline 1347 & 0.00643 & 0.00190606 \\
\hline 1348 & 0.00642 & 0.00190075 \\
\hline 1349 & 0.0064 & 0.00189546 \\
\hline 1350 & 0.00639 & 0.00189019 \\
\hline 1351 & 0.00638 & 0.00188493 \\
\hline 1352 & 0.00637 & 0.00187968 \\
\hline 1353 & 0.00636 & 0.00187445 \\
\hline 1354 & 0.00635 & 0.00186924 \\
\hline 1355 & 0.00633 & 0.00186404 \\
\hline 1356 & 0.00632 & 0.00185886 \\
\hline 135 & 0.00631 & 0.00185369 \\
\hline
\end{tabular}

0.00489681 0.00487665 0.00485658 0.0048366 0.0048167
0.00479688

0.00477716

0.00475751

0.00473795

0.00471848

0.00469909
0.00467978
0.00466055

0.00466055

0.00464141

0.00462235

0.00460337

0.00458447

0.00456565

0.00452826

0.00450969

0.00449119

0.00447278

0.00445444
0.00443618
0.004418

0.004418

0.0043999

0.00438188

0.00436393

0.00434606

0.00432827

0.00429291

0.00427535

0.00425786

0.00424045

0.00422311

0.00420584

0.00417154

0.00415449

0.00413752

0.00412063

0.0041038

0.00408705

0.00405376

0.00403722

0.00402076

0.00400436

0.00398804

0.00395559

0.00393948

0.00392343

0.00390745

0.00389154

0.0038757

0.00385993

0.00384422

0.00382858

0.00381301

0.0037975

0.00378207

0.0037667

0.00375139

0.00373615
0.00372098

0.00370587

0.00369082

0.00367584

0.00366093

0.00364607

0.00363129

0.00361656

0.0036019

0.0035873

0.00357276

0.00355829

0.00354388

0.00352952

.00351524

0.00350101

0.00348684

0.00347273

0.0034447

0.00343078

0.00341691

0.0034031

0.00338936

0.00337567

0.00336204

0.00333495

Page 15 of 33
0.12242

0.121916

0.121415

0.120915
0.120417

0.119922

0.119429

0.118938

0.118449

0.117962
0.117477

0.116994

0.116514

0.116035

0.115559

0.114612

0.114141

0.113207

0.112742

0.11228

0.111819

0.111361

0.11045
0.109998

0.109998

0.109547

0.109098

0.108652

0.108207

0.107323

0.10688

0.106447

0.106011

0.105578

0.105146

0.104716

0.104288

0.103862

0.103438
0.103016

0.102595

0.102176

0.101759

0.101344

0.100931

0.100519

0.100109

0.0997009

0.0992945

0.0984869

0.0980858

0.0976863

0.0972886

0.0964982

0.0957146

0.0953253

0.0949377

0.0945518

0937848

0.0934038

0.0930244

0.0926467
0.0922706

0.0922706

0.0915232

0.0907821

0.090414
0.0900474

0.0900474

0.089319

0.0889572

0.0885969

0.0882381

.0878809

0.0875252

0.087171

0.0868184

0.0864672

0.0861176

0.0857694

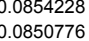

0.0847339

0.0843917

0.0840509

0.0837116

0.0833738
Printed 10/2/05

\begin{tabular}{l|l|l|}
1.10178 & 1.95872 \\
1.09725 & 1.95066
\end{tabular}

1.09725
1.09273
1.08823

$\begin{array}{ll}1.08823 & 1.94263 \\ 1.08376\end{array}$

\begin{tabular}{r|r}
1.08376 & 1.92668 \\
1.0793 & 1.91875 \\
1.07486 & 1.91086
\end{tabular}

1.07486

\begin{tabular}{r|r}
1.07044 \\
1.06604 \\
1.06166
\end{tabular}

\begin{tabular}{l|l|}
1.06604 & 1.89518 \\
1.06166 & 1.88739 \\
1.05729 & 1.87963
\end{tabular}

1.05729
1.05295 $\quad \begin{aligned} & 1.87963 \\ & 1.04862\end{aligned}$

$1.04862 \quad 1.86422$

1.04432

$1.04003 \quad 1.84894$

1.03576

$1.03151 \quad 1.83379$

\begin{tabular}{l|l}
1.02727 \\
1.02306
\end{tabular}$\quad \begin{aligned} & 1.82626 \\
& 1.01886\end{aligned}$

1.01886

$1.01468 \quad 1.80388$

$1.01052 \quad 1.79648$

\begin{tabular}{l|l}
1.00638 & 1.78911 \\
1.00225
\end{tabular}

\begin{tabular}{r|r}
1.00225 & 1.78178 \\
0.998141 & 1.77447 \\
0.994051
\end{tabular}

$0.994051 \quad 1.7672$

\begin{tabular}{l}
$0.989978 \quad 1.75996$ \\
\hline
\end{tabular}

0.985923
0.981885

0.981885
0.977864

$\begin{array}{ll}0.977864 & 1.73843 \\ 0.973861 & 1.73131 \\ 0.969875 & 1.72422\end{array}$

$\begin{array}{ll}0.969875 & 1.72422 \\ 0.905906\end{array}$

$\begin{array}{ll}0.965906 & 1.71717 \\ 0.961954 & 1.71014\end{array}$

$\begin{array}{ll}0.961954 & 1.71014 \\ 0.958019 & 1.70314 \\ 0.954101\end{array}$

$\begin{array}{ll}0.954101 & 1.69618 \\ 0.9502 & 1.68924\end{array}$

$0.9502 \quad 1.68924$

0.946315
0.942447

0.938596

$0.934761 \quad 1.6618$

$0.930943 \quad 1.65501$

$0.927141 \quad 1.64825$

\begin{tabular}{l|l}
0.923356 & 1.64152 \\
0.919586 & 1.63482 \\
0.915833
\end{tabular}

$0.915833 \quad 1.62815$

0.912096

0.908375

$0.90467 \quad 1.6083$

$0.900981 \quad 1.60174$

0.897308
0.89365

0.890009

$0.886383 \quad 1.57579$

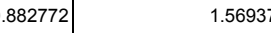

0.879177
0.875597

$0.875597 \quad 1.55662$

\begin{tabular}{l|l}
0.875597 & 1.55028 \\
0.868484 & 1.54397 \\
0.86495 & 1.53769
\end{tabular}

0.86495

$0.861431 \quad 1.53143$

0.857928
0.854439

\begin{tabular}{r|r}
0.854439 & 1.519 \\
0.850966 & 1.51283 \\
0.847507
\end{tabular}

$0.847507 \quad 1.50668$

0.844063

0.840634

0.83722

0.83382

$0.830435 \quad 1.47633$

\begin{tabular}{l|l}
0.827065 \\
0.823708 \\
0.820367
\end{tabular}

0.820367

$0.817039 \quad 1.45251$

0.813726
0.810427

$0.810427 \quad 1.44076$

0.807142
0.803871
0.800615 $\quad \begin{array}{r}1.43492 \\ 1.4291\end{array}$

0.800615

0.797372

$\begin{array}{ll}0.794143 & 1.41181\end{array}$

0.790928
0.78727

$0.787727 \quad 1.4004$

\begin{tabular}{l|l}
0.787727 \\
0.784539 \\
0.781365
\end{tabular}

0.778205

0.775058

0.771925
0.768805
-1.37231

0.768805

$0.765698 \quad 1.36124$

\begin{tabular}{l|l}
0.762605 \\
0.759525
\end{tabular}$\quad 1.35574$

0.756458

0.753405

$\begin{array}{ll}0.753405 & 1.33939 \\ 0.750364 & 1.33398\end{array}$ 


\begin{tabular}{|c|c|c|}
\hline 1358 & 0.0063 & 0.00184853 \\
\hline 1359 & 0.00628 & 0.0018434 \\
\hline 1360 & 0.00627 & 0.00183827 \\
\hline 1361 & 0.00626 & 0.00183316 \\
\hline 1362 & 0.00625 & 0.00182807 \\
\hline 1363 & 0.00623 & 0.00182299 \\
\hline 1364 & 0.00622 & 0.00181792 \\
\hline 1365 & 0.00621 & 0.00181287 \\
\hline 1366 & 0.0062 & 0.00180783 \\
\hline 1367 & 0.00619 & 0.00180281 \\
\hline 1368 & 0.00618 & 0.0017978 \\
\hline 1369 & 0.00616 & 0.00179281 \\
\hline 1370 & 0.00615 & 0.00178783 \\
\hline 1371 & 0.00614 & 0.00178287 \\
\hline 1372 & 0.00613 & 0.00177792 \\
\hline 1373 & 0.00611 & 0.00177298 \\
\hline 1374 & 0.0061 & 0.00176806 \\
\hline 1375 & 0.00609 & 0.00176316 \\
\hline 1376 & 0.00608 & 0.00175826 \\
\hline 1377 & 0.00607 & 0.00175338 \\
\hline 1378 & 0.00606 & 0.00174852 \\
\hline 1379 & 0.00604 & 0.00174367 \\
\hline 1380 & 0.00603 & 0.00173883 \\
\hline 1381 & 0.00602 & 0.00173401 \\
\hline 1382 & 0.00601 & 0.0017292 \\
\hline 1383 & 0.006 & 0.00172441 \\
\hline 1384 & 0.00599 & 0.00171963 \\
\hline 1385 & 0.00597 & 0.00171486 \\
\hline 1386 & 0.00596 & 0.00171011 \\
\hline 1387 & 0.00595 & 0.00170537 \\
\hline 1388 & 0.00594 & 0.00170064 \\
\hline 1389 & 0.00592 & 0.00169593 \\
\hline 1390 & 0.00592 & 0.00169123 \\
\hline 1391 & 0.0059 & 0.00168655 \\
\hline 1392 & 0.00589 & 0.00168188 \\
\hline 1393 & 0.00588 & 0.00167722 \\
\hline 1394 & 0.00587 & 0.00167257 \\
\hline 1395 & 0.00586 & 0.00166794 \\
\hline 1396 & 0.00585 & 0.00166333 \\
\hline 1397 & 0.00583 & 0.00165872 \\
\hline 1398 & 0.00582 & 0.00165413 \\
\hline 1399 & 0.00581 & 0.00164955 \\
\hline 1400 & 0.0058 & 0.00164499 \\
\hline 1401 & 0.00579 & 0.00164044 \\
\hline 1402 & 0.00578 & 0.0016359 \\
\hline 1403 & 0.00576 & 0.00163138 \\
\hline 1404 & 0.00575 & 0.00162687 \\
\hline 1405 & 0.00574 & 0.00162237 \\
\hline 1406 & 0.00573 & 0.00161788 \\
\hline 1407 & 0.00572 & 0.00161341 \\
\hline 1408 & 0.00571 & 0.00160895 \\
\hline 1409 & 0.00569 & 0.0016045 \\
\hline 1410 & 0.00569 & 0.00160007 \\
\hline 1411 & 0.00567 & 0.00159565 \\
\hline 1412 & 0.00566 & 0.00159124 \\
\hline 1413 & 0.00565 & 0.00158684 \\
\hline 1414 & 0.00564 & 0.00158246 \\
\hline 1415 & 0.00563 & 0.00157809 \\
\hline 1416 & 0.00562 & 0.00157374 \\
\hline 1417 & 0.0056 & 0.00156939 \\
\hline 1418 & 0.00559 & 0.00156506 \\
\hline 1419 & 0.00558 & 0.00156074 \\
\hline 1420 & 0.00557 & 0.00155643 \\
\hline 1421 & 0.00556 & 0.00155214 \\
\hline 1422 & 0.00555 & 0.00154786 \\
\hline 1423 & 0.00554 & 0.00154359 \\
\hline 1424 & 0.00553 & 0.00153933 \\
\hline 1425 & 0.00551 & 0.00153508 \\
\hline 1426 & 0.0055 & 0.00153085 \\
\hline 1427 & 0.00549 & 0.00152663 \\
\hline 1428 & 0.00548 & 0.00152242 \\
\hline 1429 & 0.00547 & 0.00151823 \\
\hline 1430 & 0.00546 & 0.00151404 \\
\hline 1431 & 0.00545 & 0.00150987 \\
\hline 1432 & 0.00544 & 0.00150571 \\
\hline 1433 & 0.00542 & 0.00150156 \\
\hline 1434 & 0.00542 & 0.00149743 \\
\hline 1435 & 0.0054 & 0.0014933 \\
\hline 1436 & 0.00539 & 0.00148919 \\
\hline 1437 & 0.00538 & 0.00148509 \\
\hline 1438 & 0.00537 & 0.001481 \\
\hline 1439 & 0.00536 & 0.00147693 \\
\hline 1440 & 0.00535 & 0.00147286 \\
\hline 1441 & 0.00534 & 0.00146881 \\
\hline 1442 & 0.00533 & 0.00146477 \\
\hline 1443 & 0.00531 & 0.00146074 \\
\hline 1444 & 0.00531 & 0.00145672 \\
\hline 1445 & 0.00529 & 0.00145272 \\
\hline 1446 & 0.00528 & 0.00144872 \\
\hline 1447 & 0.00527 & 0.00144474 \\
\hline 1448 & 0.00526 & 0.00144077 \\
\hline 1449 & 0.00525 & 0.00143681 \\
\hline 1450 & 0.00524 & 0.00143286 \\
\hline 1451 & 0.00523 & 0.00142893 \\
\hline 1452 & 0.00522 & 0.001425 \\
\hline
\end{tabular}

\begin{tabular}{|c|c|}
\hline 0.0033215 & 0.0830374 \\
\hline 0.0033081 & 0.0827024 \\
\hline 0.00329476 & 0.0823689 \\
\hline 0.00328147 & 0.0820368 \\
\hline 0.00326825 & 0.0817061 \\
\hline 0.00325507 & 0.0813769 \\
\hline 0.00324196 & 0.081049 \\
\hline 0.0032289 & 0.0807226 \\
\hline 0.0032159 & 0.0803975 \\
\hline 0.00320295 & 0.0800738 \\
\hline 0.00319006 & 0.0797516 \\
\hline 0.00317723 & 0.0794307 \\
\hline 0.00316444 & 0.0791111 \\
\hline 0.00315172 & 0.078793 \\
\hline 0.00313905 & 0.0784761 \\
\hline 0.00312643 & 0.0781607 \\
\hline 0.00311386 & 0.0778466 \\
\hline 0.00310135 & 0.0775338 \\
\hline 0.0030889 & 0.0772224 \\
\hline 0.00307649 & 0.0769123 \\
\hline 0.00306414 & 0.0766035 \\
\hline 0.00305184 & 0.0762961 \\
\hline 0.0030396 & 0.0759899 \\
\hline 0.0030274 & 0.0756851 \\
\hline 0.00301526 & 0.0753816 \\
\hline 0.00300317 & 0.0750793 \\
\hline 0.00299114 & 0.0747784 \\
\hline 0.00297915 & 0.0744787 \\
\hline 0.00296721 & 0.0741804 \\
\hline 0.00295533 & 0.0738832 \\
\hline 0.0029435 & 0.0735874 \\
\hline 0.00293171 & 0.0732928 \\
\hline 0.00291998 & 0.0729995 \\
\hline 0.0029083 & 0.0727074 \\
\hline 0.00289666 & 0.0724166 \\
\hline 0.00288508 & 0.072127 \\
\hline 0.00287355 & 0.0718387 \\
\hline 0.00286206 & 0.0715516 \\
\hline 0.00285063 & 0.0712657 \\
\hline 0.00283924 & 0.070981 \\
\hline 0.0028279 & 0.0706975 \\
\hline 0.00281661 & 0.0704153 \\
\hline 0.00280537 & 0.0701342 \\
\hline 0.00279417 & 0.0698543 \\
\hline 0.00278303 & 0.0695757 \\
\hline 0.00277193 & 0.0692982 \\
\hline 0.00276088 & 0.0690219 \\
\hline 0.00274987 & 0.0687468 \\
\hline 0.00273891 & 0.0684728 \\
\hline 0.002728 & 0.0682001 \\
\hline 0.00271714 & 0.0679284 \\
\hline 0.00270632 & 0.067658 \\
\hline 0.00269555 & 0.0673887 \\
\hline 0.00268482 & 0.0671205 \\
\hline 0.00267414 & 0.0668535 \\
\hline 0.0026635 & 0.0665876 \\
\hline 0.00265291 & 0.0663228 \\
\hline 0.00264237 & 0.0660592 \\
\hline 0.00263187 & 0.0657966 \\
\hline 0.00262141 & 0.0655352 \\
\hline 0.002611 & 0.065275 \\
\hline 0.00260063 & 0.0650158 \\
\hline 0.00259031 & 0.0647577 \\
\hline 0.00258003 & 0.0645007 \\
\hline 0.00256979 & 0.0642448 \\
\hline 0.0025596 & 0.06399 \\
\hline 0.00254945 & 0.0637363 \\
\hline 0.00253935 & 0.0634836 \\
\hline 0.00252928 & 0.0632321 \\
\hline 0.00251926 & 0.0629816 \\
\hline 0.00250928 & 0.0627321 \\
\hline 0.00249935 & 0.0624837 \\
\hline 0.00248946 & 0.0622364 \\
\hline 0.0024796 & 0.0619901 \\
\hline 0.0024698 & 0.0617449 \\
\hline 0.00246003 & 0.0615007 \\
\hline 0.0024503 & 0.0612575 \\
\hline 0.00244062 & 0.0610154 \\
\hline 0.00243097 & 0.0607743 \\
\hline 0.00242137 & 0.0605342 \\
\hline 0.00241181 & 0.0602951 \\
\hline 0.00240228 & 0.0600571 \\
\hline 0.0023928 & 0.05982 \\
\hline 0.00238336 & 0.059584 \\
\hline 0.00237396 & 0.059349 \\
\hline 0.0023646 & 0.0591149 \\
\hline 0.00235527 & 0.0588819 \\
\hline 0.00234599 & 0.0586498 \\
\hline 0.00233675 & 0.0584187 \\
\hline 0.00232754 & 0.0581886 \\
\hline 0.00231838 & 0.0579595 \\
\hline 0.00230925 & 0.0577313 \\
\hline 0.00230016 & 0.0575041 \\
\hline 0.00229111 & 0.0572779 \\
\hline & \\
\hline
\end{tabular}

\begin{tabular}{|c|c|}
\hline 0.747337 & 1.3286 \\
\hline 0.744322 & 1.32324 \\
\hline 0.74132 & 1.3179 \\
\hline 0.738331 & 1.31259 \\
\hline 0.735355 & 1.3073 \\
\hline 0.732392 & 1.30203 \\
\hline 0.729441 & 1.29678 \\
\hline 0.726503 & 1.29156 \\
\hline 0.723578 & 1.28636 \\
\hline 0.720665 & 1.28118 \\
\hline 0.717764 & 1.27602 \\
\hline 0.714876 & 1.27089 \\
\hline 0.712 & 1.26578 \\
\hline 0.709137 & 1.26069 \\
\hline 0.706285 & 1.25562 \\
\hline 0.703446 & 1.25057 \\
\hline 0.700619 & 1.24555 \\
\hline 0.697804 & 1.24054 \\
\hline 0.695002 & 1.23556 \\
\hline 0.692211 & 1.2306 \\
\hline 0.689432 & 1.22566 \\
\hline 0.686665 & 1.22074 \\
\hline 0.683909 & 1.21584 \\
\hline 0.681166 & 1.21096 \\
\hline 0.678434 & 1.20611 \\
\hline 0.675714 & 1.20127 \\
\hline 0.673006 & 1.19645 \\
\hline 0.670309 & 1.19166 \\
\hline 0.667623 & 1.18689 \\
\hline 0.664949 & 1.18213 \\
\hline 0.662287 & 1.1774 \\
\hline 0.659635 & 1.17269 \\
\hline 0.656996 & 1.16799 \\
\hline 0.654367 & 1.16332 \\
\hline 0.65175 & 1.15867 \\
\hline 0.649143 & 1.15403 \\
\hline 0.646548 & 1.14942 \\
\hline 0.643964 & 1.14482 \\
\hline 0.641391 & 1.14025 \\
\hline 0.638829 & 1.1357 \\
\hline 0.636278 & 1.13116 \\
\hline 0.633737 & 1.12664 \\
\hline 0.631208 & 1.12215 \\
\hline 0.628689 & 1.11767 \\
\hline 0.626181 & 1.11321 \\
\hline 0.623684 & 1.10877 \\
\hline 0.621197 & 1.10435 \\
\hline 0.618721 & 1.09995 \\
\hline 0.616256 & 1.09557 \\
\hline 0.613801 & 1.0912 \\
\hline 0.611356 & 1.08685 \\
\hline 0.608922 & 1.08253 \\
\hline 0.606498 & 1.07822 \\
\hline 0.604084 & 1.07393 \\
\hline 0.601681 & 1.06966 \\
\hline 0.599288 & 1.0654 \\
\hline 0.596905 & 1.06116 \\
\hline 0.594532 & 1.05695 \\
\hline 0.59217 & 1.05275 \\
\hline 0.589817 & 1.04856 \\
\hline 0.587475 & 1.0444 \\
\hline 0.585142 & 1.04025 \\
\hline 0.582819 & 1.03612 \\
\hline 0.580506 & 1.03201 \\
\hline 0.578203 & 1.02792 \\
\hline 0.57591 & 1.02384 \\
\hline 0.573627 & 1.01978 \\
\hline 0.571353 & 1.01574 \\
\hline 0.569089 & 1.01171 \\
\hline 0.566834 & 1.0077 \\
\hline 0.564589 & 1.00371 \\
\hline 0.562354 & 0.99974 \\
\hline 0.560128 & 0.995782 \\
\hline 0.557911 & 0.991842 \\
\hline 0.555704 & 0.987918 \\
\hline 0.553506 & 0.984011 \\
\hline 0.551318 & 0.98012 \\
\hline 0.549139 & 0.976246 \\
\hline 0.546969 & 0.972389 \\
\hline 0.544808 & 0.968547 \\
\hline 0.542656 & 0.964722 \\
\hline 0.540514 & 0.960913 \\
\hline 0.53838 & 0.957121 \\
\hline 0.536256 & 0.953344 \\
\hline 0.534141 & 0.949583 \\
\hline 0.532034 & 0.945839 \\
\hline 0.529937 & 0.94211 \\
\hline 0.527848 & 0.938397 \\
\hline 0.525768 & 0.934699 \\
\hline 0.523697 & 0.931018 \\
\hline 0.521635 & 0.927351 \\
\hline 0.519582 & 0.923701 \\
\hline 0.517537 & 0.920065 \\
\hline 0.515501 & 0.916446 \\
\hline 0.513473 & 0.912841 \\
\hline
\end{tabular}




\begin{tabular}{|c|c|c|}
\hline 1453 & 0.00521 & 0.00142109 \\
\hline 1454 & 0.0052 & 0.00141719 \\
\hline 1455 & 0.00518 & 0.00141329 \\
\hline 1456 & 0.00518 & 0.00140941 \\
\hline 1457 & 0.00516 & 0.00140555 \\
\hline 1458 & 0.00515 & 0.00140169 \\
\hline 1459 & 0.00514 & 0.00139784 \\
\hline 1460 & 0.00513 & 0.00139401 \\
\hline 1461 & 0.00512 & 0.00139018 \\
\hline 1462 & 0.00511 & 0.00138637 \\
\hline 1463 & 0.0051 & 0.00138257 \\
\hline 1464 & 0.00509 & 0.00137878 \\
\hline 1465 & 0.00508 & 0.001375 \\
\hline 1466 & 0.00507 & 0.00137123 \\
\hline 1467 & 0.00505 & 0.00136747 \\
\hline 1468 & 0.00505 & 0.00136372 \\
\hline 1469 & 0.00503 & 0.00135999 \\
\hline 1470 & 0.00503 & 0.00135626 \\
\hline 1471 & 0.00501 & 0.00135255 \\
\hline 1472 & 0.005 & 0.00134884 \\
\hline 1473 & 0.00499 & 0.00134515 \\
\hline 1474 & 0.00498 & 0.00134147 \\
\hline 1475 & 0.00497 & 0.0013378 \\
\hline 1476 & 0.00496 & 0.00133413 \\
\hline 1477 & 0.00495 & 0.00133048 \\
\hline 1478 & 0.00494 & 0.00132684 \\
\hline 1479 & 0.00493 & 0.00132321 \\
\hline 1480 & 0.00492 & 0.0013196 \\
\hline 1481 & 0.00491 & 0.00131599 \\
\hline 1482 & 0.0049 & 0.00131239 \\
\hline 1483 & 0.00489 & 0.0013088 \\
\hline 1484 & 0.00488 & 0.00130522 \\
\hline 1485 & 0.00487 & 0.00130166 \\
\hline 1486 & 0.00486 & 0.0012981 \\
\hline 1487 & 0.00484 & 0.00129456 \\
\hline 1488 & 0.00484 & 0.00129102 \\
\hline 1489 & 0.00482 & 0.00128749 \\
\hline 1490 & 0.00482 & 0.00128398 \\
\hline 1491 & 0.0048 & 0.00128047 \\
\hline 1492 & 0.0048 & 0.00127698 \\
\hline 1493 & 0.00478 & 0.00127349 \\
\hline 1494 & 0.00477 & 0.00127002 \\
\hline 1495 & 0.00476 & 0.00126655 \\
\hline 1496 & 0.00475 & 0.0012631 \\
\hline 1497 & 0.00474 & 0.00125965 \\
\hline 1498 & 0.00473 & 0.00125622 \\
\hline 1499 & 0.00472 & 0.00125279 \\
\hline 1500 & 0.00471 & 0.00124938 \\
\hline 1501 & 0.0047 & 0.00124597 \\
\hline 1502 & 0.00469 & 0.00124258 \\
\hline 1503 & 0.00468 & 0.00123919 \\
\hline 1504 & 0.00467 & 0.00123582 \\
\hline 1505 & 0.00466 & 0.00123245 \\
\hline 1506 & 0.00465 & 0.0012291 \\
\hline 1507 & 0.00464 & 0.00122575 \\
\hline 1508 & 0.00463 & 0.00122241 \\
\hline 1509 & 0.00462 & 0.00121909 \\
\hline 1510 & 0.00461 & 0.00121577 \\
\hline 1511 & 0.0046 & 0.00121246 \\
\hline 1512 & 0.00459 & 0.00120916 \\
\hline 1513 & 0.00458 & 0.00120588 \\
\hline 1514 & 0.00457 & 0.0012026 \\
\hline 1515 & 0.00456 & 0.00119933 \\
\hline 1516 & 0.00455 & 0.00119607 \\
\hline 1517 & 0.00454 & 0.00119282 \\
\hline 1518 & 0.00453 & 0.00118958 \\
\hline 1519 & 0.00452 & 0.00118634 \\
\hline 1520 & 0.00451 & 0.00118312 \\
\hline 1521 & 0.0045 & 0.00117991 \\
\hline 1522 & 0.00449 & 0.0011767 \\
\hline 1523 & 0.00448 & 0.00117351 \\
\hline 1524 & 0.00447 & 0.00117032 \\
\hline 1525 & 0.00446 & 0.00116715 \\
\hline 1526 & 0.00445 & 0.00116398 \\
\hline 1527 & 0.00444 & 0.00116082 \\
\hline 1528 & 0.00443 & 0.00115767 \\
\hline 1529 & 0.00442 & 0.00115454 \\
\hline 1530 & 0.00441 & 0.0011514 \\
\hline 1531 & 0.0044 & 0.00114828 \\
\hline 1532 & 0.00439 & 0.00114517 \\
\hline 1533 & 0.00438 & 0.00114207 \\
\hline 1534 & 0.00437 & 0.00113897 \\
\hline 1535 & 0.00436 & 0.00113589 \\
\hline 1536 & 0.00435 & 0.00113281 \\
\hline 1537 & 0.00434 & 0.00112974 \\
\hline 1538 & 0.00433 & 0.00112669 \\
\hline 1539 & 0.00432 & 0.00112364 \\
\hline 1540 & 0.00431 & 0.00112059 \\
\hline 1541 & 0.0043 & 0.00111756 \\
\hline 1542 & 0.00429 & 0.00111454 \\
\hline 1543 & 0.00428 & 0.00111152 \\
\hline 1544 & 0.00427 & 0.00110852 \\
\hline 1545 & 0.00426 & 0.00110552 \\
\hline 1546 & 0.00426 & 0.00110253 \\
\hline 1547 & 0.00424 & 0.00109955 \\
\hline
\end{tabular}

\begin{tabular}{|c|c|}
\hline 0.00227313 & 0.0568282 \\
\hline 0.00226419 & 0.0566049 \\
\hline 0.0022553 & 0.0563824 \\
\hline 0.00224644 & 0.0561609 \\
\hline 0.00223761 & 0.0559404 \\
\hline 0.00222883 & 0.0557207 \\
\hline 0.00222008 & 0.055502 \\
\hline 0.00221137 & 0.0552842 \\
\hline 0.00220269 & 0.0550674 \\
\hline 0.00219406 & 0.0548514 \\
\hline 0.00218546 & 0.0546364 \\
\hline 0.00217689 & 0.0544223 \\
\hline 0.00216836 & 0.054209 \\
\hline 0.00215987 & 0.0539967 \\
\hline 0.00215141 & 0.0537853 \\
\hline 0.00214299 & 0.0535747 \\
\hline 0.0021346 & 0.0533651 \\
\hline 0.00212625 & 0.0531563 \\
\hline 0.00211794 & 0.0529484 \\
\hline 0.00210966 & 0.0527414 \\
\hline 0.00210141 & 0.0525353 \\
\hline 0.0020932 & 0.05233 \\
\hline 0.00208502 & 0.0521256 \\
\hline 0.00207688 & 0.051922 \\
\hline 0.00206877 & 0.0517193 \\
\hline 0.0020607 & 0.0515175 \\
\hline 0.00205266 & 0.0513165 \\
\hline 0.00204466 & 0.0511164 \\
\hline 0.00203668 & 0.0509171 \\
\hline 0.00202874 & 0.0507186 \\
\hline 0.00202084 & 0.050521 \\
\hline 0.00201297 & 0.0503242 \\
\hline 0.00200513 & 0.0501282 \\
\hline 0.00199732 & 0.0499331 \\
\hline 0.00198955 & 0.0497387 \\
\hline 0.00198181 & 0.0495452 \\
\hline 0.0019741 & 0.0493525 \\
\hline 0.00196642 & 0.0491606 \\
\hline 0.00195878 & 0.0489695 \\
\hline 0.00195117 & 0.0487793 \\
\hline 0.00194359 & 0.0485898 \\
\hline 0.00193604 & 0.0484011 \\
\hline 0.00192853 & 0.0482132 \\
\hline 0.00192104 & 0.0480261 \\
\hline 0.00191359 & 0.0478397 \\
\hline 0.00190617 & 0.0476542 \\
\hline 0.00189878 & 0.0474694 \\
\hline 0.00189142 & 0.0472854 \\
\hline 0.00188409 & 0.0471022 \\
\hline 0.00187679 & 0.0469198 \\
\hline 0.00186952 & 0.0467381 \\
\hline 0.00186229 & 0.0465571 \\
\hline 0.00185508 & 0.046377 \\
\hline 0.0018479 & 0.0461975 \\
\hline 0.00184075 & 0.0460189 \\
\hline 0.00183364 & 0.0458409 \\
\hline 0.00182655 & 0.0456638 \\
\hline 0.00181949 & 0.0454873 \\
\hline 0.00181247 & 0.0453116 \\
\hline 0.00180547 & 0.0451367 \\
\hline 0.0017985 & 0.0449624 \\
\hline 0.00179156 & 0.0447889 \\
\hline 0.00178465 & 0.0446161 \\
\hline 0.00177776 & 0.0444441 \\
\hline 0.00177091 & 0.0442727 \\
\hline 0.00176408 & 0.0441021 \\
\hline 0.00175729 & 0.0439322 \\
\hline 0.00175052 & 0.043763 \\
\hline 0.00174378 & 0.0435945 \\
\hline 0.00173707 & 0.0434267 \\
\hline 0.00173038 & 0.0432596 \\
\hline 0.00172373 & 0.0430932 \\
\hline 0.0017171 & 0.0429275 \\
\hline 0.0017105 & 0.0427625 \\
\hline 0.00170393 & 0.0425982 \\
\hline 0.00169738 & 0.0424345 \\
\hline 0.00169086 & 0.0422715 \\
\hline 0.00168437 & 0.0421093 \\
\hline 0.00167791 & 0.0419477 \\
\hline 0.00167147 & 0.0417867 \\
\hline 0.00166506 & 0.0416265 \\
\hline 0.00165867 & 0.0414669 \\
\hline 0.00165232 & 0.0413079 \\
\hline 0.00164599 & 0.0411496 \\
\hline 0.00163968 & 0.040992 \\
\hline 0.0016334 & 0.0408351 \\
\hline 0.00162715 & 0.0406788 \\
\hline 0.00162092 & 0.0405231 \\
\hline 0.00161472 & 0.0403681 \\
\hline 0.00160855 & 0.0402137 \\
\hline 0.0016024 & 0.04006 \\
\hline 0.00159628 & 0.0399069 \\
\hline 0.00159018 & 0.0397544 \\
\hline 0.0015841 & 0.0396026 \\
\hline 0.00157806 & 0.0394514 \\
\hline
\end{tabular}

\begin{tabular}{|c|c|}
\hline 0.511454 & 0.909252 \\
\hline 0.509444 & 0.905678 \\
\hline 0.507442 & 0.902119 \\
\hline 0.505448 & 0.898575 \\
\hline 0.503463 & 0.895046 \\
\hline 0.501486 & 0.891532 \\
\hline 0.499518 & 0.888032 \\
\hline 0.497558 & 0.884548 \\
\hline 0.495606 & 0.881078 \\
\hline 0.493663 & 0.877623 \\
\hline 0.491727 & 0.874182 \\
\hline 0.4898 & 0.870756 \\
\hline 0.487881 & 0.867345 \\
\hline 0.48597 & 0.863947 \\
\hline 0.484067 & 0.860564 \\
\hline 0.482173 & 0.857196 \\
\hline 0.480286 & 0.853841 \\
\hline 0.478407 & 0.850501 \\
\hline 0.476536 & 0.847175 \\
\hline 0.474673 & 0.843863 \\
\hline 0.472817 & 0.840564 \\
\hline 0.47097 & 0.83728 \\
\hline 0.46913 & 0.834009 \\
\hline 0.467298 & 0.830753 \\
\hline 0.465474 & 0.82751 \\
\hline 0.463658 & 0.82428 \\
\hline 0.461849 & 0.821064 \\
\hline 0.460047 & 0.817862 \\
\hline 0.458254 & 0.814673 \\
\hline 0.456467 & 0.811498 \\
\hline 0.454689 & 0.808336 \\
\hline 0.452918 & 0.805187 \\
\hline 0.451154 & 0.802051 \\
\hline 0.449398 & 0.798929 \\
\hline 0.447649 & 0.79582 \\
\hline 0.445907 & 0.792723 \\
\hline 0.444173 & 0.78964 \\
\hline 0.442446 & 0.78657 \\
\hline 0.440726 & 0.783513 \\
\hline 0.439013 & 0.780468 \\
\hline 0.437308 & 0.777436 \\
\hline 0.43561 & 0.774417 \\
\hline 0.433919 & 0.771411 \\
\hline 0.432235 & 0.768417 \\
\hline 0.430558 & 0.765436 \\
\hline 0.428888 & 0.762467 \\
\hline 0.427225 & 0.759511 \\
\hline 0.425569 & 0.756567 \\
\hline 0.42392 & 0.753635 \\
\hline 0.422278 & 0.750716 \\
\hline 0.420643 & 0.747809 \\
\hline 0.419014 & 0.744914 \\
\hline 0.417393 & 0.742031 \\
\hline 0.415778 & 0.739161 \\
\hline 0.41417 & 0.736302 \\
\hline 0.412569 & 0.733455 \\
\hline 0.410974 & 0.73062 \\
\hline 0.409386 & 0.727797 \\
\hline 0.407805 & 0.724986 \\
\hline 0.40623 & 0.722187 \\
\hline 0.404662 & 0.719399 \\
\hline 0.4031 & 0.716623 \\
\hline 0.401545 & 0.713858 \\
\hline 0.399997 & 0.711105 \\
\hline 0.398455 & 0.708364 \\
\hline 0.396919 & 0.705634 \\
\hline 0.39539 & 0.702915 \\
\hline 0.393867 & 0.700208 \\
\hline 0.392351 & 0.697512 \\
\hline 0.39084 & 0.694827 \\
\hline 0.389336 & 0.692154 \\
\hline 0.387839 & 0.689491 \\
\hline 0.386348 & 0.68684 \\
\hline 0.384862 & 0.6842 \\
\hline 0.383383 & 0.68157 \\
\hline 0.381911 & 0.678952 \\
\hline 0.380444 & 0.676345 \\
\hline 0.378983 & 0.673748 \\
\hline 0.377529 & 0.671162 \\
\hline 0.37608 & 0.668588 \\
\hline 0.374638 & 0.666023 \\
\hline 0.373202 & 0.66347 \\
\hline 0.371771 & 0.660927 \\
\hline 0.370347 & 0.658394 \\
\hline 0.368928 & 0.655872 \\
\hline 0.367516 & 0.653361 \\
\hline 0.366109 & 0.65086 \\
\hline 0.364708 & 0.648369 \\
\hline 0.363313 & 0.645889 \\
\hline 0.361923 & 0.643419 \\
\hline 0.36054 & 0.64096 \\
\hline 0.359162 & 0.63851 \\
\hline 0.35779 & 0.636071 \\
\hline 0.356423 & 0.633642 \\
\hline 0.355063 & 0.631222 \\
\hline
\end{tabular}




\begin{tabular}{|c|c|c|}
\hline 1548 & 0.00424 & 0.00109658 \\
\hline 1549 & 0.00422 & 0.00109362 \\
\hline 1550 & 0.00422 & 0.00109066 \\
\hline 1551 & 0.0042 & 0.00108772 \\
\hline 1552 & 0.0042 & 0.00108478 \\
\hline 1553 & 0.00418 & 0.00108185 \\
\hline 1554 & 0.00418 & 0.00107893 \\
\hline 1555 & 0.00417 & 0.00107602 \\
\hline 1556 & 0.00416 & 0.00107312 \\
\hline 1557 & 0.00415 & 0.00107022 \\
\hline 1558 & 0.00414 & 0.00106733 \\
\hline 1559 & 0.00413 & 0.00106446 \\
\hline 1560 & 0.00412 & 0.00106159 \\
\hline 1561 & 0.00411 & 0.00105872 \\
\hline 1562 & 0.0041 & 0.00105587 \\
\hline 1563 & 0.00409 & 0.00105303 \\
\hline 1564 & 0.00408 & 0.00105019 \\
\hline 1565 & 0.00407 & 0.00104736 \\
\hline 1566 & 0.00406 & 0.00104454 \\
\hline 1567 & 0.00405 & 0.00104173 \\
\hline 1568 & 0.00404 & 0.00103892 \\
\hline 1569 & 0.00403 & 0.00103613 \\
\hline 1570 & 0.00403 & 0.00103334 \\
\hline 1571 & 0.00401 & 0.00103056 \\
\hline 1572 & 0.00401 & 0.00102779 \\
\hline 1573 & 0.00399 & 0.00102502 \\
\hline 1574 & 0.00399 & 0.00102227 \\
\hline 1575 & 0.00398 & 0.00101952 \\
\hline 1576 & 0.00397 & 0.00101678 \\
\hline 1577 & 0.00396 & 0.00101405 \\
\hline 1578 & 0.00395 & 0.00101132 \\
\hline 1579 & 0.00394 & 0.0010086 \\
\hline 1580 & 0.00393 & 0.0010059 \\
\hline 1581 & 0.00392 & 0.00100319 \\
\hline 1582 & 0.00391 & 0.0010005 \\
\hline 1583 & 0.0039 & 0.000997816 \\
\hline 1584 & 0.00389 & 0.000995139 \\
\hline 1585 & 0.00388 & 0.000992469 \\
\hline 1586 & 0.00388 & 0.000989807 \\
\hline 1587 & 0.00386 & 0.000987152 \\
\hline 1588 & 0.00386 & 0.000984505 \\
\hline 1589 & 0.00385 & 0.000981866 \\
\hline 1590 & 0.00384 & 0.000979234 \\
\hline 1591 & 0.00383 & 0.00097661 \\
\hline 1592 & 0.00382 & 0.000973994 \\
\hline 1593 & 0.00381 & 0.000971385 \\
\hline 1594 & 0.0038 & 0.000968784 \\
\hline 1595 & 0.00379 & 0.00096619 \\
\hline 1596 & 0.00378 & 0.000963603 \\
\hline 1597 & 0.00377 & 0.000961024 \\
\hline 1598 & 0.00377 & 0.000958453 \\
\hline 1599 & 0.00375 & 0.000955889 \\
\hline 1600 & 0.00375 & 0.000953332 \\
\hline 1601 & 0.00374 & 0.000950783 \\
\hline 1602 & 0.00373 & 0.000948241 \\
\hline 1603 & 0.00372 & 0.000945706 \\
\hline 1604 & 0.00371 & 0.000943178 \\
\hline 1605 & 0.0037 & 0.000940658 \\
\hline 1606 & 0.00369 & 0.000938145 \\
\hline 1607 & 0.00368 & 0.00093564 \\
\hline 1608 & 0.00367 & 0.000933141 \\
\hline 1609 & 0.00366 & 0.00093065 \\
\hline 1610 & 0.00366 & 0.000928166 \\
\hline 1611 & 0.00364 & 0.000925689 \\
\hline 1612 & 0.00364 & 0.000923219 \\
\hline 1613 & 0.00363 & 0.000920757 \\
\hline 1614 & 0.00362 & 0.000918301 \\
\hline 1615 & 0.00361 & 0.000915853 \\
\hline 1616 & 0.0036 & 0.000913411 \\
\hline 1617 & 0.00359 & 0.000910977 \\
\hline 1618 & 0.00359 & 0.000908549 \\
\hline 1619 & 0.00357 & 0.000906129 \\
\hline 1620 & 0.00357 & 0.000903715 \\
\hline 1621 & 0.00356 & 0.000901309 \\
\hline 1622 & 0.00355 & 0.000898909 \\
\hline 1623 & 0.00354 & 0.000896516 \\
\hline 1624 & 0.00353 & 0.000894131 \\
\hline 1625 & 0.00352 & 0.000891752 \\
\hline 1626 & 0.00351 & 0.000889379 \\
\hline 1627 & 0.0035 & 0.000887014 \\
\hline 1628 & 0.0035 & 0.000884656 \\
\hline 1629 & 0.00348 & 0.000882304 \\
\hline 1630 & 0.00348 & 0.000879959 \\
\hline 1631 & 0.00347 & 0.00087762 \\
\hline 1632 & 0.00346 & 0.000875289 \\
\hline 1633 & 0.00345 & 0.000872964 \\
\hline 1634 & 0.00344 & 0.000870646 \\
\hline 1635 & 0.00343 & 0.000868334 \\
\hline 1636 & 0.00343 & 0.00086603 \\
\hline 1637 & 0.00341 & 0.000863731 \\
\hline 1638 & 0.00341 & 0.00086144 \\
\hline 1639 & 0.0034 & 0.000859155 \\
\hline 1640 & 0.00339 & 0.000856876 \\
\hline 1641 & 0.00338 & 0.000854604 \\
\hline 1642 & 0.00337 & 0.000852339 \\
\hline
\end{tabular}

\begin{tabular}{|c|c|}
\hline 0.00157203 & 0.0393008 \\
\hline 0.00156604 & 0.0391509 \\
\hline 0.00156006 & 0.0390016 \\
\hline 0.00155411 & 0.0388529 \\
\hline 0.00154819 & 0.0387048 \\
\hline 0.00154229 & 0.0385573 \\
\hline 0.00153642 & 0.0384104 \\
\hline 0.00153057 & 0.0382642 \\
\hline 0.00152474 & 0.0381185 \\
\hline 0.00151894 & 0.0379735 \\
\hline 0.00151316 & 0.037829 \\
\hline 0.00150741 & 0.0376852 \\
\hline 0.00150168 & 0.0375419 \\
\hline 0.00149597 & 0.0373993 \\
\hline 0.00149029 & 0.0372572 \\
\hline 0.00148463 & 0.0371157 \\
\hline 0.00147899 & 0.0369748 \\
\hline 0.00147338 & 0.0368345 \\
\hline 0.00146779 & 0.0366948 \\
\hline 0.00146222 & 0.0365556 \\
\hline 0.00145668 & 0.036417 \\
\hline 0.00145116 & 0.036279 \\
\hline 0.00144566 & 0.0361415 \\
\hline 0.00144019 & 0.0360046 \\
\hline 0.00143473 & 0.0358683 \\
\hline 0.0014293 & 0.0357326 \\
\hline 0.0014239 & 0.0355974 \\
\hline 0.00141851 & 0.0354627 \\
\hline 0.00141315 & 0.0353287 \\
\hline 0.0014078 & 0.0351951 \\
\hline 0.00140249 & 0.0350621 \\
\hline 0.00139719 & 0.0349297 \\
\hline 0.00139191 & 0.0347978 \\
\hline 0.00138666 & 0.0346665 \\
\hline 0.00138143 & 0.0345357 \\
\hline 0.00137622 & 0.0344054 \\
\hline 0.00137103 & 0.0342757 \\
\hline 0.00136586 & 0.0341465 \\
\hline 0.00136071 & 0.0340178 \\
\hline 0.00135559 & 0.0338896 \\
\hline 0.00135048 & 0.033762 \\
\hline 0.0013454 & 0.0336349 \\
\hline 0.00134033 & 0.0335084 \\
\hline 0.00133529 & 0.0333823 \\
\hline 0.00133027 & 0.0332568 \\
\hline 0.00132527 & 0.0331318 \\
\hline 0.00132029 & 0.0330073 \\
\hline 0.00131533 & 0.0328833 \\
\hline 0.00131039 & 0.0327598 \\
\hline 0.00130547 & 0.0326368 \\
\hline 0.00130057 & 0.0325143 \\
\hline 0.00129569 & 0.0323923 \\
\hline 0.00129083 & 0.0322709 \\
\hline 0.001286 & 0.0321499 \\
\hline 0.00128118 & 0.0320294 \\
\hline 0.00127638 & 0.0319094 \\
\hline 0.0012716 & 0.0317899 \\
\hline 0.00126684 & 0.0316709 \\
\hline 0.00126209 & 0.0315524 \\
\hline 0.00125737 & 0.0314343 \\
\hline 0.00125267 & 0.0313168 \\
\hline 0.00124799 & 0.0311997 \\
\hline 0.00124332 & 0.0310831 \\
\hline 0.00123868 & 0.030967 \\
\hline 0.00123405 & 0.0308513 \\
\hline 0.00122945 & 0.0307361 \\
\hline 0.00122486 & 0.0306214 \\
\hline 0.00122029 & 0.0305072 \\
\hline 0.00121574 & 0.0303934 \\
\hline 0.0012112 & 0.0302801 \\
\hline 0.00120669 & 0.0301672 \\
\hline 0.00120219 & 0.0300549 \\
\hline 0.00119772 & 0.0299429 \\
\hline 0.00119326 & 0.0298314 \\
\hline 0.00118882 & 0.0297204 \\
\hline 0.00118439 & 0.0296098 \\
\hline 0.00117999 & 0.0294997 \\
\hline 0.0011756 & 0.0293901 \\
\hline 0.00117123 & 0.0292808 \\
\hline 0.00116688 & 0.029172 \\
\hline 0.00116255 & 0.0290637 \\
\hline 0.00115823 & 0.0289558 \\
\hline 0.00115393 & 0.0288483 \\
\hline 0.00114965 & 0.0287413 \\
\hline 0.00114539 & 0.0286347 \\
\hline 0.00114114 & 0.0285286 \\
\hline 0.00113691 & 0.0284228 \\
\hline 0.0011327 & 0.0283175 \\
\hline 0.00112851 & 0.0282127 \\
\hline 0.00112433 & 0.0281082 \\
\hline 0.00112017 & 0.0280042 \\
\hline 0.00111602 & 0.0279006 \\
\hline 0.0011119 & 0.0277974 \\
\hline 0.00110779 & 0.0276946 \\
\hline 0.00110369 & \\
\hline
\end{tabular}

\begin{tabular}{|c|c|}
\hline 0.353708 & 0.628813 \\
\hline 0.352358 & 0.626414 \\
\hline 0.351014 & 0.624025 \\
\hline 0.349676 & 0.621646 \\
\hline 0.348343 & 0.619277 \\
\hline 0.347016 & 0.616917 \\
\hline 0.345694 & 0.614567 \\
\hline 0.344378 & 0.612227 \\
\hline 0.343067 & 0.609897 \\
\hline 0.341761 & 0.607576 \\
\hline 0.340461 & 0.605265 \\
\hline 0.339167 & 0.602963 \\
\hline 0.337877 & 0.600671 \\
\hline 0.336593 & 0.598388 \\
\hline 0.335315 & 0.596115 \\
\hline 0.334041 & 0.593851 \\
\hline 0.332773 & 0.591597 \\
\hline 0.33151 & 0.589352 \\
\hline 0.330253 & 0.587116 \\
\hline 0.329 & 0.584889 \\
\hline 0.327753 & 0.582672 \\
\hline 0.326511 & 0.580464 \\
\hline 0.325274 & 0.578265 \\
\hline 0.324042 & 0.576074 \\
\hline 0.322815 & 0.573893 \\
\hline 0.321593 & 0.571721 \\
\hline 0.320376 & 0.569558 \\
\hline 0.319165 & 0.567404 \\
\hline 0.317958 & 0.565259 \\
\hline 0.316756 & 0.563122 \\
\hline 0.315559 & 0.560994 \\
\hline 0.314367 & 0.558875 \\
\hline 0.31318 & 0.556765 \\
\hline 0.311998 & 0.554663 \\
\hline 0.310821 & 0.552571 \\
\hline 0.309649 & 0.550486 \\
\hline 0.308481 & 0.548411 \\
\hline 0.307318 & 0.546343 \\
\hline 0.30616 & 0.544285 \\
\hline 0.305007 & 0.542234 \\
\hline 0.303858 & 0.540192 \\
\hline 0.302714 & 0.538159 \\
\hline 0.301575 & 0.536134 \\
\hline 0.300441 & 0.534117 \\
\hline 0.299311 & 0.532109 \\
\hline 0.298186 & 0.530108 \\
\hline 0.297065 & 0.528116 \\
\hline 0.295949 & 0.526132 \\
\hline 0.294838 & 0.524156 \\
\hline 0.293731 & 0.522189 \\
\hline 0.292629 & 0.520229 \\
\hline 0.291531 & 0.518277 \\
\hline 0.290438 & 0.516334 \\
\hline 0.289349 & 0.514398 \\
\hline 0.288265 & 0.51247 \\
\hline 0.287185 & 0.51055 \\
\hline 0.286109 & 0.508638 \\
\hline 0.285038 & 0.506734 \\
\hline 0.283971 & 0.504838 \\
\hline 0.282909 & 0.502949 \\
\hline 0.281851 & 0.501068 \\
\hline 0.280797 & 0.499195 \\
\hline 0.279748 & 0.497329 \\
\hline 0.278703 & 0.495471 \\
\hline 0.277662 & 0.493621 \\
\hline 0.276625 & 0.491778 \\
\hline 0.275593 & 0.489943 \\
\hline 0.274565 & 0.488115 \\
\hline 0.273541 & 0.486294 \\
\hline 0.272521 & 0.484481 \\
\hline 0.271505 & 0.482676 \\
\hline 0.270494 & 0.480878 \\
\hline 0.269486 & 0.479087 \\
\hline 0.268483 & 0.477303 \\
\hline 0.267484 & 0.475527 \\
\hline 0.266489 & 0.473758 \\
\hline 0.265498 & 0.471996 \\
\hline 0.26451 & 0.470241 \\
\hline 0.263527 & 0.468493 \\
\hline 0.262548 & 0.466753 \\
\hline 0.261573 & 0.465019 \\
\hline 0.260602 & 0.463293 \\
\hline 0.259635 & 0.461573 \\
\hline 0.258672 & 0.459861 \\
\hline 0.257712 & 0.458155 \\
\hline 0.256757 & 0.456457 \\
\hline 0.255805 & 0.454765 \\
\hline 0.254858 & 0.45308 \\
\hline 0.253914 & 0.451402 \\
\hline 0.252974 & 0.449731 \\
\hline 0.252038 & 0.448067 \\
\hline 0.251105 & 0.446409 \\
\hline 0.250177 & 0.444758 \\
\hline 0.249252 & 0.443114 \\
\hline 0.248331 & 0.441476 \\
\hline
\end{tabular}


$1643|\quad 0.00336|-0.00085008$

\begin{tabular}{l|l|l|}
1644 & 0.00336 & 0.000847827 \\
1645 & 0.00334 & 0.000845581 \\
1646 & 0.00334 & 0.000843342 \\
\hline
\end{tabular}

\begin{tabular}{l|l|l|}
1646 & 0.00334 & 0.000845581 \\
1647 & 0.00334 & 0.000843342 \\
\hline
\end{tabular}

$1647 \quad 0.00333 \quad 0.000841108$

\begin{tabular}{ll|l|}
1648 & 0.00332 & 0.000838882 \\
1649 & 0.00331 & 0.000836661 \\
1650 & 0.0033 & 0.000834447
\end{tabular}

$0.0033-0.000834447$

0.00329
0.00329 $\quad 0.00083224$

$0.00329 \quad 0.000830038$

$\begin{array}{ll}0.00327 & 0.000827843 \\ 0.00327 & 0.000825655 \\ 0.00326 & 0.000823472\end{array}$

$0.00326 \quad 0.000823472$

$0.00325 \quad 0.000821296$

$0.00324 \quad 0.000819126$

$0.00323 \quad 0.000816963$

$0.00322 \quad 0.000814805$

0.00322
0.00321 $\quad 0.000812654$

$0.0032 \quad 0.000808369$

0.00319

$0.00318 \quad 0.00080411$

$0.00317 \quad 0.000801989$

$0.00317 \quad 0.000799874$

0.00315
0.00315
0.00314 $\quad 0.000797766$

$0.00314 \quad 0.000793567$

$0.00313=0.000791476$

$0.00312 \quad 0.000789392$

$0.00312 \quad 0.000787313$

\begin{tabular}{r|r}
0.0031 & 0.00078524 \\
0.0031 & 0.000783174 \\
0.00309
\end{tabular}

$0.00309 \quad 0.000783114$

$0.00308 \quad 0.000779058$

$0.00307 \quad 0.000777009$

$0.00306 \quad 0.000774966$

0.00305
0.00305 $\quad \begin{aligned} & 0.000772929 \\ & 0.00304\end{aligned} \quad 0.000770897$

$0.00304 \quad 0.000768871$

$0.00303 \quad 0.000766851$

$0.00302 \quad 0.000764837$

$0.00301 \quad 0.000762829$

$0.003 \quad 0.000760826$

0.000758829

$\begin{array}{ll}0.00299 & 0.000756838 \\ 0.00298 & 0.000754853 \\ 0.00297 & 0.000752873\end{array}$

0.00297

$0.00296 \quad 0.000750898$

$0.00295 \quad 0.00074893$

$0.00295 \quad 0.000746967$

0.00294
0.00293
0.00292574501

$\begin{array}{ll}0.00293 & 0.000743058 \\ 0.00292 & 0.000741112\end{array}$

0.00291

0.0029

0.0029

0.00289

0.00288

0.00287

0.00287

0.00285

0.00285

0.00284
0.00283

0.00283

0.00282

0.0028

0.0028
0.0028

0.00279

0.00278

0.00277

0.00277

0.00276

0.00275

0.00274

0.00273
0.00272

0.00272

0.00271

0.0027

0.00269

0.00269

0.00267

0.00267

0.00266

0.00265

0.00264

0.00264

0.00263

0.00262

0.00261

0.00261

0.00259

0.00259
0.00258
0.00109961

0.00109555

0.0010915

0.00108748

0.00108347
0.00107947

0.00107549

0.00107153

0.00106758

0.00106365
0.00105974

0.00105584

0.00105195

0.00104808

0.00104423

0.00104039

0.00103657

0.00103277

0.0010252

0.00102144

0.00101769

0.00101396

0.00101025
0.00100655

0.00100286

0.000999189

0.000995533

0.000991892

0.000988265

0.000984653

0.000981055

0.000977472

0.000973903

0.000970349

0.000963282

0.00095977

0.000956272

0.000952788

0.000949318

0.000945862

0.00094242

0.000938991

0.000935576

0.000928788

0.000925413

0.000922053

0.000918706

0.000915372

0.00091205

0.000908744
0.00090545

0.000902169

0.000898901

0.000895646

0.000892404

0.000889175

0.000885959

0.000879565

0.000876387

0.000873222

0.000870069

0.000866929

0.000863801

0.000860686

0.000857583

0.000854492

0.000851414

0.000848348

0.000845294

0.000842252

0.000839222

0.000836204
0.000833198

0.000830204

0.000827222

0.000824252

0.000821293

0.000818347

0.000815411

0.000812488

0.000809576

0.000806675

0.000803786

0.000800908

0.000798042

0.000795187

0.000792343

0.00078951

0.000783878

0.000781079

Page 19 of 33
0.0274903

0.0273888

0.0272877

0.027187
0.0270867

0.0269868

0.0268873

0.0267882

0.0266896

0.0265913

0.0264934

0.0262988

0.0262021

0.0261058

0.0260098

0.0259143

0.0258191

0.02563

0.0255359

0.0254423

0.025349

0.0252562

0.0251636

.0250715

0.0249797

0.0247973

0.0247066

0.0246163

0.0244368

0.0242587

0241702

0.0240821

0.0239943

0.0239068

0.0238197

0.023733

0.0236465

0.0235605

0.0234748

0.0233894

0.0231353

0.0230513

0.0229676

0.0228843

0.0228013

0.0227186

0.0226362

0.0225542

0.0224725

0.0223912

0.0223101

0222294

0.022149

0.0220689

0.0219891

0.0219097

0.0218305

0.0217517

0.0216732

0.021595

0.0215171

0.0214396

0.0213623

0.0212854

0.0210563

.0209806

.0209051
0.02083

0.0207551

0.0206806

0.0206063

0.0205323

0.0204587

0.0203853

0.0203122

0.0202394

0.0201669

0.0200946

0.0200227

0.019951

0.0198797

0.0198086

0.0196672

0.019597

Printed 10/2/05,4:24 PM

$0.247413 \mid \quad 0.439846$
0.246499

\begin{tabular}{l}
0.438221 \\
\hline
\end{tabular}

\begin{tabular}{ll}
0.244683 & 0.436603 \\
0.24378 & 0.434992 \\
\hline
\end{tabular}

$\begin{array}{ll}0.24378 & 0.433387 \\ 0.242881 & 0.431789\end{array}$

$0.241986 \quad 0.430197$

$0.241094 \quad 0.428612$

$0.240206 \quad 0.427033$

\begin{tabular}{r|r}
0.239322 & 0.42546 \\
0.23844 & 0.423894 \\
0.23753 & 0.422334
\end{tabular}

$0.237563 \quad 0.422334$
0.236689

\begin{tabular}{l}
0.236689 \\
0.420781 \\
\hline
\end{tabular}

0.234952

\begin{tabular}{l|l}
0.234088 & 0.416157 \\
0.233229 & 0.414629
\end{tabular}

0.233229
0.232372
0.231519

0.233229
0.232372
0.231519
0.23067 $\quad \begin{array}{r}0.413106 \\ \quad 0.41159 \\ \hline\end{array}$

$0.23067 \quad 0.410079$

$0.229823 \quad 0.408575$

$0.228981 \quad 0.407077$

0.228141
0.227305 $\quad \begin{aligned} & 0.405585 \\ & 0.226473\end{aligned}$

$0.226473 \quad 0.402618$

0.225644
0.224818
0.223995

0.223995

$0.223176 \quad 0.396757$
0.22236

$0.22236 \quad 0.395306$

0.221547
0.220737
0.219931

$0.219931 \quad 0.390989$

$0.219128 \quad 0.389561$

$0.218328 \quad 0.388139$

$0.217532 \quad 0.386723$
0.216738

$0.216738 \quad 0.385313$

0.215948

0.214377

$0.213597-0.379727$ 


\begin{tabular}{|c|c|c|}
\hline 1738 & 0.00257 & 0.000662465 \\
\hline 1739 & 0.00256 & 0.000660748 \\
\hline 1740 & 0.00256 & 0.000659036 \\
\hline 1741 & 0.00255 & 0.000657328 \\
\hline 1742 & 0.00254 & 0.000655626 \\
\hline 1743 & 0.00253 & 0.000653928 \\
\hline 1744 & 0.00253 & 0.000652236 \\
\hline 1745 & 0.00251 & 0.000650548 \\
\hline 1746 & 0.00251 & 0.000648865 \\
\hline 1747 & 0.0025 & 0.000647186 \\
\hline 1748 & 0.00249 & 0.000645513 \\
\hline 1749 & 0.00248 & 0.000643844 \\
\hline 1750 & 0.00248 & 0.00064218 \\
\hline 1751 & 0.00247 & 0.00064052 \\
\hline 1752 & 0.00246 & 0.000638866 \\
\hline 1753 & 0.00245 & 0.000637216 \\
\hline 1754 & 0.00245 & 0.00063557 \\
\hline 1755 & 0.00244 & 0.000633929 \\
\hline 1756 & 0.00243 & 0.000632293 \\
\hline 1757 & 0.00242 & 0.000630662 \\
\hline 1758 & 0.00242 & 0.000629035 \\
\hline 1759 & 0.0024 & 0.000627413 \\
\hline 1760 & 0.0024 & 0.000625795 \\
\hline 1761 & 0.00239 & 0.000624182 \\
\hline 1762 & 0.00238 & 0.000622574 \\
\hline 1763 & 0.00237 & 0.00062097 \\
\hline 1764 & 0.00237 & 0.00061937 \\
\hline 1765 & 0.00236 & 0.000617775 \\
\hline 1766 & 0.00235 & 0.000616185 \\
\hline 1767 & 0.00234 & 0.000614599 \\
\hline 1768 & 0.00234 & 0.000613017 \\
\hline 1769 & 0.00232 & 0.00061144 \\
\hline 1770 & 0.00232 & 0.000609868 \\
\hline 1771 & 0.00231 & 0.0006083 \\
\hline 1772 & 0.00231 & 0.000606736 \\
\hline 1773 & 0.00229 & 0.000605177 \\
\hline 1774 & 0.00229 & 0.000603622 \\
\hline 1775 & 0.00228 & 0.000602072 \\
\hline 1776 & 0.00227 & 0.000600526 \\
\hline 1777 & 0.00226 & 0.000598984 \\
\hline 1778 & 0.00226 & 0.000597447 \\
\hline 1779 & 0.00225 & 0.000595913 \\
\hline 1780 & 0.00224 & 0.000594385 \\
\hline 1781 & 0.00223 & 0.00059286 \\
\hline 1782 & 0.00223 & 0.00059134 \\
\hline 1783 & 0.00222 & 0.000589824 \\
\hline 1784 & 0.00221 & 0.000588313 \\
\hline 1785 & 0.0022 & 0.000586806 \\
\hline 1786 & 0.0022 & 0.000585303 \\
\hline 1787 & 0.00218 & 0.000583804 \\
\hline 1788 & 0.00218 & 0.000582309 \\
\hline 1789 & 0.00217 & 0.000580819 \\
\hline 1790 & 0.00217 & 0.000579333 \\
\hline 1791 & 0.00215 & 0.00057785 \\
\hline 1792 & 0.00215 & 0.000576373 \\
\hline 1793 & 0.00214 & 0.000574899 \\
\hline 1794 & 0.00213 & 0.000573429 \\
\hline 1795 & 0.00212 & 0.000571964 \\
\hline 1796 & 0.00212 & 0.000570503 \\
\hline 1797 & 0.00211 & 0.000569046 \\
\hline 1798 & 0.0021 & 0.000567592 \\
\hline 1799 & 0.00209 & 0.000566143 \\
\hline 1800 & 0.00209 & 0.000564699 \\
\hline 1801 & 0.00208 & 0.000563258 \\
\hline 1802 & 0.00207 & 0.000561821 \\
\hline 1803 & 0.00206 & 0.000560388 \\
\hline 1804 & 0.00206 & 0.000558959 \\
\hline 1805 & 0.00205 & 0.000557535 \\
\hline 1806 & 0.00204 & 0.000556114 \\
\hline 1807 & 0.00203 & 0.000554697 \\
\hline 1808 & 0.00203 & 0.000553284 \\
\hline 1809 & 0.00201 & 0.000551875 \\
\hline 1810 & 0.00201 & 0.000550471 \\
\hline 1811 & 0.002 & 0.00054907 \\
\hline 1812 & 0.002 & 0.000547673 \\
\hline 1813 & 0.00198 & 0.00054628 \\
\hline 1814 & 0.00198 & 0.00054489 \\
\hline 1815 & 0.00197 & 0.000543505 \\
\hline 1816 & 0.00197 & 0.000542124 \\
\hline 1817 & 0.00195 & 0.000540746 \\
\hline 1818 & 0.00195 & 0.000539373 \\
\hline 1819 & 0.00194 & 0.000538003 \\
\hline 1820 & 0.00193 & 0.000536637 \\
\hline 1821 & 0.00192 & 0.000535275 \\
\hline 1822 & 0.00192 & 0.000533916 \\
\hline 1823 & 0.00191 & 0.000532562 \\
\hline 1824 & 0.0019 & 0.000531211 \\
\hline 1825 & 0.00189 & 0.000529864 \\
\hline 1826 & 0.00189 & 0.000528521 \\
\hline 1827 & 0.00188 & 0.000527181 \\
\hline 1828 & 0.00187 & 0.000525846 \\
\hline 1829 & 0.00186 & 0.000524514 \\
\hline 1830 & 0.00186 & 0.000523185 \\
\hline 1831 & 0.00185 & 0.000521861 \\
\hline 1832 & 0.00184 & 0.00052054 \\
\hline
\end{tabular}

\begin{tabular}{|c|c|}
\hline 0.000778291 & 0.0194573 \\
\hline 0.000775514 & 0.0193878 \\
\hline 0.000772747 & 0.0193187 \\
\hline 0.000769992 & 0.0192498 \\
\hline 0.000767247 & 0.0191812 \\
\hline 0.000764513 & 0.0191128 \\
\hline 0.00076179 & 0.0190448 \\
\hline 0.000759078 & 0.018977 \\
\hline 0.000756376 & 0.0189094 \\
\hline 0.000753685 & 0.0188421 \\
\hline 0.000751005 & 0.0187751 \\
\hline 0.000748335 & 0.0187084 \\
\hline 0.000745675 & 0.0186419 \\
\hline 0.000743026 & 0.0185756 \\
\hline 0.000740387 & 0.0185097 \\
\hline 0.000737759 & 0.018444 \\
\hline 0.000735141 & 0.0183785 \\
\hline 0.000732533 & 0.0183133 \\
\hline 0.000729935 & 0.0182484 \\
\hline 0.000727348 & 0.0181837 \\
\hline 0.00072477 & 0.0181193 \\
\hline 0.000722203 & 0.0180551 \\
\hline 0.000719646 & 0.0179911 \\
\hline 0.000717099 & 0.0179275 \\
\hline 0.000714561 & 0.017864 \\
\hline 0.000712034 & 0.0178009 \\
\hline 0.000709517 & 0.0177379 \\
\hline 0.000707009 & 0.0176752 \\
\hline 0.000704511 & 0.0176128 \\
\hline 0.000702023 & 0.0175506 \\
\hline 0.000699545 & 0.0174886 \\
\hline 0.000697076 & 0.0174269 \\
\hline 0.000694617 & 0.0173654 \\
\hline 0.000692167 & 0.0173042 \\
\hline 0.000689727 & 0.0172432 \\
\hline 0.000687297 & 0.0171824 \\
\hline 0.000684876 & 0.0171219 \\
\hline 0.000682464 & 0.0170616 \\
\hline 0.000680062 & 0.0170015 \\
\hline 0.000677669 & 0.0169417 \\
\hline 0.000675285 & 0.0168821 \\
\hline 0.000672911 & 0.0168228 \\
\hline 0.000670546 & 0.0167637 \\
\hline 0.00066819 & 0.0167048 \\
\hline 0.000665843 & 0.0166461 \\
\hline 0.000663506 & 0.0165876 \\
\hline 0.000661177 & 0.0165294 \\
\hline 0.000658858 & 0.0164714 \\
\hline 0.000656547 & 0.0164137 \\
\hline 0.000654246 & 0.0163561 \\
\hline 0.000651953 & 0.0162988 \\
\hline 0.000649669 & 0.0162417 \\
\hline 0.000647394 & 0.0161849 \\
\hline 0.000645128 & 0.0161282 \\
\hline 0.000642871 & 0.0160718 \\
\hline 0.000640622 & 0.0160156 \\
\hline 0.000638383 & 0.0159596 \\
\hline 0.000636151 & 0.0159038 \\
\hline 0.000633929 & 0.0158482 \\
\hline 0.000631715 & 0.0157929 \\
\hline 0.000629509 & 0.0157377 \\
\hline 0.000627313 & 0.0156828 \\
\hline 0.000625124 & 0.0156281 \\
\hline 0.000622944 & 0.0155736 \\
\hline 0.000620773 & 0.0155193 \\
\hline 0.000618609 & 0.0154652 \\
\hline 0.000616455 & 0.0154114 \\
\hline 0.000614308 & 0.0153577 \\
\hline 0.00061217 & 0.0153042 \\
\hline 0.00061004 & 0.015251 \\
\hline 0.000607918 & 0.0151979 \\
\hline 0.000605804 & 0.0151451 \\
\hline 0.000603699 & 0.0150925 \\
\hline 0.000601601 & 0.01504 \\
\hline 0.000599512 & 0.0149878 \\
\hline 0.000597431 & 0.0149358 \\
\hline 0.000595357 & 0.0148839 \\
\hline 0.000593292 & 0.0148323 \\
\hline 0.000591235 & 0.0147809 \\
\hline 0.000589185 & 0.0147296 \\
\hline 0.000587144 & 0.0146786 \\
\hline 0.00058511 & 0.0146277 \\
\hline 0.000583084 & 0.0145771 \\
\hline 0.000581066 & 0.0145266 \\
\hline 0.000579055 & 0.0144764 \\
\hline 0.000577052 & 0.0144263 \\
\hline 0.000575057 & 0.0143764 \\
\hline 0.00057307 & 0.0143267 \\
\hline 0.00057109 & 0.0142773 \\
\hline 0.000569118 & 0.0142279 \\
\hline 0.000567153 & 0.0141788 \\
\hline 0.000565196 & 0.0141299 \\
\hline 0.000563246 & 0.0140812 \\
\hline 0.000561304 & 0.0140326 \\
\hline 0.000559369 & 0.0139842 \\
\hline
\end{tabular}

\begin{tabular}{|c|c|}
\hline 0.175115 & 0.311316 \\
\hline D.174491 & 0.310205 \\
\hline 0.173868 & 0.309099 \\
\hline 0.173248 & 0.307997 \\
\hline D.172631 & 0.306899 \\
\hline 0.172016 & 0.305805 \\
\hline 0.171403 & 0.304716 \\
\hline 0.170793 & 0.303631 \\
\hline 0.170185 & 0.302551 \\
\hline 0.169579 & 0.301474 \\
\hline 0.168976 & 0.300402 \\
\hline .168375 & 0.299334 \\
\hline 0.167777 & 0.29827 \\
\hline 0.167181 & 0.29721 \\
\hline D.166587 & 0.296155 \\
\hline 0.165996 & 0.295103 \\
\hline 0.165407 & 0.294056 \\
\hline 0.16482 & 0.293013 \\
\hline .164235 & 0.291974 \\
\hline 0.163653 & 0.290939 \\
\hline 0.163073 & 0.289908 \\
\hline D.162496 & 0.288881 \\
\hline 0.16192 & 0.287858 \\
\hline 0.161347 & 0.286839 \\
\hline 0.160776 & 0.285825 \\
\hline .160208 & 0.284814 \\
\hline 0.159641 & 0.283807 \\
\hline 0.159077 & 0.282804 \\
\hline 0.158515 & 0.281804 \\
\hline 0.157955 & 0.280809 \\
\hline 0.157398 & 0.279818 \\
\hline 0.156842 & 0.27883 \\
\hline 0.156289 & 0.277847 \\
\hline 0.155738 & 0.276867 \\
\hline 0.155189 & 0.275891 \\
\hline D.154642 & 0.274919 \\
\hline 0.154097 & 0.27395 \\
\hline 0.153554 & 0.272986 \\
\hline 0.153014 & 0.272025 \\
\hline 0.152476 & 0.271068 \\
\hline 0.151939 & 0.270114 \\
\hline 0.151405 & 0.269164 \\
\hline D.150873 & 0.268218 \\
\hline 0.150343 & 0.267276 \\
\hline 0.149815 & 0.266337 \\
\hline 0.149289 & 0.265402 \\
\hline 0.148765 & 0.264471 \\
\hline 0.148243 & 0.263543 \\
\hline 0.147723 & 0.262619 \\
\hline 0.147205 & 0.261698 \\
\hline 0.146689 & 0.260781 \\
\hline 0.146176 & 0.259868 \\
\hline 0.145664 & 0.258958 \\
\hline 0.145154 & 0.258051 \\
\hline 0.144646 & 0.257148 \\
\hline 0.14414 & 0.256249 \\
\hline 0.143636 & 0.255353 \\
\hline 0.143134 & 0.254461 \\
\hline 0.142634 & 0.253572 \\
\hline 0.142136 & 0.252686 \\
\hline 0.14164 & 0.251804 \\
\hline 0.141145 & 0.250925 \\
\hline 0.140653 & 0.25005 \\
\hline 0.140162 & 0.249178 \\
\hline 0.139674 & 0.248309 \\
\hline 0.139187 & 0.247444 \\
\hline 0.138702 & 0.246582 \\
\hline 0.138219 & 0.245723 \\
\hline 0.137738 & 0.244868 \\
\hline 0.137259 & 0.244016 \\
\hline 0.136782 & 0.243167 \\
\hline 0.136306 & 0.242322 \\
\hline 0.135832 & 0.24148 \\
\hline 0.13536 & 0.240641 \\
\hline 0.13489 & 0.239805 \\
\hline 0.134422 & 0.238972 \\
\hline 0.133955 & 0.238143 \\
\hline 0.133491 & 0.237317 \\
\hline 0.133028 & 0.236494 \\
\hline 0.132567 & 0.235674 \\
\hline 0.132107 & 0.234857 \\
\hline 0.13165 & 0.234044 \\
\hline 0.131194 & 0.233234 \\
\hline 0.13074 & 0.232426 \\
\hline 0.130287 & 0.231622 \\
\hline D.129837 & 0.230821 \\
\hline 0.129388 & 0.230023 \\
\hline 0.128941 & 0.229228 \\
\hline 0.128495 & 0.228436 \\
\hline 0.128052 & 0.227647 \\
\hline D.127609 & 0.226861 \\
\hline .127169 & 0.226078 \\
\hline 0.12673 & 0.225299 \\
\hline .126293 & 0.224522 \\
\hline & \\
\hline
\end{tabular}




\begin{tabular}{|c|c|c|}
\hline 1833 & 0.00183 & 0.000519223 \\
\hline 1834 & 0.00183 & 0.000517909 \\
\hline 1835 & 0.00182 & 0.0005166 \\
\hline 1836 & 0.00181 & 0.000515294 \\
\hline 1837 & 0.0018 & 0.000513991 \\
\hline 1838 & 0.0018 & 0.000512692 \\
\hline 1839 & 0.00178 & 0.000511397 \\
\hline 1840 & 0.00178 & 0.000510105 \\
\hline 1841 & 0.00177 & 0.000508817 \\
\hline 1842 & 0.00177 & 0.000507533 \\
\hline 1843 & 0.00175 & 0.000506252 \\
\hline 1844 & 0.00175 & 0.000504975 \\
\hline 1845 & 0.00174 & 0.000503701 \\
\hline 1846 & 0.00174 & 0.000502431 \\
\hline 1847 & 0.00172 & 0.000501165 \\
\hline 1848 & 0.00172 & 0.000499902 \\
\hline 1849 & 0.00171 & 0.000498642 \\
\hline 1850 & 0.0017 & 0.000497386 \\
\hline 1851 & 0.00169 & 0.000496133 \\
\hline 1852 & 0.00169 & 0.000494884 \\
\hline 1853 & 0.00168 & 0.000493639 \\
\hline 1854 & 0.00167 & 0.000492397 \\
\hline 1855 & 0.00166 & 0.000491158 \\
\hline 1856 & 0.00166 & 0.000489923 \\
\hline 1857 & 0.00165 & 0.000488691 \\
\hline 1858 & 0.00164 & 0.000487463 \\
\hline 1859 & 0.00163 & 0.000486238 \\
\hline 1860 & 0.00163 & 0.000485016 \\
\hline 1861 & 0.00161 & 0.000483798 \\
\hline 1862 & 0.00161 & 0.000482584 \\
\hline 1863 & 0.0016 & 0.000481372 \\
\hline 1864 & 0.0016 & 0.000480164 \\
\hline 1865 & 0.00158 & 0.000478959 \\
\hline 1866 & 0.00158 & 0.000477758 \\
\hline 1867 & 0.00157 & 0.00047656 \\
\hline 1868 & 0.00157 & 0.000475366 \\
\hline 1869 & 0.00155 & 0.000474174 \\
\hline 1870 & 0.00155 & 0.000472986 \\
\hline 1871 & 0.00154 & 0.000471802 \\
\hline 1872 & 0.00153 & 0.00047062 \\
\hline 1873 & 0.00152 & 0.000469442 \\
\hline 1874 & 0.00152 & 0.000468267 \\
\hline 1875 & 0.00151 & 0.000467096 \\
\hline 1876 & 0.0015 & 0.000465927 \\
\hline 1877 & 0.00149 & 0.000464762 \\
\hline 1878 & 0.00149 & 0.0004636 \\
\hline 1879 & 0.00147 & 0.000462442 \\
\hline 1880 & 0.00147 & 0.000461286 \\
\hline 1881 & 0.00146 & 0.000460134 \\
\hline 1882 & 0.00146 & 0.000458985 \\
\hline 1883 & 0.00144 & 0.000457839 \\
\hline 1884 & 0.00144 & 0.000456696 \\
\hline 1885 & 0.00143 & 0.000455557 \\
\hline 1886 & 0.00142 & 0.00045442 \\
\hline 1887 & 0.00141 & 0.000453287 \\
\hline 1888 & 0.00141 & 0.000452157 \\
\hline 1889 & 0.0014 & 0.00045103 \\
\hline 1890 & 0.00139 & 0.000449906 \\
\hline 1891 & 0.00138 & 0.000448785 \\
\hline 1892 & 0.00138 & 0.000447668 \\
\hline 1893 & 0.00136 & 0.000446553 \\
\hline 1894 & 0.00136 & 0.000445442 \\
\hline 1895 & 0.00135 & 0.000444333 \\
\hline 1896 & 0.00135 & 0.000443228 \\
\hline 1897 & 0.00133 & 0.000442126 \\
\hline 1898 & 0.00133 & 0.000441026 \\
\hline 1899 & 0.00132 & 0.00043993 \\
\hline 1900 & 0.00131 & 0.000438837 \\
\hline 1901 & 0.0013 & 0.000437747 \\
\hline 1902 & 0.0013 & 0.00043666 \\
\hline 1903 & 0.00128 & 0.000435575 \\
\hline 1904 & 0.00128 & 0.000434494 \\
\hline 1905 & 0.00127 & 0.000433416 \\
\hline 1906 & 0.00126 & 0.000432341 \\
\hline 1907 & 0.00125 & 0.000431268 \\
\hline 1908 & 0.00125 & 0.000430199 \\
\hline 1909 & 0.00123 & 0.000429133 \\
\hline 1910 & 0.00123 & 0.000428069 \\
\hline 1911 & 0.00122 & 0.000427009 \\
\hline 1912 & 0.00122 & 0.000425951 \\
\hline 1913 & 0.0012 & 0.000424897 \\
\hline 1914 & 0.0012 & 0.000423845 \\
\hline 1915 & 0.00118 & 0.000422796 \\
\hline 1916 & 0.00118 & 0.00042175 \\
\hline 1917 & 0.00117 & 0.000420707 \\
\hline 1918 & 0.00117 & 0.000419667 \\
\hline 1919 & 0.00115 & 0.000418629 \\
\hline 1920 & 0.00115 & 0.000417595 \\
\hline 1921 & 0.00113 & 0.000416563 \\
\hline 1922 & 0.00113 & 0.000415534 \\
\hline 1923 & 0.00112 & 0.000414508 \\
\hline 1924 & 0.00111 & 0.000413485 \\
\hline 1925 & 0.0011 & 0.000412465 \\
\hline 1926 & 0.0011 & 0.000411447 \\
\hline 1927 & 0.00108 & 0.000410432 \\
\hline
\end{tabular}

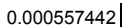
0.000555522 0.000553609 0.000551704 0.000549805 0.000546031 0.000544154 0.000542285 0.000540423 0.000538568 0.000536719 0.000534878

0.000533044 0.000531217 0.000529397 0.000527584 0.000525778 0.000523978 0.000522186

0.0005204 0.000518621 0.000516848 0.000515083 0.000513324 0.000511572 0.000509826 0.000508087 0.000506355 0.000504629 0.00050291 0.000501197 0.000499491 0.000497791 0.000496098 0.000494411 0.00049273 0.000491056 0.000489388 0.000487726

0.00048607 0.000484421 0.000482778 0.000481142 0.000479511 0.000477886 0.000476268 0.000474656 0.00047305 0.000471449 0.000468267 0.000466685 0.000465109 0.000463538 0.000461974 0.000460415 0.000458863 0.000457316 0.000455775 0.000454239 0.0004527 0.000451186 0.000449668 0.000448155 0.000446648 0.000445147 0.000443652 0.000442162 0.000440677 0.000439198 0.000437725 0.000436257 0.000434795 0.000433338 0.000431886 0.00043044 0.000428999 0.000427564 0.000426134 0.00042471 0.00042329 0.000421876 0.000420467 0.000419064 0.000417665 0.000416272 0.000414884 0.000413501 0.000412123 0.000410751 0.000409383 0.000408021 0.000406663 0.00040531
0.013936

0.013888

0.0138402

0.0137926

0.0137451
0.0136979

0.0136508

0.0136039

0.0135571

0.0135106

0.0134642

0.013418
0.013372

0.0133261

0.0132804

0.0132349

0.0131896

0.0131444

0.0130546

0.01301

0.0129655

0.0129212

0.0128771
0.0128331

0.0127893

0.0127457

0.0127022

0.0126589

0.0126157

0.0125727

0.0124873

0.0124024

0.0123603

0.0123182

0.0122764

0.0122347

0.0121931

0.0121518

0.0121105

0.0120695

0.0120285

0.0119878

0.0118664

0.0118262

0.0117862

0.0117464

0.0117067

0.0116671

0.0116277

0.0115885

0.0115493

0.0115104

0.0114716

0.0114329

0.0113944

0.011356

0.0113177

0.0112796

0.0112417

0.0112039

0.0111287

0.0110913

0.011054
0.0110169

0.01098

0.0109431

0.0109064

0.0108699

0.0108334

0.0107972

0.010761

0.010725

0.0106177

0.0105823

0.0105469

0.0105117

0.0104766

0.0104416

0.0104068

0.0103721

0.0103375

0.0103031

0.0102688

.

0.0101666
0.0101328
0.125424

0.124992

0.124562

0.124133

0.123706
0.123281

0.122857

0.122435

0.122014

0.121595
0.121178

0.120762

0.120348

0.119935

0.119114

0.118706

0.1183

0.117895

0.11709

0.11669

0.116291

0.115894

0.115498

0.115104

0.11432

0.11393

0.113542

0.113155

0.112769

0.112385

0.112003

0.111622

0.111242

0.110864

0.110488

0.110112

0.109738

0.108995

0.108625

0.108257

0.10789

0.107524

0.10716

0.106798

0.106436

0.106076
0.105717

0.10536

0.105004

0.104649

0.104296

0.103944

0.103593

0.103244

0.102896

0.102549

0.102204

0.10186

0.101517

0.101175

0.100835

0.100496

0.100158

0.0998216

0.0994864

0.0991524

0.0988196

0.0984881

0.0978288

0.097501
0.0971744
0.096849

0.0971744
0.096849

0.0965249

0.0962019

0.0958802

0.0955596

0.0952403

0.0949221

0.0946051

0.0942893

0.0936612

0.0933489

0.0930378
0.0927278

0.0924189

0.0921112

0.0918047

0.0914993
0.091195

Printed 10/2/05,4

0.222977

0.222209

0.221444
0.220681

0.219922

0.219166
0.218412

0.217662

0.216914

0.216169
0.215427

0.214688

0.213951

0.213218

0.212487

0.211759

0.211034

0.210311
0.209591

0.208874

0.20816

0.207448

0.206739
0.206033

0.20533

0.204629

0.203235
0.202542

0.201852

0.201164 


\begin{tabular}{|c|c|c|}
\hline 1928 & 0.00108 & 0.00040942 \\
\hline 1929 & 0.00106 & 0.000408411 \\
\hline 1930 & 0.00106 & 0.000407405 \\
\hline 1931 & 0.00105 & 0.000406401 \\
\hline 1932 & 0.00105 & 0.0004054 \\
\hline 1933 & 0.00103 & 0.000404402 \\
\hline 1934 & 0.00103 & 0.000403406 \\
\hline 1935 & 0.00101 & 0.000402413 \\
\hline 1936 & 0.00101 & 0.000401423 \\
\hline 1937 & 0.000994 & 0.000400436 \\
\hline 1938 & 0.000992 & 0.000399452 \\
\hline 1939 & 0.000976 & 0.00039847 \\
\hline 1940 & 0.000974 & 0.00039749 \\
\hline 1941 & 0.000957 & 0.000396514 \\
\hline 1942 & 0.000956 & 0.00039554 \\
\hline 1943 & 0.000939 & 0.000394569 \\
\hline 1944 & 0.000937 & 0.0003936 \\
\hline 1945 & 0.00092 & 0.000392634 \\
\hline 1946 & 0.000918 & 0.000391671 \\
\hline 1947 & 0.000901 & 0.000390711 \\
\hline 1948 & 0.0009 & 0.000389753 \\
\hline 1949 & 0.000882 & 0.000388797 \\
\hline 1950 & 0.00088 & 0.000387845 \\
\hline 1951 & 0.000863 & 0.000386894 \\
\hline 1952 & 0.000861 & 0.000385947 \\
\hline 1953 & 0.000843 & 0.000385002 \\
\hline 1954 & 0.000841 & 0.000384059 \\
\hline 1955 & 0.000823 & 0.00038312 \\
\hline 1956 & 0.000822 & 0.000382182 \\
\hline 1957 & 0.000803 & 0.000381248 \\
\hline 1958 & 0.000801 & 0.000380316 \\
\hline 1959 & 0.000782 & 0.000379386 \\
\hline 1960 & 0.000781 & 0.000378459 \\
\hline 1961 & 0.000761 & 0.000377534 \\
\hline 1962 & 0.00076 & 0.000376612 \\
\hline 1963 & 0.00074 & 0.000375693 \\
\hline 1964 & 0.000739 & 0.000374776 \\
\hline 1965 & 0.000718 & 0.000373861 \\
\hline 1966 & 0.000717 & 0.000372949 \\
\hline 1967 & 0.000696 & 0.00037204 \\
\hline 1968 & 0.000695 & 0.000371133 \\
\hline 1969 & 0.000673 & 0.000370228 \\
\hline 1970 & 0.000672 & 0.000369326 \\
\hline 1971 & 0.00065 & 0.000368426 \\
\hline 1972 & 0.000649 & 0.000367529 \\
\hline 1973 & 0.000626 & 0.000366634 \\
\hline 1974 & 0.000625 & 0.000365742 \\
\hline 1975 & 0.000601 & 0.000364852 \\
\hline 1976 & 0.0006 & 0.000363965 \\
\hline 1977 & 0.000576 & 0.000363079 \\
\hline 1978 & 0.000575 & 0.000362197 \\
\hline 1979 & 0.00055 & 0.000361317 \\
\hline 1980 & 0.000549 & 0.000360439 \\
\hline 1981 & 0.000522 & 0.000359563 \\
\hline 1982 & 0.000521 & 0.00035869 \\
\hline 1983 & 0.000493 & 0.000357819 \\
\hline 1984 & 0.000493 & 0.000356951 \\
\hline 1985 & 0.000463 & 0.000356085 \\
\hline 1986 & 0.000463 & 0.000355221 \\
\hline 1987 & 0.000431 & 0.00035436 \\
\hline 1988 & 0.000431 & 0.000353501 \\
\hline 1989 & 0.000397 & 0.000352644 \\
\hline 1990 & 0.000397 & 0.00035179 \\
\hline 1991 & 0.00036 & 0.000350938 \\
\hline 1992 & 0.00036 & 0.000350088 \\
\hline 1993 & 0.000319 & 0.00034924 \\
\hline 1994 & 0.000319 & 0.000348395 \\
\hline 1995 & 0.000273 & 0.000347552 \\
\hline 1996 & 0.000273 & 0.000346712 \\
\hline 1997 & 0.000218 & 0.000345873 \\
\hline 1998 & 0.000218 & 0.000345037 \\
\hline 1999 & 0.000145 & 0.000344204 \\
\hline 2000 & & 0.000343372 \\
\hline 2001 & & 0.000342543 \\
\hline 2002 & & 0.000341716 \\
\hline 2003 & & 0.000340891 \\
\hline 2004 & & 0.000340069 \\
\hline 2005 & & 0.000339248 \\
\hline 2006 & & 0.00033843 \\
\hline 2007 & & 0.000337614 \\
\hline 2008 & & 0.0003368 \\
\hline 2009 & & 0.000335989 \\
\hline 2010 & & 0.00033518 \\
\hline 2011 & & 0.000334372 \\
\hline 2012 & & 0.000333567 \\
\hline 2013 & & 0.000332765 \\
\hline 2014 & & 0.000331964 \\
\hline 2015 & & 0.000331165 \\
\hline 2016 & & 0.000330369 \\
\hline 2017 & & 0.000329575 \\
\hline 2018 & & 0.000328783 \\
\hline 2019 & & 0.000327993 \\
\hline 2020 & & 0.000327205 \\
\hline 2021 & & 0.000326419 \\
\hline 2022 & & 0.000325636 \\
\hline
\end{tabular}

0.000403964 0.000402621 0.000401284 0.000399951 0.000398624 0.000397301 0.000395983 0.000394671 0.000393363 0.000392059 0.000390761 0.000389467 0.000388178 0.000386894 0.000385615 0.00038434 0.00038307 0.000381805 0.000380545 0.000379289 0.000378037 0.000376791 0.000375548 0.000374311 0.000373078 0.000371849 0.000370625 0.000369406 0.000368191

0.00036698 0.000365774 0.000364572 0.000363375 0.000362182 0.000360993 0.000359809 0.000358629 0.000357453 0.000356282 0.000355115 0.000353952 0.000352794

0.00035164 0.000350489 0.000349344 0.000348202 0.000347064 0.000345931 0.000344802 0.000343677 0.000342556 0.000341439 0.000340326 0.000339217 0.000338112 0.000337011 0.000335914 0.000334822 0.000333733 0.000332648 0.000331567 0.00033049 0.000329417 0.000328347 0.000327282 0.000326221 0.000325163 0.000324109 0.000323059 0.000322013 0.00032097 0.000319932 0.000318897 0.000317865 0.000316838 0.000315814 0.000314794 0.000313778 0.000312765 0.000311756 0.000310751 0.000309749 0.000308751 0.000307756 0.000306765 0.000305777 0.000304794 0.000303813 0.000302836 0.000301863 0.000300893 0.000299927 0.000298964 0.000298004 0.00029704
0.0100991 0.0100655 0.0100321 0.00999878 0.00993253 0.00989959 0.00986676 0.00983406 0.00980148 0.00976902 0.00973668 0.00970446 0.00967236 0.00964038 0.00960851 0.00957676 0.00954513 0.00951361 0.00948222 0.00945093 0.00941976 0.00938871 0.00935777 0.00932694 0.00929623 0.00926563 0.00923514 0.00920476 0.0091745 0.00914434 0.0091143 0.00905454 0.00902483 0.00899522 0.00896572 0.00893633 0.00890705 0.00887788 0.00884881 0.00881985 0.00879099 0.00876224 0.00873359 0.00870505 0.00867661 0.00864828 0.00862004 0.00859192 0.00856389 0.00853596 0.00850814 0.00848042 0.0084528 0.00842528 0.00839786 0.00837054 0.00834332

0.0083162 0.00828917 0.00826225 0.00823542 0.00820869 0.00818205 0.00815551 0.00812907 0.00810273 0.00807647 0.00805032 0.00802426 0.00799829 0.00797242 0.00794664 0.00792095 0.00789536 0.00786986 0.00784445 0.00781913

0.0077939 0.00776877 0.00774372 0.00771876 0.0076939 0.00766912 0.00764444 0.00761984 0.00759533 0.00757091 0.00754657 0.00752233 0.00749817

0.0074741 0.00745011 0.00742621
Printed 10/2/0

$\begin{array}{ll}0.0908918 & 0.161585 \\ 0.0905898\end{array}$

0.0905898
0.0902889 $\quad 0.161049$

$0.0902889 \quad 0.160514$

$0.0899891 \quad 0.159981$

\begin{tabular}{r|r}
0.0896904 & 0.15945 \\
0.0893928 & 0.15892 \\
0.0890963
\end{tabular}

0.0890963

0.0888009

$0.0885066 \quad 0.157345$
0.0882133

$0.0882133 \quad 0.156824$
0.0879212

\begin{tabular}{l|l}
0.0879212 & 0.156304 \\
\hline & 0.0876301
\end{tabular}

$\begin{array}{ll}0.0876301 & 0.155787 \\ 0.0873402 & 0.155271\end{array}$

0.155271

0.0870512

$0.0864766 \quad 0.153736$

$0.0861909 \quad 0.153228$

0.0859062
0.0856225 $\quad \begin{aligned} & 0.152722 \\ & 0.0853399\end{aligned}$

0.0853399

$0.0850584 \quad 0.151215$

$0.0847779 \quad 0.150716$

$0.0844984 \quad 0.150219$

\begin{tabular}{l|l}
0.0842199 & 0.149724 \\
0.0839425 & 0.149231 \\
0.0836661 & 0.14874
\end{tabular}

$0.0836661 \quad 0.14874$
0.0833907

0.0833907

$0.0831163 \quad 0.147762$

$0.0828429 \quad 0.147276$

0.0825705
0.0822991

\begin{tabular}{r|r}
0.0822991 & 0.14631 \\
0.0820287 & 0.145829 \\
0.0817593 & 0.14535
\end{tabular}

0.0817593

$0.0814909 \quad 0.144873$

$\begin{array}{ll}0.0812235 & 0.144397 \\ 0.08095 & 0.143924\end{array}$

$0.080957 \quad 0.143924$

0.0806915
0.080427 $\quad 0.143452$

0.080427

0.0799009

$0.0796393 \quad 0.141581$

$0.0793786 \quad 0.141118$

0.0791189
0.0788601

0.0788601
0.0786023 $\quad \begin{aligned} & 0.140196 \\ & 0.0783454\end{aligned}$

0.0783454

$0.0780895 \quad 0.138826$

$0.0778345 \quad 0.138372$

0.0775804
0.0773272
0.137921

\begin{tabular}{r|r|}
0.0773272 & 0.137471 \\
0.077075 & 0.137022 \\
0.0768237 & 0.136575
\end{tabular}

0.0768237
0.076573 $\quad 0.136575$

$0.0765733 \quad 0.13613$
0.0763238

0.0763238
0.0760752

$0.0760752 \quad 0.135245$

0.0758275

0.0755807
0.0753349

\begin{tabular}{l|l}
0.0755807 & 0.133929 \\
0.0753349 & 0.133493 \\
0.0750899 & 0.133059 \\
\hline
\end{tabular}

0.0748458

0.0746025
0.0743602

$0.0743602 \quad 0.132196$
0.0741188

0.0741188

$\begin{array}{ll}0.0738782 & 0.131339 \\ 0.0736385 & 0.130913\end{array}$

0.0736385

0.0731616

$0.0729245 \quad 0.129644$

$\begin{array}{ll}0.0726883 & 0.129224 \\ 0.072529 & 0.12880\end{array}$

0.0724529

\begin{tabular}{l|l}
0.0724528 \\
0.0722183 & 0.128388 \\
0.0719846 & 0.127973 \\
0.0717518
\end{tabular}

0.0717518

$0.0715197 \quad 0.127146$

$0.0712886 \quad 0.126735$

0.0710582

\begin{tabular}{r|r|}
0.0708287 & 0.125918 \\
0.0706 & 0.125511
\end{tabular}

0.0703721

$0.0701451 \quad 0.124702$
0.0699189

$0.0699189+0.1243$

0.0696935

0.0694689

\begin{tabular}{l|l}
0.0692451 & 0.123102 \\
0.0690221 & 0.122706 \\
0.0687999 & 0.122311
\end{tabular}

0.0687999

$0.0685786 \quad 0.121917$

$0.068358 \quad 0.121525$

0.0681382

0.0679192

0.067701
0.0674835 $\quad 0.120357$

0.0674835

0.067051
0.0668359 $\quad 0.119202$

0.067051
0.0668359 $\quad \begin{aligned} & 0.119202 \\ & 0.118819\end{aligned}$ 
0.000324854 0.000324075 0.000323298 0.000322523 0.00032175 0.000320979
0.00032021 0.000319443 0.000318678 0.000317915 0.000317155 0.000316396 0.000315639 0.000314885 0.000314132 0.000313382 0.000312633 0.000311887

0.0003104 0.000309659 0.00030892 0.000308184 0.000307449 0.000306717 0.000305986 0.000305257 0.00030453 0.000303805 0.000303083 0.000302362 0.000301643 0.000300925 0.00030021 0.000299497 0.000298786 0.000298076 0.000297368 0.000296663 0.000295959 0.000295257 0.000294557 0.000293859 0.000293162 0.000292468 0.000291775 0.000291084 0.000290395 0.000289708 0.000289023 0.000288339 0.000287658 0.000286978 0.0002863 0.000285623 0.000284949 0.000284276 0.000283606 0.000282936 0.000282269 0.000281604 0.00028094 0.000280278 0.000279618 0.000278959 0.000278302 0.000277647 0.000276994 0.000276342 0.000275693 0.000275044 0.000274398 0.000273753 0.00027311 0.000272469 0.000271829 0.000271192 0.000270555 0.000269921 0.000269288 0.000268657 0.000268027 0.000267399 0.000266773 0.000266149 0.000265526 0.000264905 0.000264285 0.000263667 0.000263051 0.000262436 0.000261823 0.000261211 0.000260601 0.000259993
0.000296096 0.000295147 0.000294201 0.000293259 0.00029232 0.000291384 0.000290452 0.000289523 0.000288597 0.000287675 0.000286756 0.0002858 0.000284927 0.000284018 0.000283112 0.000282209

0.0002813 0.000280414 0.00027952 0.000278631 0.00027686 0.00027598 0.000275102 0.000274228 0.000273357 0.000272489 0.000271624 0.000270762 0.000269903 0.000269047 0.000268195 0.000267345 0.000266498 0.000265655 0.000264814 0.000263976 0.000263141 0.00026231 0.000261481 0.000260655 0.000259832 0.000259012 0.000258194 0.00025738 0.000256569

0.00025576 0.000254954 0.000254151 0.000253351 0.000252554 0.00025176

0.000250968 0.000250179 0.000249393 0.0002486 0.000247829 0.000247051 0.000246276 0.000245504 0.000244734 0.000243967 0.000243203 0.000242442 0.000241683 0.000240926 0.000240173 0.000239422 0.000238674 0.000237928 0.000237185 0.000236444 0.000235706 0.000234971 0.000234238 0.000233508 0.00023278
0.000232055 0.000232055 0.000231333 0.000230613 0.000229895 0.00022918 0.000228468 0.000227757 0.00022705 0.000226345 0.000225642 0.000224942 0.000224244 0.000223549 0.000222856 0.000222165 0.000221477 0.000220791 0.000220108

0.0074024 0.00737867 0.00735502 0.00733146 0.00730799
0.0072846 0.00726129 0.00723807 0.00719187 0.00716889 0.007146 0.00712319 0.00710045 0.00707781 0.00705524 0.00703275 0.00701034 0.00698801 0.00694359 0.0069215 0.00689949 0.00687756 0.0068557 0.00683392

0.00681222

0.0067906 0.00676905 0.00674758 0.00670487 0.00668363 0.00666246 0.00664137 0.00662035 
0.000259387 0.000258781 0.000258178 0.000257576 0.000256976 0.000256377

0.00025578

0.000255184

0.00025459

0.000253998

0.000253407

0.000252817

0.000251643

0.000251059

0.000250475

0.000249894

0.000249314

0.000248735

0.000247582

0.000247008

0.000246436

0.000245865

0.000245295

0.000244727

0.00024416

0.000243595

0.000243032

0.000242469

0.000241909

0.000241349

0.000240792

0.000240235

0.00023968

0.000239127

0.000238575

0.000238024

0.000237475

0.000236928

0.000236381

0.000235837

0.000235293

0.000234751

0.000234211

0.000232597

0.000232062

0.000231529

0.000230997

0.000230466

0.000229936

0.000229408

0.000228882

0.000228356

0.000227832

0.00022731
0.000226789

0.000226269

0.00022575

0.000225233

0.000224717

0.000224203

0.00022369

0.000223178

0.000222667

0.000222158

0.00022165

0.000221143

0.000220638

0.000220134

0.000219632

0.00021913

0.00021863

0.000218132

0.000217634

0.000216643

0.000216149

0.000215657

0.000215166

0.000214676

0.000214188

0.0002137

0.000213214

0.00021273

0.000212246

0.000211764
0.000211283

0.000210803

0.000210324

0.000209847

0.000209371
0.000219427 0.000218748 0.000218072 0.000217398 0.000216726 0.000216057

0.00021539 0.000214725 0.000214063 0.000213403 0.000212745 0.00021209 0.000211436 0.000210785 0.000210137 0.00020949 0.000208846 0.000208204 0.000207564 0.000206926 0.000206291 0.000205658 0.000205027 0.000204398 0.000203771 0.000203146 0.000202524 0.000201904 0.000201285 0.000200669 0.000200055 0.000199444 0.000198834 0.000198226 0.000197621 0.000197017 0.000196416 0.000195816 0.000195219

0.000194624 0.000194031

0.00019344

0.00019285

0.000192263 0.000191678 0.000191095 0.000190514 0.000189935 0.000189357 0.000188782 0.000188209 0.000187638

0.000187068 0.000186501 0.000185935 0.000185372 0.00018481 0.00018425 0.000183693 0.000183137 0.000182583

0.00018203

0.00018148 0.000180932 0.000180385 0.00017928 0.000178757 0.000178217 0.00017768 0.000177144 0.000176611 0.000176079 0.000175549 0.00017502 0.000174494 0.000173969 0.000173446 0.000172925 0.000172405 0.000171888 0.000171372 0.000170857 0.000170345 0.000169834 0.000169325 0.000168818 0.000168312 0.000167808 0.000167306 0.000166805 0.000166307 0.000165809 0.000165314 0.00016482

Page 24 of 33 0.0054687 0.0054518 0.00543495 0.00541816 0.00540143 0.00538475 0.00536814 0.00535158 0.00533507 0.00531863 0.00530224 0.00528591 0.00526963 0.00525342 0.00523725 0.00522115 0.00520509 0.0051891 0.00517316 0.00515727 0.00514144 0.00512566 0.00510994 0.00509427 0.00507866

0.0050631

0.00504759 0.00503214 0.00501673 0.00500139 0.00498609 0.00494052 0.00492543 0.0049104 0.00489541 0.00488048 
0.000208422 0.00020795 0.000207479 0.000207009 0.00020654 0.000206072 0.000205606 0.000205141 0.000204677 0.000204214 0.000203752 0.000203292 0.000202833 0.000202375 0.000201918 0.000201462 0.000201007 0.00020055 0.000200102 0.000199651 0.000199201 0.000198752 0.000198304 0.000197858 0.000197412 0.000196968 0.000196525 0.000196083 0.000195642 0.000195203 0.000194764 0.000194327

0.00019389 0.000193455 0.000193021 0.000192588 0.000192156 0.000191725 0.000191295 0.000190867 0.000190439 0.000190013 0.00018958 0.000189163 0.00018874 0.000188318 0.000187897 0.000187477 0.000187058 0.00018664 0.000186223 0.000185808 0.000185393 0.00018498 0.000184567 0.00018415 0.000183745 0.000183336 0.000182927 0.00018252 0.000182113 0.000181708 0.000181304 0.000180901 0.000180498 0.000180097 0.000179697 0.000179298 0.0001789 0.000178503 0.000178106 0.000177711 0.000177317 0.000176924 0.000176532 0.000176141 0.00017575 0.000175361 0.000174586 0.000174199 0.000173814 0.00017343 0.000173046 0.000172664 0.000172283 0.000171902 0.000171523 0.000171144 0.000170766 0.00017039 0.000170014 0.000169639 0.000169265 0.000168892
0.000164328 0.000163837 0.000163348 0.000162861 0.000162376 0.000161892 0.000161409 0.000160928 0.000160449 0.000159972 0.000159496 0.000159021 0.000158549 0.000158077 0.000157608 0.00015714 0.000156673 0.000156208 0.000155745 0.000155283 0.000154823 0.000154364 0.000153907 0.000153451 0.000152997 0.000152544 0.000152093 0.000151643 0.000151195 0.000150749 0.000150303 0.00014986 0.000149417 0.000148977 0.000148537 0.000148099 0.000147663 0.000147228 0.000146795 0.000146362 0.000145932 0.000145503 0.000145075 0.000144648 0.000144223

0.0001438 0.000143378 0.000142957 0.000142537 0.000142119 0.000141703 0.000141287 0.000140874 0.000140461 0.00014005 0.00013964 0.000139232 0.000138824 0.000138419 0.000138014 0.000137611 0.000137209 0.000136809 0.00013641 0.000136012 0.000135615 0.00013522 0.000134826 0.000134433 0.000134042 0.000133652 0.000133263 0.000132876 0.000132489 0.000132104 0.000131721 0.000131338 0.000130957 0.000130577 0.000130198 0.000129821 0.000129444 0.000129069 0.000128695 0.000128323 0.000127951 0.000127581 0.000127212 0.000126844 0.000126478 0.000126112 0.000125748 0.000125385 0.000125023 0.000124663

0.0041082 0.00409593 0.00408371 0.00407153 0.00405939 0.00404729 0.00402321 0.00401123 0.00399929
0.00398739 0.00397554 0.00396372 0.00395194 0.0039402 0.0039285 0.00391683 0.00390521 0.00389363 0.00388208 0.00387057 0.0038591 0.00384767 0.00383628 0.00382492 0.00381361 0.00380233 0.00379109 0.00377988 0.00376871 0.00375758 0.00374649 0.00373544 0.00372442 0.00371343 0.00370249 0.00369158 
0.000168521 0.000168149 0.000167779 0.00016741 0.000167042 0.000166675 0.000166308 0.000165943 0.000165578 0.000165215 0.000164852 0.00016449 0.000164129 0.000163769 0.00016341 0.000163052 0.000162694 0.000162338 0.000161982 0.000161628 0.000161274 0.000160921 0.000160569 0.000160218 0.000159868 0.000159518 0.00015917 0.000158822 0.000158475 0.000158129 0.000157784 0.00015744 0.000157097 0.000156754 0.000156413 0.000156072 0.000155732 0.000155393 0.000155055 0.000154717 0.000154381 0.000154045 0.00015371 0.000153376 0.000153043 0.00015271 0.000152379 0.000152048 0.000151718 0.000151389 0.000151061 0.000150733 0.000150407 0.000150081 0.000149756 0.00014943 0.000149108 0.000148785 0.000148463 0.000148142 0.000147822 0.000147503 0.000147184 0.000146866 0.000146549 0.000146233 0.000145917 0.000145602 0.000145288 0.000144975 0.000144663 0.000144351 0.00014404 0.00014373 0.00014342 0.000143112 0.000142804 0.000142497 0.000142191 0.000141885 0.00014158 0.00014127 0.000140973 0.00014067 0.000140368 0.000140067 0.000139767 0.000139467 0.000139168 0.00013887 0.000138572 0.000138276 0.00013798 0.000137684 0.000123945 0.000123588 0.000123232 0.000122877 0.000122171 0.000121819 0.000121469 0.00012112 0.000120772 0.000120425 0.000120079 0.000119734 0.000119391 0.000119049 0.000118707 0.000118367 0.000118028

0.00011769 0.000117353 0.000117017 0.000116682 0.000116349 0.000116016 0.000115685 0.000115354 0.000115025 0.000114696 0.000114369 0.000114043 0.000113718 0.000113394 0.00011307 0.000112748 0.000112427 0.000112107 0.000111788

0.00011147

0.000111153 0.000110523 0.000110209 0.000109896 0.000109584 0.000109273 0.000108963 0.000108654 0.000108346 0.000108039 0.000107734 0.000107429 0.000107125 0.000106821 0.000106519 0.000106218 0.000105918 0.000105619 0.000105321 0.000105023 0.000104727 0.000104432 0.000104137 0.000103844 0.000103551 0.000103259 0.000102969 0.000102679 0.00010239 0.000102102 0.000101815 0.000101529 0.000101244 0.000100959 0.000100676 0.000100393 0.000100112

$9.98 \mathrm{E}-05$

9.96E-05

9.93E-05

9.90E-05

9.87E-05

9.84E-05

9.82E-05

$0.76 \mathrm{E}-05$

9.73E-05

9.71E-05

9.68E-05

9.65E-05

9.63E-05

$9.60 \mathrm{E}-05$

9.55E-05 9.52E-05

Page 26 of 33

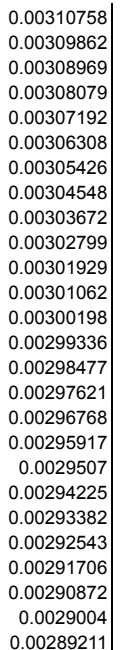

0.0279682 0.0278876 0.0278072 0.0277271 0.0276473 0.0275677 0.0274884 0.0274093 0.0273305 0.0272519 0.0271736 0.0270956 0.0270178

0.0269403 0.0267859 0.0267091 0.0266326 0.0265563

0.0264802

0.0264044 0.0263289 0.0262535
0.0261785 0.0261036 0.026029

0.0259547 0.0258806 0.0258067

0.025733 0.0256596 0.0255865

0.0254408 0.0253684 0.0252961 0.0252242 0.0251524 0.0250808 

0.000136803 0.000136511 0.000136219 0.000135928 0.000135348 0.000135059 0.000134771 0.000134484 0.000134197 0.000133911 0.000133626 0.000133341 0.000133057 0.000132774 0.000132491 0.00013221 0.000131928 0.000131089 0.00013081 0.000130533 0.000130256 0.000129979 0.000129703 0.000129428 0.000129154 0.00012888 0.000128607 0.000128063 0.000127792 0.000127521 0.000127252 0.000126983 0.000126714 0.000126179 0.000125913 0.000125647 0.000125382 0.000125117 0.000124853 0.00012459 0.000124327 0.000124065 0.000123804 0.000123543 0.000123283 0.000123023 0.000122764 0.000122506 0.000122248 0.000121991 0.000121735 0.000121479 0.000121224 0.000120969 0.000120715 0.000120462 0.000120209 0.000119957 0.000119706 0.000119455 0.000119205 0.000118955 0.000118706 0.000118457 0.000118209 0.000117962 0.000117715 0.000117469 0.000117224 0.000116979 0.000116735 0.000116491 0.000116248 0.000116005 0.000115763 0.000115522 0.000115281 0.000115041 0.000114801 0.000114562 0.000114323 0.000114085 0.000113848 0.000113611 0.000113375 0.000113139 0.000112904 0.000112669 0.000112435 9.47E-05 9.44E-05 9.41E-05 9.39E-05 9.36E-05

9.34E-05

9.31E-05

9.28E-05

9.26E-05

9.23E-05

$9.21 \mathrm{E}-05$

9.16E-05

9.13E-05

9.10E-05

9.08E-05

9.05E-05

9.00E-05

$8.98 \mathrm{E}-05$

8.95E-05

8.93E-05

8.90E-05

8.88E-05

8.86E-05

$8.83 \mathrm{E}-05$

8.78E-05

8.76E-05

$8.73 \mathrm{E}-05$
$8.71 \mathrm{E}-05$

8.69E-05

8.66E-05

8.64E-05

8.61E-05

8.59E-05

8.57E-05

8.54E-05

$8.52 \mathrm{E}-05$

8.47E-05

8.45E-05

8.43E-05

8.40E-05

8.

8.34E-05

8.31E-05

8.29E-05

8.27E-05

.24E-05

8.20 -05

8.18E-05

8.15E-05

8.13E-05

8.11E-05

$8.09 \mathrm{E}-05$

8.07E-05

8.04E-05

$8.02 \mathrm{E}-05$

$8.00 \mathrm{E}-05$

$7.98 \mathrm{E}-05$

7.96E-05

$7.91 E-05$

7.89E-05

$7.87 \mathrm{E}-05$

7.85E-05

7.83E-05

$7.81 \mathrm{E}-05$

$7.79 \mathrm{E}-05$

$7.76 \mathrm{E}-05$

$7.74 \mathrm{E}-05$

$7.72 \mathrm{E}-05$
$7.70 \mathrm{E}-05$

$7.68 \mathrm{E}-05$

7.66E-05

7.64E-05

7.62E-05

$7.60 \mathrm{E}-05$

7.58E-05

7.56E-05

$7.54 \mathrm{E}-05$

$7.52 \mathrm{E}-05$

$7.47 \mathrm{E}-05$

7.45E-05

7.43E-05

7.41E-05

7.39E-05

7.37E-05

7.35E-05
7.33E-05

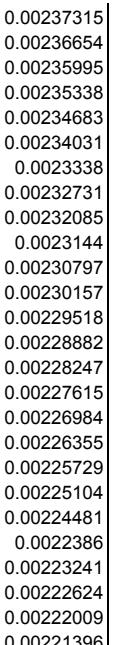
0.0212989

0.0212396

0.0211804
0.0211215

0.0211215

0.0210042

0.0209458

0.0208876

0.0208296
0.0207718

0.0207141

0.0206566

0.0205994

0.0205422
0.0204853

0.0204285

0.020372

0.0203156

0.0202593

0.0202033

0.0201474

0.0200917

0.0200362

0.0199257

0.0198706

0.0198158

0.0197611

0.0197066

0.0196523

0.0195982

0.0195442

0.0194904

0.0193832

0.0193299

0.0192767

0.0192237

0.0191183

0.0190658

0.0190134

0.0189612

0.0189092

0.0188057

0.0188057

0.0187028

0.0186516

0.0186005

0.0185496

0.0184989

0.0184483
0.0183979 

0.000111969 0.000111736 0.000111505 0.000111043 0.000110812 0.000110583 0.000110354 0.000110125 0.000109897 0.00010967 0.000109443 0.000109217 0.000108991 0.000108766 0.000108541 0.000108317 0.000108093 0.00010787 0.000107647 0.000107425 0.000107204 0.000106983 0.000106762 0.000106542 0.000106323 0.000106104 0.000105885 0.000105667 0.00010545 0.000105017 0.000104801 0.000104586 0.000104371 0.000104156 0.000103943 0.000103729 0.000103516 0.000103304 0.000103092 0.000102881 0.00010267 0.00010246 0.00010225 0.000102041 0.000101832 0.000101624 0.000101416 0.000101209 0.000101002 0.000100589 0.000100384 0.000100179 1.00E-04 9.98E-05 7.30E-05

$7.28 \mathrm{E}-05$

7.26E-05

$7.24 \mathrm{E}-05$
$7.22 \mathrm{E}-05$

7.20E-05

7.18E-05

7.16E-05

7.14E-05

$7.12 \mathrm{E}-05$
$7.10 \mathrm{E}-05$

7.08E-05

7.06E-05

7.04E- -05

7.03E-05

$7.01 \mathrm{E}-05$

$6.99 \mathrm{E}-05$
$6.97 \mathrm{E}-05$

6.95E-05

6.93E-05

6.91E-05

6.89E-05

$6.88 \mathrm{E}-05$

$6.86 \mathrm{E}-05$

$6.82 \mathrm{E}-05$

6.80E-05

6.79E-05

6.77E-05

$6.75 \mathrm{E}-05$
$6.73 \mathrm{E}-05$

6.71E-05

6.70E-05

6.68E-05

6.66E-05

6.64E-05

6.62E-05

$6.59 \mathrm{E}-05$

6.57E-05

6.55E-05

6.54E-05

6.52E-05

$6.50 \mathrm{E}-05$

$6.47 E-05$

6.45E-05

6.43E-05

6.42E-05

6.40E-05

$6.38 \mathrm{E}-05$

$6.35 \mathrm{E}-05$

6.33E-05

6.32E-05

6.30E-05

$6.28 \mathrm{E}-05$

$6.25 \mathrm{E}-05$

6.22E-05

6.20E-05

6.18E-05

6.17E-05

$6.13 E-05$

6.12E-05

6.10E-05

6.09E-05

6.07E-05

$6.05 \mathrm{E}-05$

6.04E-05

6.02E-05

6.01E-05

5.99E-05

$5.98 \mathrm{E}-05$
$5.96 \mathrm{E}-05$

5.96E-05

$5.93 \mathrm{E}-05$

5.91E-05

5.90E-05

5.88E-05

5.87E-05

$5.85 \mathrm{E}-05$

$5.84 \mathrm{E}-05$

$5.82 \mathrm{E}-05$
$5.81 \mathrm{E}-05$

5.79E-05

5.77E-05

5.76E-05

5.74E-05

5.73E-05

5.71E-05

$5.70 \mathrm{E}-05$

0.00182872 0.00182379 0.00181888 0.00181399 0.00180911 0.00180424 0.00179939 0.00179455 0.00178973 0.00178492
0.00178013 0.00177535 0.00177059 0.00176584 0.00176111 0.00175639 0.00175168 0.00174699 0.00174231

0.001733 0.00172837 0.00172375
0.00171914 0.00171455 0.00170997

0.0017054 0.00170085 0.00169631 0.00169179 0.00168728 0.00168278

0.00167383 0.00166937 0.00166493
0.0016605 0.00165608 0.00165168 0.00164729 0.00164291 0.00163855

0.0016342 0.00162986 0.00162554 0.00162122 0.00161693 0.00161264 0.00160837 0.00160411 0.00159986 0.00159563

0.0015914 0.00158719 0.001583 0.00157881
0.00157464 

$9.22 \mathrm{E}-05$ 5.67E-05 $5.66 \mathrm{E}-05$ 5.64E-05 $5.63 \mathrm{E}-05$
$5.61 \mathrm{E}-05$

5.60E-05

$5.58 \mathrm{E}-05$

5.57E-05

5.55E-05

$5.54 \mathrm{E}-05$
$5.52 \mathrm{E}-05$

5.51E-05

5.50E-05

$5.48 \mathrm{E}-05$

5.47E-05

5.45E-05

$5.44 \mathrm{E}-05$
$5.42 \mathrm{E}-05$

$5.41 \mathrm{E}-05$

$5.40 \mathrm{E}-05$

$5.38 \mathrm{E}-05$

5.37E-05

$5.36 \mathrm{E}-05$
$5.34 \mathrm{E}-05$

5.33E-05

5.31E-05

5.30E-05

5.29E-05

5.27E-05

$5.26 \mathrm{E}-05$
$5.25 \mathrm{E}-05$

5.23E-05

5.22E-05

5.21E-05

5.19E-05

5.18E-05

$5.16 \mathrm{E}-05$
$5.15 \mathrm{E}-05$

5.14E-05

5.13E-05

5.11E-05

5.10E-05

$5.09 \mathrm{E}-05$
$5.07 \mathrm{E}-05$

5.06E-05

$5.05 E-05$

5.03E-05

5.02E-05

$5.01 \mathrm{E}-05$

5.00E-05

4.97E-05

$4.96 \mathrm{E}-05$

4.94E-05

4.93E-05

4.92E-05

4.91E-05

4.89E-05

4.88E-05

4.87E-05

4.86E-05

4.84E-05

4.83E-05

$4.82 \mathrm{E}-05$
$4.81 \mathrm{E}-05$

$4.79 \mathrm{E}-05$

4.78E-05

4.77E-05

4.76E-05

4.75E-05

$4.73 \mathrm{E}-05$

$4.72 \mathrm{E}-05$
$4.71 \mathrm{E}-05$

4.70E-05

4.69E-05

4.67E-05

4.66E-05

4.65E-05

$4.64 \mathrm{E}-05$
$4.63 \mathrm{E}-05$

4.62E-05

4.60E-05

4.59E-05

$4.58 \mathrm{E}-05$

4.57E-05

4.55E-05

4.53E-05

4.52E-05

4.51E-05

4.50E-05

4.49E-05

$4.48 \mathrm{E}-05$
$4.47 \mathrm{E}-05$

Page 29 of 33

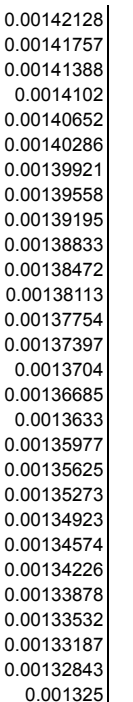

0.0127915 0.0127581

0.0127249

0.0126918

0.0126587
0.0126258

0.0125929

0.0125602

0.0125275

0.0124625

0.0124301

0.0123979

0.0123657

0.0123336
0.0123016

0.0122697

0.0122379

0.0122062

0.0121431

0.0121116

0.0120803

0.0120491
0.0120179

0.0119868

$\begin{array}{r}0.0119559 \\ 0.011925 \\ \hline\end{array}$

0.0118942

0.0118635

0.0118329

0.0118024
0.0117719

0.0117416

0.0117113

0.0116812

0.0116511

0.0116211
0.0115912

0.0115614

0.0115316

0.011502

0.0114724
0.0114429

0.0114136

0.0113843

0.0113259

0.0112969

0.0112679

0.011239
0.0112102

0.0111815

0.0111529

0.0111243

0.0110959
0.0110675

0.0110392

0.011011

0.0109828

0.0109548

0.0109268

0.0108989
0.0108711

0.0108434

0.0108157

0.0107882

0.0107607

0.0107333
0.0107059

0.0106787

0.0106515

0.0106244

0.0105974 

7.63E-05 4.44E-05 4.43E-05 4.42E-05 $4.41 \mathrm{E}-05$
$4.40 \mathrm{E}-05$

4.39E-05

4.38E-05

4.37E-05

4.35E-05

$4.34 \mathrm{E}-05$
$4.33 \mathrm{E}-05$

4.32E-05

4.31E-05

4.30E-05

4.29E-05

4.28E-05

$4.27 \mathrm{E}-05$
$4.26 \mathrm{E}-05$

4.25E-05

4.24E-05

4.22E-05

$4.21 \mathrm{E}-05$

4.20E-05

$4.19 \mathrm{E}-05$
$4.18 \mathrm{E}-05$

4.17E-05

4.16E-05

4.15E-05

4.14E-05

$4.13 \mathrm{E}-05$
$4.12 \mathrm{E}-05$

4.11E-05

4.10E-05

4.09E-05

4.08E-05

4.07E-05

$4.06 \mathrm{E}-05$
$4.05 \mathrm{E}-05$

4.04E-05

4.03E-05

4.02E-05

4.01E-05

4.00E-05

3.99E-05

$3.97 \mathrm{E}-05$

3.96E-05

3.95E-05

3.94E-05

3.93E-05

3.92E-05

$3.91 \mathrm{E}-05$

3.89E-05

3.88E-05

3.87E-05

$3.86 \mathrm{E}-05$
$3.85 \mathrm{E}-05$

$3.84 \mathrm{E}-05$

3.83E-05

3.82E-05

3.81E-05

$3.81 \mathrm{E}-05$

$3.80 \mathrm{E}-05$
$3.79 \mathrm{E}-05$

$3.78 \mathrm{E}-05$

3.77E-05

3.76E-05

3.75E-05

$3.74 \mathrm{E}-05$
$3.73 \mathrm{E}-05$

$3.72 \mathrm{E}-05$

$3.72 \mathrm{E}-05$
$3.71 \mathrm{E}-05$

3.70E-05

3.69E-05

3.68E-05

$3.68 \mathrm{E}-05$

3.67E-05

3.66E-05

3.64E-05

3.63E-05

3.62E-05

3.61E-05

3.60E-05

3.60E-05

3.59E-05

$3.58 \mathrm{E}-05$
$3.57 \mathrm{E}-05$

3.56E-05

3.55E-05

3.54E-05

3.53E-05 3.53E-05

Page 30 of 33

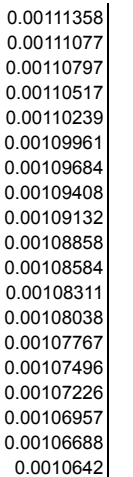

0.0010642
0.00106154
0.00105887

0.00105887

0.00105622

0.00105357

0.00105093
0.0010483

0.00104568

0.00104306

0.00104045

0.00103785
0.00103525

0.00103267

0.00103009

0.00102495

0.00102239

0.00101984

0.0010173
0.00101476

0.00101223

0.00100971

0.00100719

0.00100468

0.00100218

0.000999689

0.000997202

0.00099225

0.000989784

0.000987325

0.000984873
0.000982428

0.000979989

0.000977558

0.000975133

0.000972716

0.000970304
0.0009679

0.0009679
0.000965503

0.000963112

0.000960728

0.000958351

0.00095598
0.000953616

0.000953616
0.000951259

0.000948908

0.000946564

0.000944226

0.000941895

0.00093957
0.000937252

0.000937252
0.000934941

0.000932635

0.000930337

0.000928044

0.000925758

0.000923479
0.000921206

0.000918939

0.000916678

0.000914424

0.000912176

0.000909935

0.000907699
0.00090547

0.00090547
0.000903247

0.00090103

0.00089882 

$6.35 \mathrm{E}-05$ 3.51E-05

3.50E-05

3.49E-05

$3.48 \mathrm{E}-05$
$3.47 \mathrm{E}-05$

3.47E-05

3.46E-05

3.45E-05

$3.44 \mathrm{E}-05$
$3.43 \mathrm{E}-05$

3.42E-05

3.42E-05

$3.41 \mathrm{E}-05$

3.39E-05

3.38E-05

3.37E-05

3.36E-05

3.35E-05

3.34E-05

3.33E-05

$3.32 \mathrm{E}-05$
3.32E-05

3.31E-05

3.30E-05

3.29E-05

3.28E-05

3.28E-05

3.27E-05

3.25E-05

3.25E-05

3.24E-05

3.23E-05

$3.22 \mathrm{E}-05$
$3.21 \mathrm{E}-05$

3.21E- 05

3. $20 \mathrm{E}-05$

3.19E-05

3.18E-05

3.18E-05

$3.17 \mathrm{E}-05$
$3.16 \mathrm{E}-05$

3.15E-05

3.14E-05

3.14E-05

3.13E-05

3.12E-05

3.11E-05

3.10E-05

3.09E-05

3.08E-05

3.08E-05

3.07E-05

3.06E-05

3.05E-05

3.04E-05

3.03E-05

3.03E-05

3.02E-05

$3.00 \mathrm{E}-05$

3.00E-05

2.99E-05

2.98E-05

$2.98 \mathrm{E}-05$

2.97E-05

$295 \mathrm{E}-05$

2.95E-05

2.94E-05

2.93E-05

2.93E-05

2.91E-05

2.91E-05

2.90E-05

2.89E-05

2.88E-05

2.88E-05

2.86E-05

2.86E-05

2.85E-05

2.84E-05

2.84E-05

2.82E-05

$2.82 \mathrm{E}-05$

2.81E-05

$2.80 \mathrm{E}-05$

Page 31 of 33

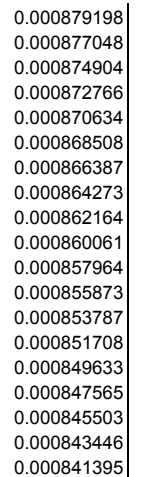

0.000839349

0.000837309

0.000835275

0.000833246

0.000831223
0.000829206

0.000827194

0.000825187

0.000823187

0.000821191

0.000819201
0.000817217

0.000817217
0.000815238

0.000813264

0.000811296

0.000809333

0.000807376

0.000805424

0.000803477
0.000801536

0.0007996

0.000797669

0.000795744
0.000793823

0.000791909

0.000789999

0.000786195

0.000784301

0.000782412

0.000780529
0.00077865

0.000776777

0.000774908

0.000773045

0.000771187

0.000769334

0.000767486
0.000765643
0.000763805

0.000763805

0.000761972

0.000760144

0.000758321

0.000756502

0.000752881

0.000751078

0.000749279

0.000747486

0.000745697

0.000743914
0.000742135

0.000740361

0.000738591

0.000736827

0.000735067

0.000733312
0.000731562

0.000729816

0.000728075

0.000726339

0.000724608

0.000722881

0.000721159
0.000719442

0.000717729

0.000714318

0.000712619

0.000710924

0.000709235
0.000707549

0.000707549
0.000705869

0.000702521 

5.32E-05

2.80E-05 2.79E-05

2.78E-05

$2.78 \mathrm{E}-05$
$2.77 \mathrm{E}-05$

2.76E-05

2.76E-05

2.75E-05

2.74E-05

$2.74 \mathrm{E}-05$
$2.73 \mathrm{E}-05$

2.72E-05

2.72E-05

2.71E-05

2.71E-05

2.69E-05

2.69E-05

2.68E-05

2.67E-05

2.67E-05

2.66E-05

2.65E-05

$2.65 \mathrm{E}-05$
$2.64 \mathrm{E}-05$

2.64E-05

2.63E-05

2.62E-05

2.62E-05

2.61E-05

$2.61 \mathrm{E}-05$

2.59E-05

2.59E-05

2.58E-05

2.57E-05

$2.57 \mathrm{E}-05$
$2.56 \mathrm{E}-05$

2.56E-05

2.55E-05

2.54E-05

$2.54 \mathrm{E}-05$

2.53E-05

2.53E-05

2.52E-05

2.51E-05

2.50E-05

2.50E-05

2.49E-05

2.49E-05

$2.48 \mathrm{E}-05$

2.47E-05

2.46E-05

2.46E-05

$2.45 \mathrm{E}-05$

$2.45 \mathrm{E}-05$
$2.44 \mathrm{E}-05$

2.43E-05

2.43E-05

2.42E-05

2.42E-05

2.41E-05

2.40E-05

2.39E-05

2.39E-05

2.38E-05

2.38E-05

2.37E-05

2.36E-05

2.36E-05

2.35E-05

2.35E-05

2.34E-05

$2.33 \mathrm{E}-05$
$2.33 \mathrm{E}-05$

2.33E-05

2.32E-05

2.31E-05

2.31E-05

2.30E-05

$2.30 \mathrm{E}-05$

2.29E-05

$2.28 \mathrm{E}-05$

2.28E-05

2.27E-05

$2.27 \mathrm{E}-05$
$2.26 \mathrm{E}-05$

$2.25 \mathrm{E}-05$

$2.25 \mathrm{E}-05$ $2.24 \mathrm{E}-05$

\subsection{1 \\ 0.000697533
0.00069588
0.0006923 \\ 0.00069423 \\ 0.000692586 \\ 0.000689309 \\ 0.000687678 \\ 0.00068605 \\ 0.000684428
0.000682809 \\ 0.000681195 \\ 0.000679585 \\ 0.00067798 \\ 0.000676379 \\ 0.000674782
0.000673189 \\ 0.000671601 \\ 0.000668437 \\ 0.000666861 \\ 0.000665289 \\ 0.000663722
0.000662159 \\ 0.0006606}

0.000659046

0.000657495

0.000655949

0.000654406

0.000652868

0.000651334
0.000649804

0.000648278

0.000646756

0.000645239

0.000643725

0.000642215
0.000640709

0.000639208

0.00063771

0.000636216

0.000634727

0.000633241

0.000631759
0.000630281

0.000628807

0.000627337

0.000625871

0.000624409

0.00062295
0.000621496

0.000620045

0.000618598

0.000617155

0.000615716

0.00061428

0.000612849

0.000611421

0.000608577

0.00060716

0.000605747

0.000604338

0.000602933

0.000600133

0.000598739

0.000597349

0.000595962

0.000594578

0.000593199

0.000590451 
Table_of_specs_Rev0.xls,CH Surface roughness

\begin{tabular}{|c|c|c|c|c|c|}
\hline 2973 & 4.48E-05 & $2.24 \mathrm{E}-05$ & 0.000559862 & 0.00503876 & 0.00895779 \\
\hline 2974 & 4.47E-05 & 2.23E-05 & 0.000558574 & 0.00502716 & 0.00893718 \\
\hline 2975 & 4.46E-05 & 2.23E- 05 & 0.000557288 & 0.00501559 & 0.00891661 \\
\hline 2976 & 4.46E-05 & 2.22E-05 & 0.000556007 & 0.00500406 & 0.00889611 \\
\hline 2977 & 4.45E-05 & 2.22E-05 & 0.000554728 & 0.00499255 & 0.00887565 \\
\hline 2978 & 4.44E-05 & 2.21E-05 & 0.000553453 & 0.00498108 & 0.00885525 \\
\hline 2979 & 4.43E-05 & 2.21E-05 & 0.000552181 & 0.00496963 & 0.0088349 \\
\hline 2980 & 4.42E-05 & $2.20 \mathrm{E}-05$ & 0.000550913 & 0.00495821 & 0.0088146 \\
\hline 2981 & 4.42E-05 & $2.20 \mathrm{E}-05$ & 0.000549647 & 0.00494683 & 0.00879436 \\
\hline 2982 & 4.41E-05 & 2.19E-05 & 0.000548386 & 0.00493547 & 0.00877417 \\
\hline 2983 & 4.40E-05 & 2.19E-05 & 0.000547127 & 0.00492414 & 0.00875403 \\
\hline 2984 & 4.39E-05 & $2.18 \mathrm{E}-05$ & 0.000545872 & 0.00491285 & 0.00873395 \\
\hline 2985 & 4.38E-05 & $2.18 \mathrm{E}-05$ & 0.00054462 & 0.00490158 & 0.00871391 \\
\hline 2986 & $4.38 \mathrm{E}-05$ & 2.17E-05 & 0.000543371 & 0.00489034 & 0.00869393 \\
\hline 2987 & 4.37E-05 & 2.17E-05 & 0.000542125 & 0.00487913 & 0.008674 \\
\hline 2988 & 4.36E-05 & $2.16 \mathrm{E}-05$ & 0.000540883 & 0.00486795 & 0.00865413 \\
\hline 2989 & 4.35E-05 & $2.16 \mathrm{E}-05$ & 0.000539644 & 0.00485679 & 0.0086343 \\
\hline 2990 & $4.34 \mathrm{E}-05$ & $2.15 \mathrm{E}-05$ & 0.000538408 & 0.00484567 & 0.00861453 \\
\hline 2991 & 4.34E-05 & $2.15 \mathrm{E}-05$ & 0.000537175 & 0.00483458 & 0.00859481 \\
\hline 2992 & 4.33E-05 & 2.14E-05 & 0.000535946 & 0.00482351 & 0.00857514 \\
\hline 2993 & 4.32E-05 & 2.14E-05 & 0.00053472 & 0.00481248 & 0.00855552 \\
\hline 2994 & 4.31E-05 & 2.13E-05 & 0.000533497 & 0.00480147 & 0.00853595 \\
\hline 2995 & 4.31E-05 & 2.13E-05 & 0.000532277 & 0.00479049 & 0.00851643 \\
\hline 2996 & 4.30E-05 & 2.12E-05 & 0.00053106 & 0.00477954 & 0.00849696 \\
\hline 2997 & 4.29E-05 & 2.12E-05 & 0.000529847 & 0.00476862 & 0.00847754 \\
\hline 2998 & 4.28E-05 & 2.11E-05 & 0.000528636 & 0.00475773 & 0.00845818 \\
\hline 2999 & $4.28 \mathrm{E}-05$ & 2.11E-05 & 0.000527429 & 0.00474686 & 0.00843886 \\
\hline 3000 & 4.27E-05 & $2.10 \mathrm{E}-05$ & 0.000526225 & 0.00473602 & 0.0084196 \\
\hline
\end{tabular}


Alternate Capsule Requirements - Uniform $\mathrm{Be}(\mathrm{Cu})$

\begin{tabular}{|c|c|c|c|c|c|c|}
\hline $\mathbf{S} / \mathbf{N}$ & Rev & WBS & Component & Title & short req't & $\begin{array}{c}\text { Requirement Text } \\
\end{array}$ \\
\hline UB1 & 0 & 1.4 .1 .1 & Be (uniform) Capsule & Applicability of other requirements & & $\begin{array}{l}\text { All requirments in table "Point Design Req'ts" except those designated for component } \\
\text { "Layered Be capsule" shall apply to the uniform Be capsule }\end{array}$ \\
\hline UB2 & 0 & I.4.1.1.2.1 & Be (uniform) capsule & Ablator composition & $\begin{array}{l}\text { Be, with } 0.30 \pm 0.05 \text { at } \% \\
\mathrm{Cu}\end{array}$ & $\begin{array}{l}\text { The ablator material shall be beryllium doped with } 0.3 \text { at } \% \mathrm{Cu}+/-0.05 \text { at } \% \text {. Oxygen shall } \\
\text { be }<0.8 \text { at } \% \text {; nominally value is } 0.3 \text { at } \%\end{array}$ \\
\hline UB3 & 0 & I.4.1.1.2.1 & Be (uniform) capsule & $\begin{array}{l}\text { Ablator total thickness - range of possible } \\
\text { requested values }\end{array}$ & $175 \mu \mathrm{m}$ & $\begin{array}{l}\text { Capsule ablator shall be producable with total ablator thickness within the range } 150-190 \mu \mathrm{m} \text {. } \\
\text { The ablator thickness for each capsule will be specified within that range. The point design } \\
\text { value shall be } 175 \mu \mathrm{m} \text {. }\end{array}$ \\
\hline UB4 & 0 & I.4.1.1.2.1 & Be (uniform) capsule & Ablator total thickness, tolerance & $\pm 3 \mu \mathrm{m}$ & The capsule ablator total thickness shall be within $\pm 3 \mu \mathrm{m}$ of the specified value \\
\hline UB5 & 0 & I.4.1.1.2.1 & Be (uniform) capsule & Ablator density & $1.88 \pm 3 \% \mathrm{~g} / \mathrm{cc}$ & The ablator density shall be $1.88 \pm 3 \% \mathrm{~g} / \mathrm{cc}$ \\
\hline UB6 & 0 & I.4.1.1.2.1 & Be (uniform) capsule & Ablator inner surface figure & see table & $\begin{array}{l}\text { The power spectrum of the deviation of the inner surface of the Be ablator from a perfect } \\
\text { sphere of the same average radius shall not exceed the spectrum in Table "Surface } \\
\text { roughness and thickness power spectra for uniformly doped Be capsule - Rev } 0 . " \text { "Shall not } \\
\text { exceed" means that the } 2 \mathrm{D} \text { power in modes } 1-12 \text { shall be less than that tabulated, and the } \\
1 \mathrm{D} \text { power in a trace, summed over any }+/-15 \% \text { bandwidth above mode } 12 \text { shall be less than } \\
\text { that of the same bandwidth for the reference power spectra. }\end{array}$ \\
\hline UB7 & 0 & I.4.1.1.2.1 & Be (uniform) capsule & Ablator thickness non-uniformity & see table & $\begin{array}{l}\text { The power spectrum of the non-uniformity of the cumulative thickness of each layer shall not } \\
\text { exceed the spectrum in Table "Surface roughness and thickness power spectra for uniformly } \\
\text { doped Be capsule - Rev } 0 . " \text { "Shall not exceed" means that the } 2 \mathrm{D} \text { power in modes } 1-12 \text { shall } \\
\text { be less than that tabulated, and the } 1 \mathrm{D} \text { power in a trace, summed over any }+/-15 \% \\
\text { bandwidth above mode } 12 \text { shall be less than that of the same bandwidth for the reference } \\
\text { power spectra. }\end{array}$ \\
\hline UB8 & 0 & I.4.1.1.2.1 & Be (uniform) capsule & Joint Plane Orientation wrt hohlraum & $\begin{array}{l}+/-10^{\circ} \text { of perpendicular to } \\
\text { fill hole and }+/-10^{\circ} \text { of the } \\
\text { LOS theta } 90 \text { phi }=340\end{array}$ & $\begin{array}{l}\text { The plane of the joint in the capsule shall be fabricated so that it is within }+/-10^{\circ} \text { of } \\
\text { perpendicular to the capsule fill hole and aligned within }+/-10^{\circ} \text { of the LOS theta }=90 \text { phi }=340\end{array}$ \\
\hline UB9 & 0 & I.4.1.1.2.1 & Be (uniform) capsule & Joint width & $<0.1 \mu \mathrm{m}$ & The width of the joint in the capsule shall be $<0.1 \mu \mathrm{m}$ \\
\hline UB10 & 0 & I.4.1.1.2.1 & Be (uniform) capsule & Bond and joint gap ablator penetration & $\begin{array}{l}\text { Depth }<1 / 3 \text { ablator } \\
\text { thickness }\end{array}$ & $\begin{array}{l}\text { The depth of the bond and joint gap shall be less than } 1 / 3 \text { of the ablator thickness to reduce } \\
\text { jet mass and penetration }\end{array}$ \\
\hline UB11 & 0 & $\mid$ I.4.1.1.2.1 & Be (uniform) capsule & Bond material composition & $\begin{array}{l}\text { Density and opacity } \\
\text { matched to ablator }\end{array}$ & $\begin{array}{l}\text { The material used for the capsule joint bond shall have the same density as the ablator } \\
\text { within }+/- \text { TBD\% and the same opacity as the ablator within }+/ \text { - TBD\% }\end{array}$ \\
\hline UB12 & 0 & I.4.1.1.2.1 & Be (uniform) capsule & Joint configuration & $\begin{array}{l}\text { see figure, } \\
\text { dimensions known to }< \\
\pm 20 \%\end{array}$ & $\begin{array}{l}\text { The joint configuration shall be as shown in Figure "Uniform Be Capsule Joint Configuration - } \\
\text { Rev 0". All dimensions shall be measured to an accuracy better than } 20 \%\end{array}$ \\
\hline
\end{tabular}




\section{Uniform Be Capsule Joint Configuration - Rev 0}

Notes:
1.All dimensions in microns
2.Assumed wall thickness of 150 microns. Dimensions would be scaled for
different wall thickness.

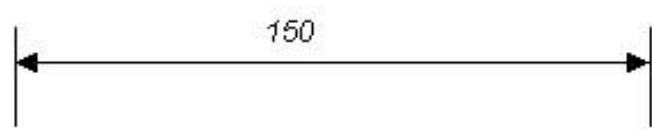

Assumed wall thickness of 150 microns Dimensions would be scaled for

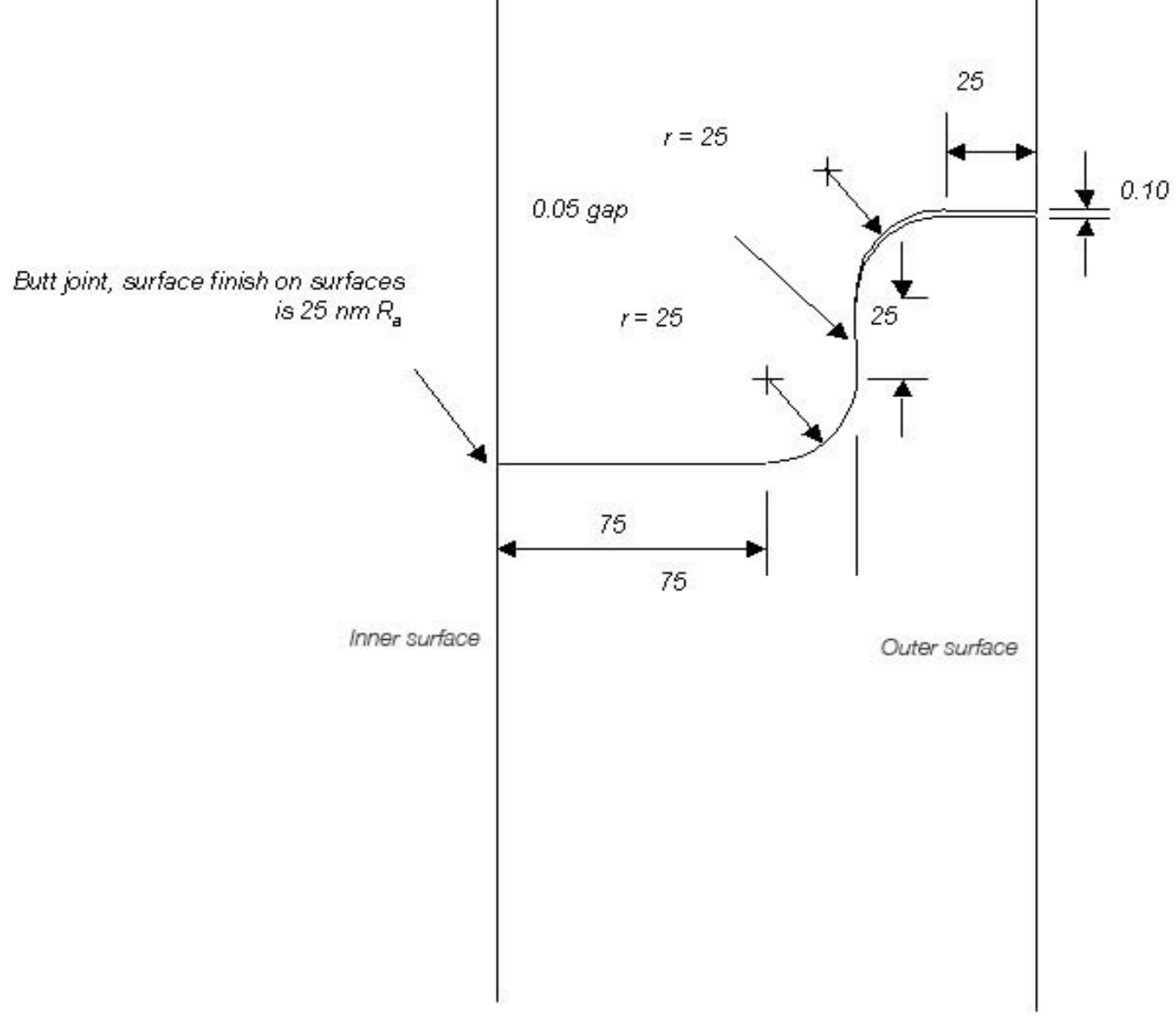




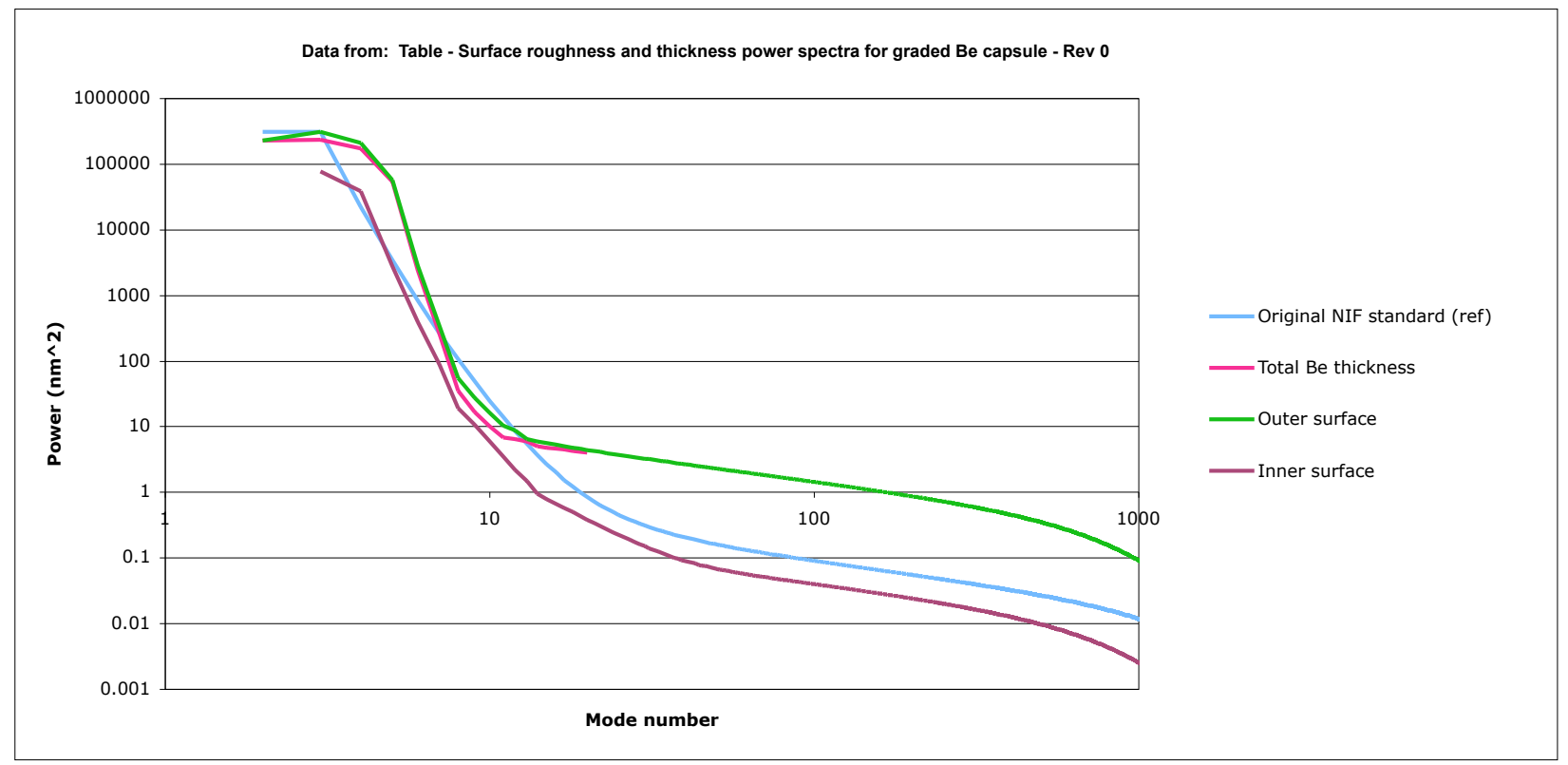

\section{Surface roughness and thickness power spectra for uniformly doped Be capsule - Rev 0}

Notes:

1. First table is $2 \mathrm{D}$ power, applies to low modes

2. Second table is the 1D power for each surface in a trace, in $\mathrm{nm}^{\wedge} 2$. Applies to modes $>12$, included for low modes for information only

3. Inner surface roughness is defined relative to an ideal sphere of the same average radius.

Other surfaces are all defined relative to inner surfaces, i.e.they are actually the variation in the thickness

4. Outer surface relative to inner surface, for modes 1-19

5. At low modes, assumes inner surface and thickness are randomly phased.

6. We have specified the outer and inner surfaces to have a factor of two lower power

at all modes compared to the graded dopant capsule. Depending upon the specific

choice of dopant concentration, perturbation growth at low, intermediate, or high modes

can be either higher or lower than the graded dopant capsule, but not uniformly lower.

\begin{tabular}{|c|c|c|c|c|}
\hline $\begin{array}{c}\text { mode } \\
\text { number }\end{array}$ & $\begin{array}{c}\text { Original NIF } \\
\text { standard, } \\
\text { reference, } \mathrm{nm}^{\wedge} \mathbf{2}\end{array}$ & $\begin{array}{c}\text { Inner surface } \\
\text { (radius), } \mathrm{nm}^{\wedge} 2\end{array}$ & \begin{tabular}{|c|} 
Total shell \\
thickness, \\
nm^2 (note 4) \\
\end{tabular} & $\begin{array}{l}\text { Outer surface, } \\
\text { nm^2 (note 5) }\end{array}$ \\
\hline \multicolumn{5}{|l|}{ 2D power } \\
\hline $1 \mid$ & not applicable & & 122500 & 122500 \\
\hline 2 & & 101667 & 275625 & 377292 \\
\hline 3 & & 61529.2 & 275625 & 337154.2 \\
\hline 4 & & 4911.74 & 99225 & 104136.74 \\
\hline 5 & & 778.152 & 4900 & 5678.152 \\
\hline 6 & & 185.31 & 566.44 & 751.75 \\
\hline 7 & & 35.5897 & 70 & 105.5897 \\
\hline 8 & & 19.7554 & 31.36 & 51.1154 \\
\hline 9 & & 10.9590367 & 20 & 30.9590367 \\
\hline 10 & & 6.182451986 & 14 & 20.18245199 \\
\hline 11 & & 3.876452247 & 13 & 16.87645225 \\
\hline 12 & & 2.588741216 & 12 & 12 \\
\hline \multicolumn{5}{|l|}{$\begin{array}{l}\text { 1D trace } \\
\text { power }\end{array}$} \\
\hline 1 & $3.13 E+05$ & & 227025 & 227025 \\
\hline 2 & 313000 & 77828.8 & 237851 & 315679.8 \\
\hline 3 & 22000 & 38678.5 & 173623 & 212301.5 \\
\hline 4 & 3440 & 2737.4 & 54411.7 & 57149.1 \\
\hline 5 & 840 & 394.719 & 2432.86 & 2827.579 \\
\hline 6 & 272 & 90.2859 & 265.775 & 356.0609 \\
\hline 7 & 107 & 19.2375 & 35.6521 & 54.8896 \\
\hline 8 & 48.8 & 10.7192 & 16.6239 & 27.3431 \\
\hline 9 & 24.9 & 6 & 10.2291 & 16.2291 \\
\hline 10 & 14 & 3.5 & 6.91169 & 10.41169 \\
\hline 11 & 8.48 & 2.2 & 6.5 & 8.7 \\
\hline 12 & 5.48 & 1.5 & 6 & 6.5 \\
\hline 13 & 3.74 & 0.962385 & 5.08165 & 6.044035 \\
\hline 14 & 2.68 & 0.78819 & 4.80045 & 5.58864 \\
\hline 15 & 2 & 0.663585 & 4.62843 & 5.292015 \\
\hline 16 & 1.55 & 0.572435 & 4.462605 & 5.03504 \\
\hline 17 & 1.24 & 0.4977295 & 4.310605 & 4.8083345 \\
\hline 18 & 1.01 & 0.435971 & 4.17062 & 4.606591 \\
\hline 19 & 0.851 & 0.384527 & 4.041155 & 4.425682 \\
\hline 20 & 0.729 & 0.3413825 & & 4.2623625 \\
\hline 21 & 0.635 & 0.3049765 & & 4.1140365 \\
\hline
\end{tabular}




\begin{tabular}{|c|c|c|}
\hline 22 & 0.561 & 0.2740845 \\
\hline 23 & 0.503 & 0.2477365 \\
\hline 24 & 0.455 & 0.2251575 \\
\hline 25 & 0.416 & 0.2057225 \\
\hline 26 & 0.384 & 0.188924 \\
\hline 27 & 0.357 & 0.174347 \\
\hline 28 & 0.333 & 0.1616495 \\
\hline 29 & 0.313 & 0.150549 \\
\hline 30 & 0.296 & 0.1408115 \\
\hline 31 & 0.281 & 0.13224 \\
\hline 32 & 0.268 & 0.1246695 \\
\hline 33 & 0.256 & 0.1179625 \\
\hline 34 & 0.245 & 0.1120005 \\
\hline 35 & 0.236 & 0.106684 \\
\hline 36 & 0.227 & 0.1019285 \\
\hline 37 & 0.219 & 0.097662 \\
\hline 38 & 0.212 & 0.0938215 \\
\hline 39 & 0.205 & 0.0903545 \\
\hline 40 & 0.199 & 0.087215 \\
\hline 41 & 0.194 & 0.084363 \\
\hline 42 & 0.189 & 0.081765 \\
\hline 43 & 0.184 & 0.079391 \\
\hline 44 & 0.179 & 0.077215 \\
\hline 45 & 0.175 & 0.075215 \\
\hline 46 & 0.171 & 0.073371 \\
\hline 47 & 0.168 & 0.071666 \\
\hline 48 & 0.164 & 0.070085 \\
\hline 49 & 0.161 & 0.0686155 \\
\hline 50 & 0.158 & 0.067245 \\
\hline 51 & 0.155 & 0.065964 \\
\hline 52 & 0.152 & 0.064763 \\
\hline 53 & 0.15 & 0.063635 \\
\hline 54 & 0.147 & 0.0625725 \\
\hline 55 & 0.145 & 0.061569 \\
\hline 56 & 0.142 & 0.0606195 \\
\hline 57 & 0.14 & 0.059719 \\
\hline 58 & 0.138 & 0.058863 \\
\hline 59 & 0.136 & 0.058048 \\
\hline 60 & 0.134 & 0.05727 \\
\hline 61 & 0.132 & 0.0565265 \\
\hline 62 & 0.13 & 0.0558145 \\
\hline 63 & 0.129 & 0.0551315 \\
\hline 64 & 0.127 & 0.054475 \\
\hline 65 & 0.125 & 0.0538435 \\
\hline 66 & 0.124 & 0.053235 \\
\hline 67 & 0.122 & 0.052648 \\
\hline 68 & 0.121 & 0.052081 \\
\hline 69 & 0.12 & 0.051532 \\
\hline 70 & 0.118 & 0.051001 \\
\hline 71 & 0.117 & 0.0504855 \\
\hline 72 & 0.116 & 0.04998585 \\
\hline 73 & 0.114 & 0.0495004 \\
\hline 74 & 0.113 & 0.0490284 \\
\hline 75 & 0.112 & 0.04856915 \\
\hline 76 & 0.111 & 0.048122 \\
\hline 77 & 0.11 & 0.0476862 \\
\hline 78 & 0.109 & 0.04726125 \\
\hline 79 & 0.108 & 0.0468466 \\
\hline 80 & 0.107 & 0.0464417 \\
\hline 81 & 0.106 & 0.0460461 \\
\hline 82 & 0.105 & 0.0456594 \\
\hline 83 & 0.104 & 0.0452812 \\
\hline 84 & 0.103 & 0.0449111 \\
\hline 85 & 0.102 & 0.04454875 \\
\hline 86 & 0.101 & 0.0441939 \\
\hline 87 & 0.1 & 0.04384615 \\
\hline 88 & 0.0992 & 0.0435053 \\
\hline 89 & 0.0984 & 0.04317105 \\
\hline 90 & 0.0976 & 0.04284315 \\
\hline 91 & 0.0968 & 0.0425214 \\
\hline 92 & 0.096 & 0.04220555 \\
\hline 93 & 0.0953 & 0.0418954 \\
\hline 94 & 0.0945 & 0.04159075 \\
\hline 95 & 0.0938 & 0.04129145 \\
\hline 96 & 0.0931 & 0.0409973 \\
\hline 97 & 0.0924 & 0.04070815 \\
\hline 98 & 0.0917 & 0.0404238 \\
\hline 99 & 0.091 & 0.04014415 \\
\hline 100 & 0.0904 & 0.039869 \\
\hline 101 & 0.0897 & 0.0395983 \\
\hline
\end{tabular}

3.9786095

3.8543565

3.7398625

3.6339375

3.535594

3.443982

3.3583845

3.278179

3.2028365

3.13189

3.0649345

3.0016175

2.9416205

2.884669

2.8305185

2.778942

2.7297515

2.6827695

2.637835

2.594803

2.553545

2.513946

2.47589

2.43929

2.404051

2.370086

2.33733

2.3057055

2.275155

2.245614

2.217033

2.189355

2.1625375

2.136534

2.1113045

2.086814

2.063028

2.039908

2.017425

1.9955565

1.9742645

1.9535315

1.93333

1.9136385

1.894435

1.875698

1.857416

1.839562

1.822126

1.8050855

1.78843085

1.7721454

1.7562184

1.74062915

1.725377

1.7104412

1.69581125

1.6814816

1.6674367

1.6536711

1.6401744

1.6269362

1.6139511

1.60120875

1.5887039

1.57642615

1.5643703

1.55253105

1.54089815

1.5294664

1.51823055

1.5071904

1.49633075

1.48565645

1.4751523

1.46482315

1.4546538

1.44464915

1.434804

1.4251083 


\begin{tabular}{|c|c|c|c|}
\hline 102 & 0.0891 & 0.0393319 & 1.4155619 \\
\hline 103 & 0.0885 & 0.0390696 & 1.4061596 \\
\hline 104 & 0.0879 & 0.0388114 & 1.3969014 \\
\hline 105 & 0.0873 & 0.0385571 & 1.3877771 \\
\hline 106 & 0.0867 & 0.03830665 & 1.37878665 \\
\hline 107 & 0.0861 & 0.03805985 & 1.36992985 \\
\hline 108 & 0.0855 & 0.03781675 & 1.36120175 \\
\hline 109 & 0.085 & 0.03757715 & 1.35259715 \\
\hline 110 & 0.0844 & 0.03734095 & 1.34411095 \\
\hline 111 & 0.0839 & 0.03710815 & 1.33574815 \\
\hline 112 & 0.0833 & 0.0368786 & 1.3274986 \\
\hline 113 & 0.0828 & 0.0366522 & 1.3193622 \\
\hline 114 & 0.0823 & 0.03642895 & 1.31133895 \\
\hline 115 & 0.0818 & 0.03620875 & 1.30341875 \\
\hline 116 & 0.0813 & 0.0359915 & 1.2956115 \\
\hline 117 & 0.0808 & 0.0357771 & 1.2879021 \\
\hline 118 & 0.0803 & 0.03556555 & 1.28029055 \\
\hline 119 & 0.0798 & 0.0353568 & 1.2727818 \\
\hline 120 & 0.0793 & 0.0351507 & 1.2653707 \\
\hline 121 & 0.0789 & 0.03494725 & 1.25805225 \\
\hline 122 & 0.0784 & 0.0347464 & 1.2508264 \\
\hline 123 & 0.078 & 0.03454805 & 1.24369305 \\
\hline 124 & 0.0775 & 0.0343522 & 1.2366422 \\
\hline 125 & 0.0771 & 0.03415875 & 1.22968375 \\
\hline 126 & 0.0766 & 0.03396765 & 1.22280765 \\
\hline 127 & 0.0762 & 0.0337789 & 1.2160139 \\
\hline 128 & 0.0758 & 0.03359235 & 1.20930235 \\
\hline 129 & 0.0754 & 0.0334081 & 1.2026681 \\
\hline 130 & 0.075 & 0.03322595 & 1.19611595 \\
\hline 131 & 0.0746 & 0.033046 & 1.189641 \\
\hline 132 & 0.0742 & 0.0328681 & 1.1832381 \\
\hline 133 & 0.0738 & 0.03269225 & 1.17690725 \\
\hline 134 & 0.0734 & 0.0325184 & 1.1706484 \\
\hline 135 & 0.073 & 0.0323465 & 1.1644615 \\
\hline 136 & 0.0726 & 0.03217655 & 1.15834655 \\
\hline 137 & 0.0723 & 0.0320085 & 1.1522985 \\
\hline 138 & 0.0719 & 0.0318423 & 1.1463123 \\
\hline 139 & 0.0715 & 0.0316779 & 1.1403979 \\
\hline 140 & 0.0712 & 0.0315153 & 1.1345453 \\
\hline 141 & 0.0708 & 0.03135445 & 1.12875445 \\
\hline 142 & 0.0705 & 0.0311953 & 1.1230253 \\
\hline 143 & 0.0701 & 0.0310379 & 1.1173579 \\
\hline 144 & 0.0698 & 0.0308821 & 1.1117521 \\
\hline 145 & 0.0694 & 0.03072795 & 1.10620295 \\
\hline 146 & 0.0691 & 0.0305754 & 1.1007104 \\
\hline 147 & 0.0688 & 0.0304244 & 1.0952744 \\
\hline 148 & 0.0684 & 0.03027495 & 1.08989495 \\
\hline 149 & 0.0681 & 0.030127 & 1.084572 \\
\hline 150 & 0.0678 & 0.02998055 & 1.07930055 \\
\hline 151 & 0.0675 & 0.0298356 & 1.0740806 \\
\hline 152 & 0.0672 & 0.02969205 & 1.06891205 \\
\hline 153 & 0.0668 & 0.02954995 & 1.06379495 \\
\hline 154 & 0.0665 & 0.0294092 & 1.0587292 \\
\hline 155 & 0.0662 & 0.0292698 & 1.0537098 \\
\hline 156 & 0.0659 & 0.0291318 & 1.0487418 \\
\hline 157 & 0.0656 & 0.0289951 & 1.0438201 \\
\hline 158 & 0.0653 & 0.0288597 & 1.0389497 \\
\hline 159 & 0.0651 & 0.02872555 & 1.03412055 \\
\hline 160 & 0.0648 & 0.02859265 & 1.02933765 \\
\hline 161 & 0.0645 & 0.02846105 & 1.02459605 \\
\hline 162 & 0.0642 & 0.0283306 & 1.0199006 \\
\hline 163 & 0.0639 & 0.0282014 & 1.0152514 \\
\hline 164 & 0.0636 & 0.02807335 & 1.01063835 \\
\hline 165 & 0.0634 & 0.0279465 & 1.0060715 \\
\hline 166 & 0.0631 & 0.02782075 & 1.00154575 \\
\hline 167 & 0.0628 & 0.02769615 & 0.99706115 \\
\hline 168 & 0.0626 & 0.02757265 & 0.99261265 \\
\hline 169 & 0.0623 & 0.0274502 & 0.9882052 \\
\hline 170 & 0.0621 & 0.02732885 & 0.98383885 \\
\hline 171 & 0.0618 & 0.0272086 & 0.9795086 \\
\hline 172 & 0.0615 & 0.02708935 & 0.97521435 \\
\hline 173 & 0.0613 & 0.0269711 & 0.9709611 \\
\hline 174 & 0.061 & 0.0268539 & 0.9667389 \\
\hline 175 & 0.0608 & 0.0267377 & 0.9625577 \\
\hline 176 & 0.0605 & 0.0266225 & 0.9584075 \\
\hline 177 & 0.0603 & 0.0265082 & 0.9542932 \\
\hline 178 & 0.0601 & 0.0263949 & 0.9502149 \\
\hline 179 & 0.0598 & 0.02628255 & 0.94617255 \\
\hline 180 & 0.0596 & 0.0261711 & 0.9421611 \\
\hline 181 & 0.0594 & 0.02606055 & 0.93818055 \\
\hline
\end{tabular}




\begin{tabular}{|c|c|c|c|}
\hline 182 & 0.0591 & 0.0259509 & 0.9342309 \\
\hline 183 & 0.0589 & 0.0258422 & 0.9303172 \\
\hline 184 & 0.0587 & 0.0257343 & 0.9264343 \\
\hline 185 & 0.0584 & 0.0256273 & 0.9225823 \\
\hline 186 & 0.0582 & 0.02552115 & 0.91876115 \\
\hline 187 & 0.058 & 0.0254158 & 0.9149708 \\
\hline 188 & 0.0578 & 0.0253113 & 0.9112063 \\
\hline 189 & 0.0576 & 0.02520765 & 0.90747765 \\
\hline 190 & 0.0573 & 0.02510475 & 0.90376975 \\
\hline 191 & 0.0571 & 0.0250027 & 0.9000977 \\
\hline 192 & 0.0569 & 0.0249014 & 0.8964514 \\
\hline 193 & 0.0567 & 0.02480085 & 0.89283085 \\
\hline 194 & 0.0565 & 0.0247011 & 0.8892411 \\
\hline 195 & 0.0563 & 0.0246021 & 0.8856771 \\
\hline 196 & 0.0561 & 0.02450385 & 0.88213885 \\
\hline 197 & 0.0559 & 0.0244063 & 0.8786263 \\
\hline 198 & 0.0557 & 0.0243095 & 0.8751395 \\
\hline 199 & 0.0555 & 0.0242134 & 0.8716834 \\
\hline 200 & 0.0553 & 0.024118 & 0.868248 \\
\hline 201 & 0.0551 & 0.0240233 & 0.8648383 \\
\hline 202 & 0.0549 & 0.0239293 & 0.8614543 \\
\hline 203 & 0.0547 & 0.023836 & 0.858096 \\
\hline 204 & 0.0545 & 0.02374335 & 0.85475835 \\
\hline 205 & 0.0543 & 0.02365135 & 0.85144635 \\
\hline 206 & 0.0541 & 0.02356 & 0.84816 \\
\hline 207 & 0.0539 & 0.0234693 & 0.8448943 \\
\hline 208 & 0.0537 & 0.02337925 & 0.84165425 \\
\hline 209 & 0.0536 & 0.0232898 & 0.8384348 \\
\hline 210 & 0.0534 & 0.023201 & 0.835236 \\
\hline 211 & 0.0532 & 0.0231128 & 0.8320628 \\
\hline 212 & 0.053 & 0.0230252 & 0.8289102 \\
\hline 213 & 0.0528 & 0.02293825 & 0.82577825 \\
\hline 214 & 0.0527 & 0.02285185 & 0.82266685 \\
\hline 215 & 0.0525 & 0.022766 & 0.819576 \\
\hline 216 & 0.0523 & 0.0226808 & 0.8165108 \\
\hline 217 & 0.0521 & 0.02259615 & 0.81346115 \\
\hline 218 & 0.052 & 0.02251205 & 0.81043205 \\
\hline 219 & 0.0518 & 0.0224285 & 0.8074285 \\
\hline 220 & 0.0516 & 0.0223455 & 0.8044405 \\
\hline 221 & 0.0514 & 0.0222631 & 0.8014731 \\
\hline 222 & 0.0513 & 0.0221812 & 0.7985212 \\
\hline 223 & 0.0511 & 0.0220998 & 0.7955948 \\
\hline 224 & 0.0509 & 0.022019 & 0.792684 \\
\hline 225 & 0.0508 & 0.02193865 & 0.78979365 \\
\hline 226 & 0.0506 & 0.02185885 & 0.78691885 \\
\hline 227 & 0.0504 & 0.02177955 & 0.78406455 \\
\hline 228 & 0.0503 & 0.02170075 & 0.78122575 \\
\hline 229 & 0.0501 & 0.0216225 & 0.7784075 \\
\hline 230 & 0.05 & 0.0215447 & 0.7756097 \\
\hline 231 & 0.0498 & 0.02146735 & 0.77282735 \\
\hline 232 & 0.0497 & 0.02139055 & 0.77006055 \\
\hline 233 & 0.0495 & 0.0213142 & 0.7673092 \\
\hline 234 & 0.0493 & 0.0212383 & 0.7645783 \\
\hline 235 & 0.0492 & 0.0211629 & 0.7618629 \\
\hline 236 & 0.049 & 0.02108795 & 0.75916795 \\
\hline 237 & 0.0489 & 0.02101345 & 0.75648345 \\
\hline 238 & 0.0487 & 0.0209394 & 0.7538194 \\
\hline 239 & 0.0486 & 0.0208658 & 0.7511658 \\
\hline 240 & 0.0484 & 0.02079265 & 0.74853265 \\
\hline 241 & 0.0483 & 0.0207199 & 0.7459149 \\
\hline 242 & 0.0481 & 0.0206476 & 0.7433126 \\
\hline 243 & 0.048 & 0.02057575 & 0.74072575 \\
\hline 244 & 0.0479 & 0.0205043 & 0.7381543 \\
\hline 245 & 0.0477 & 0.0204333 & 0.7355983 \\
\hline 246 & 0.0476 & 0.0203627 & 0.7330577 \\
\hline 247 & 0.0474 & 0.0202925 & 0.7305325 \\
\hline 248 & 0.0473 & 0.0202227 & 0.7280177 \\
\hline 249 & 0.0471 & 0.02015335 & 0.72551835 \\
\hline 250 & 0.047 & 0.02008435 & 0.72303435 \\
\hline 251 & 0.0469 & 0.02001575 & 0.72056575 \\
\hline 252 & 0.0467 & 0.01994755 & 0.71811255 \\
\hline 253 & 0.0466 & 0.01987975 & 0.71566975 \\
\hline 254 & 0.0465 & 0.0198123 & 0.7132423 \\
\hline 255 & 0.0463 & 0.01974525 & 0.71083025 \\
\hline 256 & 0.0462 & 0.0196786 & 0.7084286 \\
\hline 257 & 0.046 & 0.0196123 & 0.7060423 \\
\hline 258 & 0.0459 & 0.01954635 & 0.70366635 \\
\hline 259 & 0.0458 & 0.0194808 & 0.7013058 \\
\hline 260 & 0.0457 & 0.01941555 & 0.69896055 \\
\hline 261 & 0.0455 & 0.0193507 & 0.6966257 \\
\hline
\end{tabular}




\begin{tabular}{|c|c|c|c|}
\hline 262 & 0.0454 & 0.0192862 & 0.6943012 \\
\hline 263 & 0.0453 & 0.01922205 & 0.69199205 \\
\hline 264 & 0.0451 & 0.01915825 & 0.68969825 \\
\hline 265 & 0.045 & 0.0190948 & 0.6874148 \\
\hline 266 & 0.0449 & 0.01903165 & 0.68514165 \\
\hline 267 & 0.0448 & 0.0189689 & 0.6828789 \\
\hline 268 & 0.0446 & 0.01890645 & 0.68063145 \\
\hline 269 & 0.0445 & 0.0188443 & 0.6783943 \\
\hline 270 & 0.0444 & 0.0187825 & 0.6761725 \\
\hline 271 & 0.0443 & 0.01872105 & 0.67395605 \\
\hline 272 & 0.0441 & 0.0186599 & 0.6717549 \\
\hline 273 & 0.044 & 0.0185991 & 0.6695691 \\
\hline 274 & 0.0439 & 0.0185386 & 0.6673886 \\
\hline 275 & 0.0438 & 0.0184784 & 0.6652234 \\
\hline 276 & 0.0437 & 0.0184185 & 0.6630635 \\
\hline 277 & 0.0435 & 0.0183589 & 0.6609189 \\
\hline 278 & 0.0434 & 0.01829965 & 0.65878465 \\
\hline 279 & 0.0433 & 0.01824065 & 0.65666565 \\
\hline 280 & 0.0432 & 0.018182 & 0.654552 \\
\hline 281 & 0.0431 & 0.0181236 & 0.6524486 \\
\hline 282 & 0.043 & 0.01806555 & 0.65036055 \\
\hline 283 & 0.0428 & 0.01800775 & 0.64827775 \\
\hline 284 & 0.0427 & 0.01795025 & 0.64621025 \\
\hline 285 & 0.0426 & 0.017893 & 0.644148 \\
\hline 286 & 0.0425 & 0.0178361 & 0.6421011 \\
\hline 287 & 0.0424 & 0.01777945 & 0.64005945 \\
\hline 288 & 0.0423 & 0.01772305 & 0.63802805 \\
\hline 289 & 0.0422 & 0.01766695 & 0.63601195 \\
\hline 290 & 0.042 & 0.01761115 & 0.63400115 \\
\hline 291 & 0.0419 & 0.0175556 & 0.6320006 \\
\hline 292 & 0.0418 & 0.0175003 & 0.6300103 \\
\hline 293 & 0.0417 & 0.0174453 & 0.6280303 \\
\hline 294 & 0.0416 & 0.01739055 & 0.62606055 \\
\hline 295 & 0.0415 & 0.01733605 & 0.62409605 \\
\hline 296 & 0.0414 & 0.0172818 & 0.6221468 \\
\hline 297 & 0.0413 & 0.01722785 & 0.62020285 \\
\hline 298 & 0.0412 & 0.01717415 & 0.61826915 \\
\hline 299 & 0.0411 & 0.01712065 & 0.61634565 \\
\hline 300 & 0.041 & 0.01706745 & 0.61442745 \\
\hline 301 & 0.0409 & 0.0170145 & 0.6125195 \\
\hline 302 & 0.0408 & 0.0169618 & 0.6106218 \\
\hline 303 & 0.0407 & 0.0169093 & 0.6087343 \\
\hline 304 & 0.0406 & 0.0168571 & 0.6068571 \\
\hline 305 & 0.0405 & 0.0168051 & 0.6049851 \\
\hline 306 & 0.0404 & 0.01675335 & 0.60312335 \\
\hline 307 & 0.0403 & 0.01670185 & 0.60126685 \\
\hline 308 & 0.0402 & 0.0166506 & 0.5994206 \\
\hline 309 & 0.04 & 0.01659955 & 0.59758455 \\
\hline 310 & 0.04 & 0.01654875 & 0.59575375 \\
\hline 311 & 0.0398 & 0.01649815 & 0.59393315 \\
\hline 312 & 0.0398 & 0.0164478 & 0.5921228 \\
\hline 313 & 0.0397 & 0.0163977 & 0.5903177 \\
\hline 314 & 0.0396 & 0.0163478 & 0.5885228 \\
\hline 315 & 0.0395 & 0.01629815 & 0.58673315 \\
\hline 316 & 0.0394 & 0.0162487 & 0.5849537 \\
\hline 317 & 0.0393 & 0.01619945 & 0.58317945 \\
\hline 318 & 0.0392 & 0.01615045 & 0.58141545 \\
\hline 319 & 0.0391 & 0.01610165 & 0.57965665 \\
\hline 320 & 0.039 & 0.01605305 & 0.57790805 \\
\hline 321 & 0.0389 & 0.0160047 & 0.5761697 \\
\hline 322 & 0.0388 & 0.0159565 & 0.5744365 \\
\hline 323 & 0.0387 & 0.01590855 & 0.57270855 \\
\hline 324 & 0.0386 & 0.01586085 & 0.57099085 \\
\hline 325 & 0.0385 & 0.0158133 & 0.5692783 \\
\hline 326 & 0.0384 & 0.01576595 & 0.56757595 \\
\hline 327 & 0.0383 & 0.01571885 & 0.56587885 \\
\hline 328 & 0.0382 & 0.0156719 & 0.5641869 \\
\hline 329 & 0.0381 & 0.0156252 & 0.5625052 \\
\hline 330 & 0.038 & 0.01557865 & 0.56083365 \\
\hline 331 & 0.038 & 0.0155323 & 0.5591623 \\
\hline 332 & 0.0379 & 0.0154862 & 0.5575012 \\
\hline 333 & 0.0378 & 0.01544025 & 0.55585025 \\
\hline 334 & 0.0377 & 0.0153945 & 0.5541995 \\
\hline 335 & 0.0376 & 0.01534895 & 0.55256395 \\
\hline 336 & 0.0375 & 0.01530355 & 0.55092855 \\
\hline 337 & 0.0374 & 0.0152584 & 0.5493034 \\
\hline 338 & 0.0373 & 0.0152134 & 0.5476834 \\
\hline 339 & 0.0372 & 0.0151686 & 0.5460686 \\
\hline 340 & 0.0372 & 0.01512395 & 0.54446395 \\
\hline 341 & 0.0371 & 0.01507955 & 0.54286455 \\
\hline
\end{tabular}




\begin{tabular}{|c|c|c|}
\hline 342 & 0.037 & 0.0150353 \\
\hline 343 & 0.0369 & 0.0149912 \\
\hline 344 & 0.0368 & 0.0149473 \\
\hline 345 & 0.0367 & 0.0149036 \\
\hline 346 & 0.0366 & 0.01486005 \\
\hline 347 & 0.0366 & 0.0148167 \\
\hline 348 & 0.0365 & 0.0147735 \\
\hline 349 & 0.0364 & 0.0147305 \\
\hline 350 & 0.0363 & 0.01468765 \\
\hline 351 & 0.0362 & 0.01464495 \\
\hline 352 & 0.0361 & 0.01460245 \\
\hline 353 & 0.0361 & 0.0145601 \\
\hline 354 & 0.036 & 0.01451795 \\
\hline 355 & 0.0359 & 0.01447595 \\
\hline 356 & 0.0358 & 0.0144341 \\
\hline 357 & 0.0357 & 0.01439245 \\
\hline 358 & 0.0357 & 0.0143509 \\
\hline 359 & 0.0356 & 0.01430955 \\
\hline 360 & 0.0355 & 0.01426835 \\
\hline 361 & 0.0354 & 0.01422735 \\
\hline 362 & 0.0353 & 0.01418645 \\
\hline 363 & 0.0353 & 0.01414575 \\
\hline 364 & 0.0352 & 0.0141052 \\
\hline 365 & 0.0351 & 0.0140648 \\
\hline 366 & 0.035 & 0.01402455 \\
\hline 367 & 0.035 & 0.01398445 \\
\hline 368 & 0.0349 & 0.0139445 \\
\hline 369 & 0.0348 & 0.0139047 \\
\hline 370 & 0.0347 & 0.0138651 \\
\hline 371 & 0.0346 & 0.0138256 \\
\hline 372 & 0.0346 & 0.01378625 \\
\hline 373 & 0.0345 & 0.01374705 \\
\hline 374 & 0.0344 & 0.013708 \\
\hline 375 & 0.0343 & 0.0136691 \\
\hline 376 & 0.0343 & 0.01363035 \\
\hline 377 & 0.0342 & 0.0135917 \\
\hline 378 & 0.0341 & 0.01355325 \\
\hline 379 & 0.034 & 0.0135149 \\
\hline 380 & 0.034 & 0.0134767 \\
\hline 381 & 0.0339 & 0.01343865 \\
\hline 382 & 0.0338 & 0.01340075 \\
\hline 383 & 0.0338 & 0.013363 \\
\hline 384 & 0.0337 & 0.01332535 \\
\hline 385 & 0.0336 & 0.01328785 \\
\hline 386 & 0.0335 & 0.0132505 \\
\hline 387 & 0.0335 & 0.01321325 \\
\hline 388 & 0.0334 & 0.01317615 \\
\hline 389 & 0.0333 & 0.0131392 \\
\hline 390 & 0.0333 & 0.01310235 \\
\hline 391 & 0.0332 & 0.0130657 \\
\hline 392 & 0.0331 & 0.0130291 \\
\hline 393 & 0.033 & 0.01299265 \\
\hline 394 & 0.033 & 0.01295635 \\
\hline 395 & 0.0329 & 0.0129202 \\
\hline 396 & 0.0328 & 0.01288415 \\
\hline 397 & 0.0328 & 0.0128482 \\
\hline 398 & 0.0327 & 0.0128124 \\
\hline 399 & 0.0326 & 0.01277675 \\
\hline 400 & 0.0326 & 0.0127412 \\
\hline 401 & 0.0325 & 0.0127058 \\
\hline 402 & 0.0324 & 0.0126705 \\
\hline 403 & 0.0324 & 0.0126353 \\
\hline 404 & 0.0323 & 0.01260025 \\
\hline 405 & 0.0322 & 0.0125653 \\
\hline 406 & 0.0322 & 0.0125305 \\
\hline 407 & 0.0321 & 0.0124958 \\
\hline 408 & 0.032 & 0.01246125 \\
\hline 409 & 0.032 & 0.0124268 \\
\hline 410 & 0.0319 & 0.01239245 \\
\hline 411 & 0.0318 & 0.0123582 \\
\hline 412 & 0.0318 & 0.0123241 \\
\hline 413 & 0.0317 & 0.0122901 \\
\hline 414 & 0.0316 & 0.01225625 \\
\hline 415 & 0.0316 & 0.0122225 \\
\hline 416 & 0.0315 & 0.01218885 \\
\hline 417 & 0.0314 & 0.0121553 \\
\hline 418 & 0.0314 & 0.0121219 \\
\hline 419 & 0.0313 & 0.0120886 \\
\hline 420 & 0.0312 & 0.0120554 \\
\hline 421 & 0.0312 & 0.0120223 \\
\hline
\end{tabular}

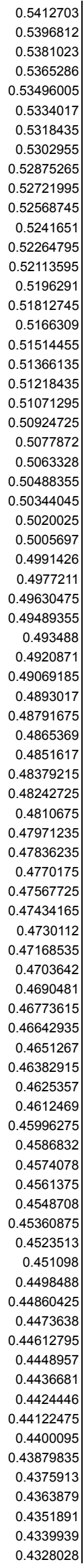




\begin{tabular}{|c|c|c|c|}
\hline 422 & 0.0311 & 0.0119893 & 0.4316158 \\
\hline 423 & 0.0311 & 0.01195645 & 0.43043245 \\
\hline 424 & 0.031 & 0.0119237 & 0.4292532 \\
\hline 425 & 0.0309 & 0.01189105 & 0.42807755 \\
\hline 426 & 0.0309 & 0.0118585 & 0.426906 \\
\hline 427 & 0.0308 & 0.01182605 & 0.42573855 \\
\hline 428 & 0.0307 & 0.01179375 & 0.42457475 \\
\hline 429 & 0.0307 & 0.0117615 & 0.4234145 \\
\hline 430 & 0.0306 & 0.0117294 & 0.4222584 \\
\hline 431 & 0.0306 & 0.0116974 & 0.4211059 \\
\hline 432 & 0.0305 & 0.0116655 & 0.4199575 \\
\hline 433 & 0.0304 & 0.0116337 & 0.4188122 \\
\hline 434 & 0.0304 & 0.011602 & 0.417671 \\
\hline 435 & 0.0303 & 0.0115704 & 0.4165334 \\
\hline 436 & 0.0303 & 0.0115389 & 0.4153999 \\
\hline 437 & 0.0302 & 0.0115075 & 0.4142695 \\
\hline 438 & 0.0301 & 0.0114762 & 0.4131427 \\
\hline 439 & 0.0301 & 0.011445 & 0.41202 \\
\hline 440 & 0.03 & 0.0114139 & 0.4109004 \\
\hline 441 & 0.03 & 0.0113829 & 0.4097849 \\
\hline 442 & 0.0299 & 0.011352 & 0.4086725 \\
\hline 443 & 0.0298 & 0.0113212 & 0.4075637 \\
\hline 444 & 0.0298 & 0.0112905 & 0.4064585 \\
\hline 445 & 0.0297 & 0.0112599 & 0.4053569 \\
\hline 446 & 0.0297 & 0.0112294 & 0.4042589 \\
\hline 447 & 0.0296 & 0.011199 & 0.403164 \\
\hline 448 & 0.0296 & 0.0111687 & 0.4020732 \\
\hline 449 & 0.0295 & 0.0111385 & 0.400985 \\
\hline 450 & 0.0294 & 0.01110835 & 0.39990085 \\
\hline 451 & 0.0294 & 0.01107835 & 0.39881985 \\
\hline 452 & 0.0293 & 0.0110484 & 0.3977424 \\
\hline 453 & 0.0293 & 0.01101855 & 0.39666805 \\
\hline 454 & 0.0292 & 0.0109888 & 0.3955973 \\
\hline 455 & 0.0292 & 0.01095915 & 0.39452965 \\
\hline 456 & 0.0291 & 0.0109296 & 0.3934656 \\
\hline 457 & 0.029 & 0.0109001 & 0.3924046 \\
\hline 458 & 0.029 & 0.01087075 & 0.39134675 \\
\hline 459 & 0.0289 & 0.01084145 & 0.39029245 \\
\hline 460 & 0.0289 & 0.01081225 & 0.38924125 \\
\hline 461 & 0.0288 & 0.01078315 & 0.38819365 \\
\hline 462 & 0.0288 & 0.01075415 & 0.38714865 \\
\hline 463 & 0.0287 & 0.0107252 & 0.3861072 \\
\hline 464 & 0.0287 & 0.01069635 & 0.38506885 \\
\hline 465 & 0.0286 & 0.0106676 & 0.3840341 \\
\hline 466 & 0.0285 & 0.01063895 & 0.38300195 \\
\hline 467 & 0.0285 & 0.01061035 & 0.38197335 \\
\hline 468 & 0.0284 & 0.0105819 & 0.3809479 \\
\hline 469 & 0.0284 & 0.0105535 & 0.379925 \\
\hline 470 & 0.0283 & 0.01052515 & 0.37890565 \\
\hline 471 & 0.0283 & 0.01049695 & 0.37788945 \\
\hline 472 & 0.0282 & 0.0104688 & 0.3768763 \\
\hline 473 & 0.0282 & 0.0104407 & 0.3758657 \\
\hline 474 & 0.0281 & 0.01041275 & 0.37485875 \\
\hline 475 & 0.0281 & 0.01038485 & 0.37385435 \\
\hline 476 & 0.028 & 0.01035705 & 0.37285355 \\
\hline 477 & 0.028 & 0.0103293 & 0.3718553 \\
\hline 478 & 0.0279 & 0.01030165 & 0.37086015 \\
\hline 479 & 0.0278 & 0.0102741 & 0.3698681 \\
\hline 480 & 0.0278 & 0.01024665 & 0.36887865 \\
\hline 481 & 0.0277 & 0.01021925 & 0.36789275 \\
\hline 482 & 0.0277 & 0.0101919 & 0.3669094 \\
\hline 483 & 0.0276 & 0.0101647 & 0.3659287 \\
\hline 484 & 0.0276 & 0.01013755 & 0.36495105 \\
\hline 485 & 0.0275 & 0.01011045 & 0.36397645 \\
\hline 486 & 0.0275 & 0.01008345 & 0.36300495 \\
\hline 487 & 0.0274 & 0.01005655 & 0.36203605 \\
\hline 488 & 0.0274 & 0.0100297 & 0.3610702 \\
\hline 489 & 0.0273 & 0.01000295 & 0.36010695 \\
\hline 490 & 0.0273 & 0.0099763 & 0.3591463 \\
\hline 491 & 0.0272 & 0.0099497 & 0.3581892 \\
\hline 492 & 0.0272 & 0.00992315 & 0.35723415 \\
\hline 493 & 0.0271 & 0.00989675 & 0.35628225 \\
\hline 494 & 0.0271 & 0.00987035 & 0.35533335 \\
\hline 495 & 0.027 & 0.0098441 & 0.3543866 \\
\hline 496 & 0.027 & 0.00981785 & 0.35344335 \\
\hline 497 & 0.0269 & 0.00979175 & 0.35250225 \\
\hline 498 & 0.0269 & 0.00976565 & 0.35156415 \\
\hline 499 & 0.0268 & 0.0097397 & 0.3506287 \\
\hline 500 & 0.0268 & 0.0097138 & 0.3496958 \\
\hline 501 & 0.0267 & 0.00968795 & 0.34876595 \\
\hline
\end{tabular}




\begin{tabular}{|c|c|c|c|}
\hline 502 & 0.0267 & 0.0096622 & 0.3478387 \\
\hline 503 & 0.0266 & 0.0096365 & 0.346914 \\
\hline 504 & 0.0266 & 0.0096109 & 0.3459919 \\
\hline 505 & 0.0265 & 0.00958535 & 0.34507235 \\
\hline 506 & 0.0265 & 0.0095599 & 0.3441559 \\
\hline 507 & 0.0264 & 0.0095345 & 0.3432415 \\
\hline 508 & 0.0264 & 0.00950915 & 0.34233015 \\
\hline 509 & 0.0263 & 0.0094839 & 0.3414214 \\
\hline 510 & 0.0263 & 0.00945875 & 0.34051475 \\
\hline 511 & 0.0262 & 0.00943365 & 0.33961115 \\
\hline 512 & 0.0262 & 0.0094086 & 0.3387101 \\
\hline 513 & 0.0262 & 0.00938365 & 0.33781165 \\
\hline 514 & 0.0261 & 0.00935875 & 0.33691525 \\
\hline 515 & 0.0261 & 0.00933395 & 0.33602195 \\
\hline 516 & 0.026 & 0.0093092 & 0.3351312 \\
\hline 517 & 0.026 & 0.0092845 & 0.3342425 \\
\hline 518 & 0.0259 & 0.0092599 & 0.3333564 \\
\hline 519 & 0.0259 & 0.00923535 & 0.33247335 \\
\hline 520 & 0.0258 & 0.0092109 & 0.3315924 \\
\hline 521 & 0.0258 & 0.0091865 & 0.330714 \\
\hline 522 & 0.0257 & 0.00916215 & 0.32983765 \\
\hline 523 & 0.0257 & 0.0091379 & 0.3289644 \\
\hline 524 & 0.0256 & 0.0091137 & 0.3280932 \\
\hline 525 & 0.0256 & 0.00908955 & 0.32722455 \\
\hline 526 & 0.0255 & 0.0090655 & 0.3263585 \\
\hline 527 & 0.0255 & 0.0090415 & 0.3254945 \\
\hline 528 & 0.0255 & 0.0090176 & 0.3246331 \\
\hline 529 & 0.0254 & 0.00899375 & 0.32377425 \\
\hline 530 & 0.0254 & 0.00896995 & 0.32291745 \\
\hline 531 & 0.0253 & 0.0089462 & 0.3220632 \\
\hline 532 & 0.0253 & 0.00892255 & 0.32121155 \\
\hline 533 & 0.0252 & 0.00889895 & 0.32036195 \\
\hline 534 & 0.0252 & 0.0088754 & 0.3195149 \\
\hline 535 & 0.0251 & 0.00885195 & 0.31866995 \\
\hline 536 & 0.0251 & 0.00882855 & 0.31782755 \\
\hline 537 & 0.025 & 0.0088052 & 0.3169877 \\
\hline 538 & 0.025 & 0.00878195 & 0.31614995 \\
\hline 539 & 0.025 & 0.00875875 & 0.31531425 \\
\hline 540 & 0.0249 & 0.0087356 & 0.3144811 \\
\hline 541 & 0.0249 & 0.0087125 & 0.31365 \\
\hline 542 & 0.0248 & 0.0086895 & 0.3128215 \\
\hline 543 & 0.0248 & 0.00866655 & 0.31199505 \\
\hline 544 & 0.0247 & 0.00864365 & 0.31117115 \\
\hline 545 & 0.0247 & 0.0086208 & 0.3103493 \\
\hline 546 & 0.0247 & 0.00859805 & 0.30952955 \\
\hline 547 & 0.0246 & 0.00857535 & 0.30871235 \\
\hline 548 & 0.0246 & 0.0085527 & 0.3078972 \\
\hline 549 & 0.0245 & 0.0085301 & 0.3070841 \\
\hline 550 & 0.0245 & 0.0085076 & 0.3062736 \\
\hline 551 & 0.0244 & 0.00848515 & 0.30546515 \\
\hline 552 & 0.0244 & 0.00846275 & 0.30465875 \\
\hline 553 & 0.0243 & 0.0084404 & 0.3038549 \\
\hline 554 & 0.0243 & 0.00841815 & 0.30305315 \\
\hline 555 & 0.0243 & 0.0083959 & 0.3022534 \\
\hline 556 & 0.0242 & 0.00837375 & 0.30145575 \\
\hline 557 & 0.0242 & 0.00835165 & 0.30066015 \\
\hline 558 & 0.0241 & 0.00832965 & 0.29986715 \\
\hline 559 & 0.0241 & 0.00830765 & 0.29907565 \\
\hline 560 & 0.0241 & 0.00828575 & 0.29828675 \\
\hline 561 & 0.024 & 0.0082639 & 0.2974999 \\
\hline 562 & 0.024 & 0.0082421 & 0.2967151 \\
\hline 563 & 0.0239 & 0.00822035 & 0.29593285 \\
\hline 564 & 0.0239 & 0.00819865 & 0.29515215 \\
\hline 565 & 0.0238 & 0.00817705 & 0.29437355 \\
\hline 566 & 0.0238 & 0.0081555 & 0.2935975 \\
\hline 567 & 0.0238 & 0.008134 & 0.292823 \\
\hline 568 & 0.0237 & 0.00811255 & 0.29205105 \\
\hline 569 & 0.0237 & 0.00809115 & 0.29128065 \\
\hline 570 & 0.0236 & 0.0080698 & 0.2905128 \\
\hline 571 & 0.0236 & 0.0080485 & 0.289747 \\
\hline 572 & 0.0236 & 0.0080273 & 0.2889828 \\
\hline 573 & 0.0235 & 0.00800615 & 0.28822115 \\
\hline 574 & 0.0235 & 0.00798505 & 0.28746105 \\
\hline 575 & 0.0234 & 0.007964 & 0.286703 \\
\hline 576 & 0.0234 & 0.007943 & 0.2859475 \\
\hline 577 & 0.0234 & 0.00792205 & 0.28519355 \\
\hline 578 & 0.0233 & 0.00790115 & 0.28444165 \\
\hline 579 & 0.0233 & 0.00788035 & 0.28369185 \\
\hline 580 & 0.0232 & 0.00785955 & 0.28294405 \\
\hline 581 & 0.0232 & 0.00783885 & 0.28219785 \\
\hline
\end{tabular}




\begin{tabular}{|c|c|c|c|}
\hline 582 & 0.0232 & 0.00781815 & 0.28145415 \\
\hline 583 & 0.0231 & 0.00779755 & 0.28071205 \\
\hline 584 & 0.0231 & 0.007777 & 0.279972 \\
\hline 585 & 0.023 & 0.0077565 & 0.279234 \\
\hline 586 & 0.023 & 0.00773605 & 0.27849805 \\
\hline 587 & 0.023 & 0.00771565 & 0.27776365 \\
\hline 588 & 0.0229 & 0.0076953 & 0.2770318 \\
\hline 589 & 0.0229 & 0.00767505 & 0.27630155 \\
\hline 590 & 0.0228 & 0.0076548 & 0.2755728 \\
\hline 591 & 0.0228 & 0.00763465 & 0.27484665 \\
\hline 592 & 0.0228 & 0.0076145 & 0.274122 \\
\hline 593 & 0.0227 & 0.00759445 & 0.27339945 \\
\hline 594 & 0.0227 & 0.0075744 & 0.2726789 \\
\hline 595 & 0.0226 & 0.00755445 & 0.27195995 \\
\hline 596 & 0.0226 & 0.00753455 & 0.27124305 \\
\hline 597 & 0.0226 & 0.00751465 & 0.27052765 \\
\hline 598 & 0.0225 & 0.00749485 & 0.26981485 \\
\hline 599 & 0.0225 & 0.0074751 & 0.2691031 \\
\hline 600 & 0.0225 & 0.0074554 & 0.2683939 \\
\hline 601 & 0.0224 & 0.00743575 & 0.26768625 \\
\hline 602 & 0.0224 & 0.0074161 & 0.2669806 \\
\hline 603 & 0.0223 & 0.00739655 & 0.26627655 \\
\hline 604 & 0.0223 & 0.00737705 & 0.26557455 \\
\hline 605 & 0.0223 & 0.0073576 & 0.2648741 \\
\hline 606 & 0.0222 & 0.0073382 & 0.2641757 \\
\hline 607 & 0.0222 & 0.00731885 & 0.26347885 \\
\hline 608 & 0.0222 & 0.00729955 & 0.26278405 \\
\hline 609 & 0.0221 & 0.0072803 & 0.2620913 \\
\hline 610 & 0.0221 & 0.0072611 & 0.2614001 \\
\hline 611 & 0.022 & 0.00724195 & 0.26071045 \\
\hline 612 & 0.022 & 0.00722285 & 0.26002285 \\
\hline 613 & 0.022 & 0.0072038 & 0.2593368 \\
\hline 614 & 0.0219 & 0.0071848 & 0.2586528 \\
\hline 615 & 0.0219 & 0.00716585 & 0.25797035 \\
\hline 616 & 0.0219 & 0.00714695 & 0.25728995 \\
\hline 617 & 0.0218 & 0.0071281 & 0.2566111 \\
\hline 618 & 0.0218 & 0.0071093 & 0.2559343 \\
\hline 619 & 0.0217 & 0.00709055 & 0.25525905 \\
\hline 620 & 0.0217 & 0.0070718 & 0.2545853 \\
\hline 621 & 0.0217 & 0.00705315 & 0.25391365 \\
\hline 622 & 0.0216 & 0.00703455 & 0.25324355 \\
\hline 623 & 0.0216 & 0.007016 & 0.2525755 \\
\hline 624 & 0.0216 & 0.00699745 & 0.25190845 \\
\hline 625 & 0.0215 & 0.006979 & 0.2512435 \\
\hline 626 & 0.0215 & 0.00696055 & 0.25058055 \\
\hline 627 & 0.0215 & 0.0069422 & 0.2499192 \\
\hline 628 & 0.0214 & 0.00692385 & 0.24925935 \\
\hline 629 & 0.0214 & 0.0069056 & 0.2486011 \\
\hline 630 & 0.0213 & 0.00688735 & 0.24794485 \\
\hline 631 & 0.0213 & 0.00686915 & 0.24729015 \\
\hline 632 & 0.0213 & 0.00685105 & 0.24663705 \\
\hline 633 & 0.0212 & 0.00683295 & 0.24598595 \\
\hline 634 & 0.0212 & 0.0068149 & 0.2453359 \\
\hline 635 & 0.0212 & 0.0067969 & 0.2446879 \\
\hline 636 & 0.0211 & 0.00677895 & 0.24404195 \\
\hline 637 & 0.0211 & 0.00676105 & 0.24339705 \\
\hline 638 & 0.0211 & 0.00674315 & 0.24275415 \\
\hline 639 & 0.021 & 0.00672535 & 0.24211285 \\
\hline 640 & 0.021 & 0.0067076 & 0.2414731 \\
\hline 641 & 0.021 & 0.00668985 & 0.24083485 \\
\hline 642 & 0.0209 & 0.0066722 & 0.2401982 \\
\hline 643 & 0.0209 & 0.00665455 & 0.23956355 \\
\hline 644 & 0.0209 & 0.00663695 & 0.23893045 \\
\hline 645 & 0.0208 & 0.0066194 & 0.2382989 \\
\hline 646 & 0.0208 & 0.0066019 & 0.2376689 \\
\hline 647 & 0.0208 & 0.00658445 & 0.23704045 \\
\hline 648 & 0.0207 & 0.00656705 & 0.23641355 \\
\hline 649 & 0.0207 & 0.00654965 & 0.23578815 \\
\hline 650 & 0.0207 & 0.00653235 & 0.23516485 \\
\hline 651 & 0.0206 & 0.00651505 & 0.23454255 \\
\hline 652 & 0.0206 & 0.00649785 & 0.23392235 \\
\hline 653 & 0.0205 & 0.00648065 & 0.23330365 \\
\hline 654 & 0.0205 & 0.0064635 & 0.232686 \\
\hline 655 & 0.0205 & 0.0064464 & 0.2320704 \\
\hline 656 & 0.0204 & 0.00642935 & 0.23145635 \\
\hline 657 & 0.0204 & 0.00641235 & 0.23084385 \\
\hline 658 & 0.0204 & 0.00639535 & 0.23023285 \\
\hline 659 & 0.0203 & 0.0063784 & 0.2296234 \\
\hline 660 & 0.0203 & 0.00636155 & 0.22901555 \\
\hline 661 & 0.0203 & 0.0063447 & 0.2284092 \\
\hline
\end{tabular}




\begin{tabular}{|c|c|c|c|}
\hline 662 & 0.0202 & 0.0063279 & 0.2278044 \\
\hline 663 & 0.0202 & 0.00631115 & 0.22720115 \\
\hline 664 & 0.0202 & 0.0062944 & 0.2265989 \\
\hline 665 & 0.0201 & 0.00627775 & 0.22599875 \\
\hline 666 & 0.0201 & 0.0062611 & 0.2254001 \\
\hline 667 & 0.0201 & 0.00624455 & 0.22480305 \\
\hline 668 & 0.02 & 0.006228 & 0.2242075 \\
\hline 669 & 0.02 & 0.0062115 & 0.223613 \\
\hline 670 & 0.02 & 0.006195 & 0.2230205 \\
\hline 671 & 0.0199 & 0.0061786 & 0.2224296 \\
\hline 672 & 0.0199 & 0.0061622 & 0.2218397 \\
\hline 673 & 0.0199 & 0.0061459 & 0.2212514 \\
\hline 674 & 0.0199 & 0.0061296 & 0.2206651 \\
\hline 675 & 0.0198 & 0.0061133 & 0.2200798 \\
\hline 676 & 0.0198 & 0.0060971 & 0.2194961 \\
\hline 677 & 0.0198 & 0.00608095 & 0.21891395 \\
\hline 678 & 0.0197 & 0.0060648 & 0.2183328 \\
\hline 679 & 0.0197 & 0.0060487 & 0.2177537 \\
\hline 680 & 0.0197 & 0.00603265 & 0.21717565 \\
\hline 681 & 0.0196 & 0.00601665 & 0.21659965 \\
\hline 682 & 0.0196 & 0.0060007 & 0.2160247 \\
\hline 683 & 0.0196 & 0.00598475 & 0.21545125 \\
\hline 684 & 0.0195 & 0.00596885 & 0.21487885 \\
\hline 685 & 0.0195 & 0.005953 & 0.2143085 \\
\hline 686 & 0.0195 & 0.0059372 & 0.2137392 \\
\hline 687 & 0.0194 & 0.00592145 & 0.21317145 \\
\hline 688 & 0.0194 & 0.0059057 & 0.2126052 \\
\hline 689 & 0.0194 & 0.00589 & 0.2120405 \\
\hline 690 & 0.0193 & 0.00587435 & 0.21147735 \\
\hline 691 & 0.0193 & 0.00585875 & 0.21091525 \\
\hline 692 & 0.0193 & 0.0058432 & 0.2103547 \\
\hline 693 & 0.0192 & 0.00582765 & 0.20979565 \\
\hline 694 & 0.0192 & 0.00581215 & 0.20923765 \\
\hline 695 & 0.0192 & 0.0057967 & 0.2086812 \\
\hline 696 & 0.0192 & 0.0057813 & 0.2081263 \\
\hline 697 & 0.0191 & 0.0057659 & 0.2075729 \\
\hline 698 & 0.0191 & 0.0057506 & 0.2070206 \\
\hline 699 & 0.0191 & 0.0057353 & 0.2064703 \\
\hline 700 & 0.019 & 0.00572 & 0.2059205 \\
\hline 701 & 0.019 & 0.0057048 & 0.2053728 \\
\hline 702 & 0.019 & 0.0056896 & 0.2048261 \\
\hline 703 & 0.0189 & 0.00567445 & 0.20428095 \\
\hline 704 & 0.0189 & 0.00565935 & 0.20373685 \\
\hline 705 & 0.0189 & 0.0056443 & 0.2031948 \\
\hline 706 & 0.0188 & 0.00562925 & 0.20265325 \\
\hline 707 & 0.0188 & 0.00561425 & 0.20211375 \\
\hline 708 & 0.0188 & 0.0055993 & 0.2015753 \\
\hline 709 & 0.0187 & 0.0055844 & 0.2010384 \\
\hline 710 & 0.0187 & 0.0055695 & 0.2005025 \\
\hline 711 & 0.0187 & 0.0055547 & 0.1999682 \\
\hline 712 & 0.0187 & 0.00553985 & 0.19943535 \\
\hline 713 & 0.0186 & 0.0055251 & 0.1989036 \\
\hline 714 & 0.0186 & 0.00551035 & 0.19837335 \\
\hline 715 & 0.0186 & 0.0054957 & 0.1978447 \\
\hline 716 & 0.0185 & 0.005481 & 0.197317 \\
\hline 717 & 0.0185 & 0.0054664 & 0.1967904 \\
\hline 718 & 0.0185 & 0.0054518 & 0.1962653 \\
\hline 719 & 0.0184 & 0.00543725 & 0.19574175 \\
\hline 720 & 0.0184 & 0.00542275 & 0.19521975 \\
\hline 721 & 0.0184 & 0.0054083 & 0.1946983 \\
\hline 722 & 0.0184 & 0.00539385 & 0.19417885 \\
\hline 723 & 0.0183 & 0.00537945 & 0.19366045 \\
\hline 724 & 0.0183 & 0.0053651 & 0.1931431 \\
\hline 725 & 0.0183 & 0.00535075 & 0.19262725 \\
\hline 726 & 0.0182 & 0.00533645 & 0.19211295 \\
\hline 727 & 0.0182 & 0.0053222 & 0.1915997 \\
\hline 728 & 0.0182 & 0.005308 & 0.191088 \\
\hline 729 & 0.0182 & 0.0052938 & 0.1905773 \\
\hline 730 & 0.0181 & 0.00527965 & 0.19006765 \\
\hline 731 & 0.0181 & 0.00526555 & 0.18955955 \\
\hline 732 & 0.0181 & 0.00525145 & 0.18905295 \\
\hline 733 & 0.018 & 0.00523745 & 0.18854745 \\
\hline 734 & 0.018 & 0.0052234 & 0.1880434 \\
\hline 735 & 0.018 & 0.00520945 & 0.18753995 \\
\hline 736 & 0.018 & 0.0051955 & 0.1870385 \\
\hline 737 & 0.0179 & 0.0051816 & 0.1865381 \\
\hline 738 & 0.0179 & 0.00516775 & 0.18603875 \\
\hline 739 & 0.0179 & 0.0051539 & 0.1855409 \\
\hline 740 & 0.0178 & 0.0051401 & 0.1850441 \\
\hline 741 & 0.0178 & 0.00512635 & 0.18454885 \\
\hline
\end{tabular}




\begin{tabular}{|c|c|c|c|}
\hline 742 & 0.0178 & 0.00511265 & 0.18405465 \\
\hline 743 & 0.0177 & 0.00509895 & 0.18356145 \\
\hline 744 & 0.0177 & 0.0050853 & 0.1830698 \\
\hline 745 & 0.0177 & 0.00507165 & 0.18257965 \\
\hline 746 & 0.0177 & 0.00505805 & 0.18209005 \\
\hline 747 & 0.0176 & 0.0050445 & 0.1816025 \\
\hline 748 & 0.0176 & 0.005031 & 0.1811155 \\
\hline 749 & 0.0176 & 0.0050175 & 0.18063 \\
\hline 750 & 0.0176 & 0.00500405 & 0.18014555 \\
\hline 751 & 0.0175 & 0.004990625 & 0.179662625 \\
\hline 752 & 0.0175 & 0.00497724 & 0.17918074 \\
\hline 753 & 0.0175 & 0.004963885 & 0.178699885 \\
\hline 754 & 0.0174 & 0.00495057 & 0.17822057 \\
\hline 755 & 0.0174 & 0.004937285 & 0.177742285 \\
\hline 756 & 0.0174 & 0.00492403 & 0.17726503 \\
\hline 757 & 0.0174 & 0.004910815 & 0.176789315 \\
\hline 758 & 0.0173 & 0.00489763 & 0.17631463 \\
\hline 759 & 0.0173 & 0.004884475 & 0.175840975 \\
\hline 760 & 0.0173 & 0.00487136 & 0.17536886 \\
\hline 761 & 0.0172 & 0.004858275 & 0.174897775 \\
\hline 762 & 0.0172 & 0.00484522 & 0.17442772 \\
\hline 763 & 0.0172 & 0.0048322 & 0.1739592 \\
\hline 764 & 0.0172 & 0.004819215 & 0.173491715 \\
\hline 765 & 0.0171 & 0.00480626 & 0.17302526 \\
\hline 766 & 0.0171 & 0.00479334 & 0.17256034 \\
\hline 767 & 0.0171 & 0.00478045 & 0.17209595 \\
\hline 768 & 0.0171 & 0.004767595 & 0.171633595 \\
\hline 769 & 0.017 & 0.00475477 & 0.17117177 \\
\hline 770 & 0.017 & 0.00474198 & 0.17071098 \\
\hline 771 & 0.017 & 0.00472922 & 0.17025172 \\
\hline 772 & 0.0169 & 0.00471649 & 0.16979349 \\
\hline 773 & 0.0169 & 0.004703795 & 0.169336795 \\
\hline 774 & 0.0169 & 0.004691135 & 0.168880635 \\
\hline 775 & 0.0169 & 0.0046785 & 0.168426 \\
\hline 776 & 0.0168 & 0.0046659 & 0.1679724 \\
\hline 777 & 0.0168 & 0.004653335 & 0.167519835 \\
\hline 778 & 0.0168 & 0.0046408 & 0.1670688 \\
\hline 779 & 0.0168 & 0.004628295 & 0.166618795 \\
\hline 780 & 0.0167 & 0.00461582 & 0.16616982 \\
\hline 781 & 0.0167 & 0.00460338 & 0.16572188 \\
\hline 782 & 0.0167 & 0.00459097 & 0.16527497 \\
\hline 783 & 0.0166 & 0.004578595 & 0.164829595 \\
\hline 784 & 0.0166 & 0.004566245 & 0.164384745 \\
\hline 785 & 0.0166 & 0.00455393 & 0.16394143 \\
\hline 786 & 0.0166 & 0.004541645 & 0.163499145 \\
\hline 787 & 0.0165 & 0.00452939 & 0.16305789 \\
\hline 788 & 0.0165 & 0.00451717 & 0.16261817 \\
\hline 789 & 0.0165 & 0.004504975 & 0.162178975 \\
\hline 790 & 0.0165 & 0.004492815 & 0.161741315 \\
\hline 791 & 0.0164 & 0.004480685 & 0.161304685 \\
\hline 792 & 0.0164 & 0.004468585 & 0.160869085 \\
\hline 793 & 0.0164 & 0.004456515 & 0.160434515 \\
\hline 794 & 0.0164 & 0.00444448 & 0.16000098 \\
\hline 795 & 0.0163 & 0.00443247 & 0.15956897 \\
\hline 796 & 0.0163 & 0.004420495 & 0.159137495 \\
\hline 797 & 0.0163 & 0.004408545 & 0.158707545 \\
\hline 798 & 0.0163 & 0.00439663 & 0.15827863 \\
\hline 799 & 0.0162 & 0.00438474 & 0.15785074 \\
\hline 800 & 0.0162 & 0.004372885 & 0.157423885 \\
\hline 801 & 0.0162 & 0.00436106 & 0.15699806 \\
\hline 802 & 0.0162 & 0.00434926 & 0.15657326 \\
\hline 803 & 0.0161 & 0.004337495 & 0.156149995 \\
\hline 804 & 0.0161 & 0.004325755 & 0.155727255 \\
\hline 805 & 0.0161 & 0.00431405 & 0.15530555 \\
\hline 806 & 0.0161 & 0.00430237 & 0.15488537 \\
\hline 807 & 0.016 & 0.004290725 & 0.154466225 \\
\hline 808 & 0.016 & 0.004279105 & 0.154047605 \\
\hline 809 & 0.016 & 0.004267515 & 0.153630515 \\
\hline 810 & 0.016 & 0.004255955 & 0.153214455 \\
\hline 811 & 0.0159 & 0.004244425 & 0.152799425 \\
\hline 812 & 0.0159 & 0.004232925 & 0.152385425 \\
\hline 813 & 0.0159 & 0.004221455 & 0.151972455 \\
\hline 814 & 0.0158 & 0.00421001 & 0.15156051 \\
\hline 815 & 0.0158 & 0.0041986 & 0.1511496 \\
\hline 816 & 0.0158 & 0.004187215 & 0.150739715 \\
\hline 817 & 0.0158 & 0.004175855 & 0.150330855 \\
\hline 818 & 0.0157 & 0.00416453 & 0.14992303 \\
\hline 819 & 0.0157 & 0.00415323 & 0.14951623 \\
\hline 820 & 0.0157 & 0.004141965 & 0.149110465 \\
\hline 821 & 0.0157 & 0.00413072 & 0.14870622 \\
\hline
\end{tabular}




\begin{tabular}{|c|c|c|c|}
\hline 822 & 0.0156 & 0.00411951 & 0.14830251 \\
\hline 823 & 0.0156 & 0.004108325 & 0.147899825 \\
\hline 824 & 0.0156 & 0.00409717 & 0.14749817 \\
\hline 825 & 0.0156 & 0.004086045 & 0.147097545 \\
\hline 826 & 0.0156 & 0.004074945 & 0.146697945 \\
\hline 827 & 0.0155 & 0.004063875 & 0.146299375 \\
\hline 828 & 0.0155 & 0.004052835 & 0.145901835 \\
\hline 829 & 0.0155 & 0.00404182 & 0.14550532 \\
\hline 830 & 0.0155 & 0.004030835 & 0.145109835 \\
\hline 831 & 0.0154 & 0.00401988 & 0.14471538 \\
\hline 832 & 0.0154 & 0.00400895 & 0.14432195 \\
\hline 833 & 0.0154 & 0.003998045 & 0.143929545 \\
\hline 834 & 0.0154 & 0.00398717 & 0.14353817 \\
\hline 835 & 0.0153 & 0.003976325 & 0.143147825 \\
\hline 836 & 0.0153 & 0.003965505 & 0.142758005 \\
\hline 837 & 0.0153 & 0.003954715 & 0.142369715 \\
\hline 838 & 0.0153 & 0.003943955 & 0.141982455 \\
\hline 839 & 0.0152 & 0.003933215 & 0.141595715 \\
\hline 840 & 0.0152 & 0.00392251 & 0.14121051 \\
\hline 841 & 0.0152 & 0.003911825 & 0.140825825 \\
\hline 842 & 0.0152 & 0.003901175 & 0.140442175 \\
\hline 843 & 0.0151 & 0.003890545 & 0.140059545 \\
\hline 844 & 0.0151 & 0.003879945 & 0.139677945 \\
\hline 845 & 0.0151 & 0.003869375 & 0.139297375 \\
\hline 846 & 0.0151 & 0.00385883 & 0.13891783 \\
\hline 847 & 0.015 & 0.00384831 & 0.13853931 \\
\hline 848 & 0.015 & 0.00383782 & 0.13816132 \\
\hline 849 & 0.015 & 0.003827355 & 0.137784855 \\
\hline 850 & 0.015 & 0.003816915 & 0.137408915 \\
\hline 851 & 0.0149 & 0.003806505 & 0.137034005 \\
\hline 852 & 0.0149 & 0.00379612 & 0.13666062 \\
\hline 853 & 0.0149 & 0.003785765 & 0.136287265 \\
\hline 854 & 0.0149 & 0.003775435 & 0.135915435 \\
\hline 855 & 0.0148 & 0.00376513 & 0.13554463 \\
\hline 856 & 0.0148 & 0.00375485 & 0.13517435 \\
\hline 857 & 0.0148 & 0.0037446 & 0.1348056 \\
\hline 858 & 0.0148 & 0.00373437 & 0.13443737 \\
\hline 859 & 0.0148 & 0.003724175 & 0.134070175 \\
\hline 860 & 0.0147 & 0.003714 & 0.133704 \\
\hline 861 & 0.0147 & 0.003703855 & 0.133338855 \\
\hline 862 & 0.0147 & 0.00369373 & 0.13297423 \\
\hline 863 & 0.0147 & 0.003683635 & 0.132611135 \\
\hline 864 & 0.0146 & 0.00367357 & 0.13224857 \\
\hline 865 & 0.0146 & 0.003663525 & 0.131887025 \\
\hline 866 & 0.0146 & 0.00365351 & 0.13152651 \\
\hline 867 & 0.0146 & 0.003643515 & 0.131166515 \\
\hline 868 & 0.0145 & 0.00363355 & 0.13080805 \\
\hline 869 & 0.0145 & 0.00362361 & 0.13045011 \\
\hline 870 & 0.0145 & 0.003613695 & 0.130093195 \\
\hline 871 & 0.0145 & 0.003603805 & 0.129736805 \\
\hline 872 & 0.0145 & 0.003593945 & 0.129381945 \\
\hline 873 & 0.0144 & 0.003584105 & 0.129027605 \\
\hline 874 & 0.0144 & 0.003574295 & 0.128674295 \\
\hline 875 & 0.0144 & 0.003564505 & 0.128322005 \\
\hline 876 & 0.0144 & 0.003554745 & 0.127970745 \\
\hline 877 & 0.0143 & 0.003545005 & 0.127620005 \\
\hline 878 & 0.0143 & 0.003535295 & 0.127270795 \\
\hline 879 & 0.0143 & 0.00352561 & 0.12692211 \\
\hline 880 & 0.0143 & 0.003515945 & 0.126573945 \\
\hline 881 & 0.0142 & 0.00350631 & 0.12622731 \\
\hline 882 & 0.0142 & 0.003496695 & 0.125881195 \\
\hline 883 & 0.0142 & 0.00348711 & 0.12553611 \\
\hline 884 & 0.0142 & 0.00347755 & 0.12519155 \\
\hline 885 & 0.0142 & 0.00346801 & 0.12484851 \\
\hline 886 & 0.0141 & 0.003458495 & 0.124505995 \\
\hline 887 & 0.0141 & 0.00344901 & 0.12416451 \\
\hline 888 & 0.0141 & 0.003439545 & 0.123823545 \\
\hline 889 & 0.0141 & 0.003430105 & 0.123483605 \\
\hline 890 & 0.014 & 0.00342069 & 0.12314469 \\
\hline 891 & 0.014 & 0.0034113 & 0.1228068 \\
\hline 892 & 0.014 & 0.003401935 & 0.122469435 \\
\hline 893 & 0.014 & 0.003392595 & 0.122133595 \\
\hline 894 & 0.014 & 0.003383275 & 0.121797775 \\
\hline 895 & 0.0139 & 0.00337398 & 0.12146348 \\
\hline 896 & 0.0139 & 0.003364715 & 0.121129715 \\
\hline 897 & 0.0139 & 0.00335547 & 0.12079697 \\
\hline 898 & 0.0139 & 0.003346245 & 0.120464745 \\
\hline 899 & 0.0138 & 0.00333705 & 0.12013355 \\
\hline 900 & 0.0138 & 0.003327875 & 0.119803375 \\
\hline 901 & 0.0138 & 0.003318725 & 0.119474225 \\
\hline
\end{tabular}




\begin{tabular}{|c|c|c|c|}
\hline 902 & 0.0138 & 0.0033096 & 0.1191456 \\
\hline 903 & 0.0138 & 0.0033005 & 0.118818 \\
\hline 904 & 0.0137 & 0.00329142 & 0.11849092 \\
\hline 905 & 0.0137 & 0.003282365 & 0.118165365 \\
\hline 906 & 0.0137 & 0.003273335 & 0.117839835 \\
\hline 907 & 0.0137 & 0.003264325 & 0.117515825 \\
\hline 908 & 0.0136 & 0.003255345 & 0.117192345 \\
\hline 909 & 0.0136 & 0.00324638 & 0.11686988 \\
\hline 910 & 0.0136 & 0.003237445 & 0.116547945 \\
\hline 911 & 0.0136 & 0.00322853 & 0.11622703 \\
\hline 912 & 0.0136 & 0.00321964 & 0.11590714 \\
\hline 913 & 0.0135 & 0.00321077 & 0.11558777 \\
\hline 914 & 0.0135 & 0.00320193 & 0.11526943 \\
\hline 915 & 0.0135 & 0.003193105 & 0.114951605 \\
\hline 916 & 0.0135 & 0.00318431 & 0.11463531 \\
\hline 917 & 0.0135 & 0.00317553 & 0.11431903 \\
\hline 918 & 0.0134 & 0.00316678 & 0.11400428 \\
\hline 919 & 0.0134 & 0.00315805 & 0.11368955 \\
\hline 920 & 0.0134 & 0.003149345 & 0.113376345 \\
\hline 921 & 0.0134 & 0.00314066 & 0.11306366 \\
\hline 922 & 0.0133 & 0.003131995 & 0.112751995 \\
\hline 923 & 0.0133 & 0.00312336 & 0.11244086 \\
\hline 924 & 0.0133 & 0.003114745 & 0.112130745 \\
\hline 925 & 0.0133 & 0.00310615 & 0.11182115 \\
\hline 926 & 0.0133 & 0.00309758 & 0.11151258 \\
\hline 927 & 0.0132 & 0.00308903 & 0.11120503 \\
\hline 928 & 0.0132 & 0.003080505 & 0.110898005 \\
\hline 929 & 0.0132 & 0.003072 & 0.110592 \\
\hline 930 & 0.0132 & 0.00306352 & 0.11028652 \\
\hline 931 & 0.0132 & 0.00305506 & 0.10998206 \\
\hline 932 & 0.0131 & 0.00304662 & 0.10967812 \\
\hline 933 & 0.0131 & 0.003038205 & 0.109375205 \\
\hline 934 & 0.0131 & 0.003029815 & 0.109073315 \\
\hline 935 & 0.0131 & 0.003021445 & 0.108771945 \\
\hline 936 & 0.0131 & 0.003013095 & 0.108471595 \\
\hline 937 & 0.013 & 0.003004765 & 0.108171765 \\
\hline 938 & 0.013 & 0.00299646 & 0.10787246 \\
\hline 939 & 0.013 & 0.00298818 & 0.10757418 \\
\hline 940 & 0.013 & 0.002979915 & 0.107276915 \\
\hline 941 & 0.0129 & 0.002971675 & 0.106980175 \\
\hline 942 & 0.0129 & 0.00296346 & 0.10668446 \\
\hline 943 & 0.0129 & 0.002955265 & 0.106389265 \\
\hline 944 & 0.0129 & 0.00294709 & 0.10609509 \\
\hline 945 & 0.0129 & 0.002938935 & 0.105801435 \\
\hline 946 & 0.0128 & 0.002930805 & 0.105508805 \\
\hline 947 & 0.0128 & 0.002922695 & 0.105217195 \\
\hline 948 & 0.0128 & 0.002914605 & 0.104925605 \\
\hline 949 & 0.0128 & 0.002906535 & 0.104635535 \\
\hline 950 & 0.0128 & 0.00289849 & 0.10434549 \\
\hline 951 & 0.0127 & 0.002890465 & 0.104056965 \\
\hline 952 & 0.0127 & 0.00288246 & 0.10376846 \\
\hline 953 & 0.0127 & 0.00287448 & 0.10348148 \\
\hline 954 & 0.0127 & 0.002866515 & 0.103194515 \\
\hline 955 & 0.0127 & 0.002858575 & 0.102908575 \\
\hline 956 & 0.0126 & 0.002850655 & 0.102623655 \\
\hline 957 & 0.0126 & 0.00284276 & 0.10233926 \\
\hline 958 & 0.0126 & 0.00283488 & 0.10205588 \\
\hline 959 & 0.0126 & 0.002827025 & 0.101773025 \\
\hline 960 & 0.0126 & 0.00281919 & 0.10149069 \\
\hline 961 & 0.0125 & 0.00281137 & 0.10120937 \\
\hline 962 & 0.0125 & 0.00280358 & 0.10092858 \\
\hline 963 & 0.0125 & 0.002795805 & 0.100648805 \\
\hline 964 & 0.0125 & 0.00278805 & 0.10037005 \\
\hline 965 & 0.0125 & 0.002780315 & 0.100091315 \\
\hline 966 & 0.0124 & 0.002772605 & 0.099813605 \\
\hline 967 & 0.0124 & 0.002764915 & 0.099536915 \\
\hline 968 & 0.0124 & 0.00275724 & 0.09926074 \\
\hline 969 & 0.0124 & 0.00274959 & 0.09898509 \\
\hline 970 & 0.0124 & 0.00274196 & 0.09871046 \\
\hline 971 & 0.0123 & 0.00273435 & 0.09843635 \\
\hline 972 & 0.0123 & 0.00272676 & 0.09816326 \\
\hline 973 & 0.0123 & 0.00271919 & 0.09789069 \\
\hline 974 & 0.0123 & 0.00271164 & 0.09761914 \\
\hline 975 & 0.0123 & 0.00270411 & 0.09734811 \\
\hline 976 & 0.0122 & 0.0026966 & 0.0970776 \\
\hline 977 & 0.0122 & 0.00268911 & 0.09680811 \\
\hline 978 & 0.0122 & 0.00268164 & 0.09653914 \\
\hline 979 & 0.0122 & 0.00267419 & 0.09627069 \\
\hline 980 & 0.0122 & 0.00266676 & 0.09600326 \\
\hline 981 & 0.0121 & 0.00265935 & 0.09573635 \\
\hline
\end{tabular}




\begin{tabular}{|c|c|c|c|}
\hline 982 & 0.0121 & 0.00265196 & 0.09547046 \\
\hline 983 & 0.0121 & 0.00264459 & 0.09520509 \\
\hline 984 & 0.0121 & 0.002637235 & 0.094940735 \\
\hline 985 & 0.0121 & 0.002629905 & 0.094676405 \\
\hline 986 & 0.012 & 0.002622595 & 0.094413095 \\
\hline 987 & 0.012 & 0.0026153 & 0.0941508 \\
\hline 988 & 0.012 & 0.002608025 & 0.093889025 \\
\hline 989 & 0.012 & 0.002600775 & 0.093627775 \\
\hline 990 & 0.012 & 0.00259354 & 0.09336754 \\
\hline 991 & 0.0119 & 0.002586325 & 0.093107825 \\
\hline 992 & 0.0119 & 0.00257913 & 0.09284863 \\
\hline 993 & 0.0119 & 0.002571955 & 0.092590455 \\
\hline 994 & 0.0119 & 0.002564795 & 0.092332795 \\
\hline 995 & 0.0119 & 0.002557655 & 0.092075655 \\
\hline 996 & 0.0118 & 0.00255054 & 0.09181954 \\
\hline 997 & 0.0118 & 0.00254344 & 0.09156394 \\
\hline 998 & 0.0118 & 0.00253636 & 0.09130886 \\
\hline 999 & 0.0118 & 0.002529295 & 0.091054795 \\
\hline 1000 & 0.0118 & 0.002522255 & 0.090801255 \\
\hline 1001 & 0.0118 & 0.00251523 & 0.09054823 \\
\hline 1002 & 0.0117 & 0.002508225 & 0.090296225 \\
\hline 1003 & 0.0117 & 0.00250124 & 0.09004474 \\
\hline 1004 & 0.0117 & 0.00249427 & 0.08979377 \\
\hline 1005 & 0.0117 & 0.00248732 & 0.08954382 \\
\hline 1006 & 0.0117 & 0.00248039 & 0.08929389 \\
\hline 1007 & 0.0116 & 0.00247348 & 0.08904548 \\
\hline 1008 & 0.0116 & 0.002466585 & 0.088797085 \\
\hline 1009 & 0.0116 & 0.00245971 & 0.08854971 \\
\hline 1010 & 0.0116 & 0.002452855 & 0.088302855 \\
\hline 1011 & 0.0116 & 0.00244602 & 0.08805652 \\
\hline 1012 & 0.0115 & 0.0024392 & 0.0878112 \\
\hline 1013 & 0.0115 & 0.0024324 & 0.0875664 \\
\hline 1014 & 0.0115 & 0.002425615 & 0.087322115 \\
\hline 1015 & 0.0115 & 0.00241885 & 0.08707885 \\
\hline 1016 & 0.0115 & 0.002412105 & 0.086835605 \\
\hline 1017 & 0.0114 & 0.00240538 & 0.08659388 \\
\hline 1018 & 0.0114 & 0.00239867 & 0.08635217 \\
\hline 1019 & 0.0114 & 0.002391975 & 0.086110975 \\
\hline 1020 & 0.0114 & 0.002385305 & 0.085870805 \\
\hline 1021 & 0.0114 & 0.00237865 & 0.08563115 \\
\hline 1022 & 0.0114 & 0.00237201 & 0.08539251 \\
\hline 1023 & 0.0113 & 0.00236539 & 0.08515389 \\
\hline 1024 & 0.0113 & 0.00235879 & 0.08491629 \\
\hline 1025 & 0.0113 & 0.002352205 & 0.084679205 \\
\hline 1026 & 0.0113 & 0.00234564 & 0.08444314 \\
\hline 1027 & 0.0113 & 0.00233909 & 0.08420709 \\
\hline 1028 & 0.0112 & 0.00233256 & 0.08397206 \\
\hline 1029 & 0.0112 & 0.00232605 & 0.08373755 \\
\hline 1030 & 0.0112 & 0.002319555 & 0.083504055 \\
\hline 1031 & 0.0112 & 0.002313075 & 0.083270575 \\
\hline 1032 & 0.0112 & 0.002306615 & 0.083038115 \\
\hline 1033 & 0.0112 & 0.00230017 & 0.08280617 \\
\hline 1034 & 0.0111 & 0.002293745 & 0.082574745 \\
\hline 1035 & 0.0111 & 0.00228734 & 0.08234434 \\
\hline 1036 & 0.0111 & 0.00228095 & 0.08211395 \\
\hline 1037 & 0.0111 & 0.002274575 & 0.081884575 \\
\hline 1038 & 0.0111 & 0.00226822 & 0.08165572 \\
\hline 1039 & 0.011 & 0.00226188 & 0.08142788 \\
\hline 1040 & 0.011 & 0.00225556 & 0.08120006 \\
\hline 1041 & 0.011 & 0.002249255 & 0.080973255 \\
\hline 1042 & 0.011 & 0.00224297 & 0.08074697 \\
\hline 1043 & 0.011 & 0.0022367 & 0.0805212 \\
\hline 1044 & 0.011 & 0.002230445 & 0.080295945 \\
\hline 1045 & 0.0109 & 0.00222421 & 0.08007171 \\
\hline 1046 & 0.0109 & 0.00221799 & 0.07984749 \\
\hline 1047 & 0.0109 & 0.00221179 & 0.07962429 \\
\hline 1048 & 0.0109 & 0.002205605 & 0.079401605 \\
\hline 1049 & 0.0109 & 0.002199435 & 0.079179435 \\
\hline 1050 & 0.0108 & 0.002193285 & 0.078958285 \\
\hline 1051 & 0.0108 & 0.00218715 & 0.07873715 \\
\hline 1052 & 0.0108 & 0.00218103 & 0.07851703 \\
\hline 1053 & 0.0108 & 0.00217493 & 0.07829743 \\
\hline 1054 & 0.0108 & 0.002168845 & 0.078078345 \\
\hline 1055 & 0.0108 & 0.002162775 & 0.077859775 \\
\hline 1056 & 0.0107 & 0.002156725 & 0.077642225 \\
\hline 1057 & 0.0107 & 0.00215069 & 0.07742469 \\
\hline 1058 & 0.0107 & 0.00214467 & 0.07720817 \\
\hline 1059 & 0.0107 & 0.00213867 & 0.07699217 \\
\hline 1060 & 0.0107 & 0.00213268 & 0.07677668 \\
\hline 1061 & 0.0107 & 0.002126715 & 0.076561715 \\
\hline
\end{tabular}




\begin{tabular}{|c|c|c|c|}
\hline 1062 & 0.0106 & 0.00212076 & 0.07634726 \\
\hline 1063 & 0.0106 & 0.002114825 & 0.076133825 \\
\hline 1064 & 0.0106 & 0.0021089 & 0.0759204 \\
\hline 1065 & 0.0106 & 0.002103 & 0.075708 \\
\hline 1066 & 0.0106 & 0.00209711 & 0.07549611 \\
\hline 1067 & 0.0105 & 0.002091235 & 0.075284735 \\
\hline 1068 & 0.0105 & 0.00208538 & 0.07507388 \\
\hline 1069 & 0.0105 & 0.00207954 & 0.07486354 \\
\hline 1070 & 0.0105 & 0.002073715 & 0.074653715 \\
\hline 1071 & 0.0105 & 0.00206791 & 0.07444491 \\
\hline 1072 & 0.0105 & 0.002062115 & 0.074236115 \\
\hline 1073 & 0.0104 & 0.00205634 & 0.07402834 \\
\hline 1074 & 0.0104 & 0.00205058 & 0.07382108 \\
\hline 1075 & 0.0104 & 0.002044835 & 0.073613835 \\
\hline 1076 & 0.0104 & 0.002039105 & 0.073407605 \\
\hline 1077 & 0.0104 & 0.00203339 & 0.07320189 \\
\hline 1078 & 0.0104 & 0.002027695 & 0.072997195 \\
\hline 1079 & 0.0103 & 0.00202201 & 0.07279251 \\
\hline 1080 & 0.0103 & 0.002016345 & 0.072588345 \\
\hline 1081 & 0.0103 & 0.002010695 & 0.072385195 \\
\hline 1082 & 0.0103 & 0.00200506 & 0.07218206 \\
\hline 1083 & 0.0103 & 0.00199944 & 0.07197994 \\
\hline 1084 & 0.0103 & 0.001993835 & 0.071778335 \\
\hline 1085 & 0.0102 & 0.001988245 & 0.071576745 \\
\hline 1086 & 0.0102 & 0.001982675 & 0.071376175 \\
\hline 1087 & 0.0102 & 0.001977115 & 0.071176115 \\
\hline 1088 & 0.0102 & 0.00197157 & 0.07097657 \\
\hline 1089 & 0.0102 & 0.001966045 & 0.070777545 \\
\hline 1090 & 0.0102 & 0.00196053 & 0.07057903 \\
\hline 1091 & 0.0101 & 0.001955035 & 0.070381035 \\
\hline 1092 & 0.0101 & 0.001949555 & 0.070184055 \\
\hline 1093 & 0.0101 & 0.001944085 & 0.069987085 \\
\hline 1094 & 0.0101 & 0.001938635 & 0.069790635 \\
\hline 1095 & 0.0101 & 0.001933195 & 0.069595195 \\
\hline 1096 & 0.0101 & 0.001927775 & 0.069399775 \\
\hline 1097 & 0.01 & 0.00192237 & 0.06920537 \\
\hline 1098 & 0.01 & 0.001916975 & 0.069010975 \\
\hline 1099 & 0.01 & 0.0019116 & 0.0688176 \\
\hline 1100 & 0.00998 & 0.001906235 & 0.068624735 \\
\hline 1101 & 0.00996 & 0.00190089 & 0.06843189 \\
\hline 1102 & 0.00995 & 0.001895555 & 0.068240055 \\
\hline 1103 & 0.00993 & 0.00189024 & 0.06804874 \\
\hline 1104 & 0.00992 & 0.001884935 & 0.067857935 \\
\hline 1105 & 0.0099 & 0.001879645 & 0.067667145 \\
\hline 1106 & 0.00988 & 0.001874375 & 0.067477375 \\
\hline 1107 & 0.00986 & 0.001869115 & 0.067288115 \\
\hline 1108 & 0.00985 & 0.00186387 & 0.06709937 \\
\hline 1109 & 0.00983 & 0.00185864 & 0.06691114 \\
\hline 1110 & 0.00982 & 0.001853425 & 0.066723425 \\
\hline 1111 & 0.0098 & 0.00184822 & 0.06653622 \\
\hline 1112 & 0.00978 & 0.001843035 & 0.066349035 \\
\hline 1113 & 0.00977 & 0.00183786 & 0.06616286 \\
\hline 1114 & 0.00975 & 0.001832705 & 0.065977205 \\
\hline 1115 & 0.00973 & 0.00182756 & 0.06579206 \\
\hline 1116 & 0.00972 & 0.00182243 & 0.06560743 \\
\hline 1117 & 0.0097 & 0.001817315 & 0.065423315 \\
\hline 1118 & 0.00969 & 0.001812215 & 0.065239715 \\
\hline 1119 & 0.00967 & 0.001807125 & 0.065056625 \\
\hline 1120 & 0.00965 & 0.001802055 & 0.064874055 \\
\hline 1121 & 0.00963 & 0.001796995 & 0.064691995 \\
\hline 1122 & 0.00962 & 0.00179195 & 0.06450995 \\
\hline 1123 & 0.0096 & 0.00178692 & 0.06432892 \\
\hline 1124 & 0.00959 & 0.0017819 & 0.0641484 \\
\hline 1125 & 0.00957 & 0.0017769 & 0.0639684 \\
\hline 1126 & 0.00956 & 0.00177191 & 0.06378891 \\
\hline 1127 & 0.00954 & 0.001766935 & 0.063609435 \\
\hline 1128 & 0.00952 & 0.001761975 & 0.063430975 \\
\hline 1129 & 0.0095 & 0.001757025 & 0.063253025 \\
\hline 1130 & 0.00949 & 0.001752095 & 0.063075595 \\
\hline 1131 & 0.00947 & 0.001747175 & 0.062898175 \\
\hline 1132 & 0.00946 & 0.001742265 & 0.062721765 \\
\hline 1133 & 0.00944 & 0.001737375 & 0.062545375 \\
\hline 1134 & 0.00943 & 0.001732495 & 0.062369995 \\
\hline 1135 & 0.00941 & 0.00172763 & 0.06219463 \\
\hline 1136 & 0.00939 & 0.00172278 & 0.06201978 \\
\hline 1137 & 0.00938 & 0.00171794 & 0.06184594 \\
\hline 1138 & 0.00936 & 0.001713115 & 0.061672115 \\
\hline 1139 & 0.00934 & 0.001708305 & 0.061498805 \\
\hline 1140 & 0.00933 & 0.001703505 & 0.061326005 \\
\hline 1141 & 0.00931 & 0.00169872 & 0.06115372 \\
\hline
\end{tabular}




\begin{tabular}{|c|c|c|c|}
\hline 1142 & 0.0093 & 0.00169395 & 0.06098195 \\
\hline 1143 & 0.00928 & 0.00168919 & 0.06081069 \\
\hline 1144 & 0.00927 & 0.001684445 & 0.060639945 \\
\hline 1145 & 0.00925 & 0.001679715 & 0.060469715 \\
\hline 1146 & 0.00924 & 0.001675 & 0.0603 \\
\hline 1147 & 0.00922 & 0.001670295 & 0.060130295 \\
\hline 1148 & 0.0092 & 0.0016656 & 0.0599616 \\
\hline 1149 & 0.00919 & 0.00166092 & 0.05979342 \\
\hline 1150 & 0.00917 & 0.001656255 & 0.059625255 \\
\hline 1151 & 0.00915 & 0.001651605 & 0.059457605 \\
\hline 1152 & 0.00914 & 0.001646965 & 0.059290965 \\
\hline 1153 & 0.00912 & 0.00164234 & 0.05912434 \\
\hline 1154 & 0.00911 & 0.001637725 & 0.058958225 \\
\hline 1155 & 0.00909 & 0.001633125 & 0.058792625 \\
\hline 1156 & 0.00908 & 0.001628535 & 0.058627535 \\
\hline 1157 & 0.00906 & 0.00162396 & 0.05846246 \\
\hline 1158 & 0.00905 & 0.0016194 & 0.0582984 \\
\hline 1159 & 0.00903 & 0.00161485 & 0.05813435 \\
\hline 1160 & 0.00902 & 0.001610315 & 0.057971315 \\
\hline 1161 & 0.009 & 0.00160579 & 0.05780829 \\
\hline 1162 & 0.00899 & 0.00160128 & 0.05764578 \\
\hline 1163 & 0.00897 & 0.00159678 & 0.05748428 \\
\hline 1164 & 0.00895 & 0.001592295 & 0.057322795 \\
\hline 1165 & 0.00894 & 0.00158782 & 0.05716132 \\
\hline 1166 & 0.00892 & 0.00158336 & 0.05700086 \\
\hline 1167 & 0.00891 & 0.00157891 & 0.05684091 \\
\hline 1168 & 0.00889 & 0.001574475 & 0.056680975 \\
\hline 1169 & 0.00888 & 0.00157005 & 0.05652205 \\
\hline 1170 & 0.00886 & 0.00156564 & 0.05636314 \\
\hline 1171 & 0.00885 & 0.001561245 & 0.056204745 \\
\hline 1172 & 0.00883 & 0.001556855 & 0.056046855 \\
\hline 1173 & 0.00881 & 0.001552485 & 0.055889485 \\
\hline 1174 & 0.0088 & 0.00154812 & 0.05573262 \\
\hline 1175 & 0.00878 & 0.00154377 & 0.05557577 \\
\hline 1176 & 0.00877 & 0.001539435 & 0.055419435 \\
\hline 1177 & 0.00875 & 0.00153511 & 0.05526411 \\
\hline 1178 & 0.00874 & 0.0015308 & 0.0551088 \\
\hline 1179 & 0.00872 & 0.001526495 & 0.054953995 \\
\hline 1180 & 0.00871 & 0.00152221 & 0.05479971 \\
\hline 1181 & 0.00869 & 0.001517935 & 0.054645435 \\
\hline 1182 & 0.00868 & 0.00151367 & 0.05449217 \\
\hline 1183 & 0.00866 & 0.001509415 & 0.054338915 \\
\hline 1184 & 0.00865 & 0.001505175 & 0.054186175 \\
\hline 1185 & 0.00863 & 0.00150095 & 0.05403395 \\
\hline 1186 & 0.00862 & 0.00149673 & 0.05388223 \\
\hline 1187 & 0.0086 & 0.001492525 & 0.053731025 \\
\hline 1188 & 0.00859 & 0.001488335 & 0.053579835 \\
\hline 1189 & 0.00857 & 0.001484155 & 0.053429655 \\
\hline 1190 & 0.00856 & 0.001479985 & 0.053279485 \\
\hline 1191 & 0.00854 & 0.001475825 & 0.053129825 \\
\hline 1192 & 0.00853 & 0.00147168 & 0.05298068 \\
\hline 1193 & 0.00851 & 0.001467545 & 0.052831545 \\
\hline 1194 & 0.0085 & 0.001463425 & 0.052683425 \\
\hline 1195 & 0.00848 & 0.001459315 & 0.052535315 \\
\hline 1196 & 0.00847 & 0.001455215 & 0.052387715 \\
\hline 1197 & 0.00845 & 0.00145113 & 0.05224063 \\
\hline 1198 & 0.00844 & 0.001447055 & 0.052094055 \\
\hline 1199 & 0.00842 & 0.00144299 & 0.05194749 \\
\hline 1200 & 0.00841 & 0.001438935 & 0.051801435 \\
\hline 1201 & 0.0084 & 0.001434895 & 0.051656395 \\
\hline 1202 & 0.00838 & 0.001430865 & 0.051511365 \\
\hline 1203 & 0.00837 & 0.001426845 & 0.051366445 \\
\hline 1204 & 0.00835 & 0.00142284 & 0.05122219 \\
\hline 1205 & 0.00834 & 0.001418845 & 0.051078345 \\
\hline 1206 & 0.00832 & 0.00141486 & 0.05093491 \\
\hline 1207 & 0.00831 & 0.001410885 & 0.050791885 \\
\hline 1208 & 0.00829 & 0.001406925 & 0.050649225 \\
\hline 1209 & 0.00828 & 0.00140297 & 0.05050702 \\
\hline 1210 & 0.00827 & 0.001399035 & 0.050365185 \\
\hline 1211 & 0.00825 & 0.001395105 & 0.050223755 \\
\hline 1212 & 0.00824 & 0.001391185 & 0.050082735 \\
\hline 1213 & 0.00822 & 0.00138728 & 0.04994213 \\
\hline 1214 & 0.00821 & 0.001383385 & 0.049801885 \\
\hline 1215 & 0.00819 & 0.0013795 & 0.04966205 \\
\hline 1216 & 0.00818 & 0.00137563 & 0.04952263 \\
\hline 1217 & 0.00816 & 0.001371765 & 0.049383615 \\
\hline 1218 & 0.00815 & 0.001367915 & 0.049244965 \\
\hline 1219 & 0.00813 & 0.001364075 & 0.049106725 \\
\hline 1220 & 0.00812 & 0.001360245 & 0.048968845 \\
\hline 1221 & 0.0081 & 0.001356425 & 0.048831375 \\
\hline
\end{tabular}




\begin{tabular}{|c|c|c|c|}
\hline 1222 & 0.00809 & 0.00135262 & 0.04869432 \\
\hline 1223 & 0.00808 & 0.001348825 & 0.048557625 \\
\hline 1224 & 0.00806 & 0.001345035 & 0.048421335 \\
\hline 1225 & 0.00805 & 0.00134126 & 0.04828541 \\
\hline 1226 & 0.00803 & 0.001337495 & 0.048149895 \\
\hline 1227 & 0.00802 & 0.001333745 & 0.048014745 \\
\hline 1228 & 0.00801 & 0.00133 & 0.04788 \\
\hline 1229 & 0.00799 & 0.00132627 & 0.04774562 \\
\hline 1230 & 0.00798 & 0.001322545 & 0.047611645 \\
\hline 1231 & 0.00796 & 0.001318835 & 0.047478035 \\
\hline 1232 & 0.00795 & 0.001315135 & 0.047344785 \\
\hline 1233 & 0.00793 & 0.001311445 & 0.047211945 \\
\hline 1234 & 0.00792 & 0.001307765 & 0.047079465 \\
\hline 1235 & 0.0079 & 0.001304095 & 0.046947395 \\
\hline 1236 & 0.00789 & 0.001300435 & 0.046815685 \\
\hline 1237 & 0.00788 & 0.001296785 & 0.046684335 \\
\hline 1238 & 0.00786 & 0.00129315 & 0.04655335 \\
\hline 1239 & 0.00785 & 0.00128952 & 0.04642277 \\
\hline 1240 & 0.00784 & 0.001285905 & 0.046292505 \\
\hline 1241 & 0.00782 & 0.001282295 & 0.046162645 \\
\hline 1242 & 0.00781 & 0.0012787 & 0.0460332 \\
\hline 1243 & 0.00779 & 0.001275115 & 0.045904065 \\
\hline 1244 & 0.00778 & 0.001271535 & 0.045775335 \\
\hline 1245 & 0.00776 & 0.00126797 & 0.04564692 \\
\hline 1246 & 0.00775 & 0.001264415 & 0.045518915 \\
\hline 1247 & 0.00774 & 0.00126087 & 0.04539127 \\
\hline 1248 & 0.00773 & 0.001257335 & 0.045263985 \\
\hline 1249 & 0.00771 & 0.001253805 & 0.045137055 \\
\hline 1250 & 0.0077 & 0.00125029 & 0.04501049 \\
\hline 1251 & 0.00768 & 0.001246785 & 0.044884285 \\
\hline 1252 & 0.00767 & 0.00124329 & 0.04475844 \\
\hline 1253 & 0.00765 & 0.001239805 & 0.044632955 \\
\hline 1254 & 0.00764 & 0.00123633 & 0.04450783 \\
\hline 1255 & 0.00763 & 0.001232865 & 0.044383065 \\
\hline 1256 & 0.00761 & 0.001229405 & 0.044258655 \\
\hline 1257 & 0.0076 & 0.00122596 & 0.04413461 \\
\hline 1258 & 0.00759 & 0.001222525 & 0.044010875 \\
\hline 1259 & 0.00757 & 0.0012191 & 0.04388755 \\
\hline 1260 & 0.00756 & 0.00121568 & 0.04376453 \\
\hline 1261 & 0.00754 & 0.001212275 & 0.043641925 \\
\hline 1262 & 0.00753 & 0.00120888 & 0.04351963 \\
\hline 1263 & 0.00752 & 0.00120549 & 0.04339764 \\
\hline 1264 & 0.00751 & 0.001202115 & 0.043276065 \\
\hline 1265 & 0.00749 & 0.001198745 & 0.043154795 \\
\hline 1266 & 0.00748 & 0.001195385 & 0.043033885 \\
\hline 1267 & 0.00746 & 0.001192035 & 0.042913335 \\
\hline 1268 & 0.00745 & 0.0011887 & 0.04279315 \\
\hline 1269 & 0.00744 & 0.00118537 & 0.04267327 \\
\hline 1270 & 0.00742 & 0.00118205 & 0.04255375 \\
\hline 1271 & 0.00741 & 0.00117874 & 0.04243454 \\
\hline 1272 & 0.0074 & 0.001175435 & 0.042315735 \\
\hline 1273 & 0.00738 & 0.001172145 & 0.042197195 \\
\hline 1274 & 0.00737 & 0.00116886 & 0.04207906 \\
\hline 1275 & 0.00735 & 0.00116559 & 0.04196124 \\
\hline 1276 & 0.00734 & 0.001162325 & 0.041843725 \\
\hline 1277 & 0.00733 & 0.00115907 & 0.04172657 \\
\hline 1278 & 0.00732 & 0.001155825 & 0.041609775 \\
\hline 1279 & 0.0073 & 0.00115259 & 0.04149329 \\
\hline 1280 & 0.00729 & 0.001149365 & 0.041377115 \\
\hline 1281 & 0.00727 & 0.001146145 & 0.041261295 \\
\hline 1282 & 0.00726 & 0.00114294 & 0.04114584 \\
\hline 1283 & 0.00725 & 0.00113974 & 0.04103069 \\
\hline 1284 & 0.00724 & 0.00113655 & 0.04091585 \\
\hline 1285 & 0.00722 & 0.00113337 & 0.04080137 \\
\hline 1286 & 0.00721 & 0.0011302 & 0.0406872 \\
\hline 1287 & 0.00719 & 0.001127035 & 0.040573335 \\
\hline 1288 & 0.00718 & 0.001123885 & 0.040459835 \\
\hline 1289 & 0.00717 & 0.00112074 & 0.04034664 \\
\hline 1290 & 0.00716 & 0.001117605 & 0.040233755 \\
\hline 1291 & 0.00714 & 0.00111448 & 0.04012123 \\
\hline 1292 & 0.00713 & 0.00111136 & 0.04000901 \\
\hline 1293 & 0.00712 & 0.001108255 & 0.039897105 \\
\hline 1294 & 0.0071 & 0.001105155 & 0.039785555 \\
\hline 1295 & 0.00709 & 0.001102065 & 0.039674315 \\
\hline 1296 & 0.00708 & 0.00109898 & 0.03956338 \\
\hline 1297 & 0.00706 & 0.00109591 & 0.03945276 \\
\hline 1298 & 0.00705 & 0.001092845 & 0.039342445 \\
\hline 1299 & 0.00704 & 0.00108979 & 0.03923244 \\
\hline 1300 & 0.00703 & 0.001086745 & 0.039122795 \\
\hline 1301 & 0.00701 & 0.001083705 & 0.039013455 \\
\hline
\end{tabular}




\begin{tabular}{|c|c|c|c|}
\hline 1302 & 0.007 & 0.00108068 & 0.03890443 \\
\hline 1303 & 0.00698 & 0.00107766 & 0.03879566 \\
\hline 1304 & 0.00697 & 0.001074645 & 0.038687245 \\
\hline 1305 & 0.00696 & 0.001071645 & 0.038579195 \\
\hline 1306 & 0.00695 & 0.00106865 & 0.0384714 \\
\hline 1307 & 0.00693 & 0.001065665 & 0.038363915 \\
\hline 1308 & 0.00692 & 0.001062685 & 0.038256735 \\
\hline 1309 & 0.00691 & 0.00105972 & 0.03814987 \\
\hline 1310 & 0.0069 & 0.00105676 & 0.03804331 \\
\hline 1311 & 0.00688 & 0.001053805 & 0.037937055 \\
\hline 1312 & 0.00687 & 0.001050865 & 0.037831115 \\
\hline 1313 & 0.00686 & 0.00104793 & 0.03772548 \\
\hline 1314 & 0.00685 & 0.001045005 & 0.037620155 \\
\hline 1315 & 0.00683 & 0.001042085 & 0.037515085 \\
\hline 1316 & 0.00682 & 0.001039175 & 0.037410375 \\
\hline 1317 & 0.0068 & 0.001036275 & 0.037305925 \\
\hline 1318 & 0.00679 & 0.001033385 & 0.037201835 \\
\hline 1319 & 0.00678 & 0.0010305 & 0.037098 \\
\hline 1320 & 0.00677 & 0.001027625 & 0.036994475 \\
\hline 1321 & 0.00675 & 0.001024755 & 0.036891205 \\
\hline 1322 & 0.00674 & 0.001021895 & 0.036788295 \\
\hline 1323 & 0.00673 & 0.001019045 & 0.036685645 \\
\hline 1324 & 0.00672 & 0.001016205 & 0.036583305 \\
\hline 1325 & 0.0067 & 0.00101337 & 0.03648127 \\
\hline 1326 & 0.00669 & 0.00101054 & 0.03637949 \\
\hline 1327 & 0.00668 & 0.001007725 & 0.036278025 \\
\hline 1328 & 0.00667 & 0.001004915 & 0.036176865 \\
\hline 1329 & 0.00665 & 0.00100211 & 0.03607596 \\
\hline 1330 & 0.00664 & 0.000999315 & 0.035975365 \\
\hline 1331 & 0.00663 & 0.00099653 & 0.03587508 \\
\hline 1332 & 0.00662 & 0.00099375 & 0.0357751 \\
\hline 1333 & 0.0066 & 0.00099098 & 0.03567533 \\
\hline 1334 & 0.00659 & 0.00098822 & 0.03557592 \\
\hline 1335 & 0.00658 & 0.000985465 & 0.035476765 \\
\hline 1336 & 0.00657 & 0.00098272 & 0.03537792 \\
\hline 1337 & 0.00655 & 0.00097998 & 0.03527933 \\
\hline 1338 & 0.00654 & 0.00097725 & 0.03518105 \\
\hline 1339 & 0.00653 & 0.00097453 & 0.03508303 \\
\hline 1340 & 0.00652 & 0.000971815 & 0.034985315 \\
\hline 1341 & 0.0065 & 0.000969105 & 0.034887855 \\
\hline 1342 & 0.00649 & 0.00096641 & 0.03479071 \\
\hline 1343 & 0.00648 & 0.000963715 & 0.034693815 \\
\hline 1344 & 0.00647 & 0.000961035 & 0.034597185 \\
\hline 1345 & 0.00645 & 0.000958355 & 0.034500855 \\
\hline 1346 & 0.00644 & 0.00095569 & 0.03440484 \\
\hline 1347 & 0.00643 & 0.00095303 & 0.03430903 \\
\hline 1348 & 0.00642 & 0.000950375 & 0.034213575 \\
\hline 1349 & 0.0064 & 0.00094773 & 0.03411833 \\
\hline 1350 & 0.00639 & 0.000945095 & 0.034023395 \\
\hline 1351 & 0.00638 & 0.000942465 & 0.033928715 \\
\hline 1352 & 0.00637 & 0.00093984 & 0.03383429 \\
\hline 1353 & 0.00636 & 0.000937225 & 0.033740175 \\
\hline 1354 & 0.00635 & 0.00093462 & 0.03364632 \\
\hline 1355 & 0.00633 & 0.00093202 & 0.03355277 \\
\hline 1356 & 0.00632 & 0.00092943 & 0.03345943 \\
\hline 1357 & 0.00631 & 0.000926845 & 0.033366395 \\
\hline 1358 & 0.0063 & 0.000924265 & 0.033273615 \\
\hline 1359 & 0.00628 & 0.0009217 & 0.0331811 \\
\hline 1360 & 0.00627 & 0.000919135 & 0.033088885 \\
\hline 1361 & 0.00626 & 0.00091658 & 0.03299688 \\
\hline 1362 & 0.00625 & 0.000914035 & 0.032905185 \\
\hline 1363 & 0.00623 & 0.000911495 & 0.032813745 \\
\hline 1364 & 0.00622 & 0.00090896 & 0.03272256 \\
\hline 1365 & 0.00621 & 0.000906435 & 0.032631635 \\
\hline 1366 & 0.0062 & 0.000903915 & 0.032541015 \\
\hline 1367 & 0.00619 & 0.000901405 & 0.032450605 \\
\hline 1368 & 0.00618 & 0.0008989 & 0.03236045 \\
\hline 1369 & 0.00616 & 0.000896405 & 0.032270605 \\
\hline 1370 & 0.00615 & 0.000893915 & 0.032180965 \\
\hline 1371 & 0.00614 & 0.000891435 & 0.032091635 \\
\hline 1372 & 0.00613 & 0.00088896 & 0.03200256 \\
\hline 1373 & 0.00611 & 0.00088649 & 0.03191369 \\
\hline 1374 & 0.0061 & 0.00088403 & 0.03182513 \\
\hline 1375 & 0.00609 & 0.00088158 & 0.03173678 \\
\hline 1376 & 0.00608 & 0.00087913 & 0.03164873 \\
\hline 1377 & 0.00607 & 0.00087669 & 0.03156094 \\
\hline 1378 & 0.00606 & 0.00087426 & 0.03147336 \\
\hline 1379 & 0.00604 & 0.000871835 & 0.031386035 \\
\hline 1380 & 0.00603 & 0.000869415 & 0.031299015 \\
\hline 1381 & 0.00602 & 0.000867005 & 0.031212205 \\
\hline
\end{tabular}




\begin{tabular}{|c|c|c|c|}
\hline 1382 & 0.00601 & 0.0008646 & 0.03112565 \\
\hline 1383 & 0.006 & 0.000862205 & 0.031039355 \\
\hline 1384 & 0.00599 & 0.000859815 & 0.030953315 \\
\hline 1385 & 0.00597 & 0.00085743 & 0.03086748 \\
\hline 1386 & 0.00596 & 0.000855055 & 0.030781955 \\
\hline 1387 & 0.00595 & 0.000852685 & 0.030696635 \\
\hline 1388 & 0.00594 & 0.00085032 & 0.03061157 \\
\hline 1389 & 0.00592 & 0.000847965 & 0.030526765 \\
\hline 1390 & 0.00592 & 0.000845615 & 0.030442165 \\
\hline 1391 & 0.0059 & 0.000843275 & 0.030357875 \\
\hline 1392 & 0.00589 & 0.00084094 & 0.03027379 \\
\hline 1393 & 0.00588 & 0.00083861 & 0.03018996 \\
\hline 1394 & 0.00587 & 0.000836285 & 0.030106335 \\
\hline 1395 & 0.00586 & 0.00083397 & 0.03002297 \\
\hline 1396 & 0.00585 & 0.000831665 & 0.029939865 \\
\hline 1397 & 0.00583 & 0.00082936 & 0.02985701 \\
\hline 1398 & 0.00582 & 0.000827065 & 0.029774365 \\
\hline 1399 & 0.00581 & 0.000824775 & 0.029691975 \\
\hline 1400 & 0.0058 & 0.000822495 & 0.029609845 \\
\hline 1401 & 0.00579 & 0.00082022 & 0.02952792 \\
\hline 1402 & 0.00578 & 0.00081795 & 0.02944625 \\
\hline 1403 & 0.00576 & 0.00081569 & 0.02936479 \\
\hline 1404 & 0.00575 & 0.000813435 & 0.029283585 \\
\hline 1405 & 0.00574 & 0.000811185 & 0.029202585 \\
\hline 1406 & 0.00573 & 0.00080894 & 0.02912189 \\
\hline 1407 & 0.00572 & 0.000806705 & 0.029041355 \\
\hline 1408 & 0.00571 & 0.000804475 & 0.028961075 \\
\hline 1409 & 0.00569 & 0.00080225 & 0.02888105 \\
\hline 1410 & 0.00569 & 0.000800035 & 0.028801235 \\
\hline 1411 & 0.00567 & 0.000797825 & 0.028721675 \\
\hline 1412 & 0.00566 & 0.00079562 & 0.02864232 \\
\hline 1413 & 0.00565 & 0.00079342 & 0.02856322 \\
\hline 1414 & 0.00564 & 0.00079123 & 0.02848433 \\
\hline 1415 & 0.00563 & 0.000789045 & 0.028405645 \\
\hline 1416 & 0.00562 & 0.00078687 & 0.02832722 \\
\hline 1417 & 0.0056 & 0.000784695 & 0.028249045 \\
\hline 1418 & 0.00559 & 0.00078253 & 0.02817103 \\
\hline 1419 & 0.00558 & 0.00078037 & 0.02809332 \\
\hline 1420 & 0.00557 & 0.000778215 & 0.028015765 \\
\hline 1421 & 0.00556 & 0.00077607 & 0.02793847 \\
\hline 1422 & 0.00555 & 0.00077393 & 0.02786138 \\
\hline 1423 & 0.00554 & 0.000771795 & 0.027784545 \\
\hline 1424 & 0.00553 & 0.000769665 & 0.027707915 \\
\hline 1425 & 0.00551 & 0.00076754 & 0.02763149 \\
\hline 1426 & 0.0055 & 0.000765425 & 0.027555325 \\
\hline 1427 & 0.00549 & 0.000763315 & 0.027479315 \\
\hline 1428 & 0.00548 & 0.00076121 & 0.02740361 \\
\hline 1429 & 0.00547 & 0.000759115 & 0.027328065 \\
\hline 1430 & 0.00546 & 0.00075702 & 0.02725277 \\
\hline 1431 & 0.00545 & 0.000754935 & 0.027177685 \\
\hline 1432 & 0.00544 & 0.000752855 & 0.027102805 \\
\hline 1433 & 0.00542 & 0.00075078 & 0.02702813 \\
\hline 1434 & 0.00542 & 0.000748715 & 0.026953665 \\
\hline 1435 & 0.0054 & 0.00074665 & 0.02687945 \\
\hline 1436 & 0.00539 & 0.000744595 & 0.026805445 \\
\hline 1437 & 0.00538 & 0.000742545 & 0.026731645 \\
\hline 1438 & 0.00537 & 0.0007405 & 0.02665805 \\
\hline 1439 & 0.00536 & 0.000738465 & 0.026584715 \\
\hline 1440 & 0.00535 & 0.00073643 & 0.02651153 \\
\hline 1441 & 0.00534 & 0.000734405 & 0.026438605 \\
\hline 1442 & 0.00533 & 0.000732385 & 0.026365885 \\
\hline 1443 & 0.00531 & 0.00073037 & 0.02629337 \\
\hline 1444 & 0.00531 & 0.00072836 & 0.02622101 \\
\hline 1445 & 0.00529 & 0.00072636 & 0.02614891 \\
\hline 1446 & 0.00528 & 0.00072436 & 0.02607706 \\
\hline 1447 & 0.00527 & 0.00072237 & 0.02600537 \\
\hline 1448 & 0.00526 & 0.000720385 & 0.025933885 \\
\hline 1449 & 0.00525 & 0.000718405 & 0.025862605 \\
\hline 1450 & 0.00524 & 0.00071643 & 0.02579153 \\
\hline 1451 & 0.00523 & 0.000714465 & 0.025720665 \\
\hline 1452 & 0.00522 & 0.0007125 & 0.02565005 \\
\hline 1453 & 0.00521 & 0.000710545 & 0.025579595 \\
\hline 1454 & 0.0052 & 0.000708595 & 0.025509345 \\
\hline 1455 & 0.00518 & 0.000706645 & 0.025439295 \\
\hline 1456 & 0.00518 & 0.000704705 & 0.025369455 \\
\hline 1457 & 0.00516 & 0.000702775 & 0.025299825 \\
\hline 1458 & 0.00515 & 0.000700845 & 0.025230395 \\
\hline 1459 & 0.00514 & 0.00069892 & 0.02516117 \\
\hline 1460 & 0.00513 & 0.000697005 & 0.025092105 \\
\hline 1461 & 0.00512 & 0.00069509 & 0.02502329 \\
\hline
\end{tabular}




\begin{tabular}{|c|c|c|c|}
\hline 1462 & 0.00511 & 0.000693185 & 0.024954635 \\
\hline 1463 & 0.0051 & 0.000691285 & 0.024886235 \\
\hline 1464 & 0.00509 & 0.00068939 & 0.02481799 \\
\hline 1465 & 0.00508 & 0.0006875 & 0.02474995 \\
\hline 1466 & 0.00507 & 0.000685615 & 0.024682115 \\
\hline 1467 & 0.00505 & 0.000683735 & 0.024614485 \\
\hline 1468 & 0.00505 & 0.00068186 & 0.02454701 \\
\hline 1469 & 0.00503 & 0.000679995 & 0.024479745 \\
\hline 1470 & 0.00503 & 0.00067813 & 0.02441273 \\
\hline 1471 & 0.00501 & 0.000676275 & 0.024345825 \\
\hline 1472 & 0.005 & 0.00067442 & 0.02427917 \\
\hline 1473 & 0.00499 & 0.000672575 & 0.024212675 \\
\hline 1474 & 0.00498 & 0.000670735 & 0.024146435 \\
\hline 1475 & 0.00497 & 0.0006689 & 0.0240803 \\
\hline 1476 & 0.00496 & 0.000667065 & 0.024014415 \\
\hline 1477 & 0.00495 & 0.00066524 & 0.02394869 \\
\hline 1478 & 0.00494 & 0.00066342 & 0.02388317 \\
\hline 1479 & 0.00493 & 0.000661605 & 0.023817855 \\
\hline 1480 & 0.00492 & 0.0006598 & 0.02375275 \\
\hline 1481 & 0.00491 & 0.000657995 & 0.023687795 \\
\hline 1482 & 0.0049 & 0.000656195 & 0.023622995 \\
\hline 1483 & 0.00489 & 0.0006544 & 0.02355845 \\
\hline 1484 & 0.00488 & 0.00065261 & 0.02349406 \\
\hline 1485 & 0.00487 & 0.00065083 & 0.02342983 \\
\hline 1486 & 0.00486 & 0.00064905 & 0.02336585 \\
\hline 1487 & 0.00484 & 0.00064728 & 0.02330198 \\
\hline 1488 & 0.00484 & 0.00064551 & 0.02323836 \\
\hline 1489 & 0.00482 & 0.000643745 & 0.023174895 \\
\hline 1490 & 0.00482 & 0.00064199 & 0.02311164 \\
\hline 1491 & 0.0048 & 0.000640235 & 0.023048535 \\
\hline 1492 & 0.0048 & 0.00063849 & 0.02298559 \\
\hline 1493 & 0.00478 & 0.000636745 & 0.022922895 \\
\hline 1494 & 0.00477 & 0.00063501 & 0.02286031 \\
\hline 1495 & 0.00476 & 0.000633275 & 0.022797975 \\
\hline 1496 & 0.00475 & 0.00063155 & 0.0227358 \\
\hline 1497 & 0.00474 & 0.000629825 & 0.022673775 \\
\hline 1498 & 0.00473 & 0.00062811 & 0.02261196 \\
\hline 1499 & 0.00472 & 0.000626395 & 0.022550295 \\
\hline 1500 & 0.00471 & 0.00062469 & 0.02248884 \\
\hline 1501 & 0.0047 & 0.000622985 & 0.022427535 \\
\hline 1502 & 0.00469 & 0.00062129 & 0.02236644 \\
\hline 1503 & 0.00468 & 0.000619595 & 0.022305495 \\
\hline 1504 & 0.00467 & 0.00061791 & 0.02224476 \\
\hline 1505 & 0.00466 & 0.000616225 & 0.022184175 \\
\hline 1506 & 0.00465 & 0.00061455 & 0.02212375 \\
\hline 1507 & 0.00464 & 0.000612875 & 0.022063525 \\
\hline 1508 & 0.00463 & 0.000611205 & 0.022003455 \\
\hline 1509 & 0.00462 & 0.000609545 & 0.021943595 \\
\hline 1510 & 0.00461 & 0.000607885 & 0.021883885 \\
\hline 1511 & 0.0046 & 0.00060623 & 0.02182433 \\
\hline 1512 & 0.00459 & 0.00060458 & 0.02176498 \\
\hline 1513 & 0.00458 & 0.00060294 & 0.02170579 \\
\hline 1514 & 0.00457 & 0.0006013 & 0.02164675 \\
\hline 1515 & 0.00456 & 0.000599665 & 0.021587915 \\
\hline 1516 & 0.00455 & 0.000598035 & 0.021529235 \\
\hline 1517 & 0.00454 & 0.00059641 & 0.02147071 \\
\hline 1518 & 0.00453 & 0.00059479 & 0.02141234 \\
\hline 1519 & 0.00452 & 0.00059317 & 0.02135417 \\
\hline 1520 & 0.00451 & 0.00059156 & 0.02129616 \\
\hline 1521 & 0.0045 & 0.000589955 & 0.021238355 \\
\hline 1522 & 0.00449 & 0.00058835 & 0.02118065 \\
\hline 1523 & 0.00448 & 0.000586755 & 0.021123155 \\
\hline 1524 & 0.00447 & 0.00058516 & 0.02106581 \\
\hline 1525 & 0.00446 & 0.000583575 & 0.021008675 \\
\hline 1526 & 0.00445 & 0.00058199 & 0.02095164 \\
\hline 1527 & 0.00444 & 0.00058041 & 0.02089481 \\
\hline 1528 & 0.00443 & 0.000578835 & 0.020838135 \\
\hline 1529 & 0.00442 & 0.00057727 & 0.02078162 \\
\hline 1530 & 0.00441 & 0.0005757 & 0.0207253 \\
\hline 1531 & 0.0044 & 0.00057414 & 0.02066909 \\
\hline 1532 & 0.00439 & 0.000572585 & 0.020613085 \\
\hline 1533 & 0.00438 & 0.000571035 & 0.020557235 \\
\hline 1534 & 0.00437 & 0.000569485 & 0.020501535 \\
\hline 1535 & 0.00436 & 0.000567945 & 0.020445995 \\
\hline 1536 & 0.00435 & 0.000566405 & 0.020390605 \\
\hline 1537 & 0.00434 & 0.00056487 & 0.02033537 \\
\hline 1538 & 0.00433 & 0.000563345 & 0.020280345 \\
\hline 1539 & 0.00432 & 0.00056182 & 0.02022542 \\
\hline 1540 & 0.00431 & 0.000560295 & 0.020170695 \\
\hline 1541 & 0.0043 & 0.00055878 & 0.02011613 \\
\hline
\end{tabular}




\begin{tabular}{|c|c|c|c|}
\hline 1542 & 0.00429 & 0.00055727 & 0.02006167 \\
\hline 1543 & 0.00428 & 0.00055576 & 0.02000741 \\
\hline 1544 & 0.00427 & 0.00055426 & 0.01995331 \\
\hline 1545 & 0.00426 & 0.00055276 & 0.01989936 \\
\hline 1546 & 0.00426 & 0.000551265 & 0.019845565 \\
\hline 1547 & 0.00424 & 0.000549775 & 0.019791925 \\
\hline 1548 & 0.00424 & 0.00054829 & 0.01973844 \\
\hline 1549 & 0.00422 & 0.00054681 & 0.01968511 \\
\hline 1550 & 0.00422 & 0.00054533 & 0.01963193 \\
\hline 1551 & 0.0042 & 0.00054386 & 0.01957891 \\
\hline 1552 & 0.0042 & 0.00054239 & 0.01952604 \\
\hline 1553 & 0.00418 & 0.000540925 & 0.019473325 \\
\hline 1554 & 0.00418 & 0.000539465 & 0.019420765 \\
\hline 1555 & 0.00417 & 0.00053801 & 0.01936836 \\
\hline 1556 & 0.00416 & 0.00053656 & 0.01931611 \\
\hline 1557 & 0.00415 & 0.00053511 & 0.01926396 \\
\hline 1558 & 0.00414 & 0.000533665 & 0.019212015 \\
\hline 1559 & 0.00413 & 0.00053223 & 0.01916023 \\
\hline 1560 & 0.00412 & 0.000530795 & 0.019108545 \\
\hline 1561 & 0.00411 & 0.00052936 & 0.01905706 \\
\hline 1562 & 0.0041 & 0.000527935 & 0.019005685 \\
\hline 1563 & 0.00409 & 0.000526515 & 0.018954465 \\
\hline 1564 & 0.00408 & 0.000525095 & 0.018903395 \\
\hline 1565 & 0.00407 & 0.00052368 & 0.01885248 \\
\hline 1566 & 0.00406 & 0.00052227 & 0.01880172 \\
\hline 1567 & 0.00405 & 0.000520865 & 0.018751065 \\
\hline 1568 & 0.00404 & 0.00051946 & 0.01870061 \\
\hline 1569 & 0.00403 & 0.000518065 & 0.018650265 \\
\hline 1570 & 0.00403 & 0.00051667 & 0.01860007 \\
\hline 1571 & 0.00401 & 0.00051528 & 0.01855003 \\
\hline 1572 & 0.00401 & 0.000513895 & 0.018500145 \\
\hline 1573 & 0.00399 & 0.00051251 & 0.01845041 \\
\hline 1574 & 0.00399 & 0.000511135 & 0.018400785 \\
\hline 1575 & 0.00398 & 0.00050976 & 0.01835131 \\
\hline 1576 & 0.00397 & 0.00050839 & 0.01830199 \\
\hline 1577 & 0.00396 & 0.000507025 & 0.018252825 \\
\hline 1578 & 0.00395 & 0.00050566 & 0.01820376 \\
\hline 1579 & 0.00394 & 0.0005043 & 0.01815485 \\
\hline 1580 & 0.00393 & 0.00050295 & 0.0181061 \\
\hline 1581 & 0.00392 & 0.000501595 & 0.018057495 \\
\hline 1582 & 0.00391 & 0.00050025 & 0.01800905 \\
\hline 1583 & 0.0039 & 0.000498908 & 0.017960708 \\
\hline 1584 & 0.00389 & 0.00049757 & 0.01791252 \\
\hline 1585 & 0.00388 & 0.000496235 & 0.017864435 \\
\hline 1586 & 0.00388 & 0.000494904 & 0.017816504 \\
\hline 1587 & 0.00386 & 0.000493576 & 0.017768726 \\
\hline 1588 & 0.00386 & 0.000492253 & 0.017721103 \\
\hline 1589 & 0.00385 & 0.000490933 & 0.017673583 \\
\hline 1590 & 0.00384 & 0.000489617 & 0.017626217 \\
\hline 1591 & 0.00383 & 0.000488305 & 0.017579005 \\
\hline 1592 & 0.00382 & 0.000486997 & 0.017531897 \\
\hline 1593 & 0.00381 & 0.000485693 & 0.017484943 \\
\hline 1594 & 0.0038 & 0.000484392 & 0.017438092 \\
\hline 1595 & 0.00379 & 0.000483095 & 0.017391395 \\
\hline 1596 & 0.00378 & 0.000481802 & 0.017344852 \\
\hline 1597 & 0.00377 & 0.000480512 & 0.017298462 \\
\hline 1598 & 0.00377 & 0.000479227 & 0.017252127 \\
\hline 1599 & 0.00375 & 0.000477945 & 0.017205995 \\
\hline 1600 & 0.00375 & 0.000476666 & 0.017159966 \\
\hline 1601 & 0.00374 & 0.000475392 & 0.017114092 \\
\hline 1602 & 0.00373 & 0.000474121 & 0.017068321 \\
\hline 1603 & 0.00372 & 0.000472853 & 0.017022703 \\
\hline 1604 & 0.00371 & 0.000471589 & 0.016977189 \\
\hline 1605 & 0.0037 & 0.000470329 & 0.016931829 \\
\hline 1606 & 0.00369 & 0.000469073 & 0.016886623 \\
\hline 1607 & 0.00368 & 0.00046782 & 0.01684152 \\
\hline 1608 & 0.00367 & 0.000466571 & 0.016796521 \\
\hline 1609 & 0.00366 & 0.000465325 & 0.016751725 \\
\hline 1610 & 0.00366 & 0.000464083 & 0.016706983 \\
\hline 1611 & 0.00364 & 0.000462845 & 0.016662395 \\
\hline 1612 & 0.00364 & 0.00046161 & 0.01661796 \\
\hline 1613 & 0.00363 & 0.000460379 & 0.016573629 \\
\hline 1614 & 0.00362 & 0.000459151 & 0.016529401 \\
\hline 1615 & 0.00361 & 0.000457927 & 0.016485327 \\
\hline 1616 & 0.0036 & 0.000456706 & 0.016441406 \\
\hline 1617 & 0.00359 & 0.000455489 & 0.016397589 \\
\hline 1618 & 0.00359 & 0.000454275 & 0.016353875 \\
\hline 1619 & 0.00357 & 0.000453065 & 0.016310315 \\
\hline 1620 & 0.00357 & 0.000451858 & 0.016266858 \\
\hline 1621 & 0.00356 & 0.000450655 & 0.016223555 \\
\hline
\end{tabular}




\begin{tabular}{|c|c|c|c|}
\hline 1622 & 0.00355 & 0.000449455 & 0.016180355 \\
\hline 1623 & 0.00354 & 0.000448258 & 0.016137308 \\
\hline 1624 & 0.00353 & 0.000447066 & 0.016094366 \\
\hline 1625 & 0.00352 & 0.000445876 & 0.016051526 \\
\hline 1626 & 0.00351 & 0.00044469 & 0.01600884 \\
\hline 1627 & 0.0035 & 0.000443507 & 0.015966257 \\
\hline 1628 & 0.0035 & 0.000442328 & 0.015923778 \\
\hline 1629 & 0.00348 & 0.000441152 & 0.015881452 \\
\hline 1630 & 0.00348 & 0.00043998 & 0.01583928 \\
\hline 1631 & 0.00347 & 0.00043881 & 0.01579716 \\
\hline 1632 & 0.00346 & 0.000437645 & 0.015755195 \\
\hline 1633 & 0.00345 & 0.000436482 & 0.015713332 \\
\hline 1634 & 0.00344 & 0.000435323 & 0.015671623 \\
\hline 1635 & 0.00343 & 0.000434167 & 0.015630017 \\
\hline 1636 & 0.00343 & 0.000433015 & 0.015588515 \\
\hline 1637 & 0.00341 & 0.000431866 & 0.015547166 \\
\hline 1638 & 0.00341 & 0.00043072 & 0.01550592 \\
\hline 1639 & 0.0034 & 0.000429578 & 0.015464778 \\
\hline 1640 & 0.00339 & 0.000428438 & 0.015423788 \\
\hline 1641 & 0.00338 & 0.000427302 & 0.015382852 \\
\hline 1642 & 0.00337 & 0.00042617 & 0.01534212 \\
\hline 1643 & 0.00336 & 0.00042504 & 0.01530144 \\
\hline 1644 & 0.00336 & 0.000423914 & 0.015260914 \\
\hline 1645 & 0.00334 & 0.000422791 & 0.015220441 \\
\hline 1646 & 0.00334 & 0.000421671 & 0.015180171 \\
\hline 1647 & 0.00333 & 0.000420554 & 0.015139954 \\
\hline 1648 & 0.00332 & 0.000419441 & 0.015099891 \\
\hline 1649 & 0.00331 & 0.000418331 & 0.015059881 \\
\hline 1650 & 0.0033 & 0.000417224 & 0.015020074 \\
\hline 1651 & 0.00329 & 0.00041612 & 0.01498032 \\
\hline 1652 & 0.00329 & 0.000415019 & 0.014940669 \\
\hline 1653 & 0.00327 & 0.000413922 & 0.014901172 \\
\hline 1654 & 0.00327 & 0.000412828 & 0.014861778 \\
\hline 1655 & 0.00326 & 0.000411736 & 0.014822486 \\
\hline 1656 & 0.00325 & 0.000410648 & 0.014783348 \\
\hline 1657 & 0.00324 & 0.000409563 & 0.014744263 \\
\hline 1658 & 0.00323 & 0.000408482 & 0.014705332 \\
\hline 1659 & 0.00322 & 0.000407403 & 0.014666503 \\
\hline 1660 & 0.00322 & 0.000406327 & 0.014627777 \\
\hline 1661 & 0.00321 & 0.000405254 & 0.014589154 \\
\hline 1662 & 0.0032 & 0.000404185 & 0.014550635 \\
\hline 1663 & 0.00319 & 0.000403119 & 0.014512269 \\
\hline 1664 & 0.00318 & 0.000402055 & 0.014473955 \\
\hline 1665 & 0.00317 & 0.000400995 & 0.014435795 \\
\hline 1666 & 0.00317 & 0.000399937 & 0.014397737 \\
\hline 1667 & 0.00315 & 0.000398883 & 0.014359783 \\
\hline 1668 & 0.00315 & 0.000397832 & 0.014321932 \\
\hline 1669 & 0.00314 & 0.000396784 & 0.014284184 \\
\hline 1670 & 0.00313 & 0.000395738 & 0.014246588 \\
\hline 1671 & 0.00312 & 0.000394696 & 0.014209046 \\
\hline 1672 & 0.00312 & 0.000393657 & 0.014171657 \\
\hline 1673 & 0.0031 & 0.00039262 & 0.01413432 \\
\hline 1674 & 0.0031 & 0.000391587 & 0.014097137 \\
\hline 1675 & 0.00309 & 0.000390557 & 0.014060057 \\
\hline 1676 & 0.00308 & 0.000389529 & 0.014023029 \\
\hline 1677 & 0.00307 & 0.000388505 & 0.013986155 \\
\hline 1678 & 0.00306 & 0.000387483 & 0.013949383 \\
\hline 1679 & 0.00305 & 0.000386465 & 0.013912715 \\
\hline 1680 & 0.00305 & 0.000385449 & 0.013876149 \\
\hline 1681 & 0.00304 & 0.000384436 & 0.013839686 \\
\hline 1682 & 0.00303 & 0.000383426 & 0.013803326 \\
\hline 1683 & 0.00302 & 0.000382419 & 0.013767069 \\
\hline 1684 & 0.00301 & 0.000381415 & 0.013730915 \\
\hline 1685 & 0.003 & 0.000380413 & 0.013694863 \\
\hline 1686 & 0.003 & 0.000379415 & 0.013658915 \\
\hline 1687 & 0.00299 & 0.000378419 & 0.013623069 \\
\hline 1688 & 0.00298 & 0.000377427 & 0.013587327 \\
\hline 1689 & 0.00297 & 0.000376437 & 0.013551687 \\
\hline 1690 & 0.00296 & 0.000375449 & 0.013516149 \\
\hline 1691 & 0.00295 & 0.000374465 & 0.013480715 \\
\hline 1692 & 0.00295 & 0.000373484 & 0.013445384 \\
\hline 1693 & 0.00294 & 0.000372505 & 0.013410155 \\
\hline 1694 & 0.00293 & 0.000371529 & 0.013375029 \\
\hline 1695 & 0.00292 & 0.000370556 & 0.013340006 \\
\hline 1696 & 0.00291 & 0.000369586 & 0.013305086 \\
\hline 1697 & 0.0029 & 0.000368618 & 0.013270268 \\
\hline 1698 & 0.0029 & 0.000367653 & 0.013235503 \\
\hline 1699 & 0.00289 & 0.000366691 & 0.013200891 \\
\hline 1700 & 0.00288 & 0.000365732 & 0.013166332 \\
\hline 1701 & 0.00287 & 0.000364775 & 0.013131925 \\
\hline
\end{tabular}




\begin{tabular}{|c|c|c|c|}
\hline 1702 & 0.00287 & 0.000363822 & 0.013097572 \\
\hline 1703 & 0.00285 & 0.00036287 & 0.01306332 \\
\hline 1704 & 0.00285 & 0.000361922 & 0.013029172 \\
\hline 1705 & 0.00284 & 0.000360976 & 0.012995126 \\
\hline 1706 & 0.00283 & 0.000360033 & 0.012961183 \\
\hline 1707 & 0.00282 & 0.000359093 & 0.012927343 \\
\hline 1708 & 0.00282 & 0.000358155 & 0.012893605 \\
\hline 1709 & 0.0028 & 0.00035722 & 0.01285992 \\
\hline 1710 & 0.0028 & 0.000356288 & 0.012826338 \\
\hline 1711 & 0.00279 & 0.000355358 & 0.012792908 \\
\hline 1712 & 0.00278 & 0.000354431 & 0.012759531 \\
\hline 1713 & 0.00277 & 0.000353507 & 0.012726257 \\
\hline 1714 & 0.00277 & 0.000352585 & 0.012693035 \\
\hline 1715 & 0.00276 & 0.000351666 & 0.012659966 \\
\hline 1716 & 0.00275 & 0.00035075 & 0.012627 \\
\hline 1717 & 0.00274 & 0.000349836 & 0.012594086 \\
\hline 1718 & 0.00273 & 0.000348924 & 0.012561274 \\
\hline 1719 & 0.00272 & 0.000348016 & 0.012528566 \\
\hline 1720 & 0.00272 & 0.00034711 & 0.01249596 \\
\hline 1721 & 0.00271 & 0.000346206 & 0.012463406 \\
\hline 1722 & 0.0027 & 0.000345305 & 0.012430955 \\
\hline 1723 & 0.00269 & 0.000344407 & 0.012398607 \\
\hline 1724 & 0.00269 & 0.000343511 & 0.012366361 \\
\hline 1725 & 0.00267 & 0.000342617 & 0.012334217 \\
\hline 1726 & 0.00267 & 0.000341727 & 0.012302127 \\
\hline 1727 & 0.00266 & 0.000340838 & 0.012270188 \\
\hline 1728 & 0.00265 & 0.000339953 & 0.012238303 \\
\hline 1729 & 0.00264 & 0.000339069 & 0.012206469 \\
\hline 1730 & 0.00264 & 0.000338189 & 0.012174789 \\
\hline 1731 & 0.00263 & 0.000337311 & 0.012143161 \\
\hline 1732 & 0.00262 & 0.000336435 & 0.012111635 \\
\hline 1733 & 0.00261 & 0.000335562 & 0.012080212 \\
\hline 1734 & 0.00261 & 0.000334691 & 0.012048841 \\
\hline 1735 & 0.00259 & 0.000333823 & 0.012017623 \\
\hline 1736 & 0.00259 & 0.000332957 & 0.011986457 \\
\hline 1737 & 0.00258 & 0.000332093 & 0.011955343 \\
\hline 1738 & 0.00257 & 0.000331233 & 0.011924383 \\
\hline 1739 & 0.00256 & 0.000330374 & 0.011893474 \\
\hline 1740 & 0.00256 & 0.000329518 & 0.011862618 \\
\hline 1741 & 0.00255 & 0.000328664 & 0.011831914 \\
\hline 1742 & 0.00254 & 0.000327813 & 0.011801263 \\
\hline 1743 & 0.00253 & 0.000326964 & 0.011770714 \\
\hline 1744 & 0.00253 & 0.000326118 & 0.011740268 \\
\hline 1745 & 0.00251 & 0.000325274 & 0.011709874 \\
\hline 1746 & 0.00251 & 0.000324433 & 0.011679583 \\
\hline 1747 & 0.0025 & 0.000323593 & 0.011649343 \\
\hline 1748 & 0.00249 & 0.000322757 & 0.011619207 \\
\hline 1749 & 0.00248 & 0.000321922 & 0.011589172 \\
\hline 1750 & 0.00248 & 0.00032109 & 0.01155924 \\
\hline 1751 & 0.00247 & 0.00032026 & 0.01152936 \\
\hline 1752 & 0.00246 & 0.000319433 & 0.011499583 \\
\hline 1753 & 0.00245 & 0.000318608 & 0.011469858 \\
\hline 1754 & 0.00245 & 0.000317785 & 0.011440285 \\
\hline 1755 & 0.00244 & 0.000316965 & 0.011410715 \\
\hline 1756 & 0.00243 & 0.000316147 & 0.011381297 \\
\hline 1757 & 0.00242 & 0.000315331 & 0.011351931 \\
\hline 1758 & 0.00242 & 0.000314518 & 0.011322618 \\
\hline 1759 & 0.0024 & 0.000313707 & 0.011293407 \\
\hline 1760 & 0.0024 & 0.000312898 & 0.011264298 \\
\hline 1761 & 0.00239 & 0.000312091 & 0.011235291 \\
\hline 1762 & 0.00238 & 0.000311287 & 0.011206337 \\
\hline 1763 & 0.00237 & 0.000310485 & 0.011177435 \\
\hline 1764 & 0.00237 & 0.000309685 & 0.011148685 \\
\hline 1765 & 0.00236 & 0.000308888 & 0.011119938 \\
\hline 1766 & 0.00235 & 0.000308093 & 0.011091343 \\
\hline 1767 & 0.00234 & 0.0003073 & 0.0110628 \\
\hline 1768 & 0.00234 & 0.000306509 & 0.011034309 \\
\hline 1769 & 0.00232 & 0.00030572 & 0.01100592 \\
\hline 1770 & 0.00232 & 0.000304934 & 0.010977634 \\
\hline 1771 & 0.00231 & 0.00030415 & 0.0109494 \\
\hline 1772 & 0.00231 & 0.000303368 & 0.010921268 \\
\hline 1773 & 0.00229 & 0.000302589 & 0.010893189 \\
\hline 1774 & 0.00229 & 0.000301811 & 0.010865211 \\
\hline 1775 & 0.00228 & 0.000301036 & 0.010837286 \\
\hline 1776 & 0.00227 & 0.000300263 & 0.010809463 \\
\hline 1777 & 0.00226 & 0.000299492 & 0.010781692 \\
\hline 1778 & 0.00226 & 0.000298724 & 0.010754024 \\
\hline 1779 & 0.00225 & 0.000297957 & 0.010726457 \\
\hline 1780 & 0.00224 & 0.000297193 & 0.010698943 \\
\hline 1781 & 0.00223 & 0.00029643 & 0.01067148 \\
\hline
\end{tabular}




\begin{tabular}{|c|c|c|c|}
\hline 1782 & 0.00223 & 0.00029567 & 0.01064412 \\
\hline 1783 & 0.00222 & 0.000294912 & 0.010616862 \\
\hline 1784 & 0.00221 & 0.000294157 & 0.010589657 \\
\hline 1785 & 0.0022 & 0.000293403 & 0.010562503 \\
\hline 1786 & 0.0022 & 0.000292652 & 0.010535452 \\
\hline 1787 & 0.00218 & 0.000291902 & 0.010508452 \\
\hline 1788 & 0.00218 & 0.000291155 & 0.010481555 \\
\hline 1789 & 0.00217 & 0.00029041 & 0.01045476 \\
\hline 1790 & 0.00217 & 0.000289667 & 0.010427967 \\
\hline 1791 & 0.00215 & 0.000288925 & 0.010401325 \\
\hline 1792 & 0.00215 & 0.000288187 & 0.010374687 \\
\hline 1793 & 0.00214 & 0.00028745 & 0.0103482 \\
\hline 1794 & 0.00213 & 0.000286715 & 0.010321715 \\
\hline 1795 & 0.00212 & 0.000285982 & 0.010295332 \\
\hline 1796 & 0.00212 & 0.000285252 & 0.010269052 \\
\hline 1797 & 0.00211 & 0.000284523 & 0.010242823 \\
\hline 1798 & 0.0021 & 0.000283796 & 0.010216646 \\
\hline 1799 & 0.00209 & 0.000283072 & 0.010190572 \\
\hline 1800 & 0.00209 & 0.00028235 & 0.01016455 \\
\hline 1801 & 0.00208 & 0.000281629 & 0.010138629 \\
\hline 1802 & 0.00207 & 0.000280911 & 0.010112761 \\
\hline 1803 & 0.00206 & 0.000280194 & 0.010086994 \\
\hline 1804 & 0.00206 & 0.00027948 & 0.01006128 \\
\hline 1805 & 0.00205 & 0.000278768 & 0.010035618 \\
\hline 1806 & 0.00204 & 0.000278057 & 0.010010057 \\
\hline 1807 & 0.00203 & 0.000277349 & 0.009984549 \\
\hline 1808 & 0.00203 & 0.000276642 & 0.009959092 \\
\hline 1809 & 0.00201 & 0.000275938 & 0.009933738 \\
\hline 1810 & 0.00201 & 0.000275236 & 0.009908486 \\
\hline 1811 & 0.002 & 0.000274535 & 0.009883235 \\
\hline 1812 & 0.002 & 0.000273837 & 0.009858087 \\
\hline 1813 & 0.00198 & 0.00027314 & 0.00983304 \\
\hline 1814 & 0.00198 & 0.000272445 & 0.009808045 \\
\hline 1815 & 0.00197 & 0.000271753 & 0.009783103 \\
\hline 1816 & 0.00197 & 0.000271062 & 0.009758212 \\
\hline 1817 & 0.00195 & 0.000270373 & 0.009733423 \\
\hline 1818 & 0.00195 & 0.000269687 & 0.009708687 \\
\hline 1819 & 0.00194 & 0.000269002 & 0.009684052 \\
\hline 1820 & 0.00193 & 0.000268319 & 0.009659469 \\
\hline 1821 & 0.00192 & 0.000267638 & 0.009634938 \\
\hline 1822 & 0.00192 & 0.000266958 & 0.009610508 \\
\hline 1823 & 0.00191 & 0.000266281 & 0.009586131 \\
\hline 1824 & 0.0019 & 0.000265606 & 0.009561806 \\
\hline 1825 & 0.00189 & 0.000264932 & 0.009537532 \\
\hline 1826 & 0.00189 & 0.000264261 & 0.009513361 \\
\hline 1827 & 0.00188 & 0.000263591 & 0.009489241 \\
\hline 1828 & 0.00187 & 0.000262923 & 0.009465223 \\
\hline 1829 & 0.00186 & 0.000262257 & 0.009441257 \\
\hline 1830 & 0.00186 & 0.000261593 & 0.009417343 \\
\hline 1831 & 0.00185 & 0.000260931 & 0.009393481 \\
\hline 1832 & 0.00184 & 0.00026027 & 0.00936972 \\
\hline 1833 & 0.00183 & 0.000259612 & 0.009346012 \\
\hline 1834 & 0.00183 & 0.000258955 & 0.009322355 \\
\hline 1835 & 0.00182 & 0.0002583 & 0.0092988 \\
\hline 1836 & 0.00181 & 0.000257647 & 0.009275297 \\
\hline 1837 & 0.0018 & 0.000256996 & 0.009251846 \\
\hline 1838 & 0.0018 & 0.000256346 & 0.009228446 \\
\hline 1839 & 0.00178 & 0.000255699 & 0.009205149 \\
\hline 1840 & 0.00178 & 0.000255053 & 0.009181903 \\
\hline 1841 & 0.00177 & 0.000254409 & 0.009158709 \\
\hline 1842 & 0.00177 & 0.000253767 & 0.009135617 \\
\hline 1843 & 0.00175 & 0.000253126 & 0.009112526 \\
\hline 1844 & 0.00175 & 0.000252488 & 0.009089538 \\
\hline 1845 & 0.00174 & 0.000251851 & 0.009066601 \\
\hline 1846 & 0.00174 & 0.000251216 & 0.009043766 \\
\hline 1847 & 0.00172 & 0.000250583 & 0.009020983 \\
\hline 1848 & 0.00172 & 0.000249951 & 0.008998251 \\
\hline 1849 & 0.00171 & 0.000249321 & 0.008975571 \\
\hline 1850 & 0.0017 & 0.000248693 & 0.008952943 \\
\hline 1851 & 0.00169 & 0.000248067 & 0.008930417 \\
\hline 1852 & 0.00169 & 0.000247442 & 0.008907942 \\
\hline 1853 & 0.00168 & 0.00024682 & 0.00888552 \\
\hline 1854 & 0.00167 & 0.000246199 & 0.008863149 \\
\hline 1855 & 0.00166 & 0.000245579 & 0.008840829 \\
\hline 1856 & 0.00166 & 0.000244962 & 0.008818612 \\
\hline 1857 & 0.00165 & 0.000244346 & 0.008796446 \\
\hline 1858 & 0.00164 & 0.000243732 & 0.008774332 \\
\hline 1859 & 0.00163 & 0.000243119 & 0.008752269 \\
\hline 1860 & 0.00163 & 0.000242508 & 0.008730308 \\
\hline 1861 & 0.00161 & 0.000241899 & 0.008708349 \\
\hline
\end{tabular}




\begin{tabular}{|c|c|c|c|}
\hline 1862 & 0.00161 & 0.000241292 & 0.008686492 \\
\hline 1863 & 0.0016 & 0.000240686 & 0.008664686 \\
\hline 1864 & 0.0016 & 0.000240082 & 0.008642932 \\
\hline 1865 & 0.00158 & 0.00023948 & 0.00862128 \\
\hline 1866 & 0.00158 & 0.000238879 & 0.008599629 \\
\hline 1867 & 0.00157 & 0.00023828 & 0.00857808 \\
\hline 1868 & 0.00157 & 0.000237683 & 0.008556583 \\
\hline 1869 & 0.00155 & 0.000237087 & 0.008535137 \\
\hline 1870 & 0.00155 & 0.000236493 & 0.008513743 \\
\hline 1871 & 0.00154 & 0.000235901 & 0.008492451 \\
\hline 1872 & 0.00153 & 0.00023531 & 0.00847116 \\
\hline 1873 & 0.00152 & 0.000234721 & 0.008449971 \\
\hline 1874 & 0.00152 & 0.000234134 & 0.008428834 \\
\hline 1875 & 0.00151 & 0.000233548 & 0.008407698 \\
\hline 1876 & 0.0015 & 0.000232964 & 0.008386714 \\
\hline 1877 & 0.00149 & 0.000232381 & 0.008365731 \\
\hline 1878 & 0.00149 & 0.0002318 & 0.0083448 \\
\hline 1879 & 0.00147 & 0.000231221 & 0.008323971 \\
\hline 1880 & 0.00147 & 0.000230643 & 0.008303143 \\
\hline 1881 & 0.00146 & 0.000230067 & 0.008282417 \\
\hline 1882 & 0.00146 & 0.000229493 & 0.008261743 \\
\hline 1883 & 0.00144 & 0.00022892 & 0.00824112 \\
\hline 1884 & 0.00144 & 0.000228348 & 0.008220548 \\
\hline 1885 & 0.00143 & 0.000227779 & 0.008200029 \\
\hline 1886 & 0.00142 & 0.00022721 & 0.00817956 \\
\hline 1887 & 0.00141 & 0.000226644 & 0.008159144 \\
\hline 1888 & 0.00141 & 0.000226079 & 0.008138829 \\
\hline 1889 & 0.0014 & 0.000225515 & 0.008118565 \\
\hline 1890 & 0.00139 & 0.000224953 & 0.008098303 \\
\hline 1891 & 0.00138 & 0.000224393 & 0.008078143 \\
\hline 1892 & 0.00138 & 0.000223834 & 0.008058034 \\
\hline 1893 & 0.00136 & 0.000223277 & 0.008037977 \\
\hline 1894 & 0.00136 & 0.000222721 & 0.008017971 \\
\hline 1895 & 0.00135 & 0.000222167 & 0.007998017 \\
\hline 1896 & 0.00135 & 0.000221614 & 0.007978114 \\
\hline 1897 & 0.00133 & 0.000221063 & 0.007958263 \\
\hline 1898 & 0.00133 & 0.000220513 & 0.007938463 \\
\hline 1899 & 0.00132 & 0.000219965 & 0.007918765 \\
\hline 1900 & 0.00131 & 0.000219419 & 0.007899069 \\
\hline 1901 & 0.0013 & 0.000218874 & 0.007879424 \\
\hline 1902 & 0.0013 & 0.00021833 & 0.00785988 \\
\hline 1903 & 0.00128 & 0.000217788 & 0.007840338 \\
\hline 1904 & 0.00128 & 0.000217247 & 0.007820897 \\
\hline 1905 & 0.00127 & 0.000216708 & 0.007801508 \\
\hline 1906 & 0.00126 & 0.000216171 & 0.007782121 \\
\hline 1907 & 0.00125 & 0.000215634 & 0.007762834 \\
\hline 1908 & 0.00125 & 0.0002151 & 0.0077436 \\
\hline 1909 & 0.00123 & 0.000214567 & 0.007724367 \\
\hline 1910 & 0.00123 & 0.000214035 & 0.007705235 \\
\hline 1911 & 0.00122 & 0.000213505 & 0.007686155 \\
\hline 1912 & 0.00122 & 0.000212976 & 0.007667126 \\
\hline 1913 & 0.0012 & 0.000212449 & 0.007648149 \\
\hline 1914 & 0.0012 & 0.000211923 & 0.007629223 \\
\hline 1915 & 0.00118 & 0.000211398 & 0.007610348 \\
\hline 1916 & 0.00118 & 0.000210875 & 0.007591525 \\
\hline 1917 & 0.00117 & 0.000210354 & 0.007572704 \\
\hline 1918 & 0.00117 & 0.000209834 & 0.007553984 \\
\hline 1919 & 0.00115 & 0.000209315 & 0.007535315 \\
\hline 1920 & 0.00115 & 0.000208798 & 0.007516698 \\
\hline 1921 & 0.00113 & 0.000208282 & 0.007498132 \\
\hline 1922 & 0.00113 & 0.000207767 & 0.007479617 \\
\hline 1923 & 0.00112 & 0.000207254 & 0.007461154 \\
\hline 1924 & 0.00111 & 0.000206743 & 0.007442743 \\
\hline 1925 & 0.0011 & 0.000206233 & 0.007424383 \\
\hline 1926 & 0.0011 & 0.000205724 & 0.007406024 \\
\hline 1927 & 0.00108 & 0.000205216 & 0.007387766 \\
\hline 1928 & 0.00108 & 0.00020471 & 0.00736956 \\
\hline 1929 & 0.00106 & 0.000204206 & 0.007351406 \\
\hline 1930 & 0.00106 & 0.000203703 & 0.0073333303 \\
\hline 1931 & 0.00105 & 0.000203201 & 0.007315201 \\
\hline 1932 & 0.00105 & 0.0002027 & 0.0072972 \\
\hline 1933 & 0.00103 & 0.000202201 & 0.007279251 \\
\hline 1934 & 0.00103 & 0.000201703 & 0.007261303 \\
\hline 1935 & 0.00101 & 0.000201207 & 0.007243457 \\
\hline 1936 & 0.00101 & 0.000200712 & 0.007225612 \\
\hline 1937 & 0.000994 & 0.000200218 & 0.007207868 \\
\hline 1938 & 0.000992 & 0.000199726 & 0.007190126 \\
\hline 1939 & 0.000976 & 0.000199235 & 0.007172435 \\
\hline 1940 & 0.000974 & 0.000198745 & 0.007154845 \\
\hline 1941 & 0.000957 & 0.000198257 & 0.007137257 \\
\hline
\end{tabular}




\begin{tabular}{|c|c|c|c|}
\hline 1942 & 0.000956 & 0.00019777 & 0.00711972 \\
\hline 1943 & 0.000939 & 0.000197285 & 0.007102235 \\
\hline 1944 & 0.000937 & 0.0001968 & 0.0070848 \\
\hline 1945 & 0.00092 & 0.000196317 & 0.007067417 \\
\hline 1946 & 0.000918 & 0.000195836 & 0.007050086 \\
\hline 1947 & 0.000901 & 0.000195356 & 0.007032806 \\
\hline 1948 & 0.0009 & 0.000194877 & 0.007015527 \\
\hline 1949 & 0.000882 & 0.000194399 & 0.006998349 \\
\hline 1950 & 0.00088 & 0.000193923 & 0.006981223 \\
\hline 1951 & 0.000863 & 0.000193447 & 0.006964097 \\
\hline 1952 & 0.000861 & 0.000192974 & 0.006947024 \\
\hline 1953 & 0.000843 & 0.000192501 & 0.006930051 \\
\hline 1954 & 0.000841 & 0.00019203 & 0.00691308 \\
\hline 1955 & 0.000823 & 0.00019156 & 0.00689616 \\
\hline 1956 & 0.000822 & 0.000191091 & 0.006879291 \\
\hline 1957 & 0.000803 & 0.000190624 & 0.006862474 \\
\hline 1958 & 0.000801 & 0.000190158 & 0.006845658 \\
\hline 1959 & 0.000782 & 0.000189693 & 0.006828943 \\
\hline 1960 & 0.000781 & 0.00018923 & 0.00681228 \\
\hline 1961 & 0.000761 & 0.000188767 & 0.006795617 \\
\hline 1962 & 0.00076 & 0.000188306 & 0.006779006 \\
\hline 1963 & 0.00074 & 0.000187847 & 0.006762447 \\
\hline 1964 & 0.000739 & 0.000187388 & 0.006745988 \\
\hline 1965 & 0.000718 & 0.000186931 & 0.006729481 \\
\hline 1966 & 0.000717 & 0.000186475 & 0.006713075 \\
\hline 1967 & 0.000696 & 0.00018602 & 0.00669672 \\
\hline 1968 & 0.000695 & 0.000185567 & 0.006680367 \\
\hline 1969 & 0.000673 & 0.000185114 & 0.006664114 \\
\hline 1970 & 0.000672 & 0.000184663 & 0.006647863 \\
\hline 1971 & 0.00065 & 0.000184213 & 0.006631663 \\
\hline 1972 & 0.000649 & 0.000183765 & 0.006615515 \\
\hline 1973 & 0.000626 & 0.000183317 & 0.006599417 \\
\hline 1974 & 0.000625 & 0.000182871 & 0.006583371 \\
\hline 1975 & 0.000601 & 0.000182426 & 0.006567326 \\
\hline 1976 & 0.0006 & 0.000181983 & 0.006551383 \\
\hline 1977 & 0.000576 & 0.00018154 & 0.00653544 \\
\hline 1978 & 0.000575 & 0.000181099 & 0.006519549 \\
\hline 1979 & 0.00055 & 0.000180659 & 0.006503709 \\
\hline 1980 & 0.000549 & 0.00018022 & 0.00648792 \\
\hline 1981 & 0.000522 & 0.000179782 & 0.006472132 \\
\hline 1982 & 0.000521 & 0.000179345 & 0.006456445 \\
\hline 1983 & 0.000493 & 0.00017891 & 0.00644076 \\
\hline 1984 & 0.000493 & 0.000178476 & 0.006425126 \\
\hline 1985 & 0.000463 & 0.000178043 & 0.006409543 \\
\hline 1986 & 0.000463 & 0.000177611 & 0.006393961 \\
\hline 1987 & 0.000431 & 0.00017718 & 0.00637848 \\
\hline 1988 & 0.000431 & 0.000176751 & 0.006363001 \\
\hline 1989 & 0.000397 & 0.000176322 & 0.006347572 \\
\hline 1990 & 0.000397 & 0.000175895 & 0.006332195 \\
\hline 1991 & 0.00036 & 0.000175469 & 0.006316869 \\
\hline 1992 & 0.00036 & 0.000175044 & 0.006301594 \\
\hline 1993 & 0.000319 & 0.00017462 & 0.00628632 \\
\hline 1994 & 0.000319 & 0.000174198 & 0.006271098 \\
\hline 1995 & 0.000273 & 0.000173776 & 0.006255926 \\
\hline 1996 & 0.000273 & 0.000173356 & 0.006240806 \\
\hline 1997 & 0.000218 & 0.000172937 & 0.006225737 \\
\hline 1998 & 0.000218 & 0.000172519 & 0.006210669 \\
\hline 1999 & 0.000145 & 0.000172102 & 0.006195652 \\
\hline 2000 & & 0.000171686 & 0.006180686 \\
\hline 2001 & & 0.000171272 & 0.006165772 \\
\hline 2002 & & 0.000170858 & 0.006150908 \\
\hline 2003 & & 0.000170446 & 0.006136046 \\
\hline 2004 & & 0.000170035 & 0.006121235 \\
\hline 2005 & & 0.000169624 & 0.006106474 \\
\hline 2006 & & 0.000169215 & 0.006091765 \\
\hline 2007 & & 0.000168807 & 0.006077057 \\
\hline 2008 & & 0.0001684 & 0.0060624 \\
\hline 2009 & & 0.000167995 & 0.006047795 \\
\hline 2010 & & 0.00016759 & 0.00603324 \\
\hline 2011 & & 0.000167186 & 0.006018686 \\
\hline 2012 & & 0.000166784 & 0.006004234 \\
\hline 2013 & & 0.000166383 & 0.005989783 \\
\hline 2014 & & 0.000165982 & 0.005975332 \\
\hline 2015 & & 0.000165583 & 0.005960983 \\
\hline 2016 & & 0.000165185 & 0.005946635 \\
\hline 2017 & & 0.000164788 & 0.005932338 \\
\hline 2018 & & 0.000164392 & 0.005918092 \\
\hline 2019 & & 0.000163997 & 0.005903897 \\
\hline 2020 & & 0.000163603 & 0.005889703 \\
\hline 2021 & & 0.00016321 & 0.00587556 \\
\hline
\end{tabular}




\begin{tabular}{|c|c|c|}
\hline 2022 & 0.000162818 & 0.005861468 \\
\hline 2023 & 0.000162427 & 0.005847377 \\
\hline 2024 & 0.000162038 & 0.005833338 \\
\hline 2025 & 0.000161649 & 0.005819349 \\
\hline 2026 & 0.000161262 & 0.005805412 \\
\hline 2027 & 0.000160875 & 0.005791475 \\
\hline 2028 & 0.00016049 & 0.00577764 \\
\hline 2029 & 0.000160105 & 0.005763755 \\
\hline 2030 & 0.000159722 & 0.005749972 \\
\hline 2031 & 0.000159339 & 0.005736189 \\
\hline 2032 & 0.000158958 & 0.005722458 \\
\hline 2033 & 0.000158578 & 0.005708778 \\
\hline 2034 & 0.000158198 & 0.005695148 \\
\hline 2035 & 0.00015782 & 0.00568152 \\
\hline 2036 & 0.000157443 & 0.005667943 \\
\hline 2037 & 0.000157066 & 0.005654366 \\
\hline 2038 & 0.000156691 & 0.005640891 \\
\hline 2039 & 0.000156317 & 0.005627417 \\
\hline 2040 & 0.000155944 & 0.005613944 \\
\hline 2041 & 0.000155571 & 0.005600571 \\
\hline 2042 & 0.0001552 & 0.0055872 \\
\hline 2043 & 0.00015483 & 0.00557388 \\
\hline 2044 & 0.00015446 & 0.00556056 \\
\hline 2045 & 0.000154092 & 0.005547292 \\
\hline 2046 & 0.000153725 & 0.005534075 \\
\hline 2047 & 0.000153359 & 0.005520909 \\
\hline 2048 & 0.000152993 & 0.005507743 \\
\hline 2049 & 0.000152629 & 0.005494629 \\
\hline 2050 & 0.000152265 & 0.005481565 \\
\hline 2051 & 0.000151903 & 0.005468503 \\
\hline 2052 & 0.000151542 & 0.005455492 \\
\hline 2053 & 0.000151181 & 0.005442531 \\
\hline 2054 & 0.000150822 & 0.005429572 \\
\hline 2055 & 0.000150463 & 0.005416663 \\
\hline 2056 & 0.000150105 & 0.005403805 \\
\hline 2057 & 0.000149749 & 0.005390949 \\
\hline 2058 & 0.000149393 & 0.005378143 \\
\hline 2059 & 0.000149038 & 0.005365388 \\
\hline 2060 & 0.000148684 & 0.005352634 \\
\hline 2061 & 0.000148332 & 0.005339932 \\
\hline 2062 & 0.00014798 & 0.00532728 \\
\hline 2063 & 0.000147629 & 0.005314629 \\
\hline 2064 & 0.000147279 & 0.005302029 \\
\hline 2065 & 0.00014693 & 0.00528943 \\
\hline 2066 & 0.000146581 & 0.005276931 \\
\hline 2067 & 0.000146234 & 0.005264434 \\
\hline 2068 & 0.000145888 & 0.005251938 \\
\hline 2069 & 0.000145542 & 0.005239492 \\
\hline 2070 & 0.000145198 & 0.005227098 \\
\hline 2071 & 0.000144854 & 0.005214754 \\
\hline 2072 & 0.000144512 & 0.005202412 \\
\hline 2073 & 0.00014417 & 0.00519012 \\
\hline 2074 & 0.000143829 & 0.005177829 \\
\hline 2075 & 0.000143489 & 0.005165589 \\
\hline 2076 & 0.00014315 & 0.0051534 \\
\hline 2077 & 0.000142812 & 0.005141222 \\
\hline 2078 & 0.000142475 & 0.005129085 \\
\hline 2079 & 0.000142138 & 0.005116973 \\
\hline 2080 & 0.000141803 & 0.005104898 \\
\hline 2081 & 0.000141468 & 0.005092853 \\
\hline 2082 & 0.000141135 & 0.005080845 \\
\hline 2083 & 0.000140802 & 0.005068862 \\
\hline 2084 & 0.00014047 & 0.005056915 \\
\hline 2085 & 0.000140139 & 0.005044999 \\
\hline 2086 & 0.000139809 & 0.005033114 \\
\hline 2087 & 0.00013948 & 0.00502126 \\
\hline 2088 & 0.000139151 & 0.005009441 \\
\hline 2089 & 0.000138824 & 0.004997649 \\
\hline 2090 & 0.000138497 & 0.004985892 \\
\hline 2091 & 0.000138171 & 0.004974161 \\
\hline 2092 & 0.000137847 & 0.004962467 \\
\hline 2093 & 0.000137522 & 0.004950797 \\
\hline 2094 & 0.000137199 & 0.004939164 \\
\hline 2095 & 0.000136877 & 0.004927557 \\
\hline 2096 & 0.000136555 & 0.004915985 \\
\hline 2097 & 0.000136235 & 0.004904445 \\
\hline 2098 & 0.000135915 & 0.00489293 \\
\hline 2099 & 0.000135596 & 0.004881446 \\
\hline 2100 & 0.000135278 & 0.004869998 \\
\hline 2101 & 0.000134961 & 0.004858576 \\
\hline
\end{tabular}




\begin{tabular}{|c|c|c|}
\hline 2102 & 0.000134644 & 0.004847184 \\
\hline 2103 & 0.000134329 & 0.004835824 \\
\hline 2104 & 0.000134014 & 0.004824494 \\
\hline 2105 & 0.0001337 & 0.00481319 \\
\hline 2106 & 0.000133387 & 0.004801917 \\
\hline 2107 & 0.000133075 & 0.004790675 \\
\hline 2108 & 0.000132763 & 0.004779463 \\
\hline 2109 & 0.000132453 & 0.004768283 \\
\hline 2110 & 0.000132143 & 0.004757128 \\
\hline 2111 & 0.000131834 & 0.004746004 \\
\hline 2112 & 0.000131526 & 0.004734911 \\
\hline 2113 & 0.000131218 & 0.004723848 \\
\hline 2114 & 0.000130912 & 0.004712812 \\
\hline 2115 & 0.000130606 & 0.004701806 \\
\hline 2116 & 0.000130301 & 0.004690826 \\
\hline 2117 & 0.000129997 & 0.004679877 \\
\hline 2118 & 0.000129694 & 0.004668959 \\
\hline 2119 & 0.000129391 & 0.004658066 \\
\hline 2120 & 0.000129089 & 0.004647204 \\
\hline 2121 & 0.000128788 & 0.004636368 \\
\hline 2122 & 0.000128488 & 0.004625563 \\
\hline 2123 & 0.000128189 & 0.004614784 \\
\hline 2124 & 0.00012789 & 0.00460404 \\
\hline 2125 & 0.000127592 & 0.004593317 \\
\hline 2126 & 0.000127295 & 0.004582625 \\
\hline 2127 & 0.000126999 & 0.004571959 \\
\hline 2128 & 0.000126704 & 0.004561324 \\
\hline 2129 & 0.000126409 & 0.004550714 \\
\hline 2130 & 0.000126115 & 0.004540135 \\
\hline 2131 & 0.000125822 & 0.004529582 \\
\hline 2132 & 0.00012553 & 0.00451906 \\
\hline 2133 & 0.000125238 & 0.004508558 \\
\hline 2134 & 0.000124947 & 0.004498092 \\
\hline 2135 & 0.000124657 & 0.004487647 \\
\hline 2136 & 0.000124368 & 0.004477233 \\
\hline 2137 & 0.000124079 & 0.004466844 \\
\hline 2138 & 0.000123791 & 0.004456481 \\
\hline 2139 & 0.000123504 & 0.004446149 \\
\hline 2140 & 0.000123218 & 0.004435843 \\
\hline 2141 & 0.000122933 & 0.004425563 \\
\hline 2142 & 0.000122648 & 0.004415313 \\
\hline 2143 & 0.000122364 & 0.004405084 \\
\hline 2144 & 0.00012208 & 0.004394885 \\
\hline 2145 & 0.000121798 & 0.004384713 \\
\hline 2146 & 0.000121516 & 0.004374571 \\
\hline 2147 & 0.000121235 & 0.00436445 \\
\hline 2148 & 0.000120955 & 0.004354355 \\
\hline 2149 & 0.000120675 & 0.00434429 \\
\hline 2150 & 0.000120396 & 0.004334251 \\
\hline 2151 & 0.000120118 & 0.004324238 \\
\hline 2152 & 0.00011984 & 0.00431425 \\
\hline 2153 & 0.000119564 & 0.004304284 \\
\hline 2154 & 0.000119288 & 0.004294353 \\
\hline 2155 & 0.000119012 & 0.004284442 \\
\hline 2156 & 0.000118738 & 0.004274558 \\
\hline 2157 & 0.000118464 & 0.004264699 \\
\hline 2158 & 0.000118191 & 0.004254866 \\
\hline 2159 & 0.000117919 & 0.004245059 \\
\hline 2160 & 0.000117647 & 0.004235277 \\
\hline 2161 & 0.000117376 & 0.004225521 \\
\hline 2162 & 0.000117106 & 0.004215791 \\
\hline 2163 & 0.000116836 & 0.004206086 \\
\hline 2164 & 0.000116567 & 0.004196407 \\
\hline 2165 & 0.000116299 & 0.004186754 \\
\hline 2166 & 0.000116031 & 0.004177126 \\
\hline 2167 & 0.000115765 & 0.00416752 \\
\hline 2168 & 0.000115499 & 0.004157939 \\
\hline 2169 & 0.000115233 & 0.004148383 \\
\hline 2170 & 0.000114968 & 0.004138858 \\
\hline 2171 & 0.000114704 & 0.004129349 \\
\hline 2172 & 0.000114441 & 0.004119871 \\
\hline 2173 & 0.000114178 & 0.004110413 \\
\hline 2174 & 0.000113916 & 0.004100986 \\
\hline 2175 & 0.000113655 & 0.004091575 \\
\hline 2176 & 0.000113395 & 0.004082195 \\
\hline 2177 & 0.000113135 & 0.004072835 \\
\hline 2178 & 0.000112875 & 0.004063505 \\
\hline 2179 & 0.000112617 & 0.004054197 \\
\hline 2180 & 0.000112359 & 0.004044909 \\
\hline 2181 & 0.000112102 & 0.004035647 \\
\hline
\end{tabular}




\begin{tabular}{|c|c|c|}
\hline 2182 & 0.000111845 & 0.00402641 \\
\hline 2183 & 0.000111589 & 0.004017199 \\
\hline 2184 & 0.000111334 & 0.004008009 \\
\hline 2185 & 0.000111079 & 0.003998844 \\
\hline 2186 & 0.000110825 & 0.0039897 \\
\hline 2187 & 0.000110572 & 0.003980582 \\
\hline 2188 & 0.000110319 & 0.003971489 \\
\hline 2189 & 0.000110067 & 0.003962417 \\
\hline 2190 & 0.000109816 & 0.003953371 \\
\hline 2191 & 0.000109565 & 0.003944345 \\
\hline 2192 & 0.000109315 & 0.003935345 \\
\hline 2193 & 0.000109066 & 0.003926366 \\
\hline 2194 & 0.000108817 & 0.003917412 \\
\hline 2195 & 0.000108569 & 0.003908484 \\
\hline 2196 & 0.000108322 & 0.003899577 \\
\hline 2197 & 0.000108075 & 0.00389069 \\
\hline 2198 & 0.000107829 & 0.003881829 \\
\hline 2199 & 0.000107583 & 0.003872988 \\
\hline 2200 & 0.000107338 & 0.003864173 \\
\hline 2201 & 0.000107094 & 0.003855379 \\
\hline 2202 & 0.00010685 & 0.003846605 \\
\hline 2203 & 0.000106607 & 0.003837857 \\
\hline 2204 & 0.000106365 & 0.003829135 \\
\hline 2205 & 0.000106123 & 0.003820428 \\
\hline 2206 & 0.000105882 & 0.003811747 \\
\hline 2207 & 0.000105642 & 0.003803092 \\
\hline 2208 & 0.000105402 & 0.003794452 \\
\hline 2209 & 0.000105162 & 0.003785837 \\
\hline 2210 & 0.000104924 & 0.003777249 \\
\hline 2211 & 0.000104686 & 0.003768676 \\
\hline 2212 & 0.000104448 & 0.003760128 \\
\hline 2213 & 0.000104211 & 0.003751601 \\
\hline 2214 & 0.000103975 & 0.0037431 \\
\hline 2215 & 0.00010374 & 0.00373462 \\
\hline 2216 & 0.000103505 & 0.00372616 \\
\hline 2217 & 0.00010327 & 0.00371772 \\
\hline 2218 & 0.000103036 & 0.003709301 \\
\hline 2219 & 0.000102803 & 0.003700908 \\
\hline 2220 & 0.000102571 & 0.003692536 \\
\hline 2221 & 0.000102339 & 0.003684184 \\
\hline 2222 & 0.000102107 & 0.003675852 \\
\hline 2223 & 0.000101876 & 0.003667541 \\
\hline 2224 & 0.000101646 & 0.003659256 \\
\hline 2225 & 0.000101417 & 0.003650987 \\
\hline 2226 & 0.000101188 & 0.003642743 \\
\hline 2227 & 0.000100959 & 0.003634519 \\
\hline 2228 & 0.000100731 & 0.003626316 \\
\hline 2229 & 0.000100504 & 0.003618129 \\
\hline 2230 & 0.000100277 & 0.003609972 \\
\hline 2231 & 0.000100051 & 0.003601831 \\
\hline 2232 & $9.98255 \mathrm{E}-05$ & 0.003593711 \\
\hline 2233 & $9.96005 \mathrm{E}-05$ & 0.003585611 \\
\hline 2234 & 0.000099376 & 0.003577531 \\
\hline 2235 & 0.000099152 & 0.003569477 \\
\hline 2236 & 0.000098929 & 0.003561439 \\
\hline 2237 & 0.000098706 & 0.003553421 \\
\hline 2238 & 0.000098484 & 0.003545429 \\
\hline 2239 & $9.82625 \mathrm{E}-05$ & 0.003537453 \\
\hline 2240 & $9.80415 \mathrm{E}-05$ & 0.003529497 \\
\hline 2241 & 0.000097821 & 0.003521561 \\
\hline 2242 & $9.76015 \mathrm{E}-05$ & 0.003513647 \\
\hline 2243 & 0.000097382 & 0.003505752 \\
\hline 2244 & $9.71635 \mathrm{E}-05$ & 0.003497879 \\
\hline 2245 & 0.000096945 & 0.003490025 \\
\hline 2246 & $9.67275 \mathrm{E}-05$ & 0.003482193 \\
\hline 2247 & $9.65105 \mathrm{E}-05$ & 0.003474376 \\
\hline 2248 & 0.000096294 & 0.003466584 \\
\hline 2249 & 0.000096078 & 0.003458808 \\
\hline 2250 & $9.58625 \mathrm{E}-05$ & 0.003451053 \\
\hline 2251 & 9.56475E-05 & 0.003443318 \\
\hline 2252 & $9.54335 \mathrm{E}-05$ & 0.003435604 \\
\hline 2253 & $9.52195 \mathrm{E}-05$ & 0.00342791 \\
\hline 2254 & $9.50065 \mathrm{E}-05$ & 0.003420232 \\
\hline 2255 & 0.000094794 & 0.003412574 \\
\hline 2256 & $9.45815 \mathrm{E}-05$ & 0.003404942 \\
\hline 2257 & 0.00009437 & 0.00339732 \\
\hline 2258 & 0.000094159 & 0.003389724 \\
\hline 2259 & 9.39485E-05 & 0.003382144 \\
\hline 2260 & $9.37385 \mathrm{E}-05$ & 0.003374589 \\
\hline 2261 & 0.000093529 & 0.003367044 \\
\hline
\end{tabular}




\begin{tabular}{|c|c|c|}
\hline 2262 & 0.00009332 & 0.003359525 \\
\hline 2263 & $9.31115 \mathrm{E}-05$ & 0.003352022 \\
\hline 2264 & 0.000092904 & 0.003344539 \\
\hline 2265 & $9.26965 \mathrm{E}-05$ & 0.003337077 \\
\hline 2266 & 0.00009249 & 0.00332963 \\
\hline 2267 & $9.22835 \mathrm{E}-05$ & 0.003322204 \\
\hline 2268 & $9.20775 \mathrm{E}-05$ & 0.003314798 \\
\hline 2269 & $9.18725 \mathrm{E}-05$ & 0.003307408 \\
\hline 2270 & 0.000091668 & 0.003300043 \\
\hline 2271 & $9.14635 \mathrm{E}-05$ & 0.003292689 \\
\hline 2272 & 0.00009126 & 0.00328536 \\
\hline 2273 & $9.10565 \mathrm{E}-05$ & 0.003278042 \\
\hline 2274 & 0.000090854 & 0.003270749 \\
\hline 2275 & 0.000090652 & 0.003263472 \\
\hline 2276 & $9.04505 \mathrm{E}-05$ & 0.003256216 \\
\hline 2277 & 0.000090249 & 0.003248974 \\
\hline 2278 & $9.00485 \mathrm{E}-05$ & 0.003241754 \\
\hline 2279 & $8.98485 \mathrm{E}-05$ & 0.003234549 \\
\hline 2280 & 0.000089649 & 0.003227364 \\
\hline 2281 & 0.00008945 & 0.003220195 \\
\hline 2282 & $8.92515 \mathrm{E}-05$ & 0.003213047 \\
\hline 2283 & 0.000089053 & 0.003205913 \\
\hline 2284 & $8.88555 \mathrm{E}-05$ & 0.003198801 \\
\hline 2285 & $8.86585 \mathrm{E}-05$ & 0.003191709 \\
\hline 2286 & 0.000088462 & 0.003184632 \\
\hline 2287 & 0.000088266 & 0.003177571 \\
\hline 2288 & $8.80705 \mathrm{E}-05$ & 0.003170531 \\
\hline 2289 & 0.000087875 & 0.003163505 \\
\hline 2290 & $8.76805 \mathrm{E}-05$ & 0.003156501 \\
\hline 2291 & $8.74865 \mathrm{E}-05$ & 0.003149512 \\
\hline 2292 & 0.000087293 & 0.003142543 \\
\hline 2293 & $8.70995 \mathrm{E}-05$ & 0.00313559 \\
\hline 2294 & 0.000086907 & 0.003128652 \\
\hline 2295 & 0.000086715 & 0.003121735 \\
\hline 2296 & 0.000086523 & 0.003114833 \\
\hline 2297 & 0.000086332 & 0.003107952 \\
\hline 2298 & $8.61415 \mathrm{E}-05$ & 0.003101087 \\
\hline 2299 & 0.000085951 & 0.003094236 \\
\hline 2300 & $8.57615 \mathrm{E}-05$ & 0.003087407 \\
\hline 2301 & 0.000085572 & 0.003080592 \\
\hline 2302 & 0.000085383 & 0.003073793 \\
\hline 2303 & 0.000085195 & 0.003067015 \\
\hline 2304 & 0.000085007 & 0.003060252 \\
\hline 2305 & $8.48195 \mathrm{E}-05$ & 0.003053505 \\
\hline 2306 & $8.46325 \mathrm{E}-05$ & 0.003046778 \\
\hline 2307 & 0.000084446 & 0.003040066 \\
\hline 2308 & $8.42605 \mathrm{E}-05$ & 0.003033371 \\
\hline 2309 & $8.40745 \mathrm{E}-05$ & 0.00302669 \\
\hline 2310 & $8.38895 \mathrm{E}-05$ & 0.00302003 \\
\hline 2311 & 0.000083705 & 0.003013385 \\
\hline 2312 & 0.000083521 & 0.003006756 \\
\hline 2313 & $8.33375 \mathrm{E}-05$ & 0.003000143 \\
\hline 2314 & 0.000083154 & 0.002993549 \\
\hline 2315 & $8.29715 \mathrm{E}-05$ & 0.002986972 \\
\hline 2316 & 0.000082789 & 0.002980409 \\
\hline 2317 & $8.26075 \mathrm{E}-05$ & 0.002973863 \\
\hline 2318 & 0.000082426 & 0.002967336 \\
\hline 2319 & 0.000082245 & 0.00296082 \\
\hline 2320 & $8.20645 \mathrm{E}-05$ & 0.002954325 \\
\hline 2321 & $8.18845 E-05$ & 0.002947845 \\
\hline 2322 & 0.000081705 & 0.00294138 \\
\hline 2323 & 0.000081526 & 0.002934931 \\
\hline 2324 & 0.000081347 & 0.002928497 \\
\hline 2325 & 0.000081169 & 0.002922084 \\
\hline 2326 & 0.000080991 & 0.002915681 \\
\hline 2327 & 0.000080814 & 0.002909299 \\
\hline 2328 & 0.000080637 & 0.002902932 \\
\hline 2329 & $8.04605 \mathrm{E}-05$ & 0.002896581 \\
\hline 2330 & $8.02845 \mathrm{E}-05$ & 0.002890245 \\
\hline 2331 & 0.000080109 & 0.002883924 \\
\hline 2332 & 0.000079934 & 0.002877619 \\
\hline 2333 & 0.000079759 & 0.002871329 \\
\hline 2334 & 0.000079585 & 0.002865055 \\
\hline 2335 & 0.000079411 & 0.002858796 \\
\hline 2336 & 7.92375E-05 & 0.002852558 \\
\hline 2337 & $7.90645 \mathrm{E}-05$ & 0.00284633 \\
\hline 2338 & 0.000078892 & 0.002840122 \\
\hline 2339 & 0.00007872 & 0.002833925 \\
\hline 2340 & 7.85485E-05 & 0.002827744 \\
\hline 2341 & 0.000078377 & 0.002821577 \\
\hline
\end{tabular}




\begin{tabular}{|c|c|c|}
\hline 2342 & 7.82065E-05 & 0.002815432 \\
\hline 2343 & 0.000078036 & 0.002809296 \\
\hline 2344 & 0.000077866 & 0.002803176 \\
\hline 2345 & 7.76965E-05 & 0.002797077 \\
\hline 2346 & $7.75275 \mathrm{E}-05$ & 0.002790988 \\
\hline 2347 & 7.73585E-05 & 0.002784914 \\
\hline 2348 & 7.71905E-05 & 0.002778856 \\
\hline 2349 & $7.70225 \mathrm{E}-05$ & 0.002772813 \\
\hline 2350 & 0.000076855 & 0.002766785 \\
\hline 2351 & 0.000076688 & 0.002760768 \\
\hline 2352 & 7.65215E-05 & 0.002754772 \\
\hline 2353 & 0.000076355 & 0.00274879 \\
\hline 2354 & $7.61895 \mathrm{E}-05$ & 0.00274282 \\
\hline 2355 & 0.000076024 & 0.002736869 \\
\hline 2356 & 0.000075859 & 0.002730929 \\
\hline 2357 & $7.56945 \mathrm{E}-05$ & 0.002725005 \\
\hline 2358 & 7.55305E-05 & 0.002719096 \\
\hline 2359 & 7.53665E-05 & 0.002713202 \\
\hline 2360 & $7.52035 \mathrm{E}-05$ & 0.002707319 \\
\hline 2361 & $7.50405 \mathrm{E}-05$ & 0.002701456 \\
\hline 2362 & 0.000074878 & 0.002695603 \\
\hline 2363 & 7.47155E-05 & 0.002689766 \\
\hline 2364 & 0.000074554 & 0.002683944 \\
\hline 2365 & $7.43925 \mathrm{E}-05$ & 0.002678138 \\
\hline 2366 & 7.42315E-05 & 0.002672342 \\
\hline 2367 & 0.000074071 & 0.002666561 \\
\hline 2368 & 0.000073911 & 0.002660801 \\
\hline 2369 & 7.37515E-05 & 0.002655047 \\
\hline 2370 & 0.000073592 & 0.002649312 \\
\hline 2371 & 0.000073433 & 0.002643588 \\
\hline 2372 & 7.32745E-05 & 0.00263788 \\
\hline 2373 & 7.31165E-05 & 0.002632187 \\
\hline 2374 & 7.29585E-05 & 0.002626509 \\
\hline 2375 & 0.000072801 & 0.002620841 \\
\hline 2376 & 0.000072644 & 0.002615189 \\
\hline 2377 & 7.24875E-05 & 0.002609553 \\
\hline 2378 & 7.23315E-05 & 0.002603927 \\
\hline 2379 & $7.21755 \mathrm{E}-05$ & 0.002598316 \\
\hline 2380 & 0.00007202 & 0.00259272 \\
\hline 2381 & 0.000071865 & 0.002587135 \\
\hline 2382 & 0.00007171 & 0.00258157 \\
\hline 2383 & 0.000071556 & 0.002576011 \\
\hline 2384 & 0.000071402 & 0.002570472 \\
\hline 2385 & $7.12485 \mathrm{E}-05$ & $2.56 \mathrm{E}-03$ \\
\hline 2386 & 7.10955E-05 & $2.56 \mathrm{E}-03$ \\
\hline 2387 & $7.09425 \mathrm{E}-05$ & $2.55 \mathrm{E}-03$ \\
\hline 2388 & 0.00007079 & $2.55 \mathrm{E}-03$ \\
\hline 2389 & 0.000070638 & $2.54 \mathrm{E}-03$ \\
\hline 2390 & $7.04865 E-05$ & $2.54 \mathrm{E}-03$ \\
\hline 2391 & 0.000070335 & $2.53 \mathrm{E}-03$ \\
\hline 2392 & 0.000070184 & $2.53 \mathrm{E}-03$ \\
\hline 2393 & 7.00335E-05 & 2.52E-03 \\
\hline 2394 & $6.98835 \mathrm{E}-05$ & $2.52 \mathrm{E}-03$ \\
\hline 2395 & $6.97335 \mathrm{E}-05$ & $2.51 \mathrm{E}-03$ \\
\hline 2396 & 0.000069584 & $2.51 \mathrm{E}-03$ \\
\hline 2397 & 0.000069435 & $2.50 \mathrm{E}-03$ \\
\hline 2398 & 0.000069286 & $2.49 \mathrm{E}-03$ \\
\hline 2399 & 0.000069138 & $2.49 \mathrm{E}-03$ \\
\hline 2400 & 0.00006899 & $2.48 \mathrm{E}-03$ \\
\hline 2401 & 0.000068842 & $2.48 \mathrm{E}-03$ \\
\hline 2402 & 0.000068695 & $2.47 \mathrm{E}-03$ \\
\hline 2403 & 0.000068548 & 2.47E-03 \\
\hline 2404 & $6.84015 \mathrm{E}-05$ & 2.46E-03 \\
\hline 2405 & $6.82555 \mathrm{E}-05$ & $2.46 \mathrm{E}-03$ \\
\hline 2406 & $6.81095 \mathrm{E}-05$ & $2.45 \mathrm{E}-03$ \\
\hline 2407 & 0.000067964 & $2.45 \mathrm{E}-03$ \\
\hline 2408 & 0.000067819 & $2.44 \mathrm{E}-03$ \\
\hline 2409 & 0.000067674 & $2.44 \mathrm{E}-03$ \\
\hline 2410 & $6.75295 \mathrm{E}-05$ & $2.43 \mathrm{E}-03$ \\
\hline 2411 & $6.73855 \mathrm{E}-05$ & 2.43E-03 \\
\hline 2412 & 0.000067242 & $2.42 \mathrm{E}-03$ \\
\hline 2413 & $6.70985 \mathrm{E}-05$ & $2.42 \mathrm{E}-03$ \\
\hline 2414 & $6.69555 \mathrm{E}-05$ & $2.41 \mathrm{E}-03$ \\
\hline 2415 & 0.000066813 & $2.41 \mathrm{E}-03$ \\
\hline 2416 & 6.66705E-05 & $2.40 \mathrm{E}-03$ \\
\hline 2417 & 6.65285E-05 & $2.40 \mathrm{E}-03$ \\
\hline 2418 & 0.000066387 & $2.39 \mathrm{E}-03$ \\
\hline 2419 & $6.62455 \mathrm{E}-05$ & $2.38 \mathrm{E}-03$ \\
\hline 2420 & 0.000066105 & $2.38 \mathrm{E}-03$ \\
\hline 2421 & 0.000065964 & 2.37E-03 \\
\hline
\end{tabular}




\begin{tabular}{|c|c|c|}
\hline 2422 & 0.000065824 & 2.37E-03 \\
\hline 2423 & 0.000065684 & 2.36E-03 \\
\hline 2424 & $6.55445 \mathrm{E}-05$ & $2.36 \mathrm{E}-03$ \\
\hline 2425 & 0.000065405 & $2.35 \mathrm{E}-03$ \\
\hline 2426 & $6.52665 \mathrm{E}-05$ & $2.35 \mathrm{E}-03$ \\
\hline 2427 & 0.000065128 & 2.34E-03 \\
\hline 2428 & $6.49895 \mathrm{E}-05$ & $2.34 \mathrm{E}-03$ \\
\hline 2429 & 6.48515E-05 & 2.33E-03 \\
\hline 2430 & 0.000064714 & $2.33 \mathrm{E}-03$ \\
\hline 2431 & 0.000064577 & $2.32 \mathrm{E}-03$ \\
\hline 2432 & 0.00006444 & $2.32 \mathrm{E}-03$ \\
\hline 2433 & $6.43035 \mathrm{E}-05$ & 2.31E-03 \\
\hline 2434 & 6.41675E-05 & 2.31E-03 \\
\hline 2435 & 6.40315E-05 & $2.31 \mathrm{E}-03$ \\
\hline 2436 & 0.000063896 & $2.30 \mathrm{E}-03$ \\
\hline 2437 & $6.37605 \mathrm{E}-05$ & $2.30 \mathrm{E}-03$ \\
\hline 2438 & 0.000063626 & $2.29 \mathrm{E}-03$ \\
\hline 2439 & $6.34915 \mathrm{E}-05$ & $2.29 \mathrm{E}-03$ \\
\hline 2440 & 0.000063357 & $2.28 \mathrm{E}-03$ \\
\hline 2441 & 0.000063223 & $2.28 \mathrm{E}-03$ \\
\hline 2442 & $6.30895 \mathrm{E}-05$ & 2.27E-03 \\
\hline 2443 & $6.29565 \mathrm{E}-05$ & $2.27 \mathrm{E}-03$ \\
\hline 2444 & $6.28235 \mathrm{E}-05$ & $2.26 \mathrm{E}-03$ \\
\hline 2445 & 0.000062691 & $2.26 \mathrm{E}-03$ \\
\hline 2446 & $6.25585 \mathrm{E}-05$ & $2.25 \mathrm{E}-03$ \\
\hline 2447 & 6.24265E-05 & $2.25 \mathrm{E}-03$ \\
\hline 2448 & 0.000062295 & $2.24 \mathrm{E}-03$ \\
\hline 2449 & $6.21635 \mathrm{E}-05$ & $2.24 \mathrm{E}-03$ \\
\hline 2450 & $6.20325 \mathrm{E}-05$ & 2.23E-03 \\
\hline 2451 & 0.000061902 & 2.23E-03 \\
\hline 2452 & $6.17715 \mathrm{E}-05$ & $2.22 \mathrm{E}-03$ \\
\hline 2453 & 6.16415E-05 & $2.22 \mathrm{E}-03$ \\
\hline 2454 & $6.15115 \mathrm{E}-05$ & $2.21 \mathrm{E}-03$ \\
\hline 2455 & 0.000061382 & $2.21 \mathrm{E}-03$ \\
\hline 2456 & 0.000061253 & $2.21 \mathrm{E}-03$ \\
\hline 2457 & 0.000061124 & $2.20 \mathrm{E}-03$ \\
\hline 2458 & 6.09955E-05 & $2.20 \mathrm{E}-03$ \\
\hline 2459 & $6.08675 \mathrm{E}-05$ & 2.19E-03 \\
\hline 2460 & $6.07395 \mathrm{E}-05$ & $2.19 \mathrm{E}-03$ \\
\hline 2461 & 0.000060612 & $2.18 \mathrm{E}-03$ \\
\hline 2462 & $6.04845 \mathrm{E}-05$ & $2.18 \mathrm{E}-03$ \\
\hline 2463 & $6.03575 \mathrm{E}-05$ & 2.17E-03 \\
\hline 2464 & 0.000060231 & 2.17E-03 \\
\hline 2465 & $6.01045 \mathrm{E}-05$ & $2.16 \mathrm{E}-03$ \\
\hline 2466 & $5.99785 \mathrm{E}-05$ & $2.16 \mathrm{E}-03$ \\
\hline 2467 & 0.000059853 & 2.15E-03 \\
\hline 2468 & $5.97275 \mathrm{E}-05$ & 2.15E-03 \\
\hline 2469 & 5.96025E-05 & $2.15 \mathrm{E}-03$ \\
\hline 2470 & 5.94775E-05 & $2.14 \mathrm{E}-03$ \\
\hline 2471 & 0.000059353 & $2.14 \mathrm{E}-03$ \\
\hline 2472 & $5.92285 \mathrm{E}-05$ & $2.13 \mathrm{E}-03$ \\
\hline 2473 & $5.91045 \mathrm{E}-05$ & $2.13 \mathrm{E}-03$ \\
\hline 2474 & 0.000058981 & 2.12E-03 \\
\hline 2475 & $5.88575 \mathrm{E}-05$ & $2.12 \mathrm{E}-03$ \\
\hline 2476 & 5.87345E-05 & $2.11 \mathrm{E}-03$ \\
\hline 2477 & 0.000058612 & $2.11 \mathrm{E}-03$ \\
\hline 2478 & $5.84895 \mathrm{E}-05$ & $2.11 \mathrm{E}-03$ \\
\hline 2479 & 5.83675E-05 & 2.10E-03 \\
\hline 2480 & $5.82455 \mathrm{E}-05$ & $2.10 \mathrm{E}-03$ \\
\hline 2481 & 0.000058124 & 2.09E-03 \\
\hline 2482 & $5.80025 \mathrm{E}-05$ & 2.09E-03 \\
\hline 2483 & $5.78815 \mathrm{E}-05$ & $2.08 \mathrm{E}-03$ \\
\hline 2484 & 0.000057761 & $2.08 \mathrm{E}-03$ \\
\hline 2485 & $5.76405 \mathrm{E}-05$ & $2.08 \mathrm{E}-03$ \\
\hline 2486 & 5.75205E-05 & 2.07E-03 \\
\hline 2487 & 5.74005E-05 & 2.07E-03 \\
\hline 2488 & 0.000057281 & 2.06E-03 \\
\hline 2489 & $5.71615 \mathrm{E}-05$ & 2.06E-03 \\
\hline 2490 & $5.70425 \mathrm{E}-05$ & $2.05 \mathrm{E}-03$ \\
\hline 2491 & 0.000056924 & 2.05E-03 \\
\hline 2492 & $5.68055 \mathrm{E}-05$ & $2.04 \mathrm{E}-03$ \\
\hline 2493 & 5.66875E-05 & $2.04 \mathrm{E}-03$ \\
\hline 2494 & 5.65695E-05 & 2.04E-03 \\
\hline 2495 & 0.000056452 & 2.03E-03 \\
\hline 2496 & $5.63345 \mathrm{E}-05$ & 2.03E-03 \\
\hline 2497 & $5.62175 \mathrm{E}-05$ & 2.02E-03 \\
\hline 2498 & 0.000056101 & $2.02 \mathrm{E}-03$ \\
\hline 2499 & $5.59845 \mathrm{E}-05$ & $2.02 \mathrm{E}-03$ \\
\hline 2500 & 0.000055868 & $2.01 \mathrm{E}-03$ \\
\hline 2501 & $5.57525 \mathrm{E}-05$ & 2.01E-03 \\
\hline
\end{tabular}




\begin{tabular}{|c|c|c|}
\hline 2502 & 5.56365E-05 & 2.00E-03 \\
\hline 2503 & 5.55215E-05 & $2.00 \mathrm{E}-03$ \\
\hline 2504 & 0.000055406 & 1.99E-03 \\
\hline 2505 & $5.52915 \mathrm{E}-05$ & $1.99 \mathrm{E}-03$ \\
\hline 2506 & 0.000055177 & $1.99 \mathrm{E}-03$ \\
\hline 2507 & 5.50625E-05 & $1.98 \mathrm{E}-03$ \\
\hline 2508 & 5.49485E-05 & $1.98 \mathrm{E}-03$ \\
\hline 2509 & 0.000054835 & $1.97 \mathrm{E}-03$ \\
\hline 2510 & $5.47215 \mathrm{E}-05$ & 1.97E-03 \\
\hline 2511 & $5.46085 \mathrm{E}-05$ & $1.97 \mathrm{E}-03$ \\
\hline 2512 & $5.44955 \mathrm{E}-05$ & $1.96 \mathrm{E}-03$ \\
\hline 2513 & 0.000054383 & $1.96 \mathrm{E}-03$ \\
\hline 2514 & 5.42705E-05 & $1.95 \mathrm{E}-03$ \\
\hline 2515 & 5.41585E-05 & $1.95 \mathrm{E}-03$ \\
\hline 2516 & $5.40465 \mathrm{E}-05$ & $1.95 \mathrm{E}-03$ \\
\hline 2517 & 0.000053935 & $1.94 \mathrm{E}-03$ \\
\hline 2518 & $5.38235 \mathrm{E}-05$ & $1.94 \mathrm{E}-03$ \\
\hline 2519 & $5.37125 \mathrm{E}-05$ & $1.93 \mathrm{E}-03$ \\
\hline 2520 & 0.000053602 & $1.93 \mathrm{E}-03$ \\
\hline 2521 & $5.34915 \mathrm{E}-05$ & $1.93 \mathrm{E}-03$ \\
\hline 2522 & 0.000053381 & 1.92E-03 \\
\hline 2523 & 0.000053271 & $1.92 \mathrm{E}-03$ \\
\hline 2524 & 5.31615E-05 & $1.91 \mathrm{E}-03$ \\
\hline 2525 & 0.000053052 & $1.91 \mathrm{E}-03$ \\
\hline 2526 & $5.29425 \mathrm{E}-05$ & $1.91 \mathrm{E}-03$ \\
\hline 2527 & $5.28335 \mathrm{E}-05$ & $1.90 \mathrm{E}-03$ \\
\hline 2528 & 0.000052725 & $1.90 \mathrm{E}-03$ \\
\hline 2529 & 5.26165E-05 & $1.89 \mathrm{E}-03$ \\
\hline 2530 & $5.25085 \mathrm{E}-05$ & $1.89 \mathrm{E}-03$ \\
\hline 2531 & $5.24005 \mathrm{E}-05$ & $1.89 \mathrm{E}-03$ \\
\hline 2532 & 0.000052293 & $1.88 \mathrm{E}-03$ \\
\hline 2533 & $5.21855 \mathrm{E}-05$ & $1.88 \mathrm{E}-03$ \\
\hline 2534 & 0.000052078 & $1.87 \mathrm{E}-03$ \\
\hline 2535 & 5.19715E-05 & $1.87 \mathrm{E}-03$ \\
\hline 2536 & 5.18645E-05 & $1.87 \mathrm{E}-03$ \\
\hline 2537 & 0.000051758 & $1.86 \mathrm{E}-03$ \\
\hline 2538 & 0.000051652 & $1.86 \mathrm{E}-03$ \\
\hline 2539 & 0.000051546 & $1.86 \mathrm{E}-03$ \\
\hline 2540 & 5.14405E-05 & $1.85 \mathrm{E}-03$ \\
\hline 2541 & 0.000051335 & $1.85 \mathrm{E}-03$ \\
\hline 2542 & 0.00005123 & $1.84 \mathrm{E}-03$ \\
\hline 2543 & 0.000051125 & $1.84 \mathrm{E}-03$ \\
\hline 2544 & 5.10205E-05 & $1.84 \mathrm{E}-03$ \\
\hline 2545 & 0.000050916 & $1.83 \mathrm{E}-03$ \\
\hline 2546 & 0.000050812 & $1.83 \mathrm{E}-03$ \\
\hline 2547 & 0.000050708 & $1.83 \mathrm{E}-03$ \\
\hline 2548 & 5.06045E-05 & $1.82 \mathrm{E}-03$ \\
\hline 2549 & 0.000050501 & $1.82 \mathrm{E}-03$ \\
\hline 2550 & 5.03975E-05 & $1.81 \mathrm{E}-03$ \\
\hline 2551 & $5.02945 \mathrm{E}-05$ & $1.81 \mathrm{E}-03$ \\
\hline 2552 & 0.000050192 & $1.81 \mathrm{E}-03$ \\
\hline 2553 & 5.00895E-05 & $1.80 \mathrm{E}-03$ \\
\hline 2554 & $5.00 \mathrm{E}-05$ & $1.80 \mathrm{E}-03$ \\
\hline 2555 & 4.99E-05 & $1.80 \mathrm{E}-03$ \\
\hline 2556 & $4.98 \mathrm{E}-05$ & $1.79 \mathrm{E}-03$ \\
\hline 2557 & 4.97E-05 & $1.79 \mathrm{E}-03$ \\
\hline 2558 & 4.96E-05 & $1.78 \mathrm{E}-03$ \\
\hline 2559 & 4.95E-05 & $1.78 \mathrm{E}-03$ \\
\hline 2560 & 4.94E-05 & $1.78 \mathrm{E}-03$ \\
\hline 2561 & 4.93E-05 & $1.77 \mathrm{E}-03$ \\
\hline 2562 & 4.92E-05 & $1.77 \mathrm{E}-03$ \\
\hline 2563 & 4.91E-05 & 1.77E-03 \\
\hline 2564 & 4.90E-05 & $1.76 \mathrm{E}-03$ \\
\hline 2565 & 4.89E-05 & $1.76 \mathrm{E}-03$ \\
\hline 2566 & $4.88 \mathrm{E}-05$ & $1.76 \mathrm{E}-03$ \\
\hline 2567 & 4.87E-05 & $1.75 \mathrm{E}-03$ \\
\hline 2568 & 4.86E-05 & $1.75 \mathrm{E}-03$ \\
\hline 2569 & 4.85E-05 & $1.75 \mathrm{E}-03$ \\
\hline 2570 & 4.84E-05 & $1.74 \mathrm{E}-03$ \\
\hline 2571 & 4.83E-05 & $1.74 \mathrm{E}-03$ \\
\hline 2572 & 4.82E-05 & $1.73 \mathrm{E}-03$ \\
\hline 2573 & 4.81E-05 & $1.73 \mathrm{E}-03$ \\
\hline 2574 & 4.80E-05 & $1.73 \mathrm{E}-03$ \\
\hline 2575 & 4.79E-05 & $1.72 \mathrm{E}-03$ \\
\hline 2576 & $4.78 \mathrm{E}-05$ & $1.72 \mathrm{E}-03$ \\
\hline 2577 & 4.77E-05 & $1.72 \mathrm{E}-03$ \\
\hline 2578 & 4.76E-05 & $1.71 \mathrm{E}-03$ \\
\hline 2579 & 4.75E-05 & $1.71 \mathrm{E}-03$ \\
\hline 2580 & 4.74E-05 & $1.71 \mathrm{E}-03$ \\
\hline 2581 & 4.73E-05 & $1.70 \mathrm{E}-03$ \\
\hline
\end{tabular}




\begin{tabular}{|c|c|c|}
\hline 2582 & 4.72E-05 & $1.70 \mathrm{E}-03$ \\
\hline 2583 & $4.71 \mathrm{E}-05$ & 1.70E-03 \\
\hline 2584 & $4.70 \mathrm{E}-05$ & $1.69 \mathrm{E}-03$ \\
\hline 2585 & 4.69E-05 & $1.69 \mathrm{E}-03$ \\
\hline 2586 & $4.68 \mathrm{E}-05$ & 1.69E-03 \\
\hline 2587 & 4.67E-05 & $1.68 \mathrm{E}-03$ \\
\hline 2588 & 4.67E-05 & $1.68 \mathrm{E}-03$ \\
\hline 2589 & 4.66E-05 & $1.68 \mathrm{E}-03$ \\
\hline 2590 & 4.65E-05 & 1.67E-03 \\
\hline 2591 & 4.64E-05 & 1.67E-03 \\
\hline 2592 & 4.63E-05 & 1.67E-03 \\
\hline 2593 & 4.62E-05 & $1.66 \mathrm{E}-03$ \\
\hline 2594 & 4.61E-05 & 1.66E-03 \\
\hline 2595 & 4.60E-05 & $1.66 \mathrm{E}-03$ \\
\hline 2596 & 4.59E-05 & $1.65 \mathrm{E}-03$ \\
\hline 2597 & 4.58E-05 & $1.65 \mathrm{E}-03$ \\
\hline 2598 & 4.57E-05 & $1.65 \mathrm{E}-03$ \\
\hline 2599 & 4.56E-05 & $1.64 \mathrm{E}-03$ \\
\hline 2600 & 4.55E-05 & $1.64 \mathrm{E}-03$ \\
\hline 2601 & 4.54E-05 & $1.64 \mathrm{E}-03$ \\
\hline 2602 & 4.54E-05 & 1.63E-03 \\
\hline 2603 & $4.53 \mathrm{E}-05$ & 1.63E-03 \\
\hline 2604 & $4.52 \mathrm{E}-05$ & $1.63 \mathrm{E}-03$ \\
\hline 2605 & 4.51E-05 & $1.62 \mathrm{E}-03$ \\
\hline 2606 & $4.50 \mathrm{E}-05$ & $1.62 \mathrm{E}-03$ \\
\hline 2607 & 4.49E-05 & $1.62 \mathrm{E}-03$ \\
\hline 2608 & 4.48E-05 & $1.61 \mathrm{E}-03$ \\
\hline 2609 & 4.47E-05 & $1.61 \mathrm{E}-03$ \\
\hline 2610 & 4.46E-05 & $1.61 \mathrm{E}-03$ \\
\hline 2611 & 4.45E-05 & $1.60 \mathrm{E}-03$ \\
\hline 2612 & 4.44E-05 & $1.60 \mathrm{E}-03$ \\
\hline 2613 & $4.44 \mathrm{E}-05$ & $1.60 \mathrm{E}-03$ \\
\hline 2614 & $4.43 \mathrm{E}-05$ & $1.59 \mathrm{E}-03$ \\
\hline 2615 & 4.42E-05 & $1.59 \mathrm{E}-03$ \\
\hline 2616 & 4.41E-05 & $1.59 \mathrm{E}-03$ \\
\hline 2617 & $4.40 \mathrm{E}-05$ & $1.58 \mathrm{E}-03$ \\
\hline 2618 & 4.39E-05 & $1.58 \mathrm{E}-03$ \\
\hline 2619 & 4.38E-05 & $1.58 \mathrm{E}-03$ \\
\hline 2620 & 4.37E-05 & 1.57E-03 \\
\hline 2621 & 4.37E-05 & $1.57 \mathrm{E}-03$ \\
\hline 2622 & 4.36E-05 & $1.57 \mathrm{E}-03$ \\
\hline 2623 & 4.35E-05 & $1.57 \mathrm{E}-03$ \\
\hline 2624 & 4.34E-05 & $1.56 \mathrm{E}-03$ \\
\hline 2625 & 4.33E-05 & $1.56 \mathrm{E}-03$ \\
\hline 2626 & $4.32 \mathrm{E}-05$ & $1.56 \mathrm{E}-03$ \\
\hline 2627 & 4.31E-05 & $1.55 \mathrm{E}-03$ \\
\hline 2628 & $4.30 \mathrm{E}-05$ & $1.55 \mathrm{E}-03$ \\
\hline 2629 & $4.30 \mathrm{E}-05$ & $1.55 \mathrm{E}-03$ \\
\hline 2630 & $4.29 \mathrm{E}-05$ & $1.54 \mathrm{E}-03$ \\
\hline 2631 & $4.28 \mathrm{E}-05$ & $1.54 \mathrm{E}-03$ \\
\hline 2632 & $4.27 \mathrm{E}-05$ & $1.54 \mathrm{E}-03$ \\
\hline 2633 & $4.26 \mathrm{E}-05$ & $1.53 \mathrm{E}-03$ \\
\hline 2634 & 4.25E-05 & $1.53 \mathrm{E}-03$ \\
\hline 2635 & 4.25E-05 & $1.53 \mathrm{E}-03$ \\
\hline 2636 & $4.24 \mathrm{E}-05$ & $1.53 \mathrm{E}-03$ \\
\hline 2637 & $4.23 \mathrm{E}-05$ & $1.52 \mathrm{E}-03$ \\
\hline 2638 & $4.22 \mathrm{E}-05$ & $1.52 \mathrm{E}-03$ \\
\hline 2639 & $4.21 \mathrm{E}-05$ & $1.52 \mathrm{E}-03$ \\
\hline 2640 & $4.20 \mathrm{E}-05$ & $1.51 \mathrm{E}-03$ \\
\hline 2641 & 4.19E-05 & $1.51 \mathrm{E}-03$ \\
\hline 2642 & 4.19E-05 & $1.51 \mathrm{E}-03$ \\
\hline 2643 & $4.18 \mathrm{E}-05$ & $1.50 \mathrm{E}-03$ \\
\hline 2644 & 4.17E-05 & $1.50 \mathrm{E}-03$ \\
\hline 2645 & $4.16 \mathrm{E}-05$ & $1.50 \mathrm{E}-03$ \\
\hline 2646 & 4.15E-05 & $1.50 \mathrm{E}-03$ \\
\hline 2647 & 4.15E-05 & $1.49 \mathrm{E}-03$ \\
\hline 2648 & 4.14E-05 & $1.49 \mathrm{E}-03$ \\
\hline 2649 & 4.13E-05 & $1.49 \mathrm{E}-03$ \\
\hline 2650 & $4.12 \mathrm{E}-05$ & $1.48 \mathrm{E}-03$ \\
\hline 2651 & 4.11E-05 & $1.48 \mathrm{E}-03$ \\
\hline 2652 & 4.10E-05 & $1.48 \mathrm{E}-03$ \\
\hline 2653 & 4.10E-05 & 1.47E-03 \\
\hline 2654 & 4.09E-05 & 1.47E-03 \\
\hline 2655 & $4.08 \mathrm{E}-05$ & 1.47E-03 \\
\hline 2656 & 4.07E-05 & 1.47E-03 \\
\hline 2657 & 4.06E-05 & $1.46 \mathrm{E}-03$ \\
\hline 2658 & 4.06E-05 & $1.46 \mathrm{E}-03$ \\
\hline 2659 & 4.05E-05 & $1.46 \mathrm{E}-03$ \\
\hline 2660 & 4.04E-05 & $1.45 \mathrm{E}-03$ \\
\hline 2661 & 4.03E-05 & $1.45 \mathrm{E}-03$ \\
\hline
\end{tabular}




\begin{tabular}{|c|c|c|}
\hline 2662 & 4.02E-05 & 1.45E-03 \\
\hline 2663 & 4.02E-05 & 1.45E-03 \\
\hline 2664 & 4.01E-05 & $1.44 \mathrm{E}-03$ \\
\hline 2665 & $4.00 \mathrm{E}-05$ & $1.44 \mathrm{E}-03$ \\
\hline 2666 & $3.99 \mathrm{E}-05$ & 1.44E-03 \\
\hline 2667 & 3.98E-05 & $1.43 \mathrm{E}-03$ \\
\hline 2668 & $3.98 \mathrm{E}-05$ & $1.43 \mathrm{E}-03$ \\
\hline 2669 & 3.97E-05 & $1.43 \mathrm{E}-03$ \\
\hline 2670 & $3.96 \mathrm{E}-05$ & $1.43 \mathrm{E}-03$ \\
\hline 2671 & 3.95E-05 & $1.42 \mathrm{E}-03$ \\
\hline 2672 & $3.95 \mathrm{E}-05$ & $1.42 \mathrm{E}-03$ \\
\hline 2673 & 3.94E-05 & $1.42 \mathrm{E}-03$ \\
\hline 2674 & 3.93E-05 & $1.41 \mathrm{E}-03$ \\
\hline 2675 & $3.92 \mathrm{E}-05$ & $1.41 \mathrm{E}-03$ \\
\hline 2676 & $3.91 \mathrm{E}-05$ & $1.41 \mathrm{E}-03$ \\
\hline 2677 & 3.91E-05 & $1.41 \mathrm{E}-03$ \\
\hline 2678 & $3.90 \mathrm{E}-05$ & $1.40 \mathrm{E}-03$ \\
\hline 2679 & $3.89 \mathrm{E}-05$ & $1.40 \mathrm{E}-03$ \\
\hline 2680 & $3.88 \mathrm{E}-05$ & 1.40E-03 \\
\hline 2681 & $3.88 \mathrm{E}-05$ & $1.40 \mathrm{E}-03$ \\
\hline 2682 & 3.87E-05 & $1.39 \mathrm{E}-03$ \\
\hline 2683 & $3.86 \mathrm{E}-05$ & $1.39 \mathrm{E}-03$ \\
\hline 2684 & 3.85E-05 & $1.39 \mathrm{E}-03$ \\
\hline 2685 & $3.85 \mathrm{E}-05$ & $1.38 \mathrm{E}-03$ \\
\hline 2686 & $3.84 \mathrm{E}-05$ & $1.38 \mathrm{E}-03$ \\
\hline 2687 & 3.83E-05 & $1.38 \mathrm{E}-03$ \\
\hline 2688 & $3.82 \mathrm{E}-05$ & $1.38 \mathrm{E}-03$ \\
\hline 2689 & $3.82 \mathrm{E}-05$ & 1.37E-03 \\
\hline 2690 & $3.81 \mathrm{E}-05$ & 1.37E-03 \\
\hline 2691 & $3.80 \mathrm{E}-05$ & 1.37E-03 \\
\hline 2692 & $3.79 \mathrm{E}-05$ & $1.37 \mathrm{E}-03$ \\
\hline 2693 & $3.79 \mathrm{E}-05$ & 1.36E-03 \\
\hline 2694 & $3.78 \mathrm{E}-05$ & 1.36E-03 \\
\hline 2695 & $3.77 \mathrm{E}-05$ & 1.36E-03 \\
\hline 2696 & $3.76 \mathrm{E}-05$ & 1.35E-03 \\
\hline 2697 & $3.76 \mathrm{E}-05$ & $1.35 \mathrm{E}-03$ \\
\hline 2698 & $3.75 \mathrm{E}-05$ & $1.35 \mathrm{E}-03$ \\
\hline 2699 & $3.74 \mathrm{E}-05$ & $1.35 \mathrm{E}-03$ \\
\hline 2700 & $3.73 \mathrm{E}-05$ & 1.34E-03 \\
\hline 2701 & 3.73E-05 & $1.34 \mathrm{E}-03$ \\
\hline 2702 & $3.72 \mathrm{E}-05$ & $1.34 \mathrm{E}-03$ \\
\hline 2703 & $3.71 \mathrm{E}-05$ & $1.34 \mathrm{E}-03$ \\
\hline 2704 & $3.71 \mathrm{E}-05$ & 1.33E-03 \\
\hline 2705 & $3.70 \mathrm{E}-05$ & 1.33E-03 \\
\hline 2706 & $3.69 \mathrm{E}-05$ & 1.33E-03 \\
\hline 2707 & $3.68 \mathrm{E}-05$ & 1.33E-03 \\
\hline 2708 & $3.68 \mathrm{E}-05$ & $1.32 \mathrm{E}-03$ \\
\hline 2709 & 3.67E-05 & $1.32 \mathrm{E}-03$ \\
\hline 2710 & 3.66E-05 & $1.32 \mathrm{E}-03$ \\
\hline 2711 & 3.66E-05 & $1.32 \mathrm{E}-03$ \\
\hline 2712 & $3.65 \mathrm{E}-05$ & $1.31 \mathrm{E}-03$ \\
\hline 2713 & $3.64 \mathrm{E}-05$ & $1.31 \mathrm{E}-03$ \\
\hline 2714 & $3.63 \mathrm{E}-05$ & $1.31 \mathrm{E}-03$ \\
\hline 2715 & 3.63E-05 & $1.31 \mathrm{E}-03$ \\
\hline 2716 & $3.62 \mathrm{E}-05$ & $1.30 \mathrm{E}-03$ \\
\hline 2717 & 3.61E-05 & $1.30 \mathrm{E}-03$ \\
\hline 2718 & $3.61 \mathrm{E}-05$ & 1.30E-03 \\
\hline 2719 & 3.60E-05 & $1.30 \mathrm{E}-03$ \\
\hline 2720 & $3.59 \mathrm{E}-05$ & $1.29 \mathrm{E}-03$ \\
\hline 2721 & $3.58 \mathrm{E}-05$ & $1.29 \mathrm{E}-03$ \\
\hline 2722 & $3.58 \mathrm{E}-05$ & $1.29 \mathrm{E}-03$ \\
\hline 2723 & $3.57 \mathrm{E}-05$ & $1.29 \mathrm{E}-03$ \\
\hline 2724 & $3.56 \mathrm{E}-05$ & $1.28 \mathrm{E}-03$ \\
\hline 2725 & $3.56 \mathrm{E}-05$ & $1.28 \mathrm{E}-03$ \\
\hline 2726 & $3.55 \mathrm{E}-05$ & $1.28 \mathrm{E}-03$ \\
\hline 2727 & $3.54 \mathrm{E}-05$ & $1.28 \mathrm{E}-03$ \\
\hline 2728 & $3.54 \mathrm{E}-05$ & $1.27 \mathrm{E}-03$ \\
\hline 2729 & $3.53 \mathrm{E}-05$ & $1.27 \mathrm{E}-03$ \\
\hline 2730 & $3.52 \mathrm{E}-05$ & $1.27 \mathrm{E}-03$ \\
\hline 2731 & $3.52 \mathrm{E}-05$ & $1.27 \mathrm{E}-03$ \\
\hline 2732 & $3.51 \mathrm{E}-05$ & $1.26 \mathrm{E}-03$ \\
\hline 2733 & 3.50E-05 & $1.26 \mathrm{E}-03$ \\
\hline 2734 & $3.50 \mathrm{E}-05$ & $1.26 \mathrm{E}-03$ \\
\hline 2735 & $3.49 \mathrm{E}-05$ & 1.26E-03 \\
\hline 2736 & $3.48 \mathrm{E}-05$ & $1.25 \mathrm{E}-03$ \\
\hline 2737 & $3.48 \mathrm{E}-05$ & $1.25 \mathrm{E}-03$ \\
\hline 2738 & 3.47E-05 & $1.25 \mathrm{E}-03$ \\
\hline 2739 & $3.46 \mathrm{E}-05$ & $1.25 \mathrm{E}-03$ \\
\hline 2740 & $3.46 \mathrm{E}-05$ & $1.24 \mathrm{E}-03$ \\
\hline 2741 & $3.45 \mathrm{E}-05$ & $1.24 \mathrm{E}-03$ \\
\hline
\end{tabular}




\begin{tabular}{|c|c|c|}
\hline 2742 & 3.44E-05 & $1.24 \mathrm{E}-03$ \\
\hline 2743 & $3.44 \mathrm{E}-05$ & $1.24 \mathrm{E}-03$ \\
\hline 2744 & 3.43E-05 & $1.23 \mathrm{E}-03$ \\
\hline 2745 & $3.42 \mathrm{E}-05$ & $1.23 \mathrm{E}-03$ \\
\hline 2746 & $3.42 \mathrm{E}-05$ & $1.23 \mathrm{E}-03$ \\
\hline 2747 & 3.41E-05 & $1.23 \mathrm{E}-03$ \\
\hline 2748 & $3.40 \mathrm{E}-05$ & $1.23 \mathrm{E}-03$ \\
\hline 2749 & $3.40 \mathrm{E}-05$ & $1.22 \mathrm{E}-03$ \\
\hline 2750 & 3.39E-05 & $1.22 \mathrm{E}-03$ \\
\hline 2751 & 3.38E-05 & $1.22 \mathrm{E}-03$ \\
\hline 2752 & $3.38 \mathrm{E}-05$ & $1.22 \mathrm{E}-03$ \\
\hline 2753 & 3.37E-05 & $1.21 \mathrm{E}-03$ \\
\hline 2754 & $3.36 \mathrm{E}-05$ & $1.21 \mathrm{E}-03$ \\
\hline 2755 & 3.36E-05 & $1.21 \mathrm{E}-03$ \\
\hline 2756 & 3.35E-05 & $1.21 \mathrm{E}-03$ \\
\hline 2757 & 3.34E-05 & $1.20 \mathrm{E}-03$ \\
\hline 2758 & 3.34E-05 & $1.20 \mathrm{E}-03$ \\
\hline 2759 & 3.33E-05 & $1.20 \mathrm{E}-03$ \\
\hline 2760 & 3.33E-05 & $1.20 \mathrm{E}-03$ \\
\hline 2761 & 3.32E-05 & 1.19E-03 \\
\hline 2762 & 3.31E-05 & $1.19 \mathrm{E}-03$ \\
\hline 2763 & 3.31E-05 & $1.19 \mathrm{E}-03$ \\
\hline 2764 & $3.30 \mathrm{E}-05$ & 1.19E-03 \\
\hline 2765 & $3.29 \mathrm{E}-05$ & 1.19E-03 \\
\hline 2766 & 3.29E-05 & $1.18 \mathrm{E}-03$ \\
\hline 2767 & $3.28 \mathrm{E}-05$ & $1.18 \mathrm{E}-03$ \\
\hline 2768 & 3.27E-05 & $1.18 \mathrm{E}-03$ \\
\hline 2769 & 3.27E-05 & 1.18E-03 \\
\hline 2770 & 3.26E-05 & 1.17E-03 \\
\hline 2771 & $3.26 \mathrm{E}-05$ & 1.17E-03 \\
\hline 2772 & 3.25E-05 & 1.17E-03 \\
\hline 2773 & $3.24 \mathrm{E}-05$ & $1.17 \mathrm{E}-03$ \\
\hline 2774 & 3.24E-05 & $1.17 \mathrm{E}-03$ \\
\hline 2775 & 3.23E-05 & 1.16E-03 \\
\hline 2776 & 3.23E-05 & 1.16E-03 \\
\hline 2777 & $3.22 \mathrm{E}-05$ & 1.16E-03 \\
\hline 2778 & $3.21 \mathrm{E}-05$ & $1.16 \mathrm{E}-03$ \\
\hline 2779 & 3.21E-05 & $1.15 \mathrm{E}-03$ \\
\hline 2780 & $3.20 \mathrm{E}-05$ & $1.15 \mathrm{E}-03$ \\
\hline 2781 & 3.19E-05 & 1.15E-03 \\
\hline 2782 & 3.19E-05 & 1.15E-03 \\
\hline 2783 & $3.18 \mathrm{E}-05$ & $1.15 \mathrm{E}-03$ \\
\hline 2784 & $3.18 \mathrm{E}-05$ & $1.14 \mathrm{E}-03$ \\
\hline 2785 & 3.17E-05 & $1.14 \mathrm{E}-03$ \\
\hline 2786 & $3.16 \mathrm{E}-05$ & $1.14 \mathrm{E}-03$ \\
\hline 2787 & 3.16E-05 & 1.14E-03 \\
\hline 2788 & 3.15E-05 & 1.13E-03 \\
\hline 2789 & 3.15E-05 & $1.13 \mathrm{E}-03$ \\
\hline 2790 & 3.14E-05 & $1.13 \mathrm{E}-03$ \\
\hline 2791 & 3.13E-05 & $1.13 \mathrm{E}-03$ \\
\hline 2792 & 3.13E-05 & $1.13 \mathrm{E}-03$ \\
\hline 2793 & 3.12E-05 & 1.12E-03 \\
\hline 2794 & 3.12E-05 & 1.12E-03 \\
\hline 2795 & $3.11 \mathrm{E}-05$ & $1.12 \mathrm{E}-03$ \\
\hline 2796 & $3.10 \mathrm{E}-05$ & 1.12E-03 \\
\hline 2797 & 3.10E-05 & $1.12 \mathrm{E}-03$ \\
\hline 2798 & $3.09 \mathrm{E}-05$ & $1.11 \mathrm{E}-03$ \\
\hline 2799 & 3.09E-05 & 1.11E-03 \\
\hline 2800 & $3.08 \mathrm{E}-05$ & $1.11 \mathrm{E}-03$ \\
\hline 2801 & $3.08 \mathrm{E}-05$ & $1.11 \mathrm{E}-03$ \\
\hline 2802 & 3.07E-05 & 1.11E-03 \\
\hline 2803 & 3.06E-05 & $1.10 \mathrm{E}-03$ \\
\hline 2804 & 3.06E-05 & 1.10E-03 \\
\hline 2805 & 3.05E-05 & 1.10E-03 \\
\hline 2806 & $3.05 \mathrm{E}-05$ & 1.10E-03 \\
\hline 2807 & 3.04E-05 & $1.09 \mathrm{E}-03$ \\
\hline 2808 & 3.04E-05 & $1.09 \mathrm{E}-03$ \\
\hline 2809 & 3.03E-05 & $1.09 \mathrm{E}-03$ \\
\hline 2810 & $3.02 \mathrm{E}-05$ & $1.09 \mathrm{E}-03$ \\
\hline 2811 & 3.02E-05 & $1.09 \mathrm{E}-03$ \\
\hline 2812 & 3.01E-05 & $1.08 \mathrm{E}-03$ \\
\hline 2813 & 3.01E-05 & $1.08 \mathrm{E}-03$ \\
\hline 2814 & $3.00 \mathrm{E}-05$ & $1.08 \mathrm{E}-03$ \\
\hline 2815 & $3.00 \mathrm{E}-05$ & $1.08 \mathrm{E}-03$ \\
\hline 2816 & $2.99 \mathrm{E}-05$ & $1.08 \mathrm{E}-03$ \\
\hline 2817 & $2.98 \mathrm{E}-05$ & 1.07E-03 \\
\hline 2818 & $2.98 \mathrm{E}-05$ & $1.07 \mathrm{E}-03$ \\
\hline 2819 & 2.97E-05 & $1.07 \mathrm{E}-03$ \\
\hline 2820 & 2.97E-05 & $1.07 \mathrm{E}-03$ \\
\hline 2821 & $2.96 \mathrm{E}-05$ & 1.07E-03 \\
\hline
\end{tabular}




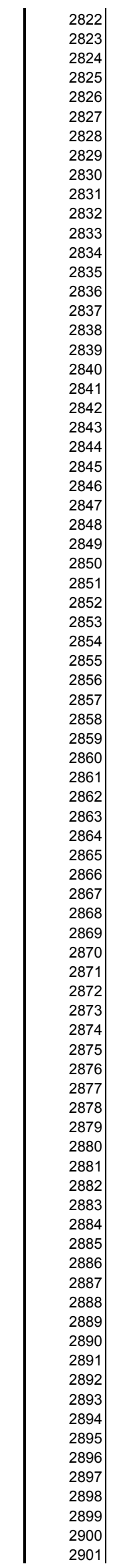

1.06E-03

$1.06 \mathrm{E}-03$

$1.06 \mathrm{E}-03$

$1.06 \mathrm{E}-03$

1.06E-03

$1.05 \mathrm{E}-03$

$1.05 \mathrm{E}-03$

$1.05 \mathrm{E}-03$

$1.05 \mathrm{E}-03$

$1.05 \mathrm{E}-03$

$1.04 \mathrm{E}-03$

$1.04 \mathrm{E}-03$

$1.04 \mathrm{E}-03$

$1.04 \mathrm{E}-03$

$1.04 \mathrm{E}-03$

$1.03 \mathrm{E}-03$

$1.03 \mathrm{E}-03$

1.03E-03

$1.03 \mathrm{E}-03$

$1.03 \mathrm{E}-03$

$1.03 \mathrm{E}-03$

$1.02 \mathrm{E}-03$

$1.02 \mathrm{E}-03$

$1.02 \mathrm{E}-03$

$1.02 \mathrm{E}-03$

$1.02 \mathrm{E}-03$

$1.01 \mathrm{E}-03$

$1.01 \mathrm{E}-03$

$1.01 \mathrm{E}-03$

1.01E-03

1.01E-03

$1.00 \mathrm{E}-03$

$1.00 \mathrm{E}-03$

$1.00 \mathrm{E}-03$

9.99E-04

9.97E-04

9.95E-04

9.93E-04

9.91E-04

9.89E-04

9.88E-04

9.86E-04

9.84E-04

9.82E-04

$9.80 \mathrm{E}-04$

$9.78 \mathrm{E}-04$

9.77E-04

9.75E-04

9.73E-04

9.71E-04

9.69E-04

9.68E-04

9.66E-04

9.64E-04

9.62E-04

9.60E-04

9.59E-04

9.57E-04

9.55E-04

9.53E-04

9.52E-04

9.50E-04

9.48E-04

9.46E-04

9.45E-04

9.43E-04

9.41E-04

9.39E-04

$9.38 \mathrm{E}-04$

9.36E-04

9.34E-04

9.32E-04

9.31E-04

9.29E-04

9.27E-04

9.26E-04

9.24E-04

9.22E-04

9.21E-04

9.19E-04 


\begin{tabular}{|c|c|c|}
\hline 2902 & $2.55 \mathrm{E}-05$ & 9.17E-04 \\
\hline 2903 & $2.54 \mathrm{E}-05$ & $9.16 \mathrm{E}-04$ \\
\hline 2904 & 2.54E-05 & $9.14 \mathrm{E}-04$ \\
\hline 2905 & $2.53 \mathrm{E}-05$ & $9.12 \mathrm{E}-04$ \\
\hline 2906 & 2.53E-05 & $9.10 \mathrm{E}-04$ \\
\hline 2907 & $2.52 \mathrm{E}-05$ & $9.09 \mathrm{E}-04$ \\
\hline 2908 & $2.52 \mathrm{E}-05$ & 9.07E-04 \\
\hline 2909 & $2.52 \mathrm{E}-05$ & $9.05 \mathrm{E}-04$ \\
\hline 2910 & $2.51 \mathrm{E}-05$ & $9.04 \mathrm{E}-04$ \\
\hline 2911 & $2.51 \mathrm{E}-05$ & $9.02 \mathrm{E}-04$ \\
\hline 2912 & $2.50 \mathrm{E}-05$ & $9.01 \mathrm{E}-04$ \\
\hline 2913 & $2.50 \mathrm{E}-05$ & 8.99E-04 \\
\hline 2914 & $2.49 \mathrm{E}-05$ & 8.97E-04 \\
\hline 2915 & $2.49 \mathrm{E}-05$ & 8.96E-04 \\
\hline 2916 & $2.48 \mathrm{E}-05$ & 8.94E-04 \\
\hline 2917 & $2.48 \mathrm{E}-05$ & $8.92 \mathrm{E}-04$ \\
\hline 2918 & $2.47 \mathrm{E}-05$ & $8.91 \mathrm{E}-04$ \\
\hline 2919 & 2.47E-05 & $8.89 \mathrm{E}-04$ \\
\hline 2920 & 2.47E-05 & 8.87E-04 \\
\hline 2921 & $2.46 \mathrm{E}-05$ & 8.86E-04 \\
\hline 2922 & $2.46 \mathrm{E}-05$ & $8.84 \mathrm{E}-04$ \\
\hline 2923 & 2.45E-05 & 8.83E-04 \\
\hline 2924 & $2.45 \mathrm{E}-05$ & $8.81 \mathrm{E}-04$ \\
\hline 2925 & $2.44 \mathrm{E}-05$ & 8.79E-04 \\
\hline 2926 & $2.44 \mathrm{E}-05$ & 8.78E-04 \\
\hline 2927 & 2.43E-05 & 8.76E-04 \\
\hline 2928 & $2.43 \mathrm{E}-05$ & $8.75 \mathrm{E}-04$ \\
\hline 2929 & 2.43E-05 & $8.73 \mathrm{E}-04$ \\
\hline 2930 & $2.42 \mathrm{E}-05$ & 8.71E-04 \\
\hline 2931 & $2.42 \mathrm{E}-05$ & $8.70 \mathrm{E}-04$ \\
\hline 2932 & $2.41 \mathrm{E}-05$ & 8.68E-04 \\
\hline 2933 & $2.41 \mathrm{E}-05$ & $8.67 \mathrm{E}-04$ \\
\hline 2934 & $2.40 \mathrm{E}-05$ & $8.65 \mathrm{E}-04$ \\
\hline 2935 & $2.40 \mathrm{E}-05$ & $8.64 \mathrm{E}-04$ \\
\hline 2936 & 2.39E-05 & $8.62 \mathrm{E}-04$ \\
\hline 2937 & 2.39E-05 & 8.60E-04 \\
\hline 2938 & $2.39 \mathrm{E}-05$ & 8.59E-04 \\
\hline 2939 & 2.38E-05 & 8.57E-04 \\
\hline 2940 & $2.38 \mathrm{E}-05$ & $8.56 \mathrm{E}-04$ \\
\hline 2941 & $2.37 \mathrm{E}-05$ & $8.54 \mathrm{E}-04$ \\
\hline 2942 & 2.37E-05 & 8.53E-04 \\
\hline 2943 & 2.36E-05 & 8.51E-04 \\
\hline 2944 & $2.36 \mathrm{E}-05$ & $8.50 \mathrm{E}-04$ \\
\hline 2945 & $2.36 \mathrm{E}-05$ & $8.48 \mathrm{E}-04$ \\
\hline 2946 & 2.35E-05 & 8.46E-04 \\
\hline 2947 & $2.35 \mathrm{E}-05$ & $8.45 \mathrm{E}-04$ \\
\hline 2948 & $2.34 \mathrm{E}-05$ & 8.43E-04 \\
\hline 2949 & $2.34 \mathrm{E}-05$ & $8.42 E-04$ \\
\hline 2950 & 2.33E-05 & $8.40 \mathrm{E}-04$ \\
\hline 2951 & 2.33E-05 & 8.39E-04 \\
\hline 2952 & 2.33E-05 & 8.37E-04 \\
\hline 2953 & 2.32E-05 & 8.36E-04 \\
\hline 2954 & 2.32E-05 & 8.34E-04 \\
\hline 2955 & $2.31 \mathrm{E}-05$ & 8.33E-04 \\
\hline 2956 & 2.31E-05 & 8.31E-04 \\
\hline 2957 & $2.31 \mathrm{E}-05$ & 8.30E-04 \\
\hline 2958 & 2.30E-05 & $8.28 \mathrm{E}-04$ \\
\hline 2959 & $2.30 \mathrm{E}-05$ & 8.27E-04 \\
\hline 2960 & $2.29 \mathrm{E}-05$ & $8.25 \mathrm{E}-04$ \\
\hline 2961 & $2.29 \mathrm{E}-05$ & $8.24 \mathrm{E}-04$ \\
\hline 2962 & $2.28 \mathrm{E}-05$ & $8.22 E-04$ \\
\hline 2963 & $2.28 \mathrm{E}-05$ & $8.21 \mathrm{E}-04$ \\
\hline 2964 & $2.28 \mathrm{E}-05$ & $8.19 \mathrm{E}-04$ \\
\hline 2965 & $2.27 \mathrm{E}-05$ & $8.18 \mathrm{E}-04$ \\
\hline 2966 & $2.27 \mathrm{E}-05$ & 8.16E-04 \\
\hline 2967 & 2.26E-05 & $8.15 \mathrm{E}-04$ \\
\hline 2968 & $2.26 \mathrm{E}-05$ & $8.14 \mathrm{E}-04$ \\
\hline 2969 & $2.26 \mathrm{E}-05$ & $8.12 \mathrm{E}-04$ \\
\hline 2970 & 2.25E-05 & 8.11E-04 \\
\hline 2971 & $2.25 \mathrm{E}-05$ & 8.09E-04 \\
\hline 2972 & $2.24 \mathrm{E}-05$ & 8.08E-04 \\
\hline 2973 & 2.24E-05 & 8.06E-04 \\
\hline 2974 & $2.24 \mathrm{E}-05$ & 8.05E-04 \\
\hline 2975 & 2.23E-05 & 8.03E-04 \\
\hline 2976 & 2.23E-05 & $8.02 \mathrm{E}-04$ \\
\hline 2977 & $2.22 \mathrm{E}-05$ & $8.00 \mathrm{E}-04$ \\
\hline 2978 & 2.22E-05 & $7.99 \mathrm{E}-04$ \\
\hline 2979 & 2.22E-05 & $7.98 \mathrm{E}-04$ \\
\hline 2980 & $2.21 \mathrm{E}-05$ & 7.96E-04 \\
\hline 2981 & $2.21 \mathrm{E}-05$ & 7.95E-04 \\
\hline
\end{tabular}




\begin{tabular}{|l|l|l|}
2982 \\
2983 \\
2984 \\
2985 \\
2986 \\
2987 \\
2988 \\
2989 \\
2990 \\
2991 \\
2992 \\
2993 \\
2994 \\
2995 & $2.20 \mathrm{E}-05$ \\
$2.20 \mathrm{E}-05$ \\
$2.20 \mathrm{E}-05$ \\
$2.19 \mathrm{E}-05$ \\
$2.19 \mathrm{E}-05$ \\
$2.18 \mathrm{E}-05$ \\
2996 \\
2998 \\
2999 & $2.18 \mathrm{E}-05$ & $7.93 \mathrm{E}-04$ \\
3000 & $2.18 \mathrm{E}-05$ & $7.92 \mathrm{E}-04$ \\
$7.90 \mathrm{E}-04$ \\
\\
\end{tabular}


Alternate Hohlraum Requirements - Foam Filled Hohlraum

\begin{tabular}{|c|c|c|c|c|c|c|}
\hline Number & $\operatorname{Rev}$ & WBS & Component & Title & short req't & Requirement Text \\
\hline $\mathrm{FH} 1$ & $\overline{0}$ & 1.4 .1 .2 & $\begin{array}{l}\text { Foam-filled } \\
\text { Hohlraum }\end{array}$ & Applicability of other requirements & & $\begin{array}{l}\text { All requirments in table "Point Design Req'ts" except those designated for component "Gas- } \\
\text { filled hohlraum" shall apply to the foam-filled hohlraum }\end{array}$ \\
\hline $\mathrm{FH} 2$ & 0 & 1.4 .1 .2 & $\begin{array}{l}\text { Foam-filled } \\
\text { Hohlraum }\end{array}$ & $\begin{array}{l}\text { Hohlraum configuration - dimensions and } \\
\text { tolerances }\end{array}$ & see dwg & $\begin{array}{l}\text { The hohlraum configuration shall be as shown in Figures TBD(a) and (b) - Foam filled } \\
\text { hohlraum point design. For parameters which may be requested over a specified range of } \\
\text { values, the tolerances shown will apply at the requested dimension }\end{array}$ \\
\hline $\mathrm{FH} 3$ & 0 & 1.4.1.2.2 & $\begin{array}{l}\text { Foam-filled } \\
\text { Hohlraum }\end{array}$ & Hohlraum foam fill - composition & $\mathrm{SiO} 2$ & $\begin{array}{l}\text { The holraum foam-fill material shall be } \mathrm{SiO} 2 \text {. } \mathrm{H} 2 \mathrm{O} \text { shall be allowed in the foam-fill material, } \\
\text { provided it still meets the density requirement.The foam composition for each hohlraum } \\
\text { shall be known with an accuracy of }<100 \text { at } \% / Z^{\wedge} 2\end{array}$ \\
\hline $\mathrm{FH} 4$ & 0 & 1.4.1.2.2 & $\begin{array}{l}\begin{array}{l}\text { Foam-filled } \\
\text { Hohlraum }\end{array} \\
\end{array}$ & Hohlraum foam fill - average density & $1 \mathrm{mg} / \mathrm{cc} \pm 15 \%$ & $\begin{array}{l}\text { The ensemble average density of the foam-fill materials for all targets shall be in the range } \\
1 \mathrm{mg} / \mathrm{cc} \pm 15 \% \text {. }\end{array}$ \\
\hline FH5 & 0 & 1.4 .1 .2 .2 & $\begin{array}{l}\text { Foam-filled } \\
\text { Hohlraum }\end{array}$ & Hohlraum foam fill - shot-shot variations & $< \pm 10 \%$ & $\begin{array}{l}\text { The density of the foam-fill materials for each shall be within }+/-10 \% \text { of the ensemble } \\
\text { average. }\end{array}$ \\
\hline FH6 & 0 & 1.4.1.2.2 & $\begin{array}{l}\text { Foam-filled } \\
\text { Hohlraum }\end{array}$ & Hohlraum foam fill - density variations & $\begin{array}{l}<10 \% \text { RMS over any } \\
200 \mu \text { m-side cube }\end{array}$ & $\begin{array}{l}\text { The foam-fill density in any } 200 \mu \mathrm{m} \text {-side cube within a specific target shall be within } 10 \% \\
\text { RMS of the average for that target }\end{array}$ \\
\hline $\mathrm{FH} 7$ & 0 & 1.4 .1 .2 .2 & $\begin{array}{l}\text { Foam-filled } \\
\text { Hohlraum }\end{array}$ & Hohlraum foam fill - cell size & $<10 \mu \mathrm{m}$ & The foam fill cell size shall be $<10 \mu \mathrm{m}$ \\
\hline FH8 & 0 & 1.4 .1 .2 .2 & $\begin{array}{l}\text { Foam-filled } \\
\text { Hohlraum }\end{array}$ & Hohlraum foam fill - gaps to hohlraum wall & $<200 \mu \mathrm{m}$ & Gaps between the foam fill and the hohlraum wall shall be $<200 \mu \mathrm{m}$ wide \\
\hline FH9 & 0 & 1.4.1.2.2 & $\begin{array}{l}\text { Foam-filled } \\
\text { Hohlraum }\end{array}$ & Hohlraum gas fill - composition & $\mathrm{He}$ & The hohlraum shall be filled with $\mathrm{He}$ \\
\hline FH10 & 0 & 1.4.1.2.2 & $\begin{array}{l}\text { Foam-filled } \\
\text { Hohlraum }\end{array}$ & Hohlraum gas fill - density & $1 . \mathrm{e}-5 \pm 0.1 \mathrm{e}-5 \mathrm{~g} / \mathrm{cm} 3$ & The density of the gas filling the hohlraum shall be $1 . e-5 \pm 0.3 \mathrm{e}-5 \mathrm{~g} / \mathrm{cm} 3$ \\
\hline FH11 & 0 & 1.4.1.2.2 & $\begin{array}{l}\text { Foam-filled } \\
\text { Hohlraum }\end{array}$ & LEH liner - composition & $\mathrm{CH}$ full density & $\begin{array}{l}\text { The lining on the LEH shall be composed of } \mathrm{CH} \text {, density } 1.08+/-5 \% \text {, composition } \\
\text { characterized to }+/-10 \% \text { on all known materials }(\mathrm{C}, \mathrm{H},<10 \% \mathrm{O}) \text { and }<100 \% / \mathrm{Z}^{\wedge} 2 \text { for all } \\
\text { impurities. }\end{array}$ \\
\hline & & & & & & \\
\hline & & & & & & \\
\hline
\end{tabular}


Foam filled hohlraum design (a)

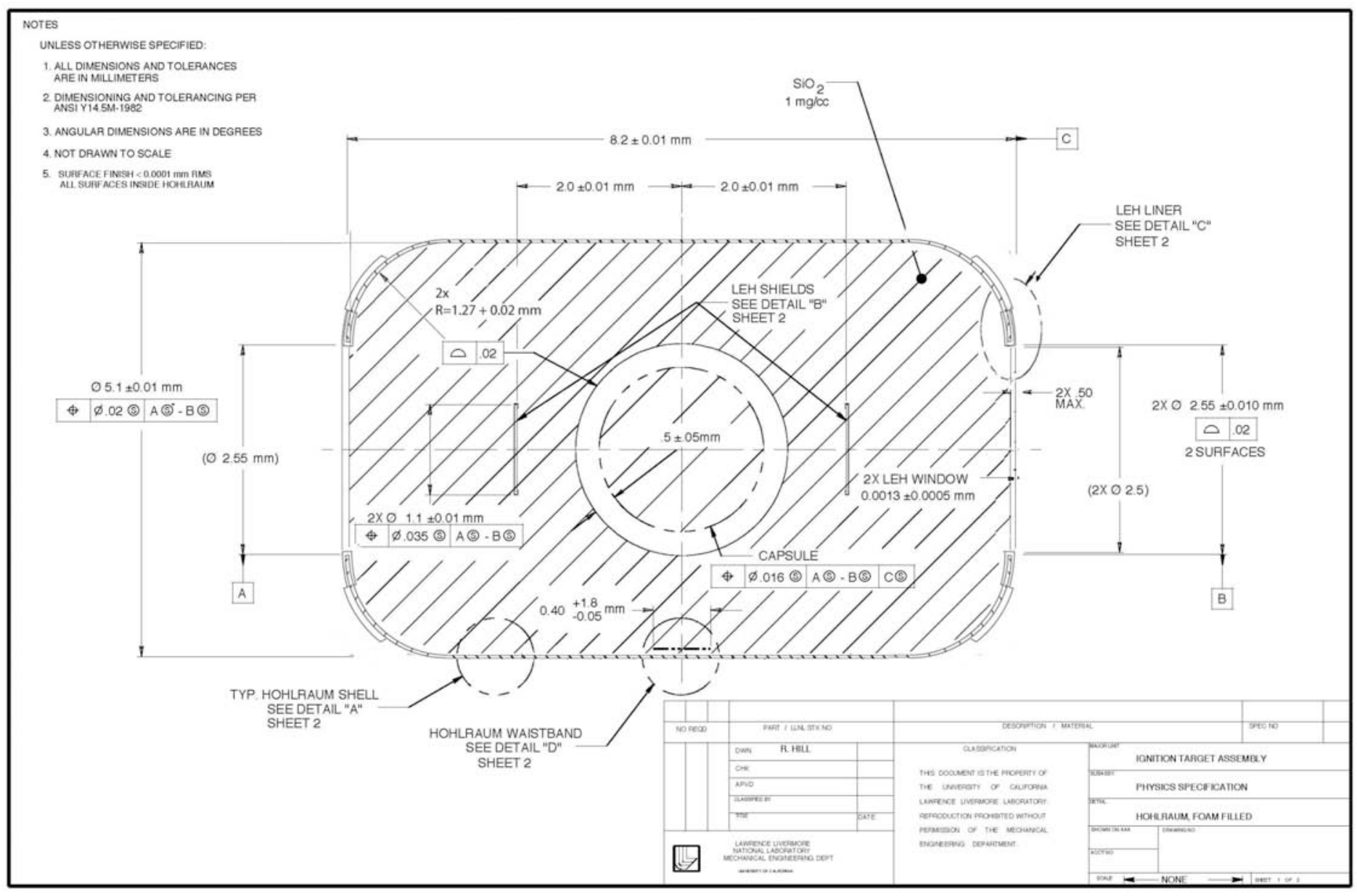


Foam filled hohlraum design (b)

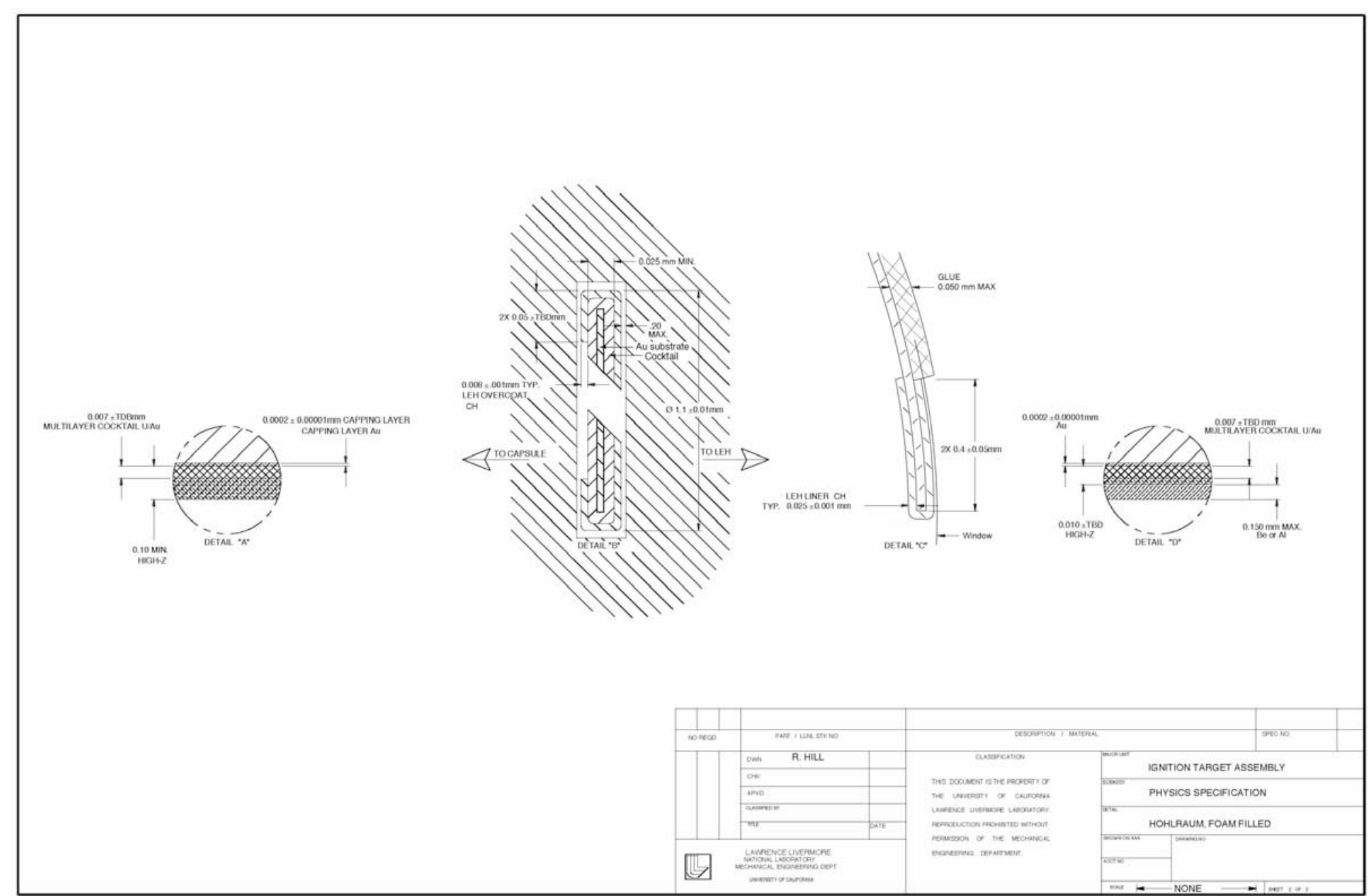




\section{Alternate Hohlraum Requirements - Lined Hohlraum}

\begin{tabular}{|c|c|c|c|c|c|c|}
\hline Number & $\operatorname{Rev}$ & WBS & Component & Title & short req't & Requirement Text \\
\hline LH1 & $\overline{0}$ & 1.4 .1 .2 & Lined Hohlraum & Applicability of other requirements & & $\begin{array}{l}\text { All requirments in table "Point Design Req'ts" except those designated for component "Gas- } \\
\text { filled hohlraum" shall apply to the lined hohlraum }\end{array}$ \\
\hline LH2 & 0 & 1.4 .1 .2 & Lined Hohlraum & $\begin{array}{l}\text { Hohlraum configuration - dimensions and } \\
\text { tolerances }\end{array}$ & see dwg & $\begin{array}{l}\text { The hohlraum configuration shall be as shown in Figures } \operatorname{TBD}(a) \text { and }(b) \text { - Lined hohlraum } \\
\text { point design. For parameters which may be requested over a specified range of values, the } \\
\text { tolerances shown will apply at the requested dimension }\end{array}$ \\
\hline LH3 & 0 & I.4.1.2.2 & Lined Hohlraum & Hohlraum liner composition & $\mathrm{SiO} 2$ & $\begin{array}{l}\text { The hohlraum liner material shall be SiO2. The impurities in the liner shall be less than } \\
100 \% / Z^{\wedge} 2 \text {. }\end{array}$ \\
\hline LH4 & 0 & 1.4 .1 .2 .2 & Lined Hohlraum & Hohlraum liner thickness & $0.5 \pm 0.05 \mu \mathrm{m}$ & The thickness of the hohlraum liner shall be $0.5 \pm 0.05 \mu \mathrm{m}$ \\
\hline LH5 & 0 & I.4.1.2.2 & Lined Hohlraum & Hohlraum liner density & $2.2 \mathrm{~g} / \mathrm{cc} \pm 10 \%$ & The density of the hohlraum liner shall be $2.2 \mathrm{~g} / \mathrm{cc} \pm 10 \%$. \\
\hline LH6 & 0 & I.4.1.2.2 & Lined Hohlraum & $\begin{array}{l}\text { Hohlraum liner density variations between } \\
\text { targets }\end{array}$ & $< \pm 5 \%$ & $\begin{array}{l}\text { The variation in liner density between all targets in a given campaign shall be less than }+/- \\
5 \%\end{array}$ \\
\hline LH7 & 0 & I.4.1.2.2 & Lined Hohlraum & $\begin{array}{l}\text { Hohlraum liner density uniformity within a } \\
\text { target }\end{array}$ & $\begin{array}{l}<10 \% \text { RMS over any } \\
200 \mu \mathrm{m} \text {-side area }\end{array}$ & $\begin{array}{l}\text { The liner density in any } 200 \mu \mathrm{m} \text {-side area within a specific target shall be within } 10 \% \text { RMS } \\
\text { of the average for that target }\end{array}$ \\
\hline LH8 & 0 & 1.4 .1 .2 .2 & Lined Hohlraum & Hohlraum gas fill - composition & $\mathrm{He}$ & $\begin{array}{l}\text { The hohlraum shall be filled with a mixture of } \mathrm{He} \text { and } \mathrm{H} \text {, with a } \mathrm{H} \text { density specified in the } \\
\text { range from } 0 \text { to } 0.65 \mathrm{mg} / \mathrm{cc} \text {. The point design value of } \mathrm{H} \text { density shall be zero. }\end{array}$ \\
\hline LH9 & 0 & 1.4 .1 .2 .2 & Lined Hohlraum & Hohlraum gas fill - density & $1 . e-5 \pm 0.2 \mathrm{e}-5 \mathrm{~g} / \mathrm{cm} 3$ & The density of the gas filling the hohlraum shall be $1 . \mathrm{e}-5 \pm 0.2 \mathrm{e}-5 \mathrm{~g} / \mathrm{cm} 3$ \\
\hline LH10 & 0 & I.4.1.2.2 & Lined Hohlraum & LEH liner - composition & same as hohlraum liner & $\begin{array}{l}\text { The LEH liner shall be composed of the same material as the hohlraum liner. The } \\
\text { specifications on composition, density, density variations, and density uniformity shall apply. }\end{array}$ \\
\hline & & & & & & \\
\hline & & & & & & \\
\hline
\end{tabular}


Lined hohlraum design (a)

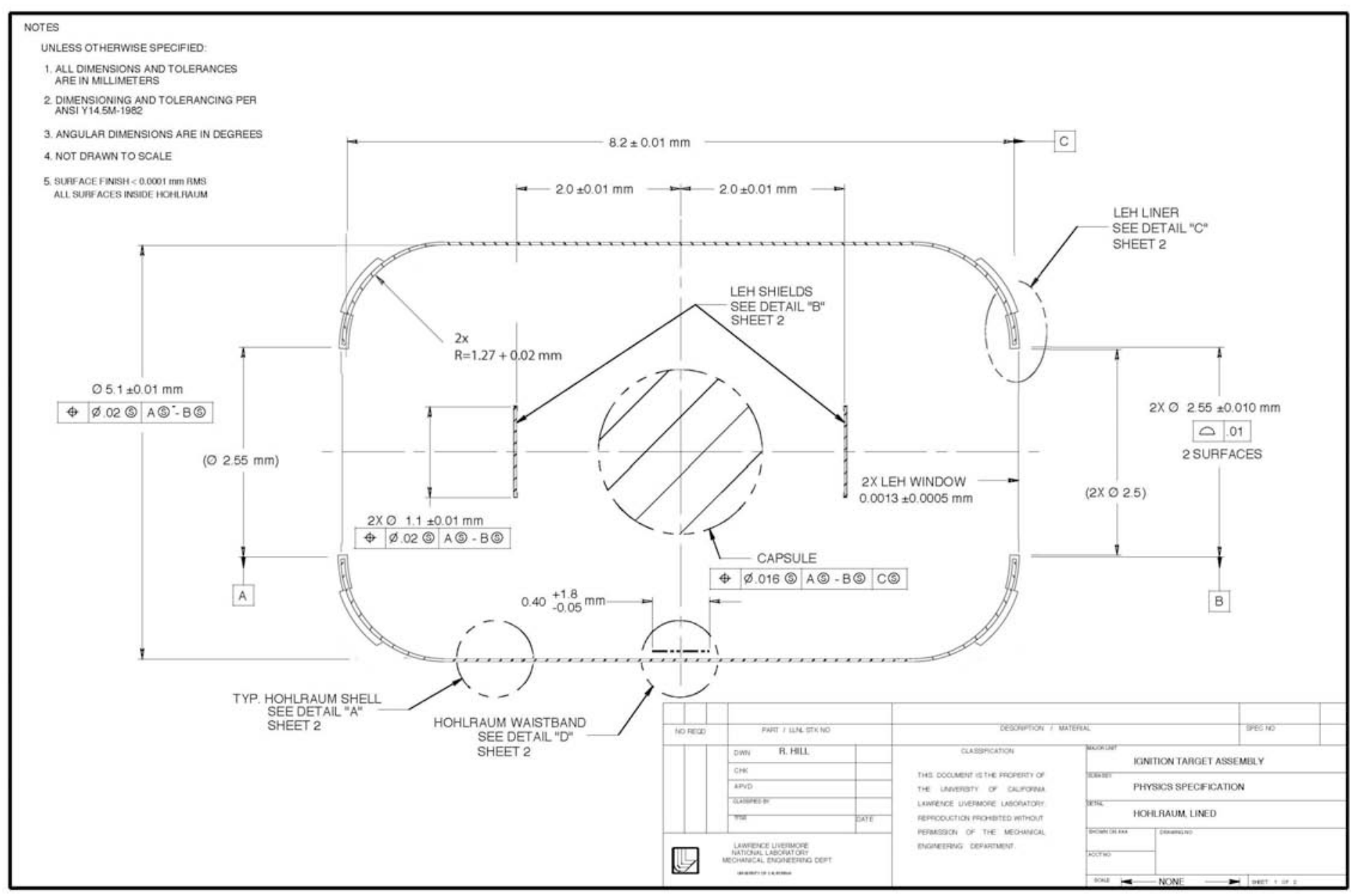




\section{Lined hohlraum design (b)}

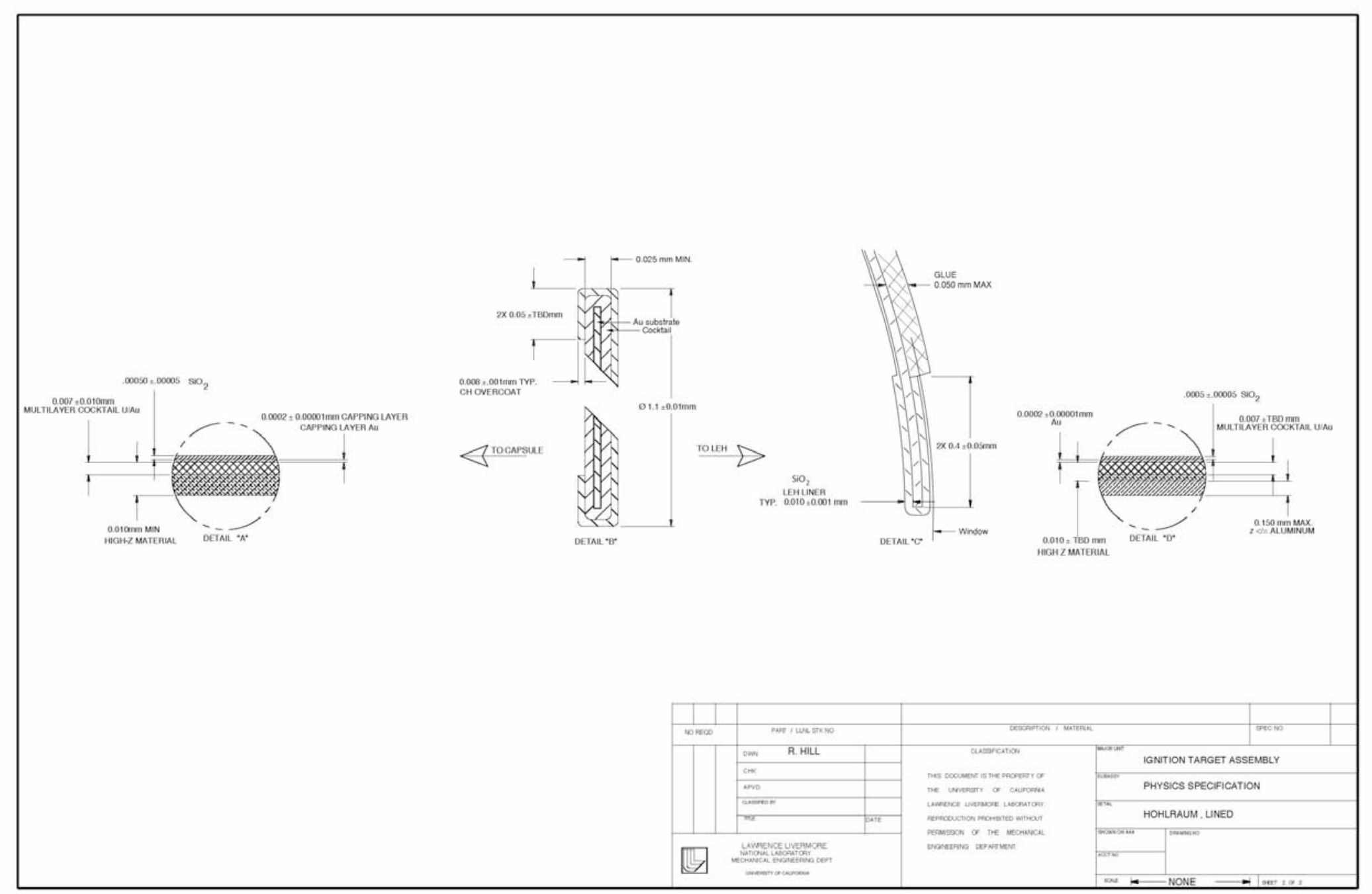

